\title{
CLOSED LOOP CHEMICAL SYSTEMS FOR ENERGY STORAGE AND TRANSMISSION (CHEMICAL HEAT PIPE)
}

\author{
ERDA CONTRACT \\ EY-76-C-02-2676 \\ FINAL REPORT \\ FEBRUARY 1978
}

NOTICE- Nount of work

This report was prepared as an accont. Neither the sponsored by the United Stales Gites Department of

United States nor the Unird

Energy, nor any of dheir employess amployees, makes

Energ, subcontractors, or their employes any legal

Conpress or implied, or asu completeness

any

a usefuness of any in fomation, app is use would no

or use ulness disclosed, or represents

process disclosed, owned rights.

Prepared By

GENERAL ELECTRIC COMPANY

POWER SYSTEMS LABORATORY

CORPORATE RESEARCH \& DEVELOPMENT

SCHENECTADY, NEW YORK 12301

HIMANSHU B. VAKIL
Principal Investigator

AND

JOHN W. FLOCK

Co-Author

Prepared for

U.S. DEPARTMENT OF ENERGY

WASHINGTON, D.C. 


\section{DISCLAIMER}

This report was prepared as an account of work sponsored by an agency of the United States Government. Neither the United States Government nor any agency Thereof, nor any of their employees, makes any warranty, express or implied, or assumes any legal liability or responsibility for the accuracy, completeness, or usefulness of any information, apparatus, product, or process disclosed, or represents that its use would not infringe privately owned rights. Reference herein to any specific commercial product, process, or service by trade name, trademark, manufacturer, or otherwise does not necessarily constitute or imply its endorsement, recommendation, or favoring by the United States Government or any agency thereof. The views and opinions of authors expressed herein do not necessarily state or reflect those of the United States Government or any agency thereof. 


\section{DISCLAIMER}

Portions of this document may be illegible in electronic image products. Images are produced from the best available original document. 


\section{ABSTRACT}

This work documents the analysis of closed loop chemical systems for energy storage and transmission, commonly referred to as the Chemical Heat Pipe (CHP). The concept has been expanded in this work beyond the original scope of heat transmission to provide district heating, as proposed by the West Germans, to uses such as high temperature process steam delivery, distributed peak generation of electricity, and cogeneration of electricity and steam. A market analysis shows that substitution for more than 1 million barrels per day oil equivalent of prime fossil fuels could be accomplished by the use of this concept.

Among the various chemical reaction systems and sources investigated, the two best systems were determined to be the high temperature methane/steam reforming reaction (HTCHP) coupled to a Very High Temperature Gas Cooled Reactor (VHTR) and the lower temperature, cyclohexane dehydrogenation reaction (LTCHP) coupled to existing sources such as coal or light water reactors. Solar and other developing technologies can best be coupled to the LTCHP.

The preliminary economic and technical analyses show that both systems could transport heat at an incremental cost of approximately $\$ 1.50 / \mathrm{GJ} / 160 \mathrm{~km}$ (in excess of the primary heat cost of $\$ 2.50 / \mathrm{GJ})$, at system efficiencies above $80 \%$. Solar heat can be transported at an incremental cost of $\$ 3 / \mathrm{GJ} / \mathrm{I} 60 \mathrm{~km}$. The use of the mixed feed evaporator concept developed in this work contributes significantly to reducing the transportation cost and increasing the efficiency of the system.

The LTCHP shows the most promise of the two systems if the technical feasibility of the cyclic closed loop chemical reaction system can be established. An experimental program for establishing this feasibility is recommended. Since the VHTR is several years away from commercial demonstration and the HTCHP chemical technology is well developed, future HTCHP programs should be aimed at VHTR and interface problems.

KEYWORDS: Chemical Heat Pipe Thermochemical Systems

Energy Storage

Energy Transmission

Nuclear Energy

Solar Energy

Coal

Process Steam Delivery

Peak Generation
Cogeneration

Thermodynamics

Exergy

Energy Efficiency

Benzene Hydrogenation

Cyclohexane Dehydrogenation

Methane Reforming

Methanation

SNG 
THIS PAGE

WAS INTENTIONALIY

LEET BLANK 


\section{LIST OF CONTRIBUTORS}

Overall technical guidance for the project was provided by Dr. Himanshu B. Vakil, who acted as the Principal Investigator.

This report was compiled and written by Drs. John W. Flock and Himanshu B. Vakil and is based on the work of the following technical contributors:

\section{General Electric Company}

Corporate Research and Development

Dr. R. A. Alpher

Dr. J. W. Flock

Mr. C. S. Herrick

Dr. P. G. Kosky

Mr. M. J. Schwedock

Dr. S. D. Silverstein

Dr. H. B. Vakil

Dr. R. H. Wentorf, Jr.
- Market Analysis

- LTCHP Analysis, Economics, Transportation Analysis

- Coal-Fired Reformers

- Nuclear Sources, HTCHP Analysis

- LTCHP Transportation Analysis

- Focused Solar Sources

- All Areas of the Study

- Alternate Reactions

\section{Engineering Consulting Services}

Mr. C. M. Young

- Market Analysis

\section{The Ralph M. Parsons Company}

Mr. T. R. Rozkowski

- Methanation Review

Mr. G. A. White

- Methanation Review

UOP, Inc. generously contributed the state-of-the-art 'reviews on hydrogenation/dehydrogenation technology (Appendix 5).

Editorial and production supervision was provided by Ms. I. H. Clark. Valuable technical assistance given by. Drs. J. B. Comly, J. J. Grimaldi and R. H. Wilson is also acknowledged.

In addition to the technical contributions listed above, this project has benefited considerably from the management expertise of Dr. R. E. Hanneman in the initial stages of the work, Dr. D. C. Golibersuch through the major part of the contract duration and Dr. J. B. Comly during the concluding phase of the project. 


\section{ACKNOWLEDGMENTS}

The work described in this report was funded by the Department of Energy (DOE); its later phases were monitored by Sandia Laboratories for the Energy Storage Division of the Department of Energy. During the period of this work the initial contract, E(11-1)-2676, was carried through for the originally planned one year period, and was subsequently extended a second year under a combined revised contract, EY-76-C-02-2676.

Various technical discussions with the team of scientists at Kernforschungsanlage, in particular $R$. Schulten, $K$. Kugeler, and H. Niessen, have led to valuable insights into the role of the Chemical Heat Pipe in this country as well as the rest of the world.

During the period of the contract, work was greatly aided by productive dialogue between the contractor and representatives of DOE headquarters: AR Landgrebe and $J$ VanDerryn in the initial phases of the contract; JH Swisher and CJ Swet during the later phases of the contract, joined by $J$ Gahimer toward the end of the contract and during the final reporting period. This work has also benefited considerably from discussions and inputs from Kermet Laughon, manager of the DOE Gas Reactor Program.

The later phases of the contract were managed for DOE by the Sandia Laboratories under the management direction of Ray Mar and Taz Bramlette, with Bill Wilson taking the place of Ray Mar at the end; their vision for and support of the CHP effort has been important in its accomplishment.

Finally, a special acknowledgment is appropriate for the continued technical and programmatic guidance of the Sandia Program Manager, Mr. James Bartel. His clear understanding of the possibilities represented by the CHP concept, his steady good judgment, and his key suggestions about the scope of this work have had a major influence on the technical balance of the work and its impact on the overall U.S. Chemical Heat Pipe program. 


\section{PREFACE}

This is the final report on the work carried out by General Electric, Corporate Research and Development, under Energy Research and Development Administration contract EY 76-C-02-2676 (originally contract $\mathrm{E}(11-1) 2676)$ to evaluate closed-loop chemical systems for energy storage and transmission, commonly referred to as chemical Heat Pipe (CHP).

The concept of CHP was first proposed in West Germany for transporting thermal energy from a high-temperature gas-cooled nuclear reactor to a district heating system. Work on CHP was initiated at GE early in 1974 and has led to a broadening of the CHP concept for on-site and distributed storage applications as well as for utility and industrial applications. Work reported here is based on the foundation laid by these two prior programs and represents the first major analysis of the CHP concept in this country.

The proposed work scope at the start of this project in June 1975 included technical and economic evaluations of the methanebased high-temperature reaction system (HTCHP) as well as an assessment of alternate reactions, thermal energy sources, and user needs. The project scope evolved considerably as the work progressed. For example, based on preliminary evaluations, a much greater emphasis was placed on the transportation aspect than on the on-site storage. Also, thermodynamic analyses of energy losses and, in particular, efficiency improvements were stressed. Results of this first-phase study under the original contract are presented in Chapters 1 through 10 .

In mid-1975, the study was extended to include technical and economic evaluation of the low-temperature chemical system (LTCHP), based on benzene/cyclohexane reactions. Results of this secondphase study are presented in Chapters 11 through 18.

The principal contributions of this study are:

- Elucidation of the flexibility, uniqueness, and importance of thermal transmission in prime fuel substitution and energy conservation

- Extension of CHP systems beyond the nuclear district heating applications envisioned in the German program to include:

- Higher temperature end uses such as process steam and distributed electrical generation

- Combined storage and transport applications

- Primary sources other than high-temperature nuclear reactors 
- Improved thermodynamic understanding of energetics of chemical conversion. processes through histograms and exergy analyses.

- Invention of a generic process modification (mixed feed evaporator) that offers the potential of increasing the overall efficiency of CHP systems.

It is hoped that these contributions constitute a first step in the formulation and eventual success of a national chp program. 


\section{EXECUTIVE SUMMARY}

\section{INTRODUCTION}

This report documents the work done by corporate Research and Development of General Electric (GE-CRD) under DOE contract on the technical and economic analysis of the transport and storage of energy by closed-loop chemical systems. These systems (shown schematically in Figure 1) are commonly referred to as Chemical Heat Pipes (CHP). In the CHP concept, thermal energy to be transported is absorbed during a high-temperature endothermic chemical reaction, and stored in the high-energy chemical products. These products are cooled to ambient temperature by heat exchange with the incoming reactants, providing the necessary specific heat to bring the reactants to an elevated temperature. The endothermic reaction products are then transported at ambient temperature to user sites $\sim 160 \mathrm{~km}$ from the location of the primary energy source. At the user site, the reverse exothermic reaction is carried out, liberating the stored energy. The reactants for the exothermic reaction are heated to the proper temperature by heat exchange with the products. The lowenergy, low-temperature products of the exothermic reaction are transported back to the site of the primary energy source to serve as reactants in the endothermic reaction, thereby completing a closedloop cycle.

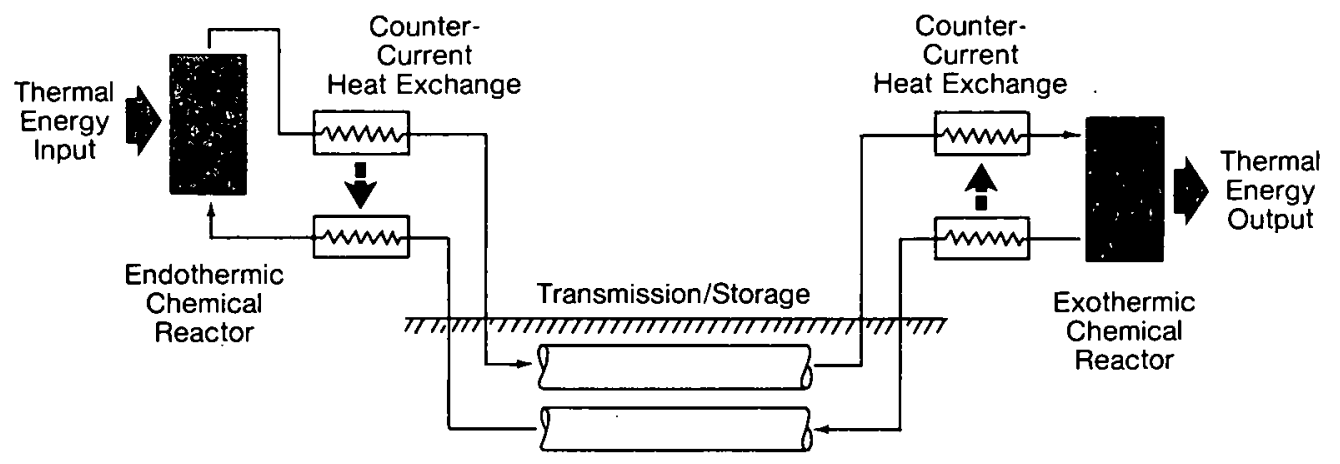

Figure 1

This concept is unique in that it makes possible the transport of high-grade thermal energy over large distances without the use of thermally insulated conduits and that these ambient temperature transmission lines can be used for storage of gases (and thus, thermal energy) by means of pressure fluctuations. Because of the cyclic operation of chemical reactions, there is no net consumption of chemical raw materials and the only exchanges between the system and the surroundings are the mechanical work required for compression work and, of course, heat. Since a greater portion of the primary heat is utilized in this concept as compared with electrical generation, primary thermal rejection is reduced.

The CHP concept suggests several new options in energy management. Most prominent among these are: 
- A thermal energy supply grid based on large nuclear or coal sources delivering process steam to small-scale industrial users, replacing scarce oil or natural gas fuels.

- The distributed generation of baseload or peak electricity, using thermal storage and transmission.

- An extension of nuclear and coal-based cogeneration (of electricity and process steam) to small-scale and intermitent users, thereby offering tremendous conservation potential for both prime fossil fuels and energy consumption as a whole.

- A possibility of a secondary heat source near populated areas for local generation of electricity and space heat without local pollution.

The development of these options is an extension of the original concept as first proposed in West Germany with the final end use of the transported heat restricted to district heating. The evolution of the CHP concept started during independent investigations conducted at General Electric Corporate Research and Development and has progressed during the course of this contract.

\section{CONCLUSIONS}

The technical and economic analyses performed during this contract have led to the following major conclusions:

- The CHP concept offers a method for reducing oil and gas consumption by as much as an equivalent of 11 million barrels of oil per day. However, there is a dramatic need for better characterization of the process steam market in the United States, by size, geographical distribution, and end-use temperature.

- The best high-temperature $(>1000 \mathrm{~K})$ chemical reaction system is based on the endothermic reforming reaction of methane and water to form hydrogen and carbon monoxide. The best potential heat source to drive this high temperature chemical heat pipe (HTCHP) is a very high temperature gas-cooled reactor (VHTR) such as the West German pebble bed reactor (PBR). Coal-and solar-based reformer technologies pose potentially insurmountable interfacing problems when coupled to the HTCHP.

- Lower temperature applications (500-800 K) based on existing sources such as light-water reactors (LWR) and coal, or on future sources such as solar, gas-cooled reactors (GCR) and liquid-metal-cooled fast breeder reactors (LMFBR) are much more desirable. The low temperature chemical heat pipe (LTCHP) based on endothermic dehydrogenation of cyclohexane is the most promising system for coupling to these sources. However, the LTCHP requires the technical demonstration of the feasibility of the cyclic operation of the chemical reaction system. 
- Efficiencies* for the transportation of heat via the HTCHP are high, ranging from 70 to $90 \%$. A process modification termed the mixed feed evaporator, (MFE) developed in the course of this investigation, improves this efficiency by 7 to $10 \%$, depending on the design parameters used.

- Typical efficiencies for the LTCHP range from $\sim 80 \%$ with a $800 \mathrm{~K}$ heat source to $\sim 50 \%$ with a $550 \mathrm{~K}$ source, both based on the delivery of $\sim 650 \mathrm{~K}$ heat.

- For a transmission distance of $160 \mathrm{~km}$ (100 miles), the HTCHP economic analysis indicates that the total cost of delivered high-grade steam is $\sim 4 / G J$ based on a primary heat cost of $\$ 2.50 / \mathrm{GJ}$. The incremental cost of HTCHP transport of heat above that of the primary heat source ranges from $\$ 1$ to $\$ 2 / G J$, depending on the temperature and somewhat on the cost of the primary heat.

- The cost of high-grade delivered steam via the LTCHP ranges from $\$ 4$ to $\$ 5 / G J$, depending on the temperature of the primary heat source based on the cost of. heat from the primary source ranging from $\$ 1.50 / G J$ to $\$ 2.50 / G J$.

- Solar application economics show a total cost of delivered steam of $\$ 9 / \mathrm{GJ}$, based on a primary heat cost of $\$ 6 / G J$. The LTCHP offers significant conservation and economic advantages for large solar plants over on-site electrical generation.

- The best CHP applications are: process steam delivery to small-scale $(<200 \mathrm{MJ} / \mathrm{s})$ and intermittent industrial users; and combined electrical/heat delivery to industrial process steam users.

- The major technical barriers for HTCHP implementation are VHTR development and the VHTR/chemical reactor interface.

- The major technical barrier for LTCHP application is the successful demonstration of closed-loop cyclic operation of the chemical reaction system.

\section{RECOMMENDATIONS}

In view of the versatility and uniqueness of the chP concept and of the enormous implications of its success on prime fuel conservation, further work in this general area is recommended.

In recommending specific projects for future work, it is recognized that three CHP options - LTCHP, HTCHP, and open CHP** - possess significant, though different, merits. In the authors'opinion,

\footnotetext{
* See Chapter 5 for the definition of efficiency

**In the open CHP, coal is gasified to intermediate Btu gas $\left(\mathrm{CO}, \mathrm{H}_{2}\right)$ which is transported to a user site, where it is methanated to produce process heat. The resulting methane is then sold as a fuel for residential use. This concept is not included in the scope of this study. $\mathbf{x i}$
} 
LTCHP offers the greatest potential benefits because of its wide range of applications in terms of future energy sources and demand;

- consequently, its development. deserves the highest priority. The open CHP, on the other hand, has the least uncertainty in technical feasibility and the shortest time scale for implementation; however, its scope is limited to coal gasification and substitute natural gas demand.

The following list of specific projects summarizes major recommendations resulting from this study:

$\underline{\text { LTCHP }}$

- An experimental program to develop and demonstrate technical capability for closed-loop operation of hydrogenation/dehydrogenation reactions should be undertaken. This program should be aimed especially at an evaluation of catalyst specificity for cyclohexane dehyarogenation at high temperatures.

- A program of reactor design analyses should also be undertaken, concurrent with and closely coupled to the above experimental program.

Open CHP

- It is recommended that a detailed design and economic evaluation of the open CHP be undertaken. Furthermore, the open CHP should be included in a comparison of current coal options to assess its economic competitiveness.

- The technical areas of the open CHP most deserving of further work include design analyses of high temperature methanation reactors for energy delivery applications and pipeline transport of intermediate Btu gases.

HTCHP

- Since the critical issue in this area appears to be whether or not there will be a successful VHTR technology in the near future, it is recommended that programs having a major impact on nuclear process heat issues (interface technology, safety, fuel cycles, etc.) be pursued with the aim of precipitating a quick resolution of VHTR technology future.

- The technologies of methane reforming and methanation are judged to be far ahead of the source and interface technology. Consequently, it is inadvisable to undertake either a more detailed technical and economic design of VHTR/HTCHP or an experimental closed-loop demonstration of HTCHP. 
- Because of its potential impact on industrial reformer applications and on energy conservation in chemical plants in general, a program to assess and implement the mixed-feed-evaporation concept should be undertaken.

- A study to obtain an accurate characterization of process heat usage should be undertaken. It should be aimed at obtaining a breakdown of process heat needs by temperature, size, duty factor, geographic location, and ease of substitution. Such a study would benefit analyses of not only CHP applications but also other alternatives for process steam delivery.

- A more detailed analysis of the economic optimization of short distance $(80$ to $320 \mathrm{~km})$ transport of gases should be performed. In addition, questions related to safety in CHP transport should be addressed.

\section{TECHNICAL HIGHLIGHTS}

In the remainder of this summary, further details from major sections of this report are presented. Chapters in the main body of the report in which further information can be found are indicated for each topic.

\section{Source/Reaction Technical Feasibility (Chapters 3, 4)}

The overall technical feasibility of a CHP system depends on two aspects: the state of readiness of a particular energy source technology, and the feasibility of operating the chemical system in closed-loop. The spectrum of sources and chemical reaction is divided into two major groups, depending on the operating temperatures for the endothermic reaction. In each group, first a preferred chemical system is selected from possible candidates and then the feasibility of coupling this reaction to potential energy sources is examined.

\section{High Temperature Group (T>875 K)}

- The best candidate in this group is the methane-steam reforming reaction:

$$
\mathrm{CH}_{4}+\mathrm{H}_{2} \mathrm{O} \neq \mathrm{CO}+3 \mathrm{H}_{2}
$$

- Major factors leading to the choice of the methane system are: (1) state of technological development and industrial know-how for the endothermic step;

(2) materials of construction and their costs; and

(3) existing development programs for CHP interface.

- VHTR is the only nuclear design capable of supplying energy to the HTCHP. The only example is the PBR being 
developed in West Germany. Major hurdles are the primary source technology and interface problems. The key issue is whether US will develop a VHTR technology.

- Coal-fired reformer technology poses insurmountable problems. The most promising coal option is a modification of gasification processes in the form of the open CHP.

- Problems of coupling a methane reformer to highconcentration solar receivers appear to be insurmountable. Major limitations are due to thermal cycling, thermal flux requirements, and economics of reformers with low duty factors.

\section{Low Temperature Group (550 to $850 \mathrm{~K}$ )}

- Chemical reactions that appear most promising are hydrogenation/dehydrogenation reactions involving benzene, toluene, and naphthalene. The preferred reactions are:

$$
\mathrm{C}_{6} \mathrm{H}_{12} \rightarrow \mathrm{C}_{6} \mathrm{H}_{6}+3 \mathrm{H}_{2}
$$

Technical feasibility is not certain. Major hurdles are catalyst specificity, carbon deposition, and hightemperature operation.

- Most of the medium and high temperature nuclear reactors are adequate sources for LTCHP. Adequacy of lower temperature water cooled reactors (LWR) for LTCHP is questionable. The major hurdle for nuclear LTCHP is that no useful set of chemical reactions is developed.

- If closed-loop operation of the chemical system can be demonstrated, the coal-fired LTCHP appears technically feasible. Interface problems are analogous to those for the utility steam systems.

- Medium and high concentration solar collectors are potentially adequate for LTCHP applications. Interface technology appears feasible. Major issues in need of resolution are the economy of scale for solar sources and economic competitiveness of solar thermal energy.

Technical Design of HTCHP (Chapters 5 and 6)

The overall HTCHP system was considered in three subsystems: the reformer plant, the methanation plant, and transportation/storage components. Details of material and enthalpy balances were illustrated by means of a reference case design based on typical operating conditions. 
The endothermic reactor design was selected from recent studies performed in West Germany on a helium-heated reformer tube with an internal pigtail. The exothermic reactor design was based on the RMProcess of the Ralph M. Parsons Company, using high temperature adiabatic methanators with intercooling.

Final conversions of the methane reforming and shift reactions were determined on the basis of thermodynamic equilibria at the following reactor exit conditions:

Reformer Plant:

$\begin{array}{ll}\text { Exit temperature } & 1100 \mathrm{~K} \\ \text { Exit pressure } & 40 \mathrm{~atm}(40.5 \mathrm{bars}) \\ \text { Stoichiometry } & 1: 3 \mathrm{CH}_{4} / \mathrm{H}_{2} \mathrm{O}\end{array}$

Methanation Plant:

$\begin{array}{ll}\text { Exit temperature } & 700 \mathrm{~K} \\ \text { Exit pressure } & 40.5 \mathrm{bars} \\ \text { Stoichiometry } & 1: 1.5 \mathrm{CH}_{4} / \mathrm{H}_{2} \mathrm{O}\end{array}$

The overall material balances, gas compositions, and $\mathrm{H}_{2} \mathrm{O}$ recycle steams based on 1 mole of carbon are shown in Figure 2 .

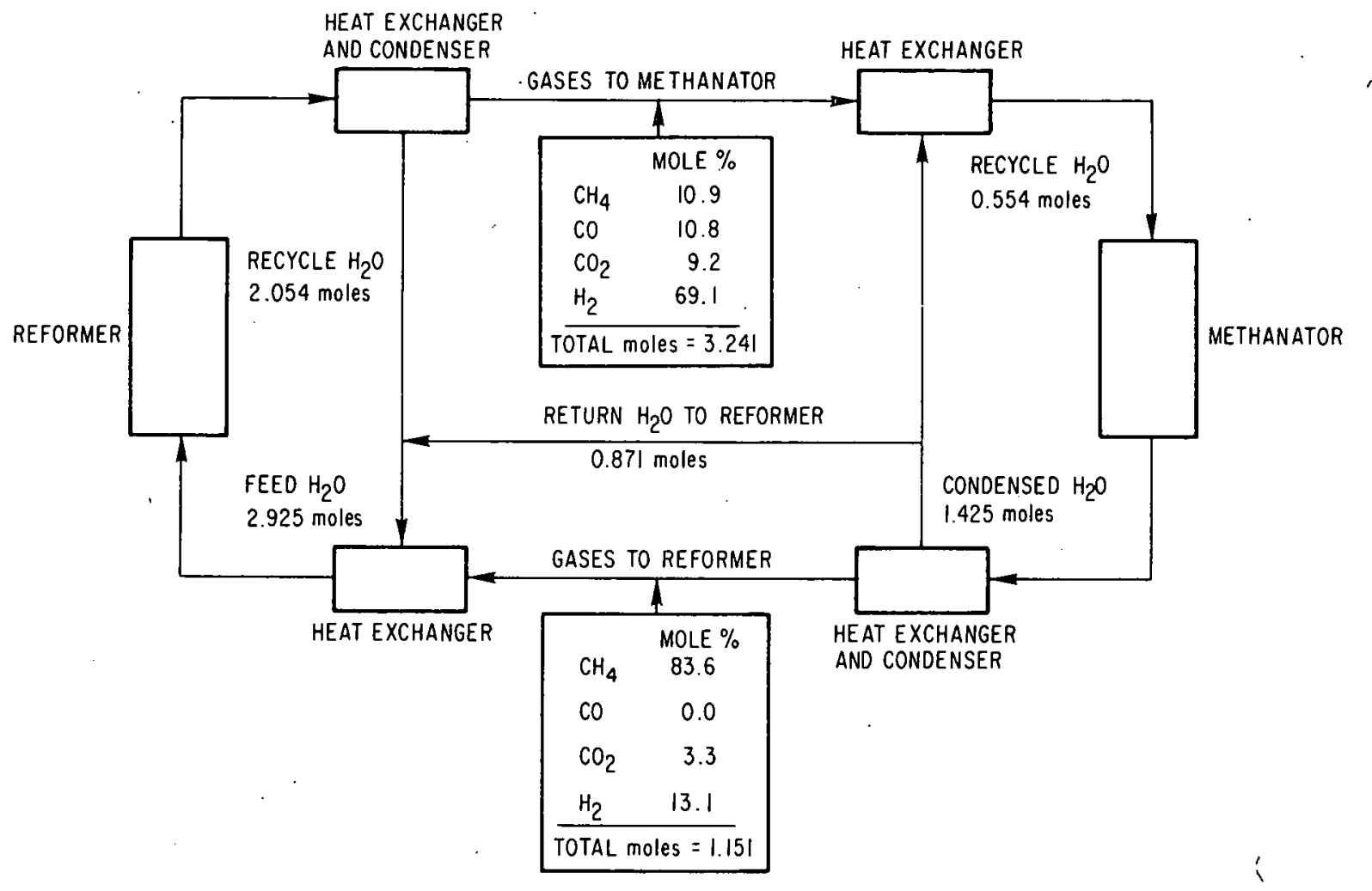

Figure 2 
Thermal duties and molar flows were calculated on the basis of a net enthalpy flow of $1000 \mathrm{MJ} / \mathrm{s}$ at the pipeline temperature of $300 \mathrm{~K}$. These are shown in Figure 3 along with the relevant stream temperatures.
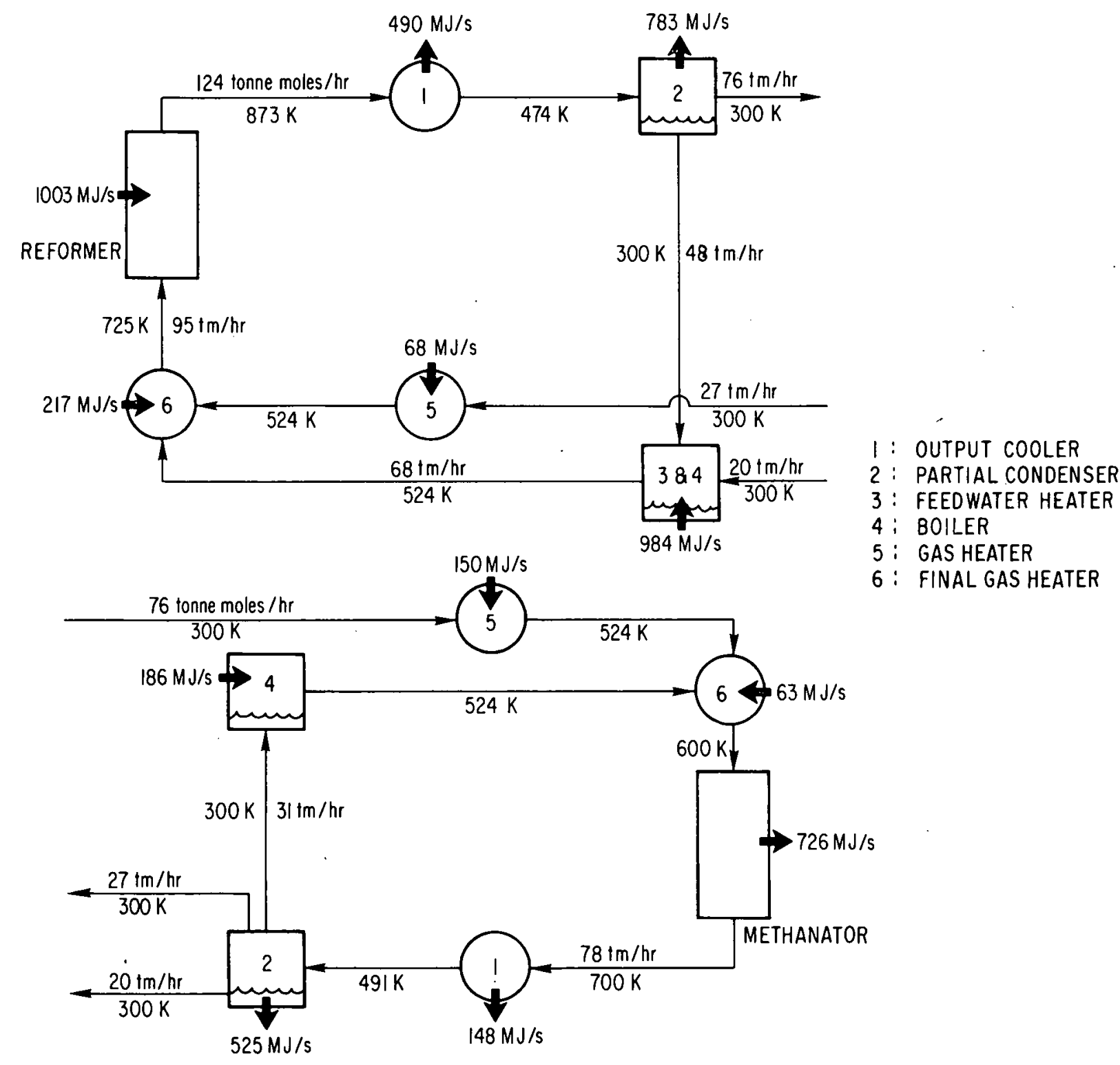

Figure 3

The total heating duty for the reformer feed $(1269 \mathrm{MJ} / \mathrm{s})$ is larger than the amount absorbed in the reformer. In order to achieve high efficiencies, it is important that a large fraction of this preheating be provided by an internal heat exchange from the cooling duty of $1273 \mathrm{MJ} / \mathrm{s}$.

To ascertain the extent to which this internal heat economy can be achieved in practice, a method of analysis based on thermal histograms was developed in the course of this study. Reformer plant histograms for an idealized case with a heat transfer temperature difference $\Delta \mathrm{T}=0$ are shown in Figure 4 . 


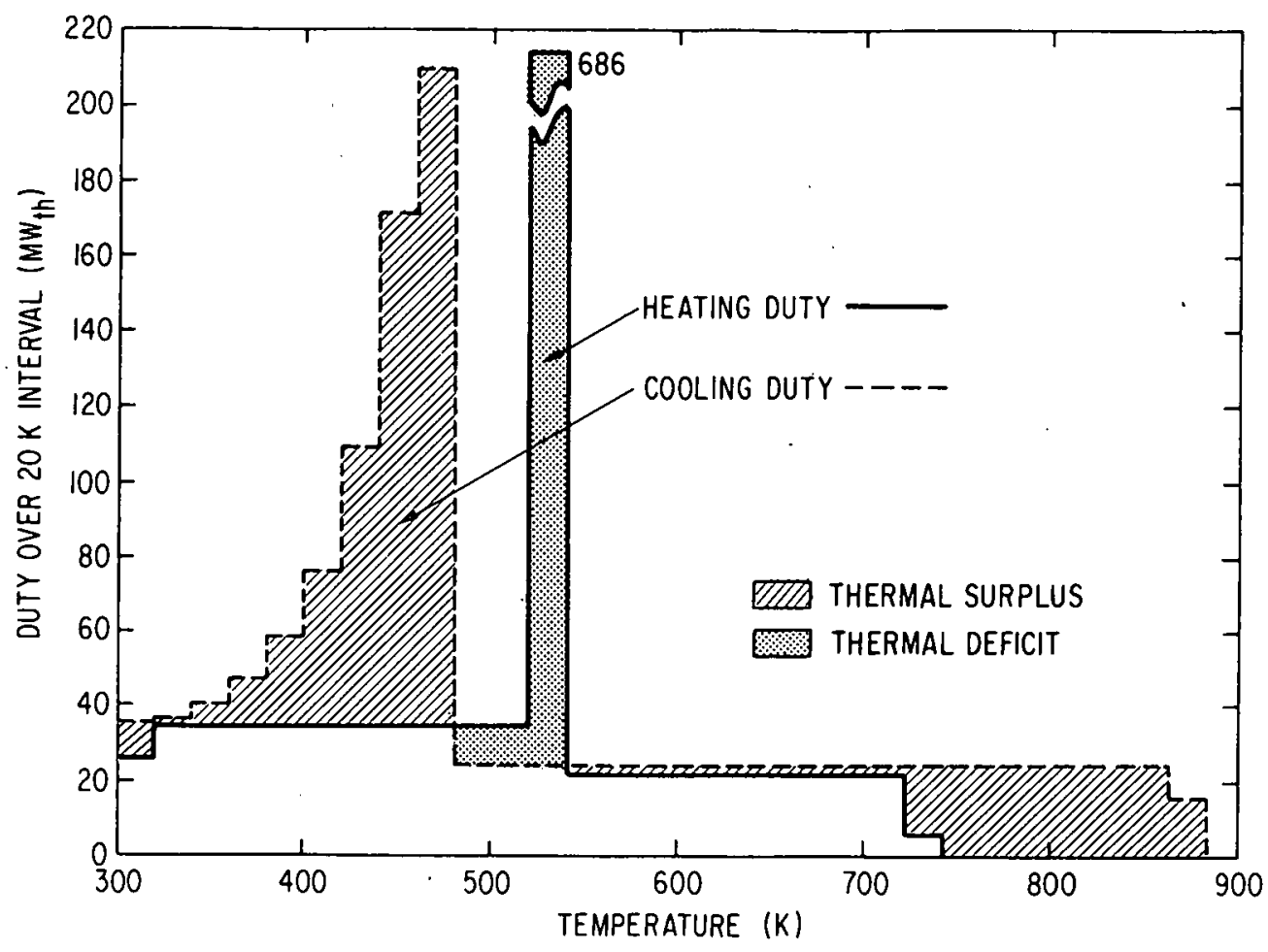

Figure 4

The following points should be noted:

- Even though the heating and cooling duties are approximately equal in magnitude, there is a significant temperature mismatch.

- An on-site powerplant must provide sufficient extraction steam to make up the large thermal deficit at $524 \mathrm{~K}$ and be able to use the surplus for steam reheat and feedwater preheating.

To evaluate the net impact of these thermal exchanges on the powerplant electrical output, a thermodynamic analysis* based on the concept of exergy flows was used. Results from such an analysis showed an indirect work penalty of 12.6 MW for this idealized case.

Based on detailed considerations of thermodynamic irreversibilities, it was concluded that the irreversible mixing of steam with feed-gas stream contributed to this temperature mismatch and therefore the exergy penalty. A process modification in the form of a mixed-feed-evaporator (MFE) was developed to eliminate this irreversibility. The effect of using MFE on the thermal mismatch is shown in Figure 5. A comparison with Figure 4 shows that the

*Details of this analysis are given in Appendix 2 


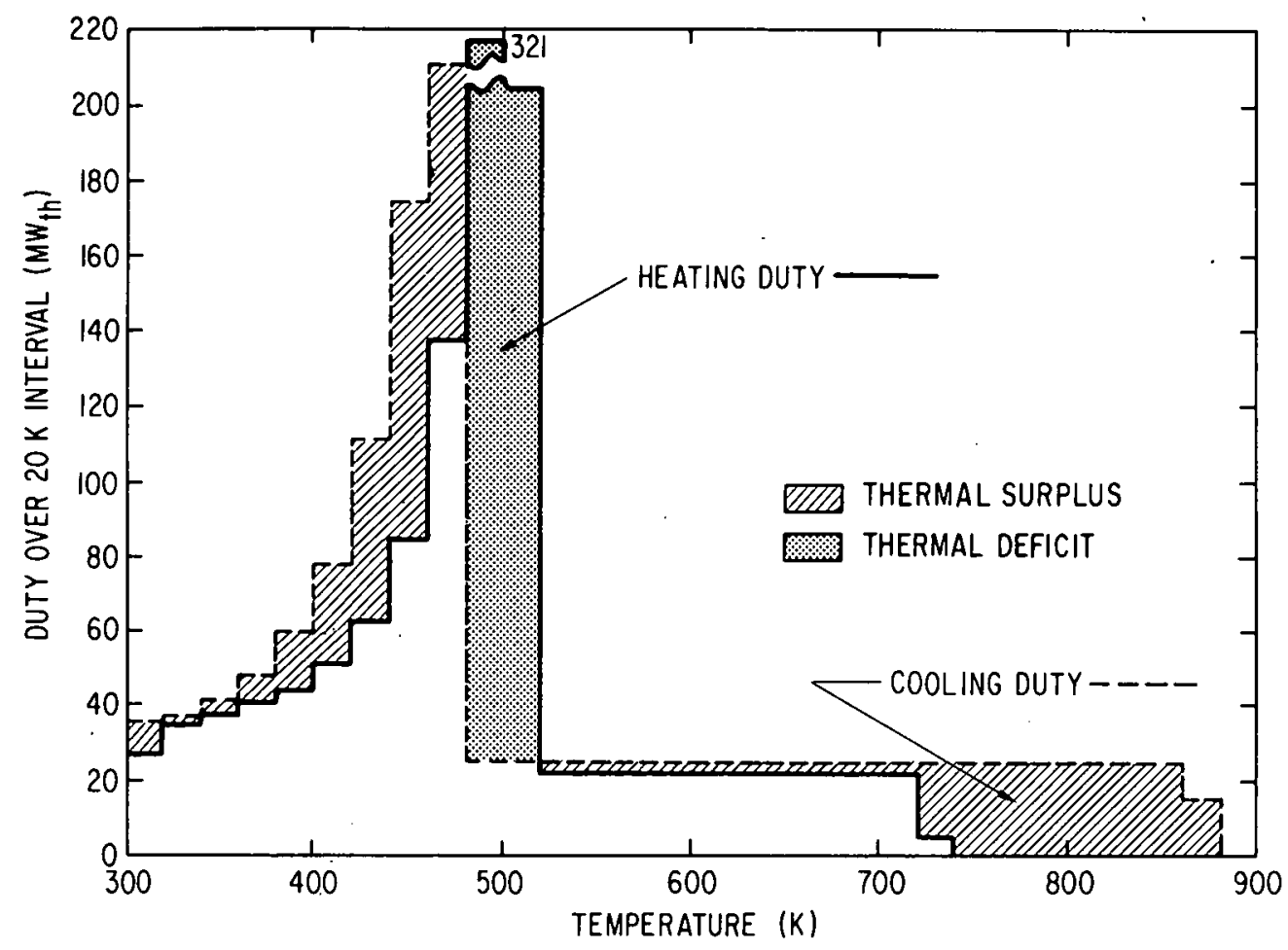

Figure 5

use of MFE could lead to a significant reduction in the amount of thermal deficit and in its temperature level. The resultant increase in powerplant electrical output was estimated by exergy analyses to be equal to $32 \mathrm{MW}$.

The overall energy efficiency for a realistic process design was obtained by calculating the thermal equivalent of all work inputs, direct and indirect. Compression work requirement was estimated to be $38.6 \mathrm{MW}$ for pipeline transport over $160 \mathrm{~km}$ and for equipment pressure drop. The indirect work penalty was calculated (assuming a heat transfor $\Delta \mathrm{T}=20 \mathrm{k}$ ) to be equal to $20.3 \mathrm{MW}$ with MFE and 54.7 MW without. The overall energy delivery efficiencies were $85.7 \%$ with $\mathrm{MFE}$ and $79.8 \%$ without MFE.

For investigating the effects of changes in design parameters, ten different case designs were selected and analyzed. Variations in peak reforming temperature, operating pressures, reactor feed stoichiometry, and methanation exit temperatures were explored. Energy flows, heat exchanger duties, and overall efficiencies were calculated both with and without MFE. The following main conclusions were reached:

- For all the cases studied, overall efficiencies ranged from 80 to $90 \%$ with $\mathrm{MFE}$ and 70 to $85 \%$ without.

- It is highly desirable to eliminate the need for $\mathrm{H}_{2} \mathrm{O}$ recycle at the methanation plant. For a majority of 
designs this can be done without catalyst deactivation due to carbon deposition.

- The use of MFE at, the reformer plant could lead to a. significant increase (5 to $10 \%$ ) in efficiency for all design cases.

- There is a significant incentive to increase the peak reforming gas temperature above $1100 \mathrm{~K}$. The lower limit on the peak temperature is in the vicinity of $975 \mathrm{~K}$ for practical designs.

\section{LTCHP Technical Design (Chapters 13 and 14)}

A technical design of the LTCHP system was carried out using an approach and guidelines similar to those used in the HTCHP technical design. The major difference was the assumption of an ideal process in the LTCHP: i.e., catalysts capable of promoting only the dehydrogenation/hydrogenation reactions with no formation of side products*; low hydrogen-to-carbon ratios; and the use of mixed feed evaporator. This assumption was made so that the economics could be evaluated to determine if the process would be competitive under ideal conditions; if not, then there would be no need to pursue the concept further.

The process flowsheet was based on the design used in the HTCHP analysis. The only major exception was the use of adiabatic fixed-bed reactors, with interheating for the endothermic reactor as opposed to the helium heat reformer used in the HTCHP. This required the use of the Liquid Hourly space Velocity (LHSV) for sizing the reactors. The heat transfer coefficients for the heat exchangers were based on analogous values in the HTCHP.

Four cases were investigated, all with the same exothermic reactor outlet conditions (589 $\mathrm{K}, 40$ bars): The difference between the cases was the variation in the endothermic (cyclohexane dehydrogenation) reactor outlet temperature and pressure, which ranged from $811 \mathrm{~K} / 40$ bars (for the reference case) to $540 \mathrm{~K} / 1$ bar. These cases were chosen to show the effect of the reaction temperature that could be expected from thermal sources such as: coal, gas cooled reactors, and solar at $811 \mathrm{~K}$; liquid metal fast breeder reactors at intermediate temperatures; and light water reactors at $540 \mathrm{~K}$.

The reactor outlet compositions and material balances for the reference case are shown in Table 1 .

High conversions are obtained at both the endothermic reactor (99\%) and the exothermic reactor (94\%) under these conditions.

\footnotetext{
*In particular, no formation of methylcyclopentane, which leads to irreversible degradation to cracking products and is detrimental to the energetics of the LTCHP system.
} 


\section{Table 1}

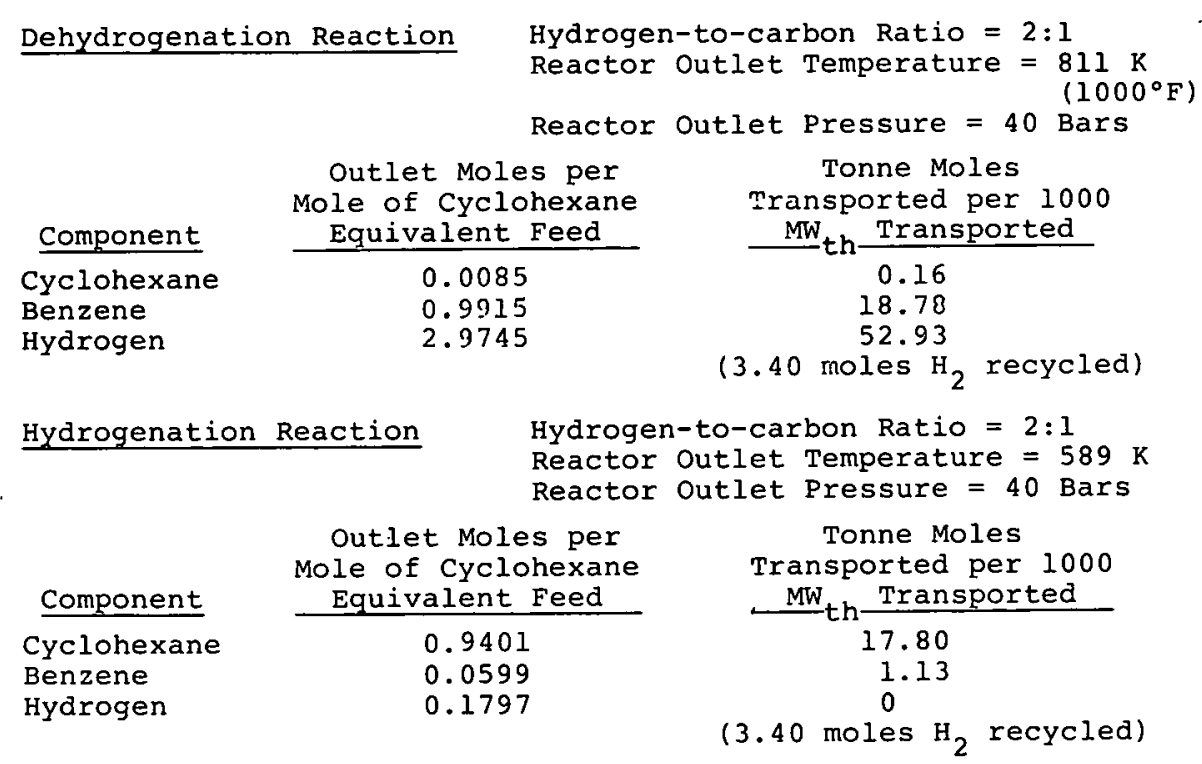

* 1 tonne mole $=10^{6} \mathrm{gram}-$ moles

However, even with the low hydrogen recycle rate, a deficiency in the heat balance was noted at the endothermic reactor as in the HTCHP design. This deficiency was again made up by an exchange of heat between the LTCHP system and an associated powerplant. The energetic analysis from the LTCHP reference case can be summarized as follows:

MW th

Heat delivered to reactor

Equivalent work input

Equivalent work loss in powerplant

Total equivalent heat input

to system

Total Heat Delivered
1110.2

70.8

6.0

1187.0

977.4

This analysis yielded a system efficiency $(\psi)$ of

$$
\psi=\frac{977.4}{1187.0}=0.82
$$

This high system efficiency is comparable to that of the HTCHP and higher than alternative applications such as electricity or SNG production. 
The system efficiencies for the other cases studied are as follows :

\begin{tabular}{|c|c|c|}
\hline Case & $\begin{array}{c}\text { Endothermic } \\
\text { Reactor Conditions } \\
\text { K/bars }\end{array}$ & $\begin{array}{r}\text { Effic } \\
\psi\end{array}$ \\
\hline $\begin{array}{l}1 \\
2 \\
3\end{array}$ & $\begin{array}{l}755 / 40 \\
673 / 40 \\
540 / 1\end{array}$ & $\begin{array}{l}0.8 \\
0.6 \\
0.5\end{array}$ \\
\hline
\end{tabular}

These analyses point out that, as the quality of endothermic heat sources decreases, an increasing amount of work (hence, a lower system efficiency) is required to deliver the exothermic reaction heat above a given temperature. The final case is of interest since all of the heat is transported and then delivered to a user at a higher temperature ( $589 \mathrm{~K}$ and above) than the heat source $(540 \mathrm{~K})$.

Economic Analysis (Chapters 7, 15, Appendix 4)

Economic analyses were performed to obtain preliminary estimates for the costs of various usage options for both the HTCHP and LTCHP. The ground rules for these analyses were as follows:

- CHP costs were separated from the primary heat source cost so that the economic effect of changes in the CHP system could be readily identified and the cost of the CHP technology could be compared with alternate technologies.

- Costs were evaluated (when appropriate) on a parametric basis to determine sensitivity to changes in key cost parameters.

- Cost and design parameters were delineated so that other values can be easily substituted if the reader so desires.

- Cost of HTCHP heat exchangers and reactors for the reference case were based on a detailed design. For other design cases, costs are varied with thermal duties.

- Standard transmission distance was assumed to be $160 \mathrm{~km}$ (linear extrapolation to other distances is acceptable).

- Capitalization factor (percent of total capital cost charged yearly) was taken as $20 \% / y r$.

- In non-baseload end-use applications (one- or two-shift process steam delivery, peak generation of electricity, and on-site storage), the endothermic reactor and pipeline were costed on a baseload basis and the exothermic reactor on a peaking basis.

- Details of the end-use applications were uncertain, therefore steam generation was assumed in most cases the exception was a brief discussion of the economics 
of peak electricity generation and cogeneration of steam and electricity.

- Optimum transportation costs were calculated based on the tradeoff between material costs (pipeline diameter) and energy costs (pumping and compressor work). Pipeline costs based on the installation of multiple lines on single right of way.

\section{HTCHP EConomics}

The technical design analysis showed that the best application of the methane reforming chemical system would be with the Very High Temperature Gas-Cooled Reactor. Therefore, the economic evaluation concentrated on this application with a cost of primary heat of $\$ 2.50 / \mathrm{GJ}$ and an electricity cost of $20 \mathrm{mills} / \mathrm{kWh}$. The following equipment costs were used:

$\begin{array}{ll}\text { Reformer } & \$ 34 / \mathrm{kW}_{\mathrm{th}} \\ \begin{array}{l}\text { Heat exchangers } \\ \text { Reformer }\end{array} & \$ 25 / \mathrm{kV} \text { th } \\ \text { Compressors } & \$ 100 / \mathrm{kW} \\ \text { Pipelines } & \text { based on diameter } \\ \text { Pumps } & \text { based on size } \\ \begin{array}{l}\text { Methanator } \\ \text { Heat exchangers } \\ \text { Methanator }\end{array} & \$ 20 / \mathrm{kW}_{\mathrm{th}} \\ \end{array}$

The total capital cost of the three major portions of the system for the reference case $(1100 \mathrm{~K} / 40$ bars, endothermic reactor outlet; $700 \mathrm{~K} / 40$ bars exothermic reactor outlet) analysis are as follows:

\begin{tabular}{lc} 
& $\$ / \mathrm{kW}_{\mathrm{th}}$ Transported \\
\cline { 2 - 2 } Reforming process & 66.3 \\
Transportation & 60.4 \\
Methanation process & $\frac{28.6}{55.3}$ \\
TOTAL & 15.6
\end{tabular}

These total capital costs and the cost of heat and work determine the total cost of the delivered heat. Figure 6 shows this cost as a function of the peaking fraction of usage at the exothermic reactor site (peak fraction equals 1.0 for baseload usage). This figure shows that, for baseload usage, if the mixed feed evaporator (MFE) concept is employed, the cost of delivered heat is $\$ 3.97 / \mathrm{GJ} ; \$ 1.47 / \mathrm{GJ}$ of that is the incremental cost of the HTCHP system (above the $\$ 2.50 / G J$ assumed as the primary heat cost). Without the MFE, the HTCHP cost becomes $\$ 1.66 / \mathrm{GJ}$. The cost of heat is fairly insensitive to peak fraction, with a total cost of $\sim \$ 4.30 / G J$ with the MFE at a peak fraction of $1 / 3$ (one-shift usage). The cost 


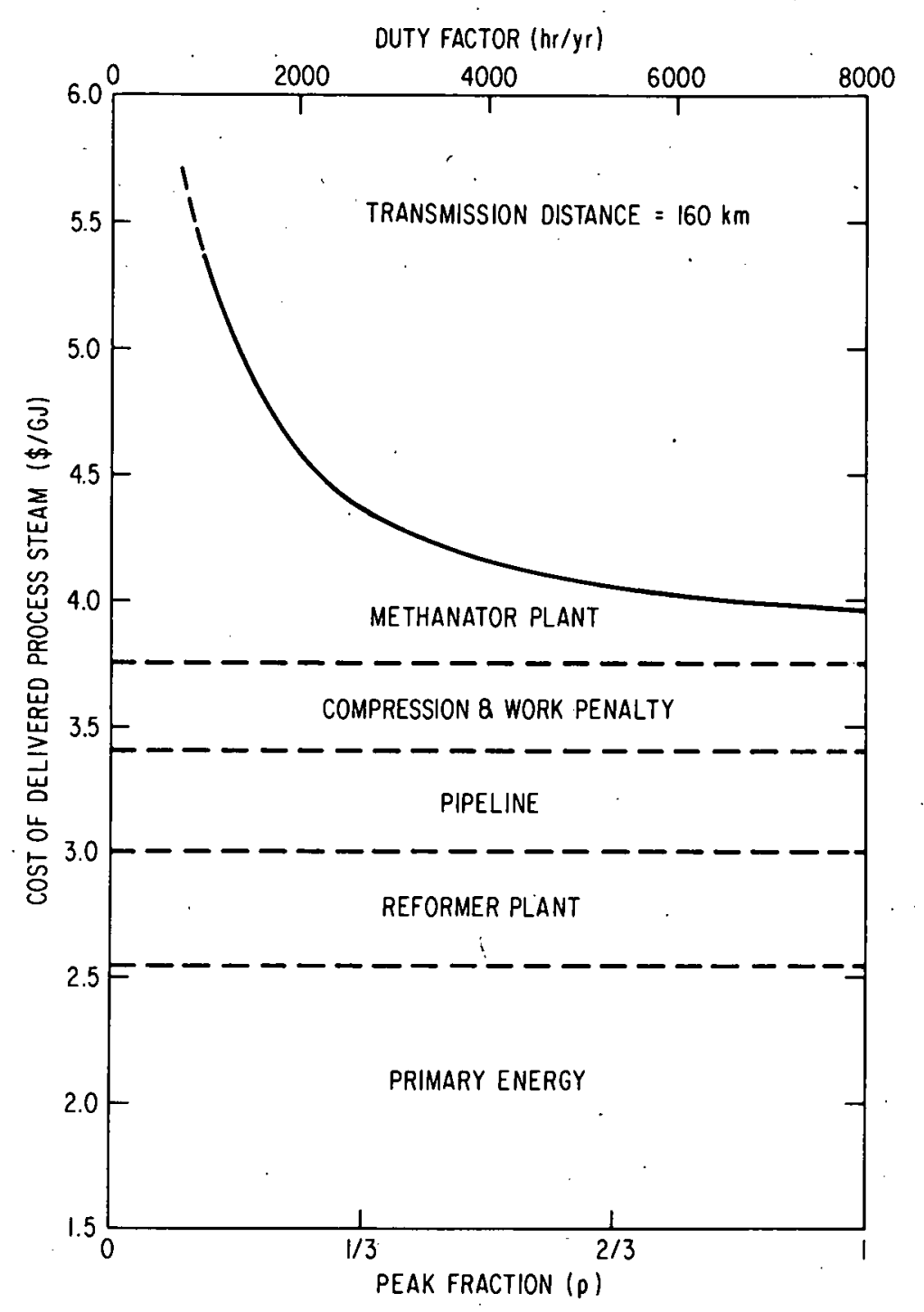

Figure 6

of the primary heat affects the HTCHP cost mainly by the effect it has on the cost of the power needed for the pumps and compressors. If the cost of power is increased to $30 \mathrm{mill} / \mathrm{s} / \mathrm{kWh}$, the incremental HTCHP transportation cost becomes $\$ 1.64 / \mathrm{GJ}$.

The economics of other HTCHP applications can be summarized as follows:

- On-site storage for peak generation with baseloaded source.

The HTCHP incremental cost ranges from $13 \mathrm{mills} / \mathrm{kWh}$ with baseload generation to $23 \mathrm{mills} / \mathrm{kWh}$ for generation at a peak fraction of $1 / 2$. The cost of caverns (at $\$ l / \mathrm{ft}^{3}$ ) for gas storage contributes only a small amount to the total cost. 
- Distributed generation of electricity.

The HTCHP incremental cost ranges from $16 \mathrm{mills} / \mathrm{kWh}$ for baseload generation to $25 \mathrm{mills} / \mathrm{kWh}$ for $1 / 3$ peak fraction generation. These costs are only slightly higher than those for on-site generation, but the electricity is distributed on a peaking basis at a distance of $160 \mathrm{~km}$ from the baseloaded primary source.

- Cogeneration of steam and electricity.

Total delivered energy costs using cogeneration are $76 \%$ of the combined separate delivery cost.

The economics of the various case designs were investigated to determine the effect of process variables on the cost of transporting heat. These incremental CHP costs all fell between $\sim \$ 1.20 / G J$ and $\$ 2.20 / \mathrm{GJ}$. The conclusions based on energetic analysis that high peak process temperatures and high conversions are favored were confirmed by these economic analyses.

\section{LTCHP Economics}

The economic analysis of the LTCHP system was carried out in a manner similar to that of the HTCHP. The major differences between the two analyses were:

- The primary heat in the LTCHP analysis was available over a range of temperatures from $550 \mathrm{~K}$ (for LWR sources) to $811 \mathrm{~K}$ (for fossil fired sources). The cost of primary heat was varied from $\$ 1.50 / \mathrm{GJ}$ for $550 \mathrm{~K}$ heat to $\$ 2.50$ for $811 \mathrm{~K}$ heat. Costs were scaled with the quality of the heat at intermediate temperatures.

- The cost of the benzene and cyclohexane inventories in the pipelines were included in the optimization calculations.

The detailed design calculations led to the following equipment costs for the base case:

\begin{tabular}{lc} 
& $\$ / k W_{t h}$ \\
\cline { 2 - 2 } $\begin{array}{c}\text { Dehydrogenation reactor } \\
\text { and catalysts }\end{array}$ & 15.4 \\
$\begin{array}{l}\text { Dehydrogenation heat } \\
\text { exchangers }\end{array}$ & 42.4 \\
$\begin{array}{l}\text { Hydrogenation reactor } \\
\text { and catalysts }\end{array}$ & 19.6 \\
$\begin{array}{l}\text { Hydrogenation heat } \\
\text { exchangers }\end{array}$ & 42.4
\end{tabular}

These costs were used to determine the total capital costs of the three major portions of the LTCHP system for the reference case analysis ( $811 \mathrm{~K}$ endothermic reaction temperature): 


\begin{tabular}{lc} 
& $\$ / \mathrm{kW}_{\mathrm{th}}$ Transported \\
\cline { 2 - 2 } Dehydrogenation process & 55.5 \\
Transportation system & 54.2 \\
Hydrogenation process & $\frac{36.5}{46.20}$ \\
$\quad$ TOTAL & $1 \frac{16.21}{4}$
\end{tabular}

The capital costs were combined with the work and energy costs to obtain the following LTCHP system costs for the four cases investigated.

\begin{tabular}{|c|c|c|c|c|c|}
\hline \multirow{2}{*}{$\begin{array}{l}\text { Case } \\
\text { (endothermic } \\
\text { reactor } \\
\text { temperature) }\end{array}$} & \multicolumn{4}{|c|}{ Yearly Cost, $\$ 10^{6} / \mathrm{yr}$} & \multirow{2}{*}{$\begin{array}{c}\text { Incremental } \\
\text { LTCHP Cost } \\
(\$ / G J) \\
\end{array}$} \\
\hline & Capital & $\begin{array}{l}\text { Unde] } \\
\text { Work }\end{array}$ & $\begin{array}{l}\text { ered } \\
\text { Heat }\end{array}$ & Total & \\
\hline $\begin{array}{cc}\operatorname{Ref} . & (811 \mathrm{~K}) \\
1 & (755 \mathrm{~K}) \\
2 & (673 \mathrm{~K}) \\
3 & (540 \mathrm{~K})\end{array}$ & $\begin{array}{l}29.2 \\
30.4 \\
56.2 \\
44.6\end{array}$ & $\begin{array}{r}4.5 \\
5.6 \\
15.9 \\
46.0\end{array}$ & $\begin{array}{r}9.6 \\
9.8 \\
13.3 \\
3.1\end{array}$ & $\begin{array}{l}43.3 \\
45.8 \\
85.4 \\
93.7\end{array}$ & $\begin{array}{l}1.54 \\
1.65 \\
3.16 \\
3.39\end{array}$ \\
\hline
\end{tabular}

It is obvious from this comparison that as the quality of the heat source decreases the LTCHP system cost increases, and that at a low source temperature a significant cost must be paid to pump the heat to a higher temperature.

Throughout this analysis, it was assumed that "ideal" catalysts would be available to prevent byproduct formation. In reality, some byproducts will form. At a formation rate of $0.1 \%$ per cycle, the cost of replacing the benzene (at $75 \% / g a l$ or $20 \% / 1$ ) would be $16 \xi / G J$ of heat transported. If the byproducts could be sold as substitute natural gas, the replacement value would drop to $3 \dot{6} / \mathrm{GJ}$.

Solar Applications (Chapters 4, 7, 16)

The mating of the CHP concept with solar heat collection technology has the obvious advantages of being able to store the periodically available heat and, at the same time, transport it to users 80 to $320 \mathrm{~km}$ from the collection site, on a baseload basis. For a CHP/solar system to be economically competitive with other solar applications, there must be a significant economy of scale to large solar collectors when compared to small "backyard" installations such that the large-scile primary heat cost and transportation cost is less than the small-scale collection cost. Siting difficulties for small-scale collectors might also favor a solar/CHP system.

The technical feasibility study undertaken has shown that the HTCHP/solar interface and limited temperature levels available pose potentially insurmountable problems. Thus, the LTCHP system offers a much higher potential for successful coupling with a solar collector. The economic analysis of a solar/LTCHP design with a peak process temperature of $811 \mathrm{~K}$ shows that the heat can be collected and transported $160 \mathrm{~km}$ for an incremental cost of $\sim \$ 3 / \mathrm{GJ}$ (using $\$ 6 / G J$ as the cost of primary heat). The cost is higher than that for a baseload source because the endothermic reactor must be oversized by a factor of 3 to account for the periodicity of the source. 
The higher primary energy cost (and, hence, the cost of electricity) also factor into the increased cost. The solar utility could take advantage of the CHP system to use cogeneration of process steam and electricity to significantly reduce the cost of delivered energy and to bring about substitution of solar energy for prime fossil fuels. This option appears more attractive than on-site storage for electricity production, since the cost of LTCHP transportation is only slightly greater than on-site storage.

\section{Market Analysis (Chapter 8)}

A market analysis was undertaken to estimate the potential market for CHP applications. This analysis was severely limited by the lack of data on the size, geographical distribution, and temperature levels of process heat users. The data that are available are not consistent; this presents a major hurdle to a rigorous analysis. These problems not withstanding, the major conclusions reached in the analysis of the existing data are as follows:

- The gross maximum potential (using 1972 data) for premium fossil fuel substitution for commercial and residential space and water heating and for industrial process heat is $29.1 \times 10^{9} \mathrm{GJ} / \mathrm{Yr}$. This compares with a total consumption of oil and gas in all uses of $53.1 \times 109 \mathrm{GJ} / \mathrm{Yr}$.

- The gross maximum potential for conservation via CHPbased cogeneration is $11 \times 10^{9} \mathrm{GJ} / \mathrm{yr}$, which is equivalent to $\sim 5 \times 106$ barrels of oil per day.

- If the maximum potential substitution is limited by including only the manufacturing portion of the industrial sector using heat at temperatures below $11000 \mathrm{~F}(870 \mathrm{~K})$, the potential is reduced to $9 \times 109 \mathrm{GJ} / \mathrm{yr}$.

- If this is further limited by including only one- or twoshift industries below $200 \mathrm{MW}$ th with no heating and air conditioning, the maximum substitution potential drops to $2.6 \times 10^{\circ} \mathrm{GJ} / \mathrm{Yr}$.

- Even though this restricted potential is only $16 \%$ of the maximum, it still represents a substantial reduction in prime fuel consumption equivalent to 1 million barrels of oil per day.

Barriers to CHP Implementation (Chapters 9 and 17)

As pointed out previously, the major technical barrier to HTCHP implementation is the source technology, and to LTCHP implementation the proof of feasibility of the cyclic closed-1oop chemical system. In addition to these technical barriers, there are significant "institutional" barriers which must be overcome. Most prominent among these are:

- Developmental barriers such as sources of funding and political and social attitudes toward possible source technologies. 
- Organizational barriers such as: who would own a combination heat and electric "utility" or how would such a technology be nucleated?

- Safety barriers such as benzene or carbon I..unoxide toxicity or large hydrogen pipelines. 


\section{TABLE OF CONTENTS}

Chapter

$\underline{\text { Page }}$

1

INTRODUCTION. . . . . . . . . . . . . . . . 1-1

1.1 The Chemical Heat Pipe Concept . . . . . . . 1-1

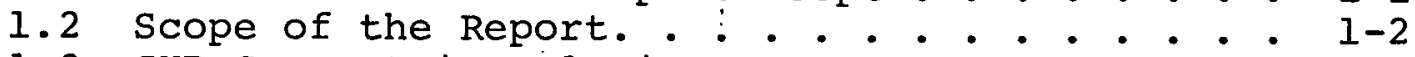

1.3 CHP Concept in Relation to Energy

Conservation and Transport... . . . . . 1-3

1.3.1 Introduction. . . . . . . . . . . 1-3

1.3.2 Prime Fossil Fuel Shortage. . . . : . . 1-4

1.3.3 Importance of Thermal Transport . . . 1-7

1.4 Historic Background of CHP Work. . . . . . . 1-11

1.4.1 West German Program . . . . . . . . 1-11

1.4.2 General Electric Program. . . . . . 1-12

1.4.3 Other CHP-Related Projects

in the U.S............. I-12

1.5 Summary. . . . . . . . . . . . . 1-13

REFERENCES. . . . . . . . . . . . . . . . . $1-14$

2 GOALS AND APPROACHES TO THE ANALYSIS OF THE HIGH

TEMPERATURE CHEMICAL HEAT PIPE. . . . . . . . 2-1

2.1 Goals of the Analysis. . . . . . . . . . . 2-1

2.2 Approaches Used in the Analysis. . . . . . 2-1

2.2.1 Technical Feasibility . . . . . . . 2-1

2.2.2 Role in the Future U.S. Energy. . . . . 2-2

2.2.3 Performance of the HTCHP system . . . 2-2

2.2.4 Barriers to Implementation. . . . . 2-5

2.3 Summary. . . . . . . . . . . . . . 2-5

REFERENCES. • • • • . • • • . . . . . . . 2-6

3 ALTERNATE CHEMICAL REACTIONS FOR CHEMICAL HEAT

PIPE APPLICATIONS . . . . . . . . . . . . . 3-1

3.1 Introduction . . . . . . . . . . . . . . 3-1

3.2 Background for Suitable CHP Reactions. . . . 3-1

3.2.1 General Requirements for CHP

Applications. . . . . . . . . 3-1

3.2.2 Thermodynamic Considerations. . . . . 3-2

3.2.3 Technology Issues . . . . . . . . 3-3

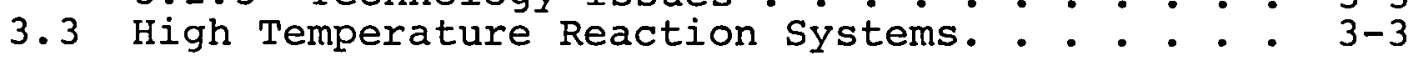

3.3.1 Background. . . . . . . . . . . 3-3

3.3.2 HTCHP Candidate Reactions . . . . . . 3-4

3.3.3 Comparison of Methane Reforming

and Sulfur Trioxide Decomposition . . 3-5

3.4 Low Temperature Reaction Systems . . . . . 3-7

3.5 Other Reactions. . . . . . . . . . . 3-8

3.6 Conclusions. . . . . . . . . . . . . 3-10

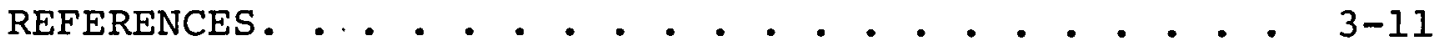




\section{TABLE OF CONTENTS (CONT'D)}

\section{Chapter}

$\underline{\text { Page }}$

PRIMARY ENERGY SOURCES. . . . . . . . . . . 4-1

4.1 Nuclear CHP Applications . . . . . . . . . . 4-1

4.1.1 Potential Sources for HTCHP . . . . .. 4-1

4.1.2 Potential Sources of LTCHP. . . . . . . 4-4

4.2 Coal-Based CHP Applications. . . . . . . . 4-5

4.2.1 Background. . . . . . . . . . . 4-5

4.2.2 Technical and Economic Feasibility

of Coal-Fired Source for HTCHP

Applications. . . . . . . . . . 4-5

4.2.3 Technical and Economic Feasibility of a Coal-Fired Source for LTCHP

Applications........... . . 4-7

4.2.4 Incentives for Coal-Fired CHP . . . . . 4-7

4.2.5 Another Coal Option: The Open Chemical Heat Pipe.............. 4-8

4.3 Solar sources. . . . . . . . . . .. . . . 4-9

4.3.1 Temperature Capabilities. . . . . . 4-9

4.3.2 Interface Problems. . . . . . . . 4-10

4.3.3 Systems Considerations. . . . . . . 4-12

4.4 Summary and Conclusions. . . . . . . . . 4-12

REFERENCES. . . . . . . . . . . . . . . 4-13

5 TECHNICAL DESIGN OF HIGH TEMPERATURE CHEMICAL

HEAT PIPE (HTCHP) . . . . . . . . . . . . 5-1

5.1 Introduction . . . . . . . . . . . . 5-1

5.2 Technological Background . . . . . . . . . 5-1

5.2.1 Methane Reforming Technology. . . . . 5-1

5.2.2. Methanation Technology. . . . . . . 5-4

5.3 Process Flowsheet. . . . . . . . . . . . . 5-5

5.3.1 Selection of Major Elements . . . . . 5-5

5.3 .2 Thermodynamic Data Base....... 5-8

5.3.3. Material Balances ......... 5-10

5.3.4 Enthalpy Balance. . . ... . . . . . 5-11

5.4 Process Efficiency . . . . . . . . . . 5-13

5.4.1 Approach to Efficiency Calculations . . 5-13

5.4.2 Thermal Histograms and Exergy Analyses. 5-15

5.4.3 Mixed Feed Evaporator (MFE) . . . . . 5-22

5.4.4 Design Efficiency . ... . . . . . 5-25

5.5 Summary and Conclusions. . . . . . . . 5-28

REFERENCES. .. • • • • • . • • • • • • • . 5-29

6 CASE DESIGN ANALYSES. - . . . . . . . . . . . 6-1

6.1 Rationale for Case Selections. . . . . . . 6-1

6.2 Results from Design Analysis . . . . . . . . 6-3

6.3 Discussion of Results. . . . . . . . . . 6-6

6.3.1 Methanation Plant . . . . . . . 6-6

6.3.2 Reformer Plant. . . . . . . . 6-7 
TABLE OF CONTENTS (CONT'D)

Chapter

Page

6.4 Summary. . . . . . . . . . . . . . 6-10

REFERENCES. . . . . . . . . . . . . . . 6-11

7 ECONOMIC ANALYSIS OF HTCHP. • • • • • • • • • • •

7.1 Introduction .. . . . . . . . . . . . . 7-1

7.2 Component Costs and Reference
Case Economics . .

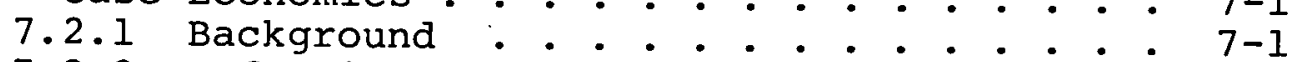

7.2.2. Reforming Process Economics... . . . 7-2

7.2.3 Transmission Line Costs....... . 7-4

7.2.4 Methanation Process . . . . . . 7-6

7.3 Base Case Economics. . . . . . . . . . . . 7-8

7.3.1 Introduction . . . . . . . . . . . . 7-8

7.3.2 Economic Calculations . . . . . . . . 7-9

7.3.3 Base Case Economic Analyses . ... . . 7-10

7.4 Application Economics. . . . . . . . . . . 7-13

7.4.1 Introduction ............. 7-13

7.4 .2 Process Steam............ . . 7-14

7.4.3 On-Site Storage for Peak

Electricity . . . . . . . . . 7-15

Distributed Generation of Peak
Electricity . . . . . . . . . 7-18

7.4.5 Combined Electricity/steam Delivery

(Cogeneration).......... 7-18

7.5 Solar Energy Applications. . . . . . . . . . . 7-20

7.5.1 Introduction. . . . . . . . . . . 7-20

7.5.2 On-Site Storage for Solar Energy

Application Utilizing HTCHP

7.5.3 Transportation of Primary Solar

7.5.4 Conclusions from Economic Analysis of Solar Energy Applications ... . . 7-24

7.6 Comparative Economics of Energy Transport

and Sources. . . . . . . . . . . 7-26

7.6 .1 Background. . . . . . . . . . . . . . 7-26

7.6.2 Determination of Economicaily

Competitive Prices of Solar and

Nuclear Heat Utilizing the HTCHP

Transportation System . . . . . . . 7-26

7.6.3 Comparative Economics for Transport of Coal and Light Water Reactor

(LWR) Heat.......... . . . 7-27

7.7 Conclusions. . . . . . . . . . . . . 7-27

REFERENCES. • • • • . . . . . . . . . . . 7-28 


\section{TABLE OF CONTENTS (CONT'D)}

Chapter

$\underline{\text { Page }}$

8 PRELIMINARY MARKET ANALYSIS FOR CHEMICAL

HEAT PIPES. . . . . . . . . . . . . . 8-1

8.1 Introduction . . . . . . . . . . . . 8-1

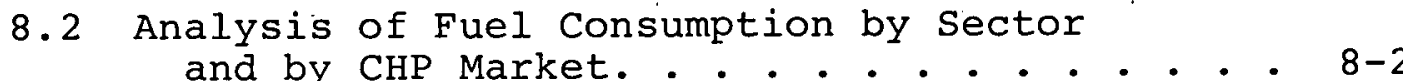

8.2.1 Total Fuel Consumption by Sector. . . 8-2

8.2.2 Types of Fuel Use Vs Function in

Various sectors........ . . 8-4

8.2.3 Upper Limits to CHP Potential for

Premium Fuel Savings and

Conservation. . . . . . . . 8-9

8.3 Analysis of the Industrial sector. . . . . . 8-11

8.3.1 Guidelines for the Analysis . . . . . 8-11

8.3.2 Premium Fuel Use in the Manufacturing

8.3.3 Fuel Usage for Process Heat at

Various Temperatures....... 8-16

8.3.4 Distribution of Plant Size Among Uses

of Process Heat.......... 8-18

8.4 Summary. . . . . . . . . . . . . . . . 8-30

REFERENCES . . . . . . . . . . . . . . . . 8-31

9 TECHNICAL AND INSTITUTIONAL BARRIERS TO

IMPLEMENTATION OF HTCHP . . . . . . . . . . . . 9-1

9.1 Introduction . . . . . . . . . . . . . 9-1

9.2 Technical Barriers . . . . . . . . . . . 9-1

9.2.1 Reformer. . . . . . . . . . . . 9-1

9.2 .2 Methanator. . . . . . . . . . 9-2

9.2.3 Transmission. . . . . . . . . . . 9-2

9.3 Institutional Barriers . . . . . . . . . 9-3

9.3.1 Introduction. . . . . . . . . . 9-3

9.3.2 Developmental Barriers. . . . . . . 9-3

9.3.3 Organizational Barriers . . . . . . . 9-4

9.3.4 Economic Barriers . . . . . . . . 9-5

9.4 Summary. . . . . . . . . . . . . . . . 9-5

REFERENCES. • • • . . . . . . . . . . . . 9-5

10 HIGH TEMPERATURE CHEMICAL HEAT PIPE ANALYSIS

CONCLUSIONS AND RECOMMENDATIONS FOR FUTURE WORK • 10-1

10.1 Introduction . . . . . . . . . . . . 10-1

10.2 Conclusions - CHP Concept. . . . . . . . . 10-1

10.3 Conclusions - Technical Feasibility. . . . . . 10-2

10.4 Conclusions - HTCHP Design and Performance - 10-3

10.5 Conclusions - Economics. . . . . . . . . 10-3

10.6 Conclusions - Market Potential . . . . . . 10-4

10.7 Recommendations for Future Work. . . . . 10-5 


\section{TABLE OF CONTENTS (CONT'D)}

Chapter

Page

11 LOW TEMPERATURE CHEMICAL HEAT PIPE -

INTRODUCTION. . . . . . . . . . . . . . . . . 11-1

11.1 Introduction. . . . . . . . . . . . 11-1

12 GOALS AND APPROACH FOR LOW TEMPERATURE CHEMICAL

HEAT PIPE . . . . . . . . . . . . . . . . 12-1

12.1 Goals of LTCHP Analysis . . . . . . . . . 12-1

12.2 Approach of LTCHP Analysis. . . . . . . . 12-1

13 TECHNICAL DESIGN OF LOW TEMPERATURE CHEMICAL

HEAT PIPE . . • • . • . . . . . . . . . . 13-1

13.1 Introduction. . . . . . . . . . . . . 13-1

13.2 Industrial Background of Hydrogenation/

Dehydrogenation Reactions . . . . . . 13-1

13.2.1 Introduction. . . . . . . . . . 13-1

13.2.2 Cyclohexane Dehydrogenation . . . . 13-1

13.2.3 Benzene Hydrogenation . . . . . . 13-2

13.3 Thermodynamic Data Base.......... . 13-3

13.3.1 Assumptions Determining the Scope

of the Data Base. . . . . . . 13-3

13.3.2 Data Base............. . 13-4

13.4 Thermodynamic Equilibria of Candidate

Reactions . . . . . . . . . . . 13-6

13.4.1 Reaction Background . . . . . . . 13-6

13.4.2 Thermodynamic Analysis . . . . . . 13-6

13.5 LTCHP Process Flowsheet........... . 13-10

13.5.1 LTCHP Flowsheet Components

and Diagrams. ....... . 13-10

13.6 Material Balances . . . . . . . . . . 13-12

13.6.1 Design Assumptions. . . . . . . . 13-12

13.6.2 Material Balance Results. . . . . 13-13

13.7 Enthalpy Balance. . . . . . . . . . . . 13-14

13.7.1 Enthalpy Balance and Histograms... 13-14

13.8 LTCHP Process Efficiency. . . . . . . . . 13-17

13.8.1 Background. . . . . . . . . . . 13-17

13.8 .2 System Efficiency . . . . . . . . 13-17

13.9 Summary and Conclusions . . . . . . . . 13-19

REFERENCES. . . . . . . . . . . . . . . . 13-19

14 BASE CASE ANALYSIS FOR LOW TEMPERATURE CHEMICAL

HEAT PIPE . . . . . . . . . . . . . . . . . . . 14-1

14.1 Introduction. . . . . . . . . . . . . 14-1

14.2 Base Case 1 Analysis. . . . . . . . . . . . . 14-1

14.3 Base Case 2. Analysis. . . . . . . . . . 14-5 


\section{TABLE OF CONTENTS (CONT'D)}

Chapter

Page

14.4 Base Case 3 Analysis. . . . . . . . . . 14-8

14.5 Summary and Conclusions . . . . . . . . 14-13

15 ECONOMICS ANALYSIS OF LTCHP . . • . . . . . • . . 15-1

15.1 Introduction. . . . . . . . . . . . 15-1

15.2 Reference Case Economics and Component

Costs . . . . . . . . . . . . . . . 15-1

15.2.1 Introduction. . . . . . . . . . 15-1

15.2.2 Cyclohexane Dehydrogenation Process - 15-1

15.2.3. Transportation System . . . . . . 15-4

15.2.4 Hydrogenation Process... . . . . 15-4

15.2.5 Total Cost of Delivered Heat. . . . 15-6

15.3 Base Case Economics . . . . . . . . . 15-8

15.3.1 Introduction. . . . . . . . . . 15-8

15.3.2 Base Case 1.. Economics. . . . . 15-9

15.3.3 Base Case 2 - Economics . . . . . 15-10

15.3.4 Base Case 3 - Economics....... . 15-12

15.4 Economics of Byproduct Formation. . . . . . . 15-12

15.5 Summary . . . . . . . . . . . . . 15-14

REFERENCES. . . . . . . . . . . . . . . 15-15

16 SOLAR APPLICATIONS OF THE LOW TEMPERATURE

CHEMICAL HEAT PIPE. . . . . . . . . . . . . 16-1

16.1 Introduction. . . . . . . . . . . . . . . 16-1

16.2 The Solar/LTCHP Interface . . . . . ... . 16-1

16.3 Economic Analysis of Solar Thermal Energy
Transport Via LTCHP . . . . . . . . . 16-2

16.4 Economic Justification of LTCHP/Solar
Applications. . . . . . . . . . . . 16-4

16.5 Summary . . . . . . . . . . . . . 16-6

REFERENCES. . . . . . . . . . . . . . . 16-7

17 TECHNICAL AND INSTITUTIONAL BARRIERS TO

IMPLEMENTATION OF THE LOW TEMPERATURE CHEMICAL

HEAT PIPE . . . . . . . . . . . . . . . . 17-1

17.1 Introduction. . . . . . . . . . . . . . . 17-1

17.2 Dehydrogenation Process Barriers. . . . . . 17-1

17.3 Hydrogenation Process Barriers. . . .. . . 17-2

17.4 Transportation System Barriers. . . . . . . 17-2

17.5 Summary . . . . . . . . . . . . . 17-3

REFERENCES. . . . . . . . . . . . . . . . 17-3 


\section{TABLE OF CONTENTS (CONT'D)}

Chapter

Page

18 LOW TEMPERATURE CHEMICAL HEAT PIPE SUMMARY, CONCLUSIONS, AND RECOMMENDATIONS FOR

FUTURE WORK. . . . . . . . . . . . . . 18-1

18.1 Introduction .. . . . . . . . . . . 18-1

18.2 Conclusions - Catalysts. . . . . . . . . 18-1

18.2.1 Dehydrogenation Catalysts. . . . . 18-1

18.2.2 Hydrogenation Catalysts. . . . . . 18-2

18.2.3 General. . . . . . . . . . . 18-2

18.3 Conclusions - Reactor Design . . . . . . . 18-2

18.4 Conclusions - Process Efficiency . . . . . 18-2

18.5 Conclusions - Process Economics. . . . . . 18-3

18.6 Recommendations For Future Work. . . . . . 18-3

APPENDIX 1

A REVIEW OF METHANATION TECHNOLOGY

1 Introduction . . . . . . . . . . . . . Al-1

2 Summary. • . . . . . . . . . . . . . . . Al-1

3 Technical Discussion . . . . . . . . . . . Al-3

4 Developmental Programs . . . . . . . . . . Al-3I

APPENDIX 2

THERMODYNAMIC ANALYSES

A2.1 Basic Definitions. . . . . . . . . . . . . A2-1

A2.2 Exergy Losses During Energy Conversion . . . . . A2-3

A2.3 Powerplant Analyses. . . . . . . . . . . . A2-7

A2.4 Irreversibilities and Entropy Production

A2 . 5 Summary. . . . . . . . . . . . . . . . A A2-27

APPENDIX 3

HEAT EXCHANGER TRAIN DESIGN FOR

$1000 \mathrm{MW}_{\text {th }}$ METHANE REFORMER FOR HTCHP

A3.1 Introduction . . . . . . . . . . . . . . . A3-1

A3.2 Design Summary for Methane Reforming Heat

Exchanges. . . . . . . . . . . . . . A3-1

A3.3 Detailed Design of Heat Exchanges. . . . . . . A3-5 


\title{
TABLE OF CONTENTS (CONT'D)
}

Section

$\underline{\text { Page }}$

\author{
APPENDIX 4 \\ ANALYSIS OF TRANSMISSION COSTS \\ FOR CHEMICAL HEAT PIPE APPLICATIONS
}

A4.1 Introduction . . . . . . . . . . . . . . . . A4-1

A4.2 Pressure Drop, Work, and Cost Relationships. . . A4-3

A4.3 Transportation Costs For Chemical Heat Pipe Materials . . . . . . . . . . . . . A4-9

A4.4 Sensitivity of Transmission Costs to Variations in Design and Economic Parameters. . . . . . A4-15

A4.5 Effect of Transportation Philosophy on Transmission Cost...................... A4-20

A4.6 Transmission Costs for Chemical Heat Pipe Applications (Multiple Lines Per Trench) . . . A4-23

A4.7 Investigation of Alternative Uses of CHP Transportation System. ............. A4-28

A4.8 Summary . . . . . . . . . . . . . . . . A4-33

\author{
APPENDIX 5 \\ REVIEW OF THE STATE-OF-THE-ART OF \\ HYDROGENATION-DEHYDROGENATION PROCESSES AND REACTOR DESIGNS \\ By UOP Inc.
}

BENZENE-CYCLOHEXANE REACTIONS REVIEW • • • • • • • • • . A5-1

HETEROGENEOUS CATALYTIC REACTOR DESIGN REVIEW • • • • . A5-9

APPENDIX 6

COMPUTER PROGRAMS

COMPUTER PROGRAMS . . . . . . . . . . . . . . . . . A6-1 


\section{LIST OF ILLUSTRATIONS}

$\underline{\text { Figure }}$

$\underline{\text { Page }}$

1-1 Chemical Heat Pipe Concept. . . . . . . . . . 1-1

5-1 KFA Design of Helium-Heated Reformer Tube . . . : 5-3

5-2 Reformer Plant Schematic. . . . . . . . . . 5-6

5-3 Methanation Plant Schematic . . . . . . . 5-7

5-4 Reformer Exit Equilibrium . . . . . . . . . . 5-11

5-5 Methanation Exit Equilibrium. . . . . . . . . 5-12

5-6 Closed-Loop Material Balance. . . . . . . . . 5-12

5-7 Enthalpy Balance. . . . . . . . . . . . 5-14

5-8 Example of a Histogram for Transfer of Heat Between Two Constant Temperature Fluids.. . . 5-17

5-9 Example of a Histogram for the. Transfer of Heat Between Fluids with Different Heat Capacities.. 5-17

5-10a Temperature Histogram - Reformer Cooling Duty . . 5-18

5-10b Temperature Histogram - Reformer Heating Duty . . 5-18

5-11 Superposition of Reformer Histograms. . . . . . 5-19

5-12 Methanation Plant Histograms. . . . . . . . . 5-21

5-14 Example Case - Reformer Plant with MFE. . . . . 5-23

5-15 Example Case - Methanation Plant with MFE . . . 5-25

7-1 Process Steam Costs as a Function of Duty Factor. 7-15

7-2 Major Parts of On-Site Storage System . . . • . 7-16

7-3 Electricity Cost for on-Site Storage. . . . . . 7-17

7-4 Cost of Distributed Electricity . . . . . . . . 7-20

7-5 Costs for Combined Steam/Electricity Delivery • • 7-21

7-6 Cost of on-Site storage and stored Heat as a Function of the Cost of Solar Heat. . . . . . 7-23

7-7 Cost of HTCHP Transportation and Transported Heat as a Function of the cost of Solar Heat 7-25

8-1 Ratio of Total Electricity to Purchased Fuel. • . 8-15

8-2 Cumulative Fraction of Fuel Energy Use vs Temperature Processing .. . . . . . . . . 8-18

8-3 SIC 20 - Food: Plant Size Distribution Based on 1971 Purchased Fuel Usage of $0.96 \times 109$ $\mathrm{GJ} / \mathrm{Yr} \mathrm{T}<11000 \mathrm{~F}$. . . . . . . . . . 8-20

8-4 SIC 26 - Paper: Plant Size Distribution Based on 1971 Purchased Fuel Usage of $1.26 \times 109$ $\mathrm{GJ} / \mathrm{Yr}$ at $\mathrm{T}<11000 \mathrm{~F}$. . . . . . . . . $8-21$ 


\section{LIST OF ILLUSTRATIONS (CONT'D)}

$\underline{\text { Figure }}$

Page

8-5 SIC 28 - Chemical: Plant Size Distribution Based on 1971 Purchased Fuel Usage of $1.72 \times 10^{9} \mathrm{GJ} / \mathrm{Yr}$ at $\mathrm{T}<11000 \mathrm{~F}$. . . . . . . . . . . . 8-22

8-6 SIC 29 - Petroleum Refining: Plant Size Distribution Based on 1971 Total Fuel Use of $2.85 \times 10^{9}$ $\mathrm{GJ} / \mathrm{Yr}$ at $\mathrm{T}<11000 \mathrm{~F}$. . . . . . . . . . . .

8-7 SIC 33 - Primary Metals: Plant Size Distribution Based on 1971 Total Fuel Use of $0.56 \times 109 \mathrm{GJ} / \mathrm{Yr}$ at $\mathrm{T}<11000 \mathrm{~F}$. . . . . . . . . . . . . .

8-8 Mean Plant Size Distribution for Manufacturing Industry, Based on 1971 Fuel Usage, for Heat

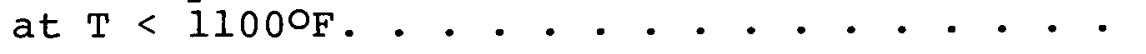

8-9 Manufacturing Industry Fuel Consumption in 1971 for $\mathrm{T}<11000 \mathrm{~F}$; Includes only Purchased Fuel Plus In-House Fuel in Petroleum Refining . . .

8-10 Comparison of Cumulative Fuel Consumption for Manufacturing Industry in 1971 per this study
(A) with DOW-1 Results (B) and (C)

13-1. Equilibrium Compositions in Cyclohexane/Benzene/
Methylcyclopentane System at 1 Bar Reaction Pressure .. . . . . . . . . . . . . . 13-8

13-2 Equilibrium Compositions in Cyclohexane/Benzene/ Methylcyclopentane system at 40 Bar Reaction Pressure • • • • • • • • • • • • • • • • • •

13-3 Equilibrium Composition in Cyclohexane/Benzene/ Methylcyclopentane system at 68 Bar Reaction Pressure . . . . . . . . . . . . . . . 13-9

13-4 Process Flowsheet for Cyclohexane Dehydrogenation Section of LTCHP System. . . . . . . . . . 13-10

13-5 Process Flowsheet for Benzene Hydrogenation Section of LTCHP System. . . . . . . . . . 13-11

13-6 Histogram of Heating and Cooling Duties for Reference Case Dehydrogenation Process . . . . 13-14

13-7 Histogram of Heating and Cooling Duties for Reference Case Hydrogenation Process . . . . . 13-15

14-1 Histogram of Heating and Cooling Duties for Dehydrogenation Process in Base Case 1; $\mathrm{T}_{\text {reaction }}=755 \mathrm{~K}, \mathrm{P}_{\text {reaction }}=40$ Bars $. . . \cdot 14-3$

14-2 Histogram of Heating and cooling Duties for Hydrogenation Process in Base Case 1 ; $\mathrm{T}_{\text {reaction }}=589 \mathrm{~K}$, $\mathrm{P}_{\text {reaction }}=40$ Bars . . . . 14-3 


\section{LIST OF ILLUSTRATIONS (CONT'D)}

Figure

$14-3$

Histogram of Heating and Cooling Duties for Dehydrogenation Process in Base Case 2; $\mathrm{T}_{\text {reaction }}=673 \mathrm{~K}, \mathrm{P}_{\text {reaction }}=40 \mathrm{Bars}$. . . . 14-6

14-4 Histogram of Heating and Cooling Duties for Hydrogenation Process in Base Case 2; $\mathrm{T}_{\text {reaction }}=589 \mathrm{~K}, \mathrm{P}_{\text {reaction }}=40$ Bars. . . . 14-7

14-5 Histogram for Heating and Cooling Duties for Dehydrogenation Process in Base Case 3; $\mathrm{T}_{\text {reaction }}=540 \mathrm{~K}, \mathrm{P}_{\text {reaction }}=1 \mathrm{Bar} . . . \cdot$.. 14-10

14-6 Histogram for Heating and Cooling Duties for Hydrogenation Process in Base Case 3; $\mathrm{T}_{\text {reaction }}=589 \mathrm{~K}, \mathrm{P}_{\text {reaction }}=40$ Bars. . . . 14-10

14-17 Gas Compression Flowsheet for $540 \mathrm{~K} / 1$ Bar in Base Case 3... . . . . . . . . . . . . 14-11

16-1 Cost of LTCHP Transportation and Cost of Delivered Heat as a Function of the cost of Solar Heat . . . . . . . . . . . . . 16-4

16-3 Solar Heat Transportation Via LTCHP and Conversion to Electricity and Process Steam. . . . 16-6

Al-1 Catalyst Temperature Profiles . . . . . . . . Al-7

Al-2 Effect of MTD on Heat Recovery to Steam . .

Al -27

A $2-1$ Energy Conversion Efficiencies.

A $2-7$

A2-2

Exergy Ratios During Energy Conversion.

A2 -8

A $2-3$

Energy and Exergy Flows in a steam Powerplant. .

A2-9

A $2-4$

A $2-5$

KFA Design; Reproduced from Nuclear Engineering and Design with Permission of the Publishers. .

A $2-11$

Energy and Exergy Flows in KFA Design . . . . . .

A $2-12$

A2 -6

Reference Case - Reformer Plant . . . . . . .

A $2-15$

A2 -7

Reference Case - Methanation Plant. .

A2- 15

A2-8

Case A - Reformer Plant.

A2-16

A 2-9

Case A - Methanation Plant.

A2-16

A2- 10

Case B - Reformer Plant

A2 -17

A2-11

Case B - Methanation Plant.

A2 -17

A $2-12$

Case C - Reformer Plant

A $2-18$

A2 -13

Case C - Methanation

A $2-18$

A2- 14

Case D - Reformer Plant.

A2-19 


\section{LIST OF ILLUSTRATIONS (CONT'D)}

Figure

A 2-15

A2-16

A2-17

A2-18

A2-19

A2-20

A2-21

A2-22

A 2-23

A 2-24

A2-25

A2-26

A2-27

A3-1

A3-2

A3-3

A 4-1

A4-2

A $4-3$

A $4-4$

A $4-5$

A $4-6$

A4-7

A 4-8

A4-9

A4-10

A4-11
Case D - Methanation Plant.

Case E - Reformer Plant

Case E - Methanation Plant.

Case F - Reformer Plant

Case F - Methanation Plant.

Case G - Reformer Plant

Case G - Methanation Plant.

Case H - Reformer Plant

Case $\mathrm{H}$ - Methanation Plant.

Case I - Reformer Plant

Case I - Methanation Plant.

Case J - Reformer Plant.

Case J - Methanation Plant.

Heat Exchanger Train.

Baffle Arrangement.

Orifice and Tube Pitch Geometry, or flow cell for a Typical Tube.

Pipeline Construction Cost Versus Pipeline

Diameter.

Transportation Cost of Reformer Exit Gases Versus Flowrate.

Transportation Cost of Methanator Exit Gases Versus Flowrate.

Transportation Cost of Water Versus Flowrate.

Transportation

Cost

Transportation Cost of Cyclohexane Versus Flowrate.

Transportation Cost of Hydrogen Versus Flowrate.

Optimum Pipeline Diameter Versus Heat Delivered for Chemical Heat Pipe Fluids . . . . . . .

Transportation Cost Versus Heat Delivered for Chemical Heat Pipe Fluids . • • . . . • .

Transportation Cost Versus Percent Increase in Cost Parameters.

Transportation Cost of Reformer Exit Gases Versus Compressor Cost.
Page

A 2-19

A $2-20$

A $2-20$

A $2-21$

A $2-21$

A $2-22$

A $2-22$

A $2-23$

A $2-23$

A2-24

A2-24

A 2-25

A2-25

A3-2

A 3-3

A3-3

A 4-7

A 4-10

A4-11

A4-11

A4-12

A4-12

A4-13

A4-13

A 4-14

A 4-17

A 4-18 


\section{LIST OF ILLUSTRATIONS (CONT'D)}

Figure

Page

A4-12 Transportation Cost of Reformer Exit Gases

Versus Electricity Cost...... ....... A4-18

A4-13 Transmission Cost of Reformer Exit Gases

Versus Maximum Transmission Line Pressure. . . A4-19

A4-14 Transportation Cost Versus Transportation

Distance Reformer Exit Gases at Optimum

Pipeline Diameter. . ... . . . . . . . A4-20

A4-15 Transportation Cost Versus Heat Delivered

for methane-Based CHP Single Line per

Trench and Multiple Lines per Trench . . . . . A4-24

A4-16 Cost per GJ Delivered Versus Heat Delivered

for Methane-Based Chemical Heat Pipe . . . . A4-24

A4-17 Incremental Cost of Water Transportation Versus

Heat Delivered for Methane-Based Chemical Heat

Pipe . . . . . . . . . . . . . . .

A4-18 Transportation Costs at Single Line per Trench

and Three Lines per Trench Versus Heat De-

livered for the Benzene/Cyclohexane-Based CHP: . A4-27

A4-19 Increase in Cost of Transported Heat and Optimum

Pipeline Diameter Versus Cost of Benzene . . . . A4-27

A4-20. Three Line per Trench Transportation Costs

Versus Heat Delivered Methane-Based and

Benzene/Cyclohexane-Based Chemical Heat

Pipes. •. . . . . . . . . . . . . . . A4-28

A4-21 Compressor/Expander System for Transporting PV Work. . . . . . . . . . . . . . A4-29

A4-22 Storage Capability of Transmission Line Versus Average Line Pressure. . . . . . . . . A4-32

A4-23 Incremental. Cost for storage Versus Transmission Line Diameter for $\mathrm{H}_{2} / \mathrm{CO}$ Storage. . . . A4-32

A5-1 Residual Benzene Concentration in Hydrogenation Reaction as a Function of Temperature and Hydrogen Partial Pressure. . . . . . A5-2

A5-2 Flow Scheme for Benzene Hydrogenation Process. . A5-3

A5-3 Modified Flow Scheme for Benzene Hydrogenation Process. . . . . . . . . . . . . . A5-3

A5-4 Typical Flow Scheme for Catalytic Reforming Process. . . . . . . . . . . . . . A5-7 


\section{LIST OF TABLES}

Table

Page

4-1 Basic Reactor Capabilities. . . . . . . . . . . 4-2

4-2 Status of Reactor Systems . . . . . . . . . . 4-3

5-1 Thermodynamic Data Base . . . . . . . . . . . 5-10

5-3 Equipment Duties for Example Case Design. • • • • 5-14

5-4 Availability Comparisons of Separate and Mixed Evaporation Example Case - Reformer Plant . . . 5-26

6-1 Parameters for Equilibria for Design Cases. • . . 6-2

6-2 Conversions and Transportation Flow Rates for Design Cases. . . . . . . . . . . 6-4

6-3 Thermal Duties for Design Cases . . . . . . . . 6-4

6-4 Process Energetics and Efficiencies . . . . 6-5

7-1 Design and Economic Parameters for Cost
Estimation of Nuclear Reformer. . . . . . . . . 7-3

7-2 Design and Economic Assumptions for Heat
Exchangers for Reference Case . . . . . . . . . 7-4

7-3 Incremental and Total Cost of Heat Delivered
by the HTCHP. . . . . . . . . . . . . . . .

7-4 Heat and Work Requirements for Base Case Process . 7-9

7-5 Capital Costs for Major Equipment for Base Cases 7-9

7-6 Components Contributing to the Cost of

Incremental Cost and Total Cost of Delivered
Heat for Base Case Economic Analysis. . . . . . 7-11

7-8 Costs and Ground Rules for Applications Analysis. - 7-13

7-9 Capital Costs for On-Site Storage for Peak
Electricity . . . . . . . . . . . . . . . . . 7-17

7-10 Capital Costs for Distributed Generation of Peak . $_{\text {7-18 }}$

7-11 Summary of Capital and operating Costs for On-Site 7-23 Storage Using a Solar Heat Source. . . . . . 7-23

7-12 Summary of Capital and operating Costs for
Transportation of Solar Heat. . . . . . . . . 7-25

8-1 Fuel and Energy Consumption: Comparison of Data
Sources for Various Years. . . . . . . . . . 8-3 


\section{LIST OF TABLES (CONT'D)}

Table

$\underline{\text { Page }}$

8-2 Residential Fuel Consumption: Fuel. Type vs Function 1968, SRI . . . . . . . . . . . 8-5

8-3 Commercial Fuel Consumption: Fuel Types vs Function 1968, SRI: . . . . . . . . . . . 8-5

8-4 Transportation Fuel Consumption: Fuel Type vs Function 1968, SRI . . . . . . . . . . . 8-6

8-5 Utility Fuel Consumption ${ }^{1}$ : Fuel Type vs Mode of Generation 1968, SRI . . . . . . . . . . 8-6

8-6 Industrial Fuel Consumption: Fuel Type vs Function 1968, SRI . . . . . . . . . . . . 8-7

8-7 Fuel Source of Energy Consumed - Industrial Sector - 1971 ECS Data . . . . . . . . . 8-13

8-8 Fuel Source of Energy Consumption - Industrial Sectorl - 1974 ECS Data. . . . . . . . . 8-14

8-9 Change in Purchased Energy in Manufacturing Industry - 1974-1971 .. . . . . . . . . . 8-15

8-10 Purchased and Generated Electricity in Manufacturing Industry - 1971, 1974. . . . . . . . . . . 8-16

8-11 Fuel Consumed for Process Heat and Steam in Manufacturing Industries - 1971, $109 \mathrm{GJ} / \mathrm{Yr}$. . . 8-17

13-1 Thermodynamic Data Base for LTCHP Calculations Units in Cals, Gram-Moles, and Kelvin. . . . . 13-5

13-2 $\Delta H^{\circ}, \Delta S^{\circ}$, and $\Delta G^{\circ}$ for. Cyclohexane Dehydrogenation and Isomerization Reactions as a Function of Temperature . . . . . . . . . . . . . 13-7

13-3 Process Parameters for LTCHP Reference Case. . . . 13-12

13-4 Equilibirum Reactor Outlet Compositions and Pipeline Flows for ITCHP Reference Case. . . . . . 13-13

13-5 Enthalpy Blance for ITCHP Reference Case for $20 \mathrm{~K}$ $\Delta \mathrm{Ts}$ in the Heat Exchangers . . . . . . . . . 13-16

14-1 Reaction Conditions for Base Case 1. . . . . . . . 14-1

14-2 Reactor Outlet Compositions for Base Case 1. . . . 14-2

14-3 Flowrates for Base Case 1. . . . . . . . . . . 14-2

14-4 Enthalpy Balance for Base Case 1 . . . . . . . . 14-4

14-5 Reaction Outlet Conditions for Base Case 2 . . . . 14-5 


\section{LIST OF TABLES (CONT'D)}

Table

$\underline{\text { Page }}$

$14-6$

Reactor Outlet Compositions for Base Case 2

$14-6$

$14-7$

Flowrates for Base Case 2.

$14-6$

$14-8$

Enthalpy Balance for Base Case 2

$14-7$

$14-9$

Reactor Outlet Conditions for Base Case 3

$14-9$

$14-10$

Reactor Outlet Compositions for Base Case 3

$14-9$

14-11

Flowrate for Base Case 3

$14-9$

14-12

Enthalpy Balance for Base Case 3

14-11

$15-1$

Design and Cost Assumptions and Reactor Size and Cost Results for LTCHP Dehydrogenation

Reactor.

$15-2$

15-2 Heat Exchanger Cost and Design Parameters for LTCHP Reference Case - Dehydrogenation

$15-3$

$15-3$

Cost and Design Parameters for LTCHP Transportation System . . . . . . . . . . . 15-5

15-4 Design and Economic Parameters for Benzene Hydrogenation Process. . . . . . . . . . 15-6

15-5 Yearly Operating Costs and Cost of Delivered Heat for Reference Case Conditions . . . . . . 15-7.

15-6 Incremental LTCHP cost and Total cost of Delivered Heat as a Function of Primary Heat Costs . . . . . . . . . . . . . . 15-7

$15-7$

Capital Cost Summary for Base Case 1 . . . . .

$15-9$

$15-8$

Yearly Operating Costs and Cost of Delivered Heat for Base Case \#1 - $755 \mathrm{~K} / 40$ Bars . . . . 15-10

15-9 Capital Cost Summary for Base Case 2 . . . . . . . 15-11

15-10 Yearly Operating Costs of Delivered Heat for Base Case 2 - $673 \mathrm{~K} / 40$ Bars. . . . . . . . . 15-11

15-11 Capital Cost Summary for Base Case 3 . . . . . . 15-13

15-12 Yearly Operating Costs and Cost of Delivered Heat for Base Case 3 - $540 \mathrm{~K} / 1$ bar . . . . . . 15-13

16-1 Summary of Cost Analysis for Solar/LTCHP Transmission of Heat . . . . . . . . . . . 16-3

Al-1 Equilibrium Constants . . . . . . . . . . . . Al-5

Al-2 Process Efficiencies . . . . . . . . . . . Al-12

Al-3 Heat of Methanation (Btu) . . . . . . . . . . Al-13 


\section{LIST OF TABLES (CONT'D)}

Table

Page

Al-

Enthalpies (Btu/lb mol

Al-14

Al-5

Methanation Mass and Energy Balance

A $1-15$

Al-6

Heat Data

Al -16

Al-7

Required Recycle Rates.

Al -17

Al-8

Al-9

AI -10

Reactor Feed Gas Preheat Duties

A1-19

Reactor Pressure Drop

A $1-21$

A1-11

Effect of Reactor Pressure on Horsepower.

Al-23

Effect of Compressor Suction Temperature on

Horsepower

Al-23

Al -12

Effect of space Velocity on Horsepower

Al-23

Al-13

Effect of Catalyst Size on Horsepower

Al -24

A1-14

Effect of Reactor Shape on Horsepower

Al-24

Al-15

Heat Exchanger Surface Areas.

Al-28

A2-I

Exergy Ratios for Various Types of Energy

Sources . . . . . . • . . . . .

A2-4

A2-2 Histogram and Exergy Analysis for Reference

Case Reformer . . . . . . . . . . . . . .

A2-13

A2 -3

Irreversibilities for the Reference Case. . . .

A2-27

A3-1

Heat Exchanger Types

A $3-2$

A3-2

Summary of Calculated Heat Transfer Coefficients, Pressure Drops, Areas, and

Costs... . . . . . . . . . . . .

A3-6

A4-1 Reactor outlet Molar Compositions for HTCHP and LTCHP Transportation Analyses . . . . . .

A4-2

A4-2 Base Case Values for Economic and Design Parameters for Chemical Heat Pipe Reactants .

A4-9

A4-3 Flowrates for CHP Materials at 1000 MW Power Delivered . . . . . . . . . . . . . . .

A4-4 Individual Components of Overall Transmission Cost at $1000 \mathrm{MW}$ Transported . . . . . . .

A4-16

A4-5 Comparison of Transmission Costs of Reformer Exit Gases for Various Compressor Locations and Capital Costs

A4-6 Comparative Economics for High and Low Pressure Liquid Transportation Philosophies. . . . . .

A4 -6

A4-7 Cost of Electricity at User site as a Function of Cost at Generation Site for Transportation via PV Work . . . . . . . . . . . . . .

A5-1 Processes for the Production of Cyclohexane: Economic Data. 


\section{Chapter 1}

\section{INTRODUCTION}

\subsection{THE CHEMICAL HEAT PIPE CONCEPT}

The closed-loop chemical systems studied in this report involve transporting and/or storing thermal energy by the use of reversible chemical reactions. The name given to this concept is the Chemical Heat Pipe (CHP) and it is illustrated in Figure 1-1. In the CHP, the primary thermal energy is converted to a chemical form by a catalyzed endothermic chemical reaction. The sensible heat required to heat the reactants from the ambient temperature to the reaction temperature is provided by a countercurrent heat exchange with the products leaving the reactor. Thus, the transport/storage of fluids takes place at ambient temperatures rather thain elevated temperatures, thereby eliminating excessive thermal loss. The invested energy is recovered by reversing these steps by a countercurrent heat exchange between reactor inlet and outlet streams and a catalyzed exothermic chemical conversion to reproduce the original chemicals.

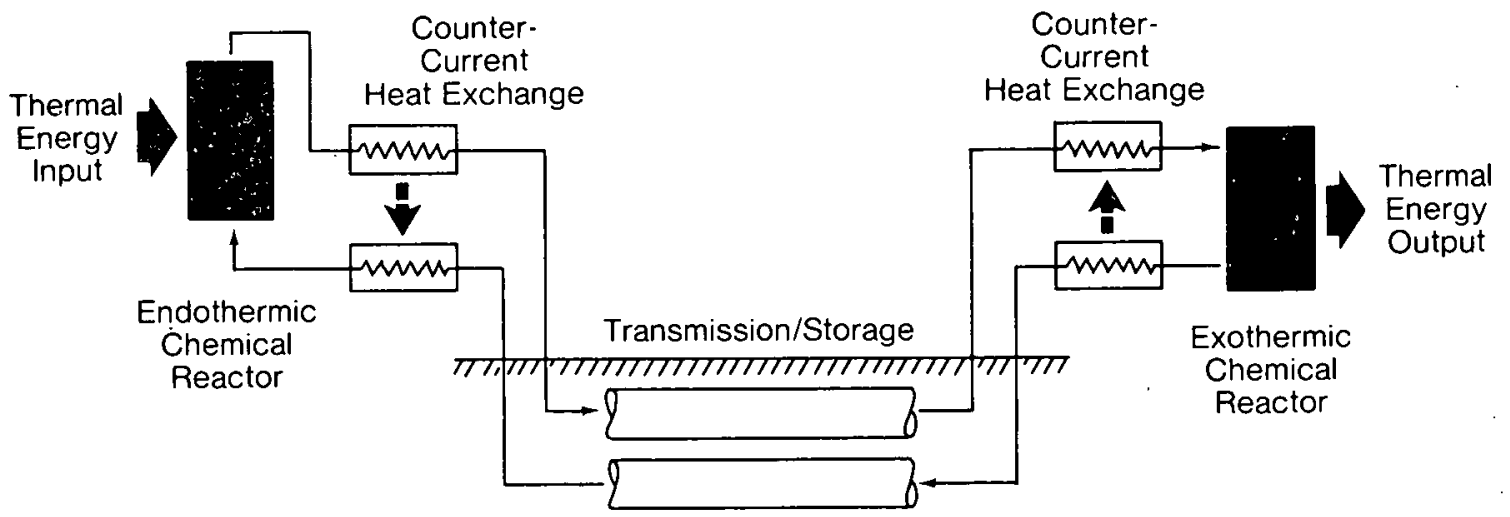

Figure 1-1. Chemical Heat Pipe Concept

The overall process is a closed cycle, the only exchanges with the environment being thermal and mechanical energy. By removing the chemicals from the catalyst bed, it is possible to prevent the reversal of the conversion in the endothermic reactor during the subsequent cooling. Thus the high energy products do not spontaneously release the stored energy at ambient temperature, in spite of the fact that this change is highly favored thermodynamically. This advantage of being able to "freeze in" the stored energy in a metastable state makes chemical conversions different from simple thermal absorption processes (phase change, heat of solution, sensible heat). For example, a mixture of co and hydrogen at ambient temperature does not spontaneously undergo a reaction to produce $\mathrm{CH}_{4}$ and $\mathrm{H}_{2} \mathrm{O}$ with a large release of thermal energy, in spite of the fact that this change is highly favorable thermodynamically. The same is not true for phase change processes; for example, steam cannot be kept in a highly subcooled (meta- 
stable) state without a spontaneous condensation and the release of stored energy.

This major difference can be readily expressed quantitatively by the large activation energy requirements for the chemical reaction, and allows for a convenient way to "turn off" the chemical change once the chemicals are out of contact with the reaction catalysts. The same is true for the exothermic change; the reaction is held off until the chemicals are heated to desired temperatures, then proceeds as they are introduced into the catalyst bed. Were it not for this convenient control, it would be impossible to contemplate a CHP.

\subsection{SCOPE OF THE REPORT}

This report documents two distinct phases of the work undertaken during the contract period. The first phase involved the investigation of the CHP concept as a whole, with particular emphasis on the evaluation of the methane-reforming-based CHP (referred to as the High Temperature Chemical Heat Pipe or HTCHP). The second (and shorter) phase was an investigation of the CHP based on cyclohexane dehydrogenation (referred to as the Low Temperature Chemical Heat Pipe, or ITCHP). The work undertaken in the first phase of the work is documented in Chapters 1 through 10 of this report; the LTCHP analysis is covered in Chapters 11 through 18.

In this chapter, the importance of the CHP concept to U.S. energy management will be outlined; it will include a summary of the major aspects of the "energy crisis" which impact this work, and a discussion of the importance of thermal energy transport in the context of improving the efficiency of energy transport and promoting prime fuel conservation. Also included at the end of this chapter is a brief summary of the history of CHP-related projects. In Chapter 2, the major goals of the HTCHP investigation and the approaches used to solve the problems encountered will be delineated. Chapter 3 provides a summary of the chemical reactions considered for use in CHP applications, along with a critical evaluation of their potential as CHP components. Chapter 4, in turn, explores the nature of possible heat sources that might be coupled to chemical heat pipes.

Chapters 5, 6, and 7 will deal with the technical design and economic evaluation of the methane reforming HTCHP. This evaluation begins in Chapter 5 with a detailed heat and material balance analysis of the HTCHP under the most likely reaction conditions and a calculation of the efficiency of the system. Chapter 6 explores variations in reactor conditions and their effect on system performance. In Chapter 7, an economic evaluation of the reference case conditions is given, and the effect of variations in reactor conditions (as given in chapter 6 ) on the economics of heat transport is investigated. Chapter 8 identifies the potential CHP market and summarizes the available data for energy usage in the United States. The potential technical and institutional barriers for implementation of the HTCHP are outlined in Chapter 9; 
Chapter 10 summarizes the HTCHP work and presents recommendations for further work.

The remainder of the main body of this report concentrates on the evaluation of the cyclohexane-dehydrogenation-based chemical heat pipe (LTCHP), with the format following that of the first ten chapters (excluding those topics common to both systems). Chapters 11 and 12 give, respectively, a brief introduction and a summary of the goals and approaches to the LTCHP analysis. Alternate reactions and source technology are not discussed again. Chapter 13 presents a reference case analysis based on the most likely endothermic reactor conditions for the.LTCHP. Chapter 14 investigates the changes in heat and material balances and system efficiency for the LTCHP as the endothermic reactor conditions are varied. The economics of the LTCHP are explored in Chapter 15 for the cases covered in Chapters 13 and 14 . Chapter 16 explores in more detail the coupling of the LTCHP with a solar collector source, giving conditions under which the two technologies would be linked and the economics of solar heat transport via the LTCHP. Technical and institutional barriers unique to the LTCHP are discussed in Chapter 17, and the major conclusions from the LTCHP analysis and recommendations for further work are summarized in Chapter 18.

In addition to the main body of the report, six appendixes are included which give technical details of the various analyses carried out during the contract. Appendix 1 documents work carried out by The Ralph M. Parsons Company analyzing high temperature methanation in the HTCHP system. Appendix 2 describes the thermodynamic analyses of energy transport in general and the west German design of the HTCHP. Appendix 3 gives a detailed analysis of the heat exchanger design for the General Electric design of an endothermic reactor for the HTCHP; this appendix serves as a basis for many of the design assumptions used in the LTCHP heat exchanger design.

The work requirements, economics, and sensitivity to design parameters of the transportation systems for the two CHP systems are investigated in Appendix 4. Appendix 5, written by UOP Inc. (without charge) for inclusion in this report, is a summary of the industrial applications of the LTCHP chemical reactions and of the evolutionary history of reactor designs in the chemical industry. The computer programs used in the technical analyses of the CHP systems are given for reference in Appendix 6 .

\subsection{CHP CONCEPT IN RELATION TO ENERGY CONSERVATION AND TRANSPORT}

\subsubsection{Introduction}

The concept of thermal energy transmission and storage through chemical heat pipes has major implications for many important en- 
ergy issues. Among these are prime fossil fuel substitution, energy conservation by cogeneration, storage, and reduction of oil imports. To provide a general framework for evaluating the merits of CHP work to be presented in this report, those aspects of the general energy crisis that are most relevant to this work are summarized in this section.

\subsubsection{Prime Fossil Fuel Shortage}

In recent years, we have become increasingly aware of the limited availability of clean fossil fuels. There have been numerous studies to predict resources of oil and natural gas. While there is considerable uncertainty as to the range of validity of different methods for estimating undiscovered natural resources (Rl-1), there is a general agreement among all such studies that the projected overall energy demand exceeds the anticipated fossil energy supplies, and even the most optimistic projections concede that clean fossil fuels will provide an ever decreasing fraction of the nation's needs (RI-2).

These two conclusions appear to be true not only for the United States, but to the rest of the world as well. These problems were demonstrated most dramatically by the oil embargo a few years ago and by the natural gas shortages in the winter of 1976-1977. The response to this projected energy deficit has been aimed at a variety of goals consisting of an increased energy production (exploration, recovery of oil, harvesting newer sources of energy), conservation, and fuel substitution.

A review of past energy consumption patterns shows that approximately $75 \%$ of the total energy needs in the U.S. are met by the use of petroleum products and natural cas (RI-3). The only possible way in which these needs can continue to be met by prime fossil fuels is through massive imports. Either for balance of trade or security reasons, this "heavy foreign imports" scenario is generally judged neither desirable nor viable in the long run. 'l'he only other alternative for the near future, apart from $a$ drastic reduction in energy consumption, appears to be a switch to alternative sources of energy consisting predominantly of coal and nuclear power. Solar, wind, and geothermal sources will play a larger role when they become technically available and economically attractive. Since there are considerable differences in the ease of substitution among the various uses of prime fuels, an examination of the nature of problems associated with this shift in energy sources from oil and gas to coal and nuclear power is in order.

The largest use of oil and gas ( $33 \%$ of total) is in the transportation sector $(\mathrm{Rl}-3)$, almost exclusively in the form of petroleum products. While there is a growing effort on storage batteries for electric vehicles, it is highly unlikely that in the near future petroleum products can be successfully replaced in this end use. Therefore, it is not surprising that the main efforts 
to reduce consumption in this sector have been restricted to conservation measures (lower speed limits, car pools) and higher efficiencies (smaller, high-mpg cars).

The next largest use of oil and gas ( $30 \%$ ) is in the industrial sector (R1-3). While $4.4 \%$ of this $30 \%$ is in the form of chemical feedstocks, and therefore very difficult to replace, the rest is consumed as fuel to provide either steam or process heat. In this category, the usage for steam is much easier to substitute than that for process heat, because the latter is often closely coupled to (and often in direct contact with) the actual processing. The question of fuel substitution can only be addressed by paying close attention to the particular requirements of each individual industrial use as is done in Chapter 8. This attempt to take a closer look at the needs and consumption of energy in the industrial sector has resulted in the realization that not only is there lack of an accurate, detailed breakdown of consumption or requirements by size, temperature levels, geographic location, and fuel type, but also there are discrepancies and int consistencies among all major studies that have attempted even a gross characterization of industrial energy consumption. These difficulties notwithstanding, industrial process steam users may be categorized into four broad groups:

- Users that require process steam in quantities large enough to accommodate on-site coal handling and cleanup equipment to produce process steam at competitive costs. The problem of prime fuel shortage is less severe for these users, for they can readily switch to a less expensive and more abundant source (e.g., coal) if they have not already done so. Some of these users are even large enough to justify a captive nuclear plant (LWR) to supply steam. (Examples are attempts by Dow Midland, U.S.A. .(R1-4) and BASF, West Germany (Rl-19) to license LWR to provide process steam.) Though it is not possible to state the size beyond which on-site generation of steam from coal combustion is the preferred, economic route, a reasonable guess would place the critical size in the vicinity of 200 $\mathrm{MW}_{\text {th }}$. Currently, this would account for approximately $20 \%$ of the fuel consumed for process steam.

- Users that are not large enough to afford large, capitalintensive coal-handling facilities but still require sufficient quantities of steam on a continuous basis to be unable to pay an excessive price for it. If they are denied the fuel currently used, as in the signifcant curtailment of natural gas already witnessed, they must either rely on imported oil or switch to more expensive alternatives such as electrode- or SNG-fired boilers. Currently, these users account for approximately 55\% of the total fuel consumption for process steam. 
- Users that need process steam on a part-time basis (either one- or two-shift operation every day). These users will be unable economically to use coal for their needs and will require some source they can útilize when needed (imported oil or SNG) or must transfer the storage need inherent in their consumption pattern to others (e.g., to utilities by the use of electrode boilers).

- Seasonal users of process steam. In the absence of a seasonal energy storage device, it appears inevitable that these users will be dependent on prime fuels at any cost, whether imported oil, coal-derived clean fuels, or electricity.

The use of oil and gas in the residential and commercial sectors ( $28 \%$ of total oil and gas consumption) is predominantly for providing space heat and hot water. These two uses alone add up to $25 \%$ of the total of $28 \%$ for these two sectors (Rl-3). In principle, any source of low-grade heat can be substituted for this usage of prime fuel. The potential benefits of using powerplant reject heat for this purpose are increasingly recognized in Europe. Whether or not district heating concepts are equally viable in this country is a matter of debate. At present, these consumers are considering primarily oil (domestic or imported), coal-derived clean fuels, heat pumps, or low-temperature solar heat for their future needs.

The last category of oil and gas users ( $10 \%$ of the total) comprises the electric utilities $(\mathrm{R} 1-3)$ that rely on these fuels primarily for the generation of peak and intermediate electricity. The rising fuel costs have led them to an increasingly intensive search for alternatives. The two main approaches to obviate the need for oil and gas are load management by regulation or pricing policies and the use of energy storage to deliver peak electricity from base load generation. While considerable work needs to be done in these areas, the problem of reducing peak electrical consumption or accommodating it with sufficient storage does not appear insurmountable in comparison with the problems described earlier.

To summarize this discussion of substitutions for prime fossil fuel, the major problem stems from the fact that, in the past, prime fossil fuels have provided a unique source of energy that could be stored inexpensively and used when and where needed, in quantities small or large, for a broad range of end uses. Their potential substitutes (coal or nuclear energy) simply cannot match these attributes. As a result, there will be a significant mismatch of characteristics of future energy sources and demands. The mismatch is most evident in three areas: 
- Sources are large in capacity (with typical sizes $3000 \mathrm{MW}(\mathrm{h})$. The demands are in smaller quantities and distributed. This requires a method for distributing the energy among various users that share the same source.

- Sources are capital-intensive and uneconomical unless operated on a continuous, round-the-clock basis. The demand is often time-dependent or intermittent. This requires storage devices to buffer the primary source against demand fluctuations.

- Siting requirements dictate that the sources be located at some distance (typically $\sim 160 \mathrm{~km}$ ) away from population centers, where the demand is. This necessitates a transmission of energy from the source to demand centers.

In light of these mismatches, one can readily appreciate why considerable emphasis has been placed on such programs as (1) coal conversion to produce synthetic fuels that match the versatility of oil and gas, (2) energy storage (electrical and thermal) to modify load characteristics to suit baseload sources, and (3) conservation, especially in those end uses where prime fuel use is seemingly inevitable (e.g., residential space heating, transportation).

Had coal conversion programs been able to produce coal-derived liquids and gas at prices comparable to the current oil and gas prices, many of the fuel shift problems would have been eliminated. Current economic projections indicate, however, that SNG prices are likely to be in excess of $\$ 4 / G J$ (Rl-5) and significantly higher than current fuel costs ( $<\$ 2.50 / \mathrm{GJ})$. If coal conversion is too expensive to be of widespread use, and if storage technology can only serve to even out such demands as that for peak electricity, the critical question appears to be: How will thermal energy be supplied to the bulk of the users in small quantities?

It would be extremely useful if the primary energy from nuclear or coal plants could be transmitted and distributed to these users. The central thrust of the chemical heat pipe concept is to develop this ability to transport useful thermal energy. Moreover, it will not only enable a significant substitution for oil and gas by alternative sources but also provide an opportunity to achieve a significant energy conservation by combined energy delivery, as discussed in the following section.

\subsubsection{Importance of Thermal Transport}

In the preceding section it was concluded that the greatest potential area for oil and gas substitutes, principally by nuclear and coal, is in such uses as process steam and space/water heating. 
In order to perform this substitution, it is necessary to convert the primary source energy to a form convenient for transmission and distribution. At present, the two options for this intermediate energy are electricity (derived from nuclear or fossil steam) or SNG or liquid fuel (derived from coal). The chemical heat pipe concept provides a third option through conversion to chemical reaction energy.

It is important to compare these possible alternatives on the basis of overall energy efficiency achievable in each case. A proper methodology for such a comparison is based on thermodynamic principles governing energy conversion and transfer in combination with practical realities of the relevant technologies. This comparison is carried out in detail in Appendix 2 (section A2.2). The important conclusions from that analysis can be summarized as follows:

- The first step in the conversion of primary heat with a high exergy ratio* is invariably the degradation of this heat to a lower exergy ratio due to the materials limitations (e.g., corrosion) of the equipment used.

- The subsequent conversion of this heat with an intermediate exergy ratio into a secondary energy carrier with a high exergy ratio (electricity, SNG, hydrogen) results in a large portion of the heat being rejected at the conversion site (i.e., a low first-law efficiency).

- The use of this secondary energy carrier with high exergy ratio to produce process steam with an intermediate or low exergy ratio, using current thermodynamically inefficient methods, invariably results in a loss in the potential work available from the secondary energy (a low second-law efficiency).

- The combination of these two steps invariably results in both heat rejection at the conversion site and loss of available work (exergy) at the user site (low firstand second-law efficiencies).

- The use of a secondary energy carrier with an intermediate exergy ratio (lower than the degraded primary energy but higher than the final process steam exergy ratio), such as the CHP, will generally result in a higher first-law efficiency and a higher-second law efficiency.

*The exergy ratio for heat equals the maximum work available from the heat divided by the quantity of heat $(W / Q)$. See Appendix 2 for a detailed explanation of this concept. 
- Using these arguments as a basis, it becomes clear that if primary thermal energy needs to be converted, transported, and reconverted to deliver lower grade heat, it is inefficient to generate an intermediate energy carrier with a very high exergy ratio.

These conclusions are graphically illustrated by the results of this work and the analysis in Appendix 2 (Section A2.2). If, in the production of process heat, a comparison is made between the transport of thermal energy from a Very High Temperature GasCooled Reactor (VHTR) through, the HTCHP and by the generation of electricity, an overall energy efficiency of $285 \%$ is obtained in the first case versus $36 \%$ in the second. Alternatively, if the heat available from coal is transported either via the LTCHP or by gasification to SNG, to produce process heat, an overall system efficiency of $\sim 72 \%$ is obtained from LTCHP transport while SNG manufacture yields only about a 52\% efficiency.

These thermodynamic arguments show why serious consideration must be given to the CHP concept of heat transport. However, thermodynamics itself is not sufficient to evaluate the real economic viability of such a scheme. In this report, a preliminary design and economic evaluation of both the HTCHP and LTCHP systems will be undertaken to allow a comparison, on an economic basis, of these systems with other alternatives to thermal energy transport.

In addition to this advantage of thermal transmission from a conversion thermodynamics viewpoint, other advantages to CHP transmission of energy should be pointed out. Since the transmitted energy has an exergy ratio less than that of the primary source energy, the undesirable thermal rejection at the primary site is eliminated. As long as a thermodynamic "uphill" climb is avoided there is no need to reject any low-grade energy at the source. This advantage can have important consequences in siting of the primary source and its effect on the environment. Similar advantages accrue at the delivery site where there are no unwanted products in addition to work and heat.

An enormous conservation potential is offered by thermal transmission combined with total energy delivery systems. Since the primary energy is at a temperature much higher than that of the demand, the option exists with thermal transmission of expanding high-pressure steam in a back-pressure turbine before delivering the process steam to the users (e.g., cogeneration). In thermodynamic terms this is equivalent to splitting the original thermal energy into two parts -- one at a much lower exergy ratio (exhaust, low-pressure steam), and the other at a much higher exergy ratio (electricity or shaft work). This method of generating electricity has been recognized generally as the most efficient since it constitutes complete utilization of energy and exergy. In view of the mismatch between future supply and demand of energy discussed earlier it is necessary that thermal energy be transported and distributed to make possible a large-scale combined delivery system. 
To illustrate the enormous conservation potential of this combination. consider the following example (see Chapter 8 , section 8.2.3 for details). If low-temperature industrial process heat. $\left(9 \times 10^{9} \mathrm{GJ} / \mathrm{Yr}\right)$, residential/commerical space heat $\left(13.6 \times 10^{9} \mathrm{GJ} / \mathrm{yr}\right)$, and electrical needs were to be supplied by the use of a combination of remotely located central gources, thermal transport, and cogeneration, approximately $11 \times 10^{\circ} \mathrm{GJ} / \mathrm{yr}$ would be saved (mostly in the form of oil and gas). The reduction in total energy consumption would be equivalent to approximately $5 \times 10^{6}$ barrels of crude per day, a figure close to the current level of total foreign oil imports. It should be emphasized, however, that while the advantages of combined generation of low-pressure steam and electricity are not new to industries, they can only be available in the future (for coal and nuclear sources) in the majority of cases if thermal transmission is a reality.

In the preceding discussions, thermal transmission has been treated generically, regardless of how it may be achieved in reality, since the advantages mentioned do not depend specifically on these details. For example, if it were practical simply to transport steam as such in pipelines over a distance of $\sim 160 \mathrm{~km}$, all of the advantages mentioned above could be realized immediately. In reality, however, because of excessive thermal losses it is not practical to pipe steam over distances exceeding a few kilometers*.

It is extremely interesting and informative to consider a hypothetical situation of a pipeline made with superinsulator (thermal) and ask the following questions: What are the most promising uses of such a pipeline? How much can one afford to pay for such a superinsulated pipeline? What impact would such a "discovery" have on the national energy situation? It is hoped that the answers to these questions will become clearer as this report progresses. The only point that should be made here is that these answers are independent of how thermal energy transport is accomplished.

It is logical at this point to raise the following question: Can conservation be promoted by converting existing process steam generators to a combined electricity and thermal delivery system without the need for thermal transmission? The answer is a qualified "yes" with a critical difference in its implication. Without thermal transport, coal or nuclear sources cannot be used for supplying the primary energy. Total energy systems, then, would use more fuel at the industrial site, with large savings in fuel consumption in the utility sector. Unfortunately, since industrial steam is generated mainly from oil and gas, this would lead to

* Recent studies on thermal transmission as steam or high-pressure hot water in insulated ducts $(\mathrm{Rl}-6,-7)$ indicate that there is a tradeoff between capital cost (insulation) and operating costs (thermal losses). This may lead to a reassessment of the distance beyond which pure thermal transport would be uneconomical; the current estimates for this distance is $50 \mathrm{~km}$. 
an increased consumption of prime fuels with large reductions in utility fuel use (mainly coal. and nuclear, in the near future). Thus, the net effect of a total energy system may well be an overall reduction in energy consumption but with an actual increase in prime fuel use and savings in coal and nuclear use. This is the exact opposite of the required fuel substitution described earlier. Therefore, a major advantage of thermal transmission is that it enables the use of more abundant future resources (coal and uranium) for supplying industrial heat needs currently served by oil and gas, and much more importantly, offers a possibility for enormous savings through total energy systems.

\subsection{HISTORIC BACKGROUND OF CHP WORK}

\subsubsection{West German Program}

While there are occasional references in the patent literature to the use of endothermic decomposition reactions for storing and transporting heat $(\mathrm{Rl}-8)$, the earliest proposals to use reversible chemical reactions for thermal energy transport as envisioned in the CHP concept originated in West Germany in the past decade $(R 1-9,-10)$. The proposed concepts were aimed at transporting thermal energy from a high-temperature, gas-cooled pebble bed reactor (PBR) under development at Kernforschungsanlage (KFA); the application was specifically aimed at providing low-grade thermal energy (as hot water) to a district heating network.

In the initial phase, chemical reactions involving dehydrogenation of ethane and propane were suggested; however these were found generally unsuitable for cyclic operation. The subsequent work with steam reforming of methane has been much more promising and as a result almost all of the German CHP work is aimed at using this reaction coupled to a PBR. The end use of the methanation thermal output was originally space heat. Until recently, the German work has been directed primarily toward lower temperature designs for the methanator.

The West German program, generally referred to as Nukleare Fern Energie (NFE), is the largest CHP research effort in the world.* To include the acronym of their first experimental test facility for helium-heated reformers (einzelrohr-versuchsanlage: EVA) their proposed scheme has become known as the EVA-ADAM concept. The single tube reformer experiments on EVA have demonstrated the technical feasibility of the convectively heated reformer concept. Plans call for the continuation of the experimental phase in the form of a 30-tube reformer bundle linked to a matching methanator to form the first "test loop." In the meantime, numerous design analyses have been performed and potential

*The NFE program is a part of the general Nuclear Process Heat Program (PNP), which also includes nuclear coal gasification projects. The dominant project in NFE is the closed-loop demonstration with a 30 tube reformer (SUPER-EVA). 
problems in the primary interface between the reactor helium and the reformer (RI-1l) have been investigated.

To summarize, the main thrust of the German program is centered around the PBR and its use for process heat. The heliumheated reformer is considered by them to be a key component for a variety of process heat applications, including NFE, and a large fraction of their efforts is devoted toward establishing the technical feasibility of coupling a methane reformer to the PBR.. Their primary interest in end-use application has been district heating; more recently, however, they have also been considering cogeneration and process steam applications as potential end uses for methanation heat.

\subsubsection{General Electric Program}

General Electric became interested in the KFA EVA-ADAM concept early in 1974. Preliminary analyses showed that the concept was inherently much more versatile than contemplated earlier. Several new concepts were developed incorporating different reactions, combined storage/transmission applications, distributed electrical generation, on-site storage for peak electricity, application to process steam delivery, and LWR topping. The generalized concept was named Chemical Heat Pipe.

Chapter 2 presents the detailed objectives of this study and the approach adopted to answer some key questions related to CHP use in the United States. Several areas of work have been carried out under GE funds in addition to this DOE-funded work; references have been made to them at different points in this report. The two most relevant to the use of CHP in the U.S. are:

1. The LWR-topping concept (R1-12), which involves the use of an exothermic chemical reaction to provide superheat for the saturated steam output from a light-water reactor (LWR) .

2. The open-CHP concept, which deals with an improved systems concept for coal gasification combined with thermal energy delivery. The improvement consists of separating the gasification step carried out centrally from the methanation step carried out at many user locations; the two are connected by an intermediate Btu gas pipeline $(\mathrm{R} 1-13)$.

\subsubsection{Other CHP-Related Projects in the U.S.}

Over the last two years, there have been several groups in the United States pursuing a variety of CHP-related proposals, It would be fair to generalize that virtually all of them have in mind the use of reversible reactions for on-site collection and storage of thermal energy from a focused solar collector. 
1. The SOLCHEM process proposed at the Naval Research Laboratories ( $\mathrm{Rl-14}$ ) aims to use the $\mathrm{SO}_{2} / \mathrm{SO}_{3}$ reaction system for harvesting and storing thermal energy from collectors for delivery to a steam generator. The main role of the CHP in this application is collection of thermal energy from several focused collectors and transportation over a short distance to a central site where the large-scale storage function is provided by a molten salt storage device.

2. Another suggestion, from stevens Institute of Technology (RI-15), involves the use of hydrogenation/dehydrogenation reactions, once again to store solar energy from focused collectors. The use of cylindrical, focused collectors is contemplated, with chemical reactions taking place in the solar tube heated at the focal line.

3. The work at the University at California at Berkely has been aimed at the use of $\mathrm{SO}_{2} / \mathrm{SO}_{3}$ reaction for on-site storage of thermal energy from a focused solar tower source $(R 1-16)$.

4. The efforts at the University of Houston have been directed at the solar "power tower" concept. The chemical reactions proposed consist of salt decompositions and methanol synthesis $(R l-14)$.

5. The work reported by Rocket Research Corporation (RI-17) involves a survey of potential chemical reactions and their thermochemical and thermodynamic properties. The work has been aimed at identifying promising candidates, but not in connection with any specific end-use.

6. Open-loop applications of methane-based CHP are currently under investigation at the Institute of Gas Technology under DOE funding (R1-18). This project is aimed at near-term (open-CHP) and mid-term CHP applications.

\subsection{SUMMARY}

In this chapter, an outline has been presented of the chemical heat pipe concept, the scope of this work, and the importance of the CHP and its role in future U.S. energy management. The latter points can be summarized as follows:

- Prime fossil fuels will be in increasingly short supply, requiring substitution of nuclear, coal, or solar sources of heat.

- In the future, sources will be large in capacity and sited at some distance from users, who will be mainly small and could require energy on an intermittent basis.

- On a thermodynamic basis, secondary energy carriers of intermediate exergy ratio are more efficient than current high-exergy-ratio secondary carriers. 
- CHP systems could alleviate a large portion of the thermal pollution at the heat source and air pollution at the user site.

- CHP systems offer, in their cogeneration potential, an enormous potential conservation of both prime fossil fuels and energy as a whole.

- Cogeneration without thermal transport would decrease total energy consumption but might actually increase consumption of prime fossil fuel.

\section{REFERENCES -- CHAPTER 1}

Rl-1 Berg, et al., "Prognosis for Expanded U.S. Production of Crude Oil," Science, 184, (1974), 331.

Rl-2 Annual Review of Energy (J.M. Hollander, ed.), Vol. 1, 1976, Annual Reviews Inc., Palo Alto, California, 1976.

Rl-3 Stanford Research Institute, "Patterns of Energy Consumption in the United States," Office of Science and Technology, Executive Office of the President, January 1972.

RI-4 Nucleonics Week, January 13, 1977; see also Nuclear News, July 1976, p. 58 .

RI-5 Hammond, O.H., and Baron, R.E., "Synthetic Fuels: Prices, Prospects, and Prior Art," American Scientist, Vol. 64, 1976, p. 407 .

R1-6 Aamot, H.W.C., and Phetteplace, G., "Long Distance Heat Transmission with steam and Hot Water," International Total Energy Congress, Copenhagen, Denmark, October 1976.

Rl-7 Meyer, C.F., et al., "Role of the Heat storage well in Future U.S. Energy Systems," Report 76-TMP-27, General Electric Company, Santa Barbara, California, December 1976.

R1-8 Lindberg, J.E., United States Patent 3,075,361 (1963).

Rl-9 Hilberath, E., and Teggers, H., West German Patent 1,298,233 (1968).

R1-10 Nuernberg, H.W., and Wolff, G., United States Patent $3,558,047(1967)$.

Rl-1l "High Temperature Reactor for Process Heat Applications," Nuclear Engineering and Design, Special Issue, Vol. 34, No. 1, 1975 .

R1-12 Vakil, H.B., and Brown, D.H., United States Patent 4,031,706 (1975). 
Rl-13 This concept is the subject of a patent application currently on file in the U.S. Patent and Trademark Office. It was included in a presentation of the GE-CHP program to ERDA on January $20,1976$.

Rl-14 Presentation at the $5 \mathrm{MW}_{\mathrm{th}}$ Solar Workshop, University of Houston, May 1976. See also Chubb, T.A., Solar Energy, Vol. 17, 1975, p. 129.

R1-15 Ritter, A., private communication, Stevens Institute of Technology, Department of Chemistry and Chemical Engineering.

Rl-16 Bhakta, M.L., "Chemical Storage of Thermal Energy Using the $\mathrm{SO}_{3} / \mathrm{SO}_{2} / \mathrm{O}_{2}$ System," MS Thesis, University of California, Berkeley, 1974 .

Rl-17 Schmidt, E.W., and Lowe, P.A., llth Intersociety Energy Conversion Engineering Conference, Lake Tahoe, California, 1976 .

Rl-18 Baker, N.R. et al., "Transmission of Energy by Open-Loop Chemical Energy Pipeline," International Conference on Alternate Energy Sources, Miami, Florida, December, 1977.

Rl-19 Chemical and Engineering News, May 31, 1976, p. 11 . 


\section{Chapter 2}

\section{GOALS AND APPROACHES TO THE ANALYSIS OF THE HIGH TEMPERATURE CHEMICAL HEAT PIPE}

\subsection{GOALS OF THE ANALYSIS}

The major goals of this analysis of the high temperature chemical heat pipe (HTCHP) are to:

- Establish the technical feasibility of the HTCHP system.

- Determine the role of this technology in future energy management in the United States.

- Determine reasonable estimates of the performance of the HTCHP system in terms of energy efficiencies and economics.

- Determine the major technical and institutional barriers to successful implementation of the HTCHP concept in the U.S. and the order in which they should be addressed.

\subsection{APPROACHES USED IN THE ANALYSIS}

\subsubsection{Technical Feasibility}

There are two major aspects of the technical feasibility question: that of operating the chemical system in a closed-loop, cyclic mode, and that related to energy source technologies and the interface between the source and the chemical system. These two aspects lead to a variation of the "chicken and egg" problem. One may either look for the best possible chemical reaction system for a particular choice of the energy source or look for the best energy source to couple to a particular choice of the chemical reaction system. The approach used in this study deals with this problem in an iterative manner by identifying promising candidate reaction systems over the whole temperature range of interest while bearing in mind simultaneously the state of readiness of each of the source technologies.

In the evaluation of chemical reactions (Chapter 3), an effort has been made to obtain a combination of pure thermodynamic criteria and industrial pragmatism. Instead of selecting esoteric chemical systems that look promising on only a thermodynamic basis, industrial background has been used to screen the selections. The ability to use current industrial technology should be a consideration because of the large scale at which these technologies would be required in CHP applications* and the advantage that existing technology offers in terms of development time, capital, and probability of success.

\footnotetext{
*Energy technologies, typically are at a scale of hundreds of megawatts (thermal), more than an order of magnitude larger than the largest of chemical technologies.
} 
In considering the state of readiness and potential availability of a particular energy source technology, the rapidly changing technological, economic, and socio-political environment has been incorporated into the analyses. In the absence of any clear-cut definition of the future energy sources, a flexible, multivariate approach that is not tied to a particular source technology is considered the most valuable. As a result, the list of potential energy sources (Chapter 4) includes coal, light water reactors (LWR), the liquid metal fast breeder (LMFBR), high-temperature gas-cooled reactor (HTGR), pebble bed reactor (PBR), and focused solar coljectors.

\section{2 .2 Role in Future U.S. Energy}

As a result of work done prior to this contract, it was concluded that CHP systems looked promising for many applications beyond that originally contemplated in West Germany -- long-distance transport of nuclear heat for district heating network (R2-1). Prominent among these other applications are those aimed at delivery of industrial process steam (continuous and part-time), onsite thermal storage for utility peaking, distributed generation of peak electricity, combined heat and work delivery from a central station, and superheating LWR steam for higher efficiency.

In the course of this work, a comparison was made between CHP and the most promising alternative technologies in each of these categories, in order to determine areas in which CHP offers only a marginal improvement and, more importantly, those in which it has a significant edge over the nearest competitor. To the extent possible, the authors have taken into account quantitative uncertainties in cost and efficiency inherent in an unproven technology such as the CHP. There is clearly considerable interaction between answers to the question of CHP's role in the U.S. and of economics in each specific application.

In recommending future roles in the U.S., judgments are needed on the relative importance of such diverse issues as energy conservation, reduction of oil imports, * economics of fuel shifts, safety, and environmental pollution. The very nature of these tradeoffs prohibits a totally objective analysis; consequently, the recommendations and priorities should be treated as somewhat subjective even though the analyses included herein are as objective as possible.

\subsubsection{Performance of HTCHP System}

\subsubsection{Efficiency Evaluation}

By necessity, questions related to $\mathrm{CHP}$ performance require a detailed investigation of the process energetics; therefore emphasis

*While issues of national security and vulnerability to abrupt changes in Mid East policies have received considerable attention since the last oil embargo, the question of large balance of trade deficits ( $\$ 20 \times 109 / y r)$ inherent in the 'heavy oil imports scenario' has only just begun to be a general concern. 
has been placed on thermodynamic analyses in the technical design of CHP. Because in CHP, unlike typical chemical processes, no net chemicals are consumed or produced and the only items purchased or sold are heat and work, this is a crucial issue. Since considerations such as entropy production and availability loss have traditionally played a small role in typical conventional chemical plant design, it was felt that CHP design should not be based simply on existing rule-of-thumb design criteria but should rely heavily: on the thermodynamics of underlying processes. As discussed in chapter 5, this viewpoint has been particularly rewarding, since it has led to an invention that may have important consequences on efficiency and economics.

For the purpose of detailed analyses, the methane-based CHP was selected as the system to be studied. The major reasons behind this selection were the precedent set by KFA work on the nuclear reformer and the IVA-ADAM design studies (R2-1); the extensive technology background on methane reforming and methanation processes (Appendix 1), and the general consensus of the suitability of methane-based reactions for use in a closed-loop cyclic reaction system such as a CHP system (R2-2). For the purpose of detailed investigation, the overall system was divided into three subsystems:

1. The endothermic chemical plant, including the reformer as well as heat exchangers for countercurrent exchanges between the reformer exit streams to be cooled and the feed stream to be preheated.

2. A transportation system including the gas compressors, pipelines, and, where needed; underground storage caverns.

3. The exothermic chemical plant, consisting of methanation reactors and intercoolers as well as the countercurrent heat exchangers.

KFA designs were chosen as a convenient starting point. Their designs were used as a reference for design checks. In view of the broader scope of this study on sources and end uses, however, several example cases were studied to map out the effect of peak source temperature, operating pressures, transmission philosophy, and reaction stoichiometry.

There are several differences between KFA's designs and those in the study reported here. On the basis of thermodynamic considerations, it was concluded early in the work that high-temperature methanation processes utilizing neither gas recycle nor feed gas quenching offered a significant efficiency advantage. The German program, which was aimed primarily at district heating end use, started with a very low-temperature methanation process involving large thermodynamic degradations. A steady trend over time in their designs toward applications aimed at higher temperatures, cogeneration, and utility applications is noted. 
The second major difference is apparent in the system boundary definitions. The CHP system has been considered as a closed* (but not isolated) system accepting thermal energy in the reformer, exchanging heat with the powerplant, drawing compression energy as electricity or shaft work, delivering heat at the methanation plant, and rejecting some heat to the environment in the form of thermal losses. The German design, on the other hand, considers the whole system (nuclear reactor, powerplant, pipelines, and methanators) as one integral system, with no effort to separate CHP from the reactor and the powerplant in efficiency and economic analyses.

The third point of difference stems from the fact that the objective of the German program is to experimentally demonstrate a particular design for implementation in the near future; as mentioned above, the goals of this study have been considerably different from this. As a result, a large part of their effort is aimed at detailed design and engineering, and at working out technical and safety issues related to nuclear reactor/reformer interface. The choice here has been to direct efforts primarily at efficiency considerations and at parametric studies to define the most promising applications of CHP in the United States.

\subsubsection{Economic Analysis}

'The CHP is a future technological option that has not yet been fully demonstrated. An important characteristic, shared by all economic analyses involving an unproven technology, is that the results from such analyses can never "prove in" the future technology in question; they can only "prove out" applications where even with optimistic predictions the economics do not appear competitive with those of existing proven alternatives. In this study, results of economic analyses have been used not to argue that CHP will definitely be cost-competitive with existing options, but to arrive at some decisions about those applications where CHP may have the greatest potential for future implementation.

It was decided to treat CHP costs as an increment that would have to be paid in addition to primary-source thermal energy, thus decoupling it from the source economics. This increment is the net cost to transport/store primary thermal energy and includes the cost of any electrical energy purchased by CHP (e.g., compression power). This approach is somewhat different from that adopted in other economic studies -- KFA Analyses (R2-1); Nuclear Process Heat Studies $(\mathrm{R} 2-3,-4)$-- where the costs of primary source, powerplant, and CHP were all lumped together. The major disadvantage of this latter approach is the fact that source economics dominate

*The term "open" and "closed" are used with the usual thermodynamic meaning, implying exchanges between the system and the environment of both material and energy in the former, and energy only in the latter. 
system cósts, and any inefficiency or improvement in CHP design would be buried by uncertainties in the source cost.

Inclusion of the powerplant in the economics poses an additional problem in that profitability of electrical generation (determined by source cost, powerplant cost, and the assumed price of electricity) is not identified separately. Consequently, if the assumed price of electricity is higher than the net cost of generation, this excess provides an internal subsidy to the remainder of the system -- in this case the CHP system. The converse is true when the price of electricity is lower than the effective generation cost. Subtle as these points may appear, the fact that it is impossible to subtract this internal subsidy is a major disadvantage and can lead to erroneous conclusions when calculating the profitability of changes in the process design.

Another related problem arises in analyzing the economics of combined delivery of work and heat, as in the case of cogeneration. The manner in which the total costs should be divided and assigned to the two forms of delivered energy is not clear, but this is a problem common to all processes producing more than one product. When appropriate, effect of various pricing options on the economics of the system has been shown.

It is impossible for any study to examine every possible parameter variation and design. An attempt has been made here to present cost estimates in a form that can be conveniently modified (different capitalization rate, electricity price, etc.) for use in further analyses as the reader chooses.

\subsubsection{Barriers to Implementation}

Identification of key technical and institutional barriers was straightforward and did not present any particular problem. In arriving at recommendations for CHP application, however, a serious obstacle was found. To ascertain the potential market for CHP use in process steam delivery it was desirable to obtain a breakdown of fuel consumption in the industrial sector as a function of size, temperature, geographic location, and duty cycle. In spite of a much greater effort than was originally planned, many questions remained unanswered because of a great lack of detailed, consistent data bases. These difficulties are discussed in Chapter 8 .

\subsection{SUMMARY}

The underlying philosophy and logic for the study has been presented in this chapter. The details of the various approaches used in different parts of the study -- starting with questions of technical feasibility, continuing with detailed efficiency and economic analyses, and ending with an evaluation of future needs -- should prove useful in understanding and interpreting the results given in chapters that follow. 


\section{REFERENCES - CHAPTER 2}

R2-1 Bonn, T., et al, , "Nukleare Fernwärme and Nukleare Fernenergie" KFA Report Jül-1077, 1974.

R2-2 Prior to the start of this contract, conversations were held concerning the CHP concept with a large number of industrial firms with background in related areas. The companies included:

- Bechtel Inc., San Francisco, California

- Haldor-Tфpsoe, Copenhagen, Denmark

- Katalco, Inc., Chicago, Illinois

- The R.M. Parsons Company, Pasadena, California

- UOP, Inc., Des Plaines, Illinois

R2-3 Spiewak, I., et al., "Assessment of Very High Temperature Reactors in Process Applications," ORNL-TM-5242, Oak Ridge National Laboratory, Tennessee.

R2-4 Kugeler, K., et al., "Transport of Nuclear Heat by Means of Chemical Energy (Nuclear Long-Distance Energy)," Nuclear Engineering and Design, Vol. 34, No. 1, 1975, p. 65. 


\section{Chapter 3}

\section{ALTERNATE CHEMICAL REACTIONS FOR CHEMICAL HEAT PIPE APPLICATIONS}

\subsection{INTRODUCTION}

The underlying philosophy and approach toward an evaluation of chemical reactions for $\mathrm{CHP}$ were outlined in chapter 2 . The background experience associated with a particular chemical technology and the scale at which it is currently practiced are vitally important criteria for CHP candidate selection; as a result, a much greater emphasis has been placed on reactions on which some of the larger scale chemical technologies are based.

The technical information in this chapter is presented in four sections: a discussion of background for suitable reactions for CHP (section 3.2); an evaluation of reactions suitable for hightemperature sources (section 3.3); relative merits of candidate reactions for lower temperature sources (section 3.4); and comments on miscellaneous chemical reactions (section 3.5 ).

\subsection{BACKGROUND FOR SUITABLE CHP REACTIONS}

\subsubsection{General Requirements for CHP Applications}

Any chemical reaction that is to be considered for large-scale use in the storage or transport of energy through the CHP must meet many requirements. Among them are:

- The main energy-carrying reaction should exhibit fairly complete reversibility with few side reactions, since reactants and products must be recycled many times without undue losses.

- It should be possible to control reaction kinetics easily and sharply by the use of catalysts. Homogeneous reaction rates (without catalysts) should be very low, to inhibit chemical changes during heating and cooling.

- Chemicals and materials of construction should be inexpensive and abundant.

- Enthalpy change associated with the principal reaction should be large enough to achieve high energy densities and reasonable flowrates.

- Thermodynamic equilibria and overall reaction rates should be suitable in the temperature range of the input thermal source and the output end use.

- Working materials should present no safety hazard.

None of the many proposed reactions can satisfy all of these requirements; only a few satisfy most of them. Consequently, each requirement needs to be assigned a different priority. In this 
study, a high priority has been placed on the technical feasibility of cyclic operation, adequate experience in large-scale operation, efficiency of operation, and reasonable. economics. Variations in energy densities have a smaller influence on overall economics than, say, a necessity to use expensive alloys to overcome corrosion.

\subsubsection{Thermodynamic Considerations}

While it is true that candidate reactions must satisfy thermodynamic requirements, this is not a sufficient condition. The thermodynamics of a particular reaction are a good initial criterion for evaluating the potential of a particular reaction for CHP application, but other criteria must also be used to screen those reactions which are thermodynamically favorable. Remarks in this section deal only with thermodynamic issues.

In preliminary screening of reaction candidates, a simplified thermodynamic test is often used to estimate the temperature range for useful operation and the energy density. For example, if at ambient temperature $T_{0}$, the standard enthalpy and entropy of the forward, endothermic reaction step are $\Delta H^{\circ}\left(\mathrm{T}_{\mathrm{O}}\right)$ and $\Delta S^{\circ}\left(\mathrm{T}_{\mathrm{O}}\right)$ respectively, then the ratio $T^{*}=\Delta H^{\circ} / \Delta S^{\circ}$ is the seesaw, or change-over temperature, roughly indicating the temperature levels of operation, since the Gibbs energy change $\left(\Delta G^{\circ}\right)$ equals zero at $T^{*}$. The ability to change $\Delta G^{\circ}$ from some negative value (endothermic reaction favored) to positive value (exothermic reaction favored) by a given temperature change requires a large entropy change ( $\Delta S^{\circ}$ ) during the reaction.

The requirement for high $\Delta S^{\circ}$ has some important implications. One may deduce immediately that the class of reactions that lead to an increase in moles in the gas phase during the endothermic reaction should meet this requirement easily. By contrast, it would be surprising if reactions where both reactants and products are liquids or solids should prove to be good candidates*. One may conclude, therefore, that a good chemical reaction should have a large $\Delta \mathrm{HO}$ for high energy density, a large $\Delta \mathrm{SO}$ for easy reversal of the reaction without a large temperature drop, with the restriction of an appropriate value of $T^{*}$ midway between the thermal source temperature and the end-use temperature.

In many instances some of the reactants or products are either liquids or solids at ambient temperature and system pressure, but the reaction takes place entirely in the gas phase. As a result, the overall change is a combination of a chemical reaction and phase changes. In these cases, the requirements for $\Delta G^{\circ}$ apply to the gas phase reaction but the transported energy ( $\Delta H^{\circ}$ ) is that corresponding to the reaction written with appropriate condensed species. The exergyt associated with the transported energy is also that corresponding to the condensed species. For example,

ॠThis general conclusion is consistent with reported lists of candidate reactions $(\mathrm{R} 3-1,-2)$.

tExergy is defined as the maximum work available from the chemicals; see Appendix 2 for a more detailed discussion of exergy. 
in the methane reforming reaction the effective exergy-to-energy ratio is lower for pipeline conditions ( 1 iquid $\mathrm{H}_{2} \mathrm{O}$ ) than for the gas phase reaction; this is also reflected by a change in $T^{*}$ by approximately $200 \mathrm{~K}$ between the reactions occuring with either liquid water or steam.

Such considerations play an important role in internal heat exchange and overall system efficiency. In addition, they may lead to erroneous conclusions about the adequacy of thermal source temperatures. In the case of methane reforming, $T^{*}$ of $\sim 750 \mathrm{~K}$ for pipeline conditions with water should not be interpreted as indication that sources above this temperature will be adequate for the reaction. The relevant reaction $\mathrm{T}^{*}$ (with steam) is $\sim 960 \mathrm{~K}$ and indicates that a source temperature in excess of $1000 \mathrm{~K}$ is needed.

\subsubsection{Technology Issues}

Any CHP application based on a chemical reaction that is not currently carried out on a large scale will need more elaborate laboratory tests, catalyst development, pilot plant operation, design scaling, and finally industrial demonstration. It is unlikely that such CHP applications will have any perceivable impact on the U.S. energy picture until at least the year 2000. Even for those candidate reactions that are currently practiced industrially on a large scale, there will be a significant time delay because of major differences in application between CHP and chemical manufacture. It seems certain that if any CHP system results in future implementation, it will be the one that is least esoteric and closest to current industrial know-how.

As was mentioned in Chapter 2, there is a strong coupling between evaluations of chemical reactions and of primary sources. Therefore, it would be appropriate to bear in mind conclusions given in Chapter 4 on the relative likelihood of various high and low temperature sources: that lower temperature applications aimed at light-water reactors, coal-fired boilers, and perhaps focused solar collectors have a higher potential in the United States than high-temperature applications requiring the development of either newer nuclear reactor designs or improved materials for handling coal combustion at elevated temperatures.

\subsection{HIGH TEMPERATURE REACTION SYSTEMS}

\subsubsection{Background}

Reactions that are suitable for source temperatures above $875 \mathrm{~K}$ are considered in this section. There are few candidate reactions in this category which meet the majority of the criteria presented in the previous section. The three reactions of most interest for high temperature energy storage and transport are first discussed briefly. A more detailed comparison of the methane reforming and sulfur trioxide decomposition reaction systems in a CHP context follows. 


\subsubsection{HTCHP Candidate Reactions}

The three reactions that appear to be of most interest for possible utilization for energy storage and transport are methane reforming, sulfur trioxide decomposition, and phosgene decomposition.

Methane Reforming:

The two reactions that have been proposed are:

$$
\begin{aligned}
& \mathrm{CH}_{4}+\mathrm{H}_{2} \mathrm{O}(\mathrm{g}) \rightleftarrows \mathrm{CO}+3 \mathrm{H}_{2} \quad \Delta \mathrm{H}_{298}{ }_{29}=206.2 \mathrm{~kJ} / \mathrm{mole} \\
& \mathrm{T}^{*}=10.9 \mathrm{~K} \\
& \mathrm{CH}_{4}+\mathrm{CO}_{2} \rightleftarrows 2 \mathrm{CO}+2 \mathrm{H}_{2} \quad \Delta \mathrm{H}_{298}=247.3 \mathrm{~kJ} / \mathrm{mole} \\
& \mathrm{T} *=962 . \mathrm{K}
\end{aligned}
$$

The former was proposed in west Germany (R3-3), and the latter was suggested as a possible alternative during earlier studies at General Electric Corporate Research and Development (R3-1,-4). At pipeline conditions, reaction $(3-1)$ is modified with $\mathrm{H}_{2} \mathrm{O}$ (liquid) used as the reactant:

$$
\begin{aligned}
& \mathrm{CH}_{4}+\mathrm{H}_{2} \mathrm{O}(\ell) \underset{t}{ } \mathrm{CO}+3 \mathrm{H}_{2} \Delta \mathrm{HO}_{298}=250.2 \mathrm{~kJ} / \mathrm{mole} \\
& \mathrm{T}^{*}=751 \mathrm{~K}
\end{aligned}
$$

In actual practice the water-gas shift reaction

$$
\begin{aligned}
& \mathrm{CO}+\mathrm{H}_{2} \mathrm{O}(\mathrm{g}) \underset{\leftarrow}{\rightarrow} \mathrm{CO}_{2}+\mathrm{H}_{2} \quad \Delta \mathrm{H}_{298} \mathrm{O}_{29}=41.2 \mathrm{~kJ} / \mathrm{mole} \\
& \mathrm{T}^{*}=979 \mathrm{~K}
\end{aligned}
$$

occurs simultaneously. Reactions (3-1), (3-2), and (3-4) are not mutually independent; any one of them can be written as a linear combination of the other two. There is not a great difference between the two proposed energy-carrying reactions, since they represent the extremes of the range of overall $\mathrm{CH}_{4} / \mathrm{H}_{2} \mathrm{O} / \mathrm{CO}_{2}$ stoichiometry.

Sulfur Trioxide Decomposition:

$$
\begin{aligned}
& 2 \mathrm{SO}_{3}(\mathrm{~g}) \underset{\leftarrow}{\rightarrow} 2 \mathrm{SO}_{2}(\mathrm{~g})+\mathrm{O}_{2} \quad \Delta \mathrm{H}_{298}=98.3 \mathrm{~kJ} / \mathrm{mole} \\
& \mathrm{T}^{*}=1036.0 \mathrm{~K}
\end{aligned}
$$

This reaction has been proposed in recent studies $(R 3-5,-6,-7)$ for energy storage. Its major advantage is a higher theoretical storage density resulting from the ability to store $\mathrm{SO}_{2}$ and $\mathrm{SO}_{3}$ as liquids. Storage densities achieved in actual designs depend on the overall conversion as well as energy efficiencies of various conversion steps.

Phosgene Decomposition $(\mathrm{R} 3-8)$ :

$$
\begin{aligned}
& \mathrm{COCl}_{2}(\mathrm{~g}) \underset{\leftarrow}{\rightarrow} \mathrm{CO}+\mathrm{Cl}_{2} \quad \Delta \mathrm{H}_{298}=109.6 \mathrm{~kJ} / \mathrm{mole} \\
& \mathrm{T}^{*}=801.1 \mathrm{~K}
\end{aligned}
$$


The source temperature requirements are lower for this reaction than for the others in this class. In view of the toxicity and corrosion potential, this system may not be suitable for transport, and therefore is probably not a promising CHP candidate. The interesting aspect of this system is the possibility of a hybrid electrochemical/ thermal system where the stored energy. can be recovered electrochemically at a low temperature as partly heat and partly electricity; or conversely, the input step can be carried out by the use of off-peak electricity.

\subsubsection{Comparison of Methane Reforming and Sulfur Trioxide Decomposition}

Since phosgene decomposition does not appear to be suitable for CHP applications, the two major candidates for the HTCHP are methane reforming and sulfur trioxide decomposition. In the remainder of section 3.3 these two candidate reactions will be compared. This comparison will be made on the basis of three criteria: thermodynamics, current technological background, and materials of construction.

\subsubsection{Thermodynamic Considerations}

Thermodynamic equilibrium calculations show that the conversion is slightly less favorable for the $\mathrm{SO}_{2} / \mathrm{SO}_{3}$ system than for the methane case. For example, assuming a temperature of $1100 \mathrm{~K}$, a pressure of 10 atmospheres, and stoichiometric mixtures, one obtains a conversion of $61.5 \%$ for the $\mathrm{CH}_{4}$ system and $52 \%$ for the $\mathrm{SO}_{3}$ system, and the relative conversion for the methane system increases at lower temperatures. However, there is another point of difference that is even more critical. In the $\mathrm{CH}_{4}$ system it is possible to drive the endothermic reaction toward higher conversions by using an excess of steam in the feed. An analog for the $\mathrm{SO}_{2} / \mathrm{SO}_{3}$ system is a continuous removal of $\mathrm{O}_{2}$ from the reaction products, which would be highly impractical.

As a matter of fact, a small excess of steam is necessary to prevent carbon deposition; typical industrial processes take advantage of this flexibility to optimize the conversion for each specific application. The normal range of operation consists of $\mathrm{H}_{2} \mathrm{O} / \mathrm{CH}_{4}$ ratios from 2 to 6 . The implication for the CHP is that a higher pressure (or lower temperature) operation is made possible with $\mathrm{H}_{2} \mathrm{O}$ recycle. Improved overall efficiency results from an optimization of this recycle. To illustrate this point, at 40 atm pressure and $1100 \mathrm{~K}$ the conversion can be increased from $37 \%$ for a $1: 1 \mathrm{CH}_{4} / \mathrm{H}_{2} \mathrm{O}$ feed to $65 \%$ for a $1: 3 \mathrm{CH}_{4} / \mathrm{H}_{2} \mathrm{O}$ feed.

\subsubsection{Technological Background}

Practically all. of the existing technological background on $\mathrm{SO}_{2} / \mathrm{SO}_{3}$ reaction engineering derives from the use of $\mathrm{SO}_{2}$ oxidation in the production of sulfuric acid. Operating temperatures are not high $(<850 \mathrm{~K})$, because the equilibrium becomes less favorable for $\mathrm{SO}_{3}$ formation at higher temperatures. There is no background on the endothermic reaction step because there is no real incentive 
to decompose the valuable $\mathrm{SO}_{3}$ to $\mathrm{SO}_{2}$ and $\mathrm{O}_{2}$. The need for transferring heat to the process across a barrier (usually metal) poses problems much more severe than those encountered in $\mathrm{SO}_{2}$ oxidation, as discussed below. By comparison, in methane reforming there is a vast amount of experience with the endothermic reaction. In fact, the methane reforming technology is better understood than the reverse reaction, the methanation of carbon monoxide. Developing a background of knowledge on materials and corrosion at temperatures of $1100 \mathrm{~K}$ is a significant problem and should be treated cautiously.

Another aspect of the technology is the scale at which these reactions are carried out. A typical sulfuric acid plant manufac-turing 1000 tons of acid per day is equivalent to only $10 \mathrm{MW}_{\mathrm{th}}$ in the CHP context, and the exothermic end of a $1000 \mathrm{MJ} / \mathrm{s} \mathrm{CHP}$ based on $\mathrm{SO}_{2} / \mathrm{SO}_{3}$ would compare favorably with the entire production of sulfuric acid in the United states. By contrast, radiantly heated methane reformers equivalent in size to 100 to $200 \mathrm{MJ} / \mathrm{s}$ have been built and operated for several years, and the current demonstration program for coal conversion to SNG involves a methanation step equivalent to $400 \mathrm{MJ} / \mathrm{s}$ reaction heat for a typical plant ( $250 \times 10^{6}$ $\mathrm{SCF} /$ day of $\mathrm{SNG}$ ).

Another major difference between the states of these two technologies is the status of the catalysts required to promote the two reactions. Methane reforming catalysts have been proven over an extended period; a similar catalyst has been used successfully to promote the high temperature methanation of carbon monoxide (Appendix 1). Vanadium pentoxide catalysts are used in the production of $\mathrm{SO}_{3}$ for sulfuric acid manufacture. However, a survey of catalyst manufacturers (R3-9) has shown that existing catalysts are unsuitable for operation at temperatures in excess of $1000 \mathrm{~K}$. In addition no data are available for the use of these or other catalysts in promoting the decomposition of $\mathrm{SO}_{3}$.

\subsubsection{Materials of Construction}

The major effect that the materials of construction have on the comparison of the methane reforming and sulfur trioxide decomposition systems is in the capital cost of the endothermic reactor. The economic analysis performed in this study (Chapter 7) shows that the reformer tubes represent a significant fraction of the endothermic plant costs, which in turn constitute a major portion of the total capital cost of the methane-reforming-based CHP. In the methane-based system, these tubes would be constructed from $\mathrm{HK}-40$ (a castable version of 310 stainless steel) or Mo-Re 1 (a high-temperature modification of $\mathrm{HK}-40$ ).

The $\mathrm{SO}_{3}$-based system would have a much more corrosive operating atmosphere and would require an alloy such as Haynes 188 or Inconel 617 (R3-9). A cost quote (R3-10) shows that the price of Inconel 617

*In this regard, the methane/steam reaction system and the benzene/ cyclohexane reaction system (to be discussed in the following section) are unique in that both are of industrial interest in forward as well as reverse reactions. 
is approximately four times as high as that of HK-40. This additional cost is a result of both a higher billet cost and a higher fabrication cost. The higher fabrication cost is a result of the fact that Inconel 617 must either be extruded or cold-finished. This additional expense would significantly increase the cost of energy storage and transmission if the $\mathrm{SO}_{2} / \mathrm{SO}_{3}$ system were employed.

Another point which should be noted is the difference in the types of materials needed for the heat exchangers for heat recovery from the reaction products. Again in the $\mathrm{SO}_{2} / \mathrm{SO}_{3}$ system, high-alloy exchangers would be required (especially if any trace amounts of water were present). In the $\mathrm{CH}_{4} / \mathrm{H}_{2} \mathrm{O}$ system, low-alloy materials would be acceptable in high-temperature exchangers and high-alloy steels would be required only below the dewpoint of the water (where carbonic acid is formed).

Both of these limitations on the materials of construction would severely influence the cost of the $\mathrm{SO}_{2} / \mathrm{SO}_{3}$ system and make it uneconomical in comparison with the methane-based system.

To summarize, $\mathrm{SO}_{2} / \mathrm{SO}_{3}$ system has significant disadvantages in comparison with the methane system. The most prominent differences pertain to technical background or lack thereof on the endothermic reaction engineering; materials of construction for high-temperature operation; and the endothermic reactor lifetimes and cost.

\subsection{LOW TEMPERATUIRE REACTION SYSTEMS}

Of the various reactions suitable for lower temperature operations considered in this study, hydrogenation/dehydrogenation reactions involving cyclic hydrocarbons appear to be the most promising. The major reasons behind this conclusion are the extensive background in the petroleum reforming industry and the greatest potential for successful cyclic operation. Efforts therefore have been concentrated on evaluating this class of reactions.

Cyclohexane Dehydrogenation:

$$
\begin{aligned}
\mathrm{C}_{6} \mathrm{H}_{12}(\mathrm{~g}) \rightleftarrows \mathrm{C}_{6} \mathrm{H}_{6}(\mathrm{~g})+3 \mathrm{H}_{2} \Delta_{\mathrm{H}^{\circ}}{ }^{298} & =206.1 \mathrm{~kJ} / \mathrm{mole} \\
\mathrm{T}^{*} & =568 \mathrm{~K}
\end{aligned}
$$

This reaction requires somewhat higher source temperatures than other candidates for dehydrogenation. The industrial background is based on catalytic reforming for production of high-octane gasoline for the endothermic step, and the production of high-purity cyclohexane for use in the petrochemical industry for the exothermic step. Major uncertainties in cyclic operation are related to isomerization and hydrocracking side reactions. Another major problem that has surfaced recently is the possible carcinogenic nature of benzene. These issues are discussed later in the LTCHP analysis (see Chapters 13 and 17 and Appendix 5). 
Methyl Cyclohexane Dehydrogenation:

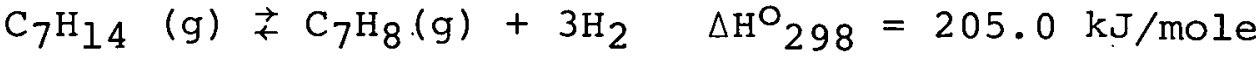

$$
\begin{aligned}
& \mathrm{T} *=549 \mathrm{~K}
\end{aligned}
$$

In comparison with reaction (3-7), the source temperature requirements are slightly lower. However, possibilities for side reactions and byproduct formation are somewhat greater for this reaction because of the methyl group. A possible advantage of this system, in comparison with the cyclohexane dehydrogenation system, is the low freezing point temperatures for toluene and methylcyclohexane in comparison with benzene and cyclohexane; this may be an important issue in transporting the liquids in cold climates. An interesting possibility may be the mixture of a small amount of toluene with benzene as an antifreeze without any detriment to the reactions. Another possible advantage is the lower toxicity of toluene as compared with benzene. However, sufficient data are not available to make final judgments on this comparison (see Chapter 17).

Tetralin Dehydrogenation:

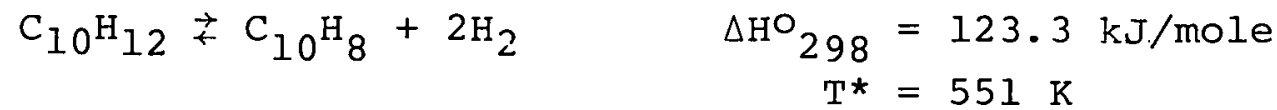

This reaction is less characterized than reactions (3-7) and (3-8), but in combination with the next reaction (3-10) it offers some interesting possibilities. Freezing of napththalene may not be as serious a problem, because small amounts of unreacted tetralin will lower the mixture melting point significantly. Though not much is known about side reactions, these molecules may be more stable toward hydrocracking than cyclohexane because of the bicyclic nature of the ring system and the lower reaction temperature.

Decalin Dehydrogenation:

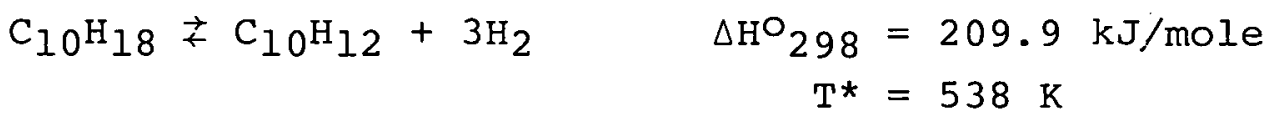

The higher heat of reaction per mole $\mathrm{H}_{2}$ than that for reaction (3-9) is the result of saturating the aromatic ring. Decalin is easily dehydrogenated (even in liquid phase) with nickel, palladium, or platinum catalysts and is often used as a hydrogen carrier for hydrogenation reactions. Very little is known about cyclic operations or side product formation of either of these reactions.

\subsection{OTHER REACTIONS}

Several additional candidate reactions have been investigated for their suitability for CHP applications. Since none of the reactions in this group appears to be highly promising the discussion is restricted to an explanation of why their use is not favored, particularly in comparison to the two preceding groups. 
Ethane Dehydrogenation:

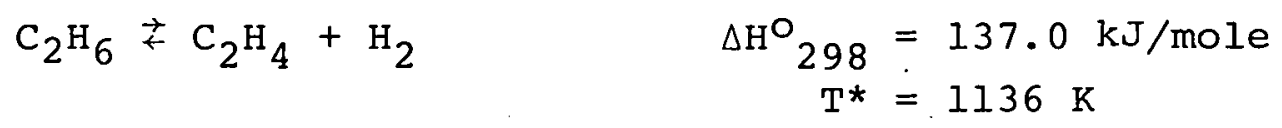

The dehydrogenation of ethane to ethylene is carried out on a large scale for plastics manufacture. One potential problem is a considerable formation of coke as a byproduct. This reaction was 'cited by the KFA group for use in a CHP (R3-11), but upon experimentation they discovered that the reactants convert mainly to coke, methane, and hydrogen after $\sim 10$ cycles of this reaction. Unless a major progress in catalyst specificity can lead to a demonstration of cyclic operation, this reaction cannot be considered a serious candidate despite its attractive energy density.

Ammonia Dissociation:

$2 \mathrm{NH}_{3}(\mathrm{~g}) \underset{\leftarrow}{\stackrel{N}{2}}+3 \mathrm{H}_{2}$

$$
\begin{aligned}
\Delta \mathrm{H}_{298}^{\circ} & =46.2 \mathrm{~kJ} / \mathrm{mole} \\
\mathrm{T}^{*} & =466 \mathrm{~K}
\end{aligned}
$$

This reaction is a good example to illustrate the shortcoming of a purely thermodynamic screening of reactions. The system looks attractive because the operating range of 400 to $600 \mathrm{~K}$ is ideally suited to light-water reactors, the enthalpy of reaction is reasonable, and the constituent materials are inexpensive and abundant. In addition, catalysts and industrial technology are available.

Unfortunately, an analysis of a typical ammonia synthesis process shows that in order to overcome poor kinetics, pressures in the range of 200 to 1000 bars and temperatures in excess of $775 \mathrm{~K}$ are required to carry out the exothermic step. To make matters worse, this energy producing step is in reality an energy loser -- instead of producing $\sim 46 \mathrm{~kJ} / \mathrm{mole}$ typical plants consume 20 times as much energy for s.team and compressor power. One could hope for a major breakthrough in ammonia synthesis catalysts, but this has not happened in the last 20 years and does not appear highly likely. If it should happen, the first impact will not be in CHP applications but in the manufacture of fertilizers, explosives, and countless other end uses for which large quantities of ammonia are currently produced.

Methanol Dissociation:

$$
\begin{aligned}
& \mathrm{CH}_{3} \mathrm{OH} \rightarrow \mathrm{CO}+2 \mathrm{H}_{2} \quad \Delta \mathrm{H}_{298}=90.7 \mathrm{~kJ} / \mathrm{mole} \\
& \mathrm{T}^{\star}=409 \mathrm{~K}
\end{aligned}
$$

Methanol synthesis is another reaction where the exothermic step should in principle deliver net energy, but does not do so in practice. Its energetics are not as bad as the case of ammonia synthesis. This reaction does not proceed as selectively as the methanation reaction; as a result, byproduct formation may be a problem. 
For these reasons this system is not considered to be as favorable as the methane-based reactions.

Decomposition of Formamide:

$$
\begin{aligned}
& \mathrm{HCONH}_{2} \stackrel{\rightarrow}{ } \mathrm{CO}+\mathrm{NH}_{3} \quad \Delta \mathrm{H}_{298}=30.0 \mathrm{~kJ} / \mathrm{mole} \\
& \mathrm{T}^{\star}=292 \mathrm{~K}
\end{aligned}
$$

The exothermic reaction of formamide formation is practiced on a commercial scale. Decomposition has been known to take place at $\sim 475 \mathrm{~K}$ and 1 bar. At higher pressures and temperatures, especially over oxide catalysts, a possible side reaction is a decomposition to yield $\mathrm{H}_{2} \mathrm{O}$ and $\mathrm{HCN}$. While this reaction does offer a potential to store liquids, it does not appear to command a high priority.

Hydrolysis of Methyl Chloride:

$$
\begin{aligned}
& \mathrm{CH}_{3} \mathrm{Cl}(\mathrm{g})+\mathrm{H}_{2} \mathrm{O}(\mathrm{g}) \stackrel{\rightarrow}{\mathrm{C}} \mathrm{CH}_{3} \mathrm{OH}(\mathrm{g})+\mathrm{HCL}(\mathrm{g}) \quad \Delta \mathrm{H}_{298}=34.7 \mathrm{~kJ} / \mathrm{mole}(3-15) \\
& \mathrm{T}^{*}=10628 \mathrm{~K}
\end{aligned}
$$

This reaction was chosen as a likely candidate because the exothermic reaction is used commercially to produce methyl chloride (R3-12). As one might guess from the stoichiometry, the entropy change is small and the $T^{*}$ is very large. Thus, the $\Delta G^{\circ}$ in the endothermic direction remains positive even at high temperatures and the reaction is difficult to reverse. This reaction does not deserve further consideration.

Ethyl Chloride Decomposition:

$$
\begin{aligned}
\mathrm{C}_{2} \mathrm{H}_{5} \mathrm{Cl} \rightarrow \mathrm{C}_{2} \mathrm{H}_{4}+\mathrm{HCl} \quad \mathrm{H}_{298} \mathrm{O}_{29} & =71.7 \mathrm{~kJ} / \mathrm{mole} \\
\mathrm{T}^{*} & =550.0 \mathrm{~K}
\end{aligned}
$$

There is no background in the decomposition of $\mathrm{C}_{2} \mathrm{H}_{5} \mathrm{Cl}$ and, in particular, no background available in possible catalysts for this reaction. In view of the KFA experience with the ethane dehydrogenation (reaction 3-11), problems with coke, methane, and hydrogen formation may limit the utility of this reaction in CHP applications.

Other reactions are mentioned in the literature (R3-2); however, none appears to have special merit. The onus is upon any advocate of an alternative system to demonstrate its technical feasibility, industrial applicability, and economic attractiveness.

\subsection{CONCLUSIONS}

The two basic conclusions from this study are:

- There are few good candidates for the high temperature reaction systems. Methane reforming reactions are judged to be far superior to the nearest competitive alternative, the $\mathrm{SO}_{2} / \mathrm{SO}_{3}$ system. 
- There are more uncertainties in the technical feasibility of cyclic operation for the lower temperature systems than for the methane-based reactions. The most promising candidates belong to the class of hydrogenation/dehydrogenation reactions involving cyclic compounds; benzene/ cyclohexane appears at the top of the list, followed by toluene/methylcyclóhexane. Naphthalene/tetralin/decalin systems appear interesting and deserve further attention.

In the discussions on source technologies that follow in the next chapter, primary attention is given to the methane reactions for high temperature CHP (HTCHP) applications and benzene/cyclohexane reaction for low temperature CHP (LTCHP) applications.

\section{REFERENCES - CHAPTER 3}

R3-1 Hanneman, R.E., 9th Intersociety Energy Conversion Engineering Conference, San Francisco, California, 1974.

R3-2 Schmidt, E.W., and Lowe, P.A., llth Intersociety Energy Conversion Engineering Conference, Lake Tahoe, California, 1976 .

R3-3 Hilberath, E., and Teggers, H., West German Patent 1,298,233 (1968).

R3-4 Wentorf, R.H., Jr., United States Patent 3,958,625 (1977).

R3-5 Presentation at the $5 \mathrm{MW}$ th Solar Workshop, University of Houston, May 1976.

R3-6 Cubb, T.A., Solar Energy, Vol. 17, 1975, p. 129.

R3-7 Bhahta, M.L., "Chemical Storage of Thermal Energy Using the $\mathrm{SO}_{3} / \mathrm{SO}_{2} / \mathrm{O}_{2}$ System," MS Thesis, University of California, Berkeley, 1976.

R3-8 Spacil, H.S., United States Patent 3,967,676 (1976).

R3-9 Gintz, J.R., "Advanced Thermal Energy Storage (TES) Systems," Vol. 1, Final Technical Report, U.S. Energy Research and Development Administration Contract EY-76-C-03-1300, Boeing Engineering and Construction, 1976.

R3-10 Market survey performed by General Electric Energy Systems Program Division, Schenectady, New York.

R3-1l Nuernberg, H.W., and Wolff, G., United States Patent 3,558,047 $(1967)$.

R3-12 Kirk-Othmer, Encyclopedia of Chemical Technology, 2d ed., Vol. 5 . 


\section{Chapter 4}

\section{PRIMARY ENERGY SOURCES}

In the preceding chapter', potential chemical reactions for chemical heat pipe (CHP) applications were discussed. Promising reactions were grouped according to the range of temperatures over which a particular reaction could operate. In this chapter, a similar classification based on useful operating temperatures will be attempted for a variety of possible nuclear, fossil, and solar energy sources.

In addition to operating temperatures, potential problems in interfacing a high-temperature CHP (HTCHP) or a low-temperature CHP (LTCHP) with these sources, as well as systems incentives, will be presented where relevant. The chapter is organized in three main sections corresponding to the type of energy sources considered: nuclear fission, coal, and solar.

\subsection{NUCLEAR CHP APPLICATIONS}

When considering an application of nuclear reactors to $\mathrm{CHP}$ systems, the range of temperatures over which the primary thermal energy is available must be compatible with the chemical reaction. From the point-of-view of overall conversion, the most critical parameter is the peak coolant temperature at the core outlet. Coolant characteristics of most of the well-known reactor types are listed in Table 4-1. The table shows three general classifications based on the type of coolant used; within each class, the reactor types are ordered according to the maximum coolant temperature at the core outlet.

The following points should be noted:

- The state of technology development ranges from wellproven, commercial experience with water-cooled reactors to the unproven and untested technology of GCBR.

- There is considerable uncertainty about the peak temperature capabilities of PBR and LMFBR technologies.

The status of representative reactor projects in each of the reactor types is shown in Table 4-2. It should be emphasized that the comments are not intended as a judgment on a particular project but, more as background on the issue of technological readiness. Further details on individual reactor projects can be found in Reference (R4-1) (references are listed at the end of this chapter).

\subsubsection{Potential Sources for HTCHP:}

The only reactor type that is currently suitable for providing heat at a temperature high enough for methane reforming is the 
Table 4-1

BASIC REACTOR CAPABILITIES

\begin{tabular}{|c|c|c|c|c|c|c|c|}
\hline \multicolumn{2}{|l|}{ Type } & \multicolumn{4}{|c|}{ Core Coolant Temperatures } & $\begin{array}{l}\text { Pressure } \\
\text { (Bars) }\end{array}$ & $\begin{array}{l}\text { Primary. } \\
\text { Coolant } \\
\text { Activity } \\
\text { Levels }\end{array}$ \\
\hline Gas-Cooled Reactor & GCR & & Outlet & & Inlet & & \\
\hline $\begin{array}{l}\text { Pebble Bed } \\
\text { Reactor }\end{array}$ & PBR & 1225 & $(1790)$ & $525-625$ & $(48,5-665)$ & 40 & Low \\
\hline $\begin{array}{l}\text { High-Temperature } \\
\text { Gas-Cooled } \\
\text { Reactor }\end{array}$ & HTGR & 1050 & $(1430)$ & 680 & $(760)$ & 49 & Low \\
\hline $\begin{array}{l}\text { Advanced Gas- } \\
\text { Cooled Reactor }\end{array}$ & AGR & 940 & $(1230)$ & 585 & $(590)$ & 42 & Low \\
\hline $\begin{array}{l}\text { Gas-Cooled } \\
\text { Breeder Reactor } \\
\text { (Proposed Type) }\end{array}$ & GCBR & 915 & $(1185)$ & 615 & $(645)$ & 69 & Low \\
\hline $\begin{array}{l}\mathrm{CO}_{2} \text {-Cooled, } \mathrm{Mg} \\
\text { Alloy }\end{array}$ & Magnox & 645 & $(700)$ & 455 & $(355)$ & 12 & Moderate \\
\hline $\begin{array}{l}\text { Molten Salt } \\
\text { Breeder Reactor } \\
\text { (Test Facility) }\end{array}$ & MSB̈K & 980 & $(1300)$ & 840 & $(1050)$ & Nominal & Very High \\
\hline $\begin{array}{l}\text { Liquid Metal Fast } \\
\text { Breeder Reactor }\end{array}$ & LMFBR & 835 & $(1045)$ & 670 & $(750)$ & Nominal & High \\
\hline Water-Cooled Reactor & & & & & & & \\
\hline $\begin{array}{l}\text { Pathfinder } \\
\text { (Integral Superheat) }\end{array}$ & ) & 715 & $(825)$ & 455 & $(360)$ & 40 & Moderate \\
\hline $\begin{array}{l}\text { Pressurized } \\
\text { Water Reactor }\end{array}$ & PWR & 590 & $(600)$ & 560 & $(550)$ & 139 & Moderate \\
\hline $\begin{array}{l}\text { Heavy-Water } \\
\text { Moderated Reactor }\end{array}$ & HWR & 565 & $(560)$ & 520 & $(480)$ & 89 & Moderate \\
\hline $\begin{array}{l}\text { Boiling water } \\
\text { Reactor }\end{array}$ & BWR & 555 & $(540)$ & 535 & $(500)$ & 69 & Moderate \\
\hline
\end{tabular}

PBR process heat reactor being developed in West Germany. The peak temperature of $1225 \mathrm{~K}$ in AVR is considered by some to be optimistic for the larger reactor designs because of uncertainties of materials capabilities in ducts, etc. Proponents of the PBR argue quite the contrary; that the peak temperatures can be as high as $1325 \mathrm{~K}$ in future designs.

Current temperature capabilities of the prismatic core HTGR are insufficient for use in HTCHP because of the large temperature drop $(\sim 125 \mathrm{~K})$ in helium-heated reformers (see chapter 5, Section 5.1.1.2) leading to too low a peak process temperature for efficient HTCHP design. As shown later, in Chapter 6, HTCHP designs become inefficient and costly if the peak process temperature falls below $950 \mathrm{~K}$. There are plans to consider a prismatic core reactor for very high temperature process heat applications.*

*These higher temperature designs have been given the acronym VHTR to distinguish them from HTGR. Currently, the only reactor. that can be called a VHTR is the Pebble Bed AVR. 
Table 4-2

STATUS OF REACTOR SYSTEMS

\begin{tabular}{|c|c|}
\hline Type & Experience \\
\hline PBR & $15 \mathrm{MWe}_{\mathrm{e}}$ AVR (FDR) \\
\hline HTGR & $330 \mathrm{MW}_{\mathrm{e}}$ Fort st. Vrain \\
\hline & $40 \mathrm{MWe}$ Peach Bottom \\
\hline AGR & $\begin{array}{l}\text { U.K. current nuclear } \\
\text { technology }\end{array}$ \\
\hline Magnox & $1960^{\prime}$ s U.K. nuclear base \\
\hline GCBR & - \\
\hline LMFBR & $\begin{array}{l}\text { USSR - } 350 \mathrm{MW} \\
\text { France }-238 \mathrm{MWe} \text { (Phenix) } \\
\text { U.K. - } 250 \mathrm{MWe} \\
\text { U.S. - } 350 \mathrm{MWe} \text { (Clinch River) }\end{array}$ \\
\hline MSBR & $\begin{array}{l}\text { MSRE - ORNL } 1960 \\
\text { Experimental }\end{array}$ \\
\hline thfinder & $40 \mathrm{MWe}$ integral superheat \\
\hline PWR & $\begin{array}{l}\text { Wany U.S. reactors (Westing- } \\
\text { house and Combustion Engi- } \\
\text { neering major vendors) }\end{array}$ \\
\hline $\mathrm{HWR}$ & Canadian program (Canäu) \\
\hline BWR & $\begin{array}{l}\text { Many U.S. reactors - Allis } \\
\text { Chalmers and GE major } \\
\text { vendors }\end{array}$ \\
\hline
\end{tabular}

\begin{abstract}
Comments
Successful operation at $1225 \mathrm{~K}$.

Fort St. Vrain - severe cost overrun and technological difficulties; has operated at 688 capacity

Peach Bottom - Shut down at $7-1 / 2$ yrs Operation.

Material-related derating from design of entire line. Capital-j.ntensive and interestrate-dominated economics. Further construction has been discontinued.

Material-related derating from design of later.models.

Extension of HTGR technology.

French demonstration reactor (Phenix) was completed on schedule in 5 years. Current plans call for a super-Phenix (1000 $\left.\mathrm{MW}_{\mathrm{e}}\right)$ construction starting now - open by 1982 . U.K. Program 2 years off scheaule; has operated at full power; suffered sporadic shutdowns and derating. U.S. program has delays and cost overruns; construction has not begun.

ORNL has $2000 \mathrm{MW}$ e MSBR reference design. Secondary He-cooled loop of low activity for power cycle.

Closed down. Economics and fuel reliability poor.

Successful type. Secondary loop for reduced activity in steam cycle.

Successful lor-temperature reactnr using natural uranium and heavy water moderator. Excellent fuel economy.

Successful type. Primary steam circuit. is active.
\end{abstract}

In discussing the interface between a VHTR and a methane reformer it is important to mention the issue of the Intermediate Heat Exhange Loop. (IHX). Recent studies (R4-2) have indicated that the use of IHX adds significantly to the cost of process heat and lowers the peak process temperature because of an additional heat transfer $\Delta \mathrm{T}$. This latter point is of particular importance in HTCHP designs. Alternative approaches to this safety issue, using a duplex steam reformer tube (R4-3) to reduce both economic and temperature penalties, are the subject of current research.

In conclusion, VHTR appears to be the only nuclear design that is capable of supplying heat to HTCHP. The German PBR design 
is the front runner as a likely source. For the United States, in the absence of a major developmental program for a U.S.-designed VHTR for process heat, the outlook for a nuclear HTCHP is pessimistic. It will be highly crucial to watch the nuclear process heat program in order to assess the likelihood of a future VHTR source.

\subsubsection{Potential Sources of LTCHP}

The preferred temperature range for LTCHP applications consists of process temperatures from 700 to $850 \mathrm{~K}$. All current gascooled reactor models (AGR, HTGR, PBR) can supply thermal energy at higher peak temperatures than 850K, and are highly suitable for LTCHP. For the LMFBR designs, the reported peak temperature of $835 \mathrm{~K}$ corresponds to the design maximum temperature in the French Phenix reactor. Some recent studies (R4-4) have proposed a lowering of the peak sodium temperature to $750 \mathrm{~K}$. The heat transfer $\Delta \mathrm{T}$ for LMFBR/LTCHP application is not expected to be lower than for helium-heated reformers. It appears, therefore, that thermal energy from LMFBR is adequate for LTCHP application. The conversion in LTCHP may not be as high for this case as for gas-cooled reactors (GCR).

The situation for water-cooled reactors is pessimistic in view of the fact that at these low temperatures primary conversion at the endothermic end is vesy low. The possibility of decreasing the operating pressure in order to improve conversion will be evaluated in Chapter 14. Another option may be to couple LWRs to lower temperature reactions such as the decalin/tetralin dehydrogenation (see Chapter 3).

If nuclear safety issues make it mandatory to use an intermediate heat exchange loop (IHX) in LTCHP applications, the penalty due to an extra heat transfer $\Delta \mathrm{T}$ is likely to be the most severe in the case of HWR and LWR sources. If peak temperatures in LMFBR are already low because of materials considerations, this added $\Delta T$ may be very detrimental to the efficiency and economics of LMFBR-based LTCHP. The use of IHX is expected to have only a minor effect on GCR-based LTCHP, particularly for the higher temperature reactor designs.

The overall outlook for nuclear CHP applications depends critically on the resolution of the following questions:

- HTCHP - Will the U.S. have a VHTR capable of coupling to a methane reformer?

Will the German program be successful in demonstrating a nuclear helium-heated methane reformer?

- LTCHP - Can the low-temperature reactions be efficiently coupled to LWR? What is the future of HTGR and LMFBR technologies in the U.S.?

The nuclear CHP appears to be facing a dilemma. The nuclear sources can be roughly categorized in decreasing order of technological readiness as LWR, HTGR, LMFBR, and VHTR. The problem is 
that this order is totally reversed if one considers the chemical reaction feasibility where VHTR-based HTCHP appears feasible: LWR-based LTCHP poses the most severe challenge.

\subsection{COAL-BASED CHP APPLICATIONS}

\subsubsection{Background}

The major driving force for investigating coal as a potential heat source for the CHP is the following plausible thesis: If coal cannot be used economically for either small-size or intermittent process steam generation (the main reasons being the largescale coal handling and cleanup facilities required) and if heat from a large, centrally located coal facility with appropriate cleanup is sufficiently inexpensive, then it may become economically attractive to transport and distribute thermal energy via CHP to several process steam/work users who need process steam in small quantities but are denied prime fossil fuels.

As was mentioned in Chapter 1 , the effective distance for economical steam transport is restricted by heat losses. It can be argued that more users can be reached if this distance can be extended by the use of a CHP.

The key problem limiting the success of such an application is again related to the interface problems between a coal-fired furnace and the chemical reactor. A secondary problem is related to the water requirements and cleanup restrictions for the coal combustion. Efforts in this section are concentrated on answering the following questions:

1. Is the concept of a coal-fired methane reformer technically feasible in either a HTCHP or LTCHP mode.

2. What are the incentives, if any, for transporting coal combustion heat in comparison with either gasifying the coal and transporting SNG or transporting the coal to an on-site coal boiler?

\subsubsection{Technical and Economic Feasibility of Coal-Fired Source} for HTCHP Applications

The question of the feasibility of coal-fired reformers can best be answered after an assessment of the various technical problems associated with high-temperature corrosion of the reformer tubes with coal combustion and the economics of proposed systems. There is published and unpublished information to assist in this assessment.

In the published domain, there is no record of a coal-fired reformer ever being built or operated. There is, however, a patented system for constructing a reformer intended for coal firing (R4-5). This system would burn coal in a conventional cyclone combustor not attached to the reformer; instead it would 
be a source of hot gas containing some ash which is fed to the reformer at about $1480 \mathrm{~K}\left(2200^{\circ} \mathrm{F}\right.$ ) (just below the ash melting temperature). A shield is interposed between the ash and reformer tubes in locations where severe corrosion might occur. Process stream outlet temperatures are 1090 to $1150 \mathrm{~K}$, the normal reformer range, while the heating gas exits at $1200 \mathrm{~K}$.

In connection with a H-coal process study (R4-6) the Foster Wheeler Company used the system mentioned above in developing a detailed design for a reformer fired by coal char to produce $58 \times 10^{6} \mathrm{std}$. ft. ${ }^{3}$ of hydrogen per day. Differences due to firing coal char rather than coal are insignificant. Cost estimates were prepared for the char-fired unit and also for a natural-gas-fired reformer of the same capacity (Foster Wheeler is an industry leader in gas-fired reformers in this size range). The installed cost of the char-fired reformer was $180 \%$ of the gas-fired unit. coal handling, ash removal/disposal, and flue-gas cleanup costs are not included in the cost.

The $80 \%$ increase in cost is due mainly to the cyclone combustor, a 78\% increase in the number of reformer tubes due to reduced heat transfer, and an overall length increase of $100 \%$ to accommodate the tubes in a single row rather than a double row for improved heat transfer and for ease of cleaning.

If coal and ash handling facilities are added, estimates by an internal GE-CRD study show, that the cost rises to $67 \%$. Again the gas cleanup cost is not included.

Starting about 1968, GE-CRD conducted design studies for coalfired reformers and coal-fired air heaters in the same temperature range as reformers. These studies incorporated a composite tube (R4-7,-8) consisting of a thin-walled metal tube fitted inside a thick-walled silicon carbide tube. During operation, the high hot strength of silicon carbide supports the inner metal tube at temperatures and pressures well beyond the normal creep-rupture limits. Preliminary experiments showed that the outer silicon carbide tube protected the inner metal tube from coal ash corrosion adjacent to a coal fire.

Sophisticated models (R4-9) were made for gas-fired and coalfired reformers to permit comparisons of the two firing methods and to explore the benefits of operating at higher temperatures using the composite tube structure. With reference to all-metal tube reformers, the models suggest that a coal-fired unit, with maximum metal temperature controlled to $1000 \mathrm{~K}$ to limit hot corrosion and ash adhesion, would have an installed cost at least $269 \%$ of the cost of a gas-fired unit of the same capacity. If the flue gas requires treatment to meet environmental standards, the cost of treatment facilities is extra.

Because the present silicon carbide technology is very uncertain with respect to its use in the reformers, estimates are uncertain. Nevertheless, if composite tube cost is conservatively estimated at ten times the cost of a metal tube, then a coal-fired 
reformer cost is $390 \%$ of a gas-fired unit, not including flue gas treatment.

Efforts were made in 1969 and 1970 to reduce the operational and economic uncertainties of composite tubes. A small reformer fired by coal and equipped with composite tubes was designed in detail and offered to industry and government; however, despite high enthusiasm, none was ever constructed.

To summarize, the technology of coal-fired reformers, with its potential advantages, poses problems that are insurmountable in view of current materials capabilities. The major hurdle is the operation of reformer tubes above ash fusion temperatures, resulting in serve corrosion limitations. Construction based on refractory, corrosion-resistant materials is unproven and poses the challenge of achieving structural integrity and adequate life span in the 10 meter tube with end connections at an economical price. If a major breakthrough (technical and economic) should occur, the impact will perhaps be felt first not so much in coal-fired cHP applications as in the conversion to coal of all prime-fuel-fired reformer plants in fertilizer, petrochemical, and other industries.

\subsubsection{Technical and Economic Feasibility of a Coal-Fired Source for LTCHP Applications}

The outlook for application of coal combustion technology to the LTCHP is much more promising. In this case, the coal combustion could take place at temperatures below the ash fusion point and the problems associated with hot corrosion of the heat exchanger tubes would be significantly reduced. In fact, this application would require a design very similar to standard designs used for steam superheaters of coal-fired powerplants.

An additional advantage of lower temperature operation would be that the heat exchanger tubes would not approach a temperature where creep rupture might be a problem. Thus, the tube design could be based only on internal pressure consideration, and the tube walls would be significantly thinner. This would result in a lower cost for the materials used in the construction and lower heat transfer resistance in the tubes. In summary, there is no reason why existing powerplant coal combustion technology cannot be applied directly to the LTCHP concept.

\subsubsection{Incentives for Coal-Fired CHP}

Keeping in mind the pessimistic outlook for coal-fired methane reformers, it is necessary to address the question of whether it would be advantageous to have a coal-based CHP anyway, based presumably on lower temperature reactions to alleviate the corrosion problems. The most plausible argument is that based on economics; namely, that transporting combustion heat by means of a CHP may lead to a lower process steam cost for small users than gasification and SNG-fired boilers. Preliminary economic analyses (Chapters 7 and 15) show that this may indeed be the case. Other 
arguments could be made based on air pollution and water usage. Were coal combustion to require less stringent pollution controls remote from heavily populated areas than near them, it would be advantageous to transport this combustion heat to the areas of need by CHP. This argument does not appear to have much merit currently, since there is no dichotomy in environmental pollution regulations with respect to populated and sparsely populated locations.

Another issue is that of water requirement. Water consumption in coal handling and cleanup facilities is not likely to be dramatically less than that for gasification. For this reason, it may not be possible to argue for coal combustion with thermal transport in favor of gasification simply on the basis of water requirements. Thus, the major incentive for coal/CHP applications is that process steam may be generated significantly more economically, particularly for small and intermittent users.

\subsubsection{Another Coal Option: The Open Chemical Heat Pipe}

While it is somewhat beyond the scope of this study, it is important to mention that instead of considering only coal gasification to SNG or a coal-based CHP, the investigation included a novel modification of the CHP concept to combine the two. The concept, tentatively called the open CHP or the Half CHP (R4-10), is based on the idea that instead of carrying out the final methanation step at the site of a large gasification plant as is currently planned in most of the proposed SNG designs, clean intermediate-Btu syngas could be transported and distributed to several sites where industrial steam is needed. The methanation would then be carried out at these smaller sites for the purpose of generating heat (and possibly work). The product SNG would be subsequently piped to its captive market, which may consist of residential users.

While it is not possible in this study to go into the details of system efficiency improvements and economic benefits of this scheme, it is more important to point out that the open CHP may represent the best existing option for delivering coal-based process steam to small-scale industrial users, under the assumption that coal conversion to SNG is a viable future technology. The closest potential competitors to this option are: small-scale fluidized bed combustion boilers (if economics at the 100 to 200 $\mathrm{MJ} / \mathrm{s}$ level are favorable), and low-temperature CHP based on central coal-fired plants of a size exemplified by current utility facilities.

In addition to General Electric's previous in-house work, this concept of open CHP is also under investigation in a study performed under a DOE contract by the Institute of Gas Technology $(R 4-11)$. 


\subsection{SOLAR SOURCES}

The major difficulty encountered in evaluating potential hightemperature solar sources results from the uncertainty in characterizing adequately the technical capabilities of the various designs, and the even greater uncertainty in their relative economics as measured by effective costs of the collected heat. In evaluating the capabilities of various solar thermal technologies this study has placed emphasis on the following three areas:

- Maximum temperature capabilities of three general classes of solar thermal devices.

- The nature of technical difficulties associated with the solar collector/chemical reactor interface.

- Relevant system efficiency and economic considerations that must be resolved in order to identify the most advantageous conditions under which to pursue a solar-based CHP technology.

\subsubsection{Temperature Capabilities}

Bearing in mind the temperature levels associated with potential CHP reaction candidates $(600$ to $800 \mathrm{~K}$ for LTCHP and 1000 to $1200 \mathrm{~K}$ for HTCHP), some of the existing and/or proposed solar thermal systems are reviewed below with brief discussion of the potential applicability of these systems as CHP sources.

\subsubsection{Flat Plate and Low Concentration Collectors}

The basic flat plate type of solar system has received widespread interest primarily as a low-temperature thermal source for implementation in climate control and service hot water systems. These systems are nonfocusing, and accordingly can utilize both the direct beam and diffuse components of the insolation. The useful temperature ranges of these systems can be extended to higher temperatures by the use of evacuated cylindrical collectors which incorporate selective absorptive surfaces to suppress both convective and thermal radiative losses.

Low concentration factors prevent these systems from achieving the temperatures required for CHp reactors. For example, at a peak insolation level of $\mathrm{l} \mathrm{km} / \mathrm{m}^{2}$, the efficiencey of an evacuated tube collector with selective surface has been estimated by empirical testing at General Electric to be $\sim 10 \%$ at $550 \mathrm{~K}$. It is clear that even the most sophisticated low concentration systems will be inadequate as sources for CHP systems requiring temperatures greater than $550 \mathrm{~K}$.

\subsubsection{Distributed Collectors}

Most typical distributed collector systems are varieties of trough-like concentrators that focus the insolation on a linear absorber/collectors. Moderate concentration factors used in most distributed systems result in peak collection temperatures from 650 to $800 \mathrm{~K}$ (R4-12). Typical power generation applications involve pumping a heat transfer fluid throughout the distributed system, 
then collecting and using it for power generation at a central location.

The temperature range of these systems is well suited to coupling to a LTCHP. Whether the endothermic reactor is located at the central collection point or is distributed to form a linear collector/reactor depends on (1) the magnitude of thermal losses from pipelines in the former option, and (2) the complexity of designing an inexpensive combination of collector chemical reactor, and heat exchanger. It is recognized that further work on conceptual designs will be needed before a definitive assessment of the technical feasibility of these systems can be made.

In concluding this section, one important exception to the moderate concentrations used by most distributed systems should be noted. The collector system used by the Solchem concept (R4-14) employs high-concentration parabolic reflectors, distributed over the collection field. Peak operating temperatures in the range 1050 to $1250 \mathrm{~K}$ are contemplated for coupling to the $\mathrm{so}_{3}$-dissociation reaction (see Chapter 3, section 3.3). These temperatures would be adequate for HTCHP systems.

\subsubsection{High-Concentration Central Receivers}

The basic concept of a central receiver solar plant (R4-13) consists of a large field of heliostats (generally flat mirrors) which reflect the solar insolation to a central receiver located on top of a high tower. Concentration ratios are typically in the vicinity of $1000 \mathrm{x}$, and peak temperature capabilities range from 900 to $1200 \mathrm{~K}$.

There is a considerable variety in the proposed thermal collection and storage systems. For example, the incident-energy may be (1) used directly at the receiver to generate and superheat steam, (2) used to heat the working fluid in a Brayton cycle, or (3) transferred via liquid metal intermediate loops to a boiler or a storage system.

There is little doubt that even the minimum temperature capabilities of the central tower should prove adequate for LTCHP and bring in line applications. If claims of a realistic operating temperature in excess of $1200 \mathrm{~K}$ are validated, then the solar tower sources will also qualify for HTCHP applications.

The next major aspect of the technical feasibility of a solar CHP involves the interface problems and will be discussed in the following section.

\subsubsection{Interface Problems}

There are two main concerns that surface when one contemplates a solar-heated CHP. One stems from the intermittent character of the primary energy source, and the other from a lack of total independent control over the heat flux and temperature level at which energy is collected. This latter point is particularly important if the chemical reactor and collector are combined in a single unit.

$$
4-10
$$




\subsubsection{HTCHP Applications}

In past industrial experience, shutting down a methane reformer more than once a year is considered highly undesirable. Reformer tubes typically operate with a metal temperature of $21200 \mathrm{~K}$. At these temperatures, the tube life is limited primarily by longterm creep and overheating. Consequently, the tubes tend to be very thick ( $15 \mathrm{~mm}$ ). Large thermal excursions are undesirable because of thermal stresses; in addition, frequent expansion/contraction of the tubes leads to a crushing of the catalyst pellets, which in turn leads to maldistribution of thermal absorption. A further complication arises from the necessity to prevent the formation of nickel-carbonyl during heatup and cooldown.

If the reformer is located inside the solar receiver cavity, then these considerations require that the cavity not be allowed to undergo daily thermal cycling. This favors systems that incorporate $a$ heat transfer loop from the collector to a chemical reactor; however, the problems associated with ducting of high-temperature $(1200 \mathrm{~K})$ fluids may also prove... severe.

Reformer tubes operate typically with a flux of $\sim 70 \mathrm{KJ} / \mathrm{m}^{2} \mathrm{sec}$, the limitation being primarily due to the poor heat transfer coefficient inside the tube. For most of the focused solar collectors that achieve high temperatures, typical incident energy fluxes are an order of magnitude higher. This mismatch poses a significant problem in directly coupling a reformer to a solar collector. Some of the proposed cavity designs (R4-15) do have a portion of the collected energy in the cavity available at this flux level. This is another reason to favor systems with an intermediate heat transfer loop. It should be noted at this point that for high-concentration distributed systems such as Solchem, this flux mismatch problem will be particularly severe and pose problems at least as severe as in coal-fired reformer designs.

\subsubsection{LTCHP Applications}

A reduction in peak operating temperature from $1200 \mathrm{~K}$ in HTCHP to $2850 \mathrm{~K}$ for LTCHP is a major factor in alleviating potential thermal cycling problems. The fact that the tubes are no longer subject to long-term creep, and therefore do not need to be very thick, reduces the ill effects of occasional shutdowns. Consequently, interface problems for LTCHP applications are not much different from those associated with high-temperature steam generation.

For endothermic reactor designs requiring a direct heating of tubes with catalysts, thermal flux mismatch will still pose problems similar to those for HTCHP. Alternate designs using a combination of adiabatic reactors and heat exchangers may prove more suitable for solar applications and deserve further study. 
Based on these considerations, it is concluded that LTCHP applications are much more favorable than HTCHP for solar sources.

\subsubsection{Systems Considerations}

Before any attempt to identify conditions under which it would be desirable to transport solar thermal energy by CHP, two questions on economics of solar thermal energy need to be answered:

1. What is the cost of high-temperature solar thermal energy as delivered to the boiler (or chemical reactor)?

2. How does this thermal energy cost vary as a function of the size in the range of 1 to $1000 \mathrm{MJ} / \mathrm{s}$ ?

Unfortunately, definitive answers to these questions do not exist at present. The economics of solar CHP based on current estimates of collected thermal energy costs will be presented in detail in chapters 7 and 16. In the context of the present discussion it is necessary only to note that if solar-based process steam is to be competitive with SNG-fired boilers, then the primary energy cost must be in the range of $\$ 4 / G J$ to $\$ 6 / G J$. The second point to note is that if there exists a significant economic advantage in favor of large sizes (greater than $200 \mathrm{MJ} / \mathrm{s}$ ), or if for reasons of siting difficulties it is not possible to locate a "backyard" collection facility for most process steam users, then it may become advantageous to transport thermal energy by CHP from large, remote solar thermal plants. A detailed discussion of the advantages of a CHP over generation of electricity at a large central solar plant will be presented in Chapter 16.

It is tentatively concluded that:

- If solar thermal energy is cost-competitive with other options in sizes below $100 \mathrm{MJ} / \mathrm{s}$, then backyard applications for smaller industrial users will be favored; or if for reasons of siting difficulty, a backyard location of competitive small solar plants is not possible, then direct thermal transport over very short distances (less than $10 \mathrm{Km}$ ) may have advantages over solar CHP.

- If, on the other hand, there exists a marked economic advantage in favor of large solar plants, then LTCHP applications may be highly desirable.

\subsection{SUMMARY AND CONCLUSIONS}

In this chapter, an assessment has been presented of the technical feasibility and systems advantages of CHP applications based on nuclear, coal, and solar sources. On the basis of conclusions from Chapter 3, methane reforming for high-temperature (HTCHP) applications and benzene/cyclohexane for low-temperature (LTCHP) applications were the two chemical reactions considered. The following main conclusions were reached: 
- The German Pebble Bed Reactor is the only current reactor design capable of supplying heat to a HTCHP. Water-cooled reactors ( $L W R, H W R$ ) are the only designs that have a temperature too low for efficient coupling for LTCHP applications.

- Major problems for nuclear HTCHP are in the areas of VHTR source technology and its future in this country, and of nuclear/chemical reactor interfaces. Major problems for nuclear LTCHP are due, on the one hand, to an unproven chemical technology and, on the other hand to uncertainties in the future of GCR and LMFBR technologies.

- The technology of coal-fired methane reformers poses problems that are apparently unsurmountable. Coalbased LTCHP applications are technically feasible if the reaction chemistry can be demonstrated.

- The concept of open CHP represents the best option for transporting coal-based thermal energy to small industrial users. .

- High-concentration central receiver designs with intermediate heat transfer loop appear to be best suited to LTCHP applications.

- Solar-heated reformer technology poses technical problems that appear to be at least as severe as those for coalfired reformers.

\section{REFERENCES -- CHAPTER 4}

R4-1 "Power Reactors, Nuclear Engineering International," special supplement, April 1977.

R4-2 "VHTR Engineering Design Study - Intermediate Heat Exchanger Program," Final Report, ERDA Contract E(11-1)-2841, General Electric Energy Systems Technology Department November 19.76.

R4-3 "Duplex Steam Reformer Project," ERDA Contract E(11-1)-2841.

R4-4 Horst, K.M., et al., "The GE Prototype Large Breeder Reactor," British Nuclear Society Meeting, London, England, November 28, 1977.

R4-5 Stotler, H.H., United States Patent 3,551,123 (1970).

R4-6 "Project H-Coal Report on Process Development," NTIS Report $\mathrm{PB}-234579$, 1968 . 
R4-7 Kydd, P.H., et al., United States Patent 3,270,780 (1966).

R4-8 "Composite Tubes Improve Steam Reforming," Chemical and Engineering News, August 18, 1969.

R4-9 Herrick, C.S., "An Analytical Model for a Steam-Methane Reformer," Report 70-C-119, General Electric Company, Schenectady, New York, March 1970.

R4-10 This concept is the subject of a General Electric patent application currently on file in the U.S. Patent and Trademark office. It was included in a presentation of the GE-CHP program to ERDA on January 20, 1976 .

R4-1l Baker, N.R., et al., "Transmission of Energy by Open-Loop Chemical Energy Pipeline," Alternative Energy Sources: A National Symposium, Univeristy of Miami, Miami Beach, Florida, December 1977.

R4-12 Russel, J.L., et al., "Development of the Fixed Mirror Solar Concentrator," Proceedings of the 12th Intersociety Energy Conversion Engineering Conference, 1977, p. 1141.

R4-13 "Report on the Symposium and Workshop on the 5MW Solar Thermal Test Facility," ALO/3701-76/1; UC-62, University of Houston, Texas, May 1976.

R4-14 Chubb, T.A., "Energy Storage Tank," ibid., p. 241.

R4-15 Powell, J.C., "Design for Solar Thermal Central Receiver Power Plants," ibid, p. 61 . 


\section{Chapter 5 \\ TECHNICAL DESIGN OF \\ HIGH TEMPERATURE CHEMICAL HEAT PIPE (HTCHP)}

\subsection{INTRODUCTION}

This chapter is concerned mainly with the procedures for carrying out technical designs of HTCHP and for evaluating the overall energy efficiency. These procedures will be used subsequently in chapters 6 and 7 for analyzing technical and economic tradeoffs for a variety of design cases.

The three major sections deal respectively with relevant technical details of reforming and methanation technologies (section 5.2), material and enthalpy balances for a typical process example (section 5.3), and the methodology for evaluating the process efficiency (section 5.4). This last section on efficiency also contains the description of a process invention to improve the energy efficiency.

\subsection{TECHNOLOGICAL BACKGROUND}

The two chemical technologies of importance in HTCHP are methane reforming at the endothermic end, and methanation at the exothermic end. In presenting a brief summary of these technologies, particular attention will be given to those details that are especially relevant to the design of HTCHP.

\subsubsection{Methane Reforming Technology}

Methane reforming is an extremely well established technology with large-size industrial reformers in operation for over 20 years. Major use of methane reforming is in ammonia production, for fertilizer and nitric acid, and in hydrogen production in the petrochemical industries. Related to this technology is the practice of naphtha reforming. Recent efforts in developing high-temperature nuclear reactors for process heat have been concerned with a modification of existing, conventional reformer technology. Given below is a description of the conventional and nuclear reforming.

\subsubsection{Conventional Reformers}

A typical steam-methane reformer consists of a refractory furnace with rows of metal tubes packed with catalyst. The endothermic heat of reaction is provided by radiative heat transfer from the refractory walls, which are heated by natural gas flames. Tube dimensions are 75 to $100 \mathrm{~mm}$ i.d., 10 to $20 \mathrm{~mm}$ wall thickness, and $\sim 10 \mathrm{~m}$ high. The most common material of construction is a modified version of 310 stainless steel suitable for centrifugal casting, known as HK-40, with a nominal composition of 25\% Cr, $20 \% \mathrm{Ni}$, balance $\mathrm{Fe}$. Because of the hicgh metal temperatures $(\geq 1150 \mathrm{~K})$ and pressures (up to 27 bars), the tube design is often 
limited by creep. More advanced alloys providing higher strength are available although at higher cost and have often been used where severe operating conditions are encountered (R5-l).

The tubes are filled with reformer catalyst, mostly nickel on alumina pellets $\sim 5 \mathrm{~mm}$ in dimension. At these high temperatures of operation, kinetics are rapid and the reaction is limited more by heat transfer than by kinetics; there is more than adequate catalytic activity in spite of a significant loss of active surface area during operation.

The reactor feed is usually a mixture of steam and methane preheated to approximately $650 \mathrm{~K}$. In order to prevent carbon deposition and drive the reaction in forward direction, an excess of steam is used. Typical ratios of feed $\mathrm{H}_{2} \mathrm{O} / \mathrm{CH}_{4}$ range from 2 to 6. Under normal conditions the exit gases are very nearly in thermodynamic equilibrium at the local gas temperature and pressure. The tube length is determined by using an average heat flux of $(70 \mathrm{~kJ} / \mathrm{s}) / \mathrm{m} 2$, based on the inside tube surface area.

\subsubsection{Nuclear Reformers (Helium-Heated)}

Since the application of methane reforming in a CHP is in-. tended for substitution of clean fossil fuels, one may conclude that the CHP reformer will not be heated by burning either natural gas or clean, low-sulfur oil. The possibilities for coupling a methane reformer to other high-temperature sources have been examined earlier in this report (Chapter 4). Work done at KFA on helium-heated reformers, intended to establish the feasibility of coupling to a PBR, provides the only example with experimental results that is suitable for immediate analyses. The results of their work and a comparison with conventional reforming has been adequately. summarized in recent publications (R5-2).

A typical design for a helium-heated reformer tube (from R5-3) is shown in Figure 5-1. Preheated steam and methane (in ratios $2: 1$ to $3: 1$ ) enter the top of the reformer tube at a temperature of $\sim 725 \mathrm{~K}$, pass through the catalyst bed as they are heated, and react. They exit from the catalyst section at a peak temperature of $\sim 1100 \mathrm{~K}$ and leave the tube through the internal coiled pigtail after cooling down to $\sim 875 \mathrm{~K}$, thereby transferring a part of the sensible heat (from 1100 to $875 \mathrm{~K}$ ) back to the catalyst bed across the pigtail wall. On the outside of the reformer tubes, hot helium from the nuclear core enters from the bottom at a temperature of $\sim 1225 \mathrm{~K}$, flows upward around the tubes, and exits from the reformer at a temperature of 875 to $975 \mathrm{~K}$.

On the basis of the experiments conducted at KFA, the expected performance of a helium-heated reformer can be summarized as follows:

1. The chemical conversion is limited by heat transfer and not by kinetics. For all practical purposes, thermodynamic equilibrium is reached for both reactions (shift and methane reforming) at all points in the catalyst bed. 


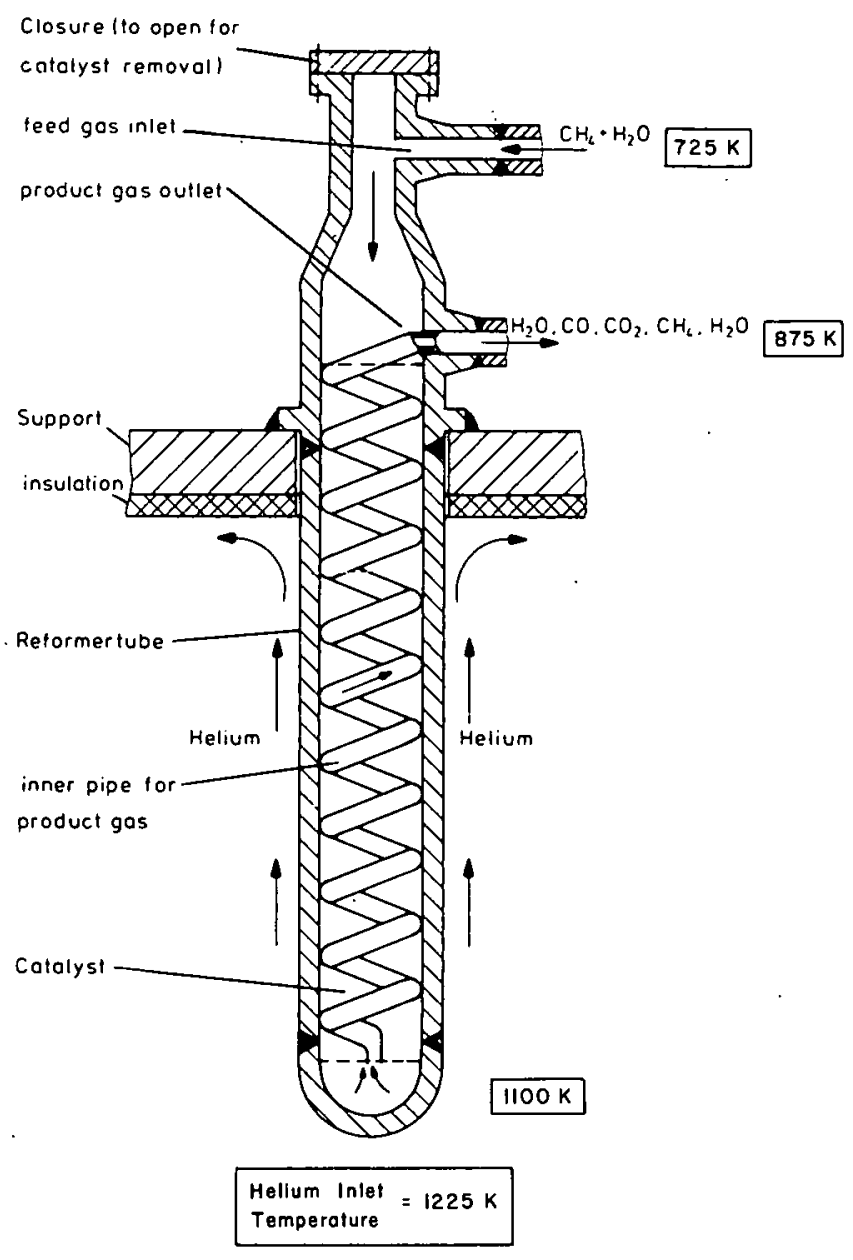

Figure 5-1. KFA Design of Helium-Heated Reformer Tube [Reproduced with permission from Nuclear Engineering and Design (R5-3)]

2. Overall heat transfer coefficients are in the neighborhood of $350 \mathrm{~W} / \mathrm{m}^{2} \mathrm{~K}$ ( $\left.260 \mathrm{Btu} / \mathrm{hr}-\mathrm{ft} \mathrm{t}^{2} \mathrm{OF}\right)$, and the average heat flux over the tube is $64 \mathrm{~kW} / \mathrm{m}^{2}$, which is nearly identical with that for conventional reformers $(\sim 20,000$ Btu/hr-ft $\left.{ }^{2}\right)$.*

3. Exit gas composition corresponds to thermodynamic equilibrium at $1100 \mathrm{~K}$ and 40 bars. No appreciable changes in composition occur in the pigtails as the exit gas stream is cooled to $875 \mathrm{~K}$.

* Correlations and measurements for these coefficients are given in References R5-4 and R5-5. In order to obtain an independent check, standard correlations were used in this study to estimate overall heat transfer coefficients. Results show a good agreement with the reported values. (An overall heat transfer coefficient of $315 \mathrm{~W} / \mathrm{m}^{2} \mathrm{~K}$ and an active length of $9.6 \mathrm{~m}$ were estimated for the operating conditions used in the KFA design.) 
4. The internal pigtail arrangement, which is necessary to permit a lower weld temperature at the exit manifold from the reformer shell, does offer a significant benefit in the form of an improved heat economy. The overall design closely resembles a countercurrent heat exchanger, with similar advantages in thermodynamic efficiency.

The implication of these conclusions in CHP design is evident in many areas. The use of chemical equilibrium to determine exit compositions greatly simplifies the overall material balance and eliminates the need to carry out a detailed reactor design. Once the reformer conversions and the associated heat transfer duties are determined, the reformer size is calculated conveniently from the average heat flux.

To a first order of approximation, the reformer heat exchange area and the cost are directly proportional to this heat transfer duty. Thus, the important design parameters that need to be fixed are the feed stoichiometry, feed flowrates, inlet temperature, catalyst bed exit temperature and pressure, and the reactor exit temperature.

\subsubsection{Methanation Technology}

A detailed review of the methanation technology is presented in Appendix 1. Rather than repeat much of the information, discussion in this section will be limited to possible implications for CHP applications.

A major difference between the use of methanation for SNG production and for CHP applications is that the heat of methanation reaction is regarded as a byproduct (and often as a problem) in SNG manufacture, while it is the only important salable product in CHP. It is for this reason that thermodynamically inefficient practices that may be acceptable as part of the SNG plant are unsuitable for CHP application. In short, it is desirable to choose a methanation plant design that consumes as little work (for compression or recycle) as possible and that generates the maximum amount of net product thermal energy at the highest possible temperature levels. For electrical and process steam end uses, the delivered thermal energy can be used at temperatures up to $800 \mathrm{~K}$.

The details presented in Appendix 1 make clear that there are considerable differences in compressor duties and temperature levels among the various processes. Numerical results in Appen-dix 1 indicate strongly that a design using high-temperature, adiabatic methanators with heat rejection between stages and without any recycle is significantly more efficient than one using low-temperature methanation or a product gas recycle, or both.

The two candidate processes are the RMProcess (1090 K peak temperature) and the Koppers/ICI process (1025 $\mathrm{K}$ peak temperature). Both of these processes use adiabatic reactors with heat exchangers (intercoolers) between stages and no product gas recycle. Depend- 
ing on the feed composition, steam may be added to the feed either for the prevention of carbon deposition or to control the maximum adiabatic temperature rise due to the strongly exothermic reaction.

As pointed out in Appendix 1, two important advantages are offered by the high temperature processes in addition to high efficiency:

- Since in such designs the reaction proceeds to equilibrium rapidly in the bed, the exit conditions are relatively insensitive to concentration and temperature disturbances in the feed. In spite of the large heat of reaction, such burned.out : reactors do not generally present difficulties in regulating the reactor operation.

- In comparison with the lower temperature operation, hightemperature methanation tends to be cleaner (no byproduct formation); any hydrocarbons higher than $\mathrm{C}_{1}$ are generally converted to $\mathrm{CH}_{4}$ and $\mathrm{CO}_{2}$. This is an important advantage for CHP application, in that cyclic operation is possible without buildup of a byproduct.

The important design parameters to be selected for the methanation end of a CHP are the maximum allowable bed temperature (specified by either catalyst or materials limitations), the reactor inlet temperatures, inlet steam ratio if needed, and the operating pressure.

\subsection{PROCESS FLOWSHEET}

\subsubsection{Selection of Major Elements}

\subsubsection{Reformer Plant}

Based on the general requirements for the reformer plant described in the previous section, the major components of the reformer plant can now be identified.

The most convenient place to start is perhaps at the exit of the reformer (see Figure 5-2, point A), where the effluents consist mostly of $\mathrm{CO}, \mathrm{CO}_{2}$, and $\mathrm{H}_{2}$ along with the excess steam and small amounts of unreacted methane. These gases are cooled as they pass through the output cooler (1) until the dewpoint is reached. The unreacted, excess steam condenses upon further cooling in the Partial Condenser (2) as the gases (and the condensate) are cooled to the exit temperature. The liquid water condensate is recycled to the feed boiler; the cooled, dried gases are then transported to the methanation plant as CO-rich gases.

The return water from the methanation plant is preheated in the Feedwater Preheater (3) and fed along with the recycled condensate from the Condenser (2) to the Boiler (4), where it is converted to steam at the appropriate pressure. In parallel, the return gases from the methanation plant, which consist mostly of 


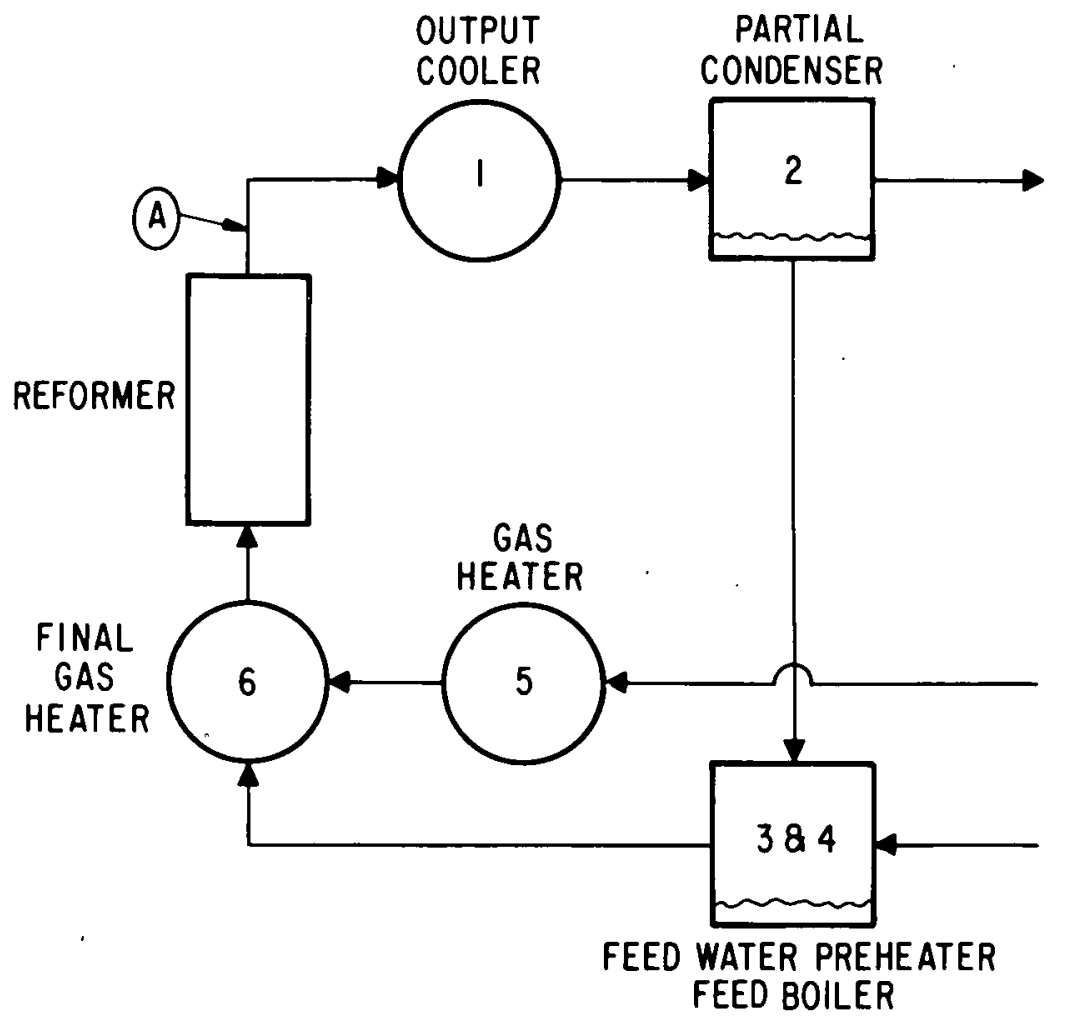

Figure 5-2. Reformer Plant Schematic

methane, are preheated in the Gas Heater (5) and then mixed with the output steam from (4) to form the reactor feed with appropriate steam/carbon ratio. The mixture then passes through a Final Gas Heater (6), where its temperature is brought up to the design value for the reactor feed. As the gases pass through the reactor bed, methane is converted to $\mathrm{CO}, \mathrm{CO}_{2}$, and $\mathrm{H}_{2}$ by the endothermic reaction of methane reforming. The required heat of reaction is provided to the reformer tubes either by convection, as in the nuclear reformer described earlier, or by radiation, as in conventional reformers.

It should be pointed out that separate equipment nomenclature has been used to indicate different steps that the streams undergo. It may be necessary to either combine consecutive steps into the same vessel (e.g., the Output cooler and the Partial Condenser may be integrated into the same piece of equipment); or they may be split into multiple units if so desired. Thus, the flowsheet in Figure 5-2 is not intended to represent actual units but rather the generic type of activity.

\subsubsection{Methanation Plant}

Since processes taking place in the methanation plant are roughly the inverse of those in the reformer plant, it is not too surprising that the plant schematic for methanation (Figure 5-3) resembles a mirror image of that for reforming. 


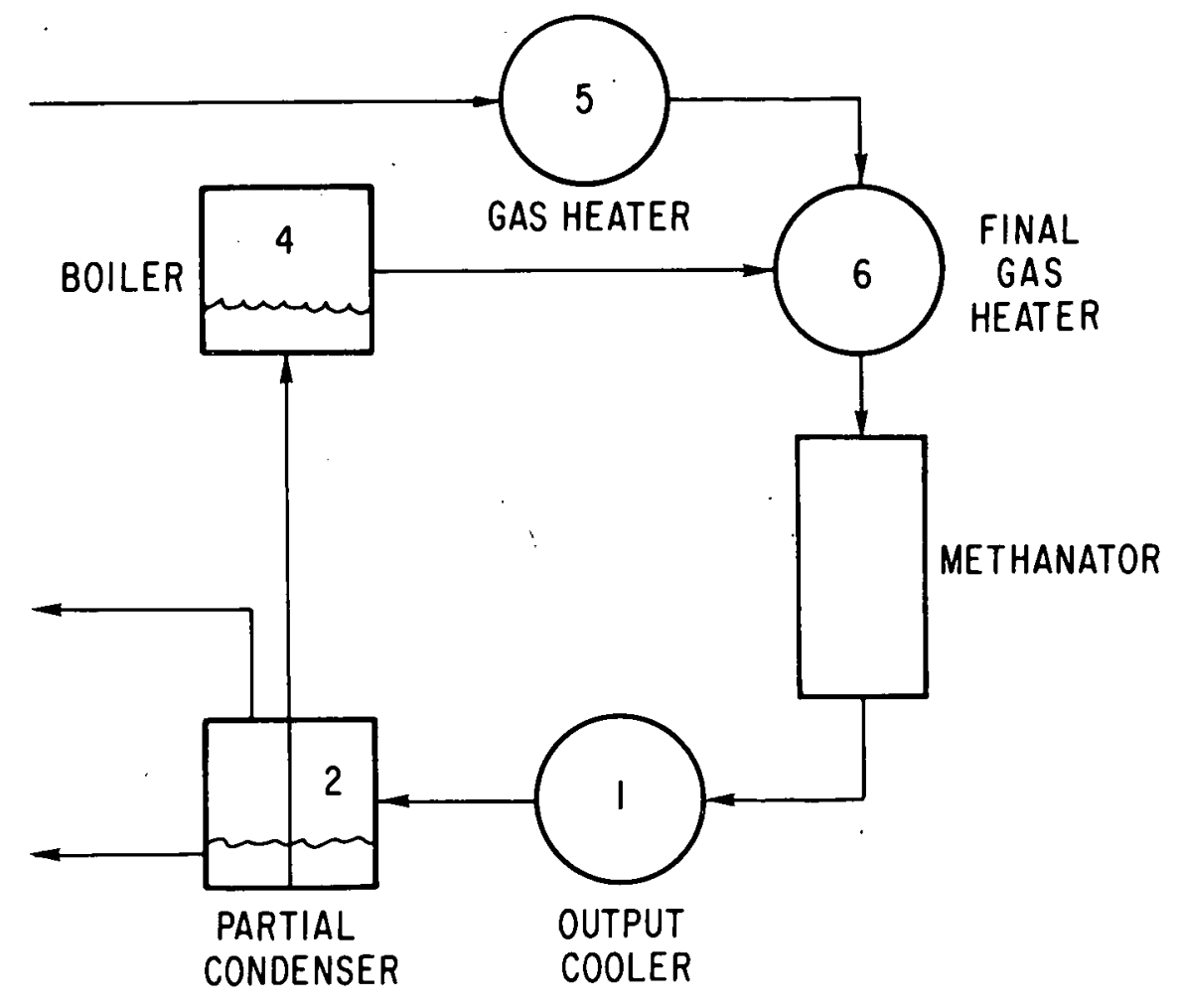

Figure 5-3. Methanation Plant Schematic

Once again, starting with the output gases from the methanation reactor, the product gases containing primarily methane and steam are cooled in the output cooler (1) until the dewpoint is reached. Further cooling results in condensation in the Partial Condenser (2) as the gases are cooled and dehumidified. The net amount of $\mathrm{H}_{2} \mathrm{O}$ formed by the methanation reactions is piped back as liquid water and the cooled, dried $\mathrm{CH}_{4}$-rich gases are transported back to the reforming plant through pipelines.

If the methanator design requires an addition of steam to feed gases, a corresponding part of the condensate water from (2) is sent to the Boiler (4), where it is converted to steam at the appropriate pressure. The Co-rich gases transported from the reformer plant go through a Gas Heater (5) and are mixed with the steam output from (4) to form a proper stoichiometric mixture. This mixture is then heated in the Final Gas Heater (6) to the design temperature for methanation reactor feed.

The methanator reactors and the intercoolers have been represented as a single block in Figure 5-3 in the interest of simplicity. In actual practice, the feed mixture flows through a repeated combination of two pieces of equipment: first an adiabatic methanator (refractory-lined cylindrical vessels filled with high-temperature methanation catalyst pellets), where chemical reaction takes place and the gases are heated; then a heat exchanger, where the gases are cooled by extracting heat to the appropriate feed temperature for the next reactor (see Appendix 1). 
The exact number of methanator-heat exchanger units in series varies from design to design from about three to six.* No detailed design of the reactors or the intercoolers has been attempted in these analyses; once a final process design is chosein it should be relatively straightforward to arrive at such a design based on standard reactor/heat exchanger design procedures and catalyst performance data.

\subsubsection{Transportation}

As mentioned in Chapter 2, the approach adopted in this study was to separate the overall design and economics into three separate sections. As a result, the transportation section includes not only the pipelines for transmission but also such components as compressors, expanders (if any), liquid pumps, and any storage components (e.g., caverns or liquid storage tanks). The input conditions for the transport are determined by the exit conditions from partial condensers in the methanation and reforming plants (units described earlier in Figures 5-2 and 5-3). The delivery conditions are also assumed to be specified by the receiving plant designs.

\subsubsection{Thermodynamic Data Base}

The calculations that needed to be performed in the analyses can be broken down into three general groups:

1. Thermodynamic equilibria for the two simultaneous reactions (reforming and shift) to determine conversions, product compositions, and overall material balances.

2. Enthalpy balances to determine the quantity and the nature of energy flow requirements for each of the system components.

3. Flow calculations to determine frictional pressure drops, heat transfer coefficients, and compression work requirements.

The necessary data base for these calculations consists of the following thermodynamic and physical properties for gasest $\left(\mathrm{CH}_{4}, \mathrm{H}_{2} \mathrm{O}, \mathrm{CO}, \mathrm{CO}_{2}\right.$, and $\left.\mathrm{H}_{2}\right)$ and liquid over the relevant range of temperatures and pressures:

\footnotetext{
*The arrangement for CHP may differ from other methanation designs (e.g., the RMProcess [R5-6]) in that all the reactors in CHP can be connected strictly in series fashion without incurring excessive temperatures in the first reactor.

tThese are derived from the standard heats of formation and specific heats.
} 
- Standard enthalpy of formation for each of the four compounds -- $\mathrm{CH}_{4}, \mathrm{H}_{2} \mathrm{O}, \mathrm{CO}, \mathrm{CO}_{2}$ at the reference temperature $\left(\mathrm{T}_{\mathrm{R}}=298.16 \mathrm{~K}\right)$

- Standard entropies of the five components at $T_{R}$

- Specific heats over the temperature range of interest for the five components in the gaseous states

- Enthalpies of the components over the temperature range of interest as well as the liquid enthalpies for water

- Equilibrium vapor pressure of $\mathrm{H}_{2} \mathrm{O}(\ell)$ and latent heat of evaporation as a function of temperature

- Physical properties such as viscosity, thermal conductivity, binary diffusivities, specific heat ratios $\left(\gamma=C_{\mathrm{p}} / \mathrm{C}_{\mathrm{v}}\right)$, and densities.

Before presenting the actual numerical values used in the analyses, it is important to note the overall objective of the quantitative analyses of the system performance. The aim here is not to perform a detailed design with given specifications but rather to arrive at first order estimates of the system performance. It is therefore not critical to work towards the best recommended data base for design purposes; a higher premium has been placed on versatility of use and compactness of the representation than on the absolute accuracy of the derived results. The most prominent example of this tradeoff is perhaps in the area of departures from ideality where ideal behavior has been assumed after having checked the magnitudes of actual nonidealities in most instances.

Table 5-1 shows the basic constants used (R5-7) to generate most of the thermodynamic data. The calculated values of specific heats were compared with reported data (R5-8, R5-9) to determine the extent of the error introduced by this simplification; the worst fit was in the case of $\mathrm{H}_{2} \mathrm{O}(\mathrm{g})$ near condensation temperatures, as would be expected. on the basis of the reduced temperatures and pressures. In any case, the resultant errors in enthalpies were not significant because of the integration of the specific heat data.

Enthalpy of liquid water was calculated according to the standard heat of evaporation $(44032 \mathrm{~J} / \mathrm{g})$ and a constant specific heat of $75.35 \mathrm{~J} / \mathrm{mole} \mathrm{K}$.

Empirical relations given by Jordan (R5-10) were used to relate the saturation partial pressures to temperatures (and vice versa). It should be pointed out that the assumption of ideal behavior introduces some errors in the distribution of thermal duties between the partial condenser and the output cooler; 
Table 5-1

\begin{tabular}{|c|c|c|c|c|c|}
\hline & $\begin{array}{c}\Delta H^{\circ} \mathrm{f}(298) \\
(\mathrm{J} / \mathrm{gmole})\end{array}$ & $\begin{array}{c}\text { THERMODYN } \\
\mathrm{S}^{\circ} 298 \\
\text { (J/gmole K) }\end{array}$ & $\begin{array}{l}\text { IIC DATA B } \\
\frac{\mathrm{C}_{\mathrm{p}}=\mathrm{a}}{\mathrm{a}} \\
(\mathrm{J} / \mathrm{mole} \mathrm{k})\end{array}$ & $\frac{\mathrm{E}}{\mathrm{P}+\mathrm{CT}^{2}}$ & $\left(\mathrm{~J} / \mathrm{mole} \mathrm{K}^{3}\right)$ \\
\hline $\mathrm{CH}_{4}$ & -74850 & 186 & 14.14 & 74.91 & -17.52 \\
\hline $\mathrm{H}_{2} \mathrm{O} \quad(\mathrm{g})$ & -241830 & 189 & 28.83 & 13.74 & -1.44 \\
\hline $\mathrm{CO}$ & -110520 & 198 & 26.15 & 8.75 & -1.92 \\
\hline $\mathrm{CO}_{2}$ & -393510 & 214 & 28.66 & 35.70 & -10.36 \\
\hline $\mathrm{H}_{2}$ & 0 & 131 & 28.79 & 0.28 & 1.17 \\
\hline
\end{tabular}

the total thermal duty is relatively independent of the departures near saturation conditions. Another point to be noted is that for the whole range of reaction conditions the reduced temperatures are high enough to justify the assumptions of ideal behavior for the equilibrium calculations.

\subsubsection{Material Balances}

Here presented is the procedure for making materials and flow balances for the closed loop under the assumptions of equilibrium conversions. The various steps in the calculations can be illustrated by a sample case with the following parameters:

Reformer Catalyst Bed

$\begin{array}{ll}\text { Exit temperature } & 1.100 \mathrm{~K} \\ \text { Exit pressure } & 40 \mathrm{~atm}(40.5 \mathrm{bars}) \\ \text { Overall stoichiometry } & 1: 3 \mathrm{CH}_{4} / \mathrm{H}_{2} \mathrm{O}\end{array}$

Methanation Catalyst Bed

$\begin{array}{ll}\text { Exit temperature } & 700 \mathrm{~K} \\ \text { Exit pressure } & 40 \mathrm{~atm} \\ \text { Overall Stoichiometry } & 1: 1.5 \mathrm{CH}_{4} / \mathrm{H}_{2} \mathrm{O}\end{array}$

Based on the catalytic bed exit conditions, the reformer reactor effluent compositions are obtained by solving for equilibrium conversions in the reforming and the shift reactions. The results are shown in Figure 5-4 in the form of molar composition of the reactor effluents and the pipeline gas mixture subsequent to the removal of unreacted $\mathrm{H}_{2} \mathrm{O}$.

*The stoichiometry selected could have been the same as that for the reformer $(1: 3)$. However, in view of the conclusion (Chapter 6) that it is advantageous to approach the minimum $\mathrm{H}_{2} \mathrm{O} / \mathrm{CH}_{4}$ ratio that can be used without endangering catalyst performance due to coke deposition, much of the work was done with reduced $\mathrm{H}_{2} \mathrm{O} / \mathrm{CH}_{4}$ ratios. 


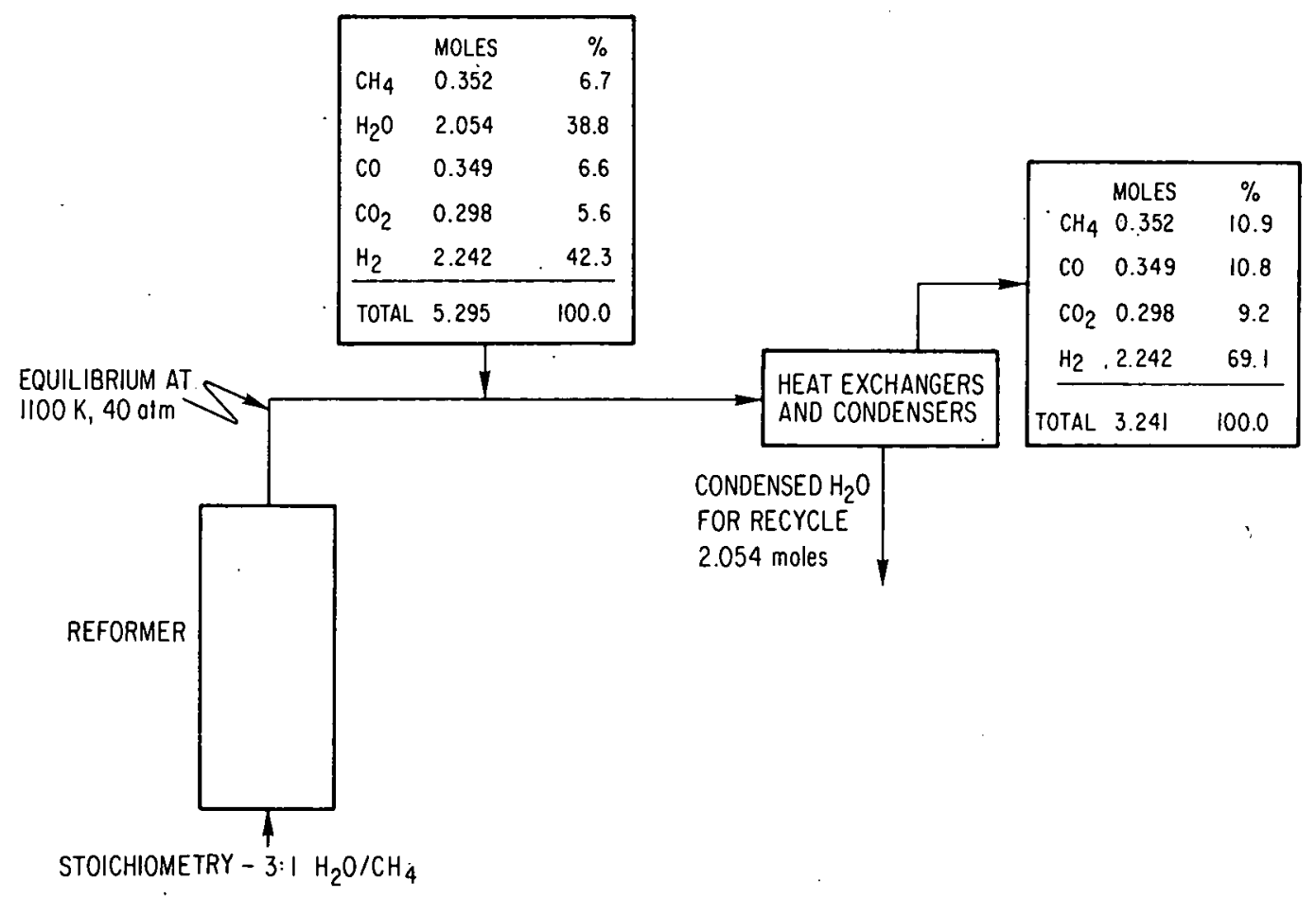

Figure 5-4. Reformer Exit Equilibrium

At the methanation end, the reactor inlet consisting of preheated pipeline gas mixture and recycle $\mathrm{H}_{2} \mathrm{O}$ (in proper proportions to achieve an overall stoichiometry equivalent to $1: 1.5 \mathrm{CH}_{4} / \mathrm{H}_{2} \mathrm{O}$ or $\mathrm{C}: \mathrm{H}: \mathrm{O}=1: 7: 1.5$ ) undergoes the reverse reactions. The equilibrium conversion once again can be calculated at $700 \mathrm{~K}$ and $40 \mathrm{~atm}$. The results are shown in Figure 5-5.

Based on these equilibrium conversion calculations, the overall closed loop material flows and conversions are determined as shown in Figure 5-6.

\subsubsection{Enthalpy Balance}

Once all the flows have been identified, it is a relatively straightforward procedure to obtain heat duties for the various components shown earlier in Figures 5-2 and 5-3.

Before presenting quantitative results, however, one important point to be discussed is the basis for standardization of flows. The enthalpy difference at the pipeline conditions was chosen as the basis for normalizing different CHP designs. As a result, flow rates (in moles $/ \mathrm{hr}$ ) are based on a net enthalpy flux corresponding to $1000 \mathrm{MJ} / \mathrm{s}$ from the reformer to the methanators. 


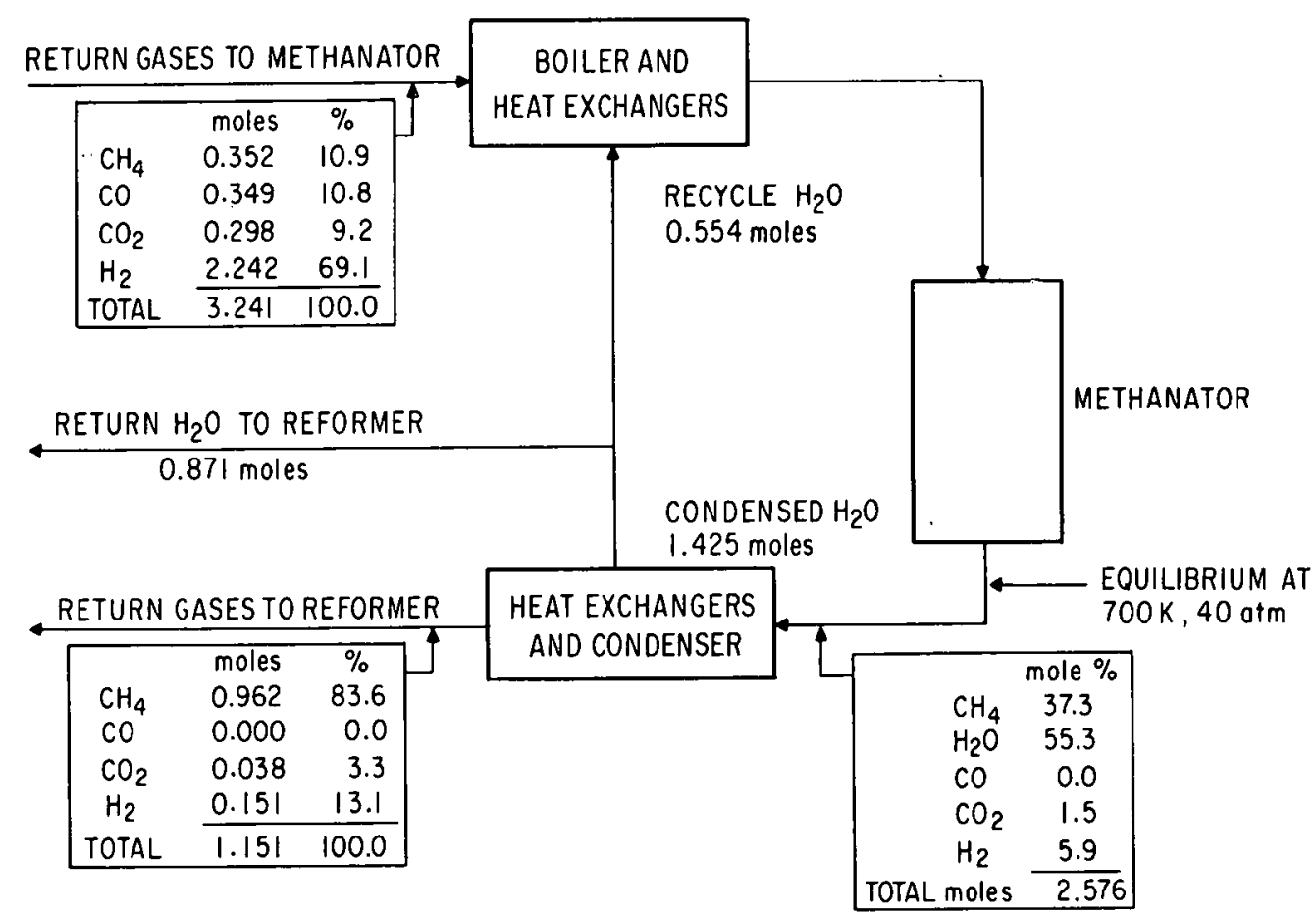

Figure 5-5. Methanation Exit Equilibrium

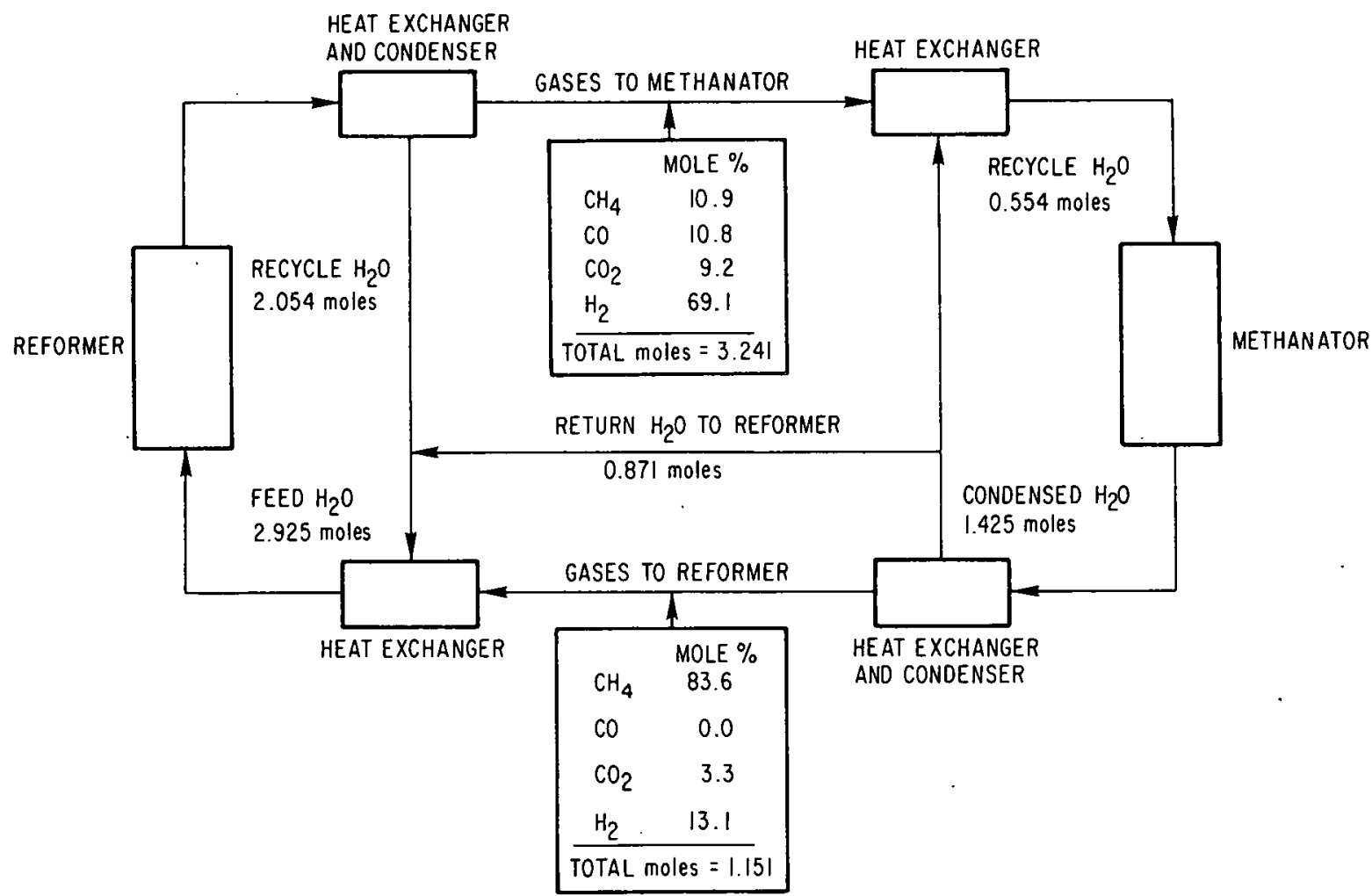

Figure 5-6. Closed-Loop Material Balance 
For the case selected, the enthalpy difference at the nominal pipeline condition of $300 \mathrm{~K}$ corresponds to $153.41 \mathrm{KJ} / \mathrm{mole}{ }^{\circ} \mathrm{C}$ (corresponding to the moles reported in Figure 5-6). Based on this enthalpy difference, molar flowrates that yield a net transmission of $1000 \mathrm{MJ} / \mathrm{s}$ are shown in Figure 5-7; also shown are the various design choices for the temperatures. It may be noted that the actual exit temperature from the reformer $\left(600^{\circ} \mathrm{C}\right)$ is much lower than the exit temperature from the peak reactor bed because of the internal pigtail arrangement. The value corresponds to the typical design for nuclear reformer used by KFA. The exit gas and recycle water temperatures were assumed to be equal to the pipeline conditions $(300 \mathrm{~K})$ in view of the previous assumption of $\Delta T=0$ in the heat exchangers. The dewpoint temperatures were determined from the partial pressure of $\mathrm{H}_{2} \mathrm{O}$ and are also shown in the figure. Based on these design values, the equipment duties can be calculated and are shown in Table 5-3. The computer programs for these calculations are shown in Appendix 6 .

\subsection{PROCESS EFFICIENCY}

\subsubsection{Approach to Efficiency Calculations}

Since the use of chemical conversions in CHP is aimed not at the production of any particular chemical product but, rather, at the transport and storage of thermal energy, the performance of any CHP system should be judged by how well it accomplishes the goals of efficient conversion and delivery of energy. Unfortunately there exists no unique definition of efficiency, because energy inputs and outputs from a given system must be measured not just quantitatively but also by their "quality" (exergy).

A common misconception is that by resorting to a second-law efficiency one can obviate this difficulty. This is not so, because it is inherently a problem related to the measure of inefficiency. In other words, while there exists a unique definition of a system that is thermodynamically $100 \%$ efficient, practical systems can never achieve this ideal. Any attempt to define the extent of inefficiency in a real system is faced with the problem of defining a measure of thermodynamic inefficiency, which is not unique. It is often more convenient and much less ambiguous to simply define entropy production rates than to list process efficiencies. These difficulties notwithstanding, it is very useful to define an overall efficiency for the purpose of comparing various competitive systems. In this report, overall energy efficiency is defined as the ratio of thermal energy output to the "net equivalent" thermal energy input, the latter being a sum of actual input of thermal energy and the thermal equivalent of work inputs. Prior to an application of this type of efficiency measure, two questions must be resolved.

1. How does one calculate the total work input for a CHP design?

2. What conversion factors does one use to obtain the thermal equivalent of this total work input? 


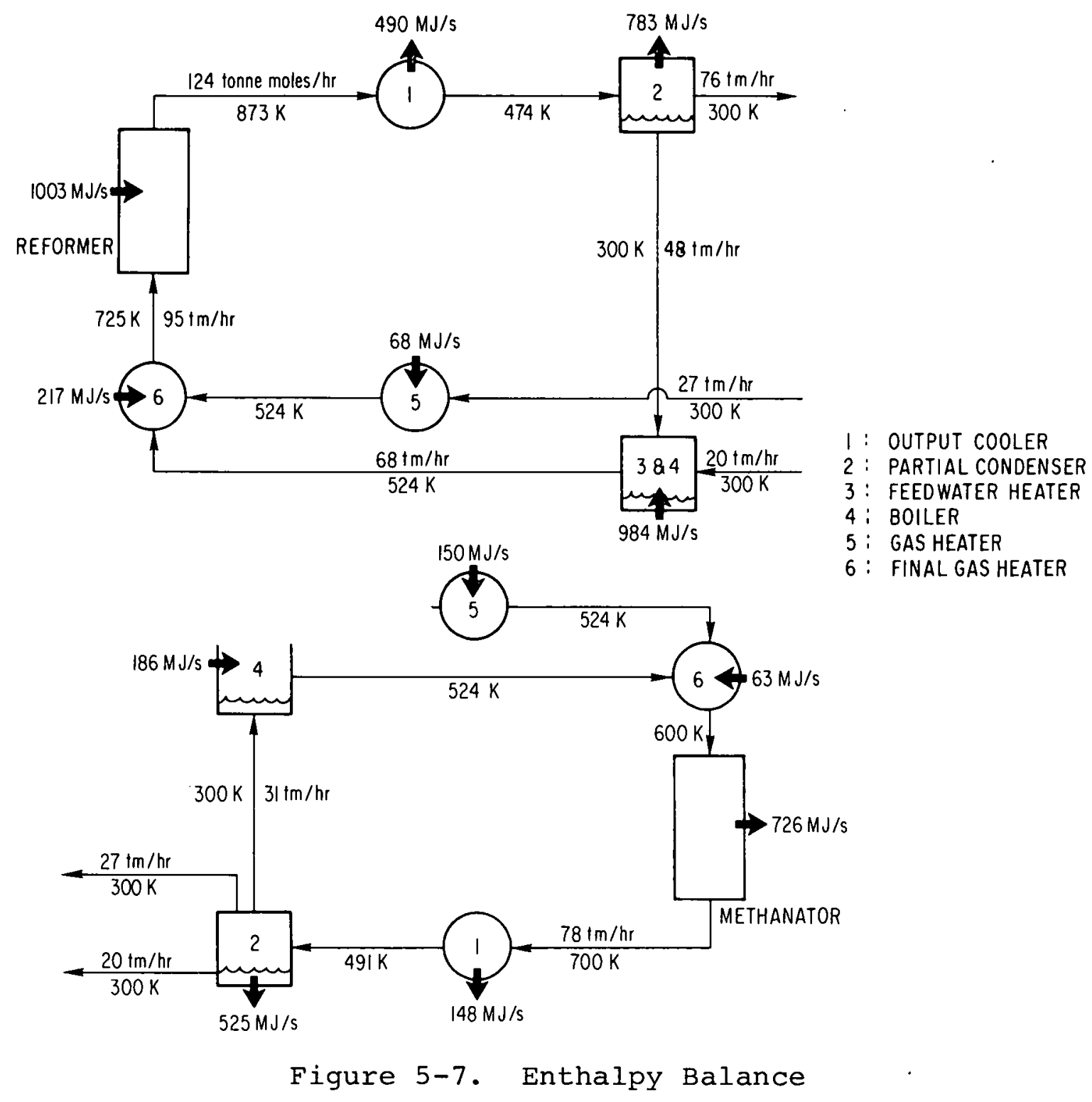

Table 5-3

EQUIPMENT DUTIES FOR EXAMPLE CASE DESIGN

\begin{tabular}{|c|c|c|c|c|}
\hline \multirow[b]{2}{*}{ Equipment } & \multicolumn{2}{|c|}{ Reformer Plant } & \multicolumn{2}{|c|}{ Methanator } \\
\hline & $\begin{array}{l}\text { I' Range } \\
(\mathrm{K})\end{array}$ & $\begin{array}{c}\text { Duty } \\
(\mathrm{MW} t h) \\
\end{array}$ & $\begin{array}{c}T \text { Range } \\
\quad(\mathrm{K}) \\
\end{array}$ & $\begin{array}{c}\text { Duty } \\
\text { (MWth })\end{array}$ \\
\hline Gas Heater (5) & $300-524$ & 67.8 & $300-524$ & 150.2 \\
\hline Feed Heater $(3 \& 4)$ & $300-524$ & 984.4 & $300-524$ & 186.3 \\
\hline Final Gas Heater $(6)$ & $524-725$ & 217.3 & $524-600$ & 63.1 \\
\hline Reactor Duty & $725-1100$ & 1002.8 & $775-600$ & 726.4 \\
\hline Output Cooler (1) & $873-474$ & 489.7 & $700-491$ & 147.9 \\
\hline Partial Condenser (2) & $474-300$ & 782.6 & $491-300$ & 525.3 \\
\hline Total Cooling Duty & & 1272.3 & & 673.2 \\
\hline Total Heating Duty & & 1269.4 & & 399.7 \\
\hline
\end{tabular}


The difficulty in answering the first question is apparent when it is realized that in addition to compression power for transmission and equipment pressure drops, CHP designs are often coupled closely to a powerplant. The net effect of these powerplant exchanges have been analyzed in a thermodynamic framework, using exergy analyses (details of this approach and an example application are given in Appendix 2, A2.3) Total work input is computed as a sum of real work inputs (i.e., compressor work) and "hidden" work penalty based on the net exergy flow to the powerplant combined with an assumed exergy efficiency.

The second question regarding a conversion factor for thermal equivalent can be resolved using typical powerplant energy efficiencies. As one would expect, powerplant efficiencies depend on the temperature level at which thermal energy is available and range from $33 \%$ at $550 \mathrm{~K}$ to $40 \%$ above $800 \mathrm{~K}$. For HTCHP applications, the primary thermal energy is always at a high temperature level $(>800 \mathrm{~K})$. Consequently, a conversion factor of 2.5 (corresponding to an efficiency of $40 \%$ ) has been used uniformly for all design cases. For low-temperature applications (see Chapters 13 and 14) an interpolation based on exergy ratios will be used in the range of temperatures from 550 to $800 \mathrm{~K}$.

The resultant efficiency can be represented by the equation

$$
\text { System efficiency }(\psi)=\frac{\text { Thermal output }}{\text { Thermal input }+\frac{\left(w_{d}+w_{i}\right)}{\eta}}
$$

where $w_{d}$ and $w_{i}$ represent the total direct and indirect work inputs respectively, and $\eta$ is the powerplant efficiency ranging from 0.33 at $550 \mathrm{~K}$ to 0.4 at $\geq 800 \mathrm{~K}$.

\subsubsection{Thermal Histograms and Exergy Analyses}

Referring to the example equipment duties in Table 5-3, it would be highiy desirable to provide as large a fraction of the heating duty as possible by the cooling duty. If, in addition to the reactor thermal input, large quantities of makeup energy are required to meet the heating demands, then the process will exhibit a poor overall performance. At first glance, a complete internal exchange appears appropriate; the heating and cooling duties are comparable in magnitude and involve similar temperature ranges. To ascertain the extent to which this heat economy can be achieved in practice, it is necessary to take a detailed look at the temperature levels of the available and the required thermal duties. This can be done most conveniently with the help of a thermal histogram in which the thermal duties are plotted to correspond to each small increment in temperature. To the best of the writers' knowledge this technique has not been used before ir. analyzing thermal systems; it has been found revealing and very helpful in making clear the thermal tradeoffs available in internal plant heat exchanger. We recommend this technique for use in other analyses, for example in thermochemical processes for hydrogen generation. 


\subsubsection{Thermal Histograms}

Before discussing the heat exchange economy of the reformer plant, it is appropriate to discuss briefly some aspects of heat transfer with reference to a histogram. A transfer of heat between two fluids at a constant temperature would be represented as shown in Figure 5-8, where $Q$ is the amount of heat transferred. The laws of thermodynamics dictate that an exchange is possible only when the source of heat (the cooling duty) is to the right of the demand (the heating duty) the horizontal separation between them being equal to the heat transfer temperature drop. A countercurrent heat exchange between two fluids with different heat capacities is shown in Figure 5-9, where the effect of a mismatch in heat capacities on the inlet and outlet temperature approaches can be observed. It should be noted that in general a transfer of heat between equal areas with height/width mismatch is less efficient than when they are matched.

In an effort to simplify the thermal analyses that follow the heat transfer temperature drop has often been neglected. For a matched transfer with a constant $\Delta T$, the same method can be applied if the source of heat (cooling duty) is moved to the left by an amount equal to the transfer $\Delta \mathrm{T}$ on the temperature axis.

\subsubsection{Reformer Plant}

For the example case, the reformer plant cooling duties shown in Table 5-3 are plotted as a histogram in Figure 5-10a. The output cooler duty of $490 \mathrm{MJ} / \mathrm{s}$ appears as a bar over the temperature range of 873 to $474 \mathrm{~K}$ (the latter being the dewpoint temperature with $\mathrm{H}_{2} \mathrm{O}$ partial pressure of $15.5 \mathrm{~atm}$ ) with an essentially constant height indicating a nearly constant specific heat. The partial condenser duty, on the other hand, is highly temperaturedependent because of a varying rate of $\mathrm{H}_{2} \mathrm{O}$ condensation upon cooling; the variation of cooling duty follows roughly the relationship between $\mathrm{H}_{2} \mathrm{O}$ vapor pressure and the temperature. In an analogous fashion, the heating duties from Table 5-3 are shown in Figure 5-10b. The final feed heater and the gas heater duties resemble the output cooler duty, showing a nearly constant specific heat, whereas the feed boiler duty shows an enormous spike of $686 \mathrm{MJ} / \mathrm{s}$ corresponding to the latent heat requirements at the boiling point of $524 \mathrm{~K}$.

It is now possible to investigate the extent to which the heating and cooling duties can be coupled by looking at the superposition of these two curves as shown in Figure 5-11. Neglecting the heat transfer temperature drop for the time being, it can be seen that the entire temperature range of 300 to $875 \mathrm{~K}$ can be broken down into four bands of somewhat different energy balances:

1. In the temperature range of 873 to $725 \mathrm{~K}$ there is heat available at a level of approximately $25 \mathrm{MJ} / \mathrm{s}$ for every $20 \mathrm{~K}$ increment. This excess energy can either be used to make up some of the deficit at lower temperatures 

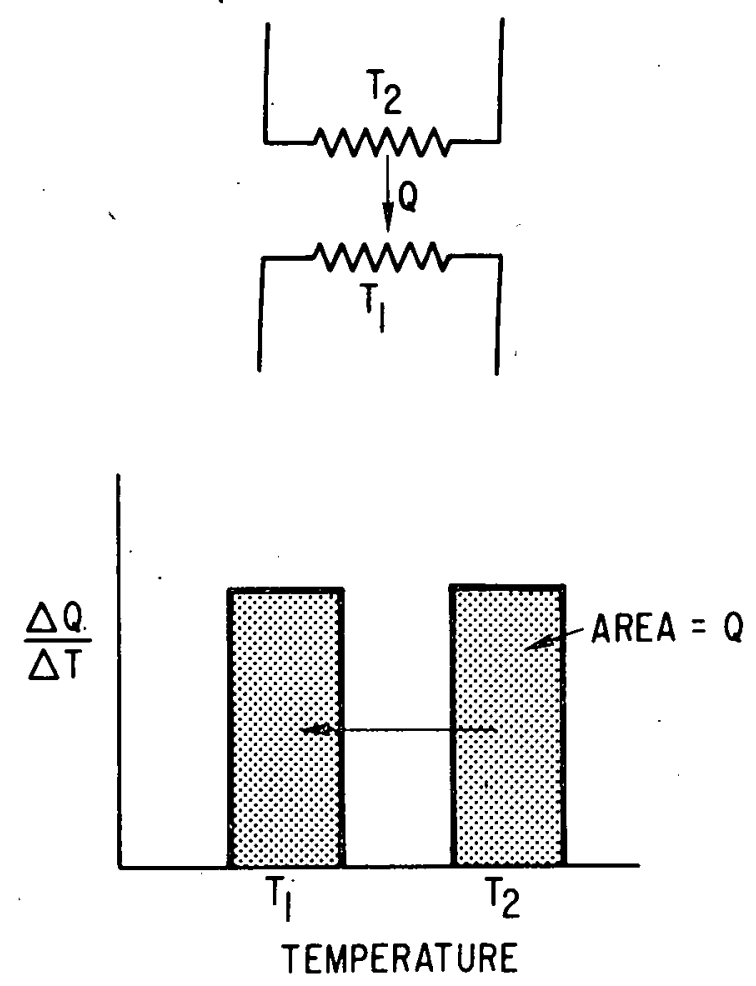

Figure 5.8. Example of a Histogram for Transfer of Heat Between Two Constant Temperature Fluids
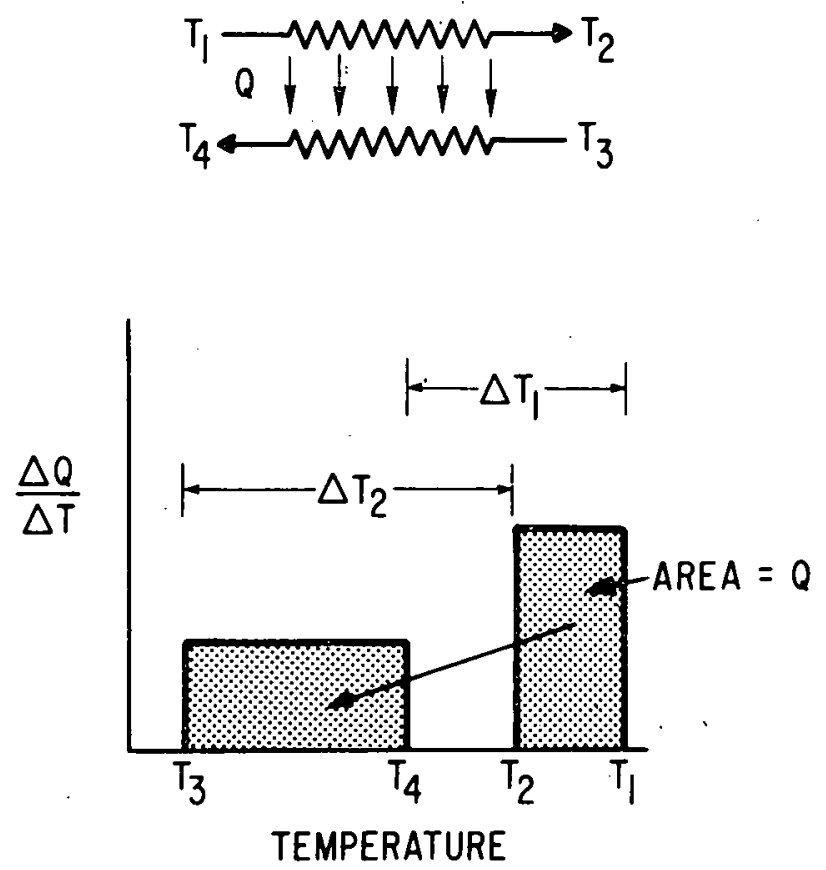

Figure 5.9. Example of a Histogram for the Transfer of Heat Between Fluids with .Different Heat Capacities 


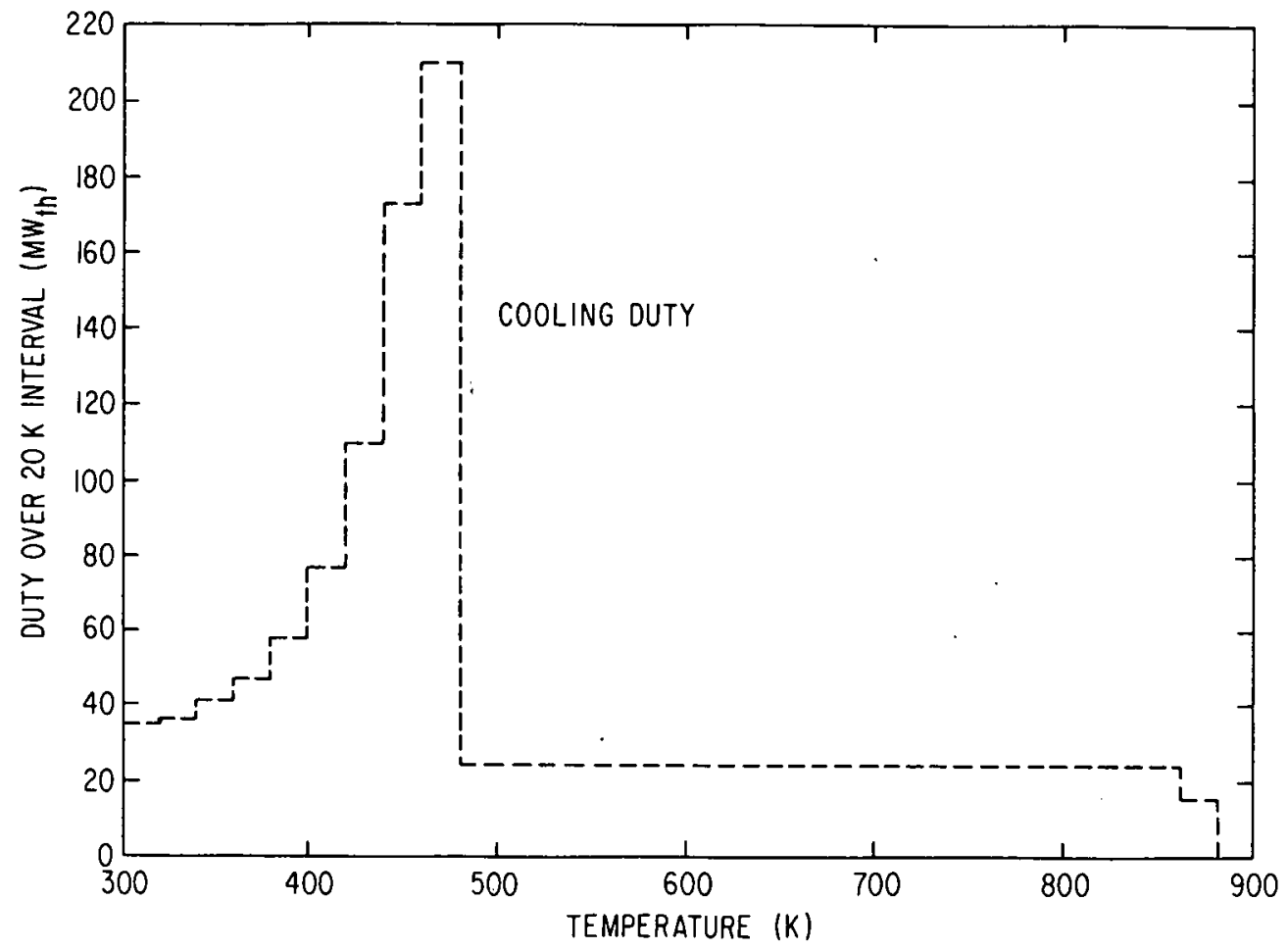

Figure 5-10a. Temperature Histogram - Reformer Cooling Duty

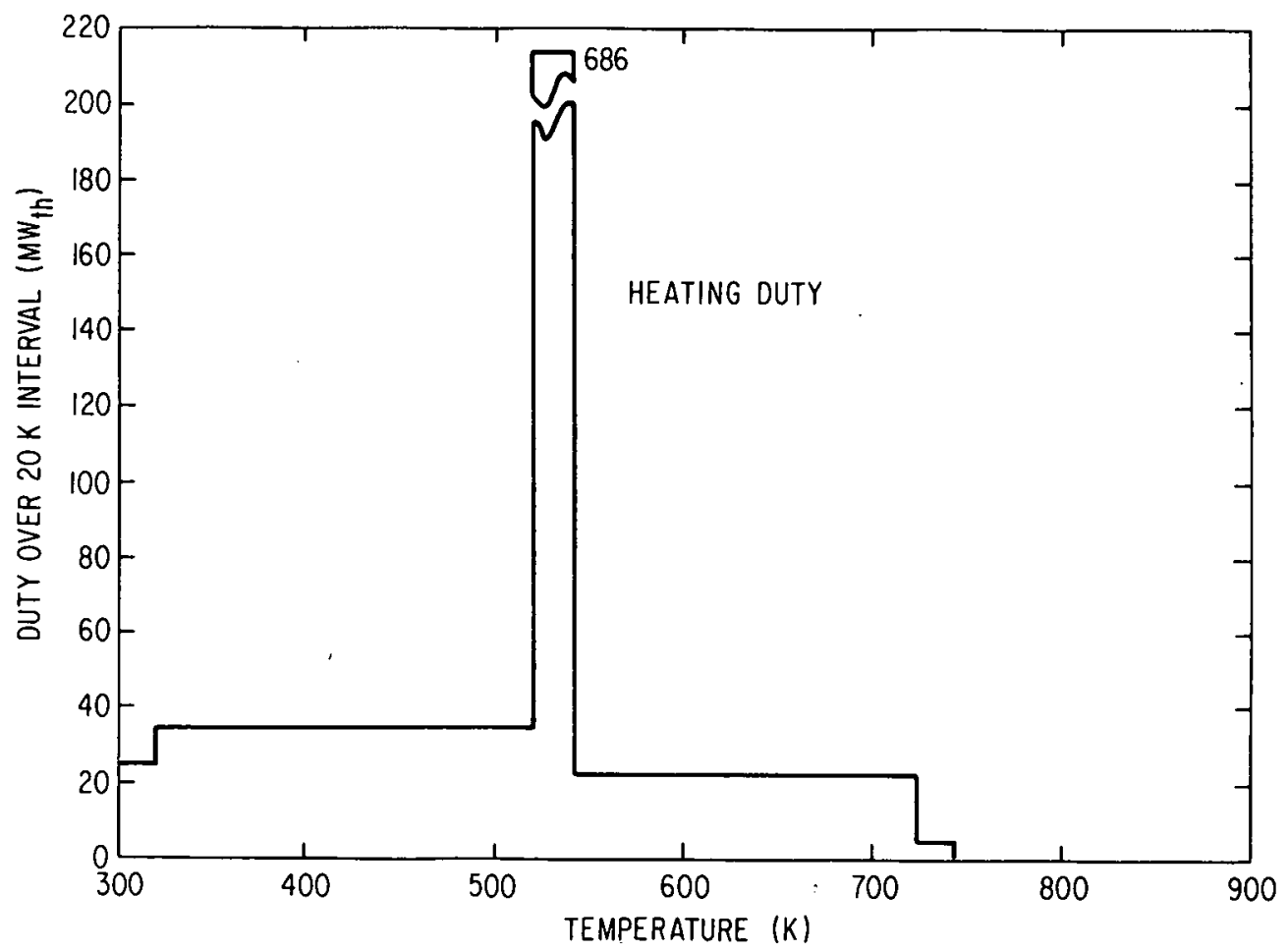

Figure 5-10b. Temperature Histogram - Reformer Heating Duty 


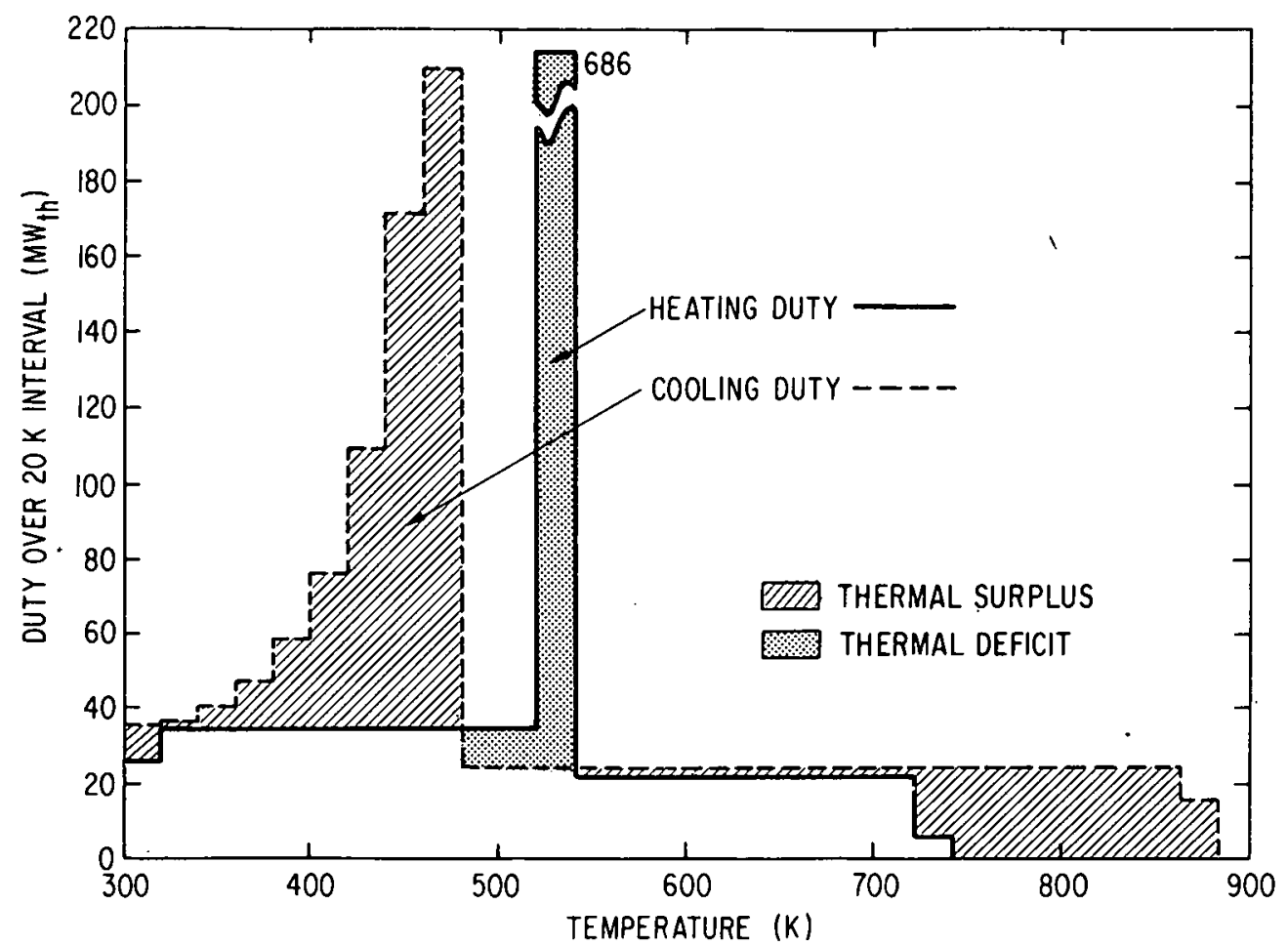

Figure 5-11. Superposition of Reformer Histograms

or be exported to the powerplant, where it would be well suited for steam reheat application.

2. In the range of 725 to $525 \mathrm{~K}$ the heating and cooling duties are very nearly equal, and good heat economy can be achieved easily by the use of countercurrent heat exchange.

3. The enormous deficit at $524 \mathrm{~K}$ is due to the heat of evaporation in the boiler, as was mentioned earlier. This spike of $690 \mathrm{MJ} / \mathrm{s}$ presents a severe problem in the heat economy, since there is no way by which it can be supplied other than by extensive extraction of steam from the process plant. The excess of $190 \mathrm{MJ} / \mathrm{s}$ between 873 and $725 \mathrm{~K}$ is insufficient to take care of this deficit, and the additional condensation heat mentioned in Item 4 is at temperatures below $525 \mathrm{~K}$. As will become increasingly clear after further analyses, the manner in which this evaporation heat is provided is a pivotal factor in determining the overall thermal efficiency.

4. At temperatures below $475 \mathrm{~K}$ a large excess of energy $(\sim 460 \mathrm{MJ} / \mathrm{s})$ is available from the partial condenser. Unfortunately there appears to be no use in the CHP design for this energy. The best use of this excess is in boiler feedwater preheating in the powerplant. It is important to note that the magnitude of this preheating heat flux is quite large and may present a problem if the powerplant is not sufficiently large to be able to absorb it. 
The conclusion one can reach from the histogram is that it is extremely difficult to envision a stand-alone reformer plant because of the gross mismatch in the surplus and deficits in the thermal balance. This presents less of a problem for some sources (VHTR) where there would be a large powerplant on site than if the total thermal output from the primary source were to be transported or stored (cf. Appendix A2,3.2).

The next step after characterizing the deficits and surplus of energy is to evaluate the net impact of these exchanges on the powerplant. Details of the exergy analysis and its application are presented in Appendix 2 and will not be given here. The results of such an analysis for this example case (with heat transfer $\Delta T=0)$ shows that over the full range of temperatures from 300 to $873 \mathrm{~K}$, the process shows a net energy deficit of $5.7 \mathrm{MJ} / \mathrm{s}$ and a net exergy deficit of $15.7 \mathrm{MW}$. A major fraction of exergy loss from the reformer process is due to the fact that the boiler heat is needed at a much higher temperature than that at which condensation heat is available. Consequently, the process is forced to accept a large amount of extraction steam with a high exergy ratio and return it as feedwater preheat at a much lower exergy ratio. This point is further discussed in section 5.4.3.

It should be noted that the heat transfer temperature drop has been neglected for the time being. This was done to simplify the discussion in this section and section 5.4.3. Exergy balances with realistic heat transfer $\Delta \mathrm{T}$ will be presented in section 5.4.4.

\subsubsection{Methanation Plant}

Heat exchanger duties for the methanation plant are plotted as a superposition of two histograms in Figure 5-12. The general character is seen to be analogous to that for the reformer plant (Figure 5-11); as a direct consequence of the reduced $\mathrm{H}_{2} \mathrm{O}$ recycle, equipment duties are generally much lower for the methanator case than those for the reformer.

One aspect of the methanation plant duties deserves a special discussion because it may lead to misinterpretation. Heat exchanger duties reported in Table 5-3 show a surplus of $274 \mathrm{MJ} / \mathrm{s}$ available from the cooling duty even after meeting the heating demand. There are two reasons for this excess:

- The last heat exchanger following the methanation reactor has been included in the cooling duty. Thus, a part of the high-temperature heat is really the reactor thermal output. 


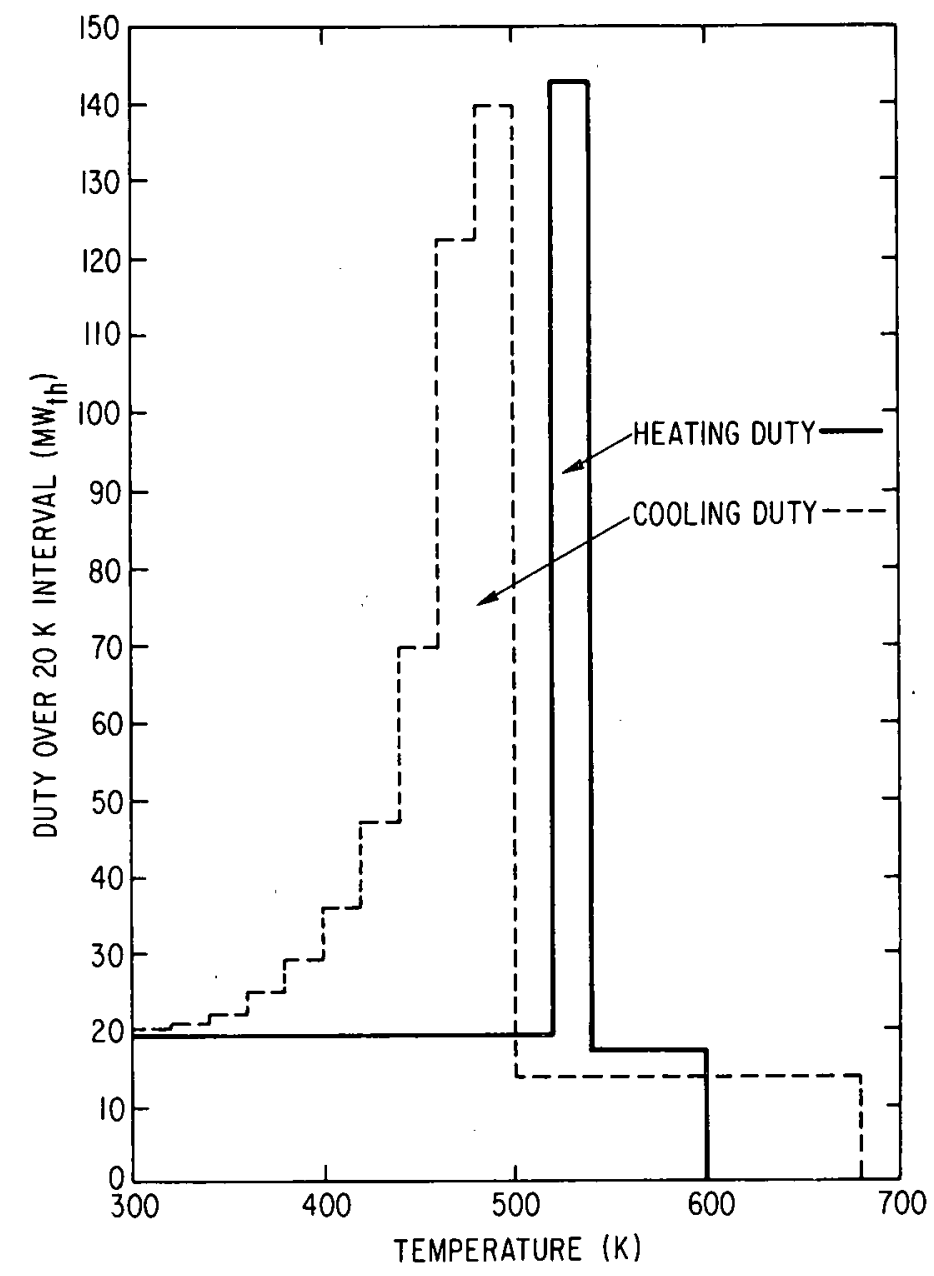

Figure 5-12. Methanation Plant Histograms

- Approximately 15\% of the total transported energy (150 $\mathrm{MJ} / \mathrm{s}$ is the latent heat of condensation of $\mathrm{H}_{2} \mathrm{O}$ formed in the methanator and is recovered only during condensation.

These two heat terms (adding up to a total of $274 \mathrm{MJ} / \mathrm{s}$ ) when added to the methanator reactor output of $726 \mathrm{MJ} / \mathrm{s}$ make up the total $1000 \mathrm{MJ} / \mathrm{s}$ transported.

The large boiler deficit at $525 \mathrm{~K}$ of $130 \mathrm{MJ} / \mathrm{s}$ poses a much different problem here than in the previous case of the reformer. In the absence of an on-site powerplant to take up the slack, it may not be easy to utilize excess energy and to provide for the deficit. A severe penalty would accrue if part of the output heat had to be used for supplying the $130 \mathrm{MJ} / \mathrm{s}$ boiler deficit. The exergy analysis for the methanation process may not be of much importance if it has to be a stand-alone design without any powerplants nearby. On the other hand, for applications involving cogeneration it would be as important to match thermal exchanges with powerplant design as in the reformer plant design.

Results of the exergy analysis (with heat transfer $\Delta \mathrm{T}=0$ ) show energy surpluses of $74 \mathrm{MJ} / \mathrm{s}$ between 700 and $600 \mathrm{~K}$ and 200 $\mathrm{MJ} / \mathrm{s}$ below $600 \mathrm{~K}$. The former can be considered as the thermal 
output from the last reactor and should be added to the reactor output of $726 \mathrm{MJ} / \mathrm{s}$. The latter, with an exergy surplus of $56 \mathrm{MW}$, is available at an effective temperature of $516 \mathrm{~K}$.

\subsubsection{Mixed Feed Evaporator (MFE)}

In the previous section, it was shown that a mismatch of temperatures in the condenser/boiler duties is responsible for a signigificant exergy penalty, particularly in the reformer plant design. In explaining the cause of this mismatch, there are two aspects to be considered -- one inherent in the chemical reaction, and the other related to the manner in which reactor feed streams are prepared.

When the reaction between $\mathrm{CH}_{4}$ and $\mathrm{H}_{2} \mathrm{O}$ proceeds to form $\mathrm{CO}$ and $\mathrm{H}_{2}$ two things happen: $\mathrm{H}_{2} \mathrm{O}$ is consumed, and the mole fraction of $\mathrm{H}_{2} \mathrm{O}$ also decreases. The consequence of the former is that condensation heat of the effluent is less than the corresponding evaporation heat for the feed; the consequence of the latter is that the temperature level of the condensation heat is lower than that for the evaporation because of the effect of $\mathrm{H}_{2} \mathrm{O}$ partial pressure. However, this effect does not imply an irreversibility; exactly the converse takes place upon methanation, where both moles and mole fraction of $\mathrm{H}_{2} \mathrm{O}$ increase, and so do the condensation heat and its temperature level.

The manner in which reactor feed streams are prepared, on the other hand, can lead to significant irreversibilities. When two gaseous streams from the boiler and the gas preheater are mixed they undergo irreversible mixing. The thermodynamic price one has to pay is not immediately evident until one has to "undo" the process by separating out the water molecules in the partial condenser. For the example case reformer plant, this irreversible entropy of mixing and the associated exergy loss have been calculated in Appendix 2, A2.4.4. The results (see Table A2-3) show an exergy loss of 39.5 MW due to the steam/gas mixing. There is a great incentive to eliminate this loss and thereby improve the overall process efficiency. A modification in the process of preparing the feed stream has been developed in the course of this contract to eliminate this irreversible step.

In the example case reformer plant, the requisite amounts of $\mathrm{H}_{2} \mathrm{O}$ were introduced into the gas stream by first evaporating (boiling) it senarately and then mixing the two gas streams. As a result, the heat of evaporation was required at the boiling point corresponding to the total system pressure of 40 bars. If, instead, the gas and liquid streams are introduced together in a heat exchanger and allowed to heat up with a continued evaporation process (much like humidification) progressive amounts of $\mathrm{H}_{2} \mathrm{O}$ can be introduced into the gas stream at much lower temperatures. The net effect is to approach conditions that are exactly the reverse of partial condensations; at each point in the heat exchanger, the amount of water vapor in the gas stream corresponds roughly to. the equilibrium vapor pressure at the local temperature. 
The reformer plant process flowsheet with this modification (Figure 5-13) is only slightly different from the previous one (Figure 5-2). The two units - feed boiler and gas heater -- are now combined into one (MFE). As mentioned earlier, this does not alter the total heating duty; however, the temperature levels at which heat is needed is changed. This shows up most clearly in the histogram shown in Figure 5-14.

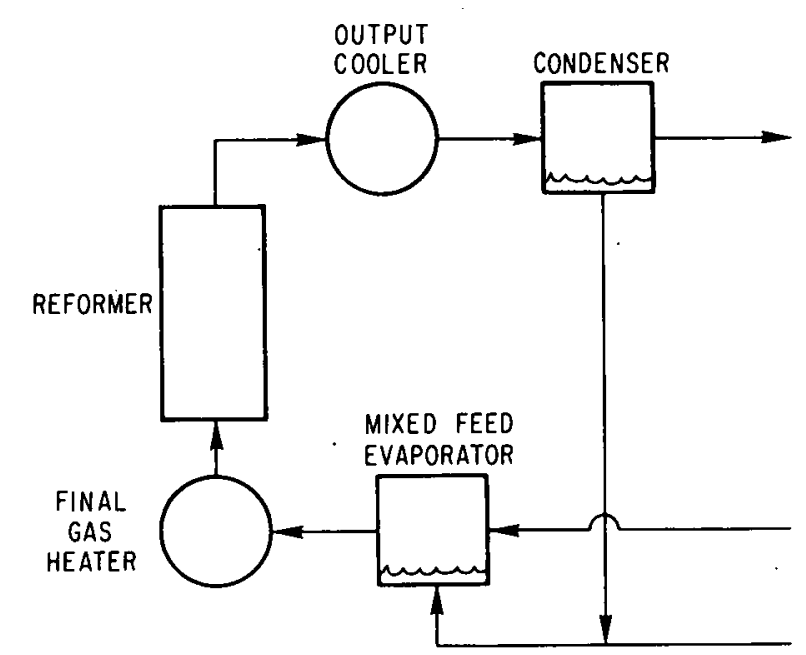

Figure 5-13. Reformer Plant Schematic with MFE

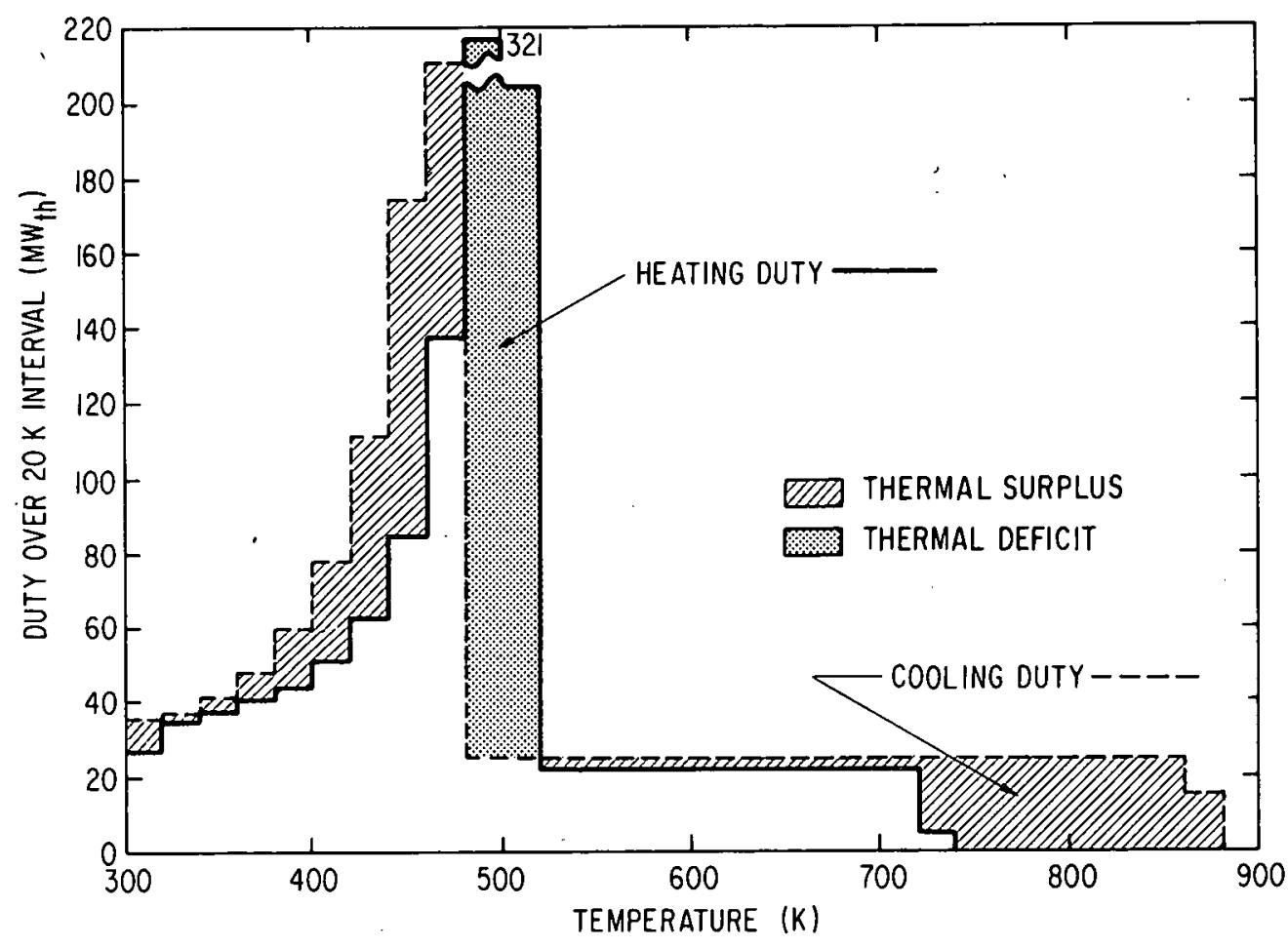

Figure 5-14. Example Case - Reformer Plant with MFE 
A comparison with Figure 5-11 shows that the large spike at $524 \mathrm{~K}$ is no longer present; the same duty, instead, is spread over a range of temperatures from 505 to $300 \mathrm{~K}$ resembling qualitatively the partial condenser. The nverall affect on the heat exchange is a reduction in the amount and the temperature level of the thermal deficit that must be made up by extraction of steam from the powerplant. The magnitude of the feedwater preheat is also reduced. This reduction in heat exchanges with the powerplant occurs because some of the condensation heat can now be used to evaporate water in a partial condenser/MFE combination.

Following the procedure used earlier, the impact of these modified exchanges can be evaluated by computing the exergy associated with thermal exchanges. The results (still with heat transfer $\Delta \mathrm{T}=0$ ) show that while the net thermal energy deficit remains at the previous level of $5.7 \mathrm{MJ} / \mathrm{s}$, the exergy trades show a surplus of $24.3 \mathrm{MW}$. It is important to note that the difference in the net exergy flow with and without $\mathrm{MFE}$ is $40 \mathrm{MW}$, which is comparable to the irreversible mixing associated loss calculated in Appendix 2, A2 . 4 .

A detailed design of heat exchangers for the reference plant is given in Appendix 3. An important feature of the MFE/partial condenser should be emphasized: the overall heat transfer coefficient is not affected adversely by the water/gas mixture but is limited by the partial condensation process. The implication is that the performance improvements mentioned above can be achieved without any significant change in the overall heat exchanger area or cost. While it is true that in the design of such two-phase heat exchangers the problems of maldistribution within the tubes and of potential instability must be considered, such exchangers are currently designed and operated.

Thus far, the application of MFE has been examined at the reformer plant, where even though its use does not completely eliminate thermal mismatches it greatly reduces the reliance on the powerplant and thereby improves the overall efficiency. The effect of the same type of modification on the methanation plant should next be examined.

Keeping in mind the possibility that certain methanation designs may required no recycle water, the intent here is to look at the effect of MFE on methanation energy balances, if it should prove to be applicable. Figure 5-15 shows the histograms for the example case methanation plant with MFE. For the first time it is possible to satisfy the entire heating need by the cooling duties; for each deficit there is an excess heat available at a higher temperature. There are no large spikes in the demand, and the heating/cooling duties are generally well matched. Results from the exergy analysis indicate an energy surplus of $200 \mathrm{MJ} / \mathrm{s}$ below $600 \mathrm{~K}$ with an associated exergy surplus of $81 \mathrm{MW}$. A comparison with the earlier analysis (section 5.4.2.3) shows the energy surplus to be unchanged; however, the exergy surplus is $25 \mathrm{MW}$ 


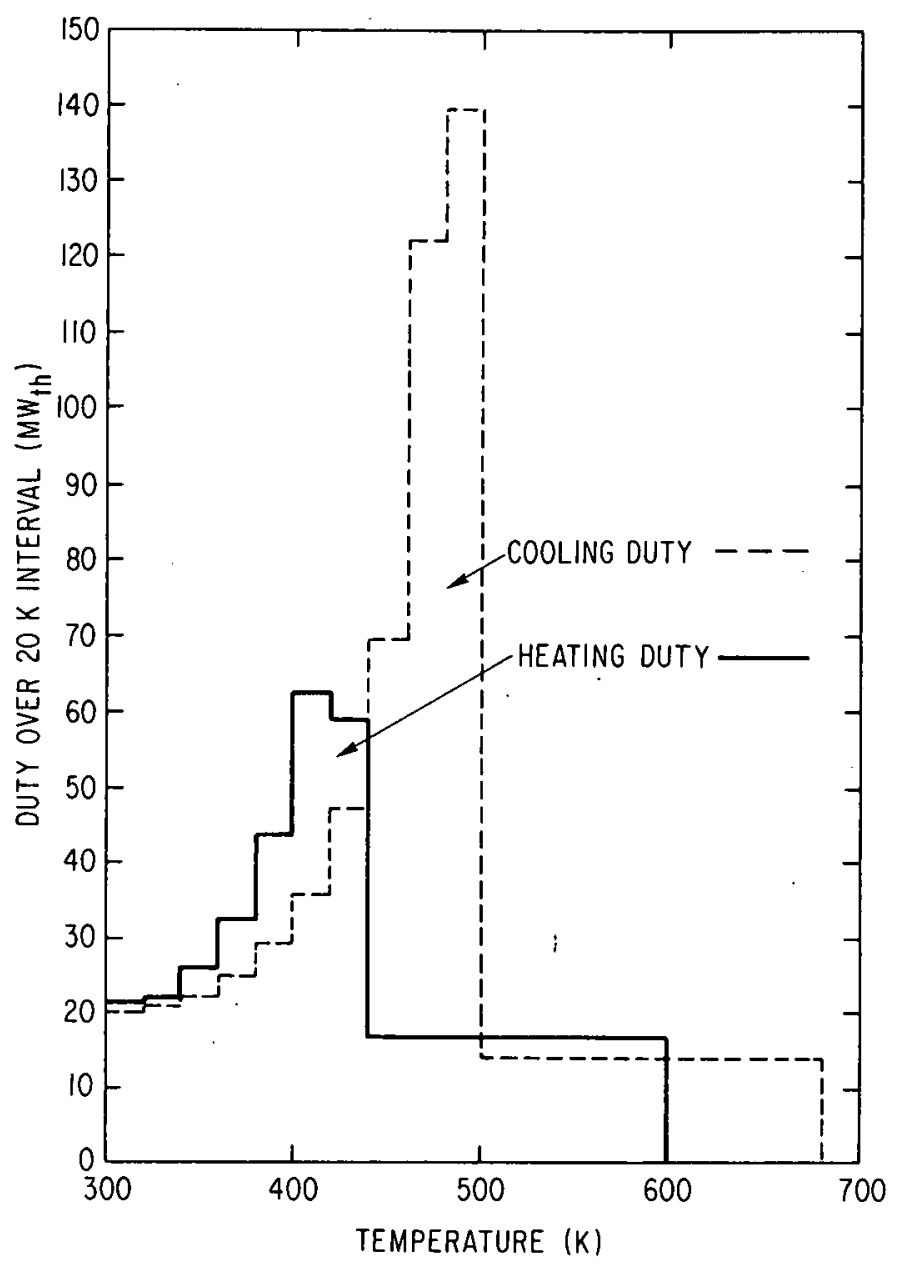

Figure 5-15. Example Case - Methanation Plant with MFE

higher, with an increase in the effective temperature from $416 \mathrm{~K}$ obtained without MFE to $504 \mathrm{~K}$ with MFE.

Once again, the use of MFE has been shown to lead to a significant improvement in process energetics. The benefits at the methanation end are different from those at the reformer plant, in that MFE enables a stand-along methanation plant design with complete internal recycle and enables the surplus energy to be delivered at higher (and therefore more useful) temperature levels.

\subsubsection{Design Efficiency}

In the interest of clarifying the thermodynamic basis for MFE, previous analyses were simplified by neglecting several irreversibilities such as frictional pressure drops and heat transfer temperature drop. These realistic energy penalties will be included in this section in order to arrive at an estimate of practical process efficiencies. 


\subsubsection{Heat Exchanger Losses}

Optimization of heat exchanger designs, based on energy efficiency or on economics, is beyond the scope of this preliminary study. It is instructive, however, to study the influence of heat transfer temperature drops on overall energetics by parametric variations. First, the sensible heat losses due to an incomplete cooling of the exit streams will be examined. This will be followed by exergy analyses with several heat transfer $\Delta \mathrm{Ts}$.

Sensible heat losses for the reformer plant amount to $13 \mathrm{MJ} / \mathrm{s}$ for a heat transfer of $\Delta T$ of $20 \mathrm{~K}$, and increase to $26 \mathrm{MJ} / \mathrm{s}$ for a $\Delta \mathrm{T}$ of $40 \mathrm{~K}$. Similar losses at the methanation plant amount to $14 \mathrm{MJ} / \mathrm{s}$ and $28 \mathrm{~mJ} / \mathrm{s}$ respectively. One may conclude, as a result, that the penalty due to incomplete heat transfer and a subsequent loss of sensible heat is not a major factor in determining the overall efficiency.

Results of exergy analysis for the reformer plant (with and without MFE) with heat transfer $\triangle \mathrm{T}$ of $0 \mathrm{~K}, 20 \mathrm{~K}$ and $40 \mathrm{~K}$ are shown in Table 5-4. Each $20 \mathrm{~K}$ increase in heat transfer $\Delta \mathrm{T}$ results in an additional exergy penalty of approximately $48 \mathrm{MW}$. Regardless of the actual heat transfer $\Delta T$, however, the use of MFE is always beneficial and improves the overall exergy flow by approximately $40 \mathrm{MW}$.

Table 5-4

AVAILABILITY COMPARISONS OF SEPARATE AND MIXED EVAPORATION EXAMPLE CASE - REFORMER PLANT

Heat
Exchanger
$\begin{aligned} & \Delta \mathrm{T} \\ & \frac{(\mathrm{K})}{0} \\ & 20 \\ & 40\end{aligned}$

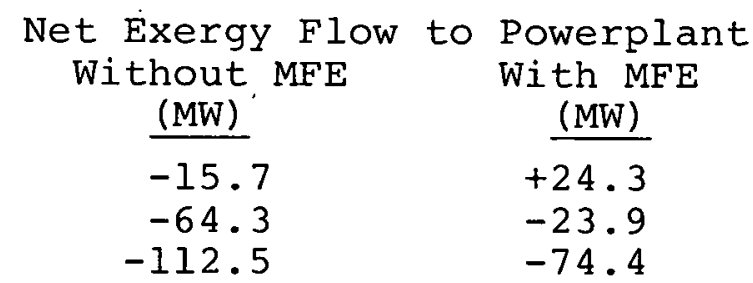

Based on component designs such as those given in Appendix 3, a temperature drop of $20 \mathrm{~K}$ appears reasonable and is used here and in Chapter 6. It should be emphasized, however, that the energy losses and the capital costs for heat exchangers are closely coupled through the choice of heat exchanger areas and, hence, of heat transfer $\Delta$ Ts. In computing the net effect of an exergy loss of $23.9 \mathrm{MW}$ a powerplant exergy efficiency of $85 \%$ is assumed (see Appendix 2) and that effect equals $20.3 \mathrm{MW}$ in the form of work input.

\subsubsection{Pumping Energy for Transportation}

An estimate of the pumping energy for transportation is based on transmission distance and pipeline diameters. The transportation distance of $160 \mathrm{~km}$ (100 miles) has been assumed. In the absence of a geographic breakdown of CHP application range based on process 
steam users, it is not possible to make a better choice of the average distance. The pipeline diameter, on the other hand, is chosen to achieve an optimal transportation cost based on a tradeoff between capital and pumping costs.

Transportation optimization for the HTCHP designs is presented in detail in Appendix 4. For the example case, the compression power requirements in the optimum design total $19.3 \mathrm{MW}$ for $160 \mathrm{~km}$. It is important to note that there is a strong coupling between compression power and pipeline capital cost.

\subsubsection{Equipment Pressure Drop}

I.t is difficult to estimate the total pressure drop through heat exchangers and reactors without first performing detailed component designs. A rough estimate of pressure drops in heat exchangers and reactor may be provided by combining total heat exchanger pressure drop of $\sim 4$ bars for a sample design (Appendix 3) and typical pressure drop in a reformer tube of $\sim 2$ bars.

With this estimate of the overall pressure drop the compressor power requirement could be computed. However, there is another important complication to be considered. The fact that the reactor effluent stream is generally at a lower pressure than the feed stream has a non-negligible impact on the thermal histograms. The evaporation process requires its energy at a higher temperature than before, while the condensation takes place at a slightly lower temperature than before. The added exergy loss represented by these shifts must be included in any assessment of frictional losses.

Since all these factors could not be accounted for in great detail a net power requirement due to equipment pressure drops was assumed equal to that for pipeline compression power. This amounts to a total of 19.3 MW for reformer and methanation plants. Calculations in Appendix 2, A2.4 indicate that this corresponds to a pressure drop of roughly 6 bars, which agrees with the previous estimate.

\subsubsection{Overall Efficiency}

The estimates of work requirements can be combined with thermal inputs and outputs to arrive at an overall energy efficiency $(\psi)$, defined in Section 5.4.1. Basic assumptions are

- Heat tránsfer $\Delta \mathrm{T}$ equal to $20 \mathrm{~K}$

- Optimal transportation design (Appendix 4)

- Overall powerplant loss estimated by the exergy analysis (Appendix 2)

- An additional power requirement (to overcome equipment pressure drop) equaling the transportation losses 
Based on these assumptions, the overall efficiency for the reference case with MFE can be calculated as follows:

Transportation work requirements

Work to overcome equipment pressure drop

Total direct work

$=19.3 \mathrm{MW}$

$=19.3 \mathrm{MW}$

$=\overline{38.6 \mathrm{MW}}$

Net exergy flow to the powerplant Indirect work penalty (@85\% exergy efficiency) Total work penalty $\left(W_{d}+W_{i}\right)$

$=-23.9 \mathrm{MW}$

$=20.3 \mathrm{MW}$

$=58.9 \mathrm{MW}$

Equivalent thermal input (@ $\eta=0.4$ )

$=147.2 \mathrm{MJ} / \mathrm{s}$

Reformer reactor thermal input

Total thermal input

Total thermal output from methanation.

plant

System Efficiency $(\psi)$

$=1003 \mathrm{MJ} / \mathrm{s}$

$=1150.2 \mathrm{MJ} / \mathrm{s}$

$=986 \mathrm{MJ} / \mathrm{s}$

$=\frac{986 \mathrm{MJ} / \mathrm{s}}{1150.2 \mathrm{MJ} / \mathrm{s}}$

$=85.7 \%$

\subsection{SUMMARY AND CONCLUSIONS}

In this chapter, the background for methane reforming and methanation technologies of relevance to the high temperature chemical heat pipe (HTCHP) was reviewed and major elements of the HTCHP process flowsheet were presented. Procedures for material and enthalpy balances were then described, using a reference case example. Finally, using a thermodynamic approach, overall efficiencies were calculated for the original and modified designs. The following major conclusions can be drawn:

- Thermodynamic considerations play a much more important role in CHP applications than in other chemical processes.

- The extent and efficiency of internal heat exchanges between the reactant and product streams should be maximized in order to improve the overall efficiency.

- Thermal histograms provide a useful and convenient tool for analyzing these exchanges and could be used to advantage in other thermal analyses.

- Inherent mismatches in heating and cooling duties at the reformer plant do not permit a stand-alone reformer design without excessive energy penalty. Thermal exchanges with an on-site powerplant are needed to deal with thermal surplus and deficits.

- There is a significant net work penalty as a result of thermal exchanges with the powerplant. The exergy analysis developed in the course of this work is a useful and effective way to evaluate this hidden work penalty without resorting to a detailed powerplant design. 
- The irreversible mixing of boiler steam with feed gases is a major source of thermodynamic degradation, leading ultimately to a thermal mismatch. The invention of mixed feed evaporation (MFE) offers significant advantages by eliminating this irreversibility.

- The use of MFE at the reformer plant improves the energy efficiency by approximately $10 \%$. If $\mathrm{H}_{2} \mathrm{O}$ recycle is required at the methanation plant, the use of MFE permits a complete internal recycle of thermal energy and an increase in the average temperature level of the delivered heat.

- The process shows a satisfactorily high efficiency. For a transmission of $1000 \mathrm{MJ} / \mathrm{s}$, major losses consist of direct work inputs $(38.6 \mathrm{MW})$ to overcome equipment and pipeline friction and indirect work penalty (23.6 MW) due to exchanges with the powerplant. Thermal losses due to incomplete heat transfer $(\sim 30 \mathrm{MJ} / \mathrm{s})$ are not very significant.

\section{REFERENCES -- CHAPTER 5}

R5-1 Anon., "Reformer Tube Alloy Sithstands Overheating," Metal Progress, Vol. 107, February 1975, p. 44.

R5-2 Nuclear Engineering and Design, Vol. 34, No. 1, 1975.

R5-3 Kugeler, K., et al., "Steam Reformers Heated by Helium from High Temperature Reactors," Nuclear Engineering and Design, Vol. 34, No. 1, 1975, p. 129 .

R5-4 Ibid, pp. 65-72 and 119-127.

R5-5 Schulten, et al., "The Pebble Bed High Temperature Reactor as a Source of Nuclear Process Heat," KFA Report, Vol. 4, 1974 .

R5-6 White, G.A., et al., Proceedings, 168th National Meeting, American Chemical Society, Div. of Fuel Chemistry, Atlantic City, New Jersey, September 1976.

R5-7 Prigogine, I., and Defay, R., Chemical Thermodynamics, Longmans Green \& Co., New York, I954.

R5-8 Stull, D.R., et al., JANAF Thermochemical Tables, 2nd Ed., National STandard Reference Data Series, No. 37, 1971.

R5-9 Hilsenrath, J. et al., "Tables of Thermodynamic \& Transport Properties, "National Bureau of Standards, Pergamon Press, New York, 1950.

R5-10 Jordan, W.B., "Fits to Thermodynamic Properties of Water," Report No. KAPL-M-6734, General Electric Knolls Atomic Power Laboratory, Schenectady, New York, 1967. 
R5-11 Vakil, H.B., "Energy Storage and Transmission by Chemical Heat Pipe," Proceedings of the Engineering Foundation Conference on Energy Storage (NTIS CONF-760212), Asilomar, California, February 1976. 


\section{Chapter 6}

\section{CASE DESIGN ANALYSES}

Throughout the previous chapter, a reference case example was used to develop, explain, and improve the process design procedures. These design procedures are used in this chapter to investigate the parametric dependence of CHP design and performance with respect to such design variables as equilibrium temperatures, reaction stoichiometrics, and operating pressures. In addition to providing information on overall reaction conversions, equipment duties, and energy efficiencies, these case designs also provide bases for economic analyses to be presented in Chapter 7 .

The selection of cases to be analyzed has been restricted to limited excursions in one or two parameters from the example case. The selected design cases are only a few of the many computer runs performed in the course of this study. Were it not for this screening, the mass of computer results would lead to more confusion than information.

The rationale behind the selection of parameters for each case is outlined in section 6.I. (Details of the design analyses are given in Appendix 2, Section A2.3.3.) Results from these analyses are summarized in section 6.2. Finally, the effect of parametric variations in important design variables on the overall energetics are discussed in Section 6.3.

\subsection{RATIONALE FOR CASE SELECTIONS}

Parameters for the ten design cases chosen for analysis are shown in Table 6-1. Each case is designed to illustrate the effect of process variations due to either source constraints or alternative design strategies. The rationale for each selection is summarized below.

Keeping in mind the example reference case, the major variables explored are the peak reforming temperature ( 1200 to 975K), reformer pressure ( 40.5 to 10 bars), stoichiometry $\left(\mathrm{H}_{2} \mathrm{O}\right.$ recycle, $\mathrm{CO}_{2}$ addition), exit methanator temperature $(600$ to $700 \mathrm{~K})$, and methanation pressure $(40.5$ to 10.1 bars). Preliminary studies led to a general conclusion that it is advantageous to use the lowest possible $\mathrm{H}_{2} \mathrm{O} / \mathrm{CH}_{4}$ ratio at the methanation end within the limitation of carbon deposition on the catalyst. As a result, in all design cases without $\mathrm{CO}_{2}$ addition (Cases $\mathrm{A}$ through $\mathrm{H}$ ) methanation stoichiometry was fixed, somewhat arbitrarily, at a value equivalent to $50 \%$ excess steam. This is considered a conservative number; in most instances it should be possible to methanate COrich gases without addition of any recycle $\mathrm{H}_{2} \mathrm{O}$ (R6-l). 
Table 6-1

PARAMETERS FOR EQUILIBRIA FOR DESIGN CASES

\begin{tabular}{|c|c|c|c|c|c|c|}
\hline \multirow[b]{2}{*}{ Case } & \multicolumn{3}{|c|}{ Reformer Plant } & \multicolumn{3}{|c|}{ Methanation Plant } \\
\hline & $\begin{array}{r}\mathrm{T} \\
(\mathrm{K}) \\
\end{array}$ & $\begin{array}{c}\mathrm{P} \\
(\mathrm{atm}) * \\
\end{array}$ & $\begin{array}{l}\text { Stoichiometry } \\
\left(\mathrm{CH}_{4} / \mathrm{H}_{2} \mathrm{O} / \mathrm{CO}_{2}\right) \\
\end{array}$ & $\begin{array}{r}\mathrm{T} \\
(\mathrm{K}) \\
\end{array}$ & $\begin{array}{c}\mathrm{P} \\
(\mathrm{atm}) * \\
\end{array}$ & $\begin{array}{l}\text { Stoichiometry } \\
\left(\mathrm{CH}_{4} / \mathrm{H}_{2} \mathrm{O} / \mathrm{CO}_{2}\right)\end{array}$ \\
\hline Example & 1100 & 40 & $1 / 3 / 0$ & 700 & 40 & $1 / 1.5 / 0$ \\
\hline A & 1100 & 40 & $1 / 3 / 0$ & 600 & 40 & $1 / 1.5 / 0$ \\
\hline B & 1100 & 40 & $1 / 4 / 0$ & 700 & 40 & $1 / 1.5 / 0$ \\
\hline C & 1100 & 40 & $1 / 2 / 0$ & 700 & 40 & $1 / 1.5 / 0$ \\
\hline $\mathrm{D}$ & 1200 & 40 & $1 / 3 / 0$ & 700 & 40 & $1 / 1.5 / 0$ \\
\hline$E$ & 1200 & 40 & $1 / 2 / 0$ & 700 & 40 & $1 / 1.5 / 0$ \\
\hline$F$ & 975 & 40 & $1 / 3 / 0$ & 700 & 40 & $1 / 1.5 / 0$ \\
\hline G & 975 & 10 & $1 / 3 / 0$ & 650 & 10 & $1 / 1.5 / 0$ \\
\hline $\mathrm{H}$ & 1100 & 25 & $1 / 3 / 0$ & 700 & 25 & $1 / 1.5 / 0$ \\
\hline I & 1100 & 40 & $1 / 2 / 1$ & 700 & 40 & $1 / 0.75 / 1$ \\
\hline $\mathrm{J}$ & 1100 & 40 & $1 / 1.433 / 2$ & 700 & 40 & $1 / 0.218 / 2$ \\
\hline
\end{tabular}

${ }^{\star} 1 \mathrm{~atm}=1.013 \mathrm{bars}$

- Case A - This case was chosen to investigate possible incentives for lowering exit methanation temperature to enhance methane formation. In practice, this would imply an inlet temperature well below $600 \mathrm{~K}$ in the last adiabatic methanator; this may present kinetic limitations and lead to larger reactor volumes than were assumed.

- Cases B and C - Effects of changes in $\mathrm{H}_{2} \mathrm{O}$ recycle at the reformer plant are investigated in these two cases. The main objective is to examine how heat exchange load and overall conversions vary as a function of $\mathrm{H}_{2} \mathrm{O}$ cycle.

- Cases D and E - As the peak process temperature is increased, a larger fraction of $\mathrm{CH}_{4}$ is converted and the energy density increases. Case D examines the effect of this increased conversion on heat exchange loads and transportation. Case $\mathrm{E}$ is designed to test whether it would be more advantageous to use the increased thermodynamic driving force to permit a lower $\mathrm{H}_{2} \mathrm{O}$ recycle without a severe conversion penalty. In terms of source technologies, a process peak temperature of $1200 \mathrm{~K}$ may represent either an optimistic design for PBR exit helium temperature $(>1300 \mathrm{~K})$ or a dramatic reduction in the temperature drop across the reformer tube. This would represent nearly a fivefold reduction in temperature drop. Possible scenarios leading to this are: 
(a) a longer active length with reduced heat flux at the hot end, or (b) improvements through catalyst innovations (e.g., alternate catalyst program currently under study) that would reduce the reformer tube diameter, leading to a reduction in heat transfer $\Delta \mathrm{T}$ and an increase in heat transfer area.

- Cases F and G - These two cases are based on a somewhat pessimistic view of the peak process temperature capabilities. They could result from the use of an intermediate heat exchange (IHX) loop or a lowering of peak helium temperature. At these low temperatures, the two cases examine the relative merits of 10 and 40 atm operation through a tradeoff between conversion and transmission efficiency.

- Case H - Effect of lowering the system operating pressure to $25 \mathrm{~atm}$ is examined in this case. For the PBR applications, 40 atm has been selected primarily for safety reasons. A relaxation of this constraint may result from the use of IHX or from higher temperature solar reformer designs.

- Cases I and J - These last two cases are aimed at evaluating effects of $\mathrm{CO}_{2}$ addition as proposed by the HYCO system (R6-2). Case I uses moderate additions of $\mathrm{CO}_{2}$ with an equivalent decrease in $\mathrm{H}_{2} \mathrm{O}$ recycle. A more complete test of the HYCO scheme is provided by Case J,. where the stoichiometry is adjusted iteratively to ensure that no $\mathrm{H}_{2} \mathrm{O}$ is being transported.

\subsection{RESULTS FROM DESIGN ANALYSIS}

The equilibrium conversions and the transportation flow rates for all of these cases are shown in Table 6-2. Following a procedure identical to the one used for the reference case, equipment heat duties were calculated for each case on the basis of a heat exchanger $\Delta \mathrm{T}$ of $20 \mathrm{~K}$, reformer reactor feed and exit temperatures of 725 and $873 \mathrm{~K}$ respectively, and the methanation reactor inlet temperature of $600 \mathrm{~K}$. These duties are summarized in Table 6-3.

Exergy analyses based on thermal histograms were performed, following the procedure outlined in Appendix 2 (section A2.3.3). The net exergy flows to the powerplants were calculated in two different ways: one with the mixed feed evaporator (MFE); the other without. While similar exergy analyses were also performed on the methanation plants, the energy flows have little relevance in the absence of a complete characterization of the end-use requirements; consequently, they have not been presented.

Table 6-4 presents the major energy and exergy flows for the various design cases. The pipeline compressor inputs correspond 
Table 6-2

CONVERSIONS AND TRANSPORTATION FLOW RATES FOR DESIGN CASES

\begin{tabular}{|c|c|c|c|c|}
\hline \multirow[b]{2}{*}{ Case } & \multirow[b]{2}{*}{$\begin{array}{l}\text { Overall } \\
\text { Conversion } \\
\left(8 \mathrm{CH}_{4}\right)\end{array}$} & \multicolumn{3}{|c|}{$\begin{array}{l}\text { Transportation Flow Rates for } 1000 \\
\qquad\left(10^{6} \mathrm{gmoles} / \mathrm{hr}\right) *\end{array}$} \\
\hline & & $\begin{array}{c}\text { CO-Rich } \\
\text { Gases }\end{array}$ & $\begin{array}{c}\mathrm{CH}_{4}-\mathrm{Rich} \\
\text { Gases } \\
\end{array}$ & $\begin{array}{c}\text { Return } \\
\mathrm{H}_{2} \mathrm{O} \quad(\ell)\end{array}$ \\
\hline Reference & 61.0 & 76 & 27 & 20 \\
\hline$A$ & 63.4 & 73 & 24 & 21 \\
\hline B & 69.8 & 73 & 24 & 21 \\
\hline C & 49.4 & 81 & 33 & 19 \\
\hline $\mathrm{D}$ & 81.9 & 67 & 20 & 18 \\
\hline E & 71.4 & 69 & 23 & 17 \\
\hline $\mathbf{F}$ & 33.3 & 102 & 49 & 24 \\
\hline$G$ & 57.6 & 80 & 29 & 22 \\
\hline $\mathrm{H}$ & 70.3 & 72 & 24 & 23 \\
\hline I & 59.0 & 88 & 50 & 9 \\
\hline $\mathrm{J}$ & 65.0 & 97 & 68 & 0 \\
\hline
\end{tabular}

$\overline{{ }^{*} 10^{6} \text { gmoles } / \mathrm{hr}}=1$ tonne moles $/ \mathrm{hr}=277.8 \mathrm{gmoles} / \mathrm{sec}$.

Table 6-3

THERMAL DUTIES FOR DESIGN CASES

(Basis: $1000 \mathrm{MW}_{\text {th }}$ Transported)

\begin{tabular}{|c|c|c|c|c|c|c|}
\hline & \multicolumn{3}{|c|}{ Reformex Plant } & \multicolumn{3}{|c|}{ Methanation Plant } \\
\hline & $\begin{array}{c}\text { Reformer } \\
\text { Reactor } \\
\text { Duty } \\
\end{array}$ & $\begin{array}{c}\text { Total } \\
\text { Cooling } \\
\text { Duty } \\
\end{array}$ & $\begin{array}{l}\text { Total } \\
\text { Heating } \\
\text { Duty } \\
\end{array}$ & $\begin{array}{l}\text { Methanator } \\
\text { Reactor } \\
\text { Duty } \\
\end{array}$ & $\begin{array}{l}\text { Total } \\
\text { Cooling } \\
\text { Duty } \\
\end{array}$ & $\begin{array}{l}\text { Total } \\
\text { Heating } \\
\text { Duty } \\
\end{array}$ \\
\hline & & & duties ar & $10^{6} \mathrm{~J} / \mathrm{sec}$ & & \\
\hline Reference & 1003 & 1239 & 1249 & 726 & 654 & 394 \\
\hline A & 992 & 1191 & 1212 & 796 & 569 & 379 \\
\hline B & 1005 & 1413 & 1420. & 726 & 571 & 311 \\
\hline c & 1010 & 1075 & 1079 & 722 & 809 & 546 \\
\hline D & 981 & 904 & 934 & 769 & 488 & 269 \\
\hline$E$ & 982 & 719 & 749 & 776 & 560 & 348 \\
\hline $\mathrm{F}$ & 1106 & 2360 & 2272 & 623 & 1192 & 835 \\
\hline G & 994 & 1300 & 1320 & 702 & 690 & 407 \\
\hline $\mathrm{H}$ & 988 & 1058 & 1082 & 744 & 565 & 322 \\
\hline I & 1148 & 1177 & 1045 & 845 & 520 & $379^{\circ}$ \\
\hline $\mathrm{J}$ & 1255 & 1108 & 871 & 939 & 397 & 350 \\
\hline
\end{tabular}


to the optimal transportation design for multiple pipelines in a trench (for details see Appendix 4). Compression energy requirements to overcome process equipment pressure drops have riot been calculated for each design case; instead, the equipment pressure drop penalty has been assumed to be equal to the total transportation work requirement. Since a detailed design and economic optimization for each case is beyond the scope of this study (and somewhat premature at this stage), this assumption seems reasonable.

Table 6-4

PROCESS ENERGETICS AND EFFICIENCIES FOR DESIGN CASES

\begin{tabular}{|c|c|c|c|c|c|c|}
\hline & $\begin{array}{l}\text { Reformer } \\
\text { Reactor } \\
\text { Thermal } \\
\text { Input } \\
\left.\text { (MW }_{\text {th }}\right) \\
\end{array}$ & $\begin{array}{c}\text { Net } \\
\text { Exergy } \\
\text { Flow to } \\
\text { the Power } \\
\text { Plant } \\
\text { (MW) }\end{array}$ & $\begin{array}{l}\text { Pipeline } \\
\text { Compressor } \\
\text { Work } \\
\text { Input } \\
\text { (MW) }\end{array}$ & $\begin{array}{c}\text { Total } \\
\text { Work } \\
\text { Input } \\
\text { (MW) }\end{array}$ & $\begin{array}{c}\text { Methanatior } \\
\text { Reactor } \\
\text { Thermal } \\
\text { Output } \\
\left(\mathrm{MW}_{\mathrm{th}}\right)\end{array}$ & $\begin{array}{c}\text { Efficiency } \\
\text { Index } \\
(\psi) \\
(8) \\
\end{array}$ \\
\hline Reference & 1003 & $-23^{\star} .9\left(-6^{\star} 4^{\star} \cdot 3\right)$ & 19.3 & $58^{\star} .9\left(93^{\star \star} .3\right)$ & 986 & $85^{\star} .7\left(79^{\star *} .8\right)$ \\
\hline A & 992 & $-29.4(-66.3)$ & 18.5 & $62.0(93.4)$ & 987 & $86.1(80.5)$ \\
\hline B & 1005 & $-34.9(-74.5)$ & 18.4 & $66.5(100.1)$ & 986 & $84.2(78.5)$ \\
\hline c & 1010 & $-6.5(-48.5)$ & 20.3 & $46.1(81.8)$ & 985 & $87.5(81.1)$ \\
\hline D & 981 & $-26.5(-56.7)$ & 16.8 & $56.1(81.8)$ & 988 & $88.1(83.3)$ \\
\hline E & 982 & $-13.9(-42.3)$ & 17.5 & $46.8(71.0)$ & 988 & $89.9(85.2)$ \\
\hline$F$ & 1106 & $+7.1(-71.5)$ & 26.1 & $46.2(113.0)$ & 980 & $80.2(70.6)$ \\
\hline G & 994 & $-16.9(-56.5)$ & 23.0 & $60.4(94.0)$ & 985 & $86.0(80.1)$ \\
\hline H & 988 & $-21.4(-50.4)$ & 18.9 & $56.0(80.6)$ & 987 & $87.5(83.0)$ \\
\hline I & 1148 & $+53.0(+5.1)$ & 27.0 & $9.0(49.7)$ & 986 & $84.2(77.5)$ \\
\hline $\mathrm{J}$ & 1255 & $+103.0(+58.9)$ & 31.8 & $-24.0(13.5)$ & 986 & $82.5(76.5)$ \\
\hline
\end{tabular}

*With mixed feed evaporator (MFE)

**Numbers in parentheses are without MFE

Also shown in Table 6-4 are overall efficiency indices (with and without MFE) based on thermal equivalent of work (see discussions in Chapters 2 and 5). In view of the high temperature of the primary source, thermal equivalent of work was obtained by using the maximum power plant efficiency of $40 \%$

Example calculations (Case F, with MFE):

Pipeline work input

Equipment $\triangle \mathrm{P}$ compression input

Net Exergy penalty ( $85 \%$ of $7.1 \mathrm{MW}$

$$
\text { exergy input) }
$$

$$
\begin{aligned}
& =26.1 \mathrm{MW} \\
& =26.1 \mathrm{MW} \dagger \\
& =\frac{-6.0 \mathrm{MW}}{\text { (credit) }}
\end{aligned}
$$$$
\text { Total 46.2 MW }
$$

Equivalent thermal input ( @ $40 \%$ ) $=115.5 \mathrm{MW}_{\text {th }}$ system efficiency $(\psi)=\frac{980}{1106+115.5}=80.2 \%$.

†Assumed to be equal to pipeline work input 


\subsection{DISCUSSION OF RESULTS}

In this section, the effects of changes in design variables on conversions, transportation flow rates, thermal duties, and overall system energetics are discussed. It is important to note that even though designs of the reformer and methanation plants are coupled to each other through material flows and compositions, this coupling is relatively weak and allows each end of CHP to be discussed independently. This simplification results mainly from the fact that at the methanation end chemical conversions are nearly complete, regardless of reformer conditions.

Another simplification results from the transportation analyses (Appendix 4) which conclude that it is undesirable to operate the methanation plant or the pipelines at a pressure significantly different from that at the reformer plant. Recovery of compression work using gas expanders is highly inefficient, and for the small distances involved there is no significant benefit in transport economics for operation at high pipeline pressures.

\subsubsection{Methanation Plant}

There are three basic decisions to be made in the methanation plant design:

\section{- Operating pressure}

- Temperature at the exit of the last catalyst bed

- Magnitude of $\mathrm{H}_{2} \mathrm{O}$ recycle

The choice of the operating pressure is tied to the conditions at the reformer plant, as mentioned above. It is true that there is a decrease in conversion as the pressure is lowered; however, for any reasonable choice of reformer pressure this decrease is insignificant for equilibrium temperature in the range from 600 to $700 \mathrm{~K}$.

The effect of lowering the exit temperature (Case A) is in general favorable but small. Overall conversion increases, flow rates decrease (particularly the $\mathrm{CH}_{4}$-rich gases), thermal exchange duties decrease, and the overall efficiency improves. The only disadvantage may be an increase in methanation reactor volume if catalyst activity and rate kinetics are too low. This is not considered a problem, since lower temperature catalysts for methanation can be designed to perform satisfactorily.

The issue of $\mathrm{H}_{2} \mathrm{O}$ recycle has not been explored in great detail because it is more of a catalyst performance question than a design tradeoff. If catalyst deactivation from carbon deposition is not a problem, it would be advantageous to have no recycle at all. This would lower the heat exchange duty and eliminate the need for MFE. 
It is the opinion of the authors that all cases with the possible exception of Cases $A$ and $\mathrm{C}$ can be operated without $\mathrm{H}_{2} \mathrm{O}$ recycle at the methanation end. In most instances $\mathrm{H}_{2} \mathrm{O}$ recycle to limit the adiabatic temperature rise would be necessary only if the methanation feed composition approaches pure $\mathrm{CO}$ and $\mathrm{H}_{2}$.

The last point deserving mention relates to the quality of delivered heat. In most of the cases studied, roughly $75 \%$ of the delivered energy is available at high temperatures $(700$ to $1000 \mathrm{~K})$ and the remainder at lower temperatures. The distribution of this latter amount shows up clearly in the histograms (see Appendix 2, section A2.3). Since even this lower temperature heat is more than adequate for most process steam users, the methanation plant has not been penalized for it. The results should provide enough information on the effects of design changes on the thermal distribution for the study of a specific application.

\subsubsection{Reformer Plant}

The various design options explored in Cases B through $\mathrm{J}$ can be grouped into the following five categories:

- Use of separate or mixed feed evaporation

- Variations in $\mathrm{H}_{2} \mathrm{O}$ recycle

- Peak process temperature variations

- Choice of different operating pressures

- Change in stoichiometry by $\mathrm{CO}_{2}$ addition

\section{3 .2 .1 Use of $\mathrm{MFE}$}

A comparison of the net exergy flows to the powerplant with MFE and without shows clearly the range of possible improvements. The net result is seen to be a reduction in the total work input by a minimum of $24 \mathrm{MW}$ (Case E) to a maximum of $67 \mathrm{MW}$. In terms of overall efficiency improvements, the use of MFE is worth a gain by 5 to $10 \%$.

It should be noted that the net reductions in exergy penalty in all cases is found to be approximately equal to the exergy loss associated with irreversible mixing of steam and methane-rich gas (see Appendix 2 section A2.4.3 and also Chapter 5).

It is concluded that the use of MFE does indeed offer a significant efficiency improvement for all the design cases. The magnitude of exergy credit depends to some extent on the overall conversion and the amount of $\mathrm{H}_{2} \mathrm{O}$ recycle.

Since the effects of design variations exhibit similar trends with or without MFE, the essential conclusions in the remainder of this section are valid in either case. 


\subsubsection{2 $\mathrm{H}_{2} \mathrm{O}$ Recycle}

Increasing the $\mathrm{H}_{2} \mathrm{O}$ recycle enhances the conversion because of a higher $\mathrm{H}_{2} \mathrm{O} / \mathrm{CH}_{4}$ ratio, thereby increasing the energy density and reducing the transport flow rates. The disadvantage of a high $\mathrm{H}_{2} \mathrm{O}$ recycle is the resultant increase in heat exchange load and associated exergy losses. The indications are that the disadvantages outweigh the advantages. As a result, it is generally advantageous to use lower $\mathrm{H}_{2} \mathrm{O} / \mathrm{CH}_{4}$ ratios. Once again the problem of carbon deposition has to be faced. It is difficult to specify the optimum $\mathrm{H}_{2} \mathrm{O}$ recycle, which should take into account economic considerations. However, it appears that for the refexence case design it would be advantageous to use a lower $\mathrm{H}_{2} \mathrm{O}$ recycle.

Another feature to be noted is that in cases where a high conversion is obtained, a reduction in $\mathrm{H}_{2} \mathrm{O}$ recycle is an important way of trading back some of the conversion for a reduction in equipment duties and irreversibilities. This is demonstrated by a comparison between Cases $\mathrm{D}$ and $\mathrm{E}$.

\subsubsection{Peak Process Temperature}

There is a significant incentive to increase the peak temperature above $1100 \mathrm{~K}$ if possible. The advantages of higher conversion are reflected in reductions in equipment duties, transportation flow rates, and reformer duty. All of these factors contribute toward an increased efficiency. The possibility of reducing $\mathrm{H}_{2} \mathrm{O}$ recycle made possible by favorable thermodynamics leads to an even greater reduction of thermal duties. Of course, the ability to reach higher process temperatures is not dependent on the design, but rather on materials, safety, and nuclear fuel design issues. What this design analysis does provide is a measure of the incentive to achieve higher process temperatures.

A counterpart of this issue is to examine possible penalties associated with lower process temperatures. Case F demonstrates the nature of the problems to be faced -- lower conversions, higher losses, and higher equipment duties. It is interesting to note that lowering the pressure helps offset much of the penalty due to a low temperature. It is important to remember that the disadvantages of low pressure operation do not show up prominently in the design analyses; this important point will be discussed in the next category. The conclusion on lower temperature operation is that $975 \mathrm{~K}$ is close to the lower limit of operation, and even then there is a stiff penalty to be paid, as shown by large heat exchange duty, pipeline work inputs, and a very low energy density.

\subsubsection{Choice of Operating Pressure}

The selection of reformer pressure may be dictated by interface problems and materials capability at elevated temperatures. This limitation aside, Cases $\mathrm{G}$ and $\mathrm{H}$ show that a lowering of pressure, especially in cases with low conversions, is generally 
beneficial in reducing equipment duties and flow rates. In fact, no specific disadvantages are indicated by the results in Tables 6-2, -3 and -4 . Since penalties associated with low pressure operation have been underestimated in this study -- a result deriving from assumptions about work needed to overcome equipment pressure drops and about pipeline cost dependence on the pressure - - this topic deserves a discussion here.

The major effect of low-pressure operation of the reformer is in reducing correspondingly the transmission and methanation pressures. This is so because it is preferable to operate the whole system at lower pressures than to take a large penalty in compression energy that cannot be recovered. The disadvantages of a low-pressure operation of reformer and methanation plants is that reactor volumes become large, heat transfer coefficients may suffer, and the pressure drops in reactors as well as heat exchangers cost much more in energy. At low pressures the transportation involves large pipelines and increased friction. Unfortunately, the assumptions of the transportation analyses do not penalize low-pressure operation sufficiently; the material cost, which is dominant for large pipes, remains approximately the same; the increase in diameter is compensated by a reduction in wall thickness. This will not be true in the limit, as some criterion other than hoop stress will determine wall thickness. Thus the pipeline compressor work is underestimated and, in view of the assumptions, so is the work to overcome equipment pressure drops. A full accounting of low-pressure designs relative to the reference case will involve extensive equipment design and optimization, which are not warranted at this stage.

\section{3 .2 .5 Addition of $\mathrm{CO}_{2}$}

Advantages of $\mathrm{CO}_{2}$ addition, which takes the system toward an overall stoichiometry representative of the HYCO system (R6-2), include a potential for eliminating water transport in cold climates, an increase in the average quality of the delivered heat, and a reduction in boiler/condenser duties. Disadvantages of $\mathrm{CO}_{2}$ addition consist of an increase in pipeline gas flow rates, an increase in reformer duties (but with corresponding decrease in extraction steam from the powerplant), and perhaps a reduction in average heat transfer coefficient.

Results show that disadvantages outweigh advantages, and the efficiency indices show this. It should be noted that work to overcome equipment pressure drops may have been overestimated in this case, since the volumetric flow rates through reformer and most of the heat exchangers do not increase as markedly as the pipeline flow rates. For example, molar flow rate at the exit of reformer reactor is $128.7 \mathrm{t}$-moles/hr for Case $\mathrm{J}$ in comparison to $124.3 \mathrm{t}$-moles/hr for the reference case, but the pipeline flowrates show a $27 \%$ increase in the co-rich gases. If work required for equipment pressure drops to be the same as that in the reference case, the efficiency index rises to $84.7 \%--$ considerably 
closer to the reference case. Purely from thermodynamic reasons, it can be argued that since Cases $I$ and $J$ deliver approximately the same methanation energy. but do so at a higher exergy ratio (cf. section $A 2.2$ ), this must be reflected in a reduction of firstlaw efficiency. A conclusion of this study is that $\mathrm{CO}_{2}$ addition is more attractive for cases where high overall conversion (high process temperature) is attainable; transportation losses and heat exchanger losses may be less important in such cases than when the conversions are low. It may also be more attractive in those applications where the delivered energy needs to be of high grade.

\subsection{SUMMARY}

In this chapter, ten design cases were selected and analyzed. Energy flows, heat exchange duties, and overall efficiencies were calculated both with and without the use of mixed feed evaporator (MFE). The following main conclusions were reached:

\section{Overail System}

- For all the cases studied, overall energy efficiencies were satisfactorily high, ranging from 80 to $90 \%$ with. $\mathrm{MFE}$, and 70 to $85 \%$ without MFE.

Methanation Plant

- There is little incentive for choosing a methanation pressure markedly different from that of the pipeline or the reformer plant.

- Lowering the exit temperature below $700 \mathrm{~K}$ is desirable if satisfactory catalyst activity can be maintained. The overall design is insensitive to a decrease in exit temperature much below $650 \mathrm{~K}$.

- It is highly desirable to eliminate the need for $\mathrm{H}_{2} \mathrm{O}$ recycle. For most of the design cases this can be achieved without the danger of catalyst deactivation due to carbon deposition.

Reformer Plant

- The use of MFE leads to a significant improvement in overall efficiency for all design cases. The net effect on the total work requirements is a reduction in the range of 24 to $67 \mathrm{MW}$. This results in a 5 to $10 \%$ increase in overall efficiency.

- It is generally advantageous to lower the $\mathrm{H}_{2} \mathrm{O} / \mathrm{CH}_{4}$ ratio especially when conversions are high. Major limitations are due to carbon deposition and a reduction in overall conversions: 
- There is a significant incentive to increase the peak process gas temperature above $1100 \mathrm{~K}$. The lower limit on the peak temperature is in the vicinity of $975 \mathrm{~K}$. Low pressure operation is required to offset the reduction in conversion at these low temperatures.

- Addition of $\mathrm{CO}_{2}$ does not appear to be an advantage, especially in cases where conversions are not very high. Pros and cons of $\mathrm{CO}_{2}$ addition involve such complex tradeoffs that it is not possible to make a definitive recommendation of the optimal stoichiometry.

\section{REFERENCES -- CHAPTER 6}

R6-1 Private communication with G.A. White of The Ralph M. Parsons Company.

R6-2 Wentorf, R.H. Jr., U.S. Patent 3,953,625 (1977). 


\section{Chapter 7}

\section{ECONOMIC ANALYSIS OF HTCHP}

\subsection{INTRODUCTION}

The technical analyses in the previous chapters have shown that the high temperature chemical heat pipe (HTCHP) should be technically feasible and should operate with a high efficiency. However, in discussing the desirability of the HTCHP concept, the system economics must also be considered, to determine the cost to the consumer of the delivered heat. This will be done in section 7.2, where the individual component costs will be presented in detail for the reference case design and these component costs will be used to determine "rule-of-thumb" cost estimates for major components. The cost of these major components will be used along with the cost of the power required by the process to determine the incremental cost for the transportation of heat via the HTCHP and the cost of delivered heat (including the cost of the primary heat). In section 7.3, similar analyses will be performed for the various base case designs, utilizing the rule-of-thumb costs for major components developed in the reference case analysis.

The economics of one- to three-shift process steam use, onsite storage for peak electricity use, distributed generation of peak electricity, and combined electricity-steam delivery will be investigated in section 7.4. Section 7.5 will discuss the economics of solar heat on-site storage and transportation; the comparative economics of different heat transport systems and heat sources will be investigated in section 7.6. Finally a summary of the major conclusions from these economic analyses will be given in section 7.7 .

\subsection{COMPONENT COSTS AND REFERENCE CASE ECONOMICS}

\section{2 .1 Background}

The individual component costs for the reference case economic evaluation are presented here. An attempt will be made to explain these costs in detail so that the effect of process design assumptions or component cost.changes on the overall system economics may be easily determined. In choosing the costs employed in this analysis, as much industrial background has been used as possible, to develop reasonable costs; however, this analysis and the resulting economics should be taken as preliminary estimates. The goal is development of order-of-magnitude costs and a reasonable procedure for quickly determining the effect of changes in process parameters on system economics. The reference case economic analysis will be divided into three major sections. 1) the reforming process, 2) the transportation system, and 3) the methanation process. 


\subsubsection{Reforming Process Economics}

The reforming process shown in Figure 5-7 consists of the nuclear reformer and the heat exchangers involved in the processgas heat exchange, the steam superheating and boilerwater preheat, and the extraction steam preheat for the process gases.

The nuclear reformer presents a costing problem, since it has not been built. However, the approach used here is to assume that the major part of the materials cost of this unit is the cost of the reformer tubes, to cost these tubes in detail, and then to apply an installation factor for assembling the tube bundles in the reformer shell. The size of the tubes is determined by catalyst dimensions, internal pigtail sizes, the size required to remove spent catalyst, and creep limits on the materials used. The current KFA design (R7-1) utilizes tubes of $100 \mathrm{~mm}$ ( 4 in.) i.d. with a wall thickness of $15 \mathrm{~mm}$ ( $0.5 \mathrm{in.})$. Creep rates of 0.0001 $\% / h r$ are used in determining. the wall thickness. Since the methane reforming reaction kinetics are fast, the tube length can be determined solely on the basis of heat transfer considerations. The design heat flux is assumed to be $20,000 \mathrm{Btu} / \mathrm{hr}-\mathrm{ft}^{2}$ (based on the inside area), which yields a tube length of 10 meters for the KFA design.

The common material used in gas-fired reformer tubes is a castable version of 310 stainless steel (25\% $\mathrm{Cr} 20 \% \mathrm{Ni}$ ) termed HK-40. Other materials* have been developed which have improved stress characteristics that allow thinner walls, resulting in smaller temperature differences between the helium heating fluid and the process gas stream. An inquiry to a tube manufacturer (R7-3) has shown that these improved materials cost somewhat more per pound; thus, the total cost of a reformer tube remains essentially constant despite the thinner walls needed by advanced alloy tubes. These costs were estimated to be approximately $\$ 1600$ per tube (10 meters long). Using a heat flux of 20,000 Btu/hr-ft ${ }^{2}$, the material costs for the tube amount to $\$ 8 / \mathrm{kW}$. Normal installation factors for heat exchangers range from 3 to 3.5 (R7-4). However, considering that this exchanger must include the internal pigtail exchangers and be installed in a confining enclosure, an installation factor of 4.25 was chosen, to yield an installed cost of $\sim \$ 34 / \mathrm{kW}$ th and the total reactor cost of $\$ 34.1 \times 10^{6}$. The design assumptions for the nuclear reforming unit are summarized in Table 7-1.

The process gas exchangers and the heat exchangers required for the heat exchange with the associated powerplant lend themselves to a more conventional analysis. A detailed evaluation of exchanger design, heat transfer coefficients, sizes, and costs are given in Appendix 3 for the reference case conditions, with $25 \mathrm{~K}$ minimum temperature differences between the exchanging streams. It is reasonable to charge the costs of only a portion of these exchangers to the HTCHP system and the remainder to the associated powerplant. The approach used in this analysis is to charge to the HTCHP the

*In particular, an alloy known as Mo-Re 1 (35\% $\mathrm{Ni}, 28 \% \mathrm{Cr}, 1.5 \% \mathrm{~W}$ ) $(\mathrm{R} 7-2)$. 
Table 7-1

DESIGN AND ECONOMIC PARAMETERS FOR COST

ESTIMATION OF NUCLEAR REFORMER

$\begin{aligned} \text { Tube Dimensions - } 100 \mathrm{~mm} & -\mathrm{i} . \mathrm{d} . \\ 15 \mathrm{~mm} & - \text { wall thickness } \\ 10 \mathrm{~m} & - \text { length }\end{aligned}$

Design Heat Flux $-20,000 \mathrm{Btu} / \mathrm{hr}-\mathrm{ft} \mathrm{t}^{2}$

$6.31 \mathrm{~W} / \mathrm{cm}^{2}$

Reformer Tube Cost - $\$ 1600 /$ tube $\left(\$ 8 / \mathrm{kW}_{\mathrm{th}}\right)$

(Uninstalled)

Installation Factor - $4.25 \mathrm{X}$

Installed Reformer Cost $-\$ 34 / \mathrm{kW}_{\mathrm{th}}$

Total Reference Case Reactor Cost - \$34.1×106

process gas exchangers and the exchanger delivering heat to the HTCHP system from extraction steam. The exchangers delivering heat to the powerplant in the form of steam superheat and boilerwater preheat are charged to it.

Some exchangers use ordinary carbon steel for the tube material and will cost on the order of $\$ 20 / \mathrm{ft}^{2}$ (FOB) (Appendix 1 , R7-5). Others, operating at a temperature below the dewpoint of water in the process gas stream, must be constructed of high alloy steel to prevent corrosion in the presence of $\mathrm{CO}_{2}$ and liquid water, and will cost on the order of $\$ 30 / \mathrm{ft}^{2}$. An average cost of $\$ 25 / \mathrm{ft}^{2}$ was assumed for this analysis. The design presented in Appendix 3 shows that those heat exchangers charged to the HTCHP system operate at an average heat flux of $3.7 \mathrm{~kW} / \mathrm{ft}^{2}$ based on a minimum temperature difference $(\Delta T)$ of $25 \mathrm{~K}$. This heat flux can be decreased by $20 \%$ (a conservative estimate) to agree with the $20-\mathrm{K} \Delta \mathrm{T}$ used in the reference case efficiency analysis. This results in a heat flux of $3 \mathrm{~kW} / \mathrm{ft}^{2}$ and a cost of $\$ 8.33 / \mathrm{kW}_{\mathrm{th}}$ (FOB). Using a factor of 3 for installation costs (R7-4), the average heat exchanger cost amounts to $\$ 25 / \mathrm{kW}_{\mathrm{th}}$. In the HTCHP reference case $1249 \mathrm{MW}$ are transferred in the exchangers, resulting in a total heat exchanger cost of $\$ 31.2 \times 10^{6}$. These design and economic assumptions are summarized in Table 7-2.

\subsubsection{Compressor and Pump Costs}

Because of the large scale of the HTCHP flowrates, centrifugal compressors are most likely to be used, as is common practice in natural gas transmission. Costs for these types of compressors in pipeline applications (R7-6) vary from $\$ 250 / \mathrm{kW}$ to $\$ 900 / \mathrm{kW}$, with an average of about $\$ 500 / \mathrm{kW}$. A major difference between natural gas 
Table 7-2

DESIGN AND ECONOMIC ASSUMPTIONS FOR HEAT

EXCHANGERS FOR REFERENCE CASE

$\begin{array}{ll}\begin{array}{l}\text { Heat Exchanger Cost } \\ \text { (Uninstalled) }\end{array} & \$ 25 / \mathrm{ft}^{2}\left(\$ 269 / \mathrm{m}^{2}\right) \\ \text { Installation Factor } & 3 \mathrm{x} \\ \text { Average Heat Flux } & 3 \mathrm{~kW} / \mathrm{ft}^{2}\left(3.2 \mathrm{~W} / \mathrm{cm}^{2}\right) \\ \text { Total Heat Transferred } & 1249 \mathrm{MW} \mathrm{th} \\ \text { Total Reference Case Heat Exchanger Cost } \$ 31.2 \times 10^{6}\end{array}$

transmission compressors and HTCHP compressors will affect the cost. Compressors on site at the powerplant might well be hooked directly to the steam turbine drive. This would allow the elimination of both the generator and the motor. Since the compressor itself is only about $10 \%$ of the above cost (the rest being the driver and installation) this approach would significantly decrease the capital cost of the centrifugal units. Other analyses (R7-7) show axial flow compressors which also might be used to cost on the order of $\$ 65 / \mathrm{kW}$. Therefore, the compressors in this analysis are costed at $\$ 100 / \mathrm{kW}$.

Pumps are somewhat more expensive than compressors for the same power rating. Pump costs (R7-4) were fitted to equations giving the cost as a function of the power requirements and updated to a mid-1976 time base (R7-8). These equations are presented in detail in Appendix 4 (section 4.2).

The compression work required to overcome the process equipment pressure drop has been estimated to be equivalent to the transportation pressure drop. Assuming that this work is divided equally between the two processes, the reformer compression work amounts to 0.5 of the transportation compression work. Thus, the compression work for the reference case reformer process amounts to $9.7 \mathrm{MW}$. At $\$ 100 / \mathrm{kW}$, the total compressor capital cost amounts to $\$ 1 \times 10^{6}$.

Combining compressor, heat exchanger, and reactor costs results in a total reformer process cost of $\$ 66.3 \times 10^{6}$, or $\$ 66 / \mathrm{kW}_{\mathrm{th}}$ transmitted. In the base case economic analyses that follow, the reforming reactors will be costed at $\$ 34 / \mathrm{kW}_{\mathrm{th}}$, the heat exchangers at $\$ 25 / \mathrm{kW}_{t h}$, and compressors at $\$ 100 / \mathrm{kW}_{e}$.

\subsubsection{Transmission Line Costs}

A detailed evaluation of gas and liquid transmission for CHP applications is included as Appendix 4 of this report. The cost parameters presented there in detail are summarized briefly here. There are two major cost components in the transportation systems: 1) the pipeline, and 2) the compressors and pumps required to overcome the pressure drops in the lines. 
Pipeline costs are highly dependent on the particular application, since installation costs vary widely with terrain and weather conditions. In this study, the most recent data available from the Federal Power Commission (R7-6) were used to determine pipeline costs as a function of line diameter. An equation was fitted to these data in the form

$$
\text { Cost }=a+b D+c P D^{2}
$$

where

$$
\begin{aligned}
& \text { Cost }=\text { pipeline capital cost, } \$ / \mathrm{kM}-\mathrm{yr} \\
& \mathrm{D} \quad=\text { pipeline diameter, meters } \\
& \mathrm{P} \quad=\text { pipeline pressure, bars }
\end{aligned}
$$

The three terms of the polynomial approximately represent rightof-way costs (independent of line diameter), installation costs (proportional to the line diameter), and materials cost (proportional to the diameter squared and the transmission pressure). Although pipelines come in only discrete diameters, this equation allows a simple optimization formula to be developed which can readily be used to determine the economically optimum line diameter, given flowrates and capital and energy costs for the pumps and compressors. Throughout these analyses, a capitalization factor* of 0.2 has been used. No attempt was made to predict future trends in pipeline costs.

Normal natural-gas transmission line costs (as represented by the FPC data) are for single lines installed in a trench. HTCHP applications will require at least two lines. The installation cost per line for multiple lines per trench should be reduced considerably, since only one trench (although bigger) must be excavated and refilled and only a single right-of-way must be purchased. To account for the decreased installation cost, the second term of the pipeline cost equation was modified to allow for an "effective" diameter:

$$
D_{e}=\left(D_{1}^{2}+D_{2}^{2}+\ldots D_{n}^{2}\right)^{1 / 2}
$$

In addition, only one right-of-way cost, independent of the number of lines used, was included in the total cost.

*The capitalization factor is defined as the portion of the capital cost of equipment charged to the operating cost. It includes factors such as depreciation, taxes, insurance, and operating and maintenance costs. 


\subsubsection{Compressor Costs}

A detailed analysis of different transportation philosophies presented in Appendix 4 shows that it is economically most attractive in compressing the gas streams at the ends of the transmission lines to use less expensive compressors hooked directly to the steam turbines. In this analysis, therefore, compressor costs were assumed equal to $\$ 100 / \mathrm{kW}_{e}$ as in the reformer plant itself. Pump costs were also calculated by the formulas presented in Appendix 4 .

\subsubsection{Total Transportation Costs}

Once both the pipeline costs and compressor and pump costs were determined, computer programs were written to evaluate the pipeline, compressor, and pump sizes and costs as a function of the composition and rate of flow of the material transported. These costs are explored in detail in Appendix 4, where costs for various flowrates and optimum line costs are given. It should be noted that the cost of electricity has an impact on total pipeline costs as well as power consumption during transportation. Because pipelines using higher power costs will tend to be larger (and cost more) power usage will be decreased. However, this power decrease is reflected in increased efficiency of transmission.

The cost coefficiencts used in this report and an electricity cost of $20 \mathrm{mills} / \mathrm{kWh}$ result in a $\$ 60.4 / \mathrm{kW}$ line cost and a power consumption of $19.2 \mathrm{MW}$ electric.

\subsubsection{Methanation Process}

No actual construction costs are available for the high temperature methanation process proposed for use in the HTCHP system. However, a combination of the costs developed for the reformer process and those presented in Appendix 1, "Review of Methanation Technology," were used to estimate the cost of the components of the process used in this design. Process-gas heat exchangers were costed at $\$ 20 / \mathrm{kW}_{\mathrm{th}}$. This is slightly lower than the reformer heat exchange costs, since higher heat fluxes can be expected in these exchangers. The methanator itself and the heat delivery exchangers were also costed at $\$ 20 / \mathrm{kW}_{\text {th }}$ of heat delivered. This is reasonable because even higher heat fluxes are expected in these exchangers as compared with the process-gas exchangers, and an additional cost must be added to account for the reactors.

These assumptions result in a cost of approximately $\$ 14 \times 10^{6}$ for the reactors and heat delivery exchangers and $\$ 13 \times 106$ for the process-gas heat exchangers (including those required to deliver the water condensation heat). Compressor costs were calculated as in the reformer section. A total of 9.7 MW of compression energy is required at $\$ 100 / \mathrm{kW}$, resulting in a total capital investment of $\$ 1 \times 10^{6}$. Thus the total methanation process cost amounts to $\$ 28.6 \times 10^{6}$, or $\$ 28.6 / \mathrm{kW}_{\mathrm{th}}$. 
To summarize, the cost estimates in this section result in the following system capital costs:

$\begin{array}{lr}\text { Reformer } & \$ 66.3 / \mathrm{kW}_{\mathrm{th}} \\ \text { Transportation } & \$ 60.4 / \mathrm{kW}_{\mathrm{th}} \\ \text { Methanation } & \frac{\$ 28.6 / \mathrm{kW}}{\mathrm{th}} \\ & \frac{\$ 155.3 / \mathrm{kW}}{\mathrm{th}}\end{array}$

To calculate the cost of the delivered energy, the capitalization factor of 0.2 can be applied to this total capital cost to obtain a capital cost component for the cost of delivered heat. In the reference case, this amounts to $\$ 31.1 \times 106 / \mathrm{yr}$. In addition to the cost capital component, the cost of the heat and electricity must be added to the delivery cost. The reformer heat cost will be assumed to be $\$ 2.50 / \mathrm{GJ}$ (see Chapters 5 and 15) and electricity will be valued at $20 \mathrm{mill} / \mathrm{s} / \mathrm{kWh}$. The work requirements for the process must include the work required by the compressors and pumps and a penalty associated with the loss (or in some cases, gain) of exergy experienced by the powerplant due to the heat exchange with the process. A powerplant utilizes the exergy delivered to it at $\sim 85 \%$ to produce work in the form of electricity (see chapter 5 and Appendix 2). Therefore, a work requirement (or credit) will be. charged to the process at the rate of $85 \%$ of the exergy loss of the powerplant. The work requirements of the reference case can be summarized as follows:

\begin{tabular}{|c|c|c|}
\hline & $\begin{array}{c}\text { With MFE } \\
\mathrm{MW}_{\mathrm{e}} \\
\end{array}$ & $\begin{array}{r}\text { Without } \\
\mathrm{MW}_{\mathrm{e}} \\
\end{array}$ \\
\hline Reformer $\triangle \mathrm{P}$ work & 9.65 & 9.7 \\
\hline Powerplant exergy loss & 20.30 & 54.7 \\
\hline Transportation work & 19.30 & 19.3 \\
\hline Methanation $\triangle \mathrm{P}$ work & 9.65 & 9.7 \\
\hline Total work requirements & $58.9 \mathrm{MW}$ & 93.3 \\
\hline
\end{tabular}

At $20 \mathrm{mills} / \mathrm{kWh}$ and $8000 \mathrm{hr} / \mathrm{yr}$, the total power cost for this case with the Mixed Feed Evaporator (MFE) is $\$ 9.4 \times 106 / \mathrm{Yr}$. Of the 1003 MW absorbed in the reformer $986 \mathrm{MW}$ is delivered at the methanator, resulting in $16 \mathrm{MW}$ undelivered by the system. The CHP must pay for this undelivered heat (although in this accounting system, a portion is recovered in the credit obtained from the powerplant for the exergy given to it). The yearly cost of undelivered heat (at $\$ 2.50 / \mathrm{GJ}$ ) is $\$ 1.2 \times 10^{6}$.

Thus the total yearly incremental cost of the delivered heat above that of the primary source is $\$ 41.7 \times 10^{6}$, or $\$ 1.47 / \mathrm{GJ}$ (for $\left.2.8410^{7} \mathrm{GJ} / \mathrm{Yr}\right)$ : To obtain the total cost of delivered heat, the primary heat cost must be added to this incremental CHP cost. At $\$ 2.50 / G J$ for primary heat, the total cost of transported heat is $\$ 3.97 / \mathrm{GJ}$. Without the MFE the power cost becomes $\$ 14.9 \times 10^{6} / \mathrm{Yr}$. 
A similar calculation yields the incremental cost of delivered heat without the MFE as $\$ 1.66 / \mathrm{GJ}$ and the total cost of delivered heat as $\$ 4.16 / G J$. These calculations are summarized in Table 7-3. Although the preliminary nature of the costing undertaken here has been repeatedly noted, it is accurate enough to state that the cost of transporting reactor heat, for this reference case, should fall somewhere between $\$ 1$ and $\$ 2 / G J$ and the cost of delivered energy (at $\$ 2.50 / \mathrm{GJ}$ at the source) should not exceed $\$ 4.50 / \mathrm{GJ}$. Also shown in Table 7-3 is the cost of HTCHP transportation as a function of electricity cost. The cost of HTCHP transport is much more a function of the electrical cost than heat cost, since only a small fraction ( $16 \mathrm{MW}(\mathrm{h})$ of the heat absorbed in the reformer is not eventually delivered at the methanator.

Table 7-3

INCREMENTAL AND TOTAL COST OF HEAT

DELIVERED BY THE HTCHP

Cost of power $10^{6} \$ / y r$

Cost of capital $10^{6} \$ / \mathrm{yr}$

Cost of undelivered heat $10^{6} \$ / y r$

Total incremental yearly cost $10^{6} \$ / y r$

Incremental cost of heat, $\$ / G J$

$987 \mathrm{MW}=(2.84 \times 107 \mathrm{GJ} / \mathrm{Yr})$

Total yearly cost of primary heat $10^{6} \$ / \mathrm{yr}$

Total yearly cost of delivered heat $10^{6} \$ / y r$

Total cost of delivered heat, $\$ / G J$

HTCHP Cost as a Function of Electricity Cost

\begin{tabular}{ccc} 
With MFE & & Without MFE \\
\cline { 1 - 1 } 9.4 & & 14.9 \\
31.1 & & 31.1 \\
1.2 & & 1.2 \\
41.7 & & 47.2 \\
& & \\
1.47 & 1.66 \\
71.06 & 71.06 \\
112.8 & & 118.3 \\
3.97 & 4.16
\end{tabular}

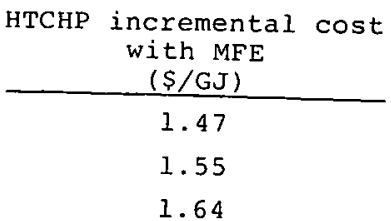

\subsection{BASE CASE ECONOMICS}

\subsubsection{Introduction}

One of the major values of this type of economic analysis is its use in measuring the impact of process changes on the cost of the delivered energy. This will be done in the following analyses for the base cases presented previously.

The procedure and major component costs developed in the reference case will also be employed here. The economics for all the base cases will be developed simultaneously and will be followed by a brief discussion of the impact of the various process design changes on the economics of heat transport. 


\subsubsection{Economic Calculations}

Table 7-4 summarizes the heat duties and work requirements for the different base cases. The base cases are lettered as in the preceding chapter, where the total work requirements are presented in Table 6-4.

Table 7-4

HEAT AND WORK REQUIREMENTS FOR BASE CASE PROCESS DESIGNS

\begin{tabular}{|c|c|c|c|c|c|c|}
\hline \multirow[b]{2}{*}{ Case } & \multirow{2}{*}{$\begin{array}{c}\text { Reformer } \\
\text { Reactnr Input } \\
\text { (MW) } \\
\end{array}$} & \multirow{2}{*}{$\begin{array}{l}\text { Reformer }{ }^{*} \\
\text { Hcat Exclaanger } \\
\text { Duty (MW) } \\
\end{array}$} & \multicolumn{2}{|c|}{$\begin{array}{l}\text { Total Process } \\
\text { Work Requirements }\end{array}$} & \multirow{2}{*}{$\begin{array}{l}\text { Methanator * } \\
\text { Heat } \\
\text { Exchanger } \\
\text { Duty (MW) } \\
\end{array}$} & \multirow{2}{*}{$\begin{array}{c}\text { Total } \\
\text { Methanition } \\
\text { Heat } \\
\text { Delivered } \\
\text { (MW) } \\
\end{array}$} \\
\hline & & & $\begin{array}{l}\text { With } \\
\text { MFE } \\
\end{array}$ & $\begin{array}{l}\text { Without } \\
\text { MFE }\end{array}$ & & \\
\hline Reference & 2003 & 1249 & 58.9 & 93.3 & 654 & 986 \\
\hline A & 992 & 1212 & 62.0 & 93.4 & 569 & 987 \\
\hline B & 1005 & 1420 & 66.5 & 100.1 & 571 & 986 \\
\hline C & 1010 & 1079 & 46.1 & 81.8 & 809 & 985 \\
\hline D & 981 & 934 & 56.1 & 81.8 & 488 & 988 \\
\hline $\mathrm{E}$ & 982 & 749 & 46.8 & 71.0 & 560 & 988 \\
\hline$F$ & 1106 & 2272 & 46.2 & 113.0 & 1192 & 980 \\
\hline G & 994 & 1320 & 60.4 & 94.0 & 690 & 985 \\
\hline $\mathrm{H}$ & 988 & 1082 & 56.0 & 80.6 & 565 & 987 \\
\hline I & 1148 & 1045 & 9.0 & 49.7 & 520 & 986 \\
\hline $\mathrm{J}$ & 1255 & 871 & $(24.0)$ & 13.5 & 397 & 986 \\
\hline
\end{tabular}

* See Table $6-4$, in Chapter 6.

** See Table 6-3, in Chapter 6 .

The capital costs for major equipment in the reforming process, transportation, and methanator process are summarized in Table 7-5 for each of the base cases. The numbers may be arrived at by combining the appropriate major component cost developed previously and the heat or work requirement. The only number that is not easy to derive from the previous data is the transmission capital cost. It has been obtained by determining the optimum pipeline diameter using computer programs documented in Appendix 6.

Table 7-5

CAPITAL COSTS FOR MAJOR EQUIPMENT FOR BASE CASES

\begin{tabular}{|c|c|c|c|c|c|c|c|c|c|c|}
\hline \multirow[b]{2}{*}{ Case } & \multicolumn{4}{|c|}{ Reformer } & \multirow{2}{*}{$\begin{array}{c}\text { Transportation } \\
\text { Total }\end{array}$} & \multicolumn{4}{|c|}{ Methanation } & \multirow[b]{2}{*}{ Total } \\
\hline & Reactor & $\begin{array}{c}\begin{array}{c}\text { Heat } \\
\text { Exchange }\end{array} \\
\end{array}$ & Compressor & Total & & Reactor & $\begin{array}{c}\begin{array}{c}\text { Heat } \\
\text { Exchange }\end{array} \\
\end{array}$ & Compression & Tota1 & \\
\hline Reference & 34.1 & 31.2 & 1.0 & 66.3 & 60.4 & 14.5 & 13.1 & 1.0 & 28.6 & 155.3 \\
\hline A & 33.7 & 30.2 & 0.9 & 64.8 & 58.1 & 15.9 & 11.4 & 0.9 & 28.2 & 151.1 \\
\hline B & 34.2 & 35.5 & 0.9 & 70.6 & 57.9 & 14.5 & 11.4 & 0.9 & 26.8 & 155.3 \\
\hline c & 34.3 & 27.0 & 1.0 & 62.3 & 65.5 & 14.4 & 16.2 & 1.0 & 31.6 & 159.4 \\
\hline D & 33.4 & 23.4 & 0.8 & 57.6 & 53.2 & 15.4 & 9.8 & 0.8 & 25.9 & 136.7 \\
\hline$E$ & 33.4 & 18.7 & 0.9 & 53.0 & 55.1 & 15.5 & 11.2 & 0.9 & 27.6 & 135.7 \\
\hline F. & 37.6 & 56.8 & 1.3 & 95.7 & 80.8 & 12.5 & 23.8 & 2.3 & 37.6 & 214.1 \\
\hline$G$ & 33.8 & 33.0 & 1.2 & 58.0 & 70.1 & 14.0 & 13.8 & 1.2 & 29.0 & 167.1 \\
\hline H & 33.6 & 27.1 & 0.9 & 61.6 & 58.9 & 14.9 & 11.3 & 0.9 & 27.1 & 147.6 \\
\hline I & 39.0 & 26.1 & 1.4 & 66.5 & 87.0 & 16.9 & 10.4 & 1.4 & 28.7 & 182.7 \\
\hline $\mathrm{J}$ & 42.7 & 21.8 & 1.6 & 66.1 & 103.9 & 18.8 & 7.9 & 1.6 & 28.3 & 198.3 \\
\hline
\end{tabular}


These capital costs are used in Table 7-6 to determine the yearly capital cost components of the cost delivered heat. Also shown are the yearly work cost component (both with and without the MFE) and the total yearly cost of undelivered and delivered heat.

Table 7-6

COMPONENTS CONTRIBUTING TO THE COST OF DELIVERED HEAT

\begin{tabular}{|c|c|c|c|c|c|c|}
\hline \multirow[t]{2}{*}{ Case } & \multirow[t]{2}{*}{$\begin{array}{c}\text { Capital Cost } \\
\text { Component } 10^{6} \$ / y x \\
\end{array}$} & \multicolumn{2}{|c|}{$\begin{array}{c}\text { Work Cost } \\
\text { Component } 10^{6} \mathrm{~S} / \mathrm{yr}\end{array}$} & \multirow[t]{2}{*}{ 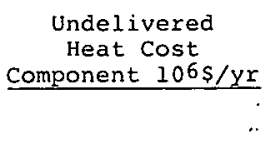 } & \multicolumn{2}{|c|}{$\begin{array}{c}\text { Total Incremental } \\
\text { Delivery Cost } \\
106 \$ / y x \\
\end{array}$} \\
\hline & & $\begin{array}{l}\text { With } \\
\text { MFE } \\
\end{array}$ & $\begin{array}{l}\text { Without } \\
\text { MFE }\end{array}$ & & $\begin{array}{l}\text { With } \\
\text { MrE } \\
\end{array}$ & $\begin{array}{l}\text { Wi. thout } \\
\text { MFE }\end{array}$ \\
\hline Reference & 31.1 & 9.4 & 14.9 & 1.2 & 41.7 & 47.2 \\
\hline A & 30.2 & 9.9 & 14.9 & 0.4 & 40.5 & 45.5 \\
\hline B & 31.1 & 10.6 & 16.0 & 1.4 & 43.1 & 48.5 \\
\hline c & 31.9 & 7.4 & .13 .1 & 1.8 & 41.1 & 46.8 \\
\hline D & 27.3 & 9.0 & 13.1 & $(0.5)$ & 35.8 & 39.9 \\
\hline E & 27.1 & 7.5 & 11.4 & $(0.4)^{\circ}$ & 34.2 & 38.1 \\
\hline$F$ & 42.8 & 7.4 & 18.1 & 9.1 & 59.3 & 70.0 \\
\hline G & 33.4 & 9.7 & 15.0 & 0.6 & 43.7 & 49.0 \\
\hline $\mathrm{H}$ & 29.5 & 9.0 & 12.9 & 0.1 & 38.6 & 42.5 \\
\hline I & 36.4 & 1.4 & 8.0 & 11.7 & 49.5 & 56.1 \\
\hline $\mathrm{J}$ & 39.7 & (3.8) & 2.2 & 19.4 & 55.3 & 61.3 \\
\hline
\end{tabular}

These total yearly costs are easily converted to incremental cost per unit of energy delivered (above that paid for the primary heat) as shown in Table 7-7. The total delivered cost, also shown, is calculated by summing the incremental cost and the cost of the heat at the reformer $(\$ 2.50 / \mathrm{GJ})$. It should be noted that this incremental transportation cost is a function of the reformer heat cost since, in almost all cases, the heat delivered at the methanator is less than that absorbed at the reforner. This excess heat is degraded and rejected as heat loss, and must be charged to the cost of CHP transmission.

\subsubsection{Base Case Economic Analyses}

In general, the economic analyses presented here confirm the general conclusions reached in the analysis of the base case energetics performed in the previous chapter. However, several points should be emphasized.

First, it is obvious that in all cases the use of the mixed feed evaporator is economically advantageous, although even without its use the incremental costs all range between $\$ 1.50 / G J$ and $\$ 2.50 / \mathrm{GJ}$. Comparison of the reference case with Case $\mathrm{C}$ and Case Dwith $\mathrm{E}$ makes clear that the economics favor low water recycle rates. Thus, it is better to achieve slightly lower conversions and pay the additional price for larger transmission pipelines than to recycle water and pay for the increased heat duty on the process gas exchangers. 
Table 7-7

INCREMENTAL COST AND TOTAL COST OF DELIVERED HEAT

FOR BASE CASE ECONOMIC ANALYSIS

\begin{tabular}{|c|c|c|c|c|}
\hline \multirow[b]{2}{*}{ Case } & \multicolumn{2}{|c|}{$\begin{array}{c}\text { Incremental Cost } \\
\text { of Delivered Heat } \\
\text { (\$/GJ) }\end{array}$} & \multicolumn{2}{|c|}{$\begin{array}{c}\text { Total Cost } \\
\text { of Delivered Heat } \\
(\$ / G J)\end{array}$} \\
\hline & $\begin{array}{l}\text { With } \\
\text { MFE } \\
\end{array}$ & $\begin{array}{l}\text { Wi thout } \\
\text { MFE }\end{array}$ & $\begin{array}{l}\text { With } \\
\text { MFE }\end{array}$ & $\begin{array}{l}\text { Wi thout } \\
\text { MFE } \\
\end{array}$ \\
\hline Reference & 1.47 & 1.66 & 3.97 & 4.16 \\
\hline A & 1.42 & 1.60 & 3.92 & 4.10 \\
\hline B & 1.52 & 1.71 & 4.02 & 4.21 \\
\hline c & 1.45 & 1.65 & 3.95 & 4.15 \\
\hline D & 1.26 & 1.40 & 3.76 & 3.90 \\
\hline $\mathrm{E}$ & 1.20 & 1.34 & 3.70 & 3.84 \\
\hline F & 2.10 & 2.48 & 4.60 & 4.98 \\
\hline G & 1.54 & 1.73 & 4.04 & 4.23 \\
\hline H & 1.36 & 1.50 & 3.86 & 4.00 \\
\hline I & 1.74 & 1.98 & 4.24 & 4.48 \\
\hline $\mathfrak{J}$ & 1.95 & 2.16 & 4.45 & 4.66 \\
\hline
\end{tabular}

The decreased conversion in the reformer plant actually shows up more in the methanation process where no decrease in water recycle was assumed; the heat loads and heat exchanger costs actually increase and offset to some extent the benefit achieved in the reformer. If it is practical to reduce or eliminate the water recycle in the methanator, the economics of low water recycle in the reformer will look even better. This type of detailed system optimization has not been undertaken here. The comparison of the high-temperature cases ( $D$ and $E$ ) also demonstrates the desirability of lowering the water recycle rate. In fact, at the higher conversions obtained at higher temperatures, the effect of lowering the water recycle rate is even more dramatic.

The advantage of high-temperature operation ( $D$ and $E$ ), discussed in the preceding chapter, is again demonstrated in these economic analyses. One might ask how much the process could afford to pay for this increased temperature. The answer could be obtained in several different forms, depending on whether the changes needed to achieve higher temperatures are in the heat source or in the reformer itself. Comparing Case $D$ and the reference case (Table 7-6), it is possible to spend an additional $\$ 5.9 \times 106 / \mathrm{yr}$ to obtain the higher process-stream temperature (with the MFE). If it is assumed that no changes are needed in the reformer, it is possible to pay an additional $20 \% / G J$ for the reactor heat $(2.70 / \mathrm{GJ}$ total) and still break even with the reference case economics. If changes could be made in the reformer, $\$ 29.5$ x106 (using a capitalization factor of 0.2 ) is available to bring about those changes. It is obvious from these quick estimates that there is incentive to obtain higher process-gas outlet temperatures. 
On the other hand, at temperatures below that of the reference case (Case $F$ ), the lower conversion causes much higher heat loads and costs for the process-gas exchangers and increased transmission costs. It appears that the 33\% conversion at $975 \mathrm{~K}$ is too low to be economically competitive with other process designs. Raising the conversion by lowering the reaction pressure helps reduce the cost; but, as mentioned previously, these low pressure costs may underestimate the actual cost as a result of underestimation of the effect of the process pressure drop and the cost of transmission at low pressure. From these analyses it appears that $65 \%$ conversion is adequate, higher conversion is desirable if it can be obtained with higher process-gas temperature rather than higher water recycle rates, and lower conversion is undesirable because of higher heat exchanger costs.

The addition of $\mathrm{CO}_{2}$ appears not to be economically beneficial. The lower heat exchanger costs are more than offset by the increased transmission costs. This option might be employed in situations where it is desirable to deliver all of the methanation heat at temperatures above $700 \mathrm{~K}$. The economics of such a case can be accurately evaluated only when some premium is placed on this high-grade heat; otherwise, $\mathrm{CO}_{2}$ addition should not be considered.

Finally, it should be noted that the HTCHP charges represent only about $40 \%$ of the final cost of delivered heat, the rest resulting from the cost of the heat at the reformer site. Thus, the relatively large changes in capital costs of the HTCHP are not dramatically reflected in the cost of HTCHP transportation. This tends to minimize the impact of process changes on the economics. However, equipment changes that greatly affect the process efficiency can dramatically alter the transportation cost. For example, the inclusion of the MFE in the reformer plant design saves approximately 40.4 MW of availability in the powerplant or $34.4 \mathrm{MW}$ of electrical output (for the reference case conditions). Without this process change the work cost component of the HTCHP transportation cost would increase by $58 \%$, or $\$ 5.5 \times 106 / \mathrm{yr}$ for the reference case (using $20 \mathrm{mills} / \mathrm{kWh}$ electricity). This amounts to $13 \%$ of the cost of HTCHP transportation, or 19\%/GJ. At an electricity cost of $30 \mathrm{mills} / \mathrm{kWh}$ and the same process design, the use of the MFE saves approximately $18 \%$ of the cost of the HTCHP transportation, or $29 \mathrm{c} / \mathrm{GJ}$. A process utilizing the MFE would thus have a notable economic advantage over one not employing it.

The other manifestation of the fact that HTCHP costs are not significantly affected by the capical cost of the process is the fact that the costs presented here should represent fairly well the actual cost of the HTCHP heat. Thus, even if the capital cost estimates are off by a significant amount, the overall HTCHP cost to the delivered heat should stay within the range of $\$ 1.00$ to $\$ 2.00 / \mathrm{GJ}$ and the cost of delivered heat should be competitive with other sources. This will be discussed in more detail in the following section on HTCHP applications. 


\subsection{APPLICATION ECONOMICS}

\subsubsection{Introduction}

In this section, the economics of process steam delivery in one to three shift, on-site storage for peak electricity, distributed generation of peak electricity, and combined electricitysteam delivery are investigated. The intent here is to demonstrate economic trends and general conclusions whose absolute values may vary only slightly with the design parameters chosen and the component costs used. As has been shown in the base case analysis in the previous section, the overall system economics are not a strong function of the various designs, and the general conclusions reached in this section should be applicable for any of the designs chosen.

The component costs, energy costs, and ground rules used in these analyses are shown in Table 7-8. The assumed cavern cost is more typical of mined hard rock caverns (R7-9) than of leached salt caverns (R7-7). In the absence of underground storage experience with hydrogen-containing gases it is not clear whether it will be practical to use the cheaper forms of underground storage, such as salt domes or aquifers. In any event, the cavern cost does not play a significant role in the overall storage economics.

Table 7-8

COSTS AND GROUND RULES FOR APPLICATIONS ANALYSIS

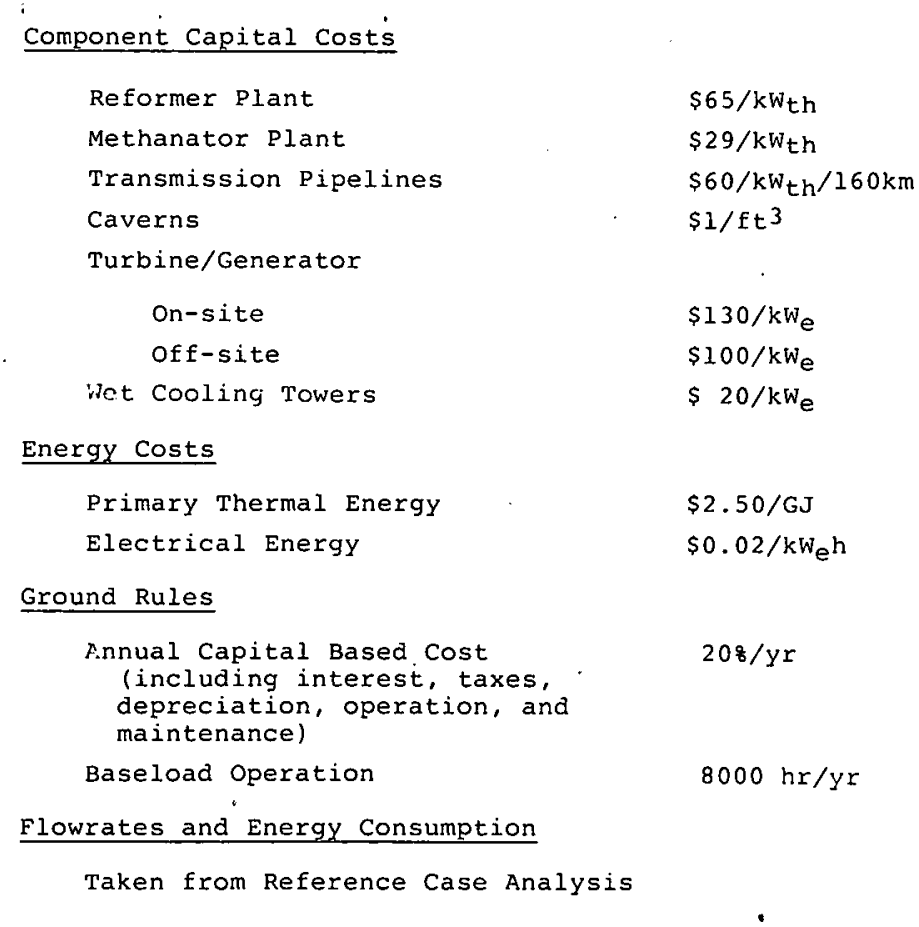




\subsubsection{Process Steam}

The following assumptions were made in calculating the delivered process steam costs. The primary energy source, the reformer plant, and the transmission lines are operated at baseload capacity $(8000 \mathrm{hr}$ ' $\mathrm{yr})$. The output methanator is sized according to energy demand and draws energy during an average* fraction (p) of total daily hours. The pipeline provides sufficient storage to absorb demand fluctuations.**

The cost of delivered process steam, broken down into constituent components, is shown in Figure 7-1. These costs are for delivered steam at conditions comparable to the highest grade boiler steam ( $10000 \mathrm{~F})$. Therefore, any comparison with alternative fuel supplies should include not only the cost of the alternate fuel but also furnace efficiencies and boiler-related capital and operating costs. The following conclusions can be drawn from this figure:

- The incremental cost (in excess of baseload steam for $\mathrm{p}=1)$ for the part-time supply of process steam is very low (only $7 \xi / G J$ for $p=2 / 3$ and $20 \% / G J$ for $p=1 / 3$ ). This makes the HTCHP particularly well suited to the supply of one- and two-shift industrial process steam.

- For the assumed nuclear heat cost, the delivered process steam cost compares quite favorably with major alternatives for small-scale process steam supplies. For example, with SNG at $\$ 4 / G J$ and $80 \%$ furnace efficiency, the delivered process steam would cost approximately \$5/GJ. At the assumed value... of electricity $(20 \mathrm{mills} /$ $\left.\mathrm{kW}_{e} \mathrm{~h}\right)$; the cost of steam from an electrode boiler would be approximately $\$ 6.33 / \mathrm{GJ}$; for larger scale coal-fired boilers, the delivered steam cost would be slightly lower than that by CHP. In view of the uncertainties in primary heat cost and the differences in the state of readiness of these technologies, it appears that coal-fired boilers would be preferable for large-scale baseload process steam supplies. It is not possible to state with any certainty what the critical size is beyond which coal-fired boilers would be preferred; a reasonable estimate is somewhere in the range of 100 to $500 \mathrm{MW}$ th.

- Transmission costs amount roughly to $0.3 \mathrm{f} / \mathrm{GJ} / \mathrm{km}$. As might be expected this is considerably more than the

*In the case of a reduced demand during the weekend, the value of $\mathrm{p}$ should be averaged over the whole week.

**In reality, as a result of these fluctuations, the pipeline capacity factor will be less than the baseload but greater than that for the methanator. A detailed design of a pipeline grid for multiple uses is beyond the scope of the present project. 
SNG transmission cost ( $20.02 \mathrm{c} / \mathrm{GJ} / \mathrm{km})$ but much less than steam transmission costs $(2.5 \mathrm{k} / \mathrm{GJ} / \mathrm{km})(\mathrm{R} 7-10)$. While it is difficult to specify the optimal range of transmission distances owing to regional disparities in pipeline costs and uncertain process steam economics, a reasonable estimate would be from 80 to 320 kilometers.

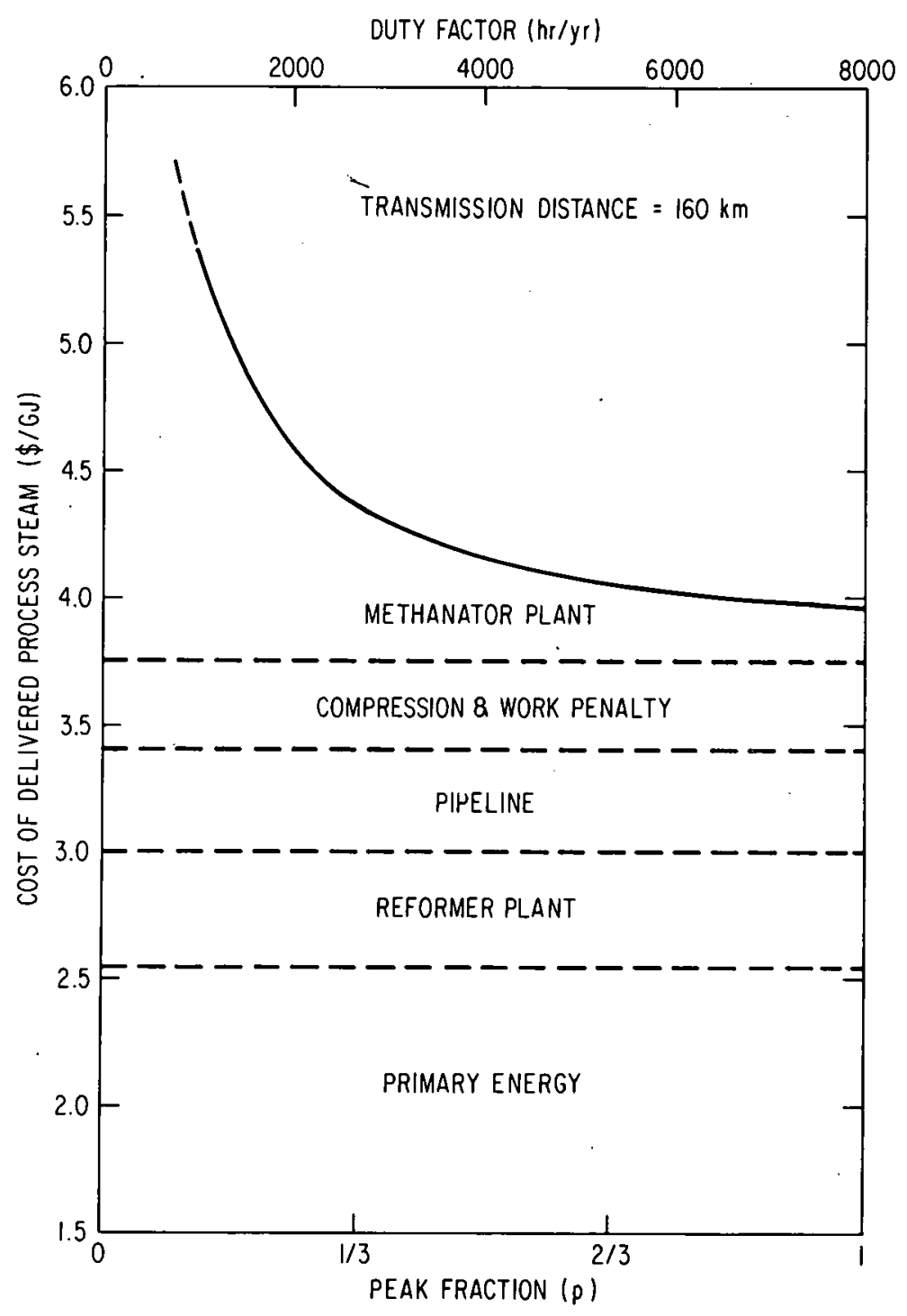

Figure 7-1. Process Steam Costs as a Function of Duty Factor

\subsubsection{On-Site Storage for Peak Electricity}

There are several different ways of accomplishing on-site storage by a $\mathrm{CH}_{4}-\mathrm{CHP}$. One approach is to operate the reformer with off-peak thermal energy during a charging period, storing the Co-rich gases in an underground cavern, and operating the methanator plant with these stored gases during peak hours for electricity generation. A second method is to operate the reformer in a continuous, baseload manner and the methanator only 
during peak hours, and to store the reformed gases in a cavern when the methanator is not in operation.

The latter approach is used in this analysis because it does not involve the somewhat arbitrary division of primary source cost into peak and off-peak thermal energy, and also because it does not require the reformer to be thermally cycled (the feasibility of which is technically questionable). Figure 7-2 shows the major parts of the on-site storage plant producing peak electricity during a fraction $p$. Without going into the details of the actual operation*, the following very approximate analysis can be used to demonstrate the major economic features of the storage application.

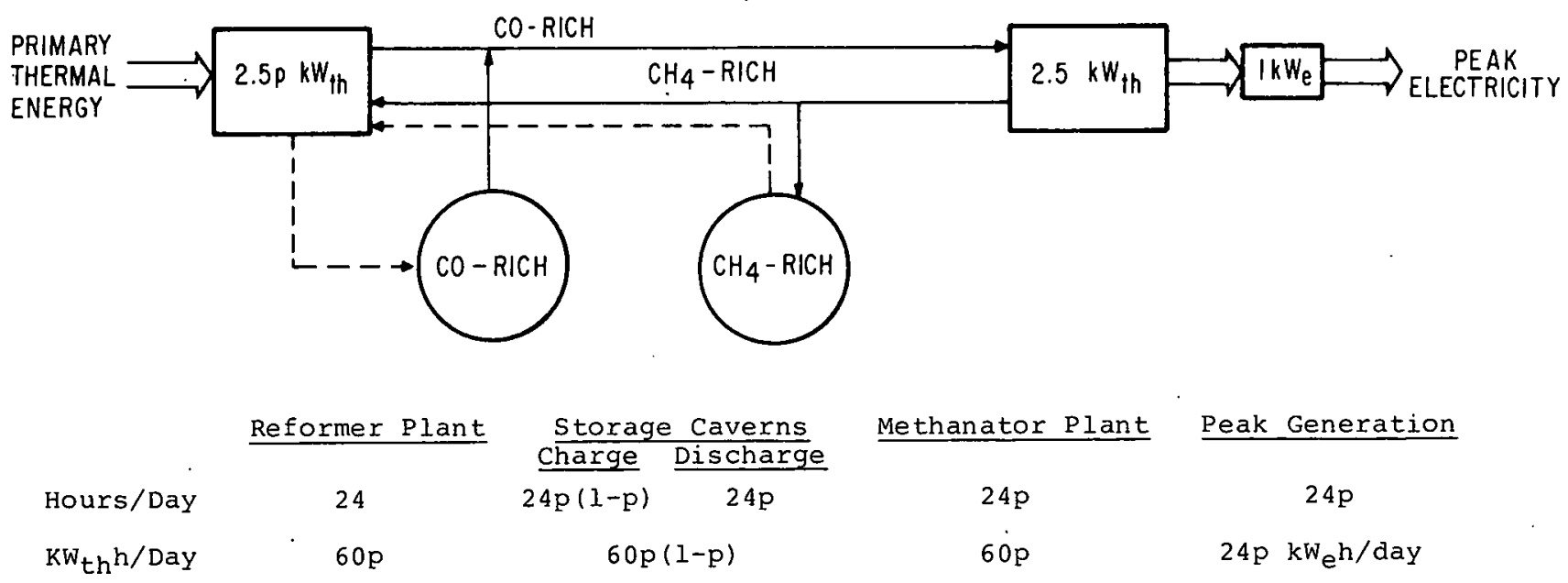

Figure 7-2. Major Parts of On-Site Storage System

A conversion efficiency from the methanator thermal output to the peak electricity is assumed to be $40 \%$. The component capital costs (from Figure $7-2$ and Table 7-8) are calculated as shown in Table 7-9.

The resultant costs for peak electricity are shown in Figure 7-3. The following points may be noted:

- The cavern contribution to the total cost is minimal even with costs representative of hard-rock caverns.

- The major item of cost for peak generation is due to, the peak generation plant operating at low capacity

*For example, whether cavern storage is compensated (isobaric) or uncompensated (isochoric), whether off-peak power is used to provide all the compression work, etc. 
Table 7-9

CAPITAL COSTS FOR ON-SITE STORAGE FOR PEAK ELECTRICITY
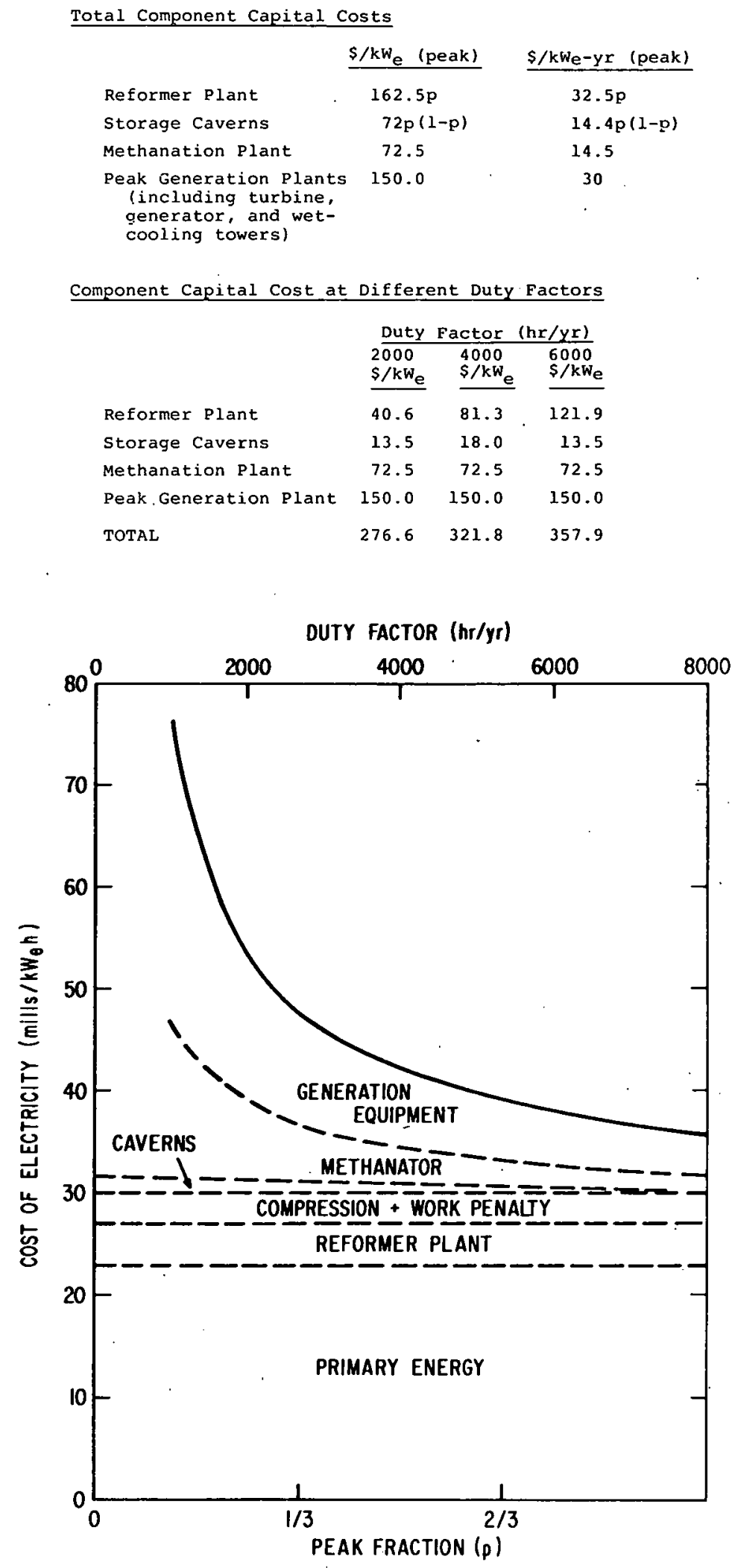

Figure 7-3. Electricity Cost for On-Site Storage 
factor. This is common to all storage systems dealing with thermal energy. If generation equipment (in particular the turbine hall) is likely to be cheaper when located off-site than on-site, then this would be another reason for favoring distributed generation.

\subsubsection{Distributed Generation of Peak Electricity}

From the results of the two previous analyses it is possible to arrive at the cost of generating peak electricity at a remote location. Such a mode of operation would be a competitor of baseload electrical transmission combined with electrical storage in batteries at substation levels; efficient thermal transmission by HTCHP offers an alternative to distributed electrical storage (batteries) and generation (fuel cells). The capital costs for CHP components are shown in Table 7-10 in terms of the peak generation fraction $\mathrm{p}$.*

Total generation costs are shown in Figure 7-4. A major point to be noted is that the costs are quite comparable to those for on-site storage. In fact, the differences are negligible compared with the uncertainties in cost estimates. Increases in cost due to the pipeline are offset to a large extent by somewhat reduced generation plant costs**; if in addition significant credits can be taken for distributed generation capability, then this option would prove to be highly attractive for the utilities. A side benefit would be the decoupling of the waste heat rejection site from the primary nuclear site. This would facilitate the siting of both units considerably.

A natural extension of the distributed generation concept is combined electricity/steam delivery by a HTCHP. Such total energy (cogeneration) systeris offer a great many advantages (conservation, reduced heat rejection, improved economics). The following section deals with the economics of such total energy systems.

\subsubsection{Combined Electricity/Steam Delivery (Cogeneration)}

The performance of a total energy system, as reflected by the ratio of delivered work to heat, deperids on the condition of primary steam and of exhaust steam given to the process. There is relatively little uncertainty about the primary steam conditions obtainable with a high temperature methanation plant: highest grade steam up to $10000^{\circ}$ and 1000 to 2400 psi can be generated. The conditions of the exhaust steam, however, are not

* Reformer and pipeline duty of $8000 \mathrm{hr} / \mathrm{yr}$, daily peak fraction of $p$ and power generation efficiency of $40 \%$.

**The main reasons for this possible reduction are (1) the added expense related to safety at the site of a nuclear reactor, and (2) the changes in construction schedules and the associated interest during construction. 
Table 7-10

CAPITAL COSTS FOR DISTRIBUTED GENERATION OF PEAK ELECTRICITY

Total Component Capital Costs

\begin{tabular}{|c|c|c|c|c|}
\hline . & & \multicolumn{2}{|c|}{$\underline{\$ k W_{e}}$ (peak) } & $\begin{array}{l}\$ / k W-k / r \text { (peak) } \\
\text { e } 208 / y r\end{array}$ \\
\hline Reformer Plant & & \multicolumn{2}{|c|}{$162.5 p$} & $32.5 p$ \\
\hline Pipeline & & \multicolumn{2}{|c|}{$150 \mathrm{p}$} & $30.0 p$ \\
\hline Methanation Plant & & \multicolumn{2}{|c|}{72.5} & 14.5 \\
\hline $\begin{array}{l}\text { Peak Generation Plant } \\
\text { (including turbine, } \\
\text { and wet-cooling towe }\end{array}$ & $\begin{array}{l}\text { Eenerato } \\
\text { (s) }\end{array}$ & \multicolumn{2}{|c|}{120} & 24.0 \\
\hline \multicolumn{5}{|c|}{ Oomponent Capital cost as a Function of Duty Factor } \\
\hline \multicolumn{5}{|c|}{ Duty Factor (hr/yr) } \\
\hline & $\begin{array}{l}2000 \\
\$ / \mathrm{kW}_{\mathrm{e}} \\
\end{array}$ & $\begin{array}{r}4000 \\
\$ / k W_{e} \\
\end{array}$ & $\begin{array}{l}6000 \\
\$ / \mathrm{kW}_{\mathrm{e}} \\
\end{array}$ & \\
\hline Reformer Plant & 40.6 & 81.3 & 121.9 & \\
\hline Pipeline & 37.5 & 75.0 & 212.5 & \\
\hline Methanation Plant & 72.5 & 72.5 & 72.5 & \\
\hline Peak Generation Plant & $\frac{120.0}{270.6}$ & $\frac{120.0}{310.8}$ & $\frac{120.0}{126.9}$ & \\
\hline TOTAL & 270.6 & 348.8 & 426.9 & \\
\hline
\end{tabular}

so easily guessed at, because of the diversity of process steam needs. Consumption statistics show that for most cases they correspond to saturated steam at temperatures in the range of 250 to $450^{\circ} \mathrm{F}$.

As an example, if process steam is delivered at $330^{\circ} \mathrm{F}$ (100 psia), the ratio of delivered work to heat is approximately equal to 0.3 . In other words, $24 \%$ of the methanation heat is converted to work and the rest is delivered to the process as $330^{\circ} \mathrm{F}$ condensation heat. Such a combined energy delivery requires only $73 \%$ of the primary energy that would be required to make the same amount of steam and electricity separately. The economic benefits that accrue in this case may be credited either to the electricity or to the process steam delivered. The total cost for supplying $1 \mathrm{~kW} \mathrm{e}^{\mathrm{h}}$ electricity and $3 \mathrm{~kW}$ thh process steam is shown in Figure 7-5.

Also shown in the figure is the cost of supplying the same energy separately as obtained by combining appropriate terms from Figures 7-1 and 7-4. The ratio of the two costs is relatively independent of the capacity factor and shows that combined energy delivery costs only $76 \%$ of what separate delivery would cost. Such savings are not atypical for total energy systems and are quite representative for on-site, coal-fired steam generators as well. The major difference is that with a CHP they can be extended to smaller scale, part-time users. In spite of the institutional difficulties, it is clear that a combined delivery of electricity and process steam through a CHP could offer significant advantages in both conservation and economics. 


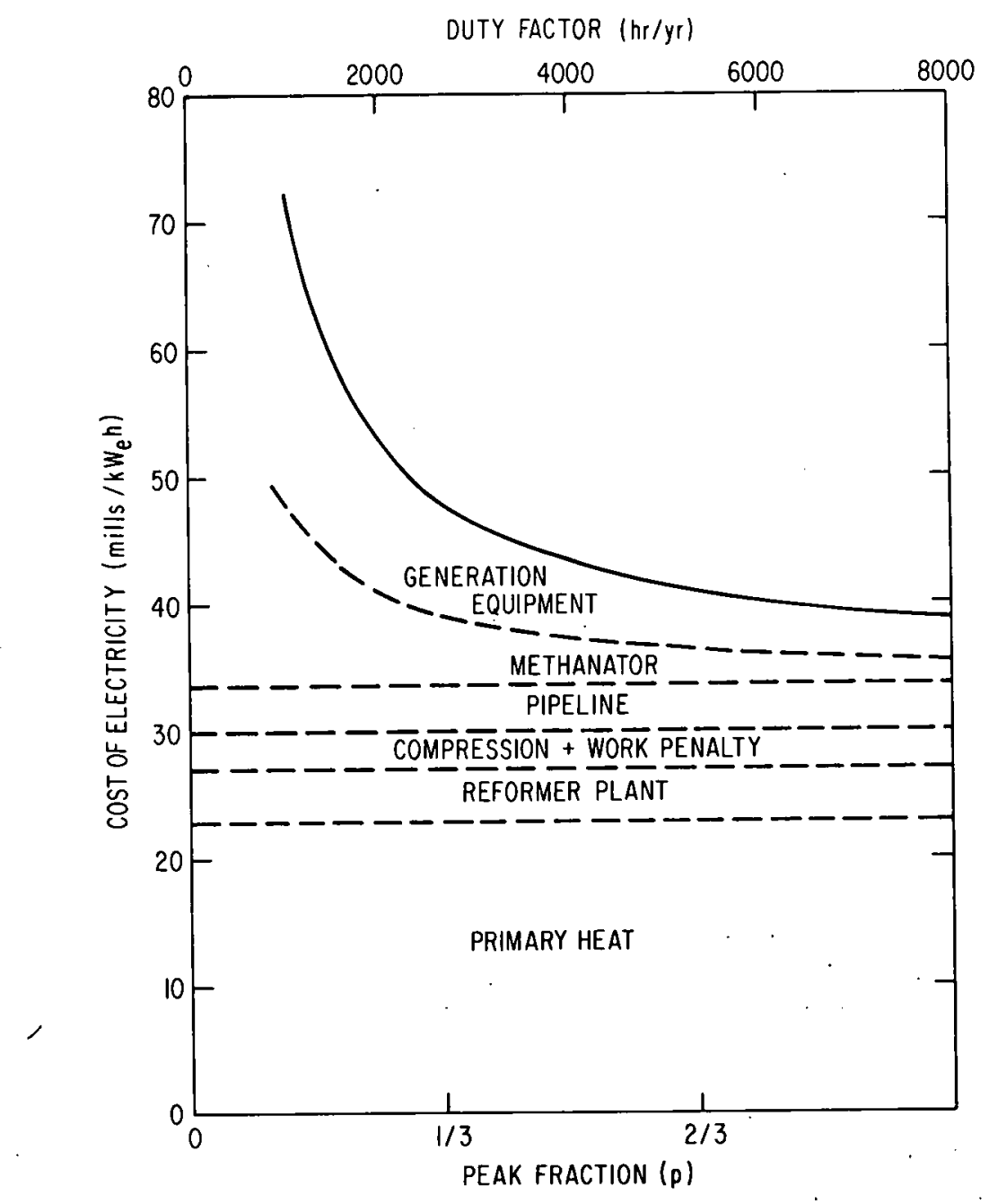

Figure 7-4. Cost of Distributed Electricity

\subsection{SOLAR ENERGY APPLICATIONS}

\subsubsection{Introduction}

The application of the HTCHP to on-site storage and to distribution of solar thermal energy will be investigated in the following paragraphs. The technical analysis of the HTCHP system has shown that the mating of these two technologies may result in a very difficult interfacing problem. The following analysis is included despite the uncertainty in the technical design so that the HTCHP economics can be compared with alternative technologies.

The mating of these two technologies has the obvious advantage that the one-shift energy collection at the reformer site can be used to supply baseload energy either on site or at remote user sites. In the transmission of solar heat, the storage available. in the transmission lines can be combined with the ability to deliver the solar energy at distances 80 to 320 kilometers from the collection site. At the present time, the cost of solar thermal energy as it would be delivered to the reformer is unknown. For purposes of this investigation, it will be assumed to be 
DUTY FACTOR $(\mathrm{hr} / \mathrm{yr})$

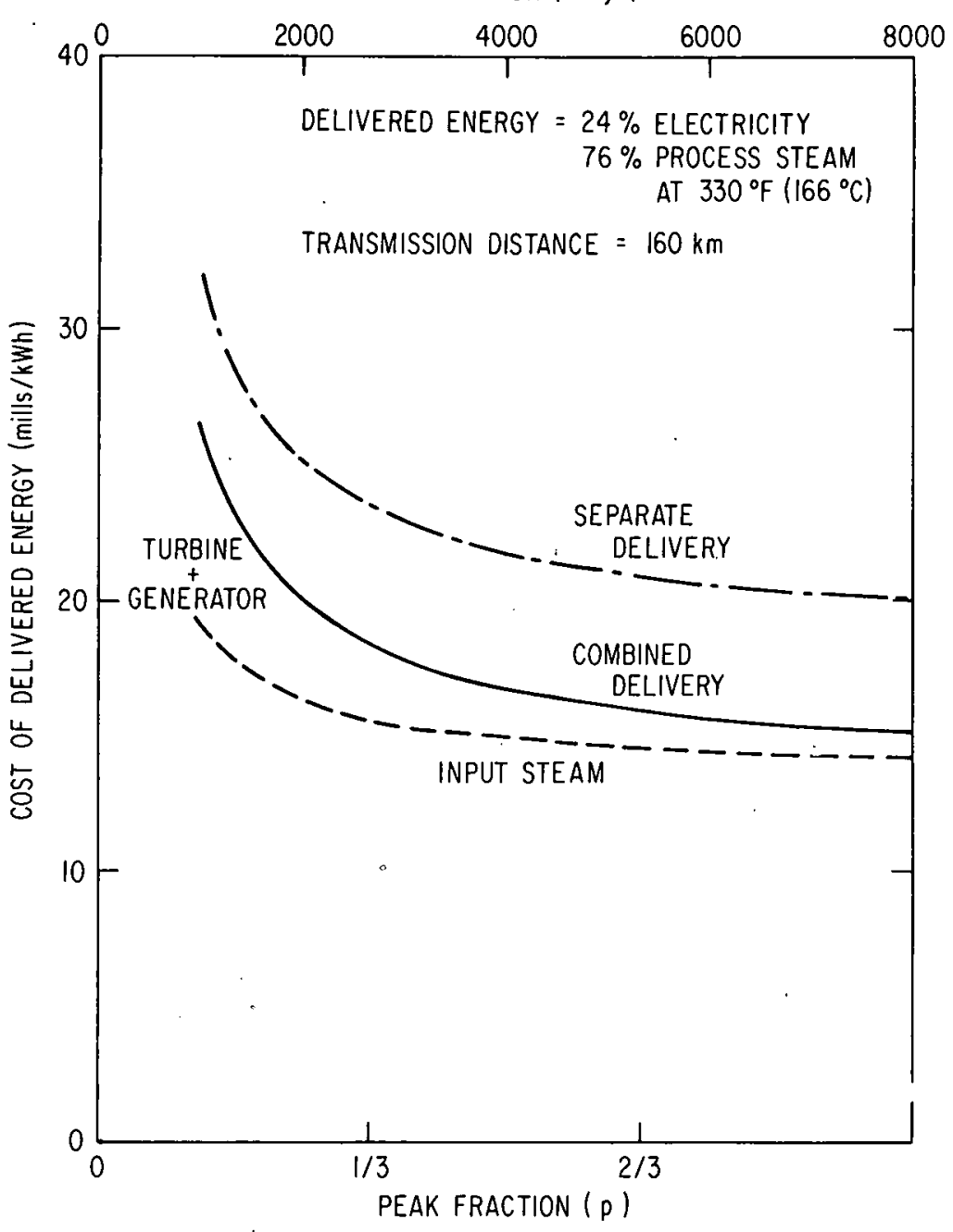

Figure 7-5. Costs for Combined Steam/Electricity Delivery

$\$ 6 / G J$ for the development of the economic calculations (R7-II). Then, in order to determine the sensitivity of the cost of delivered heat to the cost of the primary solar heat, the primary heat cost will be varied. Of course, electricity generated from this heat will be more expensive than the $20 \mathrm{mills} / \mathrm{kWh}$ used in the. previous analyses.

The approach used in this analysis will be to assume that the cost of electricity is proportional to the cost of solar heat, with a $40 \%$ efficiency for the conversion of heat to electricity. This means that one unit of electrical work costs 2.5 times that of a unit of heat. No attempt will be made to reoptimize the transmission lines.

In these analyses, reference case HTCHP conditions from chapter 5 will be used. This may be an optimistic choice in view of possible temperature limitations for energy available from solar sources (see Chapter 4), but the general conclusions reached will also be applicable to other reaction conditions. 


\subsubsection{On-Site Storage for Solar Energy Applications Utilizing HTCHP Reactions}

The major impact of a solar thermal heat source on the HTCHP system is that the reformer could operate only on the order of 8 hours per day ( 2667 hours per year). If the system is still to produce an average of $1000 \mathrm{MW}$ th over 24 hours, equipment must be oversized by a factor of 3 to account for the periodicity of the solar heat source.

Since the reformer and associated heat exchangers are already very large in the baseload cases in comparison with industrial units, it is likely that the additional equipment required will be added in modules. If this were the case, no benefit from economy of scale would be expected, and the capital cost would scale directly with capacity. However, the possibility exists in the on-site storage design of combining the methanation and reformer functions in an integrated unit since similar catalysts of low nickel content will be used in both applications. In addition to the reformer, storage caverns must be utilized to store the gases. In this preliminary analysis, it will be assumed that the work and compression losses associated with storage of the gases in the caverns are comparable to those of transmission as given in the reference case.

A summary of the capital and operating costs for the on-site storage of thermal energy based on the HTCHP reactions is given in Table 7-11 (for a solar heat cost of $\$ 6 / \mathrm{GJ}$ ); cost of storage and of the stored heat is shown in Figure 7-6 as a function of the cost of the primary solar energy.

Several points should be highlighted from this analysis. First, the capital cost component of the storage cost is significantly higher than the previous reference case analysis. This is mainly due to the increased capital cost of the reformer. Since only $20 \%$ of the capital costs are allocated each year to the cost of storing the energy, there is not a dramatic increase in storage costs for this case as compared with the transportation costs for the previous analyses ( $20 \% / G J)$. A second point -- since the HTCHP process operates at a relatively high thermal efficiency, only a small portion of the solar heat absorbed into the system is lost (assuming losses comparable to the transportation scheme). This means that the cost of storage is not highly dependent on the cost of the primary solar heat.

\subsubsection{Transportation of Primary Solar Heat Via HTCHP}

As in the on-site storage analysis, the major difference in a HTCHP transportation system utilizing solar energy is the fact that the reforming plant must be oversized by a factor of 3 in order to deliver the same rate of heat as a constant heat source. 
Table 7-11

SUMMARY OF CAPITAL AND OPERATING COSTS

FOR ON-SITE STORAGE USING A SOLAR HEAT SOURCE

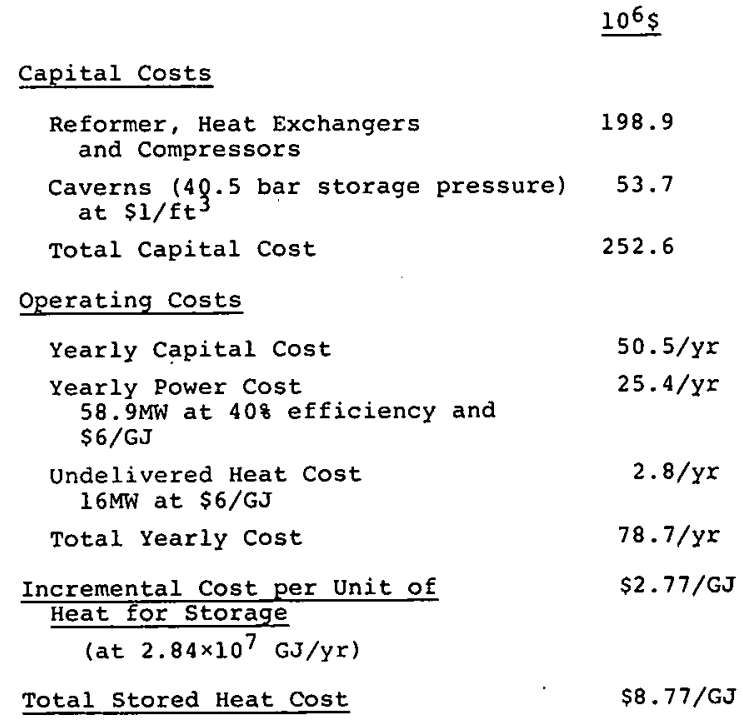

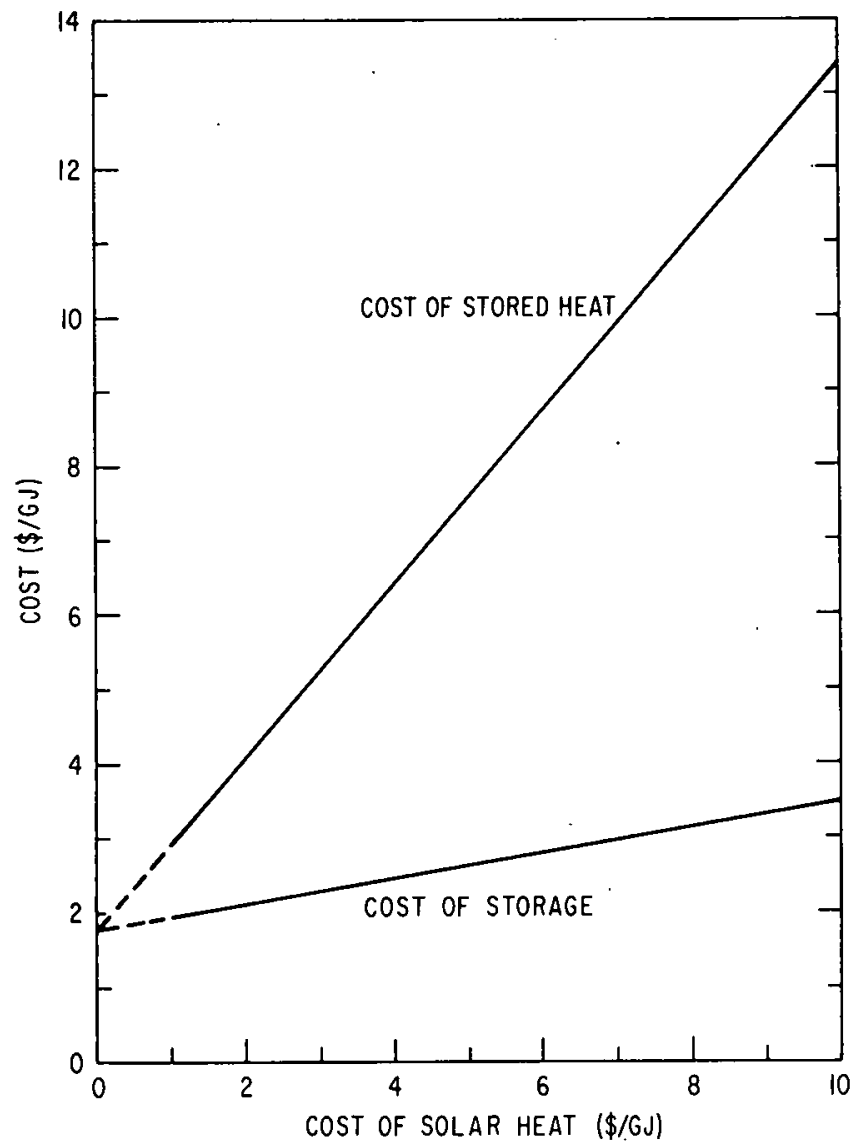

Figure 7-6. Cost of On-Site Storage and Stored Heat as a Function of the cost of Solar Heat 
In this case, however, the methanation plant must be included, since the heat is transported away from the reformer to individual user sites some distance from the solar collector. In this analysis, it will be assumed that the methanation plant and the pipeline are baseloaded, producing heat continually from the solar source which collects heat only 8 hours per day. Again the reference case conditions will be used. The costs associated with the thermal transmission are summarized in Table 7-12 using a cost of $\$ 6 / G J$ for the primary solar heat(R7-11). The transportation costs and the total cost of the delivered heat are then presented graphically as a function of the primary solar energy cost in Figure 7-7.

As in the on-site storage analysis, the capital cost contribution to the transportation cost is higher than in the baseload reformer analysis. The total transportation cost ranges from $\sim \$ 2$ to $3.50 / \mathrm{GJ}$ over the range of solar costs investigated (as compared with $\sim \$ 1.50 / G J$ for the baseload case). Since the HTCHP operates with a relatively high efficiency, the undelivered heat is a small fraction of the heat absorbed and the cost of transportation is a weak function of the cost of the primary solar energy as compared with lower efficiency processes (R7-12).

\subsubsection{Conclusions from Economic Analysis of Solar Energy Applications}

Since the technology for collecting high-grade solar energy on a large scale has not been developed, it is difficult to predict the cost of this energy. However, it is apparent from these analyses that the HTCHP system can either store on site or transport this energy at a cost of $\$ 2$ to $\$ 4 / G J$ above that of the primary enersy.

The cost breakdowns for these two analyses show that a major contributor to both the on-site storage cost and the transportation cost is the cost of the endothermic reactor. Any method which could reduce the cost of this unit would have a major impact on reducing the cost of solar heat storage or transportation through chemical reactions. This is a major reason why possible applications of the cyclohexane-dehydrogenation LTCHP (possibly with a lower reactor cost) have been investigated in the second section of this report (see Chapter 16 for details of solar applications).

The on-site storage costs calculated here do not appear as attractive as those for conventional storage schemes (high pressure steam) (R7-13). However, the main comparison that should be made is with a combined storage and transmission system. Comparison of Figures $7-6$ and $7-7$ shows that the primary solar heat can be transported to users $160 \mathrm{~km}$ away from the collection point for only a small incremental cost above that for on-site storage. This distribution is very important when the end use of the heat is considered. 
Table 7-12

SUMMARY OF CAPITAL AND OPERATING COSTS

FOR TRANSPORTATION OF SOLAR HEAT

\begin{tabular}{lc} 
& $10^{6} \$$ \\
Capital Costs & \\
\hline Reformer Plant & 198.9 \\
Transportation & 60.4 \\
Methanator Plant & 28.6 \\
Total Capital Cost & 287.9 \\
Operating Costs & \\
\hline $\begin{array}{l}\text { Yearly Capital Cost } \\
\text { Yearly Power Cost }\end{array}$ & $57.6 / \mathrm{yr}$ \\
Yearly Cost of Undelivered Heat & $25.4 / \mathrm{Yr}$ \\
$\quad$ at $\$ 6 / \mathrm{GJ}$ & $2.8 / \mathrm{Yr}$ \\
Total Yearly Cost & $85.8 / \mathrm{Yr}$ \\
Incremental Cost per Unit of Heat & $\$ 3.02 / \mathrm{GJ}$ \\
\hline Delivered & \\
$\quad$ iat $\left.2.84 \times 10^{7} \mathrm{GJ} / \mathrm{yr}\right)$ & $\$ 9.02 / \mathrm{GJ}$
\end{tabular}

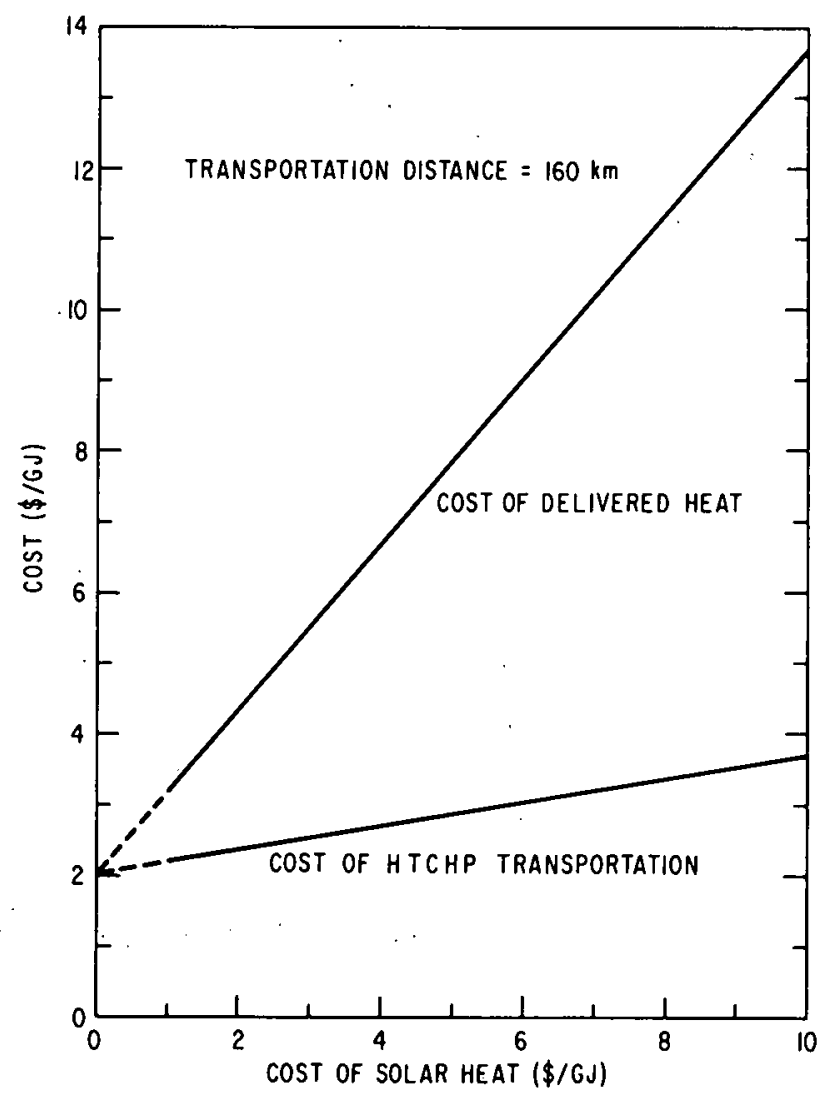

Figure 7-7. Cost of HTCHP Transportation and Transported Heat as a Function of the Cost of Solar Heat 
One example is the generation of electricity. At the collector site, thermal energy is stored and then regenerated, with consequent higher cost, and a portion (33 to $40 \%)$ is converted to electricity (the remainder being rejected to the environment). Instead, if the primary heat is transported to a process steam user site at essentially the same incremental cost as on-site storage, it can be used to produce a slightly smaller amount of electricity and the reject heat can be used as process steam. The economics of this application will be explored in more detail in Chapter 16, but even without detailed costs it is obvious that the use of transportation and cogeneration allows the solar utility to sell both the electricity and valuable reject heat rather than just the electricity from the on-site generation. This would not only increase the revenues of the utility but also decrease the amount of capital required for cooling towers at the collector/generator site.

\subsection{COMPARATIVE ECONOMICS OF ENERGY TRANSPORT AND SOURCES}

\subsubsection{Background}

The following is a brief quantitative comparison of the economics of transport of various types of energy, based on a comparison with the use of substitute natural gas (SNG). In this comparison, reasonable estimates will be used for the cost quantities; however, the same arguments can be carried out for other cost assumptions if desired. The basis for this cost comparison will be SNG delivered to a process steam user at $\$ 4 / G J$. This cost includes coal feed, conversion to SNG, and SNG transportation. The cost of process steam is then $\$ 5 / G J$, assuming an $80 \%$ boiler efficiency (see Appendix 2). This number is the basis with which other fuel costs and associated transportation costs can be compared.

\subsubsection{Determination of Economically Competitive Prices of Solar and Nuclear. Heat Utilizing the HTCHP Transportation System}

Using $\$ 5 / G J$ as the cost of SNG-derived process steam, the economically competitive price for solar and VHTR nuclear heat when used with the HTCHP can be determined by simply subtracting the HTCHP transportation cost from $\$ 5 / G J$. For the solar case, the minimum transportation cost is about $\$ 2 / \mathrm{GJ} / 160 \mathrm{kms}$. This implies that solar heat must be collected at approximately $\$ 3 / G J$ to supply process steam. For the VHTR heat source, the cost of transportation through the HTCHP system is approximately $\$ 1.50 / \mathrm{GJ} /$ $160 \mathrm{~km}$. This implies that if high-temperature nuclear heat can be generated for $\sim \$ 3.50 / \mathrm{GJ}$ it may be competitive with SNG for supplying process steam when coupled to a HTCHP.

One other situation that might be encountered is the use of nuclear or solar HTCHP in competition with small electrode boilers. Heat from these units might cost as:much as $\$ 9 / G J$. Compared with this end use, solar heat might be competitive at $\sim \$ 6 / G J$ and VHTR heat at $\$ 7 / G J$. 
7.6.3 Comparative Economics for Transport of Coal and Light Water Reactor (LWR) Heat

Looking at the same comparison from the other end, the cost of coal and LWR heat is about $\$ 2 / G J$. Since these sources do not supply heat at a high enough temperature (see Chapter 4) to be coupled to the HTCHP system, the question might be asked: how much could one afford to pay to transport this heat by other systems and still be competitive with SNG process steam? The obvious answer is $\sim \$ 3 / G J$. This number should be kept in mind throughout the analyses performed in the second portion of this report on the low temperature chemical heat pipe. In comparison with electrode boiler process steam at $\$ 9 / G J$, this allowable transportation cost increases to $\$ 7 / \mathrm{GJ}$. In either case, the low cost of these two heat sources allows considerable leeway in the cost of the transportation system used to move the heat.

Finally, it should be noted that other technologies, such as atmospheric fluidized bed combustors, may allow direct use of coal heat at the user site without transportation. Until these technologies can be developed and proved economical, the question of the development of a low-cost transportation system for coal and LWR heat remains open to investigation.

\subsection{CONCLUSIONS}

In this chapter, the economics for various reaction conditions and applications of the high temperature chemical heat pipe have been investigated. The major conclusions can be summarized as follows :

- The HTCHP system can deliver thermal energy over a distance of $160 \mathrm{~km}$ at a cost between $\$ 1.50$ and $\$ 2.00$ per GJ above the cost of the primary heat in nuclear applications.

- The cost of the HTCHP is increased by approximately 13\% if the mixed feed evaporator is not used.

- The economics favor low water recycle and the highest temperature available.

- The HTCHP is economically competitive with current alternatives (pumped hydro, compressed air) for on-site storage for peak electricity for nuclear applications.

- The incremental cost for process steam delivery, to small-scale or part-time users as well as for distributed generation of peak electricity, is very small. This makes the HTCHP superior to other alternatives in these categories. 
- Significant economic benefits can be derived by combining electrical generation with process steam delivery, either on a continuous basis (baseload) or on a peaking basis (one- or two-shift industries).

- If the technical barriers summarized in section 4.4 could be overcome, on-site storage of solar thermal energy could be accomplished at an incremental cost of $\$ 2.00$ to $\$ 3.50 / \mathrm{GJ}$, depending on the cost of the primary energy. The same energy could be transported a distance of $160 \mathrm{~km}$ at an incremental cost of $\$ 2.10$ to $\$ 3.60 / \mathrm{GJ}$, again depending on the cost of the primary energy. Considering the advantages of distribution of the energy, the best application of the HTCHP system to solar energy is transport and storage rather than simply on-site storage.

- To be competitive with SNG at $\$ 4 / G J$, the primary energy cost in a solar/HTCHP system should not exceed $\$ 3 / G J$ and in a VHTR nuclear/HTCHP system should not exceed $\$ 3.50 / \mathrm{GJ}$.

\section{REFERENCES -- CHAPTER 7}

R7-1 Nuclear Engineering and Design, Vol. 34, No. 1, 1975.

R7-2 Anon., "Reformer Tube Alloy Withstands Overheating," Metal Progress, Vol. 107, February 1975, p. 44.

R7-3 Private communication, Blaw-Knox Equipment Company, Duraloy Division, Pittsburgh, Pa.

R7-4 Guthrie, K.M., "Data and Techniques for Preliminary Capital Cost Estimating," Chemical Engineering, Vol 76, No. 6, 1969, p. 114 .

R7-5 Private communication, UOP Inc., Process Division, Des Plaines, Illinois.

R7-6 Federal Power Commission, Bureau of Natural Gas, "Cost of Pipeline and Compressor Station Construction Under Non-Budget Pipeline and Certificate Authorizations as Reported by Pipeline Companies in Fiscal Year 1976," Staff Report, Washington. D.C., 1976.

R7-7 Vosburgh, K.G., "Conceptual Design for a Pilot/Demonstration Compressed Air Storage Facility Employing a SolutionMined Salt Cavern," Final Report EPRI-EM-391, General Electric Corporate Research and Development, June 1977.

R7-8 Anon., "Economic Indicators," Chemical Engineering, Vol. 80, No. 23,1976 .

R7-9 Private communication, Fenix and Sisson, Inc., Tulsa, Oklahoma. 
R7-10 Aamot, H.W.C., and Phetteplace, G., "Long Distance Heat Transmission with Steam and Hot Water," International Total Energy Conference, Copenhagen, Denmark, October 1976.

R7-11 In view of the status of the developing technology of solar heat collection, the cost of solar thermal energy is still in doubt. The value of $\$ 6 / \mathrm{GJ}$ was recommended to General Electric by the monitors of this contract at Sandia Laboratories, Livermore, California, as a preliminary working number.

R7-12 Gintz, J.R., "Advanced Thermal Energy Storage Concept Definition Study for Solar Brayton Power Plants," Final Technical Report, ERDA Contract No. EY-76-C-03-1300, Engineering and Construction, Boeing, 1976.

R7-13 Kalhammer, F.R., and Schneider, T.R., "Energy Storage," Annual Review of Energy - 1976 (J.M. Hollander, ed.), Annual Reviews, Inc. Palo Alto, California, 1976. 


\section{Chapter 8}

\section{PRELIMINARY MARKET ANALYSIS FOR CHEMICAL HEAT PIPES}

\subsection{INTRODUCTION}

The basic question to be addressed in this chapter is the potential size of the market for a chemical heat pipe technology. The nature and size of the market depends in a detailed way on the characteristics of a chemical heat pipe system. Some of the relevant characteristics of a CHP system are that it

- Can deliver high-grade heat to users distant by as much as 160 kilometers from the source of heat.

- Provides energy storage capability associated with the transit time of unreacted material through a pipeline, which together with modulation of pressure in that pipeline leads to the possibility of as much as eight hours of energy storage per 100 kilometers of pipeline.*

- Provides the possibility of replacing heat generated by premium fuels with a reactor or coal-fired system feeding a chemical heat pipe.

- Offers opportunities for energy conservation, particularly with a total energy system in which the relative amounts of electricity and heat delivered can be adjusted according to the demand.

An unexpectedly major problem in dealing with the question of market potential has been the inadequacy of data on the use of thermal energy by industry. As will be seen in this chapter, a considerable amount of data reconciliation and reconstruction has been necessary, with consequent uncertainties in results derived. The necessity for a reliable and timely data base for energy systems studies such as are represented here cannot be overemphasized.

Data for this analysis were obtained from several sources (R8-1 through R8-10). These references have been given acronyms, which will be used throughout this chapter to aid the reader in identifying the sources.

In section 8.2 of this chapter the annual rates of fuel consumption by each of the major segments of the U.S. economy are examined. To the extent possible, the type of fuel used as a function of the nature of the end usage of the energy snown. A gross maximum potential market for a CHP system is considered. Then the gross potential saving of premium fuel (oil and natural gas) which might be expected with a CHP system is suggested. Finally the gross potential for energy conservation with a CHP-based total energy system is explored.

* See Appendix 4 of this report. 
A considerable refinement to these gross estimates is required to arrive at even rough quantitative estimates of prime fuel substitution and energy conservation resulting from the use of CHP.

In section 8.3 the industrial sector is considered in some detail. This sector appears to be the most sensible and fruitful point of entry for a new CHP technology in the near term. The nature and the limitations of the data available for this sector are discussed. With the available data, fuel consumption in the industrial sector is characterized according to a number of factors, including temperature of direct heat or process steam developed and size of plant for the several major segments of the industrial sector. The duty cycle within these segments (continuous operation, one- or two-shift operation, seasonal operation), the consumption for various types of end use within the major segments, and the geographical distribution of energy consumption patterns are considered when appropriate or feasible. Einally, some remarks are made about the problem of trend extrapolations for determining the future market for a CHP system. The results of this chapter are summarized in section 8.4 .

\subsection{ANALYSIS OF FUEL CONSUMPTION BY SECTOR AND BY CHP MARKET}

\subsubsection{Total Fuel Consumption by Sector}

Institutional barriers and the nature of energy consumption in the various sectors of society suggest that the first application of the chemical heat pipe will be in the industrial sector, particularly the manufacturing sector of industry. To place the industrial sector in perspective, as well as to verify the ECS (R8-1) analysis of the industrial sector in relation to other studies, a number of the available studies on fuel and energy consumption by sector of the United States economy have been examined in detail. This situation is uncertain in that seemingly authoritative sources are at variance, and sources on which national energy policies presumably are based are at variance with one another and sometimes internally inconsistent. The variances are not enormous, but neither are they trivial.

Table 8-1 presents consumption data by sector of the American enterprise, in units of $10^{9} \mathrm{GJ}$ per year. Part (a) presents fuel consumption, including feedstocks, with electricity use presented separately and assigned to utilities at the gross fuel value (i.e., including waste heat). Electricity generated by hydropower, geothermal, and nuclear is generally included in the utility counting, with some equivalent fuel value assigned by the particular analysis. Differences in these assignments should be of second order importance. However, in the Federal Energy Administration (FEA) analysis (R8-2), no assignment was provided for nonfossil fuel utility generation; hence no entry is provided. Part (b) again presents energy consumption by sector, but this time with electricity allocated to each sector use at the gross fuel value. Again the fuel value of feedstocks are included in the data tabulated. The data in both parts (a) and (b) are thought to include captive and 
Table 8-1

FUEL AND ENERGY CONSUMPTION: COMPARISON OF DATA SOURCES FOR VARIOUS YEARS

$1960(\mathrm{SRI}) \quad \underline{1960(\mathrm{EPRI})} \quad \underline{1968(\mathrm{SRI})} 1970$ (PIB-1) ${ }^{1} \quad \underline{1970(\mathrm{EPRI})} \quad \underline{1971(\mathrm{PIB}-3)} \quad \underline{1972(\mathrm{BNL})} \quad \underline{1974(\mathrm{BTC})} \quad \underline{1974(\mathrm{FEA})}$

(a) Fuel Consumption by Sector, Including Feedstock, in $10^{9} \mathrm{GJ} / \mathrm{Yr}$

\begin{tabular}{|c|c|c|c|c|c|c|c|c|c|}
\hline Residential & 6.25 & 6.42 & 8.23 & 10.3 & 9.38 & & 9.25 & & \\
\hline Commercial & $\underline{4.54}$ & $\underline{5.72}$ & 6.13 & 2.59 & 8.33 & 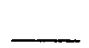 & 4.88 & & \\
\hline $\begin{array}{r}\text { Residential/ } \\
\text { Commercial }\end{array}$ & 10.8 & 12.1 & 14.4 & 12.9 & 17.7 & 15.1 & 14.1 & 14.6 & 14.6 \\
\hline Industrial 2,3 & 15.5 & 16.9 & 20.4 & & 23.6 & 21.4 & 25.2 & 24.3 & 22.3 \\
\hline Transportation & 11.6 & 11.5 & 16.0 & & 17.1 & 17.9 & 17.4 & 19.3 & 19.3 \\
\hline Utility (Gross) & 7.56 & 5.91 & 13.1 & $-{ }_{-1}$ & 11.6 & 18.4 & $17.5^{5}$ & 20.8 & \\
\hline TOTAL & 45.5 & 46.4 & 63.9 & & 70.0 & 72.8 & 74.2 & 79.0 & \\
\hline
\end{tabular}

(b) Energy Consumption by Sector, Including Electricity at Gross Fuel value and Feedstock, in $10^{9}$ GJ/Yr

\begin{tabular}{|c|c|c|c|c|c|}
\hline Residential & 8.41 & 12.3 & 15.1 & & 15.7 \\
\hline Commercial & $\underline{6.06}$ & 9.26 & 5.74 & - & 9.20 \\
\hline $\begin{array}{r}\text { Residential/ } \\
\text { Commercial }\end{array}$ & 14.5 & 21.6 & 20.8 & 25.6 & 24.9 \\
\hline Industrial & 19.4 & 26.4 & & 29.2 & 34.0 \\
\hline Trarsportation & 11.6 & 16.0 & & 18.0 & 17.4 \\
\hline TOTAL & 45.5 & 64.0 & & 72.8 & $76.3^{5}$ \\
\hline
\end{tabular}

1. PIB takes considerable exception to the SRI analysis of residential and commercial.

2. Care is required in comparing these data (see text).

3. The BTC data include "exports."

4. The tabulation for FEA is incomplete because the contribution to electricity from hydropower, geothermal and nuclear, has been omitted. Hence utility is left blank. It is interesting to note that in the face of this omission the report chooses to correct electric comsumption by 98 for transmission losses.

5. BNL in one section of their report converted hydroelectricity at an average heat rate of 10,748 Btu/kWh, obtaining $2.89 \times 10^{15} \mathrm{Btu} / \mathrm{yr}$ for 1972 . We have reduced hydro by $3413 / 10748$ in obtaining the total 17.5 for utility rather than 19.6 . However, there seems to be no easy way to correct the allocated hydroelectricity in part (b). In another section of the BNI report they state an average heat rate of $9224 \mathrm{Btu} / \mathrm{kWh}$ for hydroelectric power with the same gross equivalent fuel value. so there is some uncertainty here. 
in-house fuel use in addition to the basic purchased fuel. Where it has been determined otherwise, the data are omitted.

The Stanford Research Institute: (SRI) study (R8-3) of the years 1960 and 1968, published in 1972, has been a more or less basic data source on patterns of energy consumption for some time. The SRI data were used critically in Project Independence Blueprint, Volume 1 (PIB-l) (R8-4) but used without comment in Volume 3 (PIB-3) (R8-5). The years shown in Table 8-1 are the years for which the data apply rather than the years of publication of the analyses. Other sources were studies by Brookhaven National Laboratory (BNL) $(R 8-6)$, Electric Power Research Institute (EPRI) (R8-7), and Bankers Trust Company (BTC) (R8-8).

Variations among data sources reporting on the industrial sector result in part from real differences in what has been included or in handling of the portions of the sector. The differences range from efficiencies assigned to utility powerplants to inclusion of one or both of the manufacturing and nonmanufacturing segments of the sector. In all but the PIB-3 data the nonmanufacturing segment was included, albeit not explicitly; it is therefore difficult to be certain that there have not been assignments of parts of this segment to nonindustrial usage. For example, there are large users of petroleum in the agriculture and construction industries whose whereabouts in the energy sorting have not been explicitly indicated. The industrial sector will be discussed further in section 8-3, where attention will be directed primarily to the manufacturing sector.

The transportation and utility sectors show similar types of variances. The segmentation of energy usage into societal sectors cannot be estimated at better than 10 to 20 percent. There is need for an analysis of present and past data to resolve these differences, in order to verify quoted present values and to use these data for extrapolation to the future. As will be seen later, there is an additional problem in extrapolating the fuel consumption for the industrial sector into the future arising from a break in historical behavior in 1973 .

\subsubsection{Types of Fuel Use Vs Function in Various Sectors}

Of the several sources of data examined, those of SRI and BNL allow, in a restricted way, consideration of the functional use made of the various fuels in each sector of the U.S. economy. SRI presents such a breakdown for the years 1960 and 1968 ; BNL presents a slightly different analysis for the year 1972. This breakdown is of considerable relevance in the CHP context because of the potential of the CHP to replace a significant portion of the use of premium fuel in one or more of the sectors.

These data are presented in matrix form in Tables 8-2 through 8-6. Table 8-2 is a breakdown of fuel consumption in the residential sector. The relative values given by SRI and BNL appear to be consistent, and the indicated $3.7 \%$ per annum growth in residential 
Table 8-2

RESIDENTIAL FUEL CONSUMPTION: FUEL TYPE VS FUNCTION 1963, SRI; (1972, BNL in parentheses)

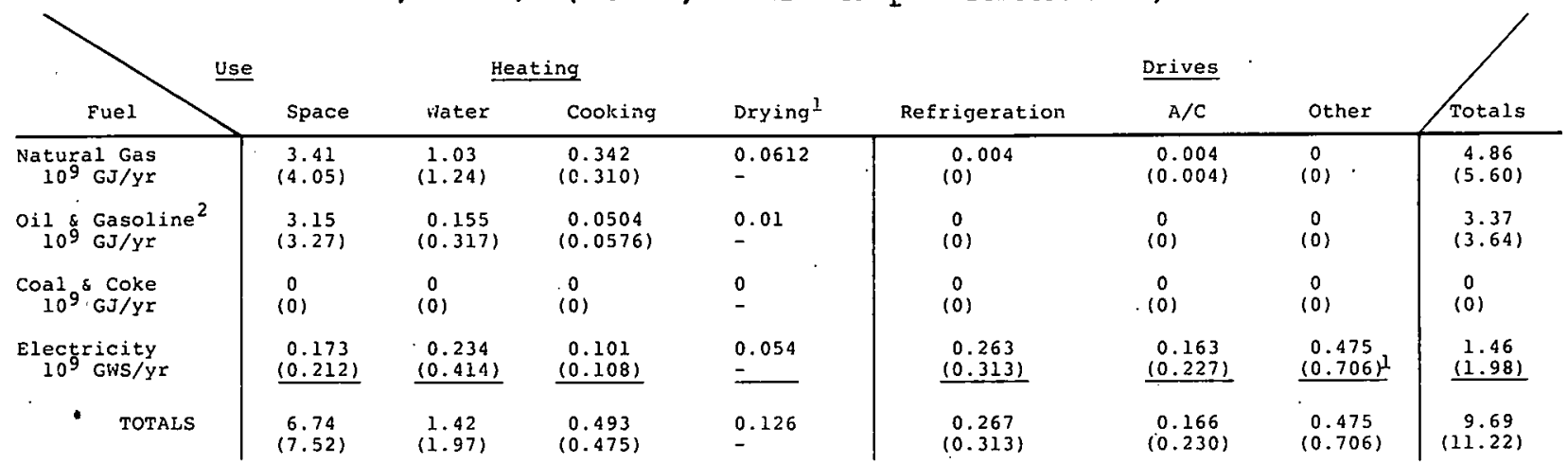

1. BNL puts $0.086 \times 10^{9}$ GWS/Yr of consumption for drying in "other."

2. LPG is included here.

Table 8-3

COMMERCIAL FUEL CONSUMPTION: FUEL TYPES VS FUNCTION 1968, SRI; (1972, BNL in parentheses)

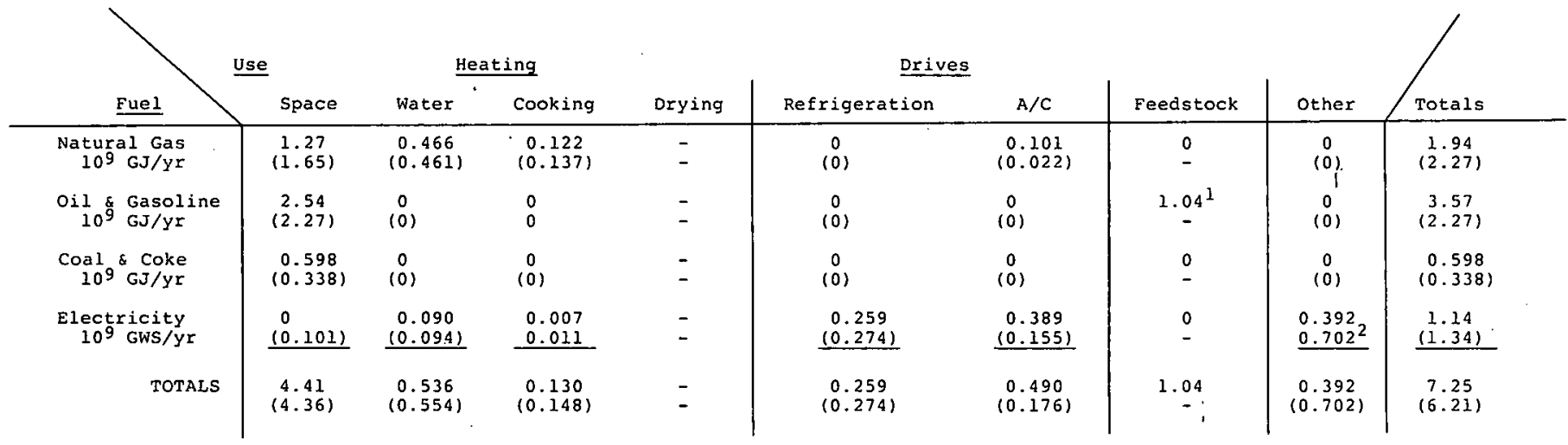

1. No such usage is identified by BNL in the comnercial sector.

2. Identified by BNL as lighting, appliances, etc. 
Table 8-4

TRANSPORTATION FUEL CONSUMPTION: FUEL TYPE VS FUNCTION 1968, SRI; (1972, BNL)

\begin{tabular}{|c|c|c|c|}
\hline Fuel & Transport & Feedstock & Totals \\
\hline $\begin{array}{l}\text { Natural Gas } \\
10^{9} \mathrm{GJ} / \mathrm{Yr}\end{array}$ & 0.644 & & 0.644 \\
\hline $\begin{array}{ll}\text { Gas } & \text { Oil } \\
10^{9} & \mathrm{GJ} / \mathrm{yr}\end{array}$ & $\begin{array}{l}15.2 \\
(17.3)\end{array}$ & $0.155^{1}$ & $\begin{array}{c}15.3 \\
(17.3)\end{array}$ \\
\hline $\begin{array}{l}\text { Coal \& Coke } \\
10^{\circ} \mathrm{GJ} / \mathrm{yr}\end{array}$ & 0.014 & & 0.014 \\
\hline $\begin{array}{l}\text { Electricity } \\
10^{9} \mathrm{GWS} / \mathrm{yr}\end{array}$ & $\begin{array}{c}0.018 \\
(0.018)\end{array}$ & & $\begin{array}{c}0.018 \\
(0.018)\end{array}$ \\
\hline TOTALS & $\begin{array}{l}15.9 \\
(17.3)\end{array}$ & $0.155^{\prime}$ & $\begin{array}{l}16.0 \\
(17.3)\end{array}$ \\
\hline
\end{tabular}

1. BNL does not identify feedstock as a category in this sector.

Table 8-5

UTILITY FUEL CONSUMPTION ${ }^{1}$ : FUEL TYPE VS MODE OF GENERATION 1968, SRI; (1972, BNL)

\begin{tabular}{|c|c|c|c|c|c|}
\hline Fuel & $\begin{array}{c}\text { Steam } \\
\text { Turbines }\end{array}$ & $\begin{array}{l}\text { Gas } \\
\text { Turbines }\end{array}$ & $\begin{array}{c}\text { Steam or } \\
\text { Gas Turbines }\end{array}$ & $\begin{array}{l}\text { Water } \\
\text { Turbines }\end{array}$ & Totals \\
\hline $\begin{array}{l}\text { Natural Gas } \\
\qquad 10^{9} \mathrm{GJ} / \mathrm{Yr}\end{array}$ & $(4.15)$ & $(0.176)$ & 3.42 & & $\begin{array}{c}3.42 \\
(4.33)\end{array}$ \\
\hline $\begin{array}{l}\text { Oil } 10^{\&} \text { Gasoline } \\
\mathrm{GJ} / \mathrm{Yr}\end{array}$ & $(3.02)$ & $(0.295)$ & 1.25 & & $\begin{array}{c}1.25 \\
(3.31)\end{array}$ \\
\hline $\begin{array}{l}\text { Coal \& Coke } \\
109^{\text {GJ }} / \mathrm{Yr} \\
\text { Nuclear } \\
109 \mathrm{GJ} / \mathrm{yr}\end{array}$ & $\begin{array}{c}7.52 \\
(8.26) \\
0.137 \\
(0.608)\end{array}$ & & & & $\begin{array}{c}7.52 \\
(8.26) \\
0.137 \\
(0.608)\end{array}$ \\
\hline $\begin{array}{l}10^{9} \mathrm{GWS} / \mathrm{Yr} \\
\simeq 10^{9} \mathrm{GJ} / \mathrm{Yr}\end{array}$ & & & & $\begin{array}{c}0.799 \\
(0.994)\end{array}$ & $\begin{array}{c}0.799 \\
(0.994)\end{array}$ \\
\hline TOTALS ${ }^{2}$ & $\begin{array}{c}7.66 \\
(16.0)\end{array}$ & $(0.472)$ & 4.67 & $\begin{array}{c}0.799 \\
(0.994)\end{array}$ & $\begin{array}{c}13.1 \\
(17.5)\end{array}$ \\
\hline
\end{tabular}

1.SRI gives hydroelectric power at its "gross fuel value" (electricity at the busbar), while BNL inflates hydro by an "efficiency" of either $1 / 0.326$ or $1 / 0.37$. Here the BNL value is converted back to delivered electricity, using the 0.326 efficiency; i.e., an equivalent heat rate of $10478 \mathrm{Btu} / \mathrm{kWh}$, whatever this may mean for hydropower. 
Table 8-6

INDUSTRIAL FUEL CONSUMPTION: FUEL TYPE VS FUNCTION 1968, SRI; (1972, BNL)

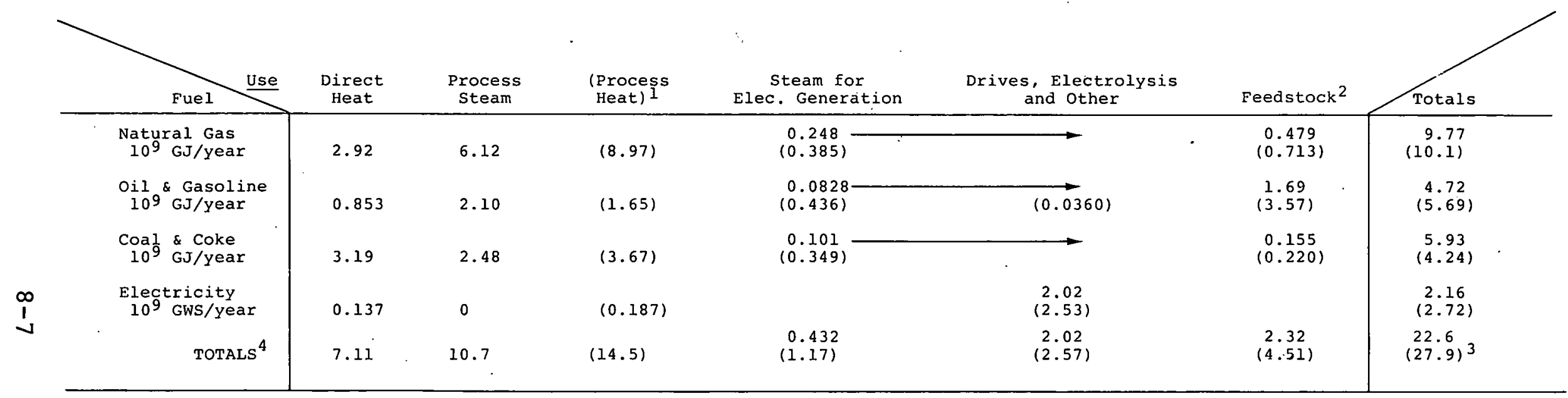

1. BNL omits the petroleum refining industry (see footnote 3 ) and does not break down this category into direct heat and process steam.

2.The BNL values here are "Petrochemical Feedstocks, Asphalt and Road Oil."

3. BNL reports the following separately

$$
\begin{array}{ll}
\text { methane production } & 2.13 \times 10^{9} \mathrm{GJ} / \mathrm{Yr} \\
\text { coal production } & 0.457 \times 10^{9} \mathrm{GJ} / \mathrm{yr} \\
\text { refinery use } & \frac{2.60 \times 10^{9} \mathrm{GJ} / \mathrm{Yr}}{} \\
& 5.19 \times 10^{9} \mathrm{GJ} / \mathrm{Yr}
\end{array}
$$

This number is added to 22.7 from the table to give the reported 27.9 . 
fuel consumption seems reasonable. The analysis of the SRI 1968 residential and commercial sector made in PIB-1 should be noted, along with the problem of adding different forms of energy.*

Table 8-3 shows a breakdown of the commercial sector. The caveat for the SRI data stated by PIB-1 should again be noted. Unlike Table 8-2, comparison of SRI and BNL data here does give cause for concern. However, the across-the-fence consumption diminished from 1968 to 1972, according to these data, which is difficult to rationalize.

Table 8-4 shows usage of the various fuels in the transportation sector. There are no great surprises or obvious inconsistencies here; It should be pointed out that the 1968 to 1972 increase of $1.3 \times 10^{9} \mathrm{GJ} / \mathrm{Yr}$, representing $2 \%$ per annum increase, is close to the year-to-year and source-to-source variances in the data in Table 8-1.

Table 8-5 shows, for the utility sector, the fuel and source distribution for the several modes by which electricity is generated. The growth shown from 1968 to 1972 is $\sim 7.5 \%$ per annum, and the increased percentage of premium fuel in the distribution during the four-year interval should be emphasized.

Table 8-6 for the industrial sector shows a $5.4 \%$ per annum growth from 1968 to 1972 of across-the-fence fuel consumption. Some comments are required concerning the internal consistency of these data, particularly the BNL data. They state that their process heat category excludes the petroleum refining industry; however, they do include as a separate category "Refinery Use," with fuel use assigned to oil. Therefore, one could include (see footnote 3 in Table 8-6) $2.60 \times 10^{9} \mathrm{GJ} / \mathrm{Yr}$ in the oil and gasoline category, making the total 8.29 rather than $5.69 \times 10^{9}$ GJ/yr. Similarly, the BNL values of fuel use for natural gas would be increased by

*The annual rates of fuel consumption are given in $10^{9}$ gigajoules per year, since the joule is a natural unit to use for energy, heat, or work. The electricity used by the sector is given in $10^{9}$ gigawatt seconds per year to obtain the net fuel value (no accounting is made for waste heat at the utility); that is, the electrical unit (power) rather than the thermal unit (energy, work, or heat). Strictly speaking, one should not add the electricity consumption to the fuel consumption. It has been done here and elsewhere in this report to demonstrate an "across the fence" energy requirement. Note that in Table 8-1 electricity generation by utilities is either displayed separately in terms of gross fuel value (thermal) or is allocated to the various sectors of the economy where it was used, still at the gross value, whence addition of fuel and electricity consumption was a legitimate addition of energy or fuel value. 
$2.13 \times 10^{9} \mathrm{GJ} / \mathrm{yr}$ and coal would be increased by $0.457 \times 10^{9}$. GJ/yr . The display in Table $8-6$ is designed to preserve the separate data reporting employed by BNL.

\subsubsection{Upper Limits to CHP Potential for Premium Fuel Savings and Conservation}

From the data in Tables 8-2 through 8-6, an upper limit to the substitution of premium fuels (oil and natural gas) by heat distributed with a CHP system can be estimated by ignoring problems of implementation and by assuming that CHP systems can be used to provide the energy for space and water heating in the residential and commercial sectors and for process heat in the industrial sector. A multiplicity of powerplant-CHP systems is envisaged, each servicing a geographically restricted region with all of its needs for space heating, hot water, and industrial process heat. According to the 1972 BNL values in Tables 8-2 through 8-6, oil and gas usage is as follows:

Residential space and water heating $\mathrm{GJ} / \mathrm{yr}$

Commercial space and water heating

$8.88 \times 10^{9}$

Industrial process heat

$4.38 \times 10^{9}$

(including refinery use, methane

$15.8 \times 10^{9}$

and coal production)

Total

$29.1 \times 109 \mathrm{GJ} / \mathrm{Yr}$

This is to be compared with a total for oil and gas of $53.1 \times 10^{9}$ GJ/yr over all sectors, including the utility sector.

The estimation of gross fuel saving which can be achieved in principle if total energy systems providing both heat and electricity are employed is not straightforward. As an example, a total energy system providing heat in the range $\dot{\leq} 3500$ to $400^{\circ} \mathrm{F}$ and electricity, in the ratio three parts thermal energy to one part electric power is considered. This has been shownt to require $\sim 73 \%$ of the fuel required for separate delivery. Tables 8-2 through 8-6 suggest the following application for 1972 data:

\section{GJ/Yr}

Residential

Space and water heating fuel

$8.88 \times 10^{9}$

Purchased electric power

$1.98 \times 10^{9}$

Commercial

Space heating fuel (incl. coal) $4.72 \times 10^{9}$

Purchased electric power $1.34 \times 10^{9}$

TThis conclusion is reached by following the exergy arguments presented in Appendix 2 of this report and is demonstrated in detail in Chapter 7 . 


\section{$\underline{G J / Y r}$}

Industrial

Process heat fuel*. (for $\leq 400 \% \mathrm{~F}$ ) $9.04 \times 10^{9}$

$(0.40 \times 22.6)$

Purchased electric power . $2.72 \times 10^{9}$

Total Fuel

$22.6 \times 10^{9}$

Purchased Electric Power

$6.04 \times 10^{9}$

For the purposes of this gross calculation, these are close enough to the ratio $3: 1$ required to apply the 73\% combined versus separately supplied process steam plus electricity. The purchased electric power is converted to thermal units by assuming a heat rate of $10,000 \mathrm{Btu} / \mathrm{kWh}$, obtaining then a total fuel requirement of $(22.6+17.7) \times 10^{9}$ or

$$
40.3 \times 10^{9} \mathrm{GJ} / \mathrm{Yr}
$$

with the potential saving

$$
(1-0.73) \times 40.3 \times 10^{9} \mathrm{GJ} / \mathrm{Yr}
$$

or

$$
11 \times 10^{9} \mathrm{GJ} / \mathrm{Yr}
$$

With 6 GJ roughly equivalent to one barrel of crude oil, this saving amounts to

$$
1.8 \times 10^{9} \text { barrels of crude per year }
$$

or.

$5 \times 10^{6}$ barrels of crude per day

This should be compared with the rate of oil import in 1975 of

$\sim 6 \times 10^{6}$ barrels of crude per day

The reader is reminded that the foregoing considerations are based on 1972 data. No extrapolations to the future are justified on the basis of available data, because of the evidence of uncertainty seen in Table 8-1 for fuel and electricity use by sectors of the economy and the relative paucity of data available for constructing Tables 8-2 through 8-6. As will be seen in the next

*This fuel figure includes oil, gas, coal, and refinery use, according to BNL. Moreover, the factor 0.40 is obtained from ECS data as the fraction of total process heat represented by process steam at $<4000 \mathrm{~F}$, for manufacturing industry in 1971 . 
section of this chapter, there was a significant change in energy consumption in the industrial sector from 1971 to 1974 , particularly vis-á-vis what a straightforward trend extrapolation would have suggested on the basis of 1971-1972 data. For purposes of this report, the potential CHP market is treated on the basis of current consumption patterns.

Determining the extent to which the potential for substitution and saving of premium fuels can be achieved is beyond the scope of this investigation, since the detailed information necessary to obtain credible results is not readily available. In the next section data available for the industrial sector are examined.

\subsection{ANALYSIS OF THE INDUSTRIAL SECTOR}

\subsubsection{Guidelines for the Analysis}

For a better characterization of the potential for a CHP system in the industrial sector, the following characteristics for each major manufacturing industrial classification at the twodigit SIC code level need to be examined:

- Temperature at which various industries require direct heat, process steam, and steam for their own energy generation and prime movers. Processes requiring temperatures above $1100 \mathrm{~F}$ will not be suitable for HTCHP application.

- Distribution of plant sizes for supplying thermal and electrical needs. Very large plants (for example, above $200 \mathrm{MiN}$ th) will probably be able to install on-site coal plants.

- Feasibility for use of a total energy system.

- Duty cycle (whether the particular manufacturing plants operate on a continuous, one-shift, or two-shift basis, or seasonally).

- Sensitivity of the industrial segment to scheduled and/ or unscheduled interruption in the delivery of heat and electricity.

- Flexibility of the industrial segment toward accepting heat and electricity delivery in a new mode (toward adapting its particular processes to a CHP system).

- The nature of both fuel and end use for the various segments within specific manufacturing industries.

- Geographical distribution of potential cHP uses, in the event that some sort of CHP utility should evolve.

It has been necessary to deal with the available data only in an approximate (and not totally satisfactory) way in order to make an evaluation of any or all of the above characteristics. 


\subsubsection{Premium Fuel Use in the Manufacturing Sector}

Tables 8-7 and 8-8 show the ECS collection of data on the distribution of types of fuel used, according to SIC code, in the manufacturing (for 1971, 1974) and nonmanufacturing (for 1971) segments of the industrial sector. In referring to Table 8-7 the following should be noted. Considering only fuel, not purchased electricity, the nonmanufacturing segment fuel use represents $20 \%$ of the total fuel used in the industrial sector. Over half of the fuel used in the nonmanufacturing segment is oil and gasoline, primarily for transportation in agriculture and the construction industries. It therefore appears that the nonmanufacturing segment is not a fruitful place in which to seek application of the CHP for reducing premium fuel use.

In the manufacturing sector for 1971, over $50 \%$ of the fuel used was premium fuel; moreover, over $45 \%$ was natural gas. The dimensions of the problem created by a natural gas shortage for manufacturing industry is indeed large. With this in mind, it is interesting to consider the shifts in fuel usage displayed by the data shown in Table 8-8 for manufacturing industry usage between 1971 and 1974. In three years premium fuel usage increased from $\sim 50 \%$ to $\sim 56 \%$ of the total fuel (again excluding purchased electricity), with natural gas usage staying constant at $\sim 44 \%$.

Table 8-9 shows some rather surprising shifts in the use of various fuels by the several segments of manufacturing industry between 1971 and 1974. There does not seem to be any consistent pattern among the six major SIC code industries as a result of oil and natural gas shortages in 1973-1974. In fact, SIC 28 - Chemicals, in particular, appears to have substantially increased its premium fuel usage at the expense of coal.

In Table 8-9 the increases or decreases from Tables 8-7 and 8-8 are shown. No overall pattern is discernible. However, premium fuel use did increase significantly, the use of coal did diminish markedly, and the slack appears to have been picked up by a large growth in purchased electricity. There are no feedstock data on these differences, nor has any change been tabulated in the use of in-house fuel. It might be inferred from these data that (a) in general, industry using petroleum products will continue to do so at increased prices (on the basis that this is cheaper and more sensible in a time of tight capital than refitting plants to use coal); (b) a long-term trend toward reduced use of coal continued through the 1973-1974 period; and (c) natural gas supplies were already short before the 1973-1974 period, and industry was already converting to oil and purchased electricity where it could or had no alternative.

It is perhaps significant for future consideration of total energy systems based on a CHP that the marked increases in purchased electricity shown in Table 8-9 between 1971 and 1974 and in Figure 8-1 as a time series for six major SIC code industries were not accompanied by any increase in the generation of electricity by industry itself. In fact, generated electricity fell 
Table 8-7

FUEL SOURCE OF ENERGY CONSUMED - INDUSTRIAL SECTOR - 1971 ECS Data (Feedstock Excluded)

$\underline{\text { Sic code Industry }}$

\section{Manufacturing}

20 Food

26 Paper

28 Chemicals

32 Stone, Clay, Glass

33 Prim. Metals

All others

in 20-39

Total without Electricity

Total Manufacturing with

electricity at net fuel value.

(Total manufacturing with elec-

$\infty$ tricity including utility

\section{Non-Manufacturing}

$\begin{aligned} 01-03 & \text { Agriculture } \\ 13 & \text { Gas Production } \\ 15-17 & \text { Construction } \\ 8-12,14 & \text { All other }\end{aligned}$

8-12,14 All other

Subtotal, without electricity

Total Industry without electricity

Total Industry, Electricity at net fuel value

(Total Industry; Electricity, including utility waste heat with $n=0.31$ )

BNL (1972), less feedstock (Table 6)

$\begin{array}{cr}\text { Nat. Gas } & \begin{array}{r}\text { Energy Val } \\ \text { Oil \& Gasol }\end{array} \\ 0.52 & \\ 0.52 & 0.13 \\ 1.56 & 0.41 \\ 1.44 & 0.22 \\ 0.77 & 0.09 \\ 1.20 & 0.13 \\ & 0.24 \\ 1.04 & \underline{ } \\ 7.05 & 1.36\end{array}$

$\frac{\text { Fuel, } 10^{9} \mathrm{GJ} / \mathrm{yr}}{\text { Coal } \& \text { Coke }}$

0.13

0.13
0.26
0.51

0.51

0.01

0.28
0.60

$\underline{0.26}$

2.05 other

0.18

0.18

0.29

0.05

0.11

0.52

1.34

0.97

1.26

1.57

$\overline{3.80}$

$\underline{0.64}$

1.86

0.13

0.13

0.09

0.09

0.44

0.64

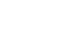

Total 1

$\left(10^{9} \mathrm{GJ} / \mathrm{yr}\right)$

$$
\begin{aligned}
& 1.09 \\
& 2.36 \\
& 2.93 \\
& 2.94 \\
& 1.38 \\
& 4.15 \\
& \\
& 2.82 \\
& 15.8 \\
& 17.7
\end{aligned}
$$

(21.8)

0.11

0.03

0.05

$\underline{0.07}$

0.26

2.12

2.11

0.08

1.23

7.40

3.74

1.42

5.03

19.7

21.8

$(26.5)$

2.7

23.4

1. Power and thermal energy again added.

2. Explanation of "in-house":

SIC 26: principally byproduct fuel - "black liquor" from process wood.

SIC 29: industry-owned petroleum or petroleum byproducts used as fuel.

SIC 33: industry-owned coal not otherwise listed, used as fuel. 
Table 8-8

FUEL SOURCE OF ENERGY CONSUMPTION - INDUS'PRIAL SECTORI - 1974 ECS Data (Feedstock Excluded)

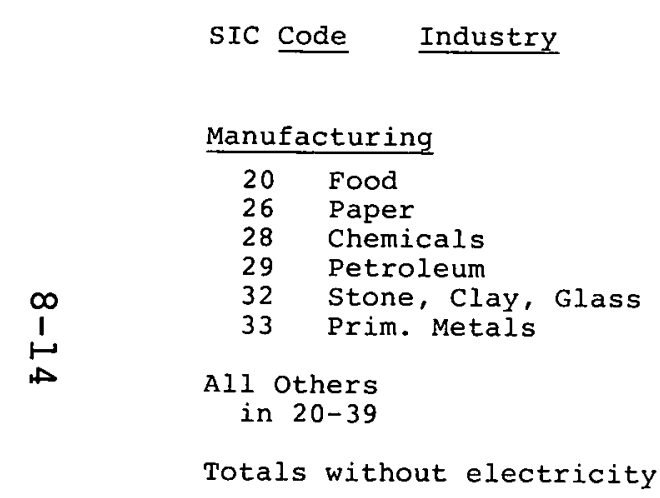

Total Manufacturing with electricity at net fuel value

Total Manufacturing with electricity including utility waste heat with $\eta=0.31$
Energy Value of Fuel, $109 \mathrm{GJ} / \mathrm{Yr}$ Oil\& Gasoline Coal \& Coke
In-House ${ }^{2}$

\begin{tabular}{l}
0.09 \\
0.23 \\
0.38 \\
0.03 \\
0.27 \\
0.54 \\
0.18 \\
\hline \\
1.72
\end{tabular}

0.15

0.08
0.23
0.12
0.15

0.15

0.16

0.97

1.26

1.57

$\underline{0.52}$

1.41

$\longrightarrow$

3.80

0.14

0.48
0.30

0.50
0.46

1.73

1. 28

0.74

0.30
0.09

0.13

$\underline{1.05}$

0.34

$\underline{0.36}$

1.84

6.89

Purch. Elec.

(109 GWS/Yr)

Total ${ }^{3}$

(1.09 GJ/Yr)

\subsection{1 \\ 2.37 \\ 3.10
2.88 \\ 2.88
1.39 \\ 4.32 \\ $\underline{2.83}$}

$\underline{0.72}$

15.7

17.9

22.9

1. Nonmanufacturing industry data not yet available.

2. 1971 values.

3. Power and thermal energy again added. 
Table 8-9

CHANGGE IN PURCHASED ENERGY IN MANUFACTURING INDUSTRY 1974-1971

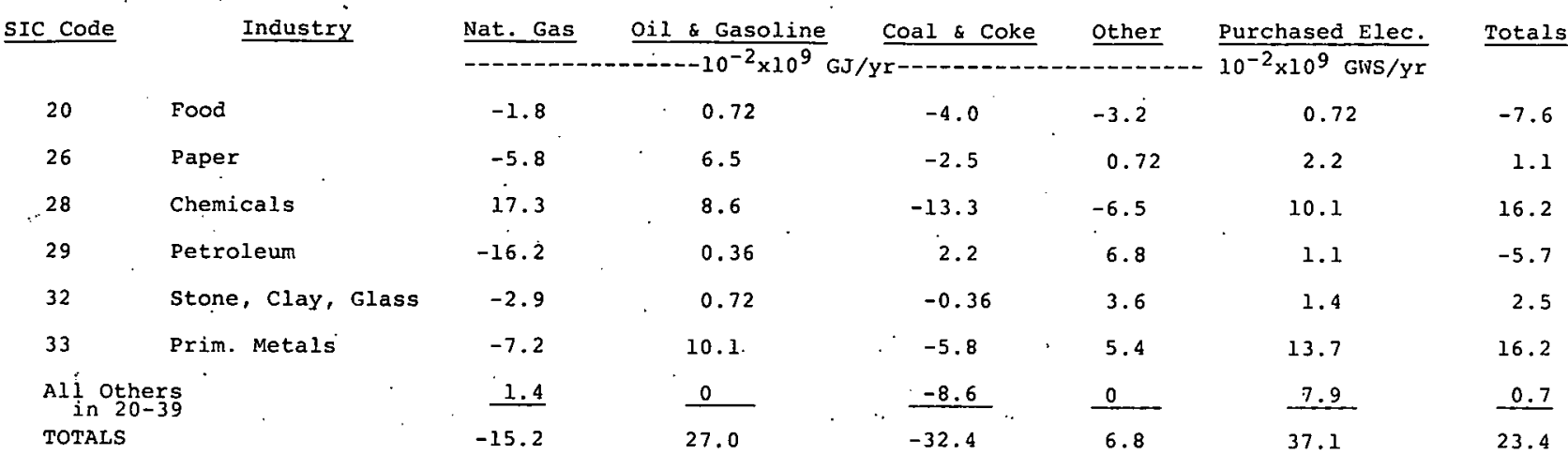

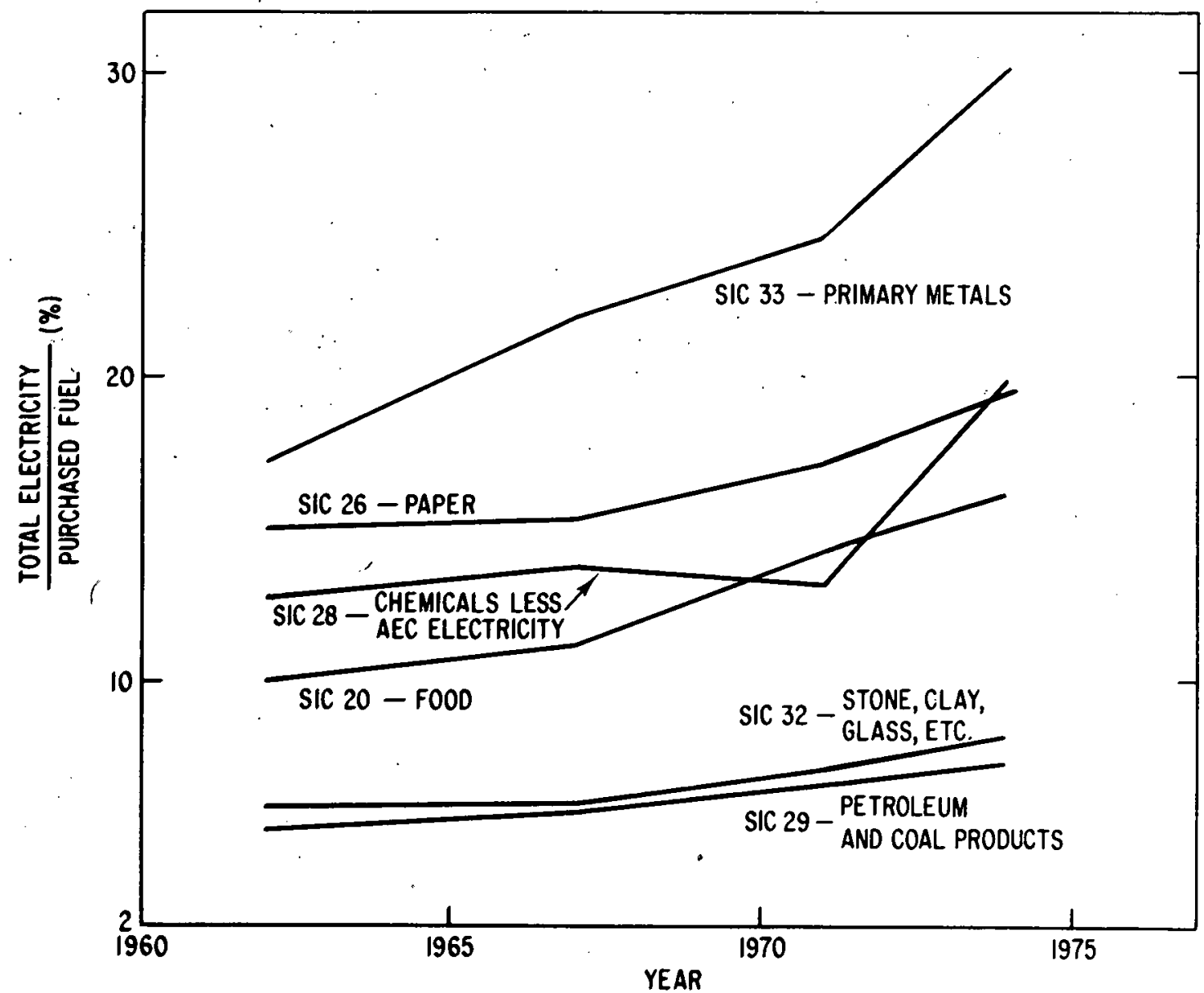

Figure 8-1. Ratio of Total Electricity (Purchased and Gen erated) to Purchased Fuel (ECS Data) 
slightly between 1971 and 1974. In Table 8-10 purchased and generated electricity are tabulated by SIC code to imphasize this result. In-plant generation has not become a popular characteristic of manufacturing industry; according to ECS the 1971-1974 downward trend is a continuation of previous trends.

It is suggested (ECS) that the ratio of generated to purchased electricity, which in the past has been as high as $20 \%$ and was down to $212 \%$ in 1974, will go up again, in part because there will be better tax write-offs for nonutility generation and in part as a result of increasing utility charges, particularly as time-of-day rate structures are adopted more widely. It should be noted that in-plant generation does not appear explicitly in Tables 8-7, 8-8, and 8-9. Rather, the fuel use for such generation is folded into the general totals of purchased fuel.

\subsubsection{Fuel Usage for Process Heat at Various Temperatures}

The competition for supplying process heat between a CHP system and other methods depends to some extent on the temperature at which the potential customer requires delivery of the process heat. In particular, with a methane-based, CHP system it appears that $\sim 11000 \mathrm{~F}$ represents an upper limit for process heat delivery (see Chapter 5 of this report). The data available on process heat needs are those given by ECS for manufacturing industry in 1971. These are collected in Table 8-11 by SIC code. Given the most liberal view of where the CHP system can operate, only that part of direct heat required at $\mathrm{T} \geq 1100^{\circ} \mathrm{F}$ and, according to the ECS study, approximately one-fourth of the energy required for mechanical drives are excluded, leaving $\sim 65 \%$ or $10.3 \times 10^{9} \mathrm{GJ} / \mathrm{yr}$ of the $15.810^{9} \mathrm{GJ} / \mathrm{Yr}$ oil and natural gas consumption by industry accessible to the CHP (see Table 8-6).

Table 8-10

PURCHASED AND GENERATED ELECTRICITY IN MANUFACTURING IN̈DUSTRY

1971,1974

\begin{tabular}{|c|c|c|c|c|c|}
\hline \multirow{3}{*}{ SIC Code } & \multirow{3}{*}{ Industry } & \multicolumn{2}{|c|}{1971} & \multicolumn{2}{|c|}{1974} \\
\hline & & Purchased & Generated & Purchased & Generated \\
\hline & & $\overline{-\ldots-\ldots-1}$ & $--10^{-2} \times 10^{9}$ & GWS/Yr---- & - \\
\hline 20 & Food & 12.6 & 1.1 & 13.4 & 0.9 \\
\hline 26 & Paper & 12.6 & 9.0 & 14.8 & 9.8 \\
\hline 28 & Chemicals & 36.0 & 7.2 & 46.1 & 6.7 \\
\hline 29 & Petroleum & 8.6 & 1.8 & 9.8 & 1.6 \\
\hline 32 & Stone, Clay, Glass & 9.0 & 0.4 & 10.5 & 0.2 \\
\hline 33 & Prim. Metals & 43.9 & 9.0 & 57.7 & 8.1 \\
\hline $\begin{array}{c}\text { All others } \\
\text { in } 20-39\end{array}$ & & 63.4 & 1.8 & 70.9 & 0.9 \\
\hline TOTALS & & 186 & 30 & 223 & 28 \\
\hline
\end{tabular}


Table 8-11

FUEL CONSUMED FOR PROCESS HEAT AND STEAM

IN MANUFACTURING INDUSTRIES - 1971

$109 \mathrm{GJ} / \mathrm{Yr}$

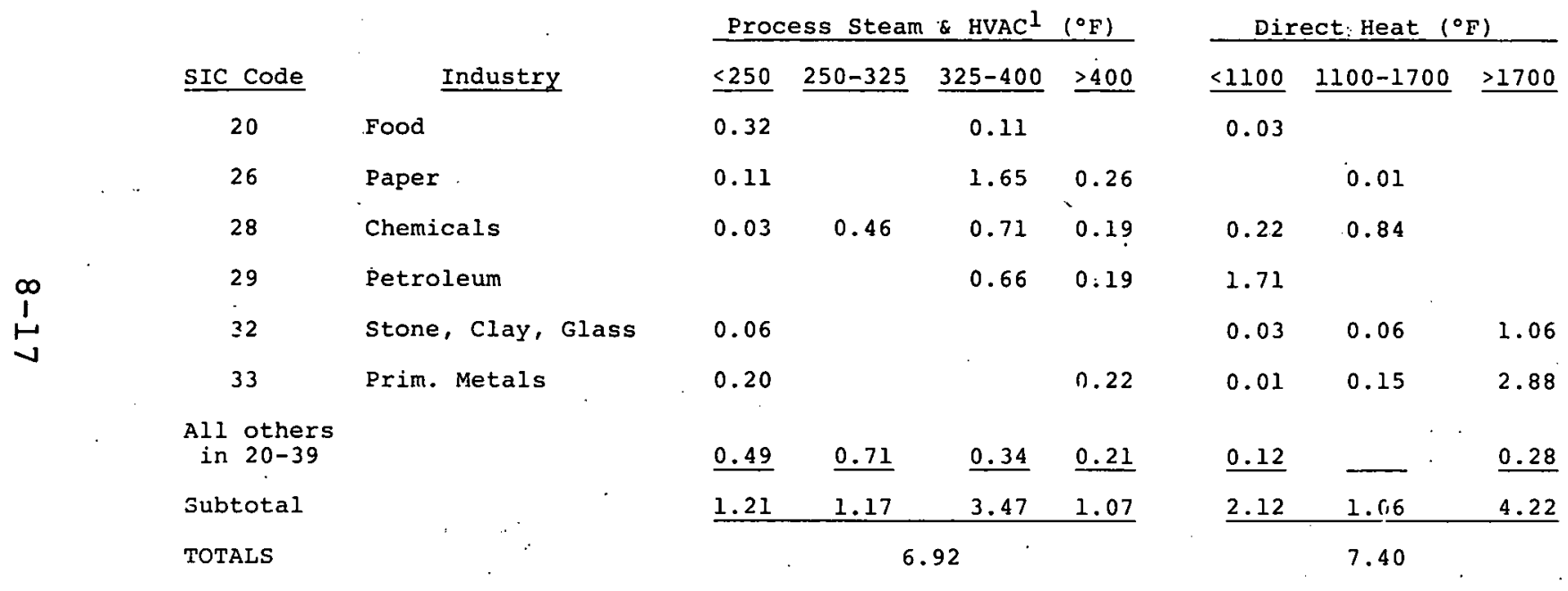

\begin{tabular}{|c|c|c|}
\hline $\begin{array}{l}\text { Mechanical } \\
\text { Drives }^{2} \\
\end{array}$ & $\begin{array}{c}\text { Electric, } \\
\text { Gen. Steam } \\
\end{array}$ & Totals \\
\hline 0.49 & 0.01 & 0.96 \\
\hline 0.06 & 0.15 & 2.24 \\
\hline 0.06 & 0.07 & 2.58 \\
\hline 0.25 & 0.04 & 2.85 \\
\hline 0.07 & 0.01 & 1.29 \\
\hline 0.12 & 0.13 & 3.71 \\
\hline 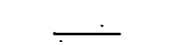 & $\underline{0.03}$ & $\underline{2.18}$ \\
\hline 1.04 & 0.43 & - \\
\hline & 15.8 \\
\hline
\end{tabular}

1. In an early study ECS estimated that heating, ventilating, and air conditioning amounted to $\sim 15 \%$ of the fuel usage in "Process steam and HVAC.

2. Includes steam and gas turbines and internal combustion engines. ECs estimates 758 of this in the range 750 to $900^{\circ} \mathrm{F}$.

3. ECS estimates this typically in the range 850 to $950^{\circ} \mathrm{F}$. 
No additional data sources which break down usage data according to the temperature at which energy is delivered have been found. The essential correctness of the data on energy usage versus temperature in Table 8-11 has been checked by comparison with a study by the Bundesministerium für Forschung und Technologies (BFT) (R8-9) showing the temperature distribution of energy usage for process heat in West Germany in 1973. To make this comparison the ECS and BFT data were normalized by plotting the fraction of total fuel usage against temperature to provide process heat at or below a given temperature range. The West German usage lies within the shaded curve shown in Figure 8-2. Also shown is the normalized ECS data. There is sufficient agreement to conclude that the shaded curve is some sort of a universal temperature distribution, given that U.S. and West German manufacturing industries are not dissimilar.

\subsubsection{Distribution of Plant Size Among Uses of Process Heat}

Determining the demand rate for fuel that obtains for the various manufacturing industries would be a formidable undertaking were it not for the easing of the constraints, mentioned earlier, on the nature of such industries in which a CHP technological substitution was deemed feasible.

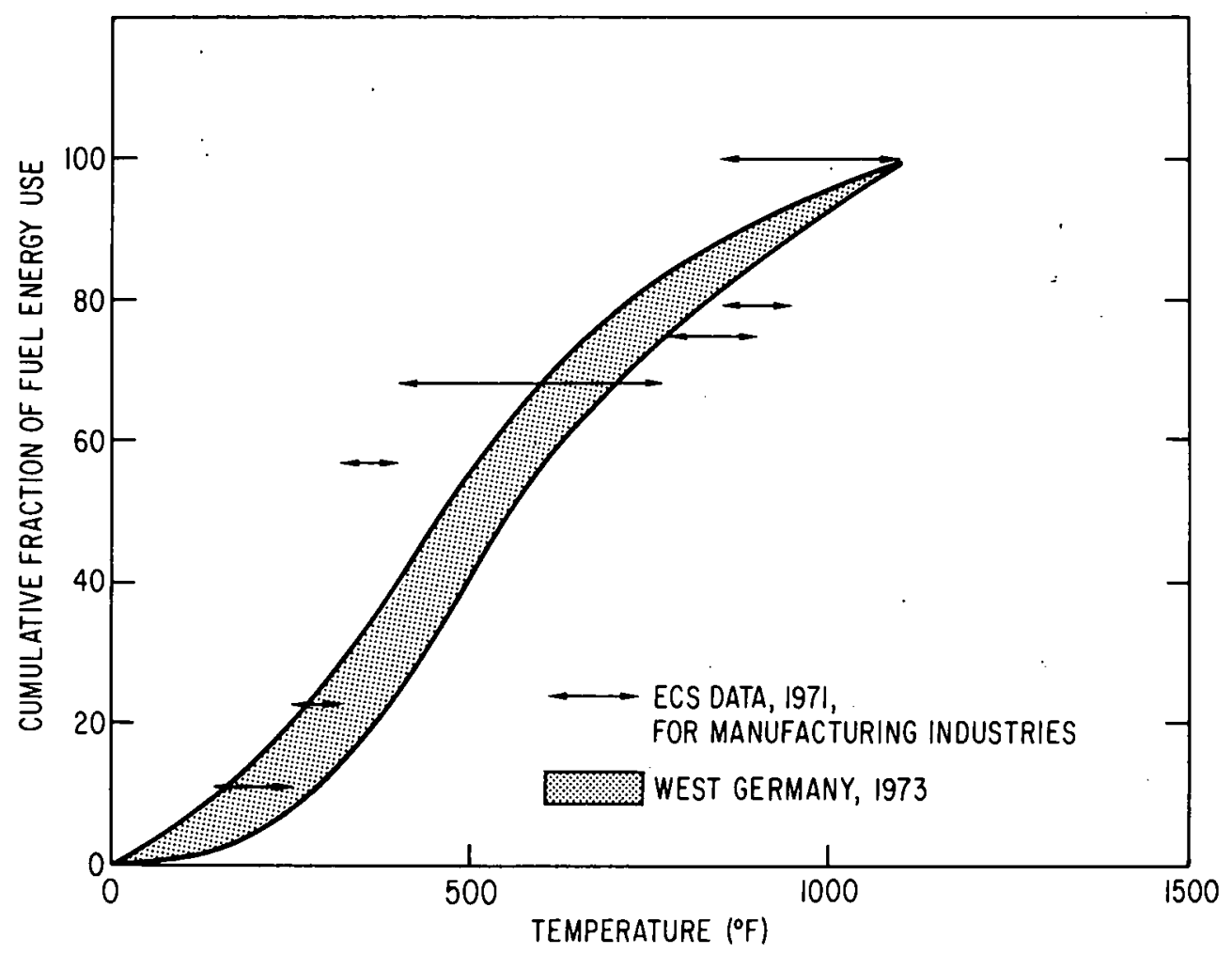

Figure 8-2. Cumulative Fraction of Fuel'Energy Use vs Temperature Processing 
Data from the year 1971 were provided by ECS as follows. Within each of the six major two-digit designations in the manufacturing sector, the fuel demand rate was determined at the level of each four-digit"SIC code and averaged. This yields a distribution for each of the six major two-digit SIC codes of mean plant size or plant size range, the number of plants of that size or within that size range, and the fraction of purchased fuel associated with the given plant size or plant size range. From these tabulations, graphical representations were constructed which are discussed in greater detail below.

To obtain a distribution for the summation of the two-digit code industries for which no distribution was supplied in the ECS study, an average curve from the six major SIC code data was constructed by normalizing each distribution by the total fuel usage and by the maximum plant size, and then assigning a weight to each distribution proportional to the total purchased fuel for each distribution. The weighted normalized distributions were then summed and averaged. This average curve was applied to the total of purchased fuel and to the maximum plant size in the "all other" SIC code category. The six curves for the major SIC code industries and the synthesized "all other" curve were then added to obtain a final curve representing the plant size distribution for the total fuel usage of manufacturing industries.

\subsubsection{SIC 20 - Food Industry}

The 1971 rate of fuel purchase (not including purchased electricity): is (summarized as follows:

$\begin{array}{ll} & \text { GJ/Year } \\ \text { Heating, ventilating, and air conditioning } & 0.07 \times 109 \\ \text { Seasonal } & 0.17 \times 109 \\ \text { Continuous } & 0.50 \times 109 \\ \text { Other duty cycles } & 0.22 \times 109 \\ \text { Total } & 0.96 \times 109\end{array}$

The heat requirements are all below $1100^{\circ} \mathrm{F}$. This industry is a mixture of continuous year-round, seasonally continuous, one- and two-shift year-round, and seasonally one- and two-shift users. The industry is heavily dominated by small users; for example, 22,400 plants require less than $0.5 \mathrm{MW}_{\text {th }}$ each, 31,100 require less than 1.0 $\mathrm{MW}_{\text {th }}$, and 33,300 require less than $5 \mathrm{MW}_{\text {th }}$, all out of a total of 35,600 plants. The 33,300 plants requiring less than $5 \mathrm{MW}_{\text {th }}$ use $261 \%$ of the total fuel energy for the industry.

It is not clear how a CHP system could be adapted to the many thousands of geographically dispersed small users with varied patterns of duty cycles. Nevertheless, with the caveat that one is dealing here with the very upper. limit of substitution for a CHP system, Figure 8-3 can show the cumulative fraction of fuel use 


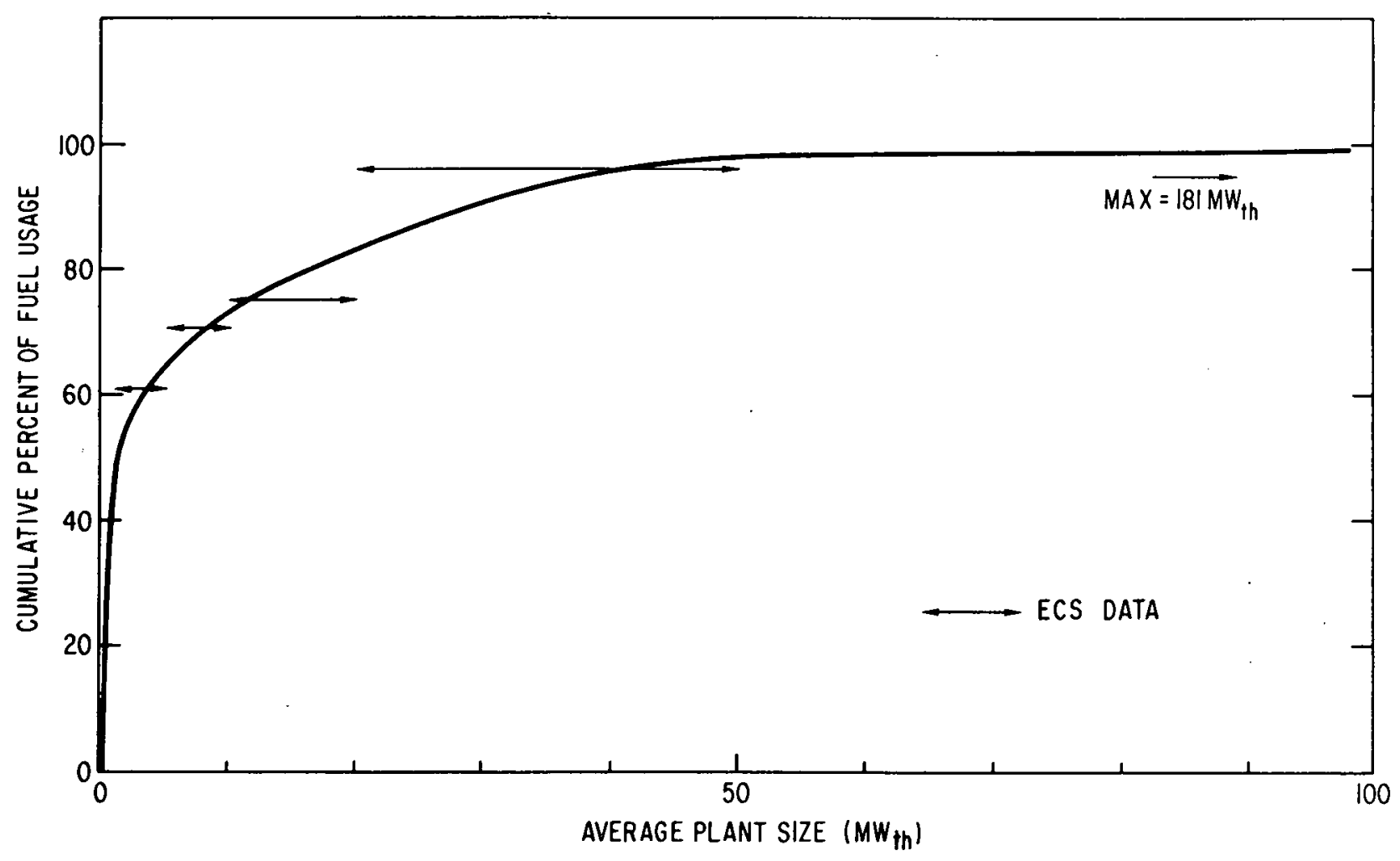

Figure 8-3. SIC 20 - Food: Plant Size Distribution Based on 1971 Purchased Fuel Usage of $0.96 \times 10^{9}$ GJ $/ \mathrm{Yr} \mathrm{T}<1100^{\circ} \mathrm{F}$

versus average plant size for the food industry. The data were provided in terms of average plant sizes within the ranges shown; the curve was drawn as an approximate fit to the data to secve later in averaging.

Referring back to Table 8-8, $\sim 73 \%$ of the fuel used by this industrial segment is premium fuel; this, coupled with the preponderance of small users, poses a real challenge for SHP development.

If, as discussed in section 8.3.1, one- or two-shift industries requiring heat at $<11000 \mathrm{~F}$ are counted, then according to ECS the net fuel usage accessible to CHP substitution is $\sim 0.44 \times 10^{9}$ GJ/yr. This is about $45 \%$ of the total fuel usage of the food segment of manufacturing industry.

\subsubsection{SIC 26 - Paper Industry}

Excluding purchased electricity, the total purchased fuel energy in the paper industry was $1.26 \times 10^{9}$ GJ/yr in 1971 . There was in addition $0.97 \times 10^{9} \mathrm{GJ} / \mathrm{yr}$ of byproduct fuel use. Since byproduct fuels are an inevitable consequence of the current processes in the paper industry, it does not seem reasonable to consider this byproduct fuel amenable to CHP substitution. In a more detailed analysis of CHP use in this industry, due attention will need to be paid to the strong geographic dependence of the use of byproduct fuel. This use amounts to approximately 10 to $15 \%$ 
of fuel energy in northeastern and north central United States, and $\sim 50 \%$ in the southeast and west.

Essentially all of the heat requirements in this industrial segment are less than $\sim 1100^{\circ} \mathrm{F}$. Virtually the entire segment is made up of continuous process plants; seasonal use, which is less than $10 \%$ of the total fuel use, is entirely accounted for by the heating, ventilating, and air conditioning (HVAC) needs of the industry.

Plant'size distribution also shows a strong geographic dependence, the largest plants being in southeastern and western U.S. As with the food industry, plant size distribution is also strongly skewed toward small plants. Of the approximately 5900 plants making up SIC 26, approximately 5000 average $\sim 1 \mathrm{MW}_{\text {th }}$ in size. In terms of potential CHP substitution, $\sim 75 \%$ of purchased fuel (excluding purchased electricity and in-house fuell is premium fuel; i.e., oil and natural gas (see Table 8-8).

The information available on plant size is shown in Figure 8-4; the curve approximating the data will be used later in averaging. The plotted points and estimated curve refer to purchased fuel energy only. If in-house fuel energy were to be included a quite different curve would result, because the larger plant sizes are also larger users of in-house fuel. However, the in-house fuel. is considered to be irrelevant in the present considerations.

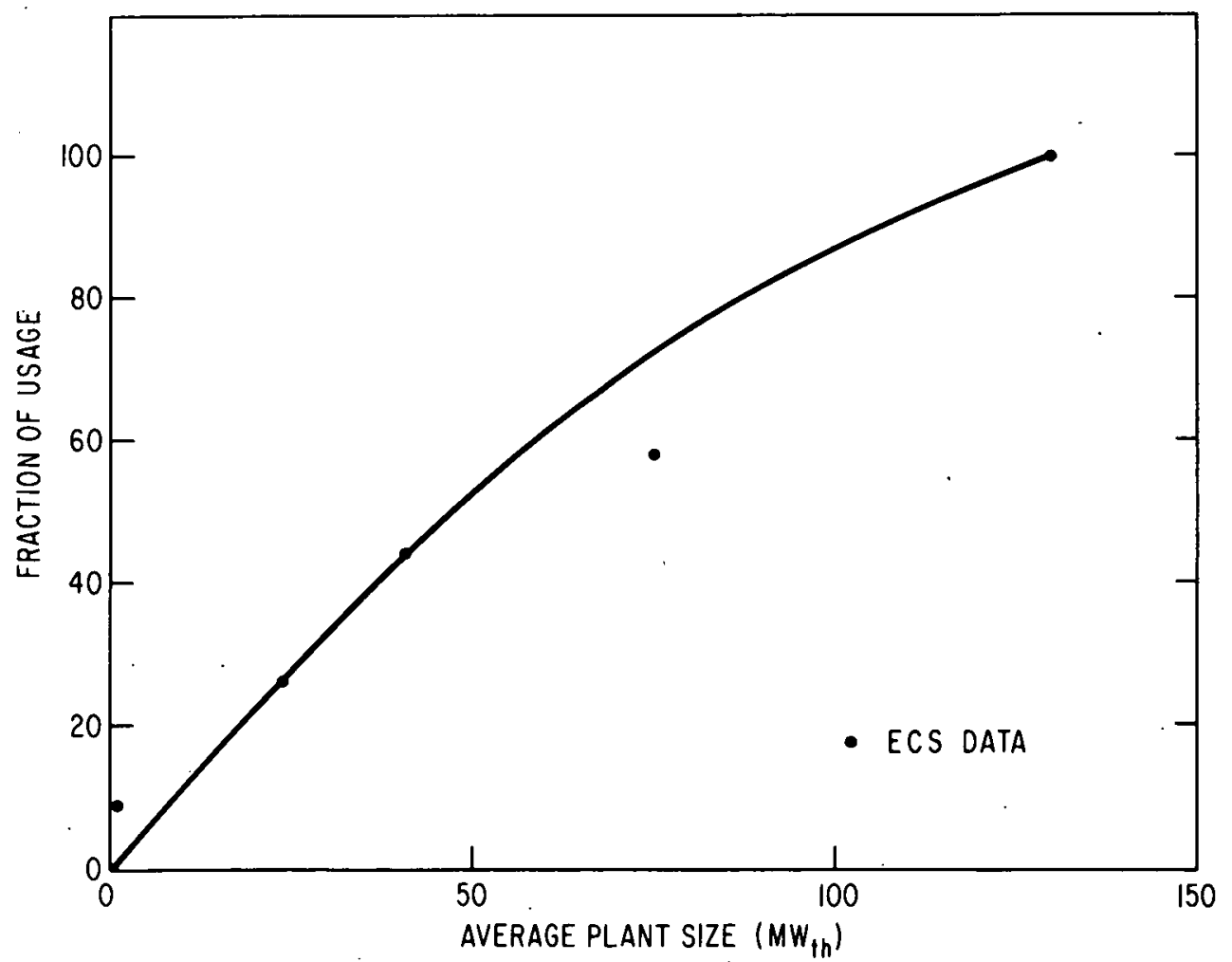

Figure 8-4. SIC 26 - Paper: Plant Size Distribution Based on 1971 Purchased Fuel Usage of $1.26 \times 10^{9} \mathrm{GJ} /$ $\mathrm{Yr}$ at $\mathrm{T}<11000 \mathrm{~F}$ 
Applying the full constraints mentioned in the introduction (ruling out usage with $\mathrm{T}>1100{ }^{\circ} \mathrm{F}$, continuous operation, operation at $>200 \mathrm{MW}_{\text {th }}$ per plant, seasonal and space heating use) little is left for CHP substitution in the paper industry, SIC 26; namely $2 \%$ of purchased fuel use or $\sim 0.12 \times 10^{9} \mathrm{GJ} / \mathrm{yr}$.

\subsubsection{SIC 28 - Chemical Industry}

The total purchased fuel in the chemical industry segment (excluding purchased electricity) was $2.57 \times 10^{9} \mathrm{GJ} / \mathrm{yr}$ in 1971 . A significant fraction of this, $33 \%$ or $0.84 \times 10^{9} \mathrm{GJ} / \mathrm{yr}$, was used for providing heat at temperatures in excess of $11000 \mathrm{~F}$, leaving $67 \%$ or $1.73 \times 10^{9} \mathrm{GJ} / \mathrm{Yr}$ as an upper limit for CHP substitution.

Obtaining the plant size distribution from the data supplied by ECS requires some assumptions. The data on distribution of plant size, given in ECS includes only $78 \%$ of the purchased fuel but contains one-third of the industries which require $\mathrm{T}>1100^{\circ} \mathrm{F}$. It is assumed that the missing $22 \%$ is the remaining two-thirds of the $T>11000 F$ demand. If it is further assumed that removing the $T>11000 F$ demand from the available data does not change the plant size distribution, then the $1.73 \times 10^{9} \mathrm{GJ} / \mathrm{yr}$ of $\mathrm{T}<11000 \mathrm{~F}$ demand can be assigned plant size according to the available data.

The resultant distribution is given in Figure 8-5. The actual upper limit in plant size is not very precise; the largest

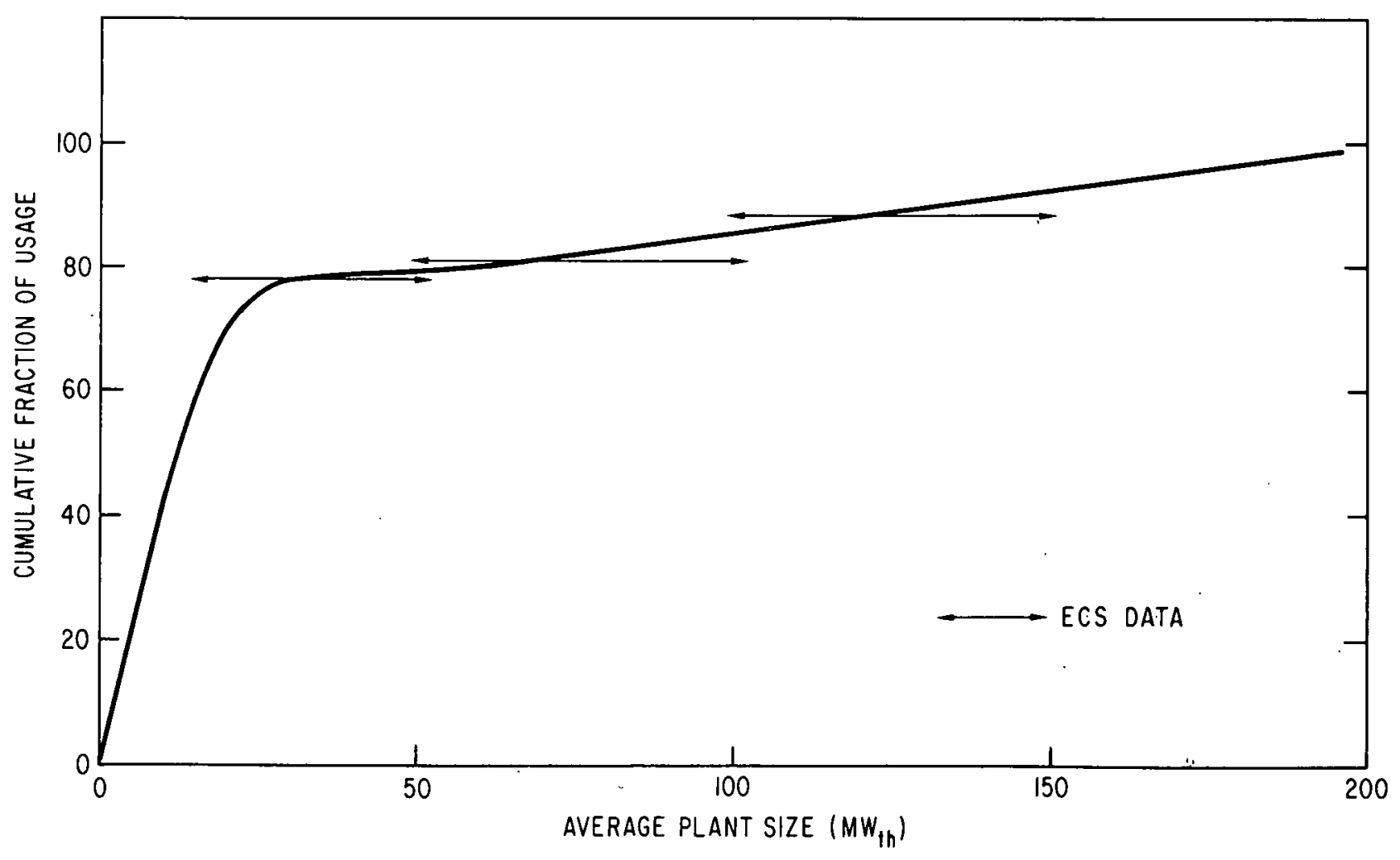

Figure 8-5. SIC 28 - Chemical: Plant Size Distribution Based on 1971. Purchased Fuel Usage of $1.72 \times 10^{9} \mathrm{GJ} / \mathrm{Yr}$ at $\mathrm{T}<11000 \mathrm{~F}$ 
plant size category, given as $>150 \mathrm{MW}_{\mathrm{th}}$, represented less than $10 \%$ of the total purchased fuel usage. The distribution data were presented as ranges; hence the appearance of the plotted data. The curve shown was sketched in to approximate the data.

If one applies the criteria mentioned earlier $(T<11000 \mathrm{~F}$, no continuous users, and no HVAC), then at most $7.4 \%$, or $0.19 \times 10^{9}$ GJ/Yr, of the 1971 usage would have been accessible to CHP application.

Table 8-8 shows that $77 \%$ of the purchased fuel usage comprised premium fuels. If the pessimistic screening of the previous paragraph is applicable, there is little chance of premium fuel relief from the use of a CHP system.

\subsubsection{SIC 29 - Petroleum Refining}

This industrial segment is unique in the high fraction of its fuel use represented by captive, in-house fuel. In 1971, of a total of $2.85 \times 10^{9} \mathrm{GJ} / \mathrm{Yr}$, some $56 \%$, or $1.60 \times 10^{9} \mathrm{GJ} / \mathrm{yr}$, was purchased fuel while $44 \%$, or $1.26 \times 10^{9} \mathrm{GJ} / \mathrm{Yr}$, was captive, in-house fuel. To within $\sim 5 \%$, the purchased fuel was virtually all natural gas; the in-house fuel use was all oil. Over the entire industry, approximately $96 \%$ of fuel usage was to produce temperatures below $11000^{\circ}$. Moreover, more than $97 \%$ of the industry operates in a continuous process mode.

The ECS data on plant size distribution are presented in Figure 8-6. The following points should be emphasized:

Purchased electricity represents only $\sim 3 \%$ of the total annual fuel use and has not been removed from the size distribution data, so far as is known. It is reported by ECS that in 1971 SIC 29 industries purchased $0.09 \times 10^{9}$ GWS/Yr and generated $0.02 \times 10^{9}$ GWS/yr of electricity.

- Captive, in-house fuel has been included for this industry, because without a more careful analysis it would seem reasonable to suppose that much or all of this $1.26 \times 10^{9} \mathrm{GJ} / \mathrm{yr}$ of oil usage might well be amenable to a CHP system substitution, with the oil thus saved being used elsewhere.

- The curve in Figure $8-6$ is carried to $100 \%$ at an average plant size of $648 \mathrm{MW}_{\mathrm{th}}$. This number is in fact the average of plants exceeding a refining capacity of $\sim 80,000$ barrels of oil per day, the largest category of classification, and the maximum plant size may be considerably greater. 


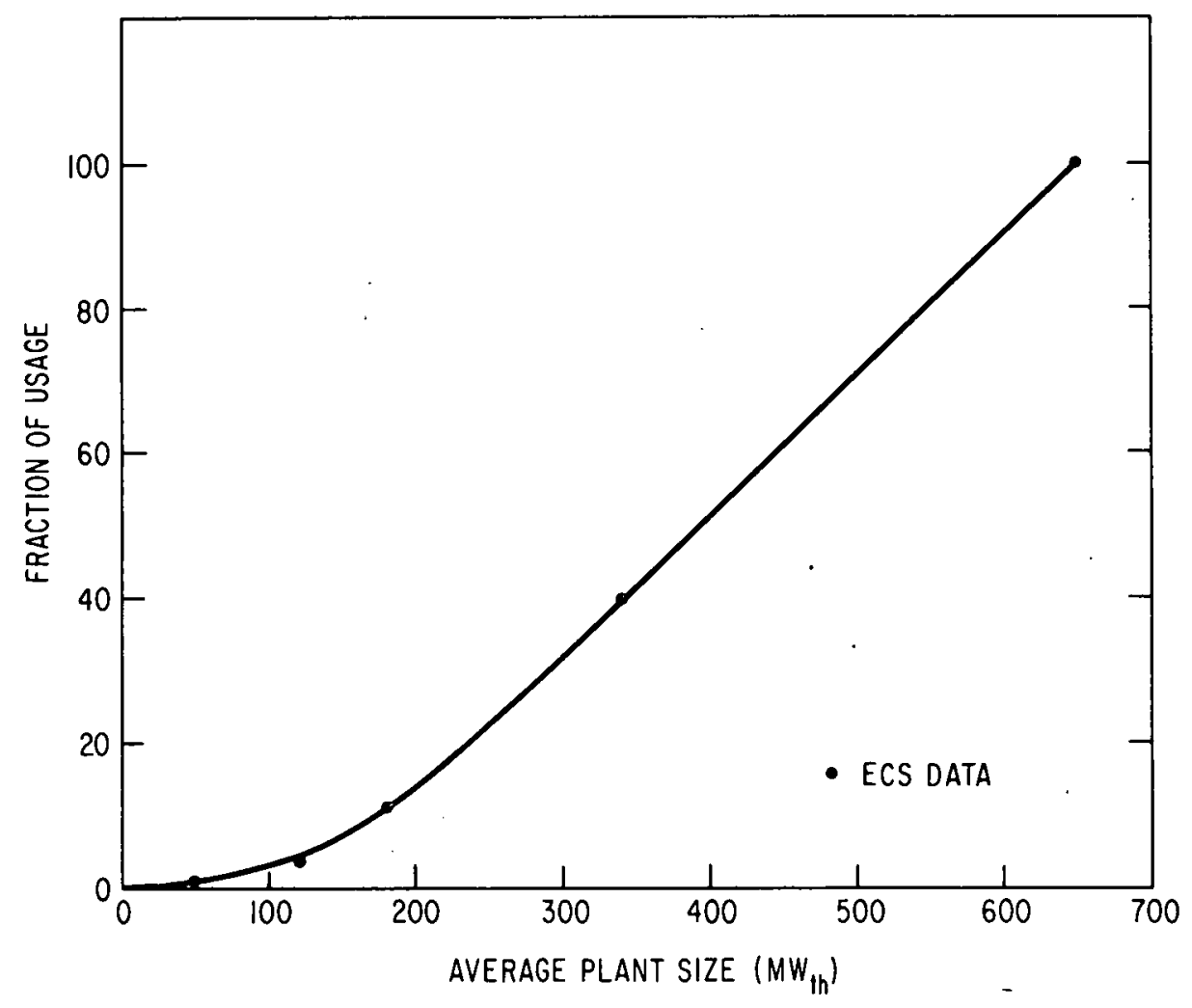

Figure 8-6. SIC 29 - Petroleum Refining: Plant Size Distribution Based on 1971 Total Fuel Use (purchased plus in-house) of $2.85 \times 109 \mathrm{GJ} / \mathrm{Yr}$ at $\mathrm{T}<11000 \mathrm{~F}$

\subsubsection{SIC 32 - Stone, Clay, and Glass}

This segment of manufacturing industries has little to offer for CHP technology substitution. Its 1971 total fuel use, all purchased, was $1.29 \times 10^{9} \mathrm{GJ} / \mathrm{yr}$; but only $0.13 \times 10^{9} \mathrm{GJ} / \mathrm{yr}$, or $\sim 10 \%$, was consumed to produce heat at $<1100^{\circ} \mathrm{F}$. "This amount is well within the uncertainties associated with fuel usage assigned to the other segments of manufacturing industry. Of the 10\%, half is fuel used directly for space heating. Therefore a meaningful statement about the plant size distribution in this industry cannot be developed from the ECS data, nor would it be particularly relevant with the $\mathrm{T}<1100^{\circ} \mathrm{F}$ constraint.

of the small amount of fuel used at $<1100^{\circ} \mathrm{F}, 0.03 \times 10^{9} \mathrm{GJ} / \mathrm{Yr}$ was for direct heat, $0.06 \times 10^{9} \mathrm{GJ} / \mathrm{yr}$ was for $\mathrm{HVAC}$, and $0.01 \times 10^{9} \mathrm{GJ} /$ yr was required to produce steam for electricity generation, all totaling $0.10 \times 10^{9} \mathrm{GJ} / \mathrm{yr}$. It is conceivable that purchased electricity used for drives $\left(0.08 \times 10^{9} \mathrm{GJ} / \mathrm{yr}\right)$ and for electrode boilers $\left(0.01 \times 10^{9} \mathrm{GJ} / \mathrm{yr}\right)$, totaling $0.09 \times 10^{9} \mathrm{GJ} / \mathrm{yr}$, could be internally generated with steam from a CHP delivery system. Finally, one could conceive of supplying the $0.07 \times 109 \mathrm{GJ} / \mathrm{yr}$ of fuel used for mechanical drives with CHP-based steam. In any event, for this first look at the potential of CHP substitution, there seems to 
be little of interest in the stone, clay, and glass industrial segment.

For the present, therefore, it is unlikely that the CHP substitution can provide national relief for the premium fuel use in this segment, where in 1971 some $69 \%\left(0.89 \times 10^{9} \mathrm{GJ} / \mathrm{Yr}\right)$ of the total fuel used $\left(1.29 \times 10^{9} \mathrm{GJ} / \mathrm{Yr}\right)$ was oil or natural gas.

\subsubsection{SIC 33 - Primary Metals}

This segment of manufacturing industry is indeed a larger user of energy - - 3.72 $\times 10^{9} \mathrm{GJ} / \mathrm{Yr}$ of purchased and captive in-house fuel. The primary steel industry develops and uses an additional $0.89 \times 10^{9}$ $\mathrm{GJ} / \mathrm{yr}$ of byproduct fuel. However, of the total $3.72 \times 10^{9} \mathrm{GJ} / \mathrm{yr}$ only $15 \%$, or $0.56 \times 10^{9} \mathrm{GJ} / \mathrm{Yr}$, of fuel use provides heat at < $11000 \mathrm{~F}$.

The 1971 data provided by ECS on plant size distribution can be corrected, approximately, for the fact that much of the fuel use is at $\mathrm{T}>1100^{\circ} \mathrm{F}$. In particular, use of primary steel and primary aluminum above $1100^{\circ} \mathrm{F}$, which accounts for $77 \%$ of the $85 \%$ of usage in this segment at $\mathrm{T}>1100^{\circ} \mathrm{F}$, was removed from the size distribution data. It is then assumed that the remaining plant size distribution applies to the $15 \%$, or $0.56 \times 10^{9} \mathrm{GJ} / \mathrm{yr}$, of $\mathrm{T}<11000^{\circ}$ usage. (The result is shown in Figure 8-7.) The ECS analysis shows that $93 \%$ of the purchased and in-house fuel is used by industry segments operating on a continuous basis. Since the size distribution data cannot be resolved into continuous and other duty cycles, it is proposed for the present that the data in Figure 8-7 be considered entirely for continuous process industries.

Of the total purchased and in-house fuel for application at $\mathrm{T}<1: 1000^{\circ}, 0.19 \times 10^{9} \mathrm{GJ} / \mathrm{Yr}$ is used for space heating. Purchased and generated electricity in this manufacturing segment is quite substantial when viewed in relation to the $15 \%$ of fuel usage at $\mathrm{T}<1100^{\circ} \mathrm{F}$. In particular, for 1971, purchased electricity was $0.44 \times 10^{9} \mathrm{GWS} / \mathrm{Yr}$, while generated electricity was $0.09 \times 10^{9} \mathrm{GWS} / \mathrm{yr}$.

If the criteria listed at the start of this section are applied, ruling out fuel usage at $\mathrm{T}>1100^{\circ} \mathrm{F}$, continuous process users, HVAC usage, etc., then according to ECS this leaves $23.7 \%$ of the purchased and in-house fuel use for 1971 , or $20.14 \times 10^{9} \mathrm{GJ} / \mathrm{yr}$.

\subsubsection{SIC - AlI Other}

Finally to be considered is the sum of all the other industries in group SIC 20 through 39 not represented by the six major users of purchased fuel already mentioned. In 1971, according to ECS, this "all other" category used purchased fuel for process heat requirements below $1100^{\circ} \mathrm{F}$ in the amount of $1.89 \times 10^{9} \mathrm{GJ} / \mathrm{yr}$, with space heating accounting for $0.49 \times 10^{9} \mathrm{GJ} / \mathrm{hr}$.

There are no data available on the plant size distribution per se for this mixture of types of industry. To make possible 


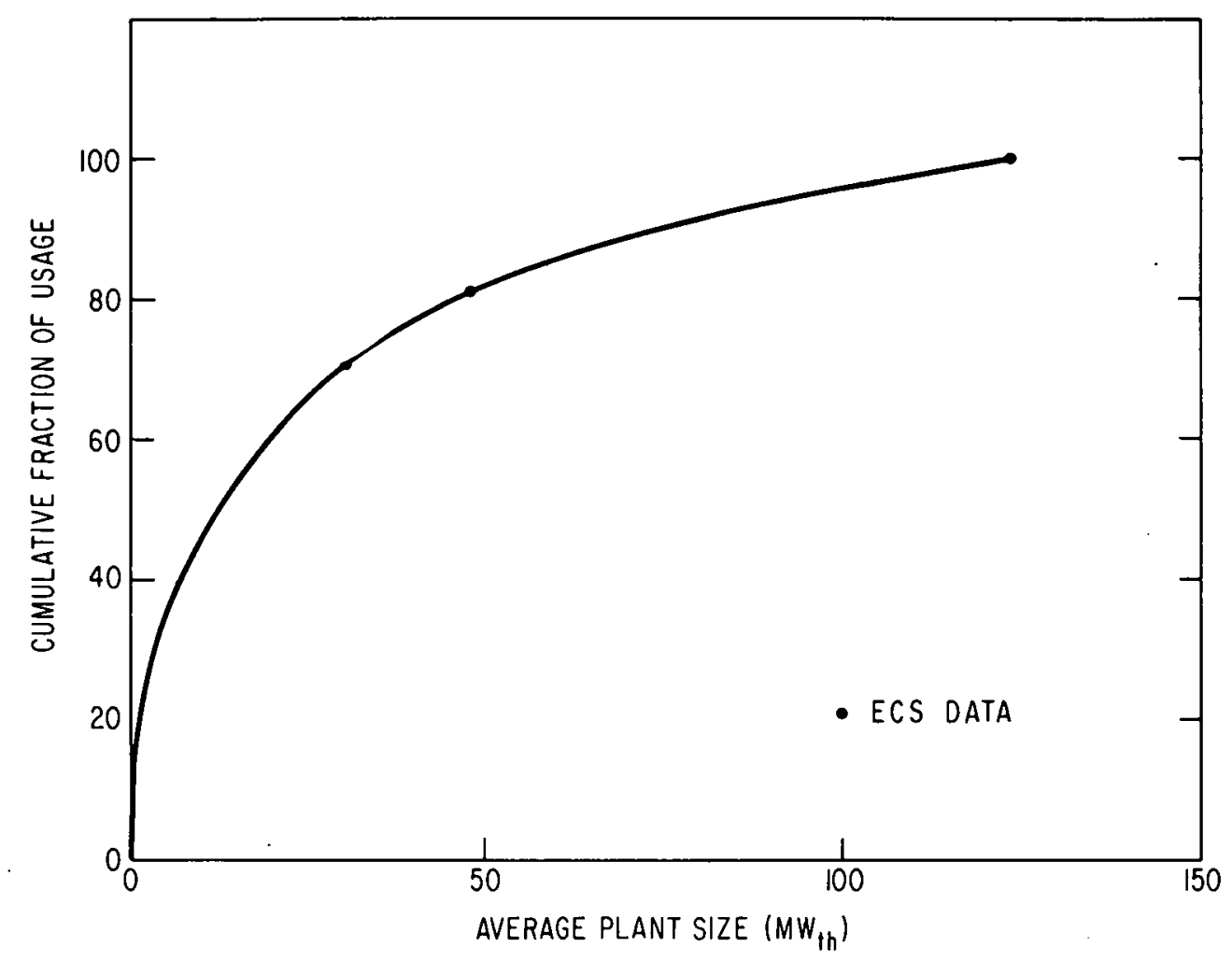

Figure 8-7. SIC 33 - Primary Metals: Plant Size Distribution Based on 1971 Total Fuel Use (purchased plus in-house, but not byproduct) of $0.56 \times 109 \mathrm{GJ} / \mathrm{Yr}$ at $\mathrm{T}<$ $11000 \mathrm{~F}$

at least a crude estimate of what such distribution might be like, two assumptions are made:

1. The ECS study suggests that the Schenectady General Electric plant represents one of the largest fuel-using installations in this category of industries (166 $\mathrm{MW}$ th for heat at $\left\langle 1100^{\circ} \mathrm{F}\right)$. This number will be taken as the maximum in the plant size distribution.

2. It will be assumed that the all-other category exhibits a plant size distribution similar to the mean of the major segments of manufacturing industry classification.

Returning now to the calculation of the mean plant size distribution for SIC 20, 26, 28, 29, and 33 (SIC 32 - stone, Clay, and Glass represented a relatively small amount of fuel usage, and no plant size distribution was available for $i t)$, the five distributions, already normalized in respect to total fuel usage, were also normalized in respect to maximum plant size. Each of the five distributions was then assigned a weight proportional to the total fuel usage of its particular industrial segment. Finally, the five distributions were averaged. The resultant mean plant size distribution is shown in Figure 8-8. As to the preci- 
sion of this curve, the band shown with the curve represents \pm 1 standard deviation about the mean curve.

Figure 8-8 can now be applied to the all-other category by noting a total purchased fuel usage of $1.89 \times 10^{9} \mathrm{GJ} / \mathrm{Yr}$ and maximum plant size of $166 \mathrm{MW}_{\mathrm{th}}$, according to ECS data.

\subsubsection{Aggregation of Manufacturing Industries}

A distribution of plant sizes for the sum total of manufacturing industries is now constructed by adding the curves of Figures 8-3 through 8-8 (where the normalization on the vertical scales of each curve is removed and the horizontal scale of Figure 8-8 converted to maximum plant size of $\left.166 \mathrm{MW}_{\mathrm{th}}\right)$. The sum curve so obtained is given in Figure 8-9. Here several points should be emphasized.

1. This represents purchased fuel consumption only for manufacturing industries. Purchased electricity is not included. Captive, in-house fuel consumption for the paper and primary metals industries is not included, on the grounds that such consumption would not be arnenable to CHP substitution. By the same token, this category of fuel consumption has been included for the petroleum refining industry.

2. Only that consumption associated with the production of temperatures below $1100^{\circ} \mathrm{F}$ has been included.

3. The curve as given is very approximate in view of the nature of its construction, and it represents an upper limit for CHP technological substitution.

Some comparison can be made between the result in Figure 8-9 and the work of others. Table II-3 of DOW-1 (R8-10) presents fuel use by size of installation for 85 corporations surveyed for a National Science Foundation study. The corporations selected were those at the top in sales in five segmemts: Paper, Chemicals, Petroleum, Stone/Clay/Glass, and Primary Metals; the data must therefore be considered as biased toward large-scale users. These survey data are presented as curve (B) in Figure 8-10 for comparison with the result from Figure 8-9 (shown on Figure 8-10 as curve (A)). Obviously the survey data can account for only a portion of industrial manufacturing fuel consumption. The survey data are presumably for 1972, for which DOW-1 reports purchased fuel use by industry (not including electricity but presumably including nonmanufacturing industry) at $21.7 \times 10^{9} \mathrm{GJ} / \mathrm{yr}$. Moreover, the bias toward large users seems quite clear.

The large difference in the maximum plant size between DOW-1 and ECS cannot be explained except to note that in several industrial segments the ECS data show the maximum values of plant size in the size distribution data as being greater than some value, and this study has possibly underestimated the maximum plant size. The resulting discrepancy in the cumulative usage will be at the 


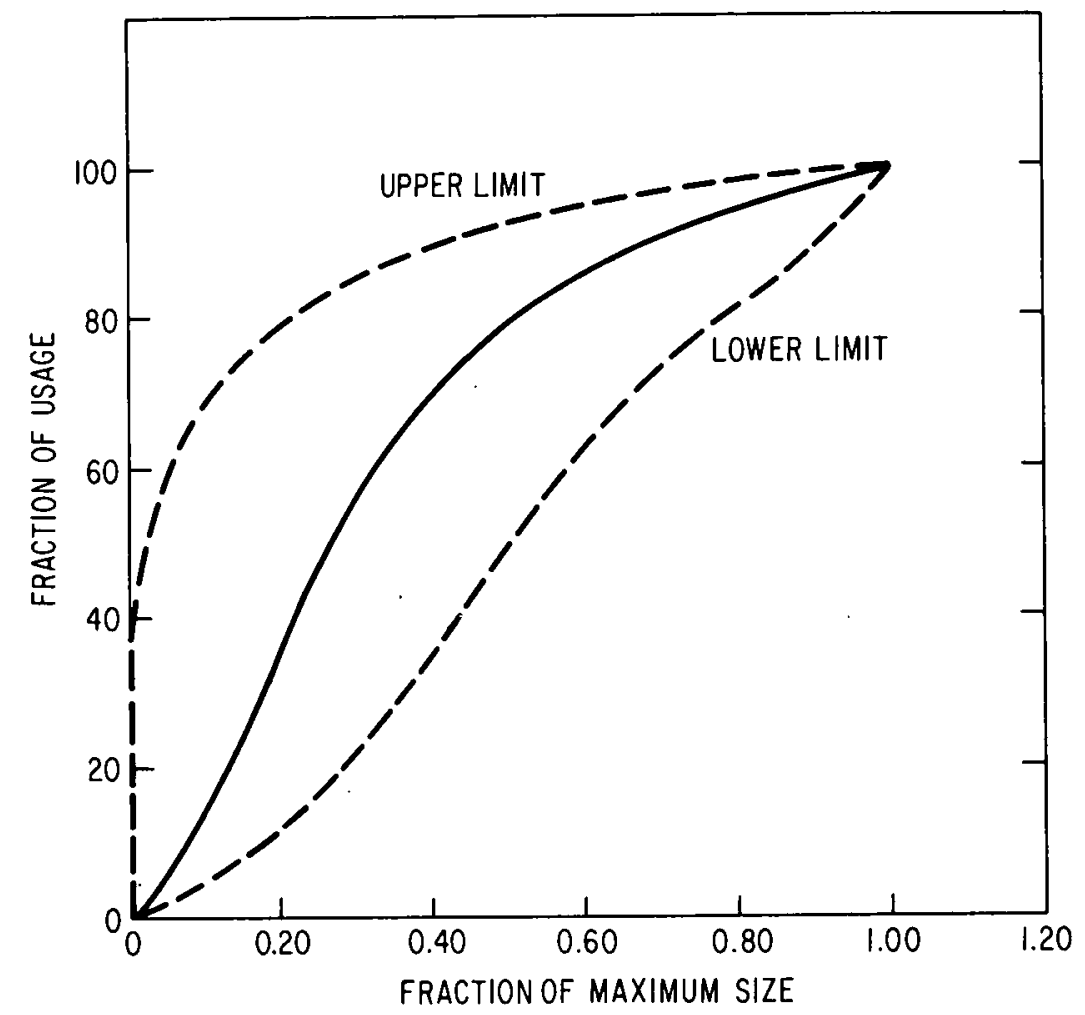

Figure 8-8. Mean Plant Size Distribution for Manufacturing Industry, Based on 1971 Fuel Usage, for Heat at $\mathrm{T}<1100^{\circ} \mathrm{F}$

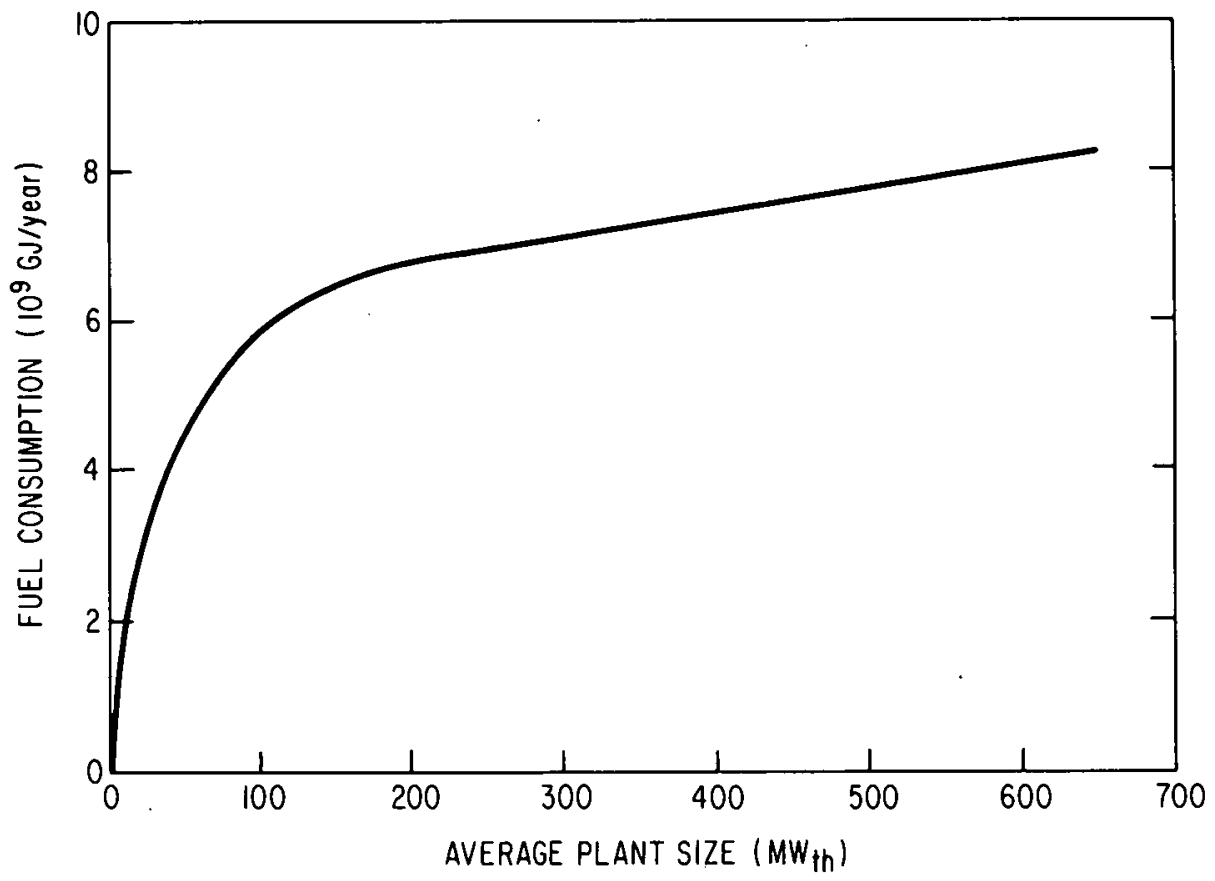

Figure 8-9. Manufacturing Industry Fuel Consumption in 1971 for $\mathrm{T}<11000 \mathrm{~F}$; Includes only Purchased Fuel Plus In-House Fuel in Petroleum Refining 


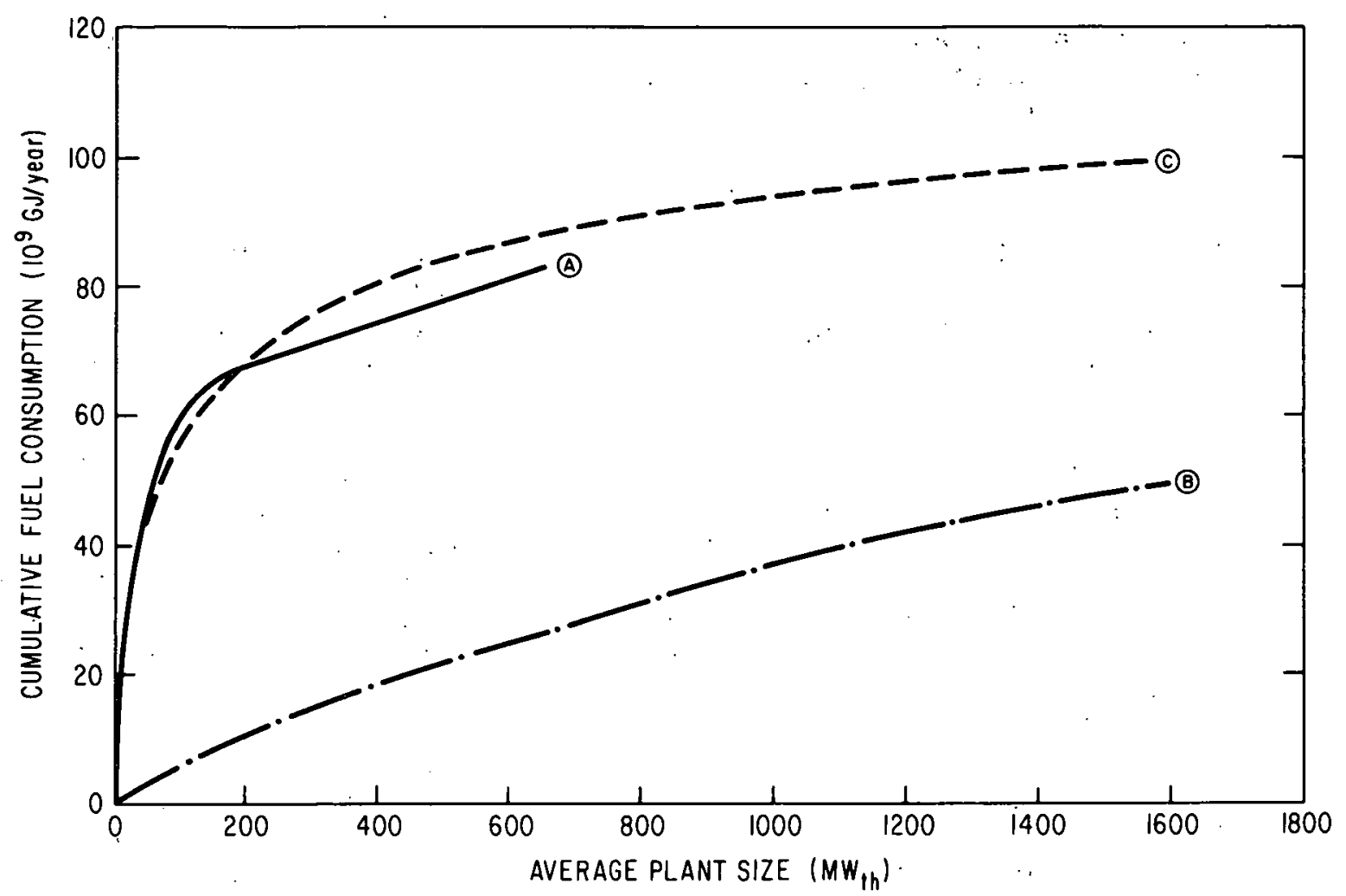

Figure 8-10. Comparison of Cumulative Fuel Consumption for Manufacturing Industry in 1971 per this Study (A) with DOW-1 Results (B) and (C) (see text)

highest fractions and is probably not significant in light of the approximations involved.

Another comparison can be made from Dow-1. Again, presumably based on the survey data, the report presents a cumulative distribution of total industrial steam load as a function of steam load per location (Figure VII-l of DOW-1). In this report, this plot of DOW-1 has been converted to cumulative fuel consumption versus average plant size, for comparison purposes, by applying their fraction of total steam load values to a total steam load calculated in the following way. The 1971 total energy consumption in the United States was obtained by an interpolation based on values used by DOW-1 for 1950, 1960, 1968, and 1972, with the result $69 \times 1015 \mathrm{Btu} / \mathrm{yr}$.

Again according to Dow-1 (Executive Summary), $17.4 \%$ of the total U.S. energy consumption in 1968 was for industrial steam; it was assumed here that this same fraction applied in 1971; the authors then multiplied by $\sim 0.8$ to remove the nonmanufacturing industry contribution. The resultant scaled steam-used distribution is shown as curve (C) in Figure 8-10. The influence of emphasis on large-system users is again seen in the DOW-1 survey as well as the problem mentioned earlier of the present authors probably not having included large users adequately. However, the curve (A) 
would seem to have some credibility for, say, evaluating the potential for CHP technological substitution at plant sizes below several hundred $\mathrm{MW}_{\mathrm{th}}$.

\subsection{SUMMARY}

In Section 8.2.3 the gross upper limits on premium fuel substitution and energy conservation were estimated to be $29.1 \times 10^{9}$ $\mathrm{GJ} / \mathrm{yr}$ and $11 \times 10^{9} \mathrm{GJ} / \mathrm{yr}$, respectively. The amounts of these totals ascribed to the industrial sector were $15.8 \times 10^{9} \mathrm{GJ} / \mathrm{Yr}$ and $4.6 \times 10^{9}$ GJ/Yr.

When consumption in the nonmanufacturing segments of this sector and the fuel used to produce temperatures greater than $1100^{\circ} \mathrm{F}$ are excluded, the potential for substitution is reduced to less than $9 \times 10^{9} \mathrm{GJ} / \mathrm{yr}$, as can be seen in Figure 8-9. If the substitution with CHP systems is further restricted to plants less than $200 \mathrm{MW}_{\mathrm{th}}$ the potential is reduced to $6.8 \times 10^{9} \mathrm{GJ} / \mathrm{yr}$, also to be seen from Figure 8-9.

If stringest limitations are applied, $\left(\mathrm{T}<1100^{\circ} \mathrm{F}\right.$, oneor two-shift industries only, no HVAC, usage at $<200 \mathrm{MW}_{\text {th only) }}$ then the following fuel consumption is found to be accessible to the CHP system substitution (derived from sections 8.3.4.1 8.3.4.7):

$$
\begin{array}{ll}
\text { SIC Code } & \frac{\text { GJ } / Y r}{14} \\
20 \text { - Food } & 0.44 \times 10^{9} \\
26 \text { - Paper } & 0.12 \times 10^{9} \\
28 \text { - Chemicals } & 0.19 \times 10^{9} \\
29 \text { - Petroleum } & 0.09 \times 10^{9} \\
32 \text { - Stone, etc. } & 0.13 \times 10^{9} \\
33 \text { - Prim. Metals } & 0.24 \times 10^{9} \\
& 1.41 \times 10^{9} \\
& 2.6 \times 10^{9} \mathrm{GJ} / \mathrm{Yr}
\end{array}
$$

Even with the stringent limitations used here, this estimate represents $16 \%$ of the gross potential in the industrial sector: it translates to the equivalent of one million barrels of oil per day. The industrial market represents a substantial opportunity for implementation of CHP systems. Once developed for the industrial market, CHP system applications are expected to grow substantially for other uses. 


\section{REFERENCES -- CHAPTER 8}

R8-1 ECS: Derived from U.S. Bureau of the Census, Census of Manufacturers, 1971 and 1974, by C.M. Young and associates of General Electric Engineering Consulting Services, in a limited distribution General Electric Report and by private communications, 1975 and 1976.

R8-2 FEA: "National Energy Outlook," Report FEZ-N-75/713, Federal Energy Administration, February 1976.

R8-3 SRI: "Patterns of Energy Consumption in the United States," Stanford Research Institute for the office of Science and Technology, Executive Office of the President, January 1972.

R8-4 PIB-1: "Residential and Commercial Energy Use Patterns 19701990," Federal Energy Administration Project Independence Blueprint, Final Task Force Report Vol. 1, November 1974.

R8-5 PIB-3: "Energy Conservation in the Manufacturing Sector," Federal Energy Administration Project Independence Blueprint, Final Task Force Report, Vol. 3, November 1974.

R8-6 BNL: "Sourcebook for Energy Assessment" (M. Beller, ed.), Report BNL 50483, Brookhaven National Laboratory for office of Assistant Administrator for Planning and Analysis, Energy Research and Development Administration, December 1975.

R8-7 EPRI: "Efficient Energy Use - A Practical Handbook for an Energy Constrained World" (Craig B. Smith, ed.), produced for Electric Power Research Institute by Applied Nucleonics Company, Inc., Pergamon Press, New York, 1976. (See Chapter 1 by C.B. Smith and Chapter 2 by R. Fazzo Lare, University of Arizona.)

R8-8 BTC: Pelley, W.E., Constable, R.W., and Krupp, H.W. "The Energy Industry and the Capital Market," Annual Review of Energy, Annual Reviews, Inc., Palo Alto, California, Vol. 1, p. 369 .

R8-9 BFT: "Einsatzmöglichkeiten neuer Energiesysteme,". Bundesministerium für Forschung und Technologies, Teil 1: Bedarfsanalyse und Strom, Seite 4. Bonn, W. Germany, 1975.

R8-10 DOW-1: "Evaluation of New Energy Sources for Process Heat," Dow Chemical Company and Environmental Research Institute of Michigan, for Office of Energy Research and Development Policy, National Science Foundation, September 1975. NTIS Report PB-245 604 . 


\section{Chapter 9}

\section{TECHNICAL AND INSTITUTIONAL BARRIERS TO IMPLEMENTATION OF HTCHP}

\subsection{INTRODUCTION}

In the preceding chapters of this report the technical and economic feasibility of the methane-based high temperature chemical heat pipe (HTCHP) system have been shown, and attractive markets for such a system have been identified. In this chapter, some of the technical and institutional barriers to implementation of the HTCHP will be delineated. "Technical barriers" refer to those technological advances which must be made before implementation can take place; "institutional barriers" are those social, economic, logistic, legal, and political obstacles that hinder implementation.

\subsection{TECHNICAL BARRIERS}

The technical barriers pertain to three main areas: the reformer, the methanator, and trasportation.

\subsection{Reformer}

The source analysis (Chapter 3) has shown that the most likely candidate for supplying heat to the HTCHP is the very high temperature gas-cooled nuclear reactor (VHTR with peak temperatures $>800^{\circ} \mathrm{C}$ ). Another source considered was the collection of solar thermal energy (Chapter 7). Each of these sources presents unique problems.

The technical barriers to using a nuclear reactor with the HTCHP lie in two areas. First, any such VHTR at best is many years from commercial implementation; second, the technology to couple the two systems in a system capable of containing accidental radioactive emissions for safety reasons is not yet available. Work in Germany and the United States is currently addressing both problems. Developmental work in Germany has progressed on the pebble bed reactor (PBR) to the extent that their system has heated helium gas for an extended period to temperatures capable of supplying heat to a methane reformer (R9-1). General Atomic has had an extended development program for a high-temperature gas reactor (HTGR) and is now obtaining some large-scale experience at the $300 \mathrm{MW}_{\text {elect }}$ Fort St. Vrain site in Colorado (R9-2). Whether the current design for the. HTGR is capable of producing temperatures needed for HTCHP applications has not been determined.

KFA (R9-l) is investigating the source coupling problems in detail; at the Adam-Eva facility the ultimate goal is mating a methane reformer with a PBR.* The potential of the HTCHP system for saving premium fuels should stimulate additional nuclear source development.

*General Electric is developing a duplex (two walled) steam reformer under DOE contract to address the safety problem. 
The solar-based source presents a similar problem in that the technology has not progressed to a state at which it could be mated to a methane reformer. Since solar energy has become a necessary source alternative for investigation in energy research and development programs, and has received increased funding from Government sources, collection progress should be forthcoming in developing a technically sound collection system. Even if this system were available, the mating of a solar collector and a methane reformer (or any other type of high temperature chemical reactor) presents a significant technical barrier. In current reformers, it is catastrophic to cycle the reformer tubes over an extended temperature range in a short time interval. This is a result of extreme metal expansion/contraction stresses placed on the tubes and the structural degradation of catalyst pellets under such cycling. Twice a year is considered too often to bring current reformers down to ambient temperatures. Current industrial practice is to feed hightemperature steam to the unit to limit thermal shock in case of fuel outage to the system.

Coupling of the source to a reformer should be considered in developing source designs.

\subsubsection{Methanator}

High-temperature methanators appear to be within the state-ofthe-art (see Appendix 1). However, despite extended pilot plant $(870 \mathrm{~kW}$ thermal output) experience in the high-temperature process (R9-3), no large industrial-scale installation exists; therefore no long-term operating experience on a high-temperature process exists. This barrier may well be eliminated in the near future if new coal-gasification plants come on stream and use hightemperature methane as part of their process design.

Thermal cycling might be thought to present a problem in the methanator analogous to that in the reformer, if used on a one or two-shift basis. However, current indications are that this thermal cycling problem is not a significant barrier. This is particularly true for designs utilizing adiabatic reactor beds and external heat exchangers. Cycling methanator duty may be less of a problem than that for normal boilers.

Another barrier which might arise is the placement of an unfamiliar technology, a chemical plant, in the process steam user facility. Although these methanators might be considered analogous to process steam boilers and are not complicated to operate, additional training for the operators and an appropriate training program would be required.

\subsubsection{Transmission}

All components of the transmission system are currently available and in use industrially. Hydrogen and, to a limited extent, carbon monoxide are transmitted by pipelines between plants. Methane, of course, is transmitted over thousands of miles in the natural-gas pipeline grid. Water pumps and gas compressors capable of 
handling any of the HTCHP gases are available. The major technological problem involved in the transmission of HTCHP components is the enormous scale of the gases produced and the resulting large pipeline required for economical transport. The transmission analyses

(see Appendix 4) have shown that the optimum pipeline diameters for $1000 \mathrm{MW}_{\mathrm{th}}$ transmitted $160 \mathrm{~km}$ often exceed one meter. Alternatively, two or more lines could be used with line diameters equivalent to those now commonly used. The economic comparison of these two cases is not available.

Since it is likely that all users of CHP-transmitted heat would not be cloistered at one geographical site $160 \mathrm{~km}$ from the heat source, actual transmission philosophies will differ from the one used (Appendix 4) and complicated grid arrangements to several load centers may in fact be required. Since existing industries may well be interested in coupling into CHP lines, the technical feasibility of installing the required pipelines in industrially congested areas may present a problem. The use of existing natural gas lines is a possibility; an analysis of this opportunity should await the selection of a specific user/geographical distribution.

A wide variety of technical barriers exist for implementation of the HTCHP, as would be expected in any developing technology. At this stage, no one barrier seems to be ultimately insurmountable, but further investigations must be conducted to remove each barrier before the HTCHP concept can be implemented.

\subsection{INSTITUTIONAL BARRIERS}

\subsubsection{Introduction}

Unlike the technical barriers, institutional barriers are not always well defined. At the same time, these barriers are as real and critical as technical barriers, and if solutions are not found they offer the same impediment to implementation. Three major types of institutional barriers are discussed here: developmental, organizational, and economic.

\subsubsection{Developmenta1 Barriers}

Developmental barriers are the economic, political, and social problems associated with the development of this technology; in particular, the possible heat sources. While being objective, one must be cognizant of the strong emotional feelings (both positive and negative) of the population toward the possible development of nuclear and solar energy sources. These popular attitudes coupled with subtle political realities may indeed dictate whether these sources are developed.

Economic constraints must also be considered when planning a variety of such large-scale programs. It is a fact that budgets for research and development are limited and only a few of the many suggested solutions to the energy problems will be implemented. 
Currently, both nuclear and solar sources are being funded; however, the future path of source technology is uncertain. Agreements on cost sharing between countries may increase the likelihood that all technologies are fully evaluated before any decision on the choice of future action is made.

\subsubsection{Organizational Barriers}

Critical to the development of any future large-scale energy conversion processes are the questions of the organization of consumers and suppliers of the technology and the funding behind that technology. That is, which institutions will invest in, construct, own, and manage the source, transportation, and delivery subsystems. Currently, some advanced energy conversion processes are of sufficient magnitude and represent sufficient business risk that individual corporations do not have the capital or R\&D budgets to pursue them singlehandedly. Rather, governmental (DOE) or private (EPRI) agencies fund them, with the understanding that in most cases licenses will be available for those who are interested in pursuing the technology.

Who will market the CHP concept, and who will own the various components of the system? Traditionally, Government-regulated utilities have supplied electricity. If a utility is to pursue the CHP concept, it will be a "new" type of utility, one which supplies heat for both process use and electricity.* The organizational difficulties of bringing the first large-scale thermal plant on-line to serve a variety of consumers with process steam will be enormous. Either the consumers must be lined up prior to startup (possibly 8 to 10 years in advance) so that plans could be made for pipelines and the geographical distribution of the heat, or a utility would have to build the plant "on speculation," hoping to line up customers during and after the plant startup.

If the latter route is followed, multiple heat sources might be required, each to be brought on-line as customers for its heat are brought under contract. Even this might prove costly, since capital equipment would have to be purchased in advance and perhaps sit idle until consumers are located. A more likely scenario would be for the utility to bleed a small portion of the heat of existing plants into small reformer units and attempt to set up a CHP pipeline grid (similar to existing electrical grids) into which the output of a new plant could be fed upon its completion. Definitive answers to this problem of nucleation of a new technology must await further study on such questions as possible location sites, consumer geographical distribution, and needs (see Chapter 8) in the areas surrounding those sites.

*Occasionally in this country and to a larger extent in some European countries, utilities have supplied both electricity and waste heat for district heating. 
The question of responsibility in case of accident must be considered for this technology as well as for the others being developed now. There is a finite danger associated with the transmission of hydrogen (fire) and CO (fire and toxicity) on a large scale through highly populated industrial areas. Where does the utility's liability end and the user's start? Will the utility own all of the equipment and assume all liability? This question of ownership forms the basis for the next section of this discussion.

\subsubsection{Economic Barriers}

The question of who owns the methanators in the HTCHP is important for reasons beyond safety or determination of responsibility. Currently, cogeneration of process steam and electricity is uneconomical for many industrial users; there is a difference in expected rate of return on capital investment between utilities and other industries. This difference results in the much higher capitalization charge in the cost of industry-generated electricity than the capital charge component in utility-generated electricity. As a result, it is cheaper for the industrial user to purchase power from the utility than to produce it himself. This difference in rate of return has developed over many years and is a result of Government regulation of utilities. It is clear, however, that this difference must be reevaluated if cogeneration (either within the framework of CHP or otherwise) is to be pursued with the aim to conserve energy. If the industrial steam user is to produce electricity along with steam, it must be profitable for him to do so.

\subsection{SUMMARY}

Several technological and institutional barriers to the implementation of HTCHP systems have been identified. None of these is insurmountable, but all of them need further consideration as plans for HTCHP development proceed.

\section{REFERENCES - CHAPTER 9}

R9-1 Nuclear Engineering and Design, Vol. 34, No. 1, 1975.

R9-2 Nuclear News, December 1977, p. 37.

R9-3 Pilot plant experience at the Ralph M. Parsons Co., Pasadena, CA. 


\section{Chapter 10}

\section{HIGH TEMPERATURE CHEMICAL HEAT PIPE ANALYSIS CONCLUSIONS AND RECOMMENDATIONS FOR FUTURE WORK}

\subsection{INTRODUCTION}

The preceding chapters have covered a range of topics, from the basic concept of the chemical heat pipe (CHP) and the potential role of thermal transmission in the United states to the details of HTCHP design and economics, with the principal aim of achieving the basic goals listed in chaptex 2. The flexibility of the CHP concept and the diversity of CHP applications, have been indicated in the course of this study. In the following summary, major conclusions have been grouped according to their relevance to each of the aspects of the CHP: 'the CHP concept as a whole, the technical feasibility of the CHP, HTCHP design and performance, economics, and market potential. In the concluding section of this chapter, recommendations are presented for future work on the CHP concept.

\subsection{CONCLUSIONS - CHP CONCEPT}

The generic concept of long-distance transport and storage of thermal energy in the CHP is novel, versatile, and unique. Its ability to transport thermal energy with high overall efficiency opens up many new options in energy management. Most prominent among these new options are:

- A thermal energy supply grid based on large central nuclear or coal sources delivering process steam to many industrial users.

- Distributed generation of electricity using thermal storäge and transmission.

- Extending a nuclear and coal-based cogeneration option to small scale and intermittent users.

- Providing a secondary source of heat near populated areas for local generation of electricity and space heat, which is free from local pollution.

Any one of these options would provide a powerful incentive to pursue CHP development; together, they could make a major impact on energy management. Among the potential advantages are: 
- Replacement of oil and gas use for industrial process steam generation by coal, nuclear, or solar thermal sources.

- Large-scale implementation of combined electricity/ heat delivery based on abundant fuel sources, resulting in enormous conservation potential of as much as five million barrels a day.

- Reduction of thermal rejection and cooling water requirements at the primary source location, resulting in increased flexibility for siting (particularly in the case of the nuclear reactors) and reduced environmental thermal pollution.

- Distributed generation of electricity with associated benefits of underground transmission, higher network reliability, and distributed storage.

\subsection{CONCLUSIONS - TECHNICAL FEASIBILITY}

The technical feasibility of a CHP system depends on the source technology as well as the chemical reaction system chosen. Based on a combination of results and conclusions given in Chapters 3 and 4 , we arrive at the following conclusions.

- The methane reforming/methanation reaction system (HTCHP) shows the greatest potential for a successful CHP application.

- It is unlikely that a practical high-temperature thermal source capable of supplying heat to the reformer (without using oil and natural gas) will be developed in the near future. The very high temperature gas-cooled nuclear reactor (VHTR) appears to be the only potential source for HTCHP; the feasibility of a nuclear reformer using the pebble bed reactor appears likely, but its demonstration is probably at least a decade away.

- The technical problems associated with interfacing either a high-temperature focused solar furnace or a coal combustion process with a reformer will be very difficult.

- As primary thermal sources, coal, light-water reactors, high-temperature gas-cooled reactors, and focused solar collectors are the most important for U.S. applications. 
- The benzene/cyclohexane reactions (LTCHP) appear to be best suited and offer the highest potential for demonstrating the technical feasibility of cyclic operation. The LTCHP faces a different problem than does the HTCHP, in that it is the chemical reaction system and not the source technology that is in question.

From these conclusions, the technical feasibility of the CHP involves the following dilemma: should the choice for development be the HTCHP, with a chemical reaction that will work but requires a source currently unavailable, or the LTCHP based on existing thermal sources (coal, LWR), but with a less proven chemical system.

\subsection{CONCLUSIONS - HTCHP DESIGN AND PERFORMANCE}

The overall energy efficiency * for HTCHP design lies in the range of $80 \%$ to $90 \%$. Technical design analyses confirm the view that thermodynamic considerations are essential in designing chemical plants for energy handling. The concept of exergy is valuable in analyzing and optimizing CHP designs.

In CHP designs, countercurrent heat exchangers play a dominant role. The methodology based on thermal histograms that was developed in the course of this work shows considerable merit in design analyses of this type.

A significant invention in this study is the mixed feed evaporator (MFE), which could improve the thermodynamic efficiency of CHP. Its impact on the overall energetics of the process, resulting from a reduction in entropy production due to mixing, demonstrates clearly the benefits of thermodvnamic analvsis.

Finally, the approach toward process efficiency developed in this study should prove valuable in pointing out some of the pitfalls of conventional efficiency definitions and caution against their improper use. The energetics of the HTCHP reference design is fairly typical of the various design cases investigated.

The overall conclusion reached is that, given an adequate source, CHP applications can achieve high efficiencies for transmission over distances on the order of $320 \mathrm{kilometers} \mathrm{(200} \mathrm{miles).}$

\subsection{CONCLUSIONS - ECONOMICS}

Bearing in mind the usual problems in estimating costs for an undemonstrated technology, the major conclusions can be summarized as follows:

* See Chapter 5 for definition. 
- Approximate costs for CHP transport are roughly in the range of $\$ 1.25 / \mathrm{GJ}$ to $\$ 2.25 / \mathrm{GJ}$ for a transport over $160 \mathrm{~km}$. When added to a primary-source thermal energy cost of $\$ 2.50 / G J$, the total cost of delivered process steam amounts to $\$ 3.75 / \mathrm{GJ}$ to $\$ 4.75 / \mathrm{GJ}$. At these total costs, CHP is competitive with such options as SNG fired boilers, electrode boilers, and perhaps small coal fired boilers with clean-up equipment.

- CHP applications that are most favorable economically include supplying process steam to small and parttime users; distributed generation of peak electricity; and cogeneration of electricity and heat for all but the largest-scale users.

- There are several applications where CHP is marginally competitive with other options. On-site storage of thermal energy and delivery of process steam to users that need large amounts on a continuous basis are examples of such applications.

- In comparison with other energy transmission alternatives, major strengths of $\mathrm{CHP}$ appear to lie in supplying heat over distances from 80 to $320 \mathrm{~km}(50$ to 200 miles).

- The main disadvantage of solar CHP applications derives from the fact that the capital costs are dominated by the reformer plant. In solar applications where the endothermic plant is operated at a capacity factor of approximately one-third, there is a heavy economic penalty. By contrast, a part-time operation of the methanation plant does not significantly increase the total cost, since its capital cost is not a major factor.

\subsection{CONCLUSIONS - MARKET POTENTIAL}

Based on available data on fuel consumption for industrial heat and process steam, it is concluded that the potential market for process steam delivery by CHP is quite large. Even with stringent technical restrictions for CHP applications $(T<800 \mathrm{~K}$, sizes below $200 \mathrm{MJ} / \mathrm{s}$ ), the maximum replacement potential is roughly $2.5 \times 10^{9} \mathrm{GJ} / \mathrm{yr}$ or $\sim 80 \mathrm{GW}_{\mathrm{th}}$ or $10^{6}$ barrels of crude per day.

Attempts in this study to obtain the dependence of process steam consumption on such variables as steam temperature and pressure, capacity or flowrates, duty cycle, types of fuel used, ease of substitution were only partly successful. Current data on industrial energy consumption are not detailed enough to permit such analyses. In the absence of such data, parametric analyses could not be performed to determine the fraction of total energy use that would be supplied by various CHP designs. In addition, the lack of detailed data on geographical distribution 
made it impossible to estimate typical distances between the thermal source and process steam uses.

There is a definite need for a data base for industrial process steam needs with sufficient detail to allow an evaluation of CHP applications and of other alternative technologies supplying process steam. Even if such a data base were available for only one geographical location, it would prove valuable in comparing various competitive technologies.

\subsection{RECOMMENDATIONS FOR FUTURE WORK}

There are three main areas of CHP in which further work is recommended. Each of them addresses a slightly different problem.

1. HTCHP: The critical issue in the HTCHP area is whether or not the U.S. will have a VHTR development program. If the German-U.S. cooperative program leads to an aggressive effort on VHTR for process heat, then significant additional work on HTCHP will be needed. Principal research areas then consist of (a) nuclear reformer designs including materials of construction, mixed feed evaporator, advanced reformer designs, and thermodynamic analyses of the reformer plant; (b) heat delivery plant including high temperature methanator design, thermodynamic analyses of process steam delivery by methanation, and process economics; (c) transportation of $\mathrm{CO}-\mathrm{rich}$ gases including economics of short distance, multiple pipeline transport, and (d) safety and institutional issues related to $\mathrm{CHP}$.

Since the time scale for VHTR development is going to be considerably longer than that of the reforming and methanation technologies a practical demonstration of a HTCHP loop is not recommended. The experimental programs most worth pursuing immediately are projects that have a major impact on nuclear process heat issues (e.g., interface safety, improvements in reformer design) and the mixedfeed-evaporator demonstration project.

2. ITCHP: Since the ITCHP would be applicable to a broad range of primary. sources (LWR, HTGR, focused solar, fossil-fired boilers) available in the U.S., a program aimed at proving its technical feasibility is strongly recommended. The first step in this program is an evaluation of the LTCHP concept along lines similar to those used in the HTCHP analysis and assuming no by-product formation. Such an evaluation has been undertaken in Chapters 11 through 18 of this report under an extension of the original CHP contract. Major conclusions from that study and recommendations for further work on the LTCHP are given in Chapter 18 . 
3. Coal Options: The emphasis on the use of coal in the national energy program dictates that near-term technologies for coal-based process steam delivery be seriously evaluated. A coal-based HTCHP is not technically viable, and LTCHP is not likely to be proven experimentally for a few years. It is the writers' opinion that the open CHP based on coal gasification to intermediate Btu gas, pipeline to the user site, methanation at the user site, and sale of the SNG, offers the best near-term option for supplying process steam to a large fraction of industrial users. Detailed recommendations for this option are not presented here because the open CHP was not.included in this study. However, in view of its role as a potential precursor of HTCHP and of many common elements between the two $\left(\mathrm{CO} / \mathrm{H}_{2}\right.$ transmission, methanation for thermal delivery, institutional issues of a thermal utility, cogeneration), it is recommended that the open CHP concept be included in the list of alternatives in future studies of process steam supply.

In addition to the three areas mentioned above, the writers feel it is important to address the institutional issues related to the CHP. Most important among these issues are: (a) the viability of a new type of utility delivering thermal energy, (b) the question of ownership of the CHP (utility or industrial), and (c) transportation issues. 


\section{Chapter 11}

\section{LOW TEMPERATURE CHEMICAL HEAT PIPE - INTRODUCTION}

\subsection{INTRODUCTION}

The analyses performed by General Electric in the initial stages of its Chemical Heat Pipe contract with ERDA (ERDA Contract EY-76-C-02-2676.*000) showed that the reactions used in the methanebased High Temperature Chemical Heat Pipe (HTCHP) are technically feasible and economically attractive. However, a heat source capable of producing the high temperatures required for the methane reforming reaction seems far from being commercially available on a large scale. Reversible chemical reactions that would match the temperature ranges of heat sources which might be available in the next two decades (fossil, light-water reactors, or perhaps breeder reactors and focused solar sources) have to date not been investigated in detail. Accordingly, an extension to the original CHP contract was granted by ERDA to allow other reactions to be investigated which might be coupled successfully to these low-temperature sources.

of the numerous chemical reactions that have been proposed for use in chemical heat pipes, those which appear to be most suited to Low Temperature Chemical Heat Pipe (LTCHP) applications, based on the studies undertaken in this contract, are of the general class of hydrogenation/dehydrogenation reactions of hydrocarbon compounds. The major advantage to this class of reactions is that a well developed background of technology exists, resulting from experience in the petroleum refining industry. In addition, the compounds involved in these reactions are relatively less corrosive and toxic* than other chemicals suggested for use in the LTCHP system.

Within the general class of hydrogenation/dehydrogenation reactions, the benzene/cyclohexane couple appears to best match the temperature requirements of the heat sources currently (or possibly shortly) available. Therefore, the LTCHP design and economic analyses performed under the current contract (and documented in the remainder of this report) concentrate on the use of cyclohexane dehydrogenation as the endothermic reaction and benzene hydrogenation as the exothermic reaction.

\footnotetext{
* See Chapter 17, "Technical and Institutional Barriers to Implementation of LTCHP."
} 


\section{Chapter 12}

\section{GOALS AND APPROACH FOR LOW TEMPERATURE CHEMICAL HEAT PIPE}

\subsection{GOALS OF LTCHP ANALYSIS}

The primary goal for this analysis is to determine the potential efficiency and cost of the LTCHP using cyclohexane ( $\mathrm{C}_{6} \mathrm{H}_{12}$ ) dehydrogenation as the endothermic reaction and benzene ( $\mathrm{C}_{6} \mathrm{H}_{6}$ ) hydrogenation as the exothermic reaction. This investigation has been restricted in that no detailed analysis of reaction kinetics or reactor design is considered. Rather, an analysis is performed which assumes optimum reaction conditions (no byproduct formation) and state-of-the-art reactor design. By using this approach, the LTCHP system could be dismissed from consideration if it is found to be economically noncompetitive under "ideal" conditions. If the LTCHP system is competitive under these conditions, then there is incentive to continue work on the system to develop catalysts which would allow the reaction to approach the ideal conditions assumed here, and to undertake a reactor design program which would allow utilization of these catalysts in the thermodynamically and economically optimum manner. As a secondary goal, this analysis will strive to lay the foundation for catalyst development and reactor design program (if they should be undertaken) by determining the important reaction, efficiency, and economic parameters of the system. The final goal of this report is to report specific recommendations for further work on the LTCHP concept.

\subsection{APPROACH OF LTCHP ANALYSIS}

The approach used in the analysis of the LTCHP parallels that of the technical analysis of the HTCHP system in the previous chapters. Discussions of topics common to the two systems such as consumer needs, alternate reactions, and source coupling are not repeated here.

To establish a background for the following development, the industrial use of the hydrogenation/dehydrogenation reactions will first be discussed in Chapter 13. Then the thermodynamic data base and a thermodynamic analysis of the LTCHP reactions will be given. A process flowsheet will be presented, and the flowsheet and reaction thermodynamics will be used to calculate detailed heat and material balances for a reference case. The system efficiency will then be determined for the reference case.

In Chapter 14, heat and material balances will be made for other example cases to show the effect of reaction temperature and pressure on the heat and mass flows and the system efficiency. In Chapter 15, equipment sizes and costs will be determined for the reference case and for the base cases. These costs will be compared with those of the HTCHP as a measure of the economic competitiveness of the LTCHP concept. Special attention will be given, in Chapter 16, to possible solar energy applications, to determine the impact of that technology on system economics. 
In order to give as complete a foundation as possible for evaluation of the LTCHP concept the various technical and institutional barriers to its implementation will be discussed in chapter 17. Those barriers discussed in chapter 9 are common to the HTCHP and LTCHP systems and wil! not be discussed in detail.

Finally, the conclusions reached during the ITCHP analysis will be presented in Chapter 18, and recommendations for further work will be made. 


\section{Chapter 13}

\section{TECHNICAL DESIGN OF LOW TEMPERATURE CHEMICAL HEAT PIPE}

\subsection{INTRODUCTION}

In this chapter, a technical design of the Low Temperature Chemical Heat Pipe (LTCHP) will be carried out for a reference case utilizing an endothermic (dehydrogenation) reactor outlet temperature of $811 \mathrm{~K}\left(1000^{\circ} \mathrm{F}\right)$ and an exothermic reactor outlet temperature of $589 \mathrm{~K}\left(600^{\circ} \mathrm{F}\right)$. Both reactors are designed to operate at 40 bars.

This technical design description will be prefaced by discussion of the industrial background of the benzene hydrogenation/ cyclohexane dehydrogenation reactions (section 13.2), the thermodynamic data base used (section 13.3), and the thermodynamic equilibrium of the candidate reactions (section 13.4). The technical design will consist of a presentation of the LTCHP process flowsheet (section 13.5), development of the material balance (section 13.6), and the energy balance (section 13.7) based on the process flowsheet. A discussion of the efficiency of the LTCHP will be presented (section 13.8), followed by a summary of the major conclusions of the analysis performed in this chapter (section 13.9).

\subsection{INDUSTRIAL BACKGROUND OF HYDROGENATION/DEHYDROGENATION REACTIONS}

\subsubsection{Introduction}

The LTCHP based on benzene/cyclohexane utilizes the following reactions for thermal energy absorption and liberation:

Endothermic step (cyclohexane dehydrogenation) -

$$
\mathrm{C}_{6} \mathrm{H}_{12} \rightarrow \mathrm{C}_{6} \mathrm{H}_{6}+3 \mathrm{H}_{2} \quad \Delta \mathrm{H}_{\text {reaction }}^{298}=206 \mathrm{Kj} / \mathrm{mole}
$$

Exothermic step (benzene hydrogenation) -

$$
\mathrm{C}_{6} \mathrm{H}_{6}+3 \mathrm{H}_{2} \rightarrow \mathrm{C}_{6} \mathrm{H}_{12} \quad \Delta \mathrm{H}_{\text {reaction }}^{298}=-206 \mathrm{Kj} / \mathrm{mole}
$$

These reactions have been used in industry -- the cyclohexane dehydrogenation reaction as part of a catalytic gasoline reforming process and the benzene hydrogenation reaction as a source of high purity cyclohexane for conversion to intermediates in polyamide (nylon) production. Detailed reviews of the industrial state-of-the-art for these two reactions and the evolution of the associated reactor designs are presented in Appendix 5 in the form of reports prepared by UOP, Inc. A brief summary of the state-ofthe-art will be presented in this section.

\subsubsection{Cyclohexane Dehydrogenation}

The isolated dehydrogenation of cyclohexane is not practiced commercially but is rather one of a series of reactions used in 
the large industry of catalytic reforming of naphthas to high octane reformates. In this process, three general types of reactions occur: initially, straight chain paraffinic molecules are cyclized into naphthalenic compounds; secondly, these cyclic compounds plus those already present in the feed are dehydrogenated to aromatics (e.g., cyclohexane to benzene); and finally, some hydrocracking and hydrodealkylation occurs.

These reactions are catalyzed by catalysts composed of noble metals (primarily platinum) on alumina/silica supports. The reaction sites on the alumina support catalyze the ring closing (and ring opening) reactions and the cracking and hydrodealkylation reactions. The noble metal sites catalyze the dehydrogenation reactions. For LTCHP applications, it is necessary that the dehydrogenation (noble metal or similar) function be retained and the isomerization and cracking (alumina) functions be eliminated. Problems of catalyst poisoning by sulfur or similar compounds should be minimal in the relatively clean LTCHP environment.

Generally, these reactions are carried out in a series of adiabatic reactors with the heat of reaction supplied by interheating between reactors. The catalysts must be regenerated to remove any carbon deposited during the cracking reactions. Prior to recent years, units were designed to be periodically taken off stream to accomplish this regeneration. Newer units employ continuous regeneration, in which a small amount of the catalyst is continuously removed from the reactors and regenerated in a separate vessel (R 13-1). A major advantage of this design is that catalyst activity is maintained at a higher level during the catalyst life, resulting in smaller reactor volumes.

LTCHP applications may not require catalyst regeneration if a highly selective/noncracking catalyst can be developed. Since the thermodynamic penalty associated with the adiabatic operation of the reactors decreases the overall efficiency of the LTCHP system, alternate reactor configurations might be considered. Other considerations such as source coupling to nuclear or solar heat sources might also dictate different approaches to the reactor design.

\subsubsection{Benzene Hydrogenation}

This reaction has long been used commercially to produce high purity (99.9+) cyclohexane for nylon manufacture. The processes currently used involve either.liquid or gas phase hydrogenation with the heat of reaction removed by vaporization of the reactant and produce liquids (with cyclohexane sometimes recycled), by intercooling between reactors, or by removal through the walls of catalyst-filled tubes. Since the conversion to cyclohexane is favored by low temperatures in this exothermic reaction, 
an attempt is made to keep the reaction temperature low by these heat removal methods. A low reaction temperature also improves catalyst performance in that the minimum of side products are formed.

Catalysts used in benzene hydrogenation processes are usually Group VIII metals (platinum, palladium, ruthenium, or nickel) on a refractory oxide support with an alkaline modifier to eliminate the acid character of the support. As in the dehydrogenation reaction, the acid sites promote formation of byproducts which are intolerable in the nylon manufacture reactions.

LTCHP application and industrial practice in the benzene hydrogenation reaction are very similar to the methanation reaction used in the High Temperature Chemical Heat Pipe (see Appendix 1, "A Review of Methanation Technology"). In both cases, current practice is to limit the reaction temperature to assure high conversions. These designs would be unacceptable in chemical heat pipe applications, since it is desirable there to recover the exothermic heat of reaction at the highest temperature possible to increase the efficiency of the process (see Appendix l). This different heat recovery philosophy has implications for both the reactor design and the type of catalyst used. The reactor design employed in LTCHP applications should allow a portion of the heat to be recovered at high temperatures, where the conversion to cyclohexane is incomplete, and then complete the reaction at lower temperatures, producing a somewhat lower quality heat. This could be accomplished by a series of adiabatic or fluidized beds, by a reactor employing catalyst-filled tubes exchanging heat countercurrently with the process steam being generated, or by some combination of these designs. In view of the economic incentive to strive for high thermodynamic efficiencies, the use of liquid phase hydrogenation (with associated thermal degradation of the available thermal energy) appears unlikely in LTCHP applications.

\subsection{THERMODYNAMIC DATA BASE}

\subsubsection{Assumptions Determining the Scope of the Data Base}

The major assumptions used in the following analyses of the LTCHP which determine the scope of the data base include:

1. No kinetic limitations on the rate of reaction for any of the reactions are considered. This is important because the material balances can be determined by the thermodynamic equilibrium composition from the outlet of the reactors and, thus, a detailed knowledge of reactor design and kinetics is not needed. Based on a survey of reaction kinetics, this appears to be a more reasonable assumption for CHP applications than for the industrial reforming process because of the high reaction rate of the dehydrogenation reaction as compared with the isomerization and cracking reactions. 
2. The reactions are "clean" in that no cracking byproducts are formed. This assumption limits the number of simultaneous equilibria that need to be considered and allows the effect of temperature and pressure on the main reactions to be more clearly demonstrated.*

3. The gases are ideal. This assumption allows the heat capacity of the gases to be represented by a simple equation.

4. The liquids are ideal mixtures. Combined with assumption 3, this assumption allows a simple relationship to be formulated between the liquid and gas mole fractions, the component vapor pressures, and the total pressure of the system (Raoult's law).

As in the HTCHP analyses, the aim of this analysis is not to perform a detailed design but rather to arrive at first order estimates of system economics and efficiencies. Therefore, the data base was chosen for versatility and compactness of representation, with some slight sacrifice made in the absolute accuracy of the results.

\subsubsection{Data Base}

The calculations that require the thermodynamic base include:

- Thermodynamic equilibria for two reactions (cyclohexane dehydrogenation and isomerization to methylcyclopentane) so that conversions, product compositions, and material balances may be determined.

- Enthalpy balances to determine magnitudes of the energy flow requirements for each of the system components.

The data required for these calculations include:

1. Standard enthalpy of formation, $\Delta \mathrm{H}_{\mathrm{f}}^{\circ}$, for each of the three compounds (cyclohexane, benzene, and methylcyclopentane) at the reference temperature, $\mathrm{T}_{\mathrm{R}}=298.16 \mathrm{~K} .\left(\Delta \mathrm{H}_{\mathrm{f}}{ }^{\circ}\right.$ for hydrogen at any temperature is zero).

2. Standard entropies, $S^{\circ}$, of the four components (including hydrogen) at $\mathrm{T}_{\mathrm{R}}$.

3. Specific heats over the required temperature range for the four components in the gas phase, and for the hydrocarbon compounds in the liquid phase.

*This is a valid assumption if the proper catalyst is used for the reactions, as demonstrated by the purity of the cyclohexane produced commercially by benzene hydrogenation (see Appendix 5). In Section 13.4 the deleterious effects of the isomerization reaction of cyclohexane to methylcyclopentane are shown. A suitable catalyst to avoid this reaction will be assumed. 
4. The heat of vaporization of the hydrocarbon compounds at the reference temperature.

5. Liquid enthalpies for the hydrocarbon compounds (these can be computed from a combination of data from 1, 3, and 4).

6. Vapor pressures for the hydrocarbon compounds.

For this study, gaseous heat capacities are represented by second degree polynomials of the form

$$
c_{p}=a+b T+c T^{2}
$$

with the constants chosen to fit the ideal gas heat capacities, ( $T$ in Kelvin, $C_{p}$ in cals/gmole-K). The liquid heat capacities in the form $a+b T+c T^{2}$ and the heat of vaporization of the compounds are combined to yield liquid enthalpies of the form

$$
\mathrm{H}_{1}=\mathrm{A}+\mathrm{BT}+\mathrm{CT}^{2}+\mathrm{DT}^{3}
$$

The vapor pressures of the hydrocarbon compounds are fitted to an equation of the form

$$
\mathrm{p}^{0}=\exp \left(\mathrm{A}^{\prime}+\mathrm{B}^{\prime} / \mathrm{T}+\mathrm{C}^{\prime} / \mathrm{T}^{2}\right)
$$

The standard enthalpies of formation, entropies, and the constants used in the above equations are shown in Table 13-1.

Table 13-1

\begin{tabular}{|c|c|c|c|c|c|c|c|c|c|}
\hline Component & $\Delta \mathrm{H}_{\mathrm{F}}^{\circ}$ & $\mathrm{s}^{\circ}$ & a & $b$ & $c$ & A & $\mathrm{B}$ & $c-$ & $\mathrm{D}$ \\
\hline Cyclohexane & -29430 & 71.28 & -12.454 & 0.14328 & $-55.143 \mathrm{E}-6$ & $-1.1502 \mathrm{E} 5$ & 574.801 & -1.41 .30 & $0.1221 \mathrm{E}-2$ \\
\hline Benzene & 19820 & 64.34 & -5.3646 & 0.09617 & $-40.936 E-6$ & $-0.5088 \mathrm{ES}$ & 450.006 & -1.076 & $0.9098 E-3$ \\
\hline Hydrogen & 0 & 31.21 & 6.88 & $0.66 \mathrm{E}-4$ & $0.279 \mathrm{E}-6$ & - & - & - & - \\
\hline \multirow[t]{2}{*}{ Methylcyclopentane } & -25500 & 81.20 & -8.4051 & 0.13179 & $-51.195 E-6$ & $-1.0743 \mathrm{E} 5$ & 554.996 & -1.3867 & $0.1227 E-2$ \\
\hline & & $A^{\prime}$ & $B^{\prime}$ & $\mathrm{C}^{\prime}$ & & & & & \\
\hline Cyclohexane & & 9.0641 & $-0.2599 \mathrm{E} 4$ & $-0.2176 \mathrm{E} 6$ & & & & & \\
\hline Benzene & & 9.2497 & $-0.2671 \mathrm{E} 4$ & $-0.2081 E 6$ & & & & & \\
\hline Hydrogen & & - & - & - & & & & & \\
\hline Methylcyclopentane & & 9.0146 & -0.2466 & $-0.2231 \mathrm{E} 6$ & & & & & \\
\hline
\end{tabular}

THERMODYNAMIC DATA BASE FOR LTCHP CALCULATIONS

Units in Cals, Gram-Moles, and Kelvin ( $\mathrm{R}$ 13-4, R 13-5)

One important difference between the LTCHP and HTCHP systems is the number of condensible components in each. In the HTCHP system, the only condensable component is water; therefore, multicomponent vapor-liquid equilibria do not have to be considered. In the LTCHP system, both benzene and cyclohexane are liquids during transportation, and multicomponent evaporation and condensation must be considered. The approach used here is an iterative technique using the individual vapor pressures and the total pressure of the system to determine the vapor and liquid mole fractions 
of both components. This procedure is standard practice in these types of application (R 13-2) and is documented in Appendix 6 in the LTCHP heat balance computer program.

\subsection{THERMODYNAMIC EQUILIBRIÀ OF CANDIDATE REACTIONS}

\subsubsection{Reaction Background}

A wide variety of products is possible in hydrocarbon reaction systems, but the equilibrium mixture of all possible products is rarely approached, because of the slow kinetics of many of the reactions. In addition, most industrial systems employ selective catalysts which increase the reaction rates for only a few desirable reaction pathways.

In the LTCHP reactions, a specific catalyst is desirable to eliminate any reactions which would lead to unwanted byproducts. Industrial experience $(\mathrm{R} 13-3)$ has shown that the major byproduct to be expected in the LTCHP system is methylcyclopentane (MCP). It may be a precursor to cracked byproducts which would build up in a cyclic system if not removed and would degrade the primary reactants into unusable products. The thermal effects of the MCP formation are different from the hydrogenation and dehydrogenation reactions and will impact the heat and material balances of the system. Therefore, the equilibrium for the cyclohexane isomerization to MCP will be explored along with the basic cyclohexane dehydrogenation/benzene hydrogenation equilibrium in order to determine the severity of these problems.

\subsubsection{Thermodynamic Analysis}

The standard enthalpy, entropy, and Gibbs free energy changes for the cyclohexane dehydrogenation and isomerization to MCP reactions are shown in Table 13-2.* These data show that the isomerization reaction has a much lower heat of reaction than the dehydrogenation reaction. Therefore any cyclohexane converted to MCP would contribute much less to the endothermicity of the reaction and would require a greater amount of cyclohexane to be circulated in the system to obtain the same rate of heat transmission.

As is normal in all endothermic reactions, the Gibbs free energy decreases with increasing temperature for both reactions with a see saw temperature (temperature at which $\Delta \mathrm{G}^{\circ}=0$ ) of $\sim 390 \mathrm{~K}$ for the isomerization reaction and $\sim 550 \mathrm{~K}$ for the dehydrogenation reaction. With only this information, it is difficult to determine the distribution of products in the combined system, since the reactions are coupled. To better understand the impact of MCP formation on the outlet compositions of the reactors, a computer program (Appendix 6) has been written to evaluate the equilibrium conversions for these reactions.

* Since the benzene hydrogenation reaction is the reverse of the hydrogenation reaction, these data can be used for it with an appropriate change in sign.

$$
13-6
$$


Table 13-2

$\triangle \mathrm{H}^{\circ}, \triangle \mathrm{S}^{\circ}$, and $\triangle \mathrm{G}^{\circ}$ FOR CYCLOHEXANE DEHYDROGENATION AND ISOMERIZATION REACTIONS AS A FUNCTION OF TEMPERATURE

Dehydrogenation $\Delta \mathrm{H}^{\circ} \quad \Delta \mathrm{S}^{\circ}$

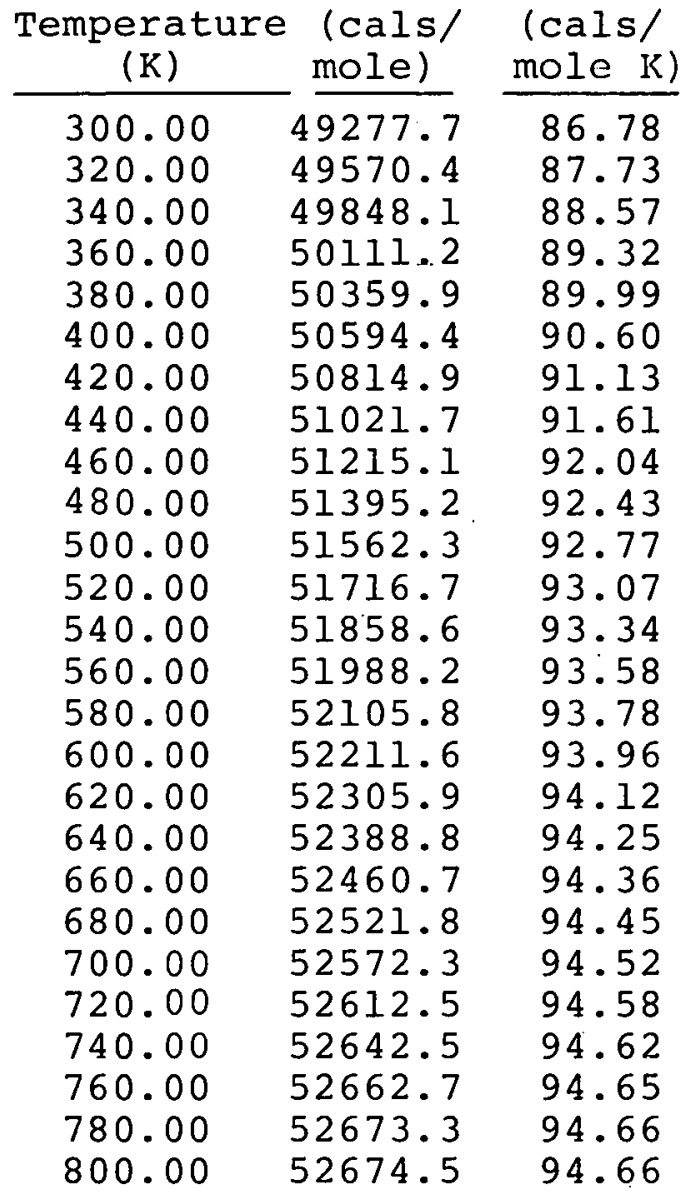

Isomerization

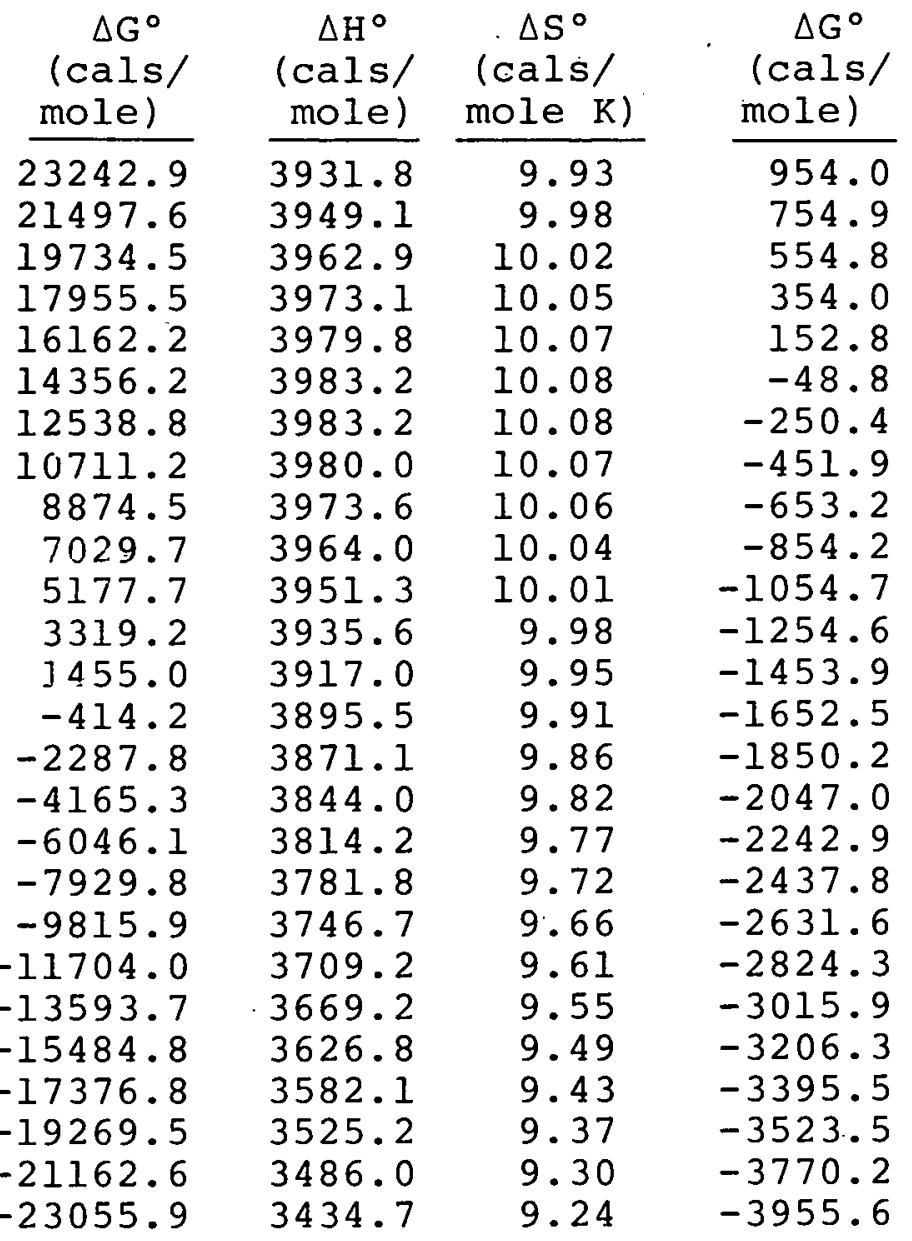

The program has the capability of calculating equilibrium conversions of both reactions in parallel or of only the dehydrogenation reaction with $\mathrm{MCP}$ formation suppressed.

This program was used to calculate the equilibrium compositions of the dehydrogenation/hydrogenation reaction with a hydrogen-to-carbon ratio in the system of 2:1 at a variety of temperatures and pressures. The results of these calculations are shown in Figure 13-1 ( $P_{\text {reaction }}=1$ bar $)$, Figure $13-2(P=40$ bars), and Figure 13-3 ( $P=68$ bars) for cases with and without the formation of MCP suppressed. The composition of the mixture is given in terms of moles of each component per mole of cyclohexane equivalent feed for the two-reaction equilibrium considering MCP formation (solid lines) and for the one-reaction equilibrium between benzene and cyclohexane (dashed lines). These curves also represent the equilibrium concentrations for the reverse reaction (benzene hydrogenation) at the same stoichiometry. 


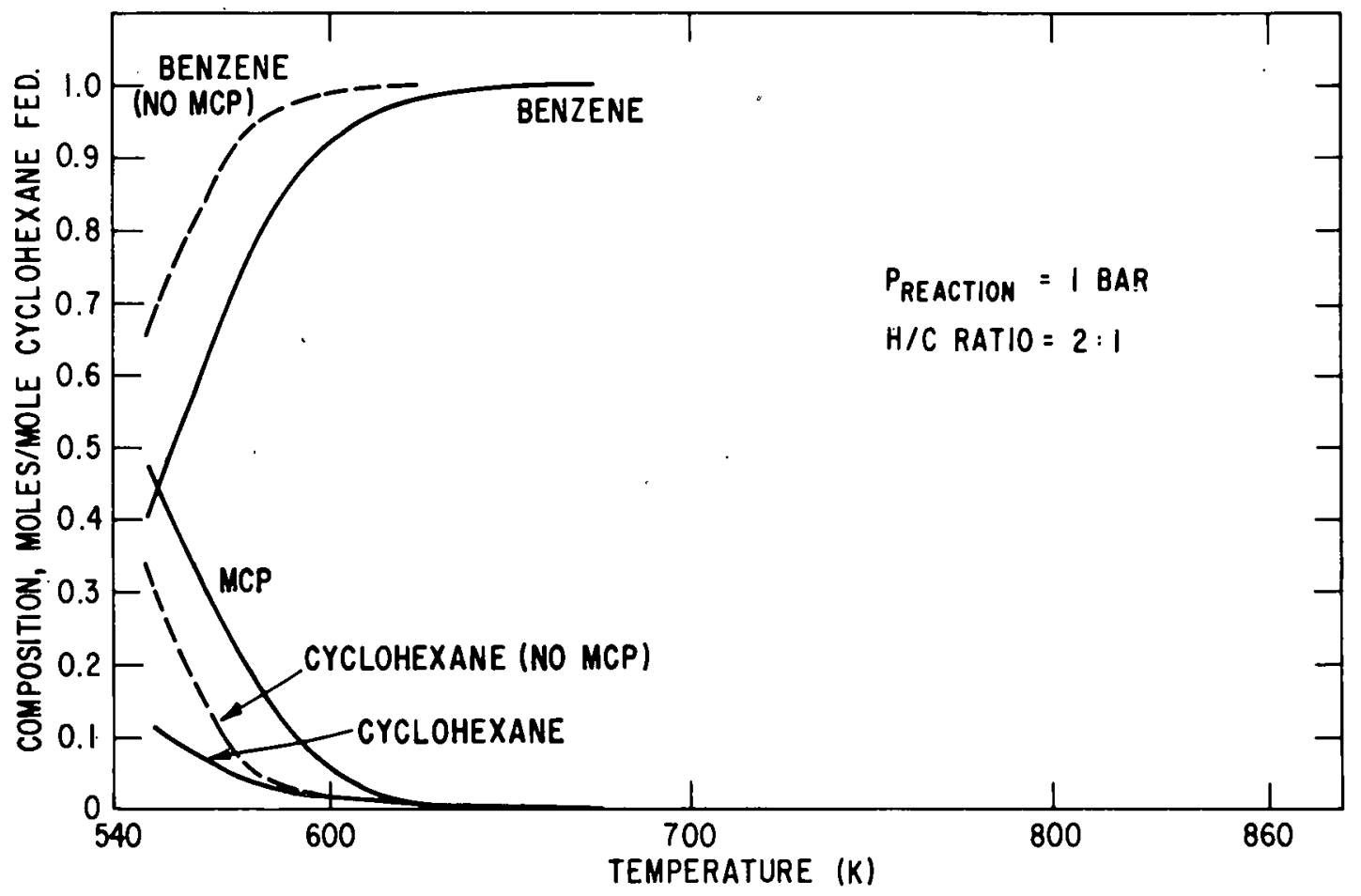

Figure 13-1. Equilibrium Compositions in Cyclohexane/ Benzene/Methylcyclopentane (MCP) System. at 1 Bar Reaction Pressure

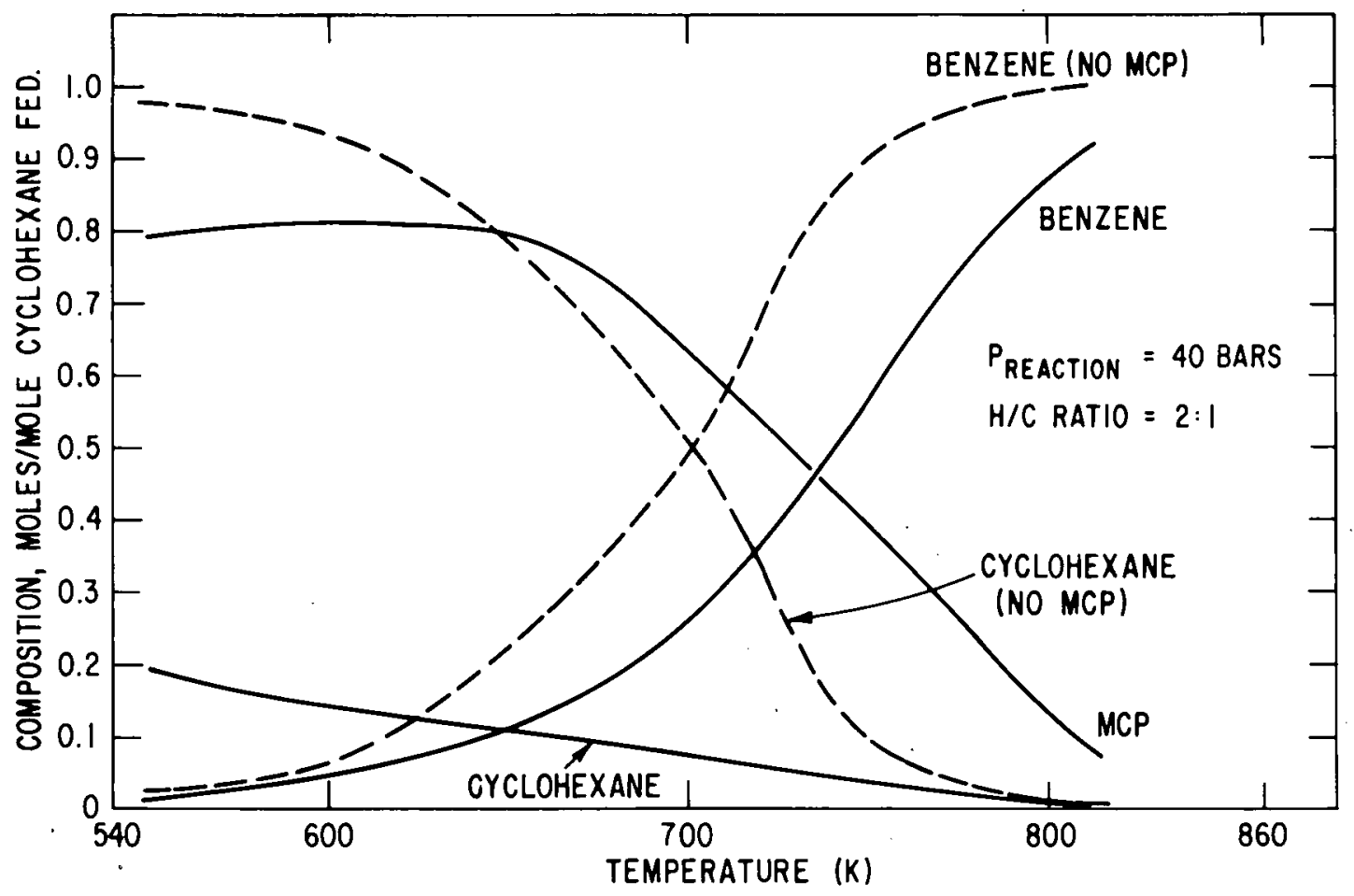

Figure 13-2. Equilibrium Compositions in Cyclohexane/ Benzene/Methylcyclopentane (MCP) System at 40 Bar Reaction Pressure 


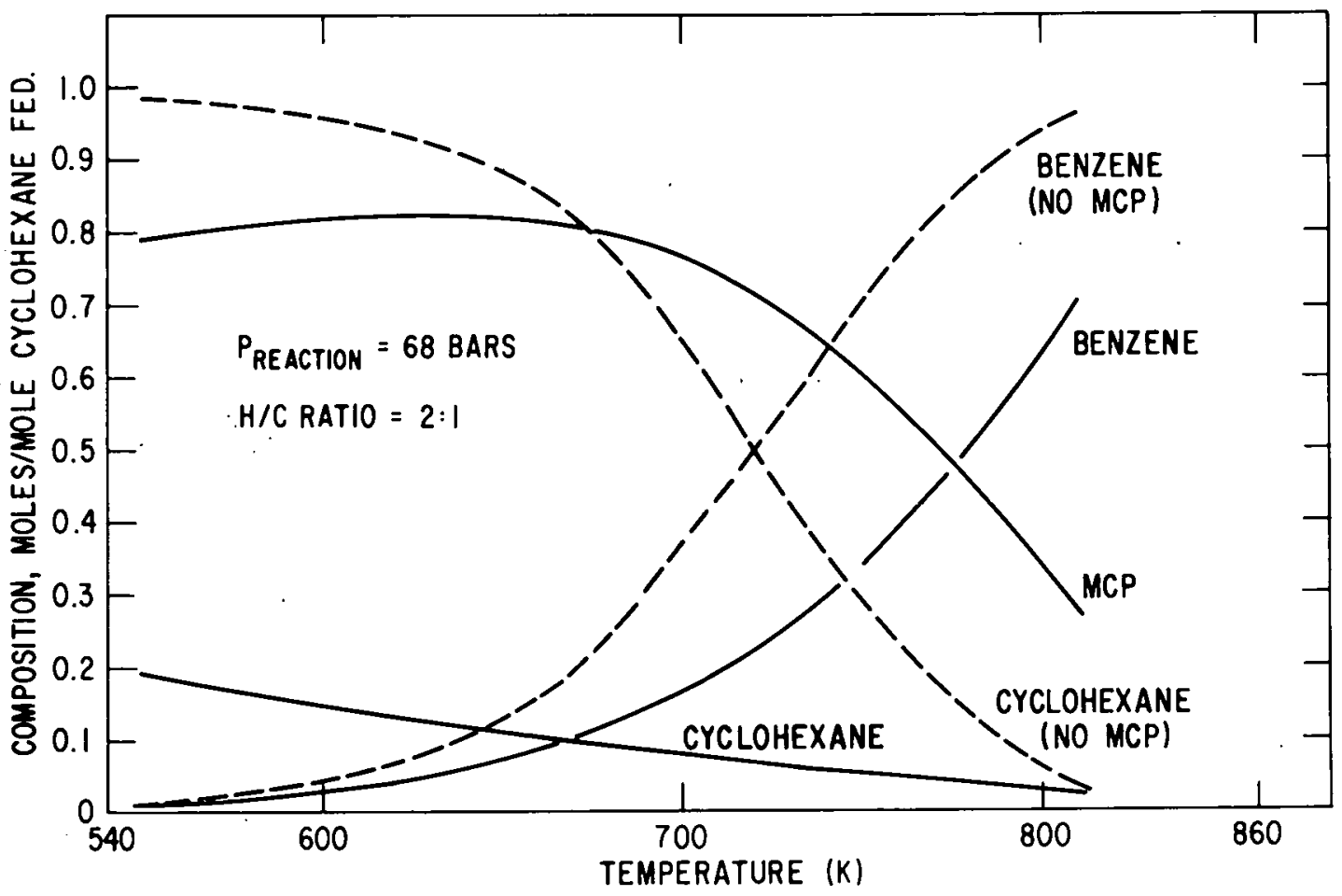

Figure 13-3. Equilibrium Composition in Cyclohexane/ Benzene/Methylcyclopentane (MCP) System at 68 Bar Reaction Pressure

The effect of temperature, pressure, and MCP suppression can be summarized as follows.

- MCP Formation Suppressed: Increasing reaction temperature tends to favor a larger mole fraction of benzene, and increased pressure tends to favor cyclohexane formation. At 1 bar, essentially all cyclohexane is converted to benzene at temperatures greater than $600 \mathrm{~K}$. At $540 \mathrm{~K}$, benzene accounts for $\sim 53 \%$ of the cyclic compounds in the product mixture. At higher pressures, higher temperatures are required to obtain a significant conversion of cyclohexane to benzene (i.e., at 40 bars, $\sim 800 \mathrm{~K}$ is needed for $99 \%$ benzene).

- With MCP Formation: The formation of MCP results in the reduction in the amount of both cyclohexane and benzene in the equilibrium mixture. For example, at 40 bars and $700 \mathrm{~K}$, the amount of cyclohexane present drops from about $0.5 \mathrm{~mole} / \mathrm{mole}$ of cyclohexane equivalent present without MCP formation to $\sim 0.09$ mole/ mole with MCP formation. Under the same conditions, benzene drops from $0.5 \mathrm{~mole} / \mathrm{mole}$ to $0.26 \mathrm{~mole} / \mathrm{mole}$. Thus, even though the conversion of cyclohexane is higher, the selectivity to benzene is decreased significantly and the total amount of heat absorbed in the endothermic reactor (per unit of cyclohexane converted) is substantially decreased with MCP formation. 
It is apparent from this analysis that the formation of MCP would seriously affect the heat balance. Therefore, in the remainder of this report it will be assumed that a selective catalyst can be formulated to suppress this MCP formation.

\subsection{LTCHP PROCESS FLOWSHEET}

\subsubsection{LTCHP Flowsheet Components and Diagrams}

The components of the low temperature CHP resemble those of the methane-based CHP design considered in Chapter 5. The endothermic reaction process flowsheet, presented in Figure 13-4 consists of a series of reactors and associated heat exchangers in which the heat of reaction is delivered to the process stream. For this analysis, it is assumed that the reactors are adiabatic fixed beds with the heat introduced by heat exchange between the beds. Although this design is not the most efficient thermodynamically, it is used commercially in the gasoline reforming industry and could probably be utilized in LTCHP applications without an extensive reactor development program. A major advantage to this design is that the components can be costed relatively accurately.

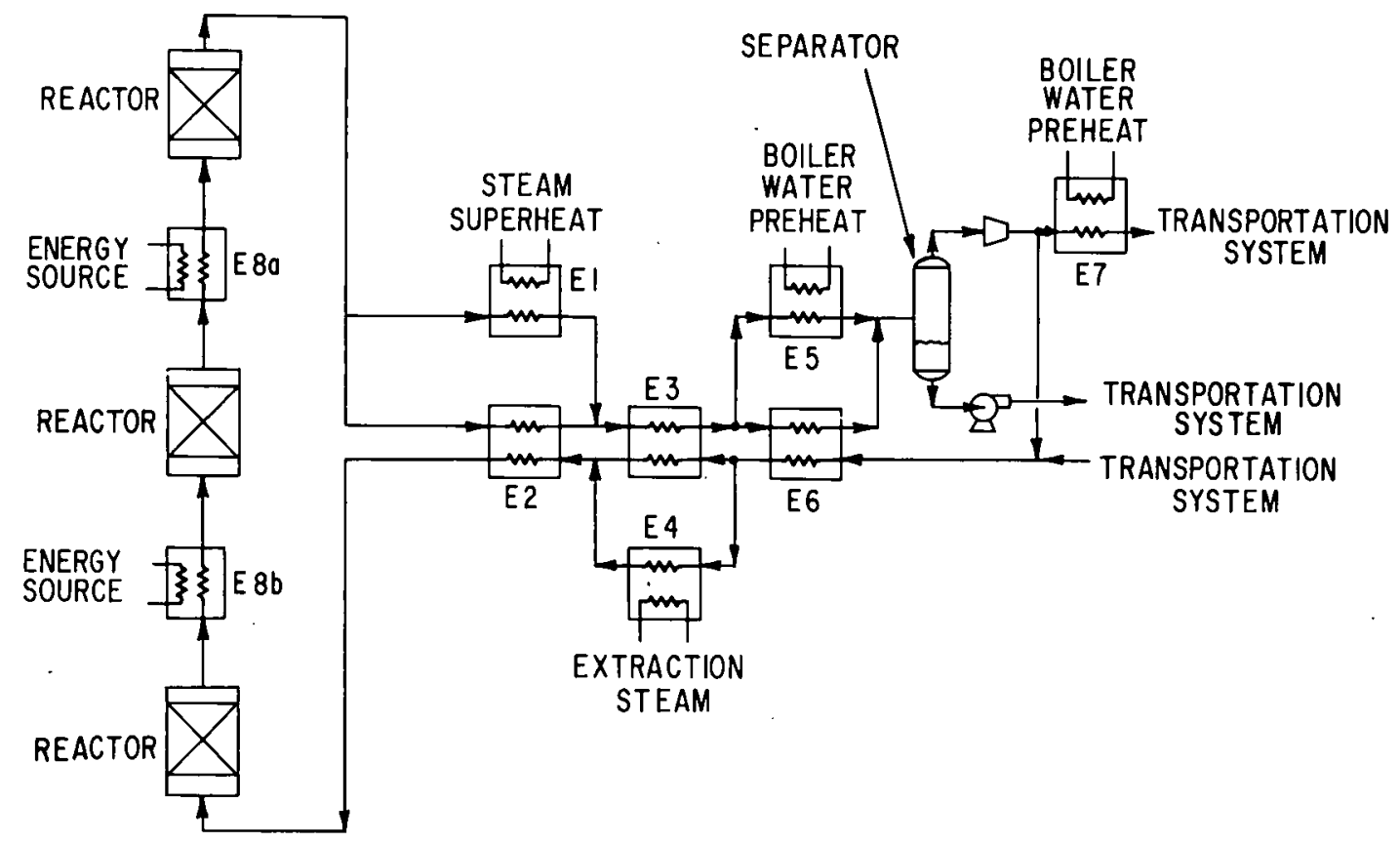

Figure 13-4. Process Flowsheet for Cyclohexane Dehydrogenation Section of LTCHP system

Additional heat exchangers are required to vaporize and preheat the reactants, and to condense and cool the products. The transfer of heat to and from an associated powerplant is utilized in this design as in the HTCHP design (see Chapter 5). The outlet gases from the dehydrogenation reactors are used as a source of steam superheat (EI) and boiler water preheat. (E5 and E7). The inlet gases are partially preheated by extraction steam from the 
powerplant cycle (E4). Other exchangers (E2, E3, and E6) are used to exchange the heat available in the product gases into the reactant stream to provide preheating.

Additional equipment required includes a vapor-liquid separator and a pump and compressor to overcome the equipment pressure drop.

Figure 13-5 shows a simplified process flowsheet for the exothermic hydrogenation reaction. Again, the reactors are assumed to be adiabatic fixed beds with the heat delivery accomplished by intercoolers. Other reactor configurations (e.g., fluidized bed with internal cooling) might be economically more attractive and more efficient, but their cost cannot be determined as accurately as the adiabatic fixed bed design. In case of the LTCHP system, the heat balance between the inlet and outlet streams at the exothermic end (see section 13.6, "Material Balances") allows this system to operate on a stand-alone basis. Thus, only one exchanger $(\mathrm{EX} 1)$ is required to recover the heat from the outlet stream in the form of preheating for the inlet stream. No attempt was made in this design to recover additional work available in the outlet stream by expansion turbines or other equipment.

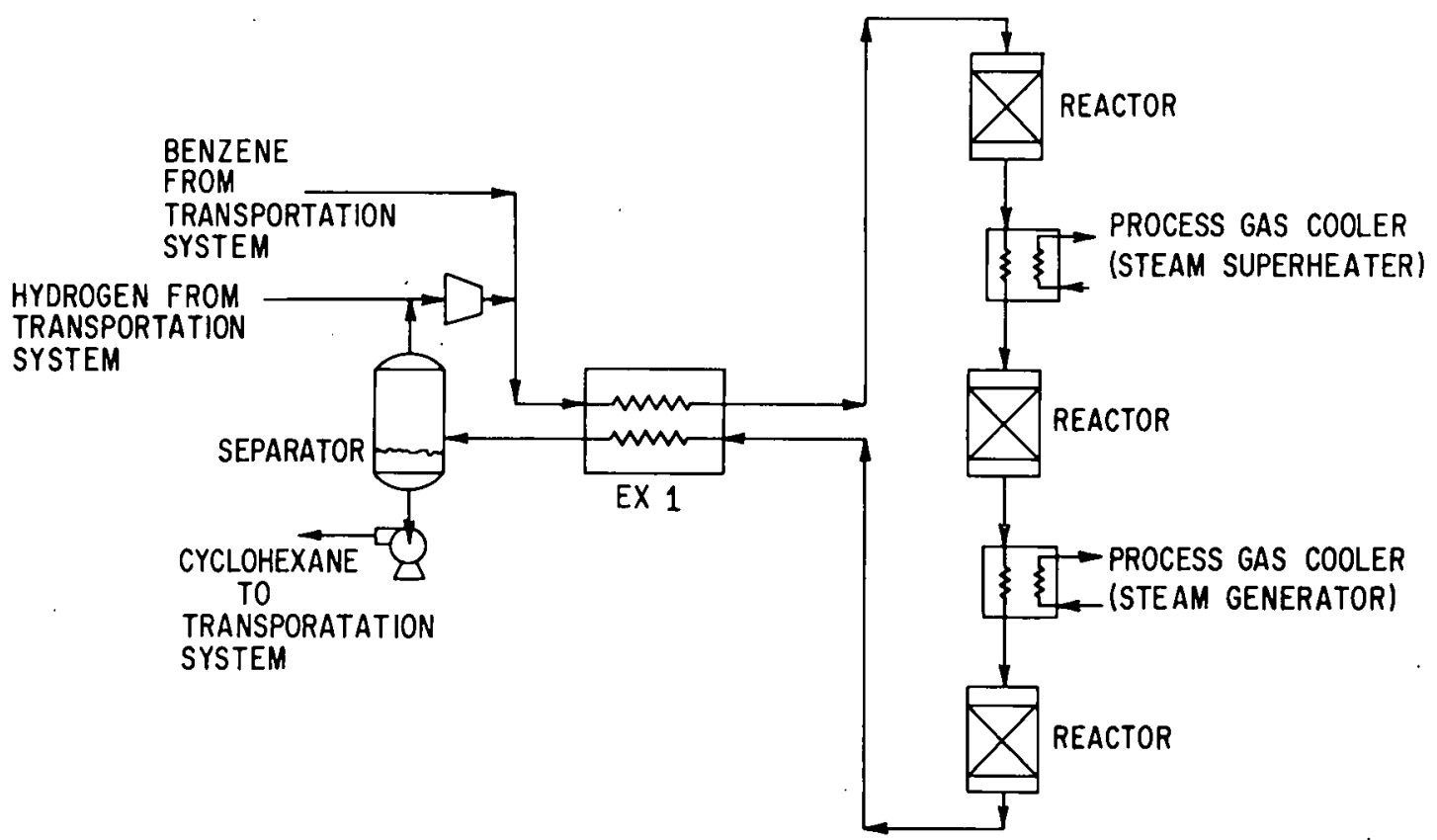

Figure 13-5. Process Flowsheet for Benzene Hydrogenation Section of LTCHP system

A vapor-liquid separator and a pump and compressor to overcome the equipment pressure drop are also included in the exothermic process.

In both of these process flowsheets, the concept of the mixed feed evaporator (MFE), developed in the HTCHP analysis, has been applied to the evaporation of the feed stream. That is, the hydrogen feed is mixed with the liquid cyclohexane/benzene stream 
prior to the evaporation of the liquids. The analysis performed in Chapter 5 has shown this approach to be thermodynamically more efficient than separate feeds; therefore it will be used here also.

\subsection{MATERIAL BALANCES}

\subsubsection{Design Assumptions}

In this section, a material balance for the LTCHP reference case will be developed. The process parameters for the reference cases are presented in Table 13.3.

Table 13.3

PROCESS PARAMETERS FOR LTCHP REFERENCE CASE

Dehydrogenation Catalyst Bed

Exit temperature

Exit pressure

$811 \mathrm{~K}\left(1000^{\circ} \mathrm{F}\right)$

40 bars

Hydrogenation Catalyst Bed

Exit temperature

Exit pressure

$589 \mathrm{~K}\left(600^{\circ} \mathrm{F}\right)$

40 bars

Hydrogen to Carbon Feed Ratio

Dehydrogenation

Hydrogenation

$2: 1$

$2: 1$

The choice of reaction conditions is based on industrial background and HTCHP process design experience. The dehydrogenation reaction temperature for this reference case was chosen to match the peak steam temperature in fossil boilers. The reaction pressure was chosen at the relatively high value because pipeline transmission of gases at low pressures tends to be more expensive and less efficient. High pressure decreases the conversion in the dehydrogenation reaction but increases it in the hydrogenation reaction. Even at this high pressure, however, the high outlet endothermic reaction temperature drives the dehydrogenation almost to completion. The exothermic reaction outlet temperature was chosen so that 1000 psia $(560 \mathrm{~K})$ steam might be generated from all the product heat.

The hydrogen/carbon ratio of $2: 1$ was chosen arbitrarily; from a conversion standpoint it is desirable to run at lower $\mathrm{H} / \mathrm{C}$ ratios in the endothermic reactor, and at higher $\mathrm{H} / \mathrm{C}$ ratios in the exothermic reactor. A major limiting factor in determining the hydrogen recycle rate in the endothermic reaction is the activity of the catalyst to promote carbon deposition reactions. If these reactions take place, then the catalyst becomes poisoned to the other reactions. This poisoning can be retarded by recycling hydrogen to maintain a high hydrogen activity. Because the characteristics of the ideal catalysts used in this study are undetermined, the arbitrary choice of $2: 1$ was made. 
No attempt was made to optimize the exothermic reaction hydrogen recycle rate. In this reaction, a tradeoff between the cost benefits associated with the increased conversion and the increased cost of the hydrogen recycle compressor must be made, to determine the optimum rate.

\subsubsection{Material. Balance. Results}

The reactor effluent compositions with MCP formation suppressed are shown in Table 13-4. These outlet compositions may be converted to the number of moles transported in the pipelines by considering that 17.63 tonne moles*/hr of benzene and cyclohexane must be converted in the reactors to transport $1000 \mathrm{MW}_{\text {th }}$ of chemical energy. The equilibrium compositions of both reactors must be matched so that the outlet composition of one reactor matches the inlet composition of the other, accounting for hydrogen recycle rates. These pipeline and hydrogen recycle flows are also shown in Table 13-4.

\section{Table 13-4 \\ EQUILIBRIUM REACTOR OUTLET COMPOSITIONS \\ AND PIPELINE FLOWS FOR LTCHP REFERENCE CASE}

Dehydrogenation Reaction

Hydrogen-to-carbon Ratio $=2: 1$

Reactor Outlet Temperature $=811 \mathrm{~K}$

Reactor Outlet Pressure $=40$ Bars

$$
\left(1000^{\circ} \mathrm{F}\right)
$$

Outlet Moles per Mole of Cycloh'exane

Component

Cyclohexane

Benzene

Hydrogen
Equivalent Feed

$$
\begin{aligned}
& 0.0085 \\
& 0.9915 \\
& 2.9745
\end{aligned}
$$

Tonne Moles/hr* Transported per 1000

$\frac{\text { MW }}{t h} \frac{\text { Transported }}{0.16} \begin{gathered}18.78 \\ 52.93\end{gathered}$

(3.40 moles $\mathrm{H}_{2}$ recycled)

Hydrogenation Reaction

Hydrogen-to-carbon Ratio $=2: 1$

Reactor Outlet Temperature $=589 \mathrm{~K}$

Reactor Outlet Pressure $=40$ Bars

Outlet Moles per Mole of Cyclohexane

Component

Cyclohexane

Benzene

Hydrogen
Equivalent Feed

$$
\begin{aligned}
& 0.9401 \\
& 0.0599 \\
& 0.1797
\end{aligned}
$$

Tonne Moles/hr*

Transported per 1000

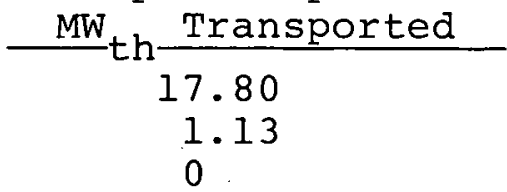

( 3.40 moles $\mathrm{H}_{2}$. recycled)

* 1 tonne mole $=10^{6} \mathrm{gram}-\mathrm{moles}$ 


\subsection{ENTHALPY BALANCE}

\subsubsection{Enthalpy Balance and Histograms}

Once the material balance is completed, calculation of the enthalpy balance and histograms for the LTCHP process design are straightforward. A computer program was written to calculate the vapor-liquid equilibrium in the evaporating and condensing streams and also the resulting enthalpy changes for these streams. This program is given in Appendix 6. Figures $13-6$ and 13-7 show the reference case histograms of heat duty in or out of the process stream (depending on whether it is vaporizing or condensing) as a function of process stream temperature for the dehydrogenation and hydrogenation processes, respectively.

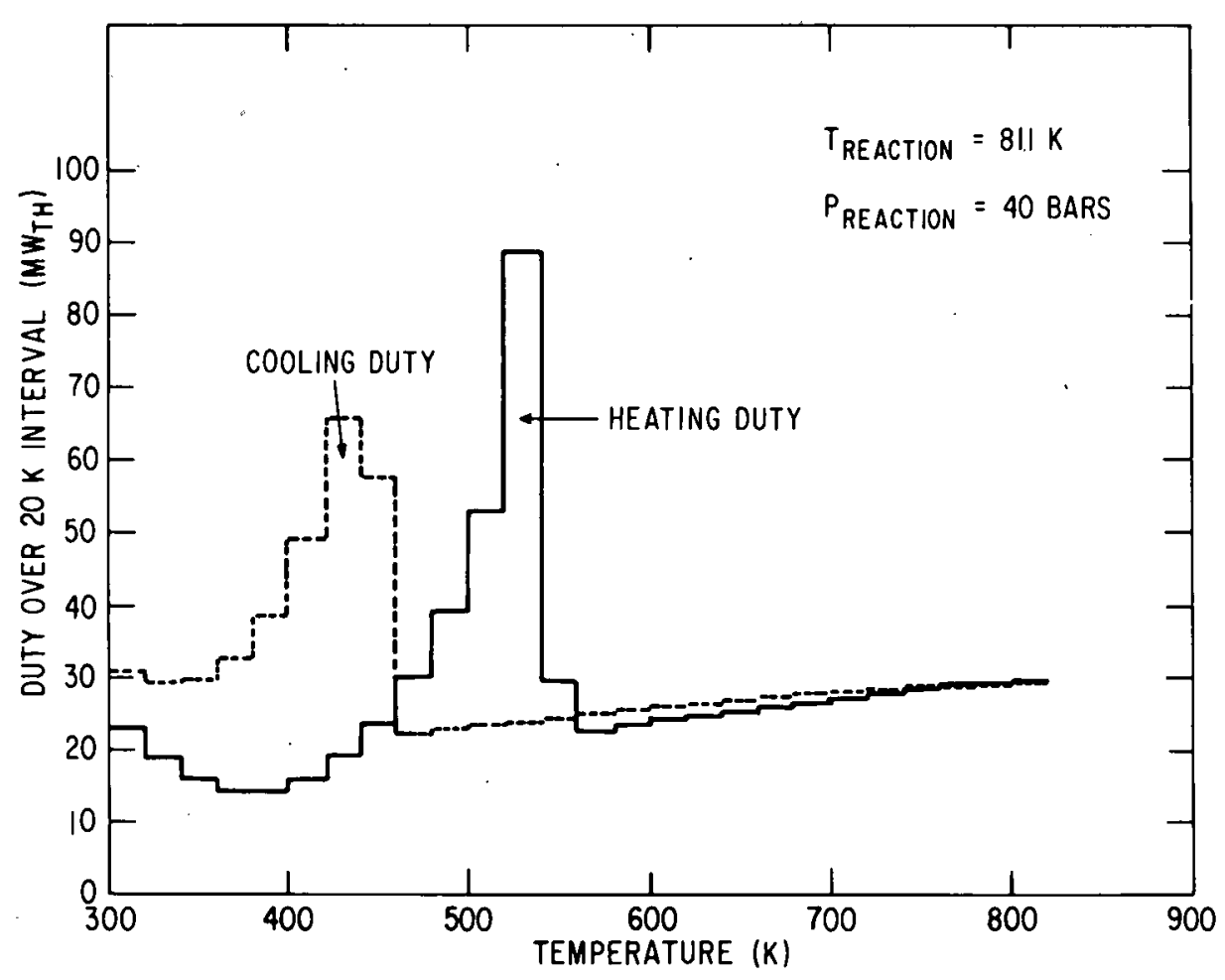

Figure 13-6. Histogram of Heating and Cooling Duties for Reference Case Dehydrogenation Process

As in the HTCHP process designs, at the endothermic site there, is a significant heat deficit at higher temperatures as the heat required by the reactant vaporization exceeds that given up by the product condensation. At lower temperatures, the situation is reversed and the product stream gives up more heat as it is cooled than is needed by the reactant stream as it is heated up. This thermal mismatch is managed by heat exchange between the LTCHP system and an associated powerplant, as was done in the HTCHP design. The discussion of the interaction between the CHP system and the powerplant presented in the HTCHP design, (Chapter 5) is generally applicable to the LTCHP system as well. 


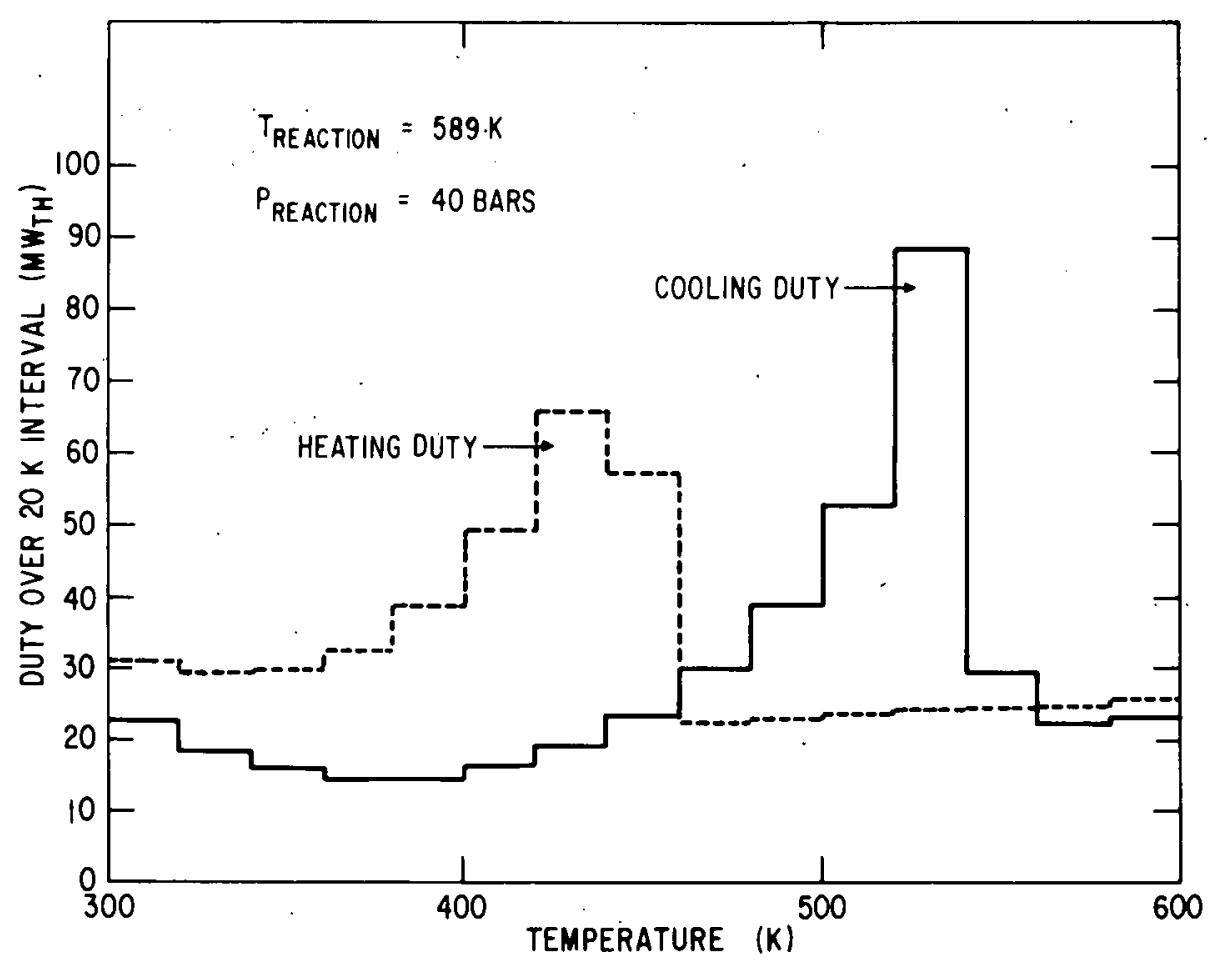

Figure 13-7. Histogram of Heating and Cooling Duties for Reference Case Hydrogenation Process

The histogram for the hydrogenation process shows that there is no thermal mismatch between the heating and cooling duties of the process streams. This is important, since it allows the exothermic reaction to take place on a "stand alone" basis at the user site. Examination of the histogram. also shows that a significant amount of exergy* is lost if the heat is transferred directly from the condensing product stream to the vaporizing reactants. Although no attempt has been made in this design to recover any of this lost work, more extensive process design development might well settle on a scheme that would utilize this energy and, hence, increase the overall efficiency of the process.

The enthalpy balance based on these histograms is shown in Table 13-5 for a $20 \mathrm{~K}$ temperature difference in the heat exchangers.

Several points from Table 13-5 should be highlighted. First, the interchange of heat with the powerplant results in a net heat gain for the powerplant (a loss to the LTCHP) $83.9 \mathrm{MW}_{\text {th }}$. In addition, since a $20 \mathrm{~K}$ minimum temperature difference in the heat exchangers was assumed for this design, the products from both reactors flow into the pipelines at $320 \mathrm{~K}$. The sensible heat available in these streams, between 320 and $300 \mathrm{~K}$, is assumed to be lost to the environment in the pipeline. This sensible heat

*Exergy can be thought of as the work available; a more complete discussion of this concept is given in Appendix 2. 
Table 13-5

ENTHALPY BALANCE FOR LTCHP REFERENCE CASE

FOR $20 \mathrm{~K} \triangle \mathrm{TS}$ IN THE HEAT EXCHANGERS

Dehydrogenation Process

Heat Exchangers

Exchanger

Number

E1 (to powerplant)

E2

E3

E4 (from powerplant)

E5 (to powerplant)

E6

E7 (to powerplant)

E8 total

Specific heat loss

Net heat to powerplant

(Sum of El, E4, E5, and E7)

Compressor Work

Pump Work

Transportation System

Compressor and Pump Work

Hydrogenation Process

Heat Exchangers

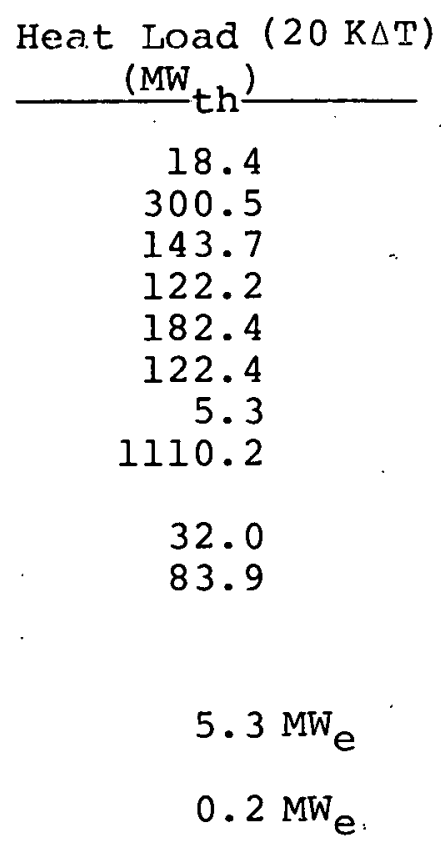

18.4

300.5

143.7

122.2

182.4

122.4

5.3

1110.2

32.0

83.9

$5.3 \mathrm{MW}_{\mathrm{e}}$

$0.2 \mathrm{MW}_{\mathrm{e}}$

$17.3 \mathrm{MW}_{\mathrm{e}}$

Heat Load $(20 \mathrm{~K} \Delta \mathrm{T})$

(MW th )

393.7

27.9

977.4

$5.3 \mathrm{MW}_{\mathrm{e}}$

$0.2 \mathrm{MW}_{\mathrm{e}}$ 
loss amounts to $32.0 \mathrm{MW}_{\mathrm{th}}$ in the dehydrogenation product stream and $27.9 \mathrm{MW}_{\mathrm{th}}$ in the hydrogenation product stream. Finally, the transportation compressor and pump work contributes $17.3 \mathrm{MW}_{\mathrm{e}}$ into the system as compression work and heating. It is assumed that this energy is also dissipated in the transmission system and lost from the LTCHP. The compression and pump work within the hydrogenation and dehydrogenation process is recovered as heat.

\subsection{LTCHP PROCESS EFFICIENCY}

\subsubsection{Background}

As discussed in the HTCHP reference case design, the process efficiency can be defined in many ways. The "first law" definition of efficiency (heat out/[work + heat in]) does not adequately account for the quality of heat at different locations during the process. The "second law" definition (exergy out/exergy in) requires detailed knowledge of the quality of heat at each interchange with the system. In this preliminary design, the quality of the heat source and the delivered process steam have not been defined in detail; therefore, a strict second law definition of efficiency is impossible. The efficiency used here is a compromise between these two traditional methods of defining efficiency.

\subsubsection{System Efficiency} i.e. :

The system efficiency is defined on a semi-first-law basis;

$$
\Psi=\text { system efficiency }=\frac{\text { total heat delivered }}{\text { total heat absorbed }}
$$

The total heat absorbed has two contributions: one from the actual heat. absorbed in the endothermic reactor $\left(1110.2 \mathrm{MW}_{\mathrm{th}}\right.$ for the reference case), and the other an amount equivalent to the heat required to produce the work needed by the system. The amount of heat required to produce a given amount of work varies with the temperature of the heat and the design of the powerplant. Typical values range from three units of heat to produce one unit of work for a LWR powerplant $(T \simeq 550 . K)$ to two and one-half units of heat to produce one unit of work in a fossil-fuel-fired plant $(T>800 \mathrm{~K})$. These values are assumed in this work, and a linear interpolation is made for intermediate temperatures used in other base designs investigated in Chapter 14 .

The situation is complicated by the interchange of heat between the LTCHP system and the associated powerplant at the endothermic reactor site. If this interchange is viewed on an exergy basis, the powerplant utilizes approximately $85 \%$ of the exergy given it, independent of the temperature at which it is given (see Appendix 2.3). Thus, it is assumed that 85\% of the net exergy given to the LTCHP (accounting for $\Delta \mathrm{Ts}$ ) in the form of heat interchange could be converted to work, and the LTCHP is charged that amount. The definition of efficiency is demonstrated in the following sample calculation for the reference case. 
- Exergy Interchange Between Powerplant and LTCHP:

To powerplant

El (steam superheat)

E5 \& E7 (boiler water preheat)

Total to powerplant

From powerplant

E4 (extraction steam)

Net exergy loss from powerplant

Net work loss from powerplant (@ 85\% of exergy loss)
Exergy, MW

9.5

41.5

51.0

53.8

2.8

2.4

- Total Work Requirement of LTCHP:

\begin{tabular}{lr} 
& \multicolumn{1}{l}{${ }^{\mathrm{MW}}$} \\
Powerplant loss (from above) & 2.4 \\
Process gas compressors & 10.6 \\
Process liquid pumps & 0.4 \\
Transportation pumps and compressors & 17.3 \\
Total & 30.7
\end{tabular}

- Heat Required to Produce Work

(e $811 \mathrm{~K}$ heat temperature):

$$
30.7 \mathrm{MW}_{\mathrm{e}} \times 2.5 \mathrm{MW}_{\mathrm{th}} / \mathrm{MW}_{\mathrm{e}}=76.8 \mathrm{MW}_{\mathrm{th}}
$$

- Total Heat into LTCHP:

Heat required to produce work Heat absorbed at endothermic reactor $76.8 \cdot \mathrm{MW}_{\mathrm{th}}$ Total heat into LTCHP $1110.2 \mathrm{MW}_{\text {th }}$ $1187.0 \mathrm{MW}_{\mathrm{th}}$

- Heat Delivered at Exothermic Reactor:

Heat Delivered $=977.4 \mathrm{MW}_{\text {th }}$

- Overall Process Efficiency:

$$
\Psi=\frac{977.4}{1187.0}=0.82
$$

Since this definition is one of several reasonable definitions, the energy requirements have been presented in detail so that other definitions can be used if desired in evaluating this system. The high efficiency calculated above is not unexpected, 
because of the match of exergy ratios between the heat source and the products from the endothermic reaction (see Appendix 2 for a more complete discussion of the thermodynamics of this situation).

\subsection{SUMMARY AND CONCLUSIONS}

The technical design for the LTCHP has been presented in this chapter for an endothermic reactor outlet temperature of $811 \mathrm{~K}$ $\left(1000^{\circ} \mathrm{F}\right)$ and an exothermic reactor outlet temperature of $589 \mathrm{~K}$ $\left(600^{\circ} \mathrm{F}\right)$, with both reactors operating at 40 bars. The following major conclusions can be drawn from this analysis:

- Methylcyclopentane formation in the hydrogenation and dehydrogenation reactions should be avoided, because of its deterious effects on the system energy balance.

- The heat loads for the exchangers used in the LTCHP are smaller than those for the HTCHP system.

- The hydrogenation reactor can be used on a stand-alone basis at the user site.

- Compression and pumping energy requirements are slightly lower in the LTCHP system than in the HTCHP system.

- The LTCHP system operates at approximately $82 \%$ efficiency.

\section{REFERENCES -- CHAPTER 13}

R 13-1 Sutton, E.A., Greenwood, A.R., and Adams, F.A., "A New Processing Concept: Continuous Platforming," The Oil and Gas Journal, May 22, 1972, p. 52 .

R 13-2 King, C.J., Separation Processes, McGraw-Hill, New York, 1971 , pp. $50 \overline{6-515 .}$

R 13-3 Private communication, UOP Process Division, Des Plaines, Illinois.

R 13-4 Technical Data Book - Petroleum Refining, American Petroleum Institute, New York, 1966.

R 13-5 Smith, J.M., and Van Ness, H.C., Introduction to Chemical Engineering Thermodynamics, 2d ed., McGraw-Hill, New York, 1959 . 


\section{Chapter 14}

\section{BASE CASE ANALYSIS FOR LOW TEMPERATURE CHEMICAL HEAT PIPE}

\subsection{INTRODUCTION}

In this chapter, several cases will be analyzed to determine the effect of the endothermic (dehydrogenation) reaction temperature and pressure on the heat and material balances of the Low Temperature Chemical Heat Pipe (LTCHP) system. These analyses will follow the pattern developed in the previous chapter for the reference case. First, reactor outlet conditions will be tabu lated, then material and heat balances formulated, and finally process efficiencies calculated. The first case evaluated (section 14.2) utilizes an endothermic reactor temperature and pressure of $755 \mathrm{~K}$ and 40 bars. These conditions might correspond to an advanced solar collector design or a high-temperature breeder reactor. The second case studied (section 14.3) drops the endothermic reaction temperature to $673 \mathrm{~K}$ with the pressure remaining at 40 bars. These conditions might correspond to intermediate technology solar collector or low-temperature breeder reactor heat sources. The final case selected (section 1.4.4) employs a reactor temperature and pressure of $540 \mathrm{~K}$ and 1 bar. These conditions correspond to those expected if the system were coupled to a Light water Reactor (LWR) or a low-temperature solar collector.

In the cases considered here, the exothermic reactor conditions are held constant at the reference case conditions ( $T_{\text {outlet }}$ $=589 \mathrm{~K}$, pressure $=40 \mathrm{bars})$. These conditions are chosen so that all the heat liberated from the exothermic reaction can be used to raise and superheat $1000 \mathrm{psia}$ of steam and at the same time obtain reasonable (94\%) conversion. Since the object of the CHP concept is to recover the transported heat at the highest possible temperature, lowering the exothermic reaction temperature by lowering the reactor pressure is not reasonable in the majority of cases. The exothermic outlet temperature of $589 \mathrm{~K}$ is conservative with respect to reaction kinetics, since it is above the temperatures currently used industrially (see Appendix 5) and, under the assumption of no byproduct formation used in this report, does not adversely affect the selectivity of the hydrogenation reaction.

\subsection{BASE CASE 1 ANALYSIS}

The reaction outlet conditions for this case are presented in Table 14-1.

Table 14-1

REACTION CONDITIONS FOR BASE CASE I

\begin{tabular}{ccccc}
\multicolumn{1}{c}{ Reaction } & $\begin{array}{c}\text { Reaction Outlet } \\
\text { Temperature }(\mathrm{K})\end{array}$ & & $\begin{array}{c}\text { Reaction Outlet } \\
\text { Pressure (bars) }\end{array}$ & $\begin{array}{c}\text { H/C } \\
\text { Ratio }\end{array}$ \\
\cline { 2 - 3 } Dehydrogenation & $755\left(900^{\circ} \mathrm{F}\right)$ & & 40 & $2: 1$ \\
Hydrogenation & $589\left(600^{\circ} \mathrm{F}\right)$ & & 40 & $2: 1$
\end{tabular}


Under these conditions, the reactor outlet composition are as shown in Table 14-2.

Table 14-2

REACTOR OUTLET COMPOSITIONS FOR BASE CASE 1

Basis: Moles/Mole Cyclohexane Equivalent Feed

\begin{tabular}{|c|c|c|c|}
\hline & $\begin{array}{l}\text { Cyclohexane } \\
\text { (moles/mole) }\end{array}$ & $\begin{array}{c}\text { Benzene } \\
\text { (moles/mole) }\end{array}$ & $\begin{array}{c}\text { Hydrogen } \\
\text { (moles/mole) } \\
\end{array}$ \\
\hline Dehydrogenation & 0.084 & 0.916 & 2.749 \\
\hline Hydrogenation & 0.940 & 0.060 & 0.180 \\
\hline
\end{tabular}

Combining these outlet compositions with the requirement of transporting 1000 MW of energy at the pipeline conditions, the flowrates shown in Table 14-3 are obtained.

$$
\begin{aligned}
& \text { Table 14-3 } \\
& \text { FLOWRATES FOR BASE CASE } 1 \\
& \text { Basis: } 1000 \text { MW Transported } \\
& \text { Cyclohexane Benzene Hydrogen } \\
& \text { (tonnemoles/hr) (tonnemoles/hr) (tonnemoles/hr) } \\
& \text { From Dehydrogenation } \\
& 1.73 \\
& 18.88 \\
& 52.93 \\
& \text { From Hydrogenation } \\
& 19.37 \\
& 1.23 \\
& (3.70 \text { recycled) } \\
& (3.70 \text { recycled })
\end{aligned}
$$

With these flowrates and the reaction temperature, the enthalpy balances for the two processes and their corresponding histograms can be determined. The histograms for the heat loads for the dehydrogenation and hydrogenation processes are shown in Figures 14-1 and 14-2 respectively. Again, a mismatch in thermal duties appears in the dehydrogenation heat exchange train, and the match in thermal duties in the hydrogenation process is present. These histograms, the material balances, and the computer analysis of the transportation system (see Appendix 4) are used to obtain the heat balance shown in Table 14-4. The heat exchanger numbers refer to the process flowsheet (Figures 13-4 and 13-5) presented in the technical analysis, and the heat balance assumes $20 \mathrm{~K} \Delta \mathrm{T}^{\prime} \mathrm{s}$ in the heat exchanger.

An exergy analysis of the heat interchange with the powerplant shows that the powerplant contributes 59.3 MW of exergy to the LTCHP system by extraction steam in exchanger E4. In turn, the LTCHP system gives back a total of 54.6 MW of exergy in exchangers El, E5, and E7. This results in a net exergy loss in the 


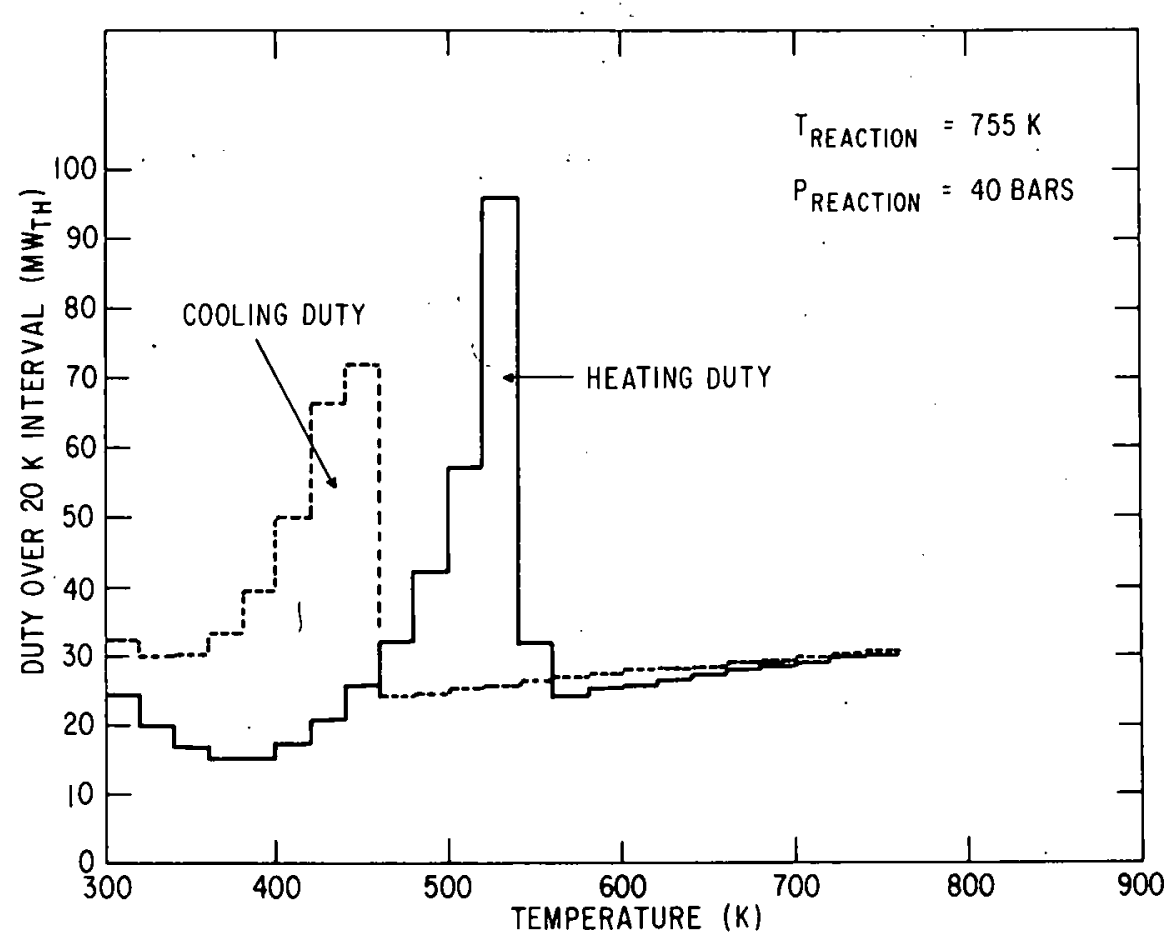

Figure 14-1. Histogram of Heating and Cooling Duties for Dehydrogenation Process in Base Case $1 ; \mathrm{T}_{\text {reaction }}=755 \mathrm{~K}, \mathrm{P}_{\text {reaction }}$
$=40 \mathrm{Bars}$

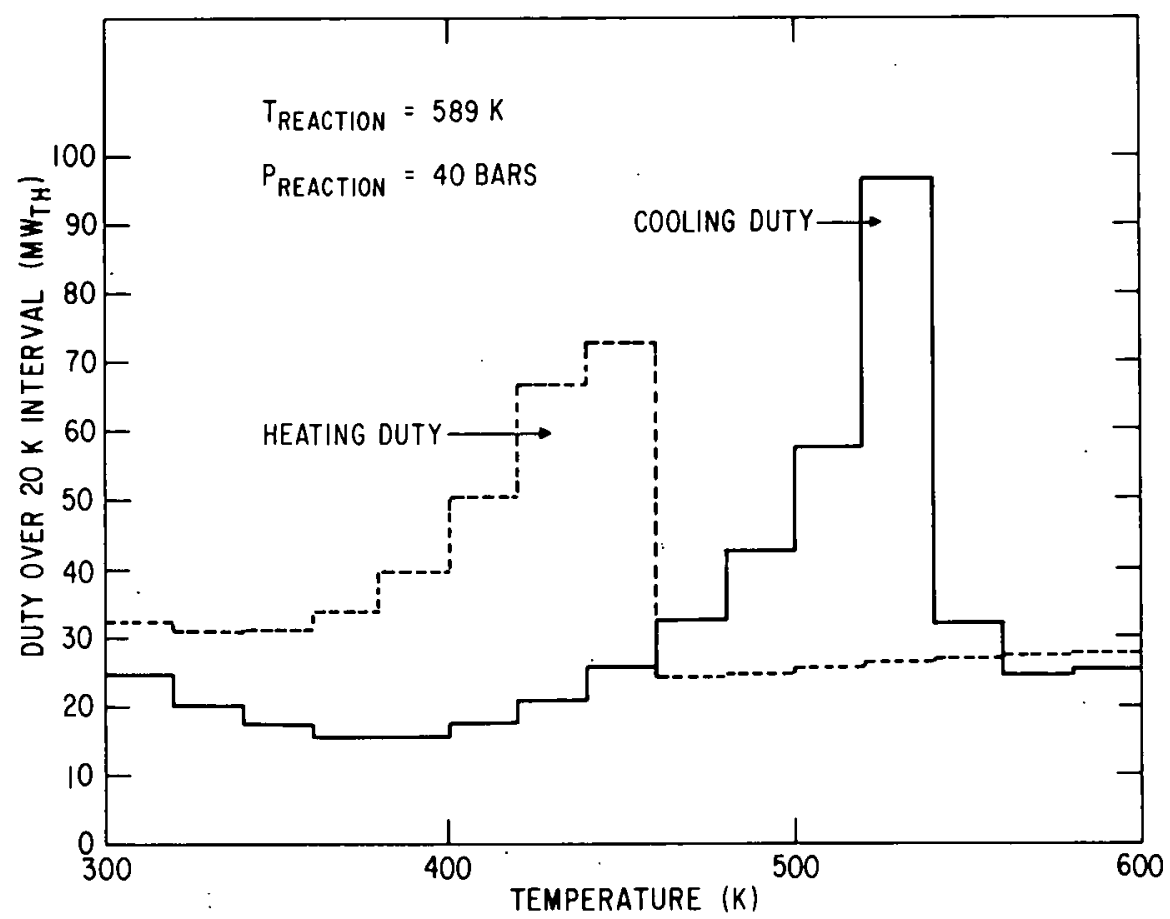

Figure 14-2. Histogram of Heating and Cooling Duties for Hydrogenation Process in Base Case 1; $\mathrm{T}_{\text {reaction }}=589 \mathrm{~K}, \mathrm{P}_{\text {reaction }}=40$ Bars 
Table 14-4

ENTHALPY BALANCE FOR BASE CASE 1

\begin{abstract}
Basis: $1000 \mathrm{MW}$ Transmitted; $20 \mathrm{~K} \Delta \mathrm{T}$ in Exchangers
Dehydrogenation Process
\end{abstract}

Heat Exchangers

Heat Load

El (to power plant)

E2

E3

E4 (from power plant)

E5 (to powerplant)

E6

E7 (to powerplant)

E8 process heaters

(MW)

17.2

240.0

154.8

134.6

194.8

133.1

5.8

1110.6

Sensible Heat Loss

33.2

Recycle Compressor

5.8

Pump

0.2

Transportation system

Compressors and Pumps

18.1

Hydrogenation Process

Heat Exchanger EX 1

428.1

Recycle Compressor

5.8

Pump

0.2

Process Gas Coolers

964.7

Sensible Heat Loss

41.1

powerplant of $4.7 \mathrm{MW}$. Assuming $85 \%$ conversion of exergy to electricity in the powerplant, the LTCHP process must be penalized 4.0 MW. Thus, the sum of the work requirements for LTCHP are:

\begin{tabular}{lr}
\multicolumn{1}{c}{ Process } & \multicolumn{1}{c}{ MW } \\
Compressor and pumps & 12.0 \\
Transportation & 18.1 \\
Powerplant loss & 4.0 \\
Total Work Requirement & 34.1
\end{tabular}

As explained in section 13.8 , the amount of heat required to produce this work is a function of the temperature of the heat. In this case it will be assumed that 2.6 units of heat are required to produce 1 unit of work (a linear interpolation between $811 \mathrm{~K}$ and $540 \mathrm{~K})$. The calculation of the system efficiency can then be summarized as follows: 
- Total Heat Required

To produce work $\left(34.1 \mathrm{MW}_{\mathrm{e}} \times 2.6 \frac{\mathrm{MW}_{\mathrm{th}}}{\mathrm{MWe}_{\mathrm{e}}}\right) \frac{\mathrm{MW}_{\mathrm{th}}}{88.7}$

Absorbed by endothermic reactor 1110.6

Total

1199.3

- Heat Delivered By System

Heat delivered. $964.7 \mathrm{MW}_{\text {th }}$

- System Efficiency

$$
\Psi=\frac{964.7}{1199.3}=0.80
$$

This efficiency is slightly lower than that of the reference case presented in the previous chapter. This can be explained by examining the effect of lower conversion $(0.92 \mathrm{vs} 0.99)$ in the endothermic reaction. As the conversion decreases, the flowrates through the system increase. As a result, higher amounts of heat must be transferred; this leads to larger irreversibilities in the heat exchangers; more heat exchange with the powerplant, and larger sensible heat losses in the streams fed to the transmission system. In addition, more energy is required to pump the larger liquid streams and compress the larger gas streams in the process and in the transportation system. It should be noted that the hydrogen transmission rate remains constant, independent of conversion, since only the hydrogen required to hydrogenate 17.64 tonne moles per hour of benzene need to transported. However, the decreased conversion does show up in the hydrogen recycle rate, which must increase at lower conversions to maintain the same hydrogen/carbon ratio in the reactor feed.

Even with the decreased efficiency, the energetics of the system at this reaction temperature still appear favorable. As the endothermic reaction temperature decreases further at constant pressure, one might expect that the decrease in conversion might adversely affect the efficiency even more. This will be explored in the next base case.

\subsection{BASE CASE 2 ANALYSIS}

The endothermic reaction outlet conditions for this case are presented in Table 14-5, the reactor outlet compositions in Table 14-6, and the transportation and process flowrates in Table 14-7.

Table 14-5

REACTION OUTLET CONDITIONS FOR BASE CASE 2

\begin{tabular}{|c|c|c|c|c|}
\hline Reaction & $\begin{array}{l}\text { Reac } \\
\text { Temp } \\
\end{array}$ & $\begin{array}{l}\text { Outlet } \\
\text { eure (K) }\end{array}$ & $\begin{array}{l}\text { Reactor Outlet } \\
\text { Pressure (bars) }\end{array}$ & $\begin{array}{r}\mathrm{H} / \mathrm{C} \\
\text { Ratio } \\
\end{array}$ \\
\hline Dehydrogenation & 673 & $\left(752^{\circ} \mathrm{F}\right)$ & 40 & $2: 1$ \\
\hline Hydrogenation & 589 & $\left(6.00^{\circ} \mathrm{F}\right)$ & 40 & $2: 1$ \\
\hline
\end{tabular}


Table 14-6

REACTOR OUTLET COMPOSITIONS FOR BASE CASE 2

\begin{tabular}{|c|c|c|c|}
\hline Reaction & $\begin{array}{l}\text { Cyclohexane } \\
\text { (moles/mole) }\end{array}$ & $\begin{array}{c}\text { Benzene } \\
\text { (moles/mole) }\end{array}$ & $\begin{array}{c}\text { Hydrogen } \\
\text { (moles/mole) }\end{array}$ \\
\hline Dehydrogenation & 0.671 & 0.329 & 0.988 \\
\hline Hydrogenation & 0.940 & 0.060 & 0.180 \\
\hline
\end{tabular}

Table 14-7

FLOWRATES FOR BASE CASE 2

Basis: $1000 \mathrm{MW}$ Transported

Cyclohexane Benzene Hydrogen

(tonnemoles/hr) (tonnemoles/hr) (tonnemoles/hr)

$\begin{array}{lccc}\text { From Dehydrogenation } & 43.92 & 21.57 & 52.93 \\ \text { From Hydrogenation } & 61.57 & 3.92 & (11.77 \text { recycled } \\ & & & (11.77 \text { recycled })\end{array}$

The histograms for the heat loads during heating and cooling for both the dehydrogenation and hydrogenation reactions are shown in Figures 14-3 and 14-4. The thermal mismatch is again present but more severe than in previous cases because of the much larger heat loads. These heat loads are presented more definitively in the enthalpy balance shown in Table 14-8.

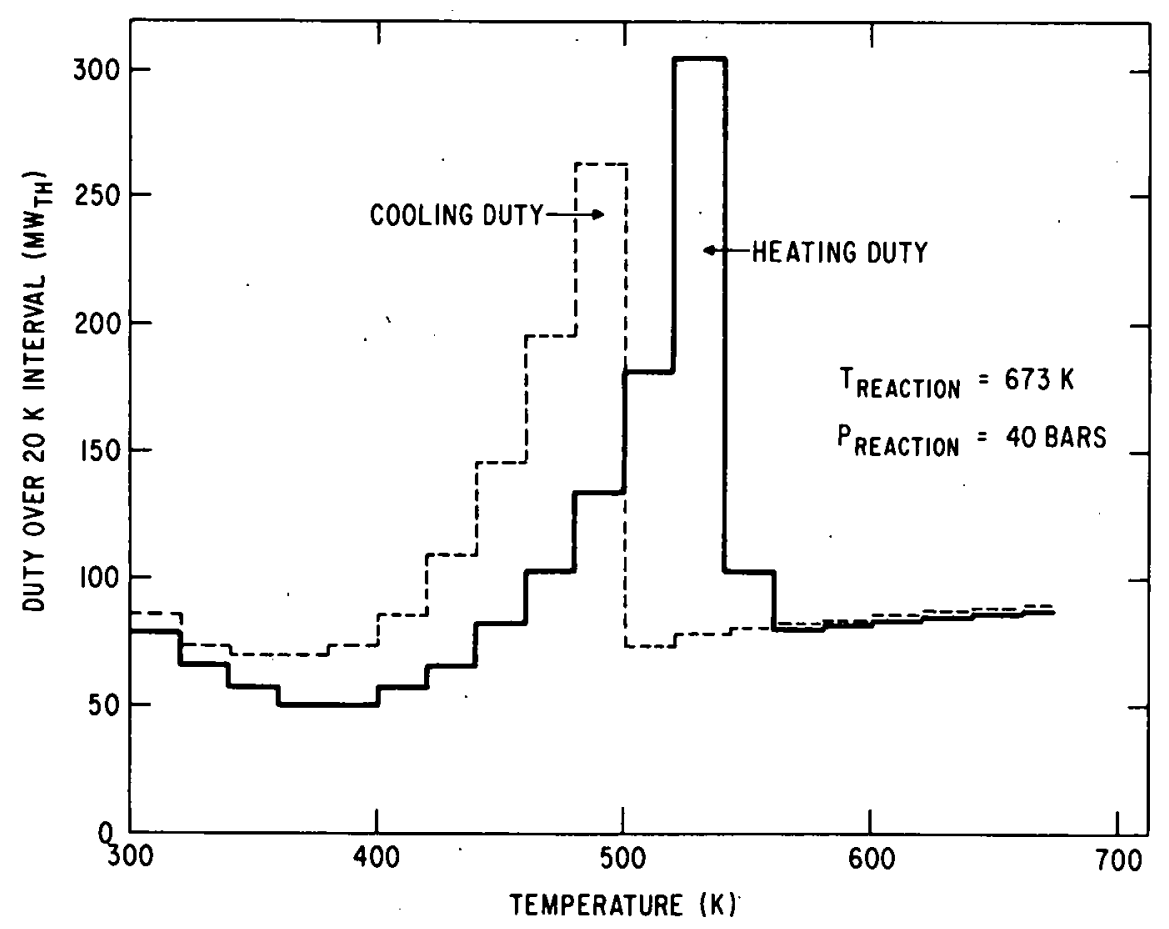

Figure 14-3. Histogram of Heating and Cooling Duties for Dehydrogenation Process in Base Case 2; $\mathrm{T}_{\text {reaction }}=673 \mathrm{~K}, \mathrm{P}_{\text {reaction }}=40$ Bars 


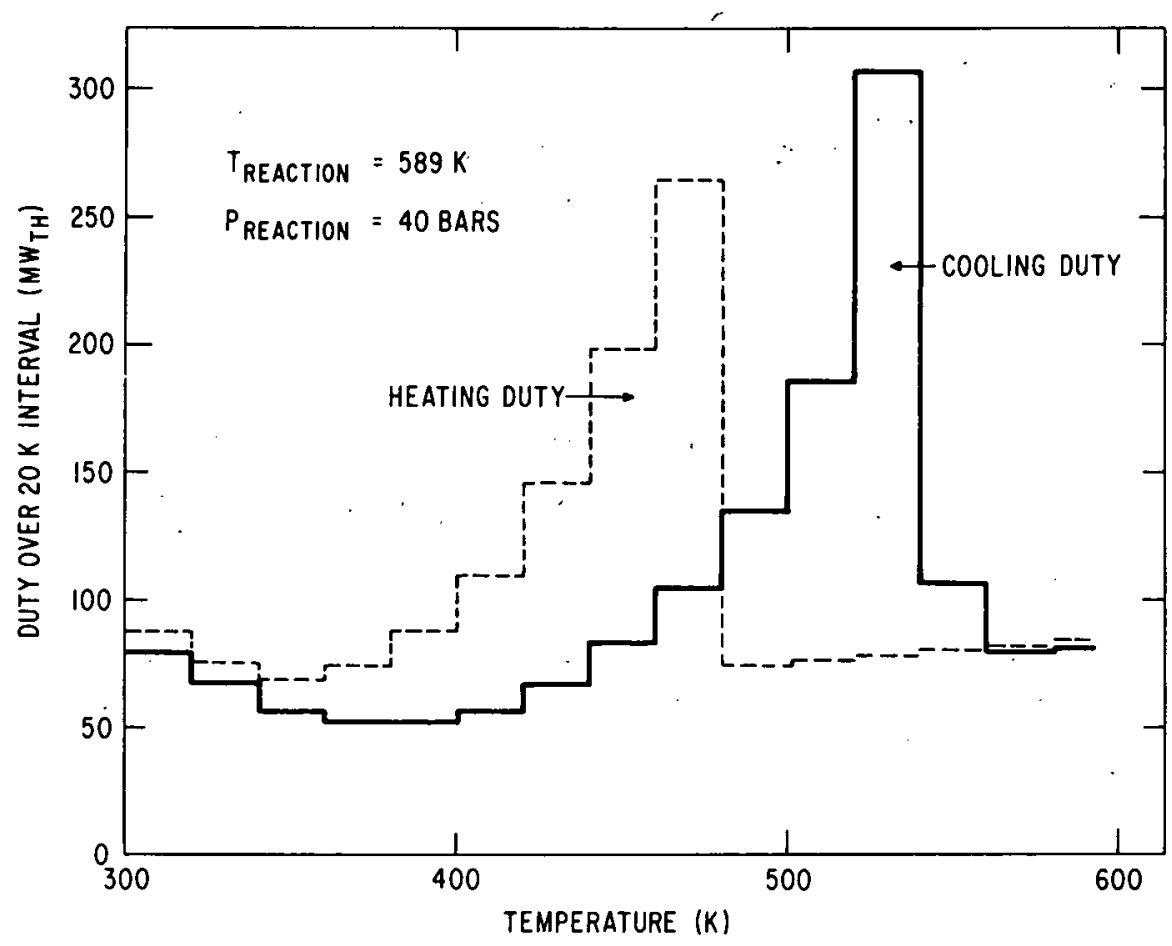

Figure 14-4. Histogram of Heating and Cooling Duties for Hydrogenation Process in Base Case 2; $\mathrm{T}_{\text {reaction }}=589 \mathrm{~K}, \mathrm{P}_{\text {reaction }}=40$ Bars

Table 14-8

ENTHALPY BALANCE FOR BASE CASE 2

Basis: $1000 \mathrm{MW}$ Transmitted; $20 \mathrm{~K} \Delta \mathrm{T}$ in Exchangers

Dehydrogenation Process

Heat Exchangers

E1 (to powerplant)
E2
E3
E4 (from powerplant)
E5 (to powerplant)
E6
E7 (to process plant)
E8 process heaters

Sensible Heat Loss

Recycle Compressor

Pump

Transportation System

Compressors and Pumps Hydrogenation Process

Heat Exchangér Ex 1

Recycle Compressor

Pump

Process Gas Coolers

Sensible Heat Loss

\begin{tabular}{c}
$\begin{array}{c}\text { Heat Load } \\
(\text { MW })\end{array}$ \\
\hline 18.5 \\
385.2 \\
310.8 \\
422.2 \\
482.2 \\
609.9 \\
18.5 \\
1165.9 \\
87.4 \\
18.5 \\
0.7
\end{tabular}

37.8

1369.9

18.5

0.7

939.2

79.3 
The exergy analysis for the process/powerplant interactions shows the powerplant loses $187.5 \mathrm{MW}$ from extract steam and gains 159.9 in superheat and boiler water preheat. This means the powerplant loses a net 27.6 MW in exergy or $23.4 \mathrm{MW}$ in electrical output. The total work input to the process is as follows:

$\begin{array}{lc} & \underline{M W} \\ \text { Powerplant loss } & 23.4 \\ \text { Recycle compressors and pumps } & 38.4 \\ \text { Transportation compressors and pumps } & \frac{37.8}{99.6} \\ \text { Total Work Requirement } & 99.6\end{array}$

Applying the same linear interpolation as used previously, 2.7 units of $673 \mathrm{~K}$ heat are required to produce 1 unit of work. Thus the heat requirements of the process and system efficiency can be summarized as follows:

- Total Heat Required

$$
\begin{aligned}
& \text { To produce work }\left(99.6 \mathrm{MW}_{\mathrm{e}} \times \frac{2.7 \mathrm{MW}_{\mathrm{th}}}{\mathrm{MW}_{\mathrm{e}}}\right) \frac{\mathrm{MW}_{\mathrm{th}}}{268.9} \\
& \text { Absorbed by endothermic reactor } 1165.9 \\
& \begin{array}{ll}
\text { Total } & 1434.8
\end{array}
\end{aligned}
$$

- Heat Delivered By System

Heat delivered

- System Efficiency

$$
\Psi=\frac{939.2}{1434.8}=0.65
$$

This low efficiency is not surprising in light of the low (33\%) conversion in the endothermic reactor. The discussion on the previous base case concerning process efficiency also applies here, where large heat exchanger and sensible heat losses tend to lower the efficiency of the process. The pressure of the endothermic reactor might be lowered to increase the conversion and, perhaps, the efficiency of the overall process. This approach will be investigated in the next case.

\subsection{BASE CASE 3 ANALYSIS}

The endothermic reaction outlet conditions for case 3 are presented in Table 14-9, the reactor outlet compositions in Table 14-10, and the transportation and process flowrate in Table 14-11. 
Table 14-9

REACTOR OUTLET CONDITIONS FOR BASE CASE 3

\begin{tabular}{|c|c|c|c|c|}
\hline Reaction & $\begin{array}{l}\text { Rea } \\
\text { Tem }\end{array}$ & $\begin{array}{l}\text { Outlet } \\
\text { zure (K) }\end{array}$ & $\begin{array}{l}\text { Reactor Outlet } \\
\text { Pressure (bars) }\end{array}$ & $\begin{array}{r}\mathrm{H} / \mathrm{C} \\
\text { Ratio }\end{array}$ \\
\hline Dehydrogenation & 540 & $\left(512^{\circ} \mathrm{F}\right)$ & 1 & $2: 1$ \\
\hline Hydrogenation & 589 & $\left(600^{\circ} \mathrm{F}\right)$ & 40 & $2: 1$ \\
\hline
\end{tabular}

Table 14-10

REACTOR OUTLET COMPOSITIONS FOR BASE CASE 3

Basis: Moles/Mole Cyclohexane Equivalent Feed

\begin{tabular}{|c|c|c|c|}
\hline Reaction & $\begin{array}{l}\text { Cyclohexane } \\
\text { (moles/mole) }\end{array}$ & $\begin{array}{c}\text { Benzene } \\
\text { (moles/mole) } \\
\end{array}$ & $\begin{array}{c}\text { Hydrogen } \\
\text { (moles!mole) }\end{array}$ \\
\hline lation & 0.4720 & 0.5280 & 1.5841 \\
\hline Hydrogenation & 0.9401 & 0.0599 & 0.1797 \\
\hline
\end{tabular}

Table 14-11

FLOWRATE FOR BASE CASE 3

Basis: 1000 MW Transported

Cyclohexane Benzene Hydrogen

(tonnemoles $/ \mathrm{hr}$ ) (tonnemoles/hr) (tonnemoles/hr)

$\begin{array}{lccc}\text { From Dehydrogenation } & 17.79 & 19.90 & 52.93 \\ \text { From Hydrogenation } & 35.43 & 2.26 & (6.77 \text { recycled }) \\ 0\end{array}$

The heat load histograms for this case are shown in Figure 14-5 for the dehydrogenation process and Figure 14-6 for the hydrogenation process. Examination of the histogram for the dehydrogenation process reveals a significant problem: because of the low pressure, a large portion ( 28 tonne moles) of the benzene and cyclohexane present in the dehydrogenator exit gases remain in the vapor phase at $320 \mathrm{~K}$ (the outlet temperature of exchanger E5). As the gases are compressed to 40 bars (the transmission and exothermic reaction pressure) and cooled, this cyclohexane and benzene condense out of the hydrogen, liberating approximately $197 \mathrm{MW}$ th in condensation heat. To accomplish this, the four stages of compression with intercooling shown in Figure 14-7 should be added to the process flowsheet, replacing exchanger $E 7$ and the recycle compressor. The enthalpy balance for this complete process is shown in Table 14-12.

Proceeding with the exergy analysis of the powerplant heat interchange as in previous cases, the powerplant gives the process. 47.1 MW of exergy from extraction steam and receives a total of 59.1 MW of exergy back in superheat and boiler water preheat. of 


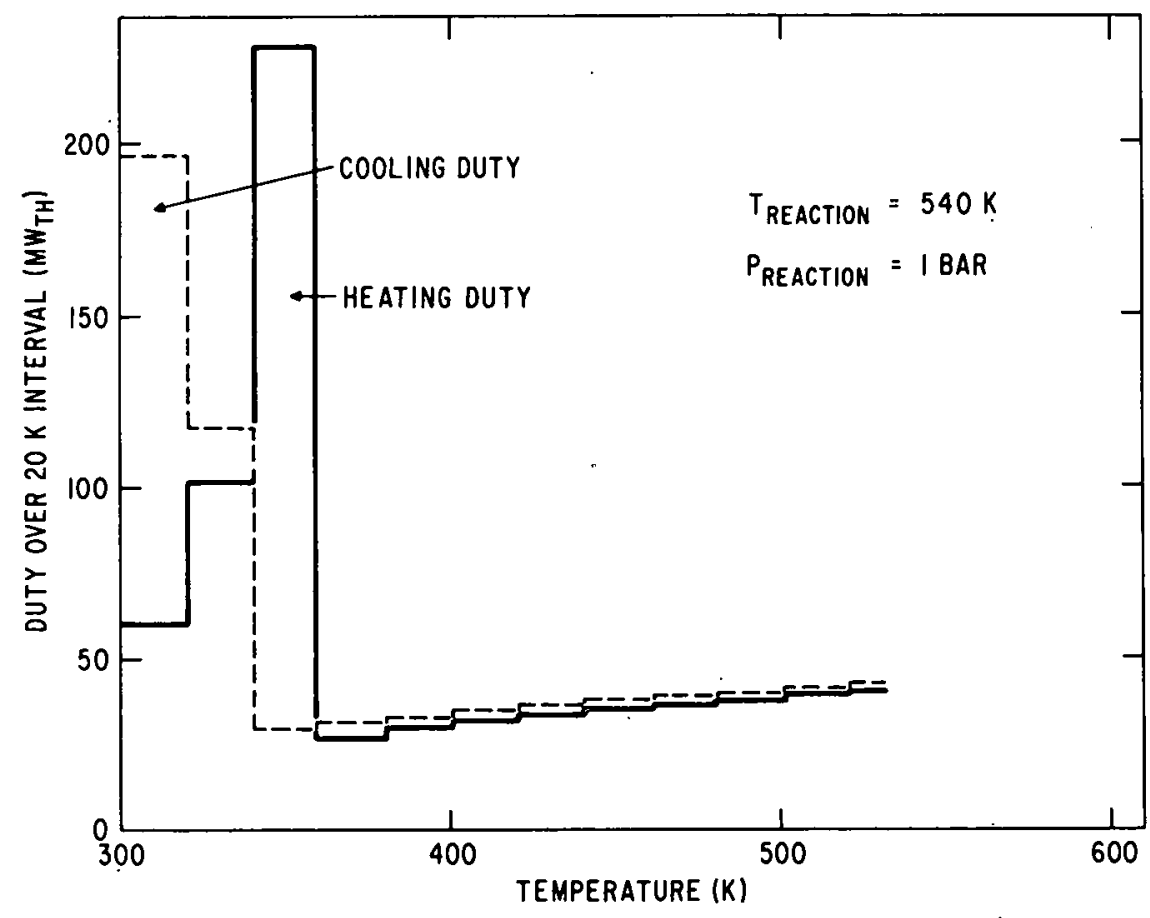

Figure 14-5. Histogram for Heating and Cooling Duties for Dehydrogenation Process in Base Case 3; $\mathrm{T}_{\text {reaction }}=540 \mathrm{~K}, \mathrm{P}_{\text {reaction }}=1 \mathrm{Bar}$

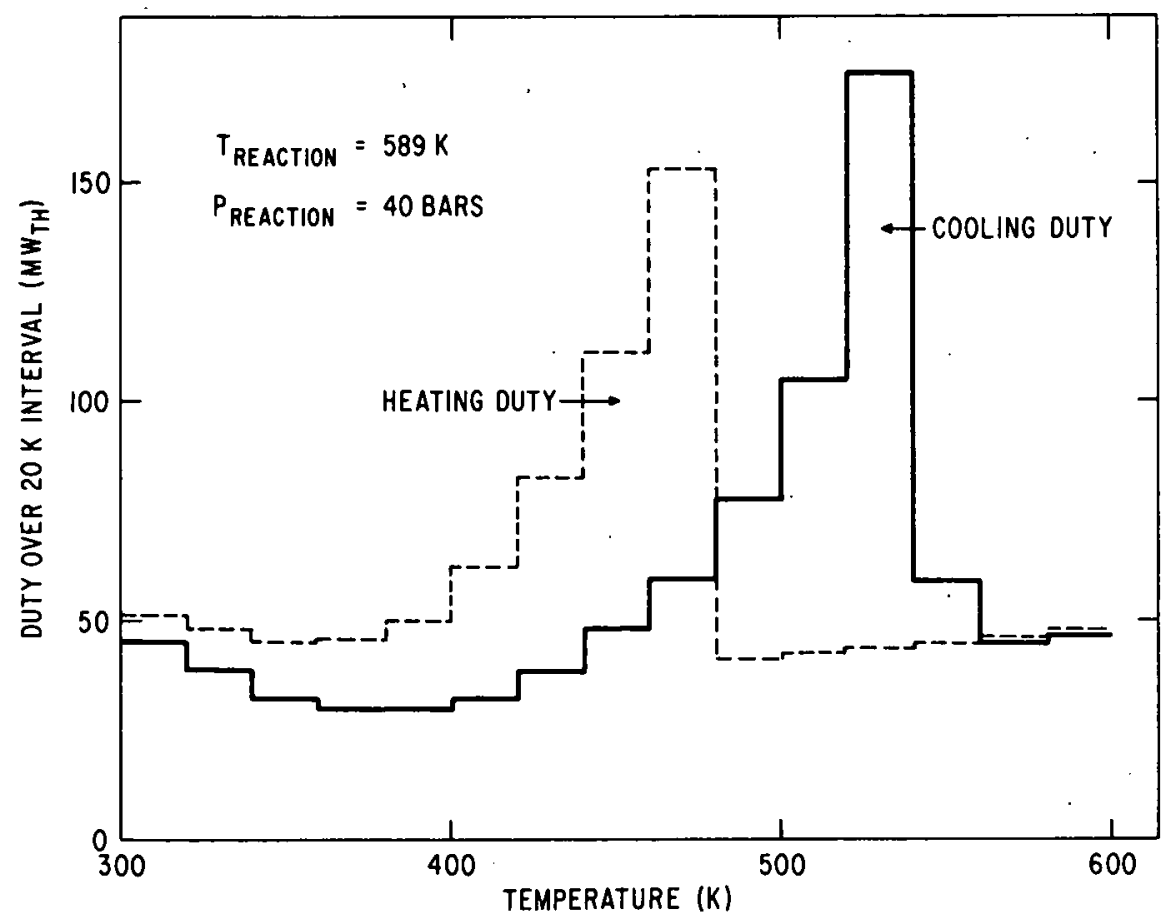

Figure 14-6. Histogram for Heating and Cooling Duties for Hydrogenation Process in Base Case 3; $\mathrm{T}_{\text {reaction }}=589 \mathrm{~K} ; \mathrm{P}_{\text {reaction }}=40 \mathrm{Bars}$ 


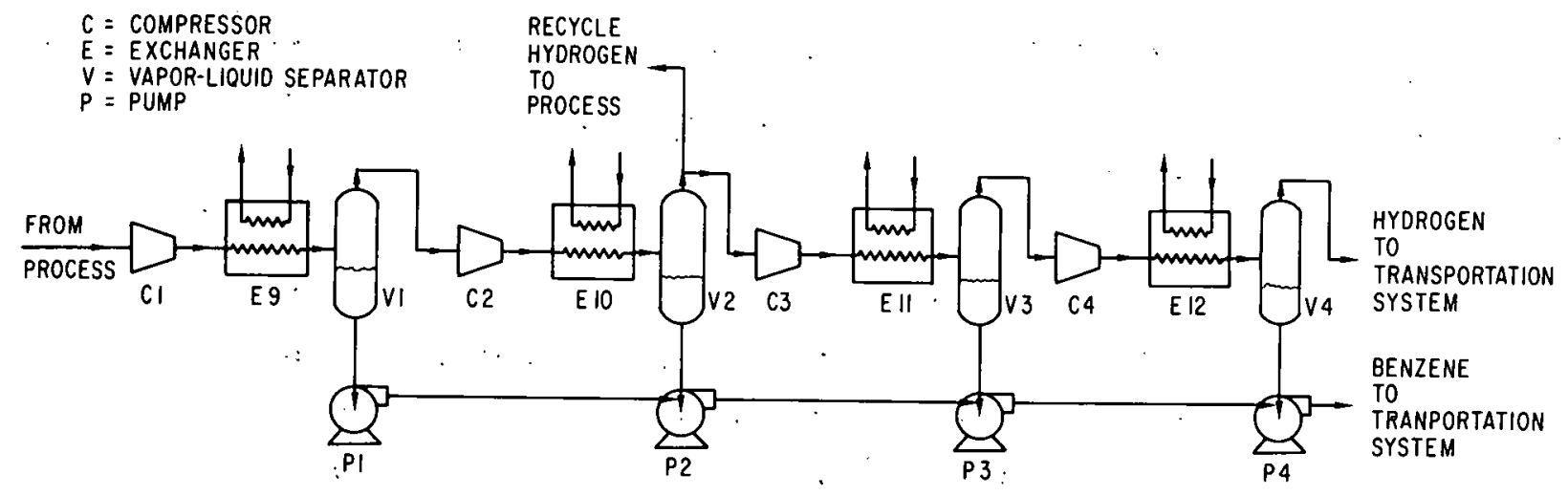

Figure 14-7. Gas Compression Flowsheet for $540 \mathrm{~K} / 1 \mathrm{Bar}$ in Base Case 3

Table 14-12

ENTHALPY BALANCE FOR BASE CASE 3

Basis:" $1000 \mathrm{MW}$ Transported; $20 \mathrm{~K} \Delta \mathrm{T}$ in Exchangers

Dehydrogenation Process

Heat Exchangers

$\begin{array}{lr}\text { E1 (to power plant) } & 43.6 \\ \text { E2 } & 283.1 \\ \text { E3 (from powerplant) } & 67.9 \\ \text { E4 (to powerplant) } & 266.2 \\ \text { E5 (to } & 57.2 \\ \text { E6 (to powerplant) } & 60.6 \\ \text { E9 (to powerplant) } & 218.8 \\ \text { E10 (to pow } & 108.4 \\ \text { El1 (to powerplant) } & 62.0 \\ \text { El2 (to powerplant) } & 52.9 \\ \text { E8 (process heaters) } & 1032.0\end{array}$

Compressors

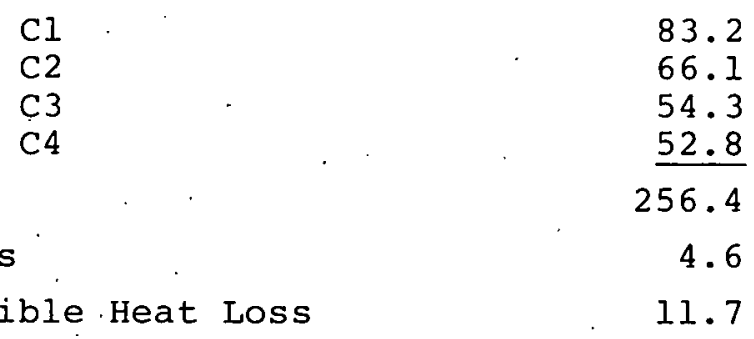

Sensible Heat Loss $\quad 11.7$

Transportation system

Compressors and Pumps 26.0

Hydrogenation Process

Heat Exchanger EX $1 \quad 788.4$

Compressor $\quad 10.6$

Pump

Process Gas Coolers

Sensible Heat Loss

0.4

960.1

50.5 
that 59.1 MW, 44.2 MW is returned in the compressor intercoolers. This is a net gain of $12 \mathrm{MW}$ of exergy for the powerplant, resulting in a credit to the process of $10.2 \mathrm{MW}$. The total work requirements of the process are then:

$\begin{array}{lc} & \underline{M W} \\ \text { Powerplant losses } & (10.2) \\ \text { Transportation compressors and pumps } & 26.0 \\ \text { Process compressors and pumps } & 272.0 \\ \text { Total Work Requirement } & 287.8\end{array}$

Using three units of heat for every unit of work required, the heat requirements of the process and the system efficiency can be summarized as follows:

- Total Heat Required To produce work $\left(287.8 \mathrm{MW}_{\mathrm{e}} \times 3 \frac{\mathrm{MW}_{\mathrm{th}}}{\mathrm{MW}}\right) \frac{\frac{\mathrm{MW}}{\mathrm{th}}}{863.4}$ Absorbed by the endothermic reactor $\frac{1032.0}{18.95 .4}$ Total 960.1 Heat delivered

- System Efficiency

$$
\Psi=\frac{960.1}{1895.4}=0.51
$$

Several comments should be made concerning the process conditions and this efficiency. First, the reaction pressure was chosen as 1 bar in order to assure reasonable conversion in the endothermic reactor. The previous case showed that the inefficiencies due to low conversion (e.g., high heat loads in the exchanger) cause a considerable lowering of the process efficiency. In this case, the conversion is higher than the previous case $(0.53$ vs 0.33$)$, but the overall thermal efficiency is still lower $(0.51$ vs 0.65$)$. This can be explained by an exergy analysis of the heat input to the endothermic reactor and the heat delivered at the exothermic reactor. The heat is absorbed at an average temperature of about $530 \mathrm{~K}$, but the heat is delivered at an average temperature of about $643 \mathrm{~K}$. The input heat has an exergy ratio* of 0.43 , while the delivered heat has an exergy ratio of 0.53 . This increase in exergy ratio must come from some source, and in this case it is supplied by the process compressors which compress the output hydrogen from the endothermic reactor from 1 to 40 bars.

\footnotetext{
*Exergy ratio = work available per unit of heat available (see Appendix 2 for complete discussion).
} 
This case is very much like the production of electricity, in that a heat source of low exergy ratio is being converted to a high exergy ratio product.* Referring to the discussion in Appendix 2 on this subject, it is inevitable that a low thermal efficiency will result. That is, to pump the heat from $\sim 530 \mathrm{~K}$ up to $\sim 640 \mathrm{~K}$, a penalty must be paid; that penalty is the consumption of additional heat.

To evaluate the merits of this case, one must consider the economics involved (as will be done in the next chapter) and be willing to value the heat according to its exergy. Of course, one has the option of recovering the transported heat at lower temperatures by using a lower exothermic reaction pressure. The evaluation of a low-pressure hydrogenation reaction must consider effects of low-pressure gas transmission (large pipelines and relatively greater pressure drops), and the transmission design must meet pipeline standards for pipeline wall thickness, etc. This type of design is beyond the scope of this contract and will not be considered here.

\subsection{SUMMARY AND CONCLUSIONS}

In this chapter, analyses of LTCHP systems were conducted to determine the effect of endothermic reaction temperature and pressure on system heat and material balances and on system efficiency. The major conclusions from this chapter are:

- Lowering the endothermic reaction temperature at a constant reference case pressure of 40 bars decreases the conversion.

- This decreased conversion decreases the overall system efficiency.

- Between $673 \mathrm{~K}$ and $755 \mathrm{~K}$ reaction temperature (most probably around $700 \mathrm{~K}$ ), the system efficiency dictates that lower reaction pressures be employed.

- At $540 \mathrm{~K}$ reaction temperature (1 bar pressure) the system efficiency approaches $50 \%$ if the exothermic reaction conditions are held constant.

- This lower efficiency is not necessarily undesirable, since $540 \mathrm{~K}$ heat is being pumped to $640 \mathrm{~K}$.

*The approach utilizing a hydrogenation reactor pressure that is significantly higher than that for dehydrogenation was arrived at on the basis of prior proprietary investigations at $\mathrm{GE}$. 


\section{Chapter 15}

\section{ECONOMIC ANALYSIS OF LTCHP}

\subsection{INTRODUCTION}

This chapter will be devoted to determining the cost of transporting heat through the Low Temperature Chemical Heat Pipe (LTCHP). Since costing of process equipment is, at this level, only estimation, and is highly dependent on both the process variables and cost parameters chosen, an attempt is made to delineate these choices and their effect on the total cost. Thereby, the effect of changes in the values of the cost parameters on the process economics may be determined. In all cases, an attempt is made to choose process and cost parameters that are consistent with current industrial practice. The approach is to examine the economics of the reference case (section 15.2) with the process cost parameters and reasoning behind their choice documented in detail. Then the base-case cost estimation is completed using these same parameters with the exceptions noted (section 15.3). Section 15.4 is devoted to the effect of byproduct formation on process economics. Finally, section 15.5 summarizes the major conclusions from this chapter.

\subsection{REFERENCE CASE ECONOMICS AND COMPONENT COSTS}

\subsubsection{Introduction}

The economic analysis of the reference case $(811 \mathrm{~K}$ endothermic reaction temperature, 40 bar operating pressure) will be divided into three areas:
- Cyclohexane dehydrogenation process
- Transportation system
- Benzene hydrogenation process

For this analysis, it will be assumed that these various components are on stream $8000 \mathrm{hr} / \mathrm{yr}$, and a capitalization factor* of 0.2 was chosen for all three sections.

\subsubsection{Cyclohexane Dehydrogenation Process}

The dehydrogenation process can be divided into four main equipment categories: reactors, exchangers in the heat exchanger train, process gas heaters, and pumps and compressors. These categories are described below.

\subsubsection{Reactors}

The reactor section offers an interesting comparison with current industrial practice in the gasoline reforming. A liquid

\footnotetext{
* Capitalization factor is defined as the yearly portion of the capital cost of equipment charged to the operating cost. This includes such factors as depreciation, taxes, and insurance.
} 
hourly space velocity (LHSV) of $1^{\text {hr }}$ is commonly used in gasoline reforming units. Since the dehydrogenation reaction rate is fast as compared with the isomerization and hydrocracking reactions which also take place in the reformer, a reactor designed for the dehydrogen reaction only can probably utilize LHSVs up to $200^{-h r}$ (Rl5-l). A conservative estimate for the LHSV of $30 \mathrm{hr}^{-1}$ is used in this analysis.

This LHSV and the given flowrates are used to calculate the required catalyst volume. The total reactor volume is then determined as 1.4 times the catalyst volume, to allow for reactor internals (Rl5-1). The volume and weight of metal used in the shell $(\mathrm{L} / \mathrm{D}=5)$ are calculated on the basis of 40 bar internal pressure and an allowable stress at $1000^{\circ} \mathrm{F}$ of 6250 psia (R15-2). The reactor cost is estimated at $\$ 2 / 1 b$ of steel with a factor of 2 used to account for installation of pressure vessels (reactors) (RI5-1). These values agree well with those used in Appendix 1 for the cost of methanation reactors. Catalyst capital costs are estimated at $\$ 10 / 1 b$ installed (R15-3).

In LTCHP applications, the rate of irreversible catalyst deactivation should be significantly less than in present commercial applications, since LTCHP reactants will be essentially poison free because of the cyclic nature of the process. A summary of the design and cost assumptions and the resultant capital costs are shown in Table 15-1 for the reactor system.

Table 15-1

DESIGN AND COST ASSUMPTIONS AND REACTOR SIZE
AND COST RESULTS FOR LTCHP DEHYDROGENATION REACTOR

Design and Cost Assumptions

$\begin{array}{ll}\text { Design Liquid Hourly Space Velocity } & 30 \mathrm{hr}^{-1} \\ \text { Reactor Pressure } & 40 \mathrm{bars} \\ \text { Reactor I/D Ratio } & 5: 1 . \\ \text { Metal Design Allowable Stress } & 6250 \text { psia } \\ \text { Reactor Cost } & \$ 2 / 1 \mathrm{~b} \\ \text { Reactor Installation Factor } & 2 \\ \text { Catalyst Cost } & \$ 10 / 1 \mathrm{~b}\end{array}$

Size and Cost Results

Total Catalyst Volume

Total Reactor Volume

Reactor Dimensions ( 3 required)

Reactor wall Thickness

Reactor Weight

( 3 required)

Total Reactor Cost

Total Catalyst cost

Total cost

$2400 \mathrm{ft}^{3}$
$3425 \mathrm{ft}^{3}$
$6.6 \mathrm{ft} \times 33 \mathrm{ft}$
$4.5 \mathrm{in}$.
$130,0001 \mathrm{~b}$
$\$ 1.6 \times 10^{6}$
$\$ 6 \times 10^{6}$
$\$ 7.6 \times 10^{6}$

*Liquid Hourly Space Velocity is defined as the ratio of the rate of volumetric liquid feed to the reactor (at standard conditions) to the volume of catalyst. 


\subsubsection{Heat Exchangers}

All heat exchanger designs are based on $20 \mathrm{~K}\left(36^{\circ} \mathrm{F}\right)$ minimum temperature differences between the hot and cold fluids in the heat exchangers. Heat transfer coefficients for the various exchangers are estimated by comparison with analogous exchangers in the HTCHP as detailed. in Appendix 3. The heat exchanger costs are estimated at $\$ 25 / \mathrm{ft}^{2}$ with a factor of 3 used to account for installation (RI5-1; Appendix 1). The cost and design parameters for the heat exchangers are shown in Table 15-2.

$$
\text { Table 15-2 }
$$

HEAT EXCHANGER COST AND DESIGN PARAMETERS

FOR LTCHP REFERENCE CASE - DEHYDROGENATION PROCESS

$$
\text { Basis: } \$ 75 / \mathrm{ft}^{2} \text { installed, } \Delta \mathrm{T}=20 \mathrm{~K}
$$

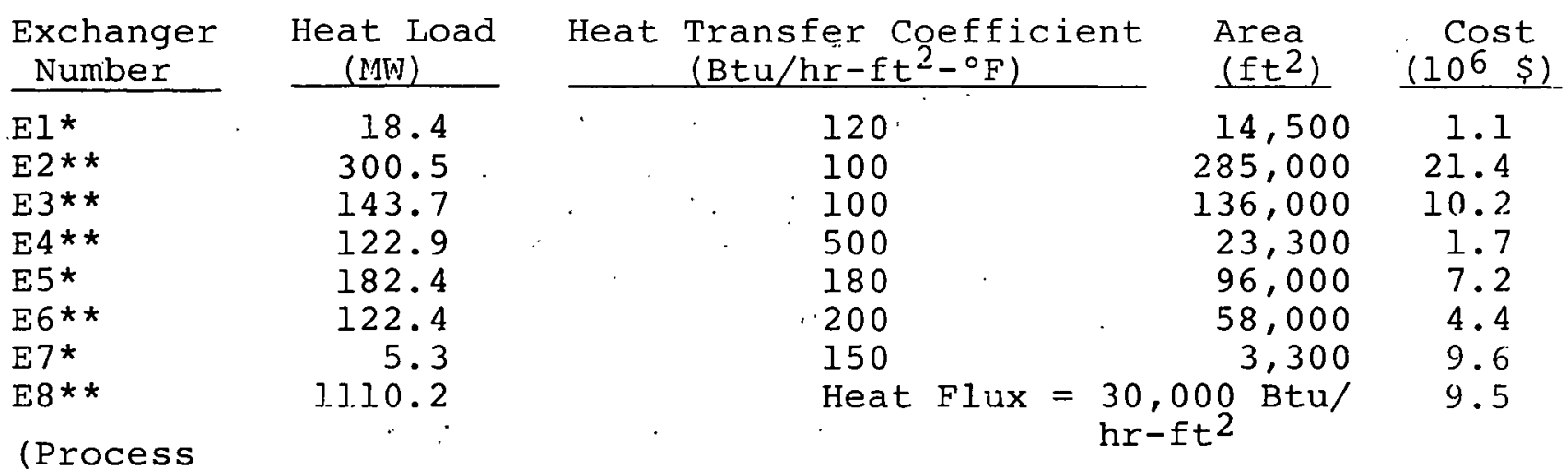

Gas Interheaters)

\footnotetext{
*Charged to powerplant **Charged to LTCHP
}

When costing the heat exchangers, it is important to note that the powerplant associated with the LTCHP should assume a portion of the costs. No attempt has been made to optimize the power plant, although general rules for the trade of exergy between the LTCHP and the powerplant derived for the HTCHP system have been employed (see Appendix 2). The basis for this interaction is that the powerplant will utilize $85 \%$ of the exergy in the steam to deliver electricity. The source of this exergy is not important; it can come from the primary heat source, as reject heat from some other source, or from the LTCHP system.

This is obviously a complicated problem which would require a detailed analysis of the powerplant in order to assure that the cost of the exchangers is allocated correctly between the various systems involved. However, since this is a preliminary analysis and experience has shown that the powerplant can utilize approxiately $85 \%$ of the exergy given it., the assumption has been made in this analysis that the cost of the heat exchangers which deliver heat to the powerplant (EI, E5, and E7, in Figure 13-1) and the 
cost of the heat exchangers which deliver heat to the LTCHP (E4) will be charged to it. Other exchangers (E2, E3, and E6), of course, are part of the LTCHP and should be charged to the cost of delivered heat.

\subsubsection{Process Gas Heaters}

The term "process gas heater" refers to the heat exchangers required to deliver the primary heat to the LTCHP reactant stream. In this design, the reactors are assumed to be adiabatic fixed beds, and the heat is delivered by heat exchange prior to and between the three reactors. The heat flux to the process gas heaters which supply the heat of reaction is assumed to be $30,000 \mathrm{Btu} / \mathrm{hr}-$ $\mathrm{ft}^{2}$ for the reference case. This value is reasonable for flamefired furnaces (RI5-I) and is varied for other cases to account for decreased $\Delta \mathrm{Ts}$.

\subsubsection{Pumps and Compressors}

Compressor capital costs are assumed to be $\$ 100 / \mathrm{kW}$. A complete discussion of the details of compressor costing is given in Appendix 4, "Analysis of Transmission Costs." Pump costs are taken to be $\$ 600 / \mathrm{kW}$. The capital cost of the compressors and pumps amounts to $\$ 750,000$. It is important to note that the capital cost component for the compressors and pumps contributes only a small fraction of the cost of the system. It is the energy cost component that is most important; here, that is assumed to be $20 \mathrm{mills} / \mathrm{kWh}$.

These economic design parameters yield the following costs for the dehydrogenation portion of the LTCHP:

$\begin{array}{lr} & \frac{106 \$}{7.6} \\ \text { Reactor + catalyst } & 9.5 \\ \text { Process gas heaters } & 37.7 \\ \text { Exchangers } & 0.7 \\ \text { Pump and compressors } & 55.5\end{array}$

\subsubsection{Transportation System}

The cost and design parameters for the transportation system are described in detail in Appendix 4 of this report. For this analysis, the cost of the benzene and cyclohexane inventory in the transmission lines has been included as a portion of the capital cost of the lines. The cost and design parameters for the transmission system are summarized in Table 15-3 for a transmission distance of $160 \mathrm{~km}$.

\subsubsection{Hydrogenation Process}

An approach similar to that used in the dehydrogenation process is used in costing the hydrogenation process. The design 
Table 15-3

COST AND DESIGN PARAMETERS FOR LTCHP

TRANSPORTATION SYSTEM

. Line Diameter

Hydrogen

1.02 meters

Benzene

0.57 meter

Cyclohexane

0.60 meter

Inventories

Benzene

$40.3 \times 10^{6}$ liters (10.5 $10.10^{6}$ gallons)

\footnotetext{
Cyclohexane

$45.7 \times 10^{6} 1$ iters$$
\text { (12.1 } 10^{6} \text { gallons) }
$$

Inventory cost At 75c/gal (Rl5-4)

Benzene

$\$ 8.0 \times 10^{6}$

Cyclohexane

$\$ 9.0 \times 10^{6}$

Total Steel Cost

Hydrogen line $\$ 9.1 \times 10^{6}$

Benzene line $\$ 2.4 \times 10^{6}$

Cyclohexane $\$ 2.8 \times 10^{6}$

line

Total Pipeline Capital Cost

$\$ 50.3 \times 10^{6}$

Including inventory, steel, installation and right of way

Compressor and Pump

Capital Cost $\$ 3.9 \times 10^{6}$

TOTAL CAPITAL COST

$\$ 54.2 \times 10^{6}$

Compressor and Pump Power
Requirements $17.3 \mathrm{MW}_{\mathrm{e}}$

and cost parameters used in this evaluation are listed in Table 15-4. The value of $15,000 \mathrm{Btu} / \mathrm{hr}-\mathrm{ft}^{2}$ used for the heat flux in the heat delivery exchangers is lower than that used in the endothermic reaction to account for the smaller $\Delta \mathrm{Ts}$ expected in this application. The liquid hourly space velocity is again taken as $30 \mathrm{hr}^{-1}$. This is somewhat higher than in the conventional benzene hydrogenation process; however, it would be expected to be, because of the significantly higher temperatures employed in this design as compared with the conventional design ( 589 to $700 \mathrm{~K}$
} versus 400 to $550 \mathrm{~K}$ ). 
Table 15-4

DESIGN AND ECONOMIC PARAMETERS. FOR BENZENE HYDROGENATION PROCESS

$\begin{array}{ll}\text { Heat delivery exchanger heat flux } & 15,000 \mathrm{Btu} / \mathrm{hr}^{-\mathrm{ft}^{2}} \\ \text { Other exchanger minimum } \Delta \mathrm{T} & 20 \mathrm{~K} \\ \text { Exchanger heat transfer coefficient } & 200 \mathrm{Btu} / \mathrm{hr}^{2} \mathrm{ft}^{2} \\ \text { Exchanger cost } & \$ 25 / \mathrm{ft}^{2} \\ \text { Exchanger installation factor } & 3 \mathrm{x} \\ \text { Liquid hourly space velocity } & 30 \mathrm{hr}^{-1} \\ \text { Reactor cost }: & \$ 2 / 1 \mathrm{~b} \\ \text { Reactor installation factor } & 2 \mathrm{X} \\ \text { Catalyst cost } & \$ 10 / 1 \mathrm{~b} \\ \text { Pump cost } & \$ 600 / \mathrm{KW} \\ \text { Compressor cost } & \$ 100 / \mathrm{KW}_{\mathrm{e}}\end{array}$

These cost and design parameters yield the following cost for the hydrogenation portion of the LTCHP:

$\begin{array}{lr} & \frac{10^{6} \$}{6.7} \\ \text { Reactor + catalyst } & 12.4 \\ \text { Process heat exchangers } & 16.7 \\ \text { Process gas coolers } & \frac{0.7}{36.5} \\ \text { Pump and compressors } & \\ \text { Cost of Delivered Heat } & \end{array}$

The total cost of the heat delivered to the user is the sum of the capital related costs, the power cost to supply work to the system, and the cost of heat at the primary source. The capital related costs are determined from the total capital costs derived in the previous section, and from an appropriate capitalization factor which accounts for the depreciation charges, taxes, insurance, operation and maintainence costs. In this study, a capitalization rate of $20 \%$ per year is assumed. The power cost can be determined from the energy balance and the cost of energy. Finally, the heat cost can again be determined from the energy balance and the cost of heat at the primary source.

To separate the costs associated with the LTCHP from the cost of the primary heat source, the heat cost is divided into two components:

- Cost of heat required by the system but not delivered; this heat is required to account for losses due to exchanges with the powerplant and sensible heat losses. 
(Credit is given to work produced by the heat transferred to the powerplant by an appropriate reduction in the work requirements for the LTCHP system).

- Heat absorbed at the primary source and subsequently delivered to the users.

A summary of the component costs and delivered heat cost is given in Table 15-5 for the reference case conditions.

\author{
Table 15-5. \\ YEARLY OPERATING COSTS AND COST OF DELIVERED HEAT \\ FOP REFERENCE CASE CONDITIONS
}

Basis: $8000 \mathrm{hr} / \mathrm{yr}$; Primary Heat Cost at $\$ 2.50 / \mathrm{GJ}$;

Electricity at $20 \mathrm{mills} / \mathrm{kWh}$

\begin{tabular}{|c|c|c|}
\hline Component & Component Value & $\begin{array}{c}\text { Contribution to } \\
\text { Delivered Heat Cost } \\
\left(10^{6} \$ / \mathrm{yr}\right) \\
\end{array}$ \\
\hline Capital & $\$ 146.2 \times 10^{6}$ at $208 / \mathrm{Yr}$ & 29.2 \\
\hline Power & $28.3 \mathrm{MW}$ & 4.5 \\
\hline Undelivered Heat. & $132.8 \mathrm{MW}_{\mathrm{th}}$ & 9.6 \\
\hline Total Incremental Cost & & 43.3 \\
\hline Delivered Heat & $977.4 \mathrm{MW}_{\mathrm{th}}$ & 70.4 \\
\hline $\begin{array}{l}\text { Yearly Cost of } \\
\text { Delivered Heat }\end{array}$ & At $977.4 \mathrm{MW}$ & 113.7 \\
\hline 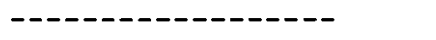 & & \\
\hline $\begin{array}{l}\text { Incremental cost } \\
\text { of LTCHP System }\end{array}$ & $977.4 \mathrm{MW}_{\mathrm{th}}$ & $1.54 / G J$ \\
\hline Cost of Delivered Heat & $977.4 \mathrm{MW}_{\mathrm{th}}$ & $4.04 / \mathrm{GJ}$ \\
\hline
\end{tabular}

The cost of the heat source is taken as $\$ 2.50 / G J$ in this analysis. This cost is uncertain, because of the variety of possible heat sources which might be used. To show the sensitivity of the LTCHP delivered heat cost to this cost of primary heat, the cost of delivered heat is given in Table 15-6 for a wide range of primary heat costs.

Table 15-6

INCREMENTAL LTCHP COST AND TOTAL COST OF DELIVERED HEAT AS A FUNCTION OF PRIMARY HEAT COSTS .

\begin{tabular}{|c|c|c|}
\hline \multirow{2}{*}{$\begin{array}{l}\text { Cost of } \\
\text { Primary Heat } \\
(\$ / G J)\end{array}$} & \multirow{2}{*}{$\begin{array}{l}\text { Incremental } \\
\text { Cost of LTCHP } \\
\text { Heat Transport } \\
(\$ / G J) \\
\end{array}$} & \multirow[b]{2}{*}{$\begin{array}{c}\text { Total Cost } \\
\text { of } \begin{array}{c}\text { Delivered Heat } \\
(\$ / G J)\end{array} \\
\end{array}$} \\
\hline & & \\
\hline $\begin{array}{l}1.0 \\
1.5 \\
2.0 \\
2.5 \\
3.0 \\
3.5 \\
4.0 \\
4.5 \\
5.0\end{array}$ & $\begin{array}{l}1.33 \\
1.40 \\
1.47 \\
1.54 \\
1.60 \\
1.67 \\
1.74 \\
1.81 \\
1.88\end{array}$ & $\begin{array}{l}2.33 \\
2.90 \\
3.47 \\
4.04 \\
4.60 \\
5.17 \\
5.74 \\
6.31 \\
6.88\end{array}$ \\
\hline
\end{tabular}


Important conclusions that can be drawn from this table are:

1. The cost of LTCHP heat transport is of the order of $\$ 1.50 / \mathrm{GJ}$.

2. The LTCHP heat transport is fairly insensitive to primary heat cost.

In addition it should be noted that if an alternate design were to be chosen (increased capital cost to improve overall process efficiency), the undelivered heat requirements would drop and the sensitivity of the cost of LTCHP heat transmission to the cost of heat from the primary source would decrease. This tradeoff between capital cost and efficiency is a traditional problem for design engineers. That is, with additional capital equipment (e.g., additional heat exchange area) irreversibilities can be decreased and the efficiency of the process can be increased.

In this LTCHP design, heat exchange $\Delta$ Ts are arbitrarily set at $20 \mathrm{~K}$. A variation in the exchanger areas to an optimum for each exchanger would decrease the total processing cost. No heat exchanger optimization is attempted in these designs, but it would be advantageous in future work. The same can be said for the reactor configurations. Adiabatic fixed bed reactors are assumed in this design; however, thermodynamic analyses show that this type of reactor contributes significantly to the irreversibility of the process. Further work in these areas would result in efficiency and cost benefits for the LTCHP system.

\subsection{BASE CASE ECONOMICS}

\subsubsection{Introduction}

A similar economic analysis can be performed on the base cases discussed in the previous chapter. In general, all cost and process design parameters remain the same. An exception is the heat flux used in the process gas heaters in the dehydrogenation process. Since these base cases explore reaction conditions at lower temperatures than the reference case, the heat fluxes in these cases are lowered to comply with the expected temperature differences between the heating fluids and the process gases. Again, no optimization is attempted because of the preliminary nature of these designs, but it should be recognized that an optimum reaction temperature (and heat flux) exists for each heat source.

Another exception to the reference case economic parameters in these analyses is the variation in cost of the primary heat, to reflect the thermodynamic value of heat at the various temperature levels. Heat at $1000^{\circ} \mathrm{F}(811 \mathrm{~K})$ (eyergy ratio $\left.=0.63\right)$ * should have a value somewhat greater than, say, light-water reactor heat at $550^{\circ} \mathrm{F}(560 \mathrm{~K})$ (exergy ratio $\left.=0.47\right)$. As an approximation to reflect this variation in value, $811 \mathrm{~K}$ heat is assumed to be worth

* See Appendix 2 for definition of exergy ratio. 
$\$ 2.50 / \mathrm{GJ}, 560 \mathrm{~K}$ heat is assumed to be worth $\$ 1.50 / \mathrm{GJ}$, and the value of intermediate temperatures is assumed proportional to its exergy ratio. Therefore, $755 \mathrm{~K}$ heat with an exergy ratio of 0.60 is valued at $\$ 2.33 / \mathrm{GJ}$, and $673 \mathrm{~K}$ heat with an exergy ratio of 0.55 is valued at assumed costs will vary in a manner similar to the reference case, the sensitivity increasing with increased inefficiencies of the process.

\subsubsection{Base Case 1 - Economics}

The endothermic reaction conditions for this case are $755 \mathrm{~K}$ and 40 bars; the exothermic reaction conditions are the same as the reference case, $589 \mathrm{~K} / 40$ bars. In this case, the design heat flux in the interheaters of the endothermic reactors is assumed to be $20,000 \mathrm{Btu} / \mathrm{hr}-\mathrm{ft} \mathrm{f}^{2}$, and the cost of primary heat is assumed to be $\$ 2.33 / G J$. Other economic and design parameters are assumed to be the same as those in the reference case (Table 15-1). The capital costs for the three sections of the LTCHP system are summarized in Table 15-7 for these reaction conditions. The work requirements for the design amount to $34.1 \mathrm{MW}$ and the undelivered heat totals $145.9 \mathrm{MW}_{t h}$. Following the procedure used in the economic analysis of the reference case, the yearly totals for these various components of the operating cost are summarized in Table 15-8.

Table 15-7

CAPITAL COST SUMMARY FOR BASE CASE 1

Reaction Conditions: $755 \mathrm{~K} / 40$ Bars

\begin{tabular}{|c|c|c|}
\hline Process & Equipment & Cost $110^{6}$ \\
\hline \multirow[t]{2}{*}{ Dehydrogenation } & $\begin{array}{l}\text { Reactors + catalyst } \\
\text { Process gas heaters } \\
\text { Heat exchangers } \\
\text { Pumps and compressors }\end{array}$ & $\begin{array}{r}7.6 \\
14.2 \\
34.6 \\
0.7 \\
\end{array}$ \\
\hline & Total & 57.1 \\
\hline \multirow[t]{2}{*}{$\begin{array}{c}\text { Transportation } \\
(160 \mathrm{~km})\end{array}$} & $\begin{array}{l}\text { Transmission line } \\
\text { Pump and compressors }\end{array}$ & $\begin{array}{r}52.4 \\
4.1 \\
\end{array}$ \\
\hline & Total & 56.5 \\
\hline \multirow[t]{3}{*}{ Hydrogenation } & $\begin{array}{l}\text { Reactors + catalyst } \\
\text { Process gas coolers } \\
\text { Heat exchangers } \\
\text { Pumps and compressors }\end{array}$ & $\begin{array}{r}6.7 \\
16.5 \\
13.5 \\
0.7 \\
\end{array}$ \\
\hline & Total & 38.4 \\
\hline & TOTAL & 152.0 \\
\hline
\end{tabular}


Table 15-8

YEARLY OPERATING COSTS AND COST OF DELIVERED HEAT: FOR BASE CASE \#l - $755 \mathrm{~K} / 40$ BARS

Basis: $8000 \mathrm{hrs} / \mathrm{yr}$; Primary Heat at $\$ 2.33 / \mathrm{GJ}$;

Electricity at $20 \mathrm{mills} / \mathrm{kWh}$

Contribution to

Component

Capital

Power

Undelivered Heat

Total Incremental Cost

Delivered Heat

Yearly Cost of Delivered Heat

$$
\begin{gathered}
\text { Component Value } \\
\$ 152.0 \times 10^{6} \\
\text { at } 20 \% / \mathrm{yr}
\end{gathered}
$$

$$
\begin{gathered}
34.1 \mathrm{MW}_{\mathrm{e}} \\
145.9 \mathrm{MW}_{\mathrm{th}}
\end{gathered}
$$$$
964.7 \mathrm{MW}_{\text {th }}
$$

$$
\begin{gathered}
964.7 \mathrm{MW}_{\mathrm{th}} \\
\left(2.8 \times 10^{7} \mathrm{GJ} / \mathrm{Yr}\right) \\
964.7 \mathrm{MW}_{\mathrm{th}}
\end{gathered}
$$

Incremental cost of LTCHP System

Cost of Delivered Heat
Delivered Heat cost $\left(10^{6} \mathrm{~s} / \mathrm{yr}\right)$ 30.4

5.6

9.8 45.8

64.7 110.4 $\$ 1.65 / \mathrm{GJ}$ $\$ 3.98 / \mathrm{GJ}$

The fact that the delivered cost of heat is less for this case than for the reference case is a direct result of the assumption of lower primary heat cost used in this analysis. At $\$ 2.50 / G J$ primary heat cost (as used in the reference case), the incremental LTCHP cost and cost of delivered heat become $\$ 1.67 / \mathrm{GJ}$ and $\$ 4.77 / \mathrm{GJ}$ respectively, as compared with $\$ 1.54 / G J$ and $\$ 4.04 / G J$ in the reference case.

These results demonstrate that the lower conversion obtained at $755 \mathrm{~K} / 40$ bars (as compared with the reference case) affects the economics by increasing the capital costs of the heat eychangers and transportation system and the operating costs by increasing the work and the amount of undelivered heat required by the process. This effect is not dramatic under the reaction conditions assumed in this case, since the decrease in cyclohexane conversion with temperature is relatively small between the reference case temperature, $811 \mathrm{~K}$, and the temperature used in this case, $755 \mathrm{~K}$ (see Figure 13-5).

\subsubsection{Base Case 2 - Economics}

The endothermic reaction conditions for this case are $673 \mathrm{~K}$ and 40 bars; the exothermic reaction conditions remain at $589 \mathrm{~K}$ and 40 bars. In this case, the heat flux in the process gas heaters in the endothermic process is assumed to be $15,000 \mathrm{Btu} / \mathrm{hr}-\mathrm{ft} 2$; and the cost of the primary heat is assumed to be $\$ 2.04 / \mathrm{GJ}$. The other 
economic and design parameters are assumed to be the same as the reference case (Table 15-1). Capital costs for this design are summarized in Table 15-9. Power requirements total $99.6 \mathrm{MWe}_{\mathrm{e}}$ and the undelivered heat amounts to $226.7 \mathrm{MW}_{\mathrm{th}}$. The yearly totals for the components of the operating costs are summarized in Table 15-10.

Table 15-9

CAPITAL COST SUMMARY FOR BASE CASE 2 Reaction Conditions: $673 \mathrm{~K} / 40$ Bars

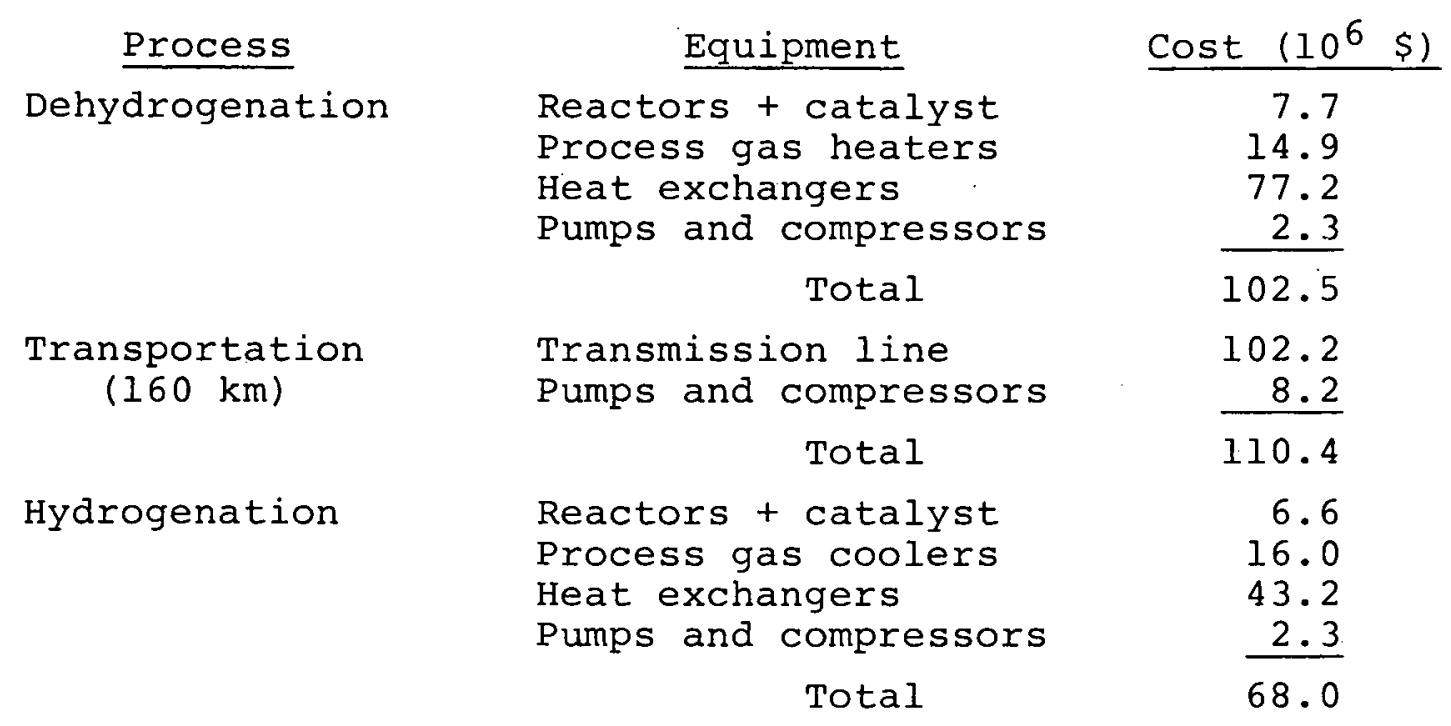

Table 15-10

YEARLY OPERATING COSTS AND COST OF DELIVERED HEAT FOR BASE CASE 2 - $673 \mathrm{~K} / 40$ BARS

Basis: $8000 \mathrm{hr} / \mathrm{yr}$; Primary Heat at $\$ 2.04 / \mathrm{GJ}$; Electricity at. $20 \mathrm{mills} / \mathrm{kWh}$

Component

Capital

Power

Undelivered Heat

Total Incremental Cost

Delivered Heat

Yearly Cost

of Delivered Heat

------------

Incremental cost

of LTCHP System

Cost of Delivered Heat
Component Value

$\$ 280.9 \times 10^{6}$

at $20 \% / \mathrm{yr}$

$99.6 \mathrm{MWe}_{\mathrm{e}}$

$226.7 \mathrm{MW}_{\mathrm{th}}$

$939.2 \mathrm{MW}_{\mathrm{th}}$

$\left(2.7 \times 10^{7} \mathrm{GJ} / \mathrm{yr} .55 .2\right.$

Contribution to Delivered Heat Cost $\left(10^{6} / \mathrm{yr}\right)$

56.2

15.9

13.3

85.4

140.6

$\$ 3.16$

$\$ 5.20$ 
The decrease in conversion in this case due to the lower endothermic reaction temperature results in a significant increase in the cost of delivered heat. The capital cost component increases primarily as a result of increased heat exchanger and pipeline costs. The higher circulation rates require a greater amount of pumping power and result in higher sensible heat losses in the transmission lines. All these factors contribute to the increased LTCHP cost and, hence, a higher delivered heat cost (even though the cost of primary energy is lower). It appears that at a 40 bar reaction pressure, the lower limit to economical LTCHP heat transmission occurs at about 700 to $725 \mathrm{~K}$ endothermic reaction temperature.

\subsubsection{Base Case 3 - Economics}

The endothermic reaction conditions for this case are $540 \mathrm{~K}$ and 1 bar. The exothermic reaction conditions remain at $589 \mathrm{~K}$ and 40 bars. As discussed in Chapter 14 , this case presents an example of increasing the exergy ratio of the heat delivered over that of the primary heat source by compression of the hydrogen and benzene. The heat flux in the process gas heaters is assumed to be $10,000 \mathrm{Btu} / \mathrm{hr}-\mathrm{ft} \mathrm{t}^{2}$ in this case. This value is more in line with the low temperature difference between the process stream and the heat source expected with LWR or, perhaps, solar applications. The primary heat cost is assumed to be $\$ 1.50 / \mathrm{GJ}$, reflecting the lower value of the heat at this temperature.

The capital costs for this case are summarized in Table 15-11 for a transmission distance of $160 \mathrm{~km}$ (100 miles). Power requirements total $287.8 \mathrm{MW}_{e}$, including the hydrogen compression power. The undelivered heat amounts to $71.9 \mathrm{MW}_{\mathrm{th}}$. The contributions of each of these factors to the cost of delivered heat are shown in Table 15-12.

The results of this economic evaluation show that although the incremental cost of the LTCHP system is greater than for previous cases, the final cost of delivered heat is less than for the previous case. In addition, the LTCHP system is used not only to transport the heat but also to upgrade the quality of the heat. This upgrading is reflected by the fact that the heat is absorbed by the system at $540 \mathrm{~K}$ but delivered by the system at temperatures in excess of $589 \mathrm{~K}$.

\subsection{ECONOMICS OF BYPRODUCT FORMATION}

The economics associated with byproduct formation in the benzene/cyclohexane system will be discussed in this section. Several approaches might be used to treat the byproducts. For an analysis of these various options, it will be assumed that cyclohexane and benzene are valued at $75 \% / g a l l o n$, and hydrogen and SNG at $\$ 5 /$ MMBtu. 
Table 15-11

CAPITAL COST SUMMARY FOR BASE CASE 3

Reaction Conditions: $540 \mathrm{~K} / 1$ bar

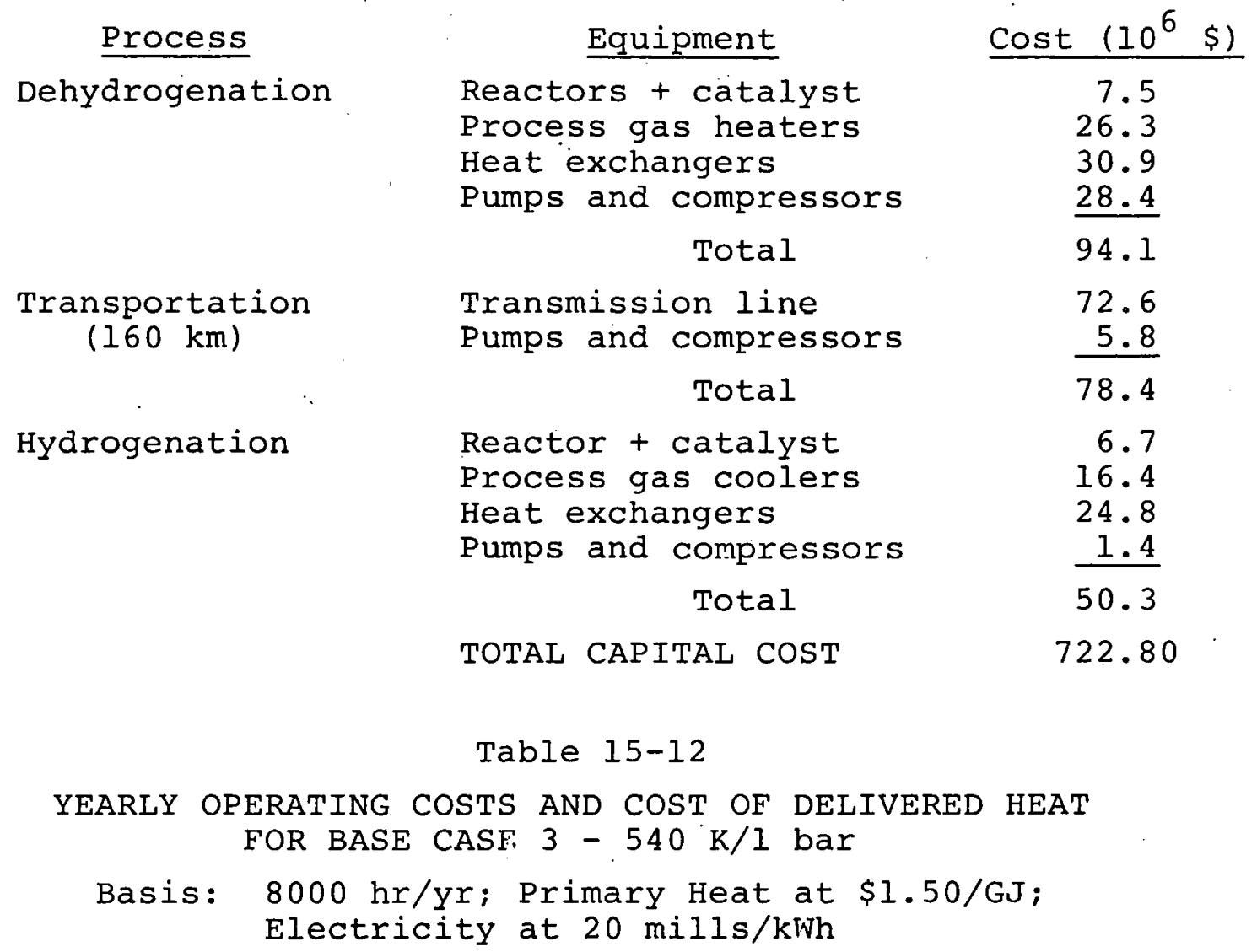

Contribution to

Component

Capital

Power

Undelivered Heat

Total Incremental cost

Delivered Heat

Yearly Cost

of Delivered Heat

Incremental cost

of LTCHP system

Cost of Delivered Heat
Delivered Heat Cost

Component value

$\$ 222.8$ at $20 \% / \mathrm{yr}$

$\left(10^{6} \$ / y r\right)$

44.6

$287.8 \mathrm{MW}_{\mathrm{e}}$

46.0

$71.9 \mathrm{MW}_{\mathrm{th}}$

3.1

93.7

$960.1 \mathrm{MW}_{\mathrm{th}}$

$2.8 \times 107^{\mathrm{GJ}} / \mathrm{Yr}$

41.5

$960.1 \mathrm{MW}_{\text {th }}$

135.2

$\$ 3.39 / \mathrm{GJ}$

$\$ 4.89 / \mathrm{GJ}$ 
A conservative approach would be to take no credit for any byproducts; then the cost to the system would be the cost of makeup cyclohexane. At $0.1 \%$ loss of the cyclic compounds per cycle for the reference case conditions, the makeup chemicals would cost about $\$ 0.16 / G J$ transported. However, a much better use of the byproducts would be to sell them as SNG. Under these circumstances, the cost to the process would be only about $\$ 0.03 /$ GJ transported. Based on these rough figures, it appears that up to $1 \%$ loss/cycle would be tolerated without a large effect on the system economics.

Other solutions to the problem of byproduct formation could also be proposed. For example, the byproducts could be fed to a naphtha reforming unit along with the naphtha feed. The products from this unit could then be hydrodealkylated to yield benzene and methane. The net effect of this processing would be to convert the byproducts and naphtha into the desired.replacement benzene and SNG. The economics of this type of solution are as yet undetermined but should be even more reasonable than the direct purchase of makeup chemicals.

Another important aspect of byproduct economics is the cost associated with separating them from the main constituents of the LTCHP system. Again, several options exist for this treatment. Gaseous cracking products could most easily be separated following the hydrogenation reaction, where they would appear in the highest concentration. This could be accomplished by drawing a drag stream at the vapor-liquid separator with little capital investment and only a small loss of hydrogen. Liquid byproducts would undoubtedly be allowed to build up to a certain extent in the system, perhaps to a steady-state value. Depending on their nature, they could be treated in a variety of ways, most probably at the dehydrogenation site, where the treatment could be centralized. However, at this point in the LTCHP development, when types and amounts of byproducts have not been identified, it is impossible to detail the type of treatment needed or the cost of that treatment.

\subsection{SUMMARY}

In this chapter, preliminary estimates for LTCHP process: economics have been given. The major conclusions from this study are:

- For reference case conditions, the incremental cost of transporting heat via the LTCHP is approximately $\$ 1.50 /$ GJ.

- This transportation cost is fairly insensitive to primary heat costs, although transportation costs for designs requiring large amounts of heat which is not ultimately delivered will be more sensitive to primary heat cost. 
- The transport of heat using high-pressure (4.0 bars) reaction conditions becomes economically less attractive at endothermic reaction temperatures below $700 \mathrm{~K}$.

- The LTCHP system can transport and upgrade low-quality heat $(540 \mathrm{~K})$ at a reasonable price.

- Byproduct formation can be tolerated up to about $1 \%$ per cycle without the cost of the LTCHP transmission becoming unreasonable.

It should be realized that this economic analysis has been performed on a simple design that has not been optimized. Every attempt was made to account for equipment and other factors that might contribute to the cost under the conditions of no byproduct formation. As work progresses on this concept, more detailed designs may be undertaken to update these costs.

\section{REFERENCES -- CHAPTER 15}

R15-1 Private communication, UOP Process Division, Des Plaines, Illinois.

R15-2 Perry, R.H., ed., Chemical Engineers' Handbook, 4th ed., McGraw-Hill, New York, 1963, 24-11.

R15-3 Kirk-Othmer, Encyclopedia of Chemical Technology, 2d ed., Wiley - Interscience.

R15-4 Anon., Chemical Marketing Reporter, Schnell Publishing Company, December 5, 1977. 


\section{Chapter 16}

\section{SOLAR APPLICATIONS OF THE LOW TEMPERATURE CHEMICAL HEAT PIPE}

\subsection{INTRODUCTION}

In this chapter, the application of the low temperature chemical heat pipe (LTCHP) to the distribution of solar thermal energy will be investigated. Three major topics will be discussed: the problems associated with the solar collector/LTCHP interface will be reviewed (section 16.2); then the economics for solar heat transport will be evaluated (section 16.3); and finally, criteria will be developed for judging when the use of a solar/LTCHP system could be economically justified (section 16.4). The major conclusions from this analysis will be presented in section 16.5 .

\subsection{THE SOLAR/LTCHP INTERFACE}

The analyses performed in the first phase of this work (Chapters 1 through 10) have shown that there are three major problems associated with the HTCHP/solar system.

- The required reaction temperature for the methane reforming reaction is higher than existing solar collector designs can achieve (Chapters 4 and 6 ).

- The problem of high temperature cycling in a solarbased HTCHP endothermic reactor presents major materials problems for the reactor and heat exchangers, and for the catalysts and refractory materials.

- The costly endothermic reactor contributes significantly to the total cost of transporting or storing on site the collected solar heat.

The lower temperature operation of the LTCHP lessens to some degree the severity of each of these problems. First, the thermodynamic analysis for the cyclohexane dehydrogenation reaction (Chapter 13) shows that this reaction is almost complete at $811 \mathrm{~K}$ (10000F) even at a pressure of 68 bars. Therefore, there should be no problem in obtaining high conversions in a solar-driven ITCHP, and the equipment sizing should be represented by the reference case design outlined in Chapter 15.

The problems of high temperature reactor cycling are threefold. First, the design of the reactor and heat exchangers must be based on an allowable creep rate rather than on the hoop stress. This results in thick walls with large material usage. The lower temperature of the LTCHP allows thinner walled exchangers and less exotic materials to be used, both resulting in a less expensive system. In addition, any thermal cycles which the system might encounter would cause less severe problems at the lower temperatures of the LTCHP. The expansion and contraction of the metals should be less; hence, the problems of maintaining mechanical integrity of welds should be lessened. 
Second, the thermal expansion of the catalyst tubes or bed allows the catalyst to settle, and as the reactor is cooled the contraction of the containing vessel or tubes tends to crush the catalyst pellets into smaller particles. These small particles tend to pack into the interstitial regions in the bed, causing a greater pressure drop in the gases flowing through the bed and requiring more compression work. Since the thermal expansion should be less in the ITCHP system, the catalyst integrity should be maintained to a greater degree during any large thermal cycles encountered in operation.

Third, the refractory material used to line high-temperature adiabatic reactors cannot withstand sudden changes in temperature; cracking will occur. This problem will not be alleviated even at LTCHP temperatures, and care must be taken to avoid sudden upsets in process stream temperatures in both cases.

The other major problem in solar applications associated with high-temperature operation is the high cost of the endothermic reactor and associated heat exchangers. This cost must essentially be tripled to account for the periodicity of the solar heat source if chemical reactions are to be employed as a means of on-site storage or of transportation of solar thermal energy. The analysis in Chapter 15 has shown that the endothermic reactors for the LTCHP reactions can be expected to be significantly less expensive than those for the HTCHP, and the total of the reactor and heat exchange cost is about 15 to $20 \%$ less in the LTCHP than the HTCHP. This is the direction in which the economics must be shifted if chemical reactions are to be used in conjunction with solar energy. These quantitative results of the economics of solar energy applications will be explored further in the following section and the effect of this lower endothermic reactor cost (and the effect of thermal efficiency) will be explored in detail.

To summarize the important points from this discussion, it can be concluded that the LTCHP can have significant benefits as opposed to the HTCHP operation of the chemical process to transport energy, and the potential cost savings of the endothermic reaction process offer an incentive for further investigation.

\subsection{ECONOMIC ANALYSIS OF SOLAR THERMAL ENERGY TRANSPORT VIA LTCHP}

In this section, an economic analysis of the potential coupling of the LTCHP to a solar thermal source will be undertaken. For this analysis, it will be assumed that the heat is available 8 hours per day; therefore, the endothermic reactor must be oversized by a factor of 3 as compared with the baseload case. In addition, liquid storage must be included for 16 hours of output and input of the hydrocarbon liquids. The pipelines and the hydrogenation reactors are designed on a baseload basis, assuming that the hydrogen pipeline can absorb capacity fluctuations. A summary of the cost analysis for a solar thermal energy cost of $\$ 6 / G J(R I 6-1)$ is given in Table 16-1. 
Table 16-1

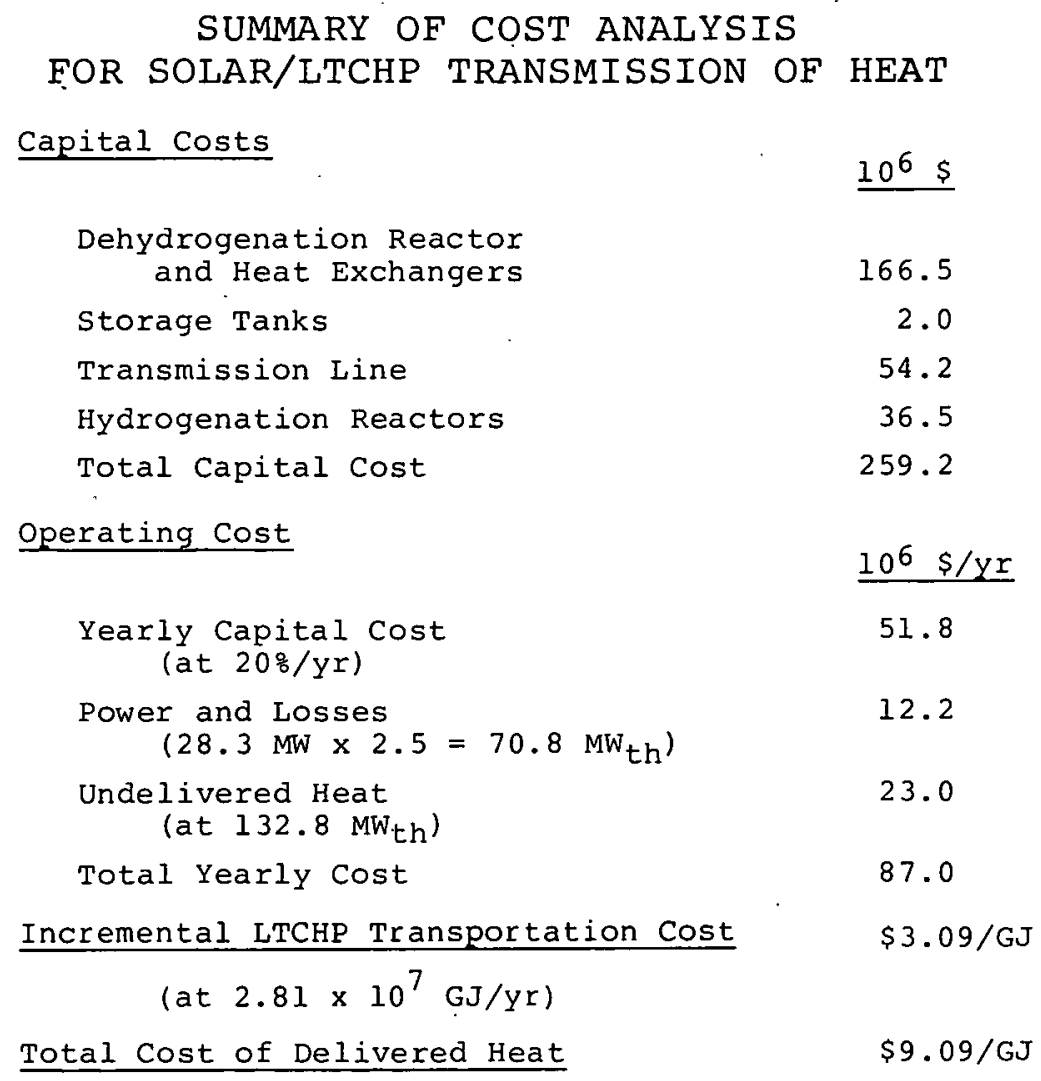

The cost of the major components are calculated as in chapter 15. The cost of the additional storage tanks is taken from Reference Rl6-2 and updated to 1976 costs (Rl6-3). The transportation and delivered heat costs calculated in a similar manner for a wide range of solar heat costs are presented graphically in Figure $16-1$.

This analysis demonstrates several important aspects of the solar design of the ITCHP. First, the smaller capital investment required for the endothermic process does lower the cost of delivered heat. This is best demonstrated in a hypothetical situation where the solar energy is supplied at no cost. In the LTCHP design, the capital charges result in a transportation expense of $\$ 1.84 / G J$ as compared with $\$ 2.03 /$ GJ with the more capital-intensive HTCHP (see Chapter 7). However, since solar energy will not be supplied at no cost, additional energy costs must be included in the cost of transporting the energy.

There are two major contributors to the energy requirements: the power required for the compressors and pump and to account for the energy losses in the associated powerplant, and the heat absorbed by the process but lost as a result of inefficiencies in the system. The power requirements are less for the LTCHP as compared with the HTCHP, but the undelivered energy is significantly increased because of the lower efficiency of the LTCHP system. 


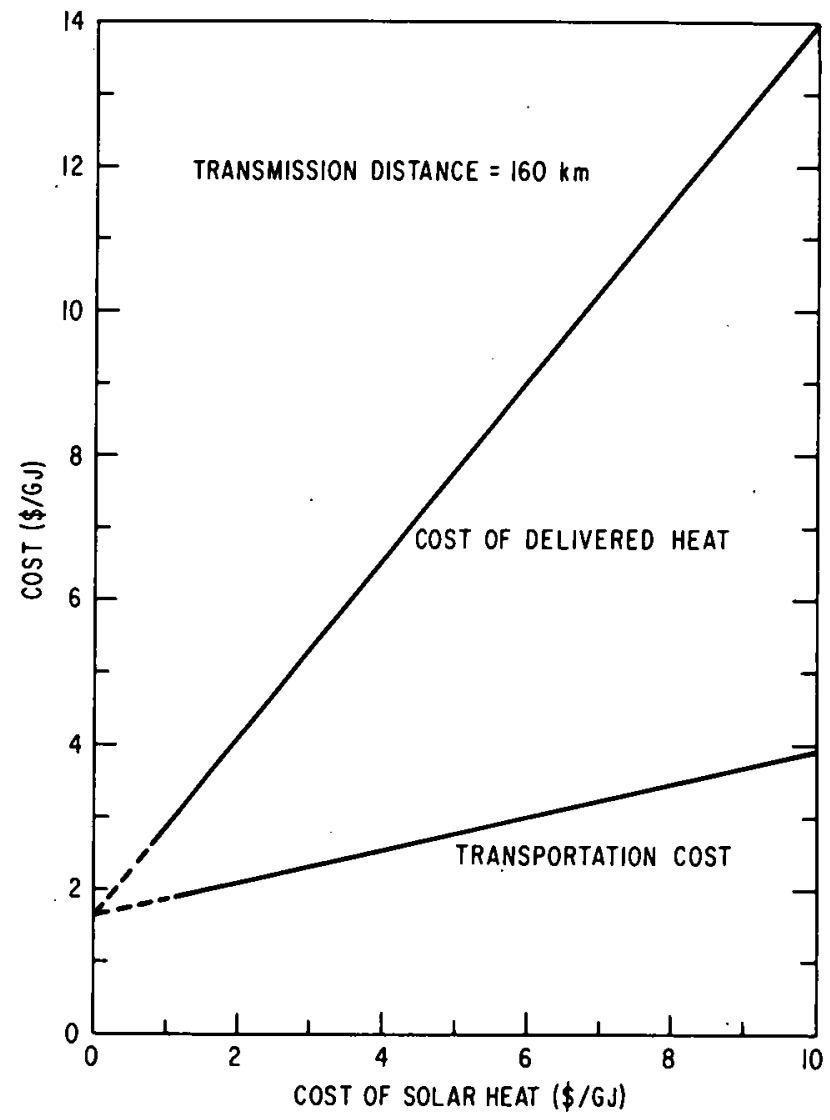

Figure 16-1. Cost of ITCHP Transportation and Cost of Delivered Heat as a Function of the cost of Solar Heat

Two major points need to be addressed. The first is the problem of increasing the efficiency of the chemical reactors without significantly increasing their cost. This is even more important when the cost of the primary energy source is high, as in the demonstration case analyzed in detail here. The second major point is the tradeoff between the optimum distribution of capital and energy costs in the design. No attempt was made in this preliminary analysis to optimize the design at various primary energy costs. As more becomes known about the reaction systems and their specific applications, a reevaluation of the process design must take place in order to determine the most economical design for the given conditions.

\subsection{ECONOMIC JUSTIFICATION OF LTCHP/SOLAR APPLICATIONS}

In the light of the previous economic analysis it is pertinent to investigate in some detail the economic situation that would justify the use of a solar/LTCHP energy transport system. Two important conclusions from previous analyses (both here and in Chapter 7) should be restated. 
- The use of these chemical reaction systems will add a cost of at least $\$ 2 / G J \cdot$ to the cost of the primary energy.

- The cost of distributing the energy over distançes up to $160 \mathrm{~km}$ is only slightly greater than simple on-site storage.

- On-site storage costs are greater for the LTCHP system than for other thermal energy systems (see section 7.5.4)

The fact that a significant incremental cost must be added to the primary heat cost if these systems are used means that the economy of scale for the solar thermal collection must be well understood before transport through the CHP can be envisioned. That is, if a solar system (including storage) can be constructed close to a potential user at essentially the same cost per unit of heat on a smaller scale than the $1000 \mathrm{MW}_{\text {th }}$ size used here, then there is little incentive to build a central system with LTCHP transportation. Under these conditions, the best approach would be to build a number of smaller "backyard" collectors and generate process steam which could be shipped a short distance to the user to supply heat and electricity.

However, if small-scale solar collectors are uneconomical, then the best approach is that of collecting the energy, transporting it via the LTCHP to a user, and there generating both electricity and process steam. The advantages of this system are shown quantitatively in the following example.

Assume, first, that solar heat is available at $\$ 6 / G J$ and that it can be converted to electricity costing $70 \mathrm{mills} / \mathrm{kWh}(\$ 19.44 / \mathrm{GJ})$. At a $36 \%$ overall system efficiency* to sell 1 GJ of electricity, $2.78 \mathrm{GJ}$ of heat must be collected at the solar site. This situation is shown in Figure 16-2. For this case, the gross income from the sale of electricity is $\$ 19.44 / G J$ of energy delivered; however, $1.78 \mathrm{GJ}$ of heat is lost during conversion and transportation. As shown in Figure 16-3, using the same amount of heat absorbed $(2.78 \mathrm{GJ})$, the LTCHP system would tranport $2.28 \mathrm{GJ}$ to a user site at a cost of $\sim \$ 9 / G J$ (see Figure 16-1). Approximately $24 \%$ of this heat could be converted to electricity (see chapter 7) and the remainder would be available at high-grade process steam.

If the utility sold the electricity at the same price as in the preceding case $(70 \mathrm{mills} / \mathrm{kWh})$, it could afford to sell the process steam at $\$ 5.71 / G J$ and still cover the cost of transportation of heat.t In addition, $82 \%$ of the primary heat collected would be used in the ITCHP system, while only $36 \%$ would be utilized in the generation of electricity. Thus, the conservation and prime fuel substitution capabilities of the LTCHP system could be fully realized.

*These numbers are calculated by considering a $40 \%$ conversion efficiency, $10 \%$ losses during transmission, and the added capital costs for generation and transmission.

$+2.28 \mathrm{GJ}$ of heat transported at $\$ 9.00 / \mathrm{GJ}=\$ 20.52$

$0.55 \mathrm{GJ}$ of electricity produced $=\$ 10.64$

Cost of process steam $=\frac{\$(20.52-10.64)}{1.73 \mathrm{GJ}}=\$ 5.71 / \mathrm{GJ}$

$$
16-5
$$




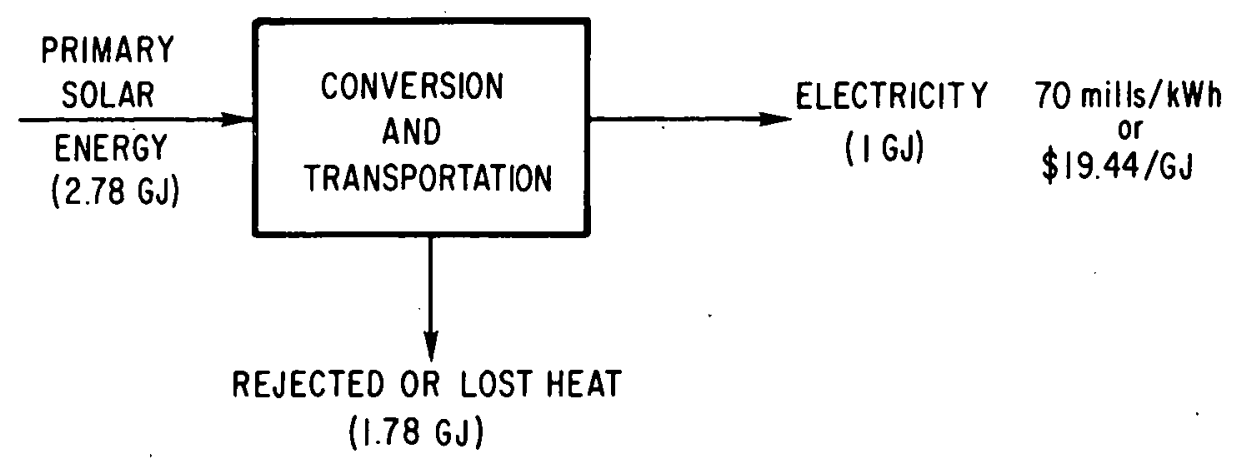

Figure 16-2. Solar Heat Conversion to Electricity

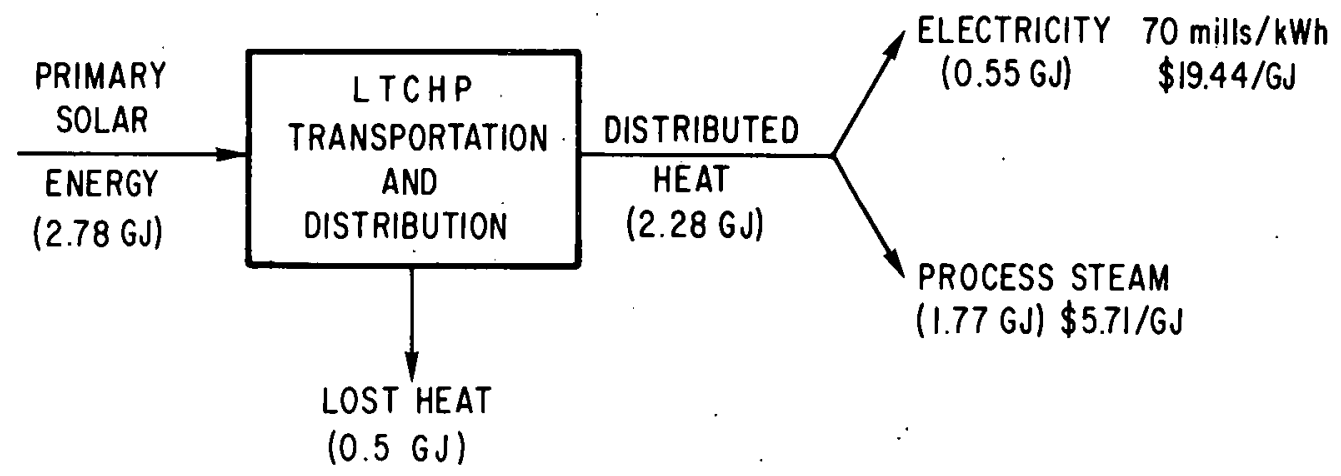

Figure 16-3. Solar Heat Transportation Via LTCHP and Conversion to Electricity and Process Steam

This same type of argument can be used to show that LTCHP cogenerated electricity could be sold at a reduced price (33 mills/ $\mathrm{kWh}$ ) if the economic burden of the heat transport is assumed by the process steam user, or that both forms of energy could be sold below that of their transported cost if cogeneration were not used. No matter what economic division is made, 2.25 times the solar heat is utilized in cogeneration when compared to the generation of electricity.

\subsection{SUMMARY}

An economic analysis of the use of the LTCHP combined with the collection of solar heat has been presented in this chapter. The following major conclusions can be drawn from this analysis:

- The lower temperature operation of the LTCHP offers operational and interfacing advantages over the HTCHP.

- The LTCHP system can transport solar heat to distributed process steam users at approximately $\$ 3.00 / \mathrm{GJ}$ for the transportation.

- The lower endothermic reactor cost results in lower capital charges; however, the lower system efficiency results in higher energy charges which become increasingly significant as the cost of the primary heat increases. 
- Because of this greater dependence on primary heat cost in the LTCHP versus the HTCHP, an economical high-efficiency design is of increased importance.

- The use of the LTCHP system to transport solar heat for use in a cogeneration system is not only more economical than producing only electricity on-site; it also offers a significant added conservation potential by its ability to substitute solar energy for the prime fossil fuels that would otherwise be used at an industrial site.

\section{REFERENCES -- CHAPTER 16}

R16-1 Because of the current status of the developing technology of solar heat collection, the cost of solar thermal energy is still unknown. The value $\$ 6 / \mathrm{GJ}$ was recommended to General Electric by the monitors of this contract at Sandia Laboratories, Livermore, California as a preliminary working number.

Rl6-2 Guthrie, K.A., "Data and Techniques for Preliminary Capital Cost Estimating," Chemical Engineering, Vol. 76, No. 6, 1969 , p. 114 .

Rl6-3 Anon., "Economic Indicators," Chemical Engineering, Vol. 80, No. 23, 1976 . 


\section{Chapter 17}

\section{TECHNICAL AND INSTITUTIONAL BARRIERS TO IMPLEMENTATION OF THE LOW TEMPERATURE CHEMICAL HEAT PIPE}

\subsection{INTRODUCTION}

While many of the technical and institutional barriers discussed in Chapter 9 are common to both the High Temperature Chemical Heat Pipe (HTCHP) and Low Temperature Chemical Heat Pipe (LTCHP), there are significant differences, which vill be enumerated in this chapter.

The technical barriers in the HTCHP center around the fact that, although the chemistry appears feasible, there is no commercial source close to demonstration which would be capable of supplying the high temperature heat required to drive the reactions.

The opposite is true in the LTCHP; several possible sources are currently or will be available, but the chemistry is unproven. The ramifications of this problem will be discussed as they apply to each of the three sections of the system -- the dehydrogenation process, the hydrogenation process, and transportation of the reaction products.

\subsection{DEHYDROGENATION PROCESS BARRIERS}

Over several decades, industry has developed a gasoline reforming process very similar to the cyclohexane dehydrogenation process proposed for the LTCHP system (see Appendix 5). However, the catalyst used in this process is bifunctional and its use would undoubtedly lead to significant unwanted byproduct formation. Therefore, the development of a single functional catalyst tailored specifically to perform the dehydrogenation function stands as a major barrier to the successful implementation of the benzene/ cyclohexane LTCHP. Industrial experience in catalyst development indicates that such a catalyst can be developed (R17-1, R17-2). The degree of selectivity of this catalyst would be a function of the operating conditions in the reactor and, until formulation is completed and the catalyst tested under a variety of possible operating conditions, can only be estimated.

Assuming that a workable catalyst can be formulated, a much more detailed process design and evaluation must be undertaken before the concept can be "proven in." This design should determine in detail the reactor design (based on actual kinetic data) and the effect of various source temperatures on process efficiency. Once these studies are carried out, some type of large-scale demonstration of the technical feasibility must be carried out. 


\subsection{HYDROGENATION PROCESS BARRIERS}

A parallel situation exists in the hydrogenation process, but it appears that the catalyst used in the current industrial benzene hydrogenation process more nearly approaches that required by the LTCHP than does the dehydrogenation catalyst. As discussed. in the HTCHP section of this report, there is a large thermodynamic incentive to recover the hydrogenation heat at as high a temperature as possible. The current design should be modified to high temperature hydrogenation in a manner similar to the high temperature methanation process proposed by R.M. Parsons (Appendix 1, R17-3). Therefore, the same type of catalyst development, process evaluation, and large-scale testing of a final design are major barriers to actual implementation of the benzene hydrogenation process in the ITCHP. As in the HTCHP, installation of a chemical process and electric generating equipment in a process steam user's facilities may offer a significant institutional barrier and a technical barrier, in that operation personnel must be trained.

\subsection{TRANSPORTATION SYSTEM BARRIERS}

Several significant barriers exist for implementing the transportation system for the LTCHP. Not mentioned previously is the fact that both benzene and cyclohexane freeze at relatively high temperatures; benzene at $5.5^{\circ} \mathrm{C}\left(42.0^{\circ} \mathrm{F}\right)$ and cyclohexane at $6.5^{\circ} \mathrm{C}$ $\left(43.8^{\circ} \mathrm{F}\right)$. This obviously limits the transportability of these compounds over extended distances in regions of even mild winters unless lines are buried below the frostline. One solution to this problem would be to add some compound to the system which would act as an anti-freeze agent. Low freezing paraffins, methylcyclopentane (MCP), and methylcyclohexane (MCH) have been suggested. However, both paraffinic compounds and MCP are subject to hydrocracking and might be irreversibly degraded in the LTCHP reactors.

$\mathrm{MCH}$ is more stable to hydrocracking than MCP and would probably undergo significant dehydrogenation to toluene in the endothermic reactor. Since the heat of reaction is similar to the cyclohexane dehydrogenation (see Chapter 3), this reaction would participate in the endothermicity in the dehydrogenation reactor. Presumably the toluene would be reconverted to $\mathrm{MCH}$ in the exothermic reactor and, thus, cycle through the system like benzene. The melting points of both $\mathrm{MCH}\left(-126.6^{\circ} \mathrm{C},-195.8^{\circ} \mathrm{F}\right)$ and toluene $\left(-94.991^{\circ} \mathrm{C},-139.0^{\circ} \mathrm{F}\right)$ are sufficiently low to serve as good antifreeze agents.

A safety problem presented by the LTCHP is the difficulty in transmitting both hydrogen (fire) and benzene (fire and toxicity). The practice of odorizing the gas as is done in the natural gas industry might also be applied in the case of hydrogen in order to detect small leaks. However, current odorizing agents (mercaptans, $\left.\mathrm{H}_{2} \mathrm{~S}\right)$ might act as a catalyst poisons. The problem of benzene toxicity has received much attention during the past year (Rl7-4), and its transport through populated areas will require a thorough evaluation once governmental exposure standards are set. If the LTCHP cannot be implemented because of this constraint, the logical 
modification to the system would be to use substituted compounds (methylcyclohexane/toluene or naphthalene/tetraline/decalin) in the hydrogenation and dehydrogenation reactions.

\subsection{SUMMARY}

The major barriers to LTCHP discussed in this chapter and in conjunction with HTCHP implementation in Chapter 9 can be summarized as follows:

- Proof of the technical feasibility of the dehydrogenation/ hydrogenation chemistry and catalysts at the elevated temperatures required for efficient LTCHP operation

- Completion of a detailed design of the process to give a better indication of process economics, once kinetic, catalyst stability, and operation characteristics are known in more detail.

- Completion of pilot scale testing to prove technical feasibility

- Evaluation of benzene exposure standards in reference to LTCHP applications

- Determination of the structure of and funding for the heat/electric utility which will operate the LTCHP system (see Chapter 9)

\section{REFERENCES -- CHAPTER 17}

R17-1. Private Communication, UOP Inc., Corporate Research and Development, Chicago, I1.

R17-2. Private Communication, Exxon Research and Engineering Company, Linden, NJ.

R17-3. White, Roszkowski, and stanbridge, "The RM Process," Proceedings, 168th National Meeting, American Chemical Society, Division of Fuel Chemistry, Atlantic City, NJ, September 1974 .

R17-4. Science News, 112, October 8, 1977, p 233. 


\section{Chapter 18}

\section{LOW TEMPERATURE CHEMICAL HEAT PIPE SUMMARY, CONCLUSIONS, AND RECOMMENDATIONS FOR FUTURE WORK}

\subsection{INTRODUCTION}

The major aim of this study was to determine whether the Low Temperature Chemical Heat Pipe (LTCHP) offers sufficient economic and energy efficiency potential to justify an experimental program aimed at establishing the technical feasibility of a CHP based on the hydrogenation/dehydrogenation chemistry. The approach adopted was to assume "ideal" catalysts to be available and carry out estimation of energy efficiencies and economics for the system. The overall conclusion reached is that the LTCHP efficiency and economics appear sufficiently attractive to warrant a program to prove its technical feasibility. The catalyst performance is the major hurdle in establishing the technical feasibility; reactor and heat exchanger design and costs are the main uncertainties in the system economics.

In this chapter, the major conclusions regarding catalysts, reactor designs, process efficiency, and system economics will be summarized. These conclusions form the basis of recommendations on the future course of action which are stated in the final section of this chapter.

\subsection{CONCLUSIONS - CATALYSTS}

To provide guidelines and priorities for the catalyst development, the most important issues have been identified for both the hydrogenation and the dehydrogenation catalysts and for the catalysts in general.

\subsubsection{Dehydrogenation Catalysts}

The desirable characteristics for the dehydrogenation catalysts can be summarized as follows:

- A high selectivity for the dehydrogenation reaction at high temperatures, including suppression of isomerization to methylcyclopentane and reactions leading to ring losses.

- Ability to operate at or slightly below a hydrogen/ carbon ratio of $2: 1$ without coke deposition, thus reducing or eliminating a hydrogen recycle and the associated energy penalties.

- Ability to operate at low temperatures $(\sim 520 \mathrm{~K})$ and low pressure (above 1 bar) with fast kinetics. 


\subsubsection{Hydrogenation Catalysts}

The desirable characteristics of the hydrogenation catalyst can be summarized as follows:

- Operation at high temperatures (up to $800 \mathrm{~K}$ ) without sacrificing the selectivity of current lower temperature catalysts.

- Operation in gas phase reactors rather than the current low temperature, liquid phase reactors.

- Operation at a hydrogen/carbon ratio of 2:1 or less without coke deposition, to eliminate hydrogen recycle.

\subsubsection{General}

Any development that reduces pressure drops, resulting in decreased compression energy requirements, without a significant reactor volume penalty should be highly advantageous.

\subsection{CONCLUSIONS - REACTOR DESIGN}

In the course of efficiency and economic analyses of the LTCHP a need was found for a better understanding of reactor design tradeoffs. The question of which generic reactor design (fixed bed, adiabatic internally cooled, or fluidized bed) offers the maximum energy efficiency within economical constraints is crucial to the LTCHP system analysis, and under the charter of this contract only a small number of possible design tradeoffs have been investigated. One thing appears certain: the reactor design for the endothermic reactor for LTCHP will be considerably different from existing. dehydrogenation reformer designs. This is not surprising, since the overall objectives, catalysts, and the heat source are all quite different in the two cases. The same is true for the hydrogenation reactor. These points are best discussed in the context of recommendations for further work, later in this chapter.

\subsection{CONCLUSIONS - PROCESS EFFICIENCY}

Preliminary conclusions are that, given desirable kinetics and a sufficiently high temperature source, the overall energy efficiencies can be as high ( $20 \%)$ as in the HTCHP. As the process peak temperature drops below $7.00 \mathrm{~K}$, losses increase as a result of a tradeoff between low conversion and high capital cost and pumping power. An example of this is the LWR application case. An efficiency penalty must be paid to pump LWR grade heat to higher temperatures that may be acceptable, and the preferred process design for use with low temperature sources, such as the LWR, are highly dependent on end-use application. In view of the availability of such sources, additional tradeoff analyses are highly important.

A final comment regarding process efficiency concerns the use of mixed feed evaporation, described earlier in the HTCHP study. 
If hydrogen recycle is not needed at the endothermic reactor, then there is no need to used mixed evaporation. At the hydrogenation end, the improvement is significant and depends on the relative molar magnitudes of the benzene-rich liquid and the hydrogen.

\subsection{CONCLUSIONS - PROCESS ECONOMICS}

A preliminary estimate of component costs indicates that the overall capital cost is lower than that for HTCHP. In particular, the endothermic reactor costs are much lower than the corresponding reformer costs in HTCHP. The primary reasons for this are a very high reaction rate, the assumption of adiabatic reactor design, and a lower cost of materials of construction (except for the catalysts).

The largest uncertainties are in the endothermic reactor costs, since the optimal design choice is uncertain and the rates of dehydrogenation are known to be much faster than those for other sequential steps (e.g., cyclization) in current industrial practice. This makes it very difficult to predict and cost the reactor volumes. The estimation of hydrogenation reactor costs posed somewhat analogous but less severe problems.

To summarize the economics, the thermal transmission cost of roughly $\$ 1.50 / \mathrm{GJ} / 160 \mathrm{~km}$ is well within the cost targets where LTCHP can compete and win against alternatives for process steam delivery. LTCHP delivery temperatures will be high enough to offer nearly the same cogeneration potential as the HTCHP, but perhaps with a greater penalty in the heat exchangers.

Economics for different sources indicate that coal-based LTCHP would be practical for distributed steam and electric generation, especially if the unit cost of clean coal combustion in units smaller than $200 \mathrm{MJ} / \mathrm{s}$ of steam increases rapidly with a decrease in size.

The economics of solar energy transport are essentially the same for both the LTCHP and the HTCHP (if it proves technically feasible) for the preliminary designs used here. If a more efficient LTCHP design is used without a significant increase in capital costs, the LTCHP would appear more economical due to the lower endothermic process cost. In Solar/CHP applications for storage and transportation are superior to on-site storage applications for the generation of peak electricity.

\subsection{RECOMMENDATIONS FOR FUTURE WORK}

It is recommended that an experimental program for the identification of improved catalysts be undertaken. This program should be aimed toward identifying catalysts with high specificity at elevated temperatures (up to $825 \mathrm{~K}$ ), low coke deposition kinetics, and low temperature activity. These catalysts should also have, if possible, attributes that permit the design of reactors that are highly energy-efficient..

It is also recommended that a simultaneous and closely coupled programs of reactor design analyses be initiated. 
Such an analytical program is of greater importance than was anticipated before this study. It should be aimed at identifying desirable design features for LTCHP applications, should provide technical performance targets for the catalyst program, and should attempt to obtain improved estimates of size, performance, and cost of reactor components, utilizing the results of best catalyst performance.

Several projects of a lower priority are also recommended: A detailed study of process design options and overall economic tradeoffs for the LTCHP system, utilizing the results of the catalyst and reactor design programs.

A better definition of the process steam market (size and temperature characteristics), which will benefit not only the LTCHP application but also any other proposed process steam delivery system.

- A study of institutional issues related to liability and ownership for process steam delivery, cogeneration, and distributed peak electric generation

- A study of LTCHP system safety.

- A technical study of the transportation system for the components of the LTCHP.

Such programs could lead to a practical LTCHP system which could be implemented in the relatively near future. The potential for conservation and premium fuel substitution with such a system justifies the proposed program. 


\title{
Appendix 1
}

\section{A REVIEW OF METHANATION TECHNOLOGY*}

\author{
Section 1 - INTRODUCTION
}

The critical condition of world reserves of petroleum and natural gas has resulted in great interest in the general field of coal conversion technology. In the United States, with the availability of natural gas declining more rapidly than that of petroleum, particular interest has centered on processes for the conversion of plentiful, indigenous coal to a substitute natural gas (SNG). In general, such processes follow a route by which coal is converted by incomplete combustion into a crude syngas, consisting mainly of hydrogen and the carbon oxides, which is then purified and thermally upgraded by methanation to pipeline quality.

Although there are no commercial operations for SNG production from coal, the methanation of small concentrations of carbon oxides in the presence of large excesses of hydrogen has been commercially exploited for at least 20 years. These applications are in purification of refinery hydrogen and in purification of synthesis gas for ammonia production. Current investigations into the reaction of hydrogen with carbon oxides for the production of SNG have concentrated on mixtures rich in carbon oxides. At the request of General Electric Corporate Research and Development, The Ralph M. Parsons Company has prepared this report covering methanation technology for SNG production and the status of the various developmental programs.

The purpose of this review is to present information on methanation in sufficient detail and in such a form that it can be used in evaluation studies by General Electric related to the transport of energy through reversible chemical reactions. One such chemical system is reforming/methanation.

\section{Section 2 - SUMMARY}

Currently, there are a large number of design concepts under development for an SNG-related methanation process. The diversity in concept is due largely to the heat release in methanation rather than to the chemistry of its formation. Approximately $28 \%$ of the heat of combustion of methane is released in its formation from hydrogen and carbon monoxide. This is a substantial quantity of heat, the recovery of which, for useful purposes, serves as a principal evaluation parameter between processes. The success of the recovery operation relates to the heat's potential energy level for further utilization, the efficiency of its recovery, and the cost of its recovery.

In most methanation processes, released heat is absorbed by an adiabatic temperature rise in the process gas itself. The size of the temperature increase is limited by considerations of the thermal stability of reactions

\footnotetext{
*Prepared by The Ralph M. Parsons Company for General Electric Corporate Research and Development, March 7, 1977
} 
such as coke formation, catalyst activity, and catalyst sintering. For control of the temperature rise, one or more of the products of the methanation reactions is recycled or added to the fresh makeup syngas. The dilution of syngas by methane, carbon dioxide, and/or steam causes both a reduction in thermodynamic potential for methane production and an increase in mass with greater potential to absorb sensible heat. In other design concepts, the heat of methanation is absorbed in a boiling liquid through indirect heat exchange or in a direct-contact, inert, liquid-phase medium, both of which approximate isothermal operations.

Although the recovery of energy in the form of heat at a high energy level is a point of comparison between concepts, other considerations may preclude the selection of such preferred operating conditions. The catalyst, for example, would be expected to perform for a reasonable commercial life in spite of deviations in operation from design temperatures, gas compositions, and contaminant levels such as sulfur content. On the basis of pilot plant demonstration work, processes based on the higher design temperatures would be more likely to survive temperature excursions or upsets in gas quality, providing the catalyst were of a suitable nature.

The development and commercial use of methanation for application in hydrogen and syngas purification is based on a low-temperature operation. Such a design temperature was selected in the purification processes because it favors, equilibrium-wise, the production of higher purity products. For SNG production, low temperatures also favor the formation of methane. In this latter application, however, because syngases from the gasification of coal generally contain high percentages of hydrogen and carbon oxides, the calculated temperature rise in the fresh syngas without dilution would exceed $1000^{\circ} \mathrm{F}$. The use of a diluent, such as a recycle product, steam, or carbon dioxide, has already been noted. Such a large overall temperature rise may also be distributed over a series of adiabatic reactors in high-temperature processes, progressively lowering the temperature to a level at which, at final equilibrium, high concentrations of methane are produced. Since at a nominal 400 psia, more than $80 \%$ of the methane could be produced at a temperature in excess of $1000^{\circ} \mathrm{F}$, the exclusive use of operating temperatures below $1000^{\circ} \mathrm{F}$ would not be a design or operating advantage in itself.

The active element is nickel in catalysts being developed for application in SNG/methanation processes. The nickel concentration ranges from approximately $15 \%$ for a high-temperature operation to $60 \%$ for bulk or polishing methanation at low temperatures. Of equal importance to the concentration of nickel is its dispersion on the support and retention thereon when operating near design temperature limits. Nickel crystallites grow in size by element migration, thereby causing a reduction in active surface area. The selection of a thermally stable support is necessary to retain a reasonable percentage of the surface area of the support when exposed to high temperatures.

As indicated, methanation at elevated temperatures requires only a relatively low concentration of nickel. All processes must operate at relatively low temperatures if residual hydrogen and carbon monoxide are to be reduced to extremely low levels; however, all must use an active, high-nickel-content 
catalyst for final methanation in such cases. Because activity depends somewhat upon particle size, catalysts that are used in fluid bed systems or dispersed in a liquid medium would probably exhibit the same activity at a lower nickel content than a pelleted catalyst sized for fixed-bed application.

All catalysts must operate on a coke-free basis to sustain design performance. Since coke formation from syngas and methane is predictable, based upon pressure, temperature, and gas composition, all processes can successfully operate under design conditions selected to avoid coke formation. Sulfur, on the other hand, is only a temporary poison for methanation catalysts, having less effect on catalytic activity at the higher temperatures.

At temperatures above $1200^{\circ} \mathrm{F}$, as much as $5 \mathrm{ppm}$ of sulfur would not have a deleterious effect on performance. At lower temperatures, sulfur would accumulate on the catalyst, causing a progressive and final loss of activity. A catalyst that has lost activity because of sulfur accumulation can be regenerated in situ, provided a temperature of approximately $1400^{\circ} \mathrm{F}$ does not otherwise cause sintering and a subsequent loss of surface area. Under nominal operation conditions, the typical operating life of the state-of-the-art catalyst is approximately 3 to 5 years.

On the basis of a rough comparison of major equipment sizes, higher capital costs are predictable from higher recycle gas rates; costs would be expected to be lowest for a design operating at higher temperatures without recycle. Increased costs with recycle are caused primarily by added heat exchange surface to interchange heat between the methanator feed and product streams. Additional costs are caused by the need for recycle compressors, although this is not a major cost item. The reactor vessels represent the major costs for equipment, and, within the accuracy of available information, all processes would require approximately the same total volume of catalyst.

\section{Section 3 - TECHNICAL DISCUSSION}

\section{CHEMICAL REACTIONS}

Practical experience indicates that catalysts that are active in promoting the methanation reaction are still more active in promoting the water-gas shift reaction. As a result, the sequence of events taking place upon exposing a synthesis gas to a suitable catalyst may be approximated by writing two equations. Water-gas shift equilibration takes place very rapidly according to

$$
\mathrm{CO}+\mathrm{H}_{2} \mathrm{O} \longrightarrow \mathrm{H}_{2}+\mathrm{CO}_{2}
$$

Methane is produced at a slower rate according to

$$
3 \mathrm{H}_{2}+\mathrm{CO} \longrightarrow \mathrm{CH}_{4}+\mathrm{H}_{2} \mathrm{O}
$$

As carbon monoxide is depleted from the system by reaction (2), the reverse of reaction (1) takes place until both hydrogen and carbon monoxide are reduced to levels corresponding to a state of chemical equilibrium for both reactions. 
While Equations (1) and (2) are useful in gaining some insight into the mechanism of methanation, they display little utility, as is, in examining stoichiometry. Linear combinations of Equations (1) and (2) give rise to equations more descriptive of the stoichiometry. For example, by combining Equations (1) and (2) in equal proportions, there results

$$
2 \mathrm{H}_{2}+2 \mathrm{CO} \longrightarrow \mathrm{CH}_{4}+\mathrm{CO}_{2}
$$

which is representative of the stoichiometry of the methanation of the type of synthesis gas normally encountered from coal gasification; namely, that in which the ratio of hydrogen to carbon monoxide is about $1: 1$. Deducting Equation (1) from Equation (2) results in

$$
4 \mathrm{H}_{2}+\mathrm{CO}_{2} \longrightarrow \mathrm{CH}_{4}+2 \mathrm{H}_{2} \mathrm{O}
$$

describing the stoichiometry of methanation of a hydrogen-rich synthesis gas. It also demonstrates the need for carbon dioxide, in the absence of carbon monoxide; for complete conversion of the hydrogen. The principal byproduct of this reaction is water vapor. If Equation (1) is weighted by a factor of 3 before addition to Equation (2), there results

$$
4 \mathrm{CO}+2 \mathrm{H}_{2} \mathrm{O} \longrightarrow \mathrm{CH}_{4}+3 \mathrm{CO}_{2}
$$

displaying the stoichiometry of the methanation of a hydrogen-lean synthesis gas. Steam must be added to the system to ensure complete conversion of the carbon monoxide with carbon dioxide being produced as the main byproduct. While Equations (2), (3), (4), and (5) serve to describe the course of the main methanation reaction, they are incomplete, because they do not include the effect of water-gas shifting of residual hydrogen, carbon monoxide; carbon dioxide, and water vapor.

In practice, conversion to methane is somewhat less than complete, being limited by the equilibrium conditions associated with Equations (1) and (2). The choice of Equations (1) and (2) is rather arbitrary. The principle of mathematical dependency, however, can be used again to prove that the final gas composition remains the same regardless of whether Equation (2), (3), (4), or (5) is used in conjunction with Equation (1).

Because the reaction system involves five molecular species combining in two independent reactions, conditions of equilibriums are too complex to be amenable to simple graphical representation. The conditions for chemical equilibrium may be expressed mathematically as follows:

$$
\begin{aligned}
\left(\mathrm{CH}_{4}\right)+(\mathrm{CO})+\left(\mathrm{CO}_{2}\right) & =(\mathrm{C}) \\
2\left(\mathrm{H}_{2}\right)+4\left(\mathrm{CH}_{4}\right)+2\left(\mathrm{H}_{2} \mathrm{O}\right) & =(\mathrm{H}) \\
(\mathrm{CO})+2\left(\mathrm{CO}_{2}\right)+\left(\mathrm{H}_{2} \mathrm{O}\right) & =(\mathrm{O}) \\
\left(\mathrm{H}_{2}\right)+\left(\mathrm{CH}_{4}\right)+(\mathrm{CO})+\left(\mathrm{CO}_{2}\right)+\left(\mathrm{H}_{2} \mathrm{O}\right) & =(\mathrm{Z})
\end{aligned}
$$




$$
\begin{aligned}
& \frac{\left(\mathrm{H}_{2}\right)^{3}(\mathrm{CO}) \mathrm{p}^{2}}{\left(\mathrm{CH}_{4}\right)\left(\mathrm{H}_{2} \mathrm{O}\right)(\mathrm{Z})^{2}}=\mathrm{Kr} \\
& \frac{\left(\mathrm{H}_{2}\right)\left(\mathrm{CO}_{2}\right)}{(\mathrm{CO})\left(\mathrm{H}_{2} \mathrm{O}\right)}=\mathrm{Ks}
\end{aligned}
$$

where $\left(\mathrm{H}_{2}\right)$. moles of hydrogen

$$
\begin{aligned}
& \left(\mathrm{CH}_{4}\right)=\text { moles of methane } \\
& (\mathrm{CO})=\text { moles of carbon monoxide } \\
& \left(\mathrm{CO}_{2}\right)=\text { moles of carbon dioxide } \\
& \left(\mathrm{H}_{2} \mathrm{O}\right)=\text { moles of water vapor } \\
& (\mathrm{C}) \quad=\text { total atoms of carbon } \\
& (\mathrm{H}) \quad=\text { total atoms of hydrogen } \\
& (\mathrm{O}) \quad=\text { total atoms of oxygen } \\
& (\mathrm{Z}) \quad=\text { total moles of gas } \\
& \mathrm{p} \quad=\text { atmospheres } \\
& \mathrm{Kr} \quad=\text { reforming equilibrium constant } \\
& \mathrm{Ks} \quad=\text { water-gas shift equilibrium constant }
\end{aligned}
$$

Equilibrium constants for the reforming and shift reactions, expressed in terms of atmospheres partial pressure, are a function of temperature only and are summarized in Table Al-1.

Table Al-1

EQUILIBRIUM CONSTANTS

\begin{tabular}{|c|l|l|}
\hline${ }^{\circ} \mathrm{F}$ & \multicolumn{1}{|c|}{$\mathrm{Kr}$} & $\mathrm{Ks}$ \\
\hline 500 & $2.178 \times 10^{-9}$ & 72.4 \\
600 & $2.181 \times 10^{-7}$ & 31.41 \\
700 & $1.019 \times 10^{-5}$ & 15.68 \\
800 & $2.649 \times 10^{-4}$ & 9.02 \\
900 & $4.34 \times 10^{-3}$ & 5.61 \\
1000 & $4.92 \times 10^{-2}$ & 3.745 \\
1100 & $4.12 \times 10^{-1}$ & 2.848 \\
1200 & $2.667 \times 10^{0}$ & 1.961 \\
1300 & $1.425 \times 10^{1}$ & 1.510 \\
1400 & $6.34 \times 10^{1}$ & 1.200 \\
1500 & $2.427 \times 10^{2}$ & 0.980 \\
1600 & $8.17 \times 10^{2}$ & 0.818 \\
\hline
\end{tabular}


Of particular interest is the accelerated rate of decrease of the reforming equilibrium constant as the temperature decreases through the range 1100 to $900^{\circ} \mathrm{F}$.

Potential side reactions include the formation of higher homologues of methane as well as low-molecular-weight unsaturates. Thermodynamic studies indicate that the quantities of these products produced at equilibrium are so small that, for all practical purposes, they can be ignored. Such components, however, have been reported in methanation effluent streams under such circumstances that they merit question as to whether their source was the synthesis gas.

Still other side reactions are relevant. Since these are chiefly associated with catalyst deactivation, they will be discussed in the section of the report dealing with this subject.

\section{$\underline{\text { KINETICS }}$}

Homogeneous gas-phase methanation requires temperatures in the vicinity of $2500^{\circ} \mathrm{F}$ to show any appreciable reaction velocity. At such temperatures, equilibrium concentrations of methane are so low as to render such regions unsuitable for methanation. Therefore, any discussion of the kinetics of methanation resolves into a discussion of the heterogeneous catalysis of the referenced reactions. Undoubtedly, by this time most elements have been' screened for catalytic activity toward methanation. The most promising candidates have been determined to be the metals of Period 4 of Group VIII of the Periodic Table, which includes iron, cobalt, and nickel in ascending order of activity. Still other metals display activity toward methanation reactions. The general consensus, however, is that nickel offers the best compromise between activity and cost.

Many mechanisms have been postulated for the methanation reactions. To date, none of these mechanisms has succeeded in accumulating a sufficient number of adherents to characterize it as representative of an appreciable portion of current thinking. Lacking a mechanism, the kinetics of methanation remain unquantized. In the evaluation of a catalyst, generally a small sample is exposed to a synthesis gas under adiabatic conditions; the temperature rises as methanation takes place and a definite temperature profile is established. At any point within the catalyst bed, the temperature may be used as an indication of the extent of reaction. Figure Al-1 shows typical shapes of such profiles in an adiabatic downflow test reactor. When a fresh catalyst is charged to the test unit, a profile such as that illustrated by curve (1.) develops. As the test continues, the profile gradually shifts to the position indicated by curve (2.). When the profile has shifted to such a position that the temperature rise is no longer equivalent to or greater than the desired conversion, the catalyst charge is no longer serviceable for the duty.

Catalyst life may be extended by charging a greater volume of catalyst to the test unit. In a sense, the lower layers of catalyst act as a reserve for the upper layers. Because the lower layers are exposed to maximum operating temperatures, however, catalyst life is not necessarily inversely proportional to the space velocity. Application of accelerated pilot plant lifetest data to commercial designs requires the use of judgment factors. Such life-test data can be provided by test runs at increased space velocities. 


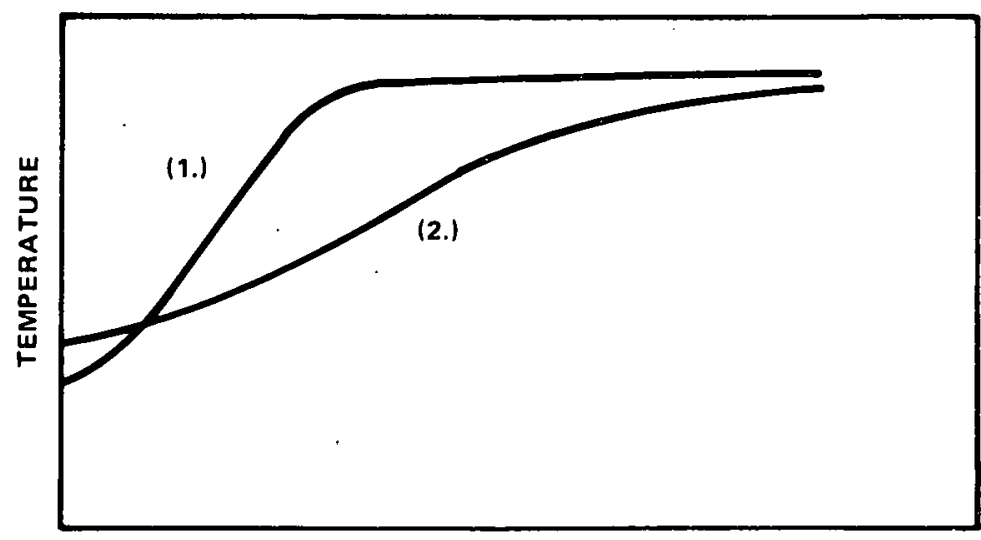

BED DEPTH

Figure Al-1. Catalyst Temperature Profiles

The movement of catalyst bed temperature profiles is generally caused by sintering. At operating temperatures, the catalyst crystallite sizes increase toward a larger equilibrium value accompanied by a decrease in catalyst surface area, which results in reduced activity. As the activity decreases, the rate of change of temperature with bed depth decreases. Also, as the activity decreases, higher inlet temperatures may be required before the reaction initiates.

The concept of space velocity is most useful in applying experimental kinetic data to commercial designs involving heterogeneous catalysis. In its most general sense, the concept introduces the simple consideration that the work accomplished for a unit of catalytic activity in the laboratory can be reproduced in a commercial unit under identical conditions of pressure, temperature, and formulation. The units of work accomplished by the catalyst are expressed as the volume of reactants being processed, while the units of catalytic activity are expressed as the volume of catalyst. The quotient of the former by the latter gives rise to space velocity which is considered a constant for the pressure, temperature, and formulation tested. However, in the application of the concept, room exists for several ambiguities. The first ambiguity rests in the pressure and temperature at which the volume of gaseous reactants is measured. Since a space velocity is specific to a pressure and temperature, and the critical factor is the mass of the reactants, any pressure and temperature may be used to express the volume of gaseous reactants. General practice is to use standard conditions.

A second ambiguity rests in precisely which components of a mixture are to be considered in arriving at the reactants' volumetric rate. With regard to steam, practice is variable, and the basis (wet or dry) must be defined. Since processes which employ steam normally operate over a limited range of steam to dry gas ratios, neither form of the space velocity is to be preferred over the other, providing the basis is properly defined. Other reactant components pose special problems. For example, several of the methanation processes moderate temperature rises by recirculating and blending product with makeup synthesis gas. In some cases, the volume of recycle is sufficient to reduce the carbon oxides concentration of makeup synthesis gas from up to 50 volume percent to about 2 to 3 vol \%. Under these circumstances, it would be unreasonable to expect that a volume of catalyst which would be adequate to methanate the 
undiluted makeup synthesis gas would still be adequate after a 20:1 dilution. For systems of this type, a space velocity defined on the basis of makeup plus recycle gases is more representative of performance.

Earliest versions of bulk methanation catalysts were modifications of the catalysts commercially employed for many years in the purification of refinery hydrogen and ammonia synthesis gas. As a general class, these catalysts are coprecipitates of nickel and alumina with high nickel content. Other ingredients may be incorporated to secure selectivity against carbon deposition. After precipitation, the catalyst is pelleted or extruded, dried, and calcined to convert the ingredients to oxides. The catalyst is activated by reduction. operating temperatures above the range 900 to $1000^{\circ} \mathrm{F}$ in these commercial catalysts cause rapid growth in crystallite size with consequent reduction in surface area and activity.

More recent versions of bulk methanation catalysts resemble the nickel catalysts employed commercially for many years in the steam reforming of light hydrocarbons. Ceramic supports are dipped in solutions of soluble nickel salts, with or without other components to impart selectivity. The catalyst is then dried and calcined to convert the soluble salts to oxides. The catalyst is activated by reduction. Nickel content is relatively low. Crystallite size is relatively coarse but stable, which permits operation at higher temperatures. The improved kinetics at elevated temperatures compensate for the low nickel content and relatively low surface area.

A variety of effects serve to reduce the activity of methanation catalysts. of primary importance, in this respect, is the deposition of carbon. Carbon may be produced by the Boudouard reaction:

$$
2 \mathrm{CO} \rightarrow \mathrm{C}+\mathrm{CO}_{2}
$$

Some divergence of opinion exists as to which of the several other carbon producing reactions may also be operative. Thus, there may be written:

$$
\begin{aligned}
& \mathrm{CO}+\mathrm{H}_{2} \longrightarrow \mathrm{C}+\mathrm{H}_{2} \mathrm{O} \\
& \mathrm{CO}_{2}+2 \mathrm{H}_{2} \longrightarrow \mathrm{C}+2 \mathrm{H}_{2} \mathrm{O} \\
& \mathrm{CH}_{4}+\longrightarrow \mathrm{C}+2 \mathrm{H}_{2}
\end{aligned}
$$

If the reaction system is in a state of water-gas shift equilibrium while at a point of incipient carbon formation, then the principle of mathematical dependency can be used to show that equilibrium conditions for reactions (12), (13), and (14) all must be satisfied. As a result, the question as to which reaction is operative becomes academic. If, in addition, the reaction system is also in a state of equilibrium relative to methane, the equilibrium constant for reaction (15) must also be satisfied. Consequently, it appears that the choice of whether carbon oxide reduction or methane pyrolysis is the causative agent of carbon deposition hinges upon whether methane is present in amounts greater than or less than that corresponding to equilibrium. If the carbon originates from reduction of carbon oxides, increasing pressures and decreasing temperatures favor its production. If the source of the carbon is methane pyrolysis, decreasing pressures and increasing temperatures favor its production. 
For the class of high-temperature methanation catalysts, inactivation by carbon deposition is reversible. Exposure of the coked catalyst to a hydrogen atmosphere at elevated pressure and temperature removes the carbon by the reverse of reaction. (15). For the low-temperature methanation catalysts, however, regeneration temperatures are so high as to promote rapid crystallite growth. Attempts to reactivate low-temperature methanation catalysts serve simply to change the inactivating mechanism from carbon to insufficient surface area.

Iron surfaces exposed to carbon monoxide in the temperature range 300 to $600{ }^{\circ}$ react to produce gaseous iron carbonyl as follows:

$$
\mathrm{Fe}+5 \mathrm{CO} \longrightarrow \mathrm{Fe}(\mathrm{CO})_{5}
$$

When iron carbonyl is a constituent of the synthesis gas entering a methanation reactor, rising temperatures associated with the methanation reaction decompose the carbonyl depositing iron at the catalyst surface. Such iron is highly active in promoting the deposition of carbon. Thus, it can deposit carbon under operating conditions that normally would not produce carbon in the iron's absence. Such transfer of iron can be prevented only by proper equipment design and metallurgy selection upstream of the methanators.

Related to catalyst inactivation by carbon deposition is the inactivation effect wherein nickel carbide is produced. The reaction may be represented as

$$
3 \mathrm{Ni}+2 \mathrm{CO} \longrightarrow \mathrm{Ni}_{3} \mathrm{C}+\mathrm{CO}_{2}
$$

The range of conditions favoring this form of catalyst inactivation over simple carbon deposition has not yet been determined.

Of equal importance to carbon in the deactivation of nickel catalysts is the element sulfur. Trace quantities of sulfur in the synthesis gas react with nickel as follows:

$$
\mathrm{Ni}+\mathrm{H}_{2} \mathrm{~S} \longrightarrow \mathrm{NiS}+\mathrm{H}_{2}
$$

At higher temperatures, higher partial pressures of hydrogen sulfide are required to maintain the nickel in its sulfided form. Consequently, high-temperature methanation catalysts are less sensitive to sulfur. Once sulfided, high-temperature methanation catalysts can be reactivated by exposing them to hydrogen-sulfide-free hydrogen atmospheres at high temperature. Sulfided low-temperature methanation catalysts cannot be regenerated because the required high temperatures would cause sintering.

To retain activity, methanation catalysts must remain reduced. An excessive ratio of oxidants to reductants in the synthesis gas can inactivate nickel catalyst by the following reactions:

$$
\begin{aligned}
& \mathrm{Ni}+\mathrm{H}_{2} \mathrm{O} \longrightarrow \mathrm{NiO}+\mathrm{H}_{2} \\
& \mathrm{Ni}+\mathrm{CO}_{2} \longrightarrow \mathrm{NiO}+\mathrm{CO}
\end{aligned}
$$


When oxidized, the activity of both low- and high-temperature methanation catalysts can be restored in most cases by adjusting the synthesis gas composition back to a reducing condition. When exposed to high partial pressures of steam at temperatures that are high relative to normal operating temperatures, however, there is a partial but permanent loss of catalyst activity when operating at the lower temperatures. This loss occurs even though there is sufficient hydrogen present to ensure a reducing atmosphere.

Finally, methanation catalyst activity can be lost by simple loss of nickel through a chemical process. Nickel reacts with carbon monoxide in the temperature range 300 to $600^{\circ} \mathrm{F}$ to produce gaseous nickel carbonyl as follows:

$$
\mathrm{Ni}+4 \mathrm{CO} \longrightarrow \mathrm{Ni}(\mathrm{CO})_{4}
$$

Increasing temperatures favor the kinetics of the reaction. Increasing temperatures, however, also decrease the amount of nickel carbonyl produced at equilibrium. Within the temperature range 300 to $600^{\circ} \mathrm{F}$, the two effects achieve such a balance that significant quantities of the metal react. The sole means of avoiding the depletion of nickel from methanation catalysts is by minimizing exposure of the catalyst to carbon monoxide at the appropriate temperatures.

While methanation catalyst inactivation mechanisms have been effectively identified, much remains to be done in arriving at quantitative relationships. primarily, the difficulty rests in relating gas phase bulk properties to catalyst surface effects. At present, the safest approach is to reproduce successful pilot plant test conditions in commercial designs.

\section{References}

Bartholomew and Barton, "Methanation Activity of Supported Nickel Alloys," Proceedings, 172nd National Meeting, American Chemical Society, Division of Fuel Chemistry (Symposium on Methanation of Synthesis Gas), San Francisco, California, August 29-September 3, 1976.

Bridger and Woodward, "Formulation and Operation of Methanation Catalysts," Proceedings, 168th National Meeting, American Chemical Society, Division of Fuel Chemistry, Atlantic City, New Jersey, September 1974.

Dalla Betta and Shelef, "Heterogeneous Methanation; Effects of Carbon, Sulfur and Water on Activity Measurements at Various Temperatures," Proceedings, 172nd National Meeting, American Chemical Society, Division of Fuel Chemistry (Symposium on Methanation of Synthesis Gas), San Francisco, California, August 29-September 3, 1976.

Strakey, Forney, and Haynes, "Methanation in Coal Gasification Processes," PERC/IC-75/1, U.S. Energy Research and Development Administration, September 1975 .

Wentrcek, McCarty, Wood, and Wise, "Formation of Surface Carbon and Methanation Catalysis on Alumina Supported Nickel," Proceedings, 172nd National Meeting, American Chemical Society, Division of Fuel Chemistry (Symposium on Methanation of Synthesis Gas), San Francisco, California, August 29-September 3, 1976. 
The heat effects accompanying methanation may be divided into two classes. The first class would include those effects that are a direct result of the thermochemistry of the reactions. The second class, by difference, would include those effects that are introduced primarily by the process configuration. Being related to the process configuration, they will be discussed in a later part of this report.

The standard heats of reaction for reactions (2) through (5) may be derived from heats of formation:

$$
\begin{aligned}
& 3 \mathrm{H}_{2}+\mathrm{CO} \longrightarrow \mathrm{CH}_{4}+\mathrm{H}_{2} \mathrm{O}(1) \\
& 3 \times 0-47549 \quad-32200-122971 \\
& \text { delta } \mathrm{H}=-107622 \mathrm{Btu} / \mathrm{lb} \text { mole } \mathrm{CH}_{4} \\
& \text { release }=283.59 \mathrm{Btu} / \mathrm{SCF} \mathrm{CH}_{4} \\
& 2 \mathrm{H}_{2}+2 \mathrm{CO} \longrightarrow \mathrm{CH}_{4}+\mathrm{CO}_{2} \\
& 2 \times 0 \quad 2 x-47549 \quad-32200 \quad-169294 \\
& \text { delta } \mathrm{H}=-106396 \mathrm{Btu} / \mathrm{lb} \text { mole } \mathrm{CH}_{4} \\
& \text { release }=280.36 \mathrm{Btu} / \mathrm{SCF} \mathrm{CH}_{4} \\
& 4 \mathrm{H}_{2}+\mathrm{CO}_{2} \longrightarrow \mathrm{CH}_{4}+2 \mathrm{H}_{2} \mathrm{O}(1) \\
& 4 \times 0-169294 \quad-32200 \quad 2 x-122971 \\
& \text { delta } \mathrm{H}=-108848 \mathrm{Btu} / \mathrm{lb} \text { mole } \mathrm{CH}_{4} \\
& \text { release }=286.82 \mathrm{Btu} / \mathrm{SCF} \mathrm{CH}_{4} \\
& 4 \mathrm{CO}+2 \mathrm{H}_{2} \mathrm{O}(\mathrm{I}) \longrightarrow \mathrm{CH}_{4}+3 \mathrm{CO}_{2} \\
& 4 x-47549 \quad 2 x-122971 \quad-32200 \quad 3 x-169294 \\
& \text { delta } \mathrm{H}=-103944 \mathrm{Btu} / \mathrm{Ib} \text { mole } \mathrm{CH}_{4} \\
& \text { release }=273.90 \mathrm{Btu} / \mathrm{SCF} \mathrm{CH}_{4}
\end{aligned}
$$

The standard heat of reaction for the water-gas shift reaction is determined in a similar manner:

$$
\mathrm{CO}+\mathrm{H}_{2} \mathrm{O}(\mathrm{l}) \longrightarrow \mathrm{H}_{2}+\mathrm{CO}_{2}
$$




$\begin{array}{llcl}-47549 & -122971 & 0 & -169294 \\ \text { delta } \mathrm{H}= & 1226 \mathrm{Btu} / \mathrm{lb} \text { mole }\end{array}$

From an examination of the standard heats of reaction, it is apparent that the standard heats of reactions (3), (4), and (5) can be derived by the same Iinear combinations as used in deriving the equations.

Based upon gross heating values of $324.04,320.81$, and $1,099.33 \mathrm{Btu} / \mathrm{SCF}$ for hydrogen, carbon monoxide, and methane respectively, process efficiencies, defined as the ratio of the heats of combustion in the product gas to the feed gas (at $100 \%$ conversion) expressed as a percentage, may be calculated and are summarized in Table Al-2. The difference between $100 \%$ and the indicated percentages represents the net heat released in the respective reactions. For convenience, the data have been arranged in increasing order of hydrogen-tocarbon-monoxide ratio.

Table Al-2

PROCESS EFFICIENCIES

\begin{tabular}{|c|c|c|c|}
\hline \multirow{2}{*}{ Reaction } & \multicolumn{3}{|c|}{ Efficiency (\%) } \\
\cline { 2 - 4 } & Product & Release & Total \\
\hline$(5)$. & 78.65 & 21.35 & 100.00 \\
$(3)$. & 78.26 & 21.74 & 100.00 \\
$(2)$. & 78.07 & 21.93 & 100.00 \\
$(4)$. & 77.87 & 22.13 & 100.00 \\
\hline
\end{tabular}

It is apparent that, ignoring process configuration for the time being, very little difference in process efficiency is to be expected as the feed gas changes from a hydrogen-rich to a hydrogen-lean feed gas. Because methane in the feed gas would contribute equally to the numerator and denominator of the efficiency expression, methanation of a methane-rich feed gas would show a higher product efficiency than those indicated in Table Al-2.

The heat of reaction of methanation should vary with the sensible heats of reactants, and with products and composition. Table Al-3 summarizes the effect of temperature and composition parameters upon heat of methanation. 
Table Al-3

HEAT OF METHANATION (Btu)

\begin{tabular}{|c|c|c|c|c|}
\hline \multirow{2}{*}{${ }^{\circ} \mathrm{F}$} & \multicolumn{4}{|c|}{ Reaction } \\
\cline { 2 - 5 } & $(5)$. & $(3)$. & $(2)$. & $(4)$. \\
\hline 600 & 143874 & 110342 & 93576 & 76810 \\
700 & 143843 & 110789 & 94262 & 77735 \\
800 & 143719 & 111155 & 94873 & 78591 \\
900 & 143513 & 111447 & 95414 & 79381 \\
1000 & 143218 & 111666 & 95890 & 80114 \\
1100 & 142892 & 111826 & 96293 & 80760 \\
1200 & 142502 & 111926 & 96638 & 81350 \\
1300 & 142067 & 111977 & 96932 & 81887 \\
1400 & 141584 & 111978 & 97175 & 82372 \\
1500 & 141080 & 111952 & 97388 & 82824 \\
1600 & 140554 & 111888 & 97555 & 83222 \\
\hline
\end{tabular}

Once again, the data are arranged in order of increasing hydrogen to carbon monoxide ratio. At 600 to $1600^{\circ} \mathrm{F}$, the heat of methanation shows almost a twofold variation with hydrogen-to-carbon monoxide ratio. Variation of the heat of reaction with temperature is not as pronounced. It would appear that the. major contribution to the variation in heat of methanation is associated with the latent heat of water and whether water is consumed or produced in the reaction. For all practical purposes, the effect of pressure upon heat of methanation may be ignored.

While the generalizations in the preceding sections are useful, they are incomplete in that they do not include the effect of water-gas shifting of residual hydrogen, carbon monoxide, carbon dioxide, and water vapor. Definitive analysis of methanation heat effect requires that the chemical equilibriums first be established. The overall heat effect is then determined by resolution of the overall reaction according to the specific reactions (2) through (5), described above, to determine the contribution of each. Such resolution, however, is not necessary if enthalpy tables are used that set the enthalpy of each component equal to its heat of formation at the datum. Table Al-4 presents such data for the molecular species involved in methanation. Since these are essentially zero-pressure data, they are not suitable for operations involving large pressure changes. 
Table Al-4

ENTHALPIES (Btu/lb mol)

\begin{tabular}{|c|c|c|c|c|c|c|}
\hline${ }^{\circ} \mathrm{F}$ & $\mathrm{H}_{2}$ & $\mathrm{CH}_{4}$ & CO & $\mathrm{CO}_{2}$ & $\mathrm{H}_{2} \mathrm{O}-\mathrm{v}$ & $\mathrm{H}_{2} \mathrm{O}-1$ \\
\hline $\begin{array}{r}77 \\
100 \\
200 \\
300 \\
400 \\
500 \\
600 \\
700 \\
800 \\
900 \\
1000 \\
1100 \\
1200 \\
1300 \\
1400 \\
1500 \\
1600\end{array}$ & $\begin{array}{r}0 \\
159 \\
853 \\
1550 \\
2248 \\
2947 \\
3646 \\
4348 \\
5051 \\
5757 \\
6464 \\
7174 \\
7887 \\
8605 \\
9327 \\
10054 \\
10786\end{array}$ & $\begin{array}{l}-32200 \\
-32002 \\
-31108 \\
-30140 \\
-29100 \\
-27979 \\
-26779 \\
-25502 \\
-24151 \\
-22729 \\
-21239 \\
-19685 \\
-18069 \\
-16396 \\
-14667 \\
-12901 \\
-11087\end{array}$ & $\begin{array}{l}-47549 \\
-47389 \\
-46691 \\
-45990 \\
-45284 \\
-44571 \\
-43850 \\
-43120 \\
-42379 \\
-41629 \\
-40870 \\
-40100 \\
-39323 \\
-38537 \\
-37743 \\
-36942 \\
-36134\end{array}$ & $\begin{array}{l}-169294 \\
-169088 \\
-168158 \\
-167176 \\
-166148 \\
-165078 \\
-163971 \\
-162831 \\
-161660 \\
-160462 \\
-159239 \\
-157993 \\
-156729 \\
-155445 \\
-154143 \\
-152827 \\
-151497\end{array}$ & $\begin{array}{r}-104047 \\
-103863 \\
-103055 \\
-102237 \\
-101407 \\
-100565 \\
-99709 \\
-98836 \\
-97948 \\
-97043 \\
-96129 \\
-95186 \\
-94231 \\
-93258 \\
-92270 \\
-91267 \\
-90244\end{array}$ & $\begin{array}{l}-122971 \\
-122549 \\
-120673 \\
-118633 \\
-116288 \\
-113427 \\
-109591\end{array}$ \\
\hline
\end{tabular}


Process configurations employed in methanation are unalterably linked to the type of methanation catalyst in service. Since current technology has not yet quantized the kinetics of the methanation reactions, reactor designs are restricted to adiabatic types. Normally, when using a high-temperature methanation process, several adiabatic reactors are employed in series with cooling between stages. High operating temperatures and the highly exothermic nature of methanation result in something less than complete conversion in a single stage; even when introducing the synthesis gas at temperatures as low as permitted by catalyst initiation temperatures. As a consequence, several contacting stages, each having up to approximately $500^{\circ} \mathrm{F}$ rise, are followed by cooling stages to achieve desired conversions. With a methane-lean feed gas, as many as six stages may be required.

Typical low-temperature methanation processes initiate the methanation reactions at about $550^{\circ} \mathrm{F}$. Temperatures above $850^{\circ} \mathrm{F}$ subject a catalyst with activity sufficient for such an operation to increasing growth rates of crystallites. Hence, a temperature rise of approximately $300^{\circ} \mathrm{F}$ is consicered the range within which the system may be allowed to operate. In the absence of cooling devices, heat released in methanation can be controlled within this range only by recirculation of product gas. Some insight may be gained into design considerations of product recirculation of low-temperature methanation processes as follows.

Leading candidates for the gasification step in SNG processes typically produce a synthesis gas wherein approximately half the product methane is produced in the gasification step and the other half is produced in methanation. In the unreacted portion, hydrogen and carbon monoxide are usually present in about equal amounts. Table Al-5 presents a simple mass and energy balance for the isothermal methanation of such a gas at $700 \mathrm{~F}$. The temperature represents the midpoint of typical operating conditions for low-temperature catalysts. Table Al-6 is a summary of heat data for the product gas that is used to determine its heat capacity.

Table Al-5

METHANATION MASS AND ENERGY BALANCE

\begin{tabular}{|c|c|c|c|c|}
\hline Component & $\begin{array}{l}\text { Feed } \\
\text { (mol) }\end{array}$ & $\begin{array}{c}\mathrm{H} \text { a } 700 \\
\mathrm{Btu} / \mathrm{lb} \text { mol }\end{array}$ & $\begin{array}{l}\text { Product } \\
\text { (mol) }\end{array}$ & $\begin{array}{c}\mathrm{H} @ 700 \\
\mathrm{Btu} / 1 \mathrm{~b} \mathrm{~mol}\end{array}$ \\
\hline $\mathrm{H}_{2}$ & 2.0 & 4348 & 0.0 & 4348 \\
\hline $\mathrm{CH}_{4}$ & 1.0 & -25502 & 2.0 & -25502 \\
\hline $\mathrm{CO}$ & 2.0 & -43120 & 0.0 & -43120 \\
\hline $\mathrm{CO}_{2}$ & $\underline{0.0}$ & -162831 & $\underline{1.0}$ & -162831 \\
\hline Total & 5.0 & -103046 & 3.0 & $\begin{array}{c}-213835 \\
-(-103046)\end{array}$ \\
\hline \multicolumn{4}{|l|}{ Delta H, Btu } & -110789 \\
\hline
\end{tabular}


Table Al-6

HEAT DATA

\begin{tabular}{|c|c|c|c|}
\hline Component & $\begin{array}{c}\text { Product } \\
\text { (mol) }\end{array}$ & $\begin{array}{c}\mathrm{H} \text { @ 500 } \\
\text { Btu/lb mol }\end{array}$ & $\begin{array}{c}\text { H @ 900 } \\
\text { Btu/1b mol }\end{array}$ \\
\hline $\mathrm{H}_{2}$ & 0.0 & 2947 & 5757 \\
$\mathrm{CH}_{4}$ & 2.0 & -27979 & -22729 \\
$\mathrm{CO}$ & 0.0 & -44571 & -41629 \\
$\mathrm{CO}_{2}$ & $\underline{1.0}$ & -165078 & -160462 \\
Total & 3.0 & -221036 & -205920 \\
\hline
\end{tabular}

$$
\begin{aligned}
\text { Heat capacity product } & =-205920-(-221036) /(900-500) \\
& =37.79 \mathrm{Btu} /{ }^{\circ} \mathrm{F} \\
& =12.60 \mathrm{Btu} / 1 \mathrm{~b} \operatorname{mol}{ }^{\circ} \mathrm{F}
\end{aligned}
$$

If $R$ represents the volumetric ratio of recycle product to makeup synthesis gas, and if dT represents the temperature rise during methanation, then an energy balance about a reactor with recycle may be expressed as

$$
110789=37.79 \times \mathrm{dT}+12.60 \times 5 \times \mathrm{R} \times \mathrm{dT}
$$

Equation (22) can be rearranged to make it explicit in $R$ :

$$
R=\frac{11078.9-37.79 \mathrm{dT}}{63.0 \mathrm{dT}}
$$

$R$ represents the ratio of recycle gas to fresh makeup syngas required to limit the methanation temperature rise of a typical synthesis gas to dT. Solutions of this equation are presented in Table Al-7. Recycle rates can also be expressed in terms of the percentage of the methanation effluent which is recycled to the reactor (the difference from $100 \%$ is product gas). These percentage figures are also included in Table Al-7.

The implications of the recycle ratios in Table Al-7 are not immediately apparent. Most SNG facilities únder consideration today call for the production of 250 MMSCFD of SNG. If a reactor temperature rise of $300^{\circ} \mathrm{F}$ were permissible, a recirculation of $250 \times 89.8 /(100-89.8)=2,200$ MMSCFD or $1,528,000$ 
Table Al-7

REQUIRED RECYCLE RATES

\begin{tabular}{|c|c|c|}
\hline $\mathrm{dT}$ & $\mathrm{R}$ (Ratio) & $\begin{array}{c}\text { \% Methanator Effluent } \\
\text { Recycled }\end{array}$ \\
\hline 200 & 8.19 & 93.2 \\
300 & 5.26 & 89.8 \\
400 & 3.797 & 86.4 \\
\hline
\end{tabular}

SCFM would be required. The problems introduced with such large flows include problems of heat transfer as well as pressure drop and recycle gas compression.

The recirculation concept selected for temperature control involves compromise between the design features of cold and hot gas recycle. With cold-gas recycle, more heat exchange surface is required; with hot-gas recycle, more horsepower is required for gas circulation. Table Al-8 sumarizes the effect of recycle ratio (R) and cold feed gas temperature (TC) upon preheat duty. The duties reflect the gas analyses and heat data summarized in Tables Al-4, -5 , and -6. The design basis is the production of 250 MMSCFD of SNG from a synthesis gas in which half the product methane is already present. Hydrogen and carbon monoxide appear in equal proportions in the balance of the synthesis gas. The duties entered are preheat duties only. The factor $F$ represents the ratio of preheat duty to process heat release. Therefore, the total cooling load is in proportion to the preheat duty as $(F+1): F$. The heat transfer surfaces quoted are for the preheat duties alone and are based upon a heat transfer coefficient of $50 \mathrm{Btu} / \mathrm{hr} / \mathrm{ft}^{2} / \mathrm{O}_{\mathrm{F}}$ and a mean temperature difference of $100^{\circ} \mathrm{F}$.

The pressure drop and recycle gas compression design considerations may also be discussed by example.

If SCFD represents the volumetric flow rate of reactants, (SV) the space velocity (makeup and recycle), $\mathrm{D}$ and $\mathrm{L}$ the catalyst bed dimensions, and $\mathrm{N}$ the number of reactors in parallel, the volume of catalyst required can be expressed as

$$
\frac{\mathrm{SCFD}}{24(\mathrm{SV})}=\mathrm{N} \times \frac{\pi}{4} \times \mathrm{D}^{2} \times \mathrm{L}
$$

Equation (24) can be rearranged to be explicit in D:

$$
\dot{D}=0.37588^{3} \frac{\mathrm{SCFD}}{\mathrm{N}(\mathrm{SV})(\mathrm{L} / \mathrm{D})}
$$


Manufacturers of catalysts have measured and correlated pressure gradient through packed catalyst beds by

$$
\frac{d P}{d I}=6.58 \times 10^{-10} \frac{G^{1.9} K(1-E) / E^{3}}{e^{D_{P}^{1.1}}}
$$

where

$$
\begin{aligned}
\mathrm{G} & =\text { mass velocity, } \mathrm{lb} / \mathrm{ft}^{2} / \mathrm{hr} \\
\mathrm{K} & =\text { a roughness factor }=\text { about } 1.5 \\
\mathrm{E} & =\text { a porosity factor and }(1-\mathrm{E}) / \mathrm{E}^{3}=\text { about } 12.2 \\
\mathrm{e} & =\text { gas density, } 1 \mathrm{~b} / \mathrm{ft}^{3} \\
\mathrm{D}_{\mathrm{P}} & =\text { particle diameter, in. }
\end{aligned}
$$

Mass velocity is determined by

$$
\mathrm{G}=\frac{(\mathrm{SV}) \mathrm{L}(\mathrm{MW})}{379}
$$

Gas density is a function of molecular weight, temperature, and pressure as follows :

$$
\mathrm{e}=\frac{(\mathrm{MW})}{379} \times \frac{520}{\mathrm{~T}} \times \frac{\mathrm{P}}{14.7}
$$

If Equations (27) and (28) are substituted in Equation (26) and the result multiplied by $L$, an expression for the pressure drop across a catalyst bed is derived. When simplified, the expression resolves into

$$
\Delta \mathrm{P}=1.626 \times 10^{-12} \times \frac{\mathrm{T}(\mathrm{MW})^{0.9}}{\mathrm{P}} \times \frac{(\mathrm{SV})^{1.9}}{\mathrm{D}_{\mathrm{p}}^{1.1}} \times \mathrm{D} \cdot \frac{\mathrm{L}^{2}}{\mathrm{D}}
$$

It is interesting to note that three independent terms result from this arrangement: the first relating to the gas, the second primarily determined by the catalyst, and the third a function of bed geometry. $N$, the number of reactors in parallel, does not appear in this expression because it has been used to size the reactors using total flow and space velocity. Mass velocity enters a calculation through the space velocity and bed geometry. Evaluation of the expression results in the pressure drop across a reactor, which is equivalent to that of each of the other parallel reactors. 
Table Al-8

REACTOR FEED GAS PREHEAT DUTIES

\begin{tabular}{|c|c|c|c|c|c|c|c|c|c|c|c|c|}
\hline \multirow[b]{2}{*}{$\mathrm{R}$} & \multicolumn{3}{|c|}{$\mathrm{Tc}=100$} & \multicolumn{3}{|c|}{$\mathrm{Tc}=200$} & \multicolumn{3}{|c|}{$\mathrm{Tc}=300$} & \multicolumn{3}{|c|}{$\mathrm{Tc}=400$} \\
\hline & MMBtu/H & $\mathrm{F}$ & $A-f t^{2}$ & MMBtu/H & $\mathrm{F}$ & $A-f t^{2}$ & MMBtu/H & $F$ & $A-f t^{2}$ & MMBtu/H & F & $A-f t^{2}$ \\
\hline $0: 1$ & 236.8 & 0.1557 & 47400 & 186.3 & 0.1225 & 37260 & 134.7 & 0.0886 & 26940 & 81.9 & 0.0539 & 16380 \\
\hline $3: 1$ & 1184 & 0.779 & 236800 & 947 & 0.623 & 189400 & 696 & 0.458 & 139200 & 429 & 0.2821 & 85800 \\
\hline $5: 1$ & 1816 & 1.194 & 363200 & 1454 & 0.956 & 290800 & 1069 & 0.703 & 213800 & 661 & 0.435 & 132200 \\
\hline $9: 1$ & 3080 & 2.026 & 616000 & 2470 & 1.624 & 494000 & 1817 & 1.195 & 363400 & 1125 & 0.740 & 225000 \\
\hline
\end{tabular}


Assuming that pressure drop is small relative to system pressure, the product of the volumetric flow rate at pressure, and temperature and pressure drop, yield the horsepower required to circulate the recycle gas:

$$
\mathrm{BHP}=\frac{\mathrm{SCFD} \times \frac{\mathrm{T}}{520} \times \frac{14.7}{\mathrm{P}} \times \Delta \mathrm{P} \times 144}{\mathrm{E} \times 24 \times 60 \times 33000}
$$

which simplifies to

$$
\mathrm{BHP}=8.57 \times 10^{-8} \times \frac{\mathrm{SCFD} \times \mathrm{T} \times \Delta \mathrm{P}}{\mathrm{E} \times \mathrm{P}}
$$

E represents compressor efficiency expressed as a decimal.

Equations (25), (29), and (31) may be used to determine reactor pressure drops and recycle gas compressor loads under the same conditions as those employed in the feed gas preheat analysis. Since a great deal of work in methanation is being done at pressures in the vicinity of 300 psia, this pressure was used in the analysis.

In recycle methanation systems, reactor temperatures normally range from 550 to $850^{\circ} \mathrm{F}$. Therefore, catalyst pressure drops were evaluated at an average $700^{\circ} \mathrm{F}$. Typical catalyst parameters were selected; namely, $3000 \mathrm{l} / \mathrm{H}$ for space velocity and $3 / 8$ inch for catalyst particle diameter. Catalyst bed length-todiameter ratio was set at unity, since practical experience indicates that this represents a lower limit beyond which the distribution of gas over a catalyst may become a problem. On a rather arbitrary basis, a compressor suction temperature of $100^{\circ} \mathrm{F}$ and a compressor efficiency of $70 \%$ were chosen. Table Al-9 summarizes the effect of recycle ratio and reactor paralleling on pressure drop and recirculation compressor horsepower.

In order to size the recirculation compressor, it was necessary to make several assumptions concerning pressure drops in the system external to the reactors. These extraneous pressure drops are associated with heat exchangers, piping, and control systems. It is obvious that if the external pressure drop exceeds the reactor pressure drop to any considerable extent, then the external pressure drop is being utilized inefficiently for the purpose of sizing and maintaining uniformity of flow in the parallel heat exchanger trains. On the other hand, if the reactor pressure drop is excessive compared to the external system, then problems are introduced in maintaining uniformity of flow. Therefore, for the purpose of constructing Table Al-9, reactor pressure drops were doubled in calculating compressor horsepowers. While this procedure represents an assumption, it is not an unreasonable one. The sensitivity of compressor load to various design parameters is reported in Tables Al-10 through -14 .

The data in Tables Al-9 through -14 reveal that the design of recycletype methanation systems will proceed by game rules that require the balancing of the complexity introduced by paralleling, large heat exchange surfaces, and large compressor loads. Various devices are being considered to minimize the total effect. 
Table Al-9

REACTOR PRESSURE DROP

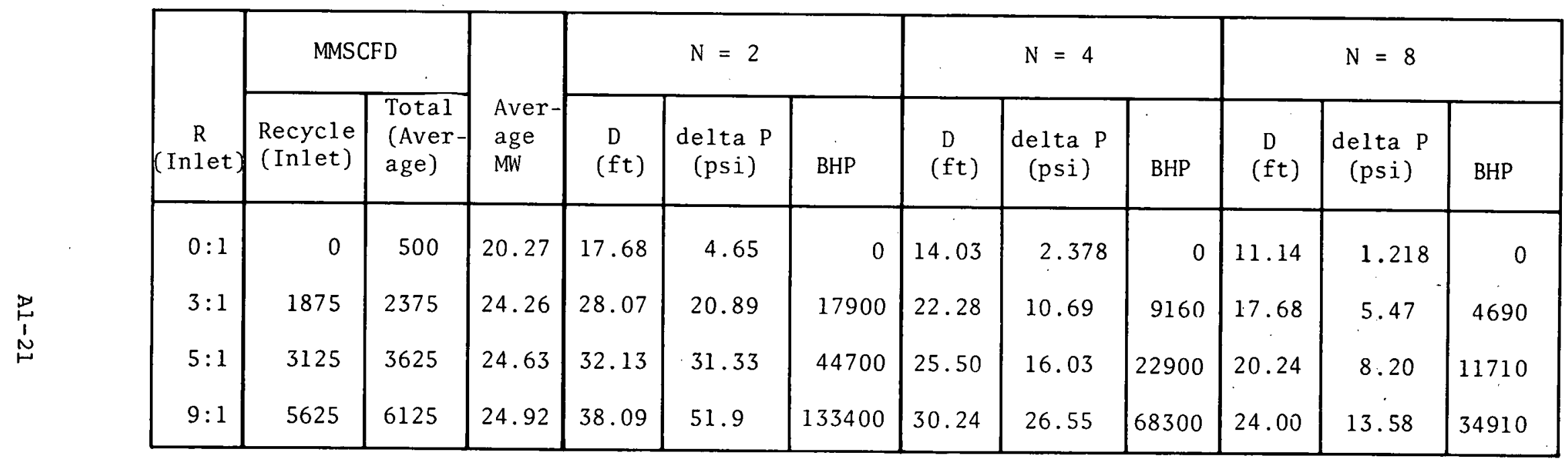


Use of hot gas recycle compressors reduces the recycle gas preheat load at the expense of compressor load. Compressor load, however, is not as sensitive to suction temperature (Table Al-11) as it is to other parameters. Use of the hot gas recycle compressor may be limited by sensitivity of the catalyst to side products of the reaction, particularly water vapor.

Makeup synthesis gas may be proportioned between two reactors if the methanation catalyst is distributed between tow vessels in series. The recirculation is then determined by the recycle ratio and the volume of makeup synthesis gas entering the first reactor. After methailation in the first reactor, the recycle may be used a second time to moderate the reaction in the second reactor. Further advantage may be taken of split feed in that the portion of the makeup synthesis gas proceeding to the second reactor need not be preheated. This is a relatively minor advantage, however, in view of the small magnitude of makeup gas preheat duty. Recycle ratio can be reduced by a factor of two with split feed.

Still another variant is the use of cold-recycle gas quench with split feed. In this variation on split feed, recirculation is increased, with the increment directed to the second reactor without preheat. Sufficient coldrecycle gas is sent to the second reactor so that the blend of first reactor effluent, cold-synthesis gas, and cold recycle yield a mix temperature equivalent to the required inlet temperature at the second reactor. Heat exchange surface is reduced at the cost of added recycle.

In all of the split-feed and cold-quench schemes, the second reactor operates at normal space velocity. The space velocity in the first reactor may be increased because of the presence of a followup reactor. The use of split feed and cold quench, however, increases catalyst volume and effective length-todiameter ratio, again increasing compressor load.

Modification of the methanation catalyst permits an entirely different approach to the problem of the exothermic nature of the methanation reaction. By use of a supported catalyst of relatively coarse crystallite size, thermal stability can be assured. The elevated temperatures improve kinetics to the point that, even with a relatively coarse crystallite size and low surface area, only low-nickel contents are required. Since a maximum temperature limit is not predetermined, product gas recycle is not required and the reaction is permitted to proceed to equilibrium temperature and conversion in each reactor.

A series of adiabatic reactors is used with interstage cooling to achieve the desired conversion. Elimination of the recycle removes the compression load as well as reducing the heat exchanger duty by a severalfold factor, since the heat load for reheating recycle gas can be substantially larger than the methanation heat load. When such heat loads are examined in the light of the limited mean-temperature differences imposed by thermal recovery efficiencies, as well as by maximum and minimum reactor temperatures, they translate into large heat exchange surfaces. When, on the other hand, a larger mean-temperature difference is permitted by the high temperature rise in recycleless methanation, heat exchange surface is reduced proportionately.

Conditions favorable to the formation of carbon by the Boudouard reaction are always present in low-temperature, recycle-type methanation processes. Its formation at the catalyst surface is suppressed by catalyst formulations for 
Table Al-10

EFFECT OF REACTOR PRESSURE ON HORSEPOWER

\begin{tabular}{|c|c|}
\hline $\begin{array}{c}\text { Pressure } \\
\text { (psia) }\end{array}$ & BHP \\
\hline 100 & $206100^{*}$ \\
300 & 22900 \\
1000 & 2061 \\
\hline
\end{tabular}

*Beyond the range of assumption.

Table A1-11

EFFECT OF COMPRESSOR SUCTION TEMPERATURE ON HORSEPOWER

\begin{tabular}{|c|c|}
\hline Temperature & BHP \\
\hline 100 & 22900 \\
200 & 26990 \\
300 & 31080 \\
400 & 35170 \\
\hline
\end{tabular}

Table Al-12

EFFECT OF SPACE VELOCITY ON HORSEPOWER

\begin{tabular}{|c|c|}
\hline $\begin{array}{c}\text { Space Velocity } \\
(1 / \mathrm{H})\end{array}$ & BPH \\
\hline 2000 & 15690 \\
3000 & 22900 \\
4000 & 29950 \\
\hline
\end{tabular}




\begin{tabular}{|c|c|}
\hline $\begin{array}{c}\text { Diameter } \\
\text { (in.) }\end{array}$ & Horsepower \\
\hline $1 / 4$ & 35770 \\
$3 / 8$ & 22900 \\
$1 / 2$ & 16690 \\
\hline
\end{tabular}

\begin{tabular}{|c|c|}
\hline L/D & Horsepower \\
\hline 0.8 & 14880 \\
1.0 & 22900 \\
1.2 & 32580 \\
\hline
\end{tabular}

proper selectivity. Its homogeneous gas phase formation is minimized by reducing the kinetics of its formation by operating within the specified maximum temperature limit. In high-temperature, recycleless methanation processes, carbon formation is avoided by methanating in the presence of steam at high temperatures.

\section{Reference}

Immartino, "Methanation Routes Ready," Chemical Engineering, October 1974.

\section{REACTOR DESIGN}

The design of nonadiabatic methanation reactors implies a quantitative understanding of the kinetics of methanation. Any such design invariably involves a multiplicity of zones within the reactor that separate into classes according to function. In one class, the function may be to conduct the reaction. In still another class, the function may be to cool the gaseous reaction mass. For example, the cross section of a packed reactor tube constitutes the reaction zone. The tube wall represents the heat transfer zone. The geometry of such configurations is related to the relative rates of reaction and of heat transfer. If the system cools more rapidly than it reacts, the gases are quenched and the reaction stops. Conversely, if the system does not cool rapidly enough, the catalyst may be exposed to such high temperatures as to cause it to sinter rapidly. In the absence of kinetic data for the methanation reactions, the development of nonadiabatic reactors has proceeded very slowly. 
Still another factor has served to deter activity in the development of nonadiabatic reactors. A freshly prepared methanation catalyst shows a rapid decline in activity over a very short initial period of operation. At the conclusion of this period, the catalyst shows what may be considered to be its normal activity, which is the basis for equipment design. Any nonadiabatic methanation reactor design would have to incorporate features to cover this characteristic, which presents problems in itself.

Thus, most of the work done to date in the development of methanation processes has centered about the use of adiabatic reactors. As such, they constitute no more than simple pressure vessel designs with internal refractory lining for heat conservation. The reaction systems handled introduce no new metallurgical considerations. If there is anything unique in the design of these reactors for methanation processes, it is probably in their size because of the large process capacities being considered. Both for purposes of reliability of operation and for control of system pressure drop, however, paralleling of trains undoubtedly places maximum reactor sizes well within the range of field fabrication techniques, if not actually within the range of shop fabrication and transportable sizes.

Other catalyst alternatives under consideration include special Raney nickel-type catalysts and fluidized bed systems where bed expansion is maintained either by the gas or a temperature-moderating inert liquid. The diversity of approaches to the problem of bulk methanation, the lack of commercial design construction experience, and the large scale-up factors from pilot plant operations all serve to relegate such systems to a next-generation development.

\section{COST ESTIMATING}

Since several methanation processes are still in an early stage of development, no capital cost information has been published. For even those processes that are considered "commercially ready" by pilot plant demonstrations, cost information is not available in published literature. However, based on previous estimates by The Ralph M. Parsons Company, the installed cost of a two-train, high-temperature, adiabatic methanation plant for producing 250 MMSCFD of methane ( $210 \mathrm{MW}$ th heat release) would be approximately $\$ 40 \times 10^{\circ}$, excluding engineering costs and fees. The cost for units of similar size would vary approximately as the 0.6 power of capacity. In an effort to establish relative costs, it is useful to list major equipment that would be the main contributors to this capital cost. Such items would include reactor vessels, heat exchange surface, and recycle gas compressors.

\section{REACTOR VESSELS}

As previously discussed, the number and size of methanation reactors depend upon many factors, of which space velocity and pressure drop are highly significant. For a nominal capacity of 250 MMSCFD of product SNG, parallel trains would be required by all processes. And for considerations of on-time service factors, an owner may wish to consider two parallel trains as minimum regardless of other considerations. 
On the basis of comparable selection conditions, it would be expected that a.ll fixed-bed, adiabatic processes would have approximately the same installed volume of catalyst. Although there may be variations in parallel and series flow, the ${ }_{3}$ overall average design space velocity would be approximately 1500 $\mathrm{scf} / \mathrm{hr} / \mathrm{ft}^{3}$ of catalyst based on dry, makeup syngas. A specific space velocity for a single reactor could be many times as large for a gas-recycle process and four to six times as large for a high-temperature process, depending on the number of reactors in series flow. As a basis to establish relative costs for reactor vessels, however, it is estimated that all fixed-bed systems would require the same total vessel volume. The cost of the vessels would depend on the number of parallel trains that are selected for use as discussed later. Operating in the range of 900 to $1500^{\circ} \mathrm{F}$ would require refractory lining, which would add a small percentage to the total installed cost of each vessel. As a first approximation, the cost of the reactors can be estimated at $\$ 300 / \mathrm{ft}^{3}$, uninstalled, with a factor of 2 used to obtain the installed cost.

\section{HEAT EXCHANGE SURFACE}

In Table A1-8, presented in a preceding subsection, the $\mathrm{F}$ factor is the fraction of heat released in methanation that must be interchanged between feed and product gases. Although this exchange of heat does not represent a thermal loss, it is required to preheat the feed gas to a temperature at which the methanation reaction will initiate. For this reason, it represents added heat exchange surface in proportion to the amount of recycle gas as well as the degrading of high-temperature-level heat to low-temperature-level heat.'

In an effort to establish a basis for comparison of heat transfer surface from one process to another, it is necessary to make assignments of transfer rates and to estimate mean temperature differences (MTD). An overall coefficient has already been assumed for gas-to-gas transfer to establish Table Al-8. An increase of $60 \%$ in this rate would be expected when transferring heat from gas to boiler feedwater preheat or to steam generation. Further, an assumed temperature difference of $100^{\circ} \mathrm{F}$ was used to establish Table Al-8. When 600psig steam is produced from the heat in the product gas above $650^{\circ} \mathrm{F}$ (leaving a $100^{\circ} \mathrm{F}$ differential to preheat the feed gas to $\left.550^{\circ} \mathrm{F}\right)$, the temperature difference for steam generation would be approximately $230^{\circ} \mathrm{F}$.

In Figure Al-2, a sample enthalpy diagram shows the effect of feed gas preheat MTD on the availability of heat for steam generation. For example, when using a 5:1 recycle ratio, approximately $30 \%$ of the heat released in methanation is required to service the interchange requirements. This leaves only $70 \%$ of the heat from methanation available for steam generation. This reduction represents a loss in operating efficiency but at the same time results in a reduction in surface required for steam generation.

The overall effect on heat transfer surface for steam generation and feedproduct gas interchange can be approximated by consideration of the relative design conditions shown in Table Al-15. This table of calculated data shows that large percentage increases in heat transfer surface accompany the use of recycle gas. Even with as low a recycle as $3: 1$, more than $50 \%$ of the total surface would be required to preheat feed gas from a hot-gas recycle compressor at $400^{\circ} \mathrm{F}$ to reactor inlet temperature. 


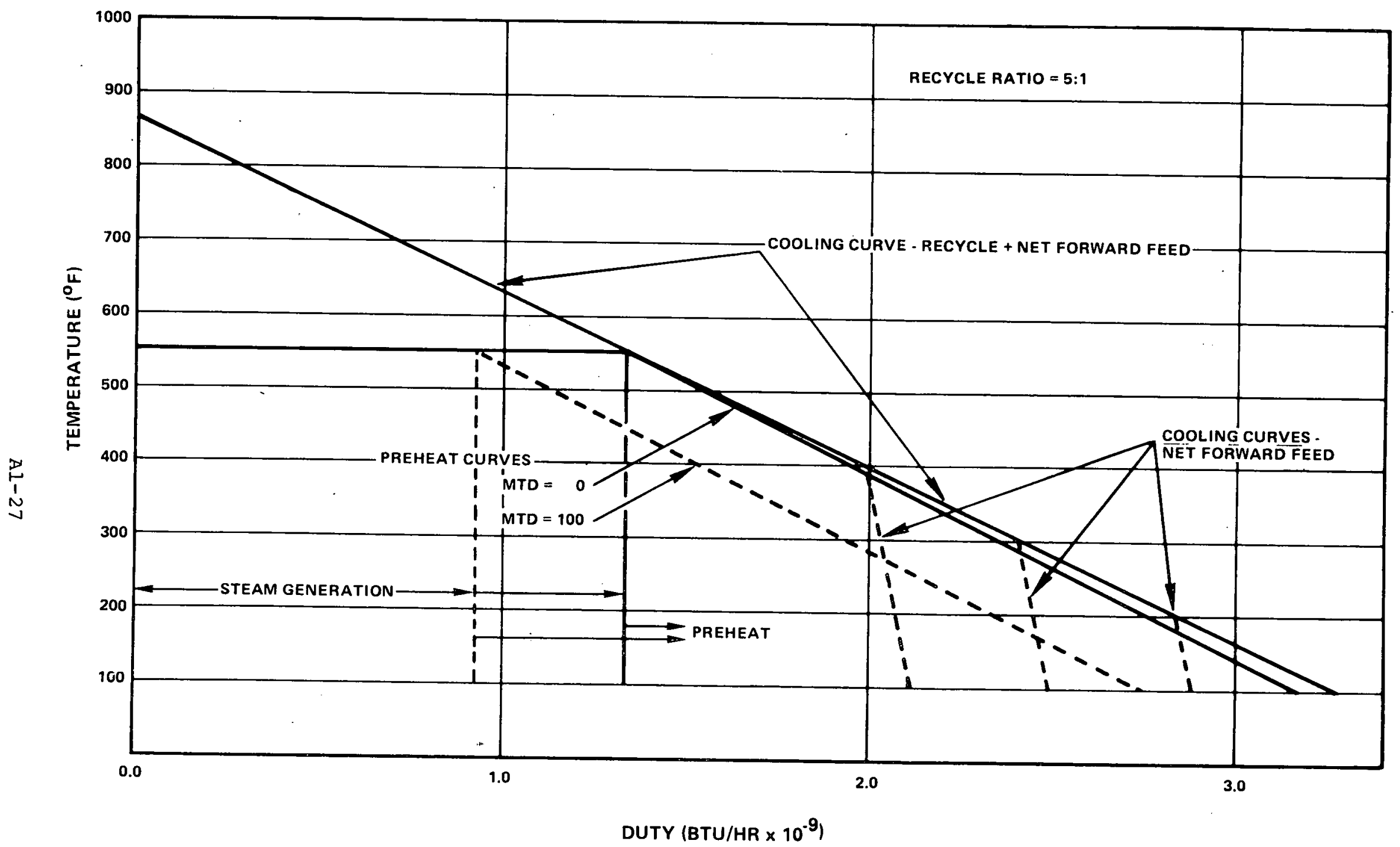

Figure A1-2. Effect of MTD on Heat Recovery to Steam 
Table Al-15

HEAT EXCHANGER SURFACE AREAS

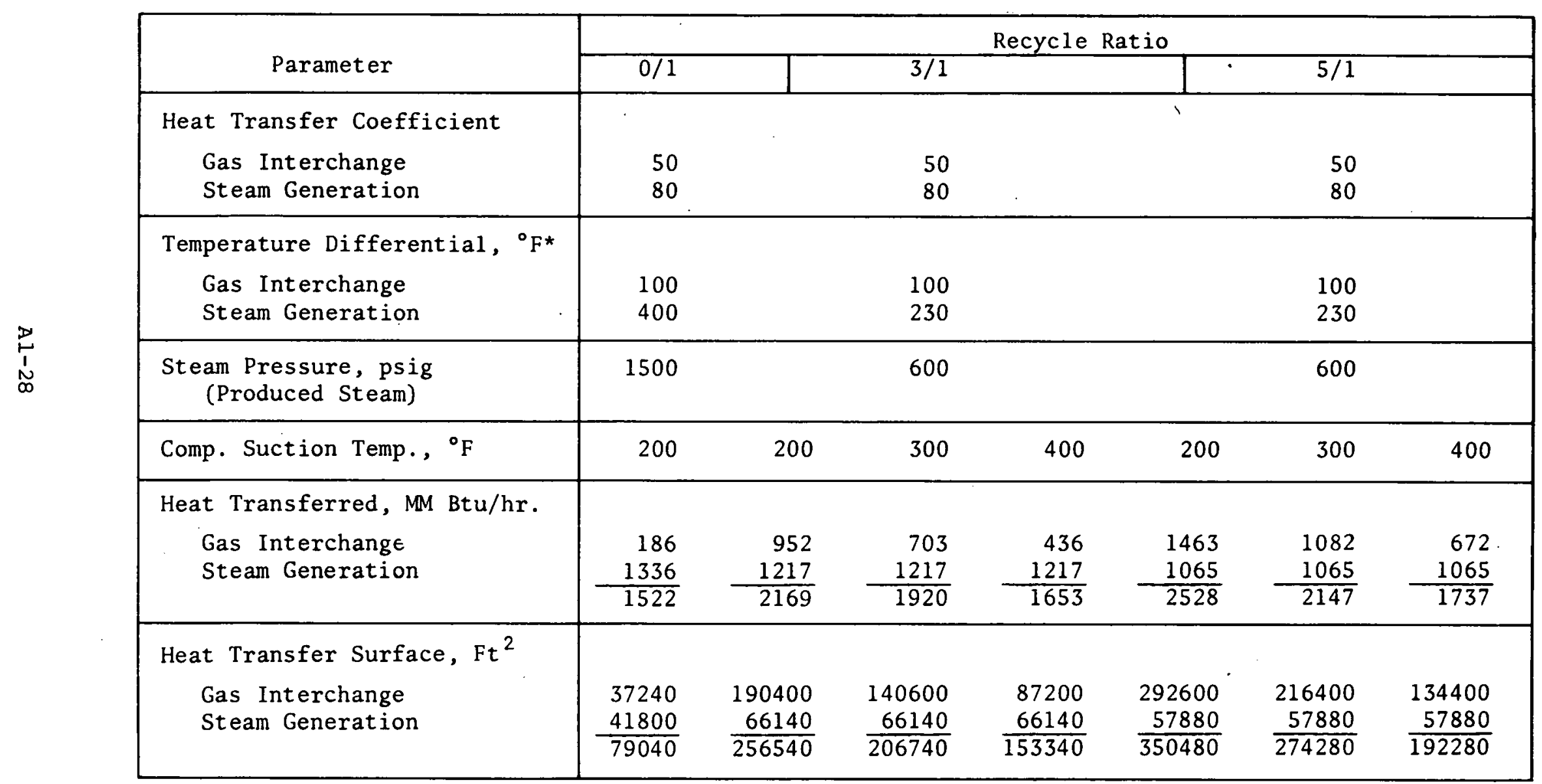

*Uncorrected for temperature approaches or crossover. 
When operating at a 3:1 recycle ratio and assuming a minimum preheat temperature of $550^{\circ} \mathrm{F}$, however, a cold-gas quench system must be employed to avoid exceeding $900^{\circ} \mathrm{F}$ reactor outlet temperature. When a quench system is used, the heat duty for feed gas preheat would be reduced proportionately. Partly. compensating for this reduction in heat duty, however, is the loss in temperature differential caused by unbalancing the mass flow rates of feed to product gas. Because of the complexity of heat exchange systems that could be employed, the calculated surface assumes no quench system has been used in this evaluation on the basis that such a modification would not have a significant impact on an overview evaluation.

The heat exchange system must be properly designed to avoid three major materials problems: (1) carbonyl formation, (2) hydrogen embrittlement, and (3) $\mathrm{CO}_{2}$ corrosion. The preheat exchangers for the raw syngas are designed with a high heat flux to ensure that the CO-rich gas passes quickly through the temperature regime where carbonyl formation is favored. In addition, these preheat exchangers must use materials containing about $0.5 \%$ Mo and $1.25 \% \mathrm{Cr}$ (costing about $\$ 20 / \mathrm{ft}^{2}$, uninstalled) in order to minimize attack by hydrogen in the feed gas. The $\mathrm{CO}_{2}$ and $\mathrm{H}_{2} \mathrm{O}$ in the methanator exit gases combine at the steam dewpoint, forming carbonic acid, which is corrosive to ordinary carbon steels. In these instances, high alloy $\left(\mathrm{Cr}, \mathrm{Ni}\right.$ ) steels (costing approximately $\$ 30 / \mathrm{ft}^{2}$, uninstalled) must be used. A factor of 3.5 may be used to obtain the installed cost of the exchanger. In a typical design, the exit gas coolers dominate the cost of the heat exchange equipment.

\section{COMPRESSOR HORSEPOWER}

Table Al-9, presented in a preceding subsection, shows that power for recirculation can be reduced by using multiple parallel trains. However, multiple trains of reactors would add to the cost of vessels. As a rough estimate, the cost of compressors at a $3: 1$ recycle ratio is approximately $20 \%$ of the cost of reactor vessels in two parallel trains. The incremental cost for the addition of twice as many vessels would far outweigh a 50\% reduction in horsepower. on a relative cost basis, use of the fewest practical number of vessels would be preferred.

Considering only bulk methanation (assuming pretreatment for sulfur removal and final methanation are the same for all processes), the cost of reaction vessels is approximately 55 to $60 \%$ of the total equipment cost and twice the cost of exchangers for a high-temperature process based upon previous estimates made by The Ralph M. Parsons Company. Since the exchanger surface required for a case using a hot-gas recycle at a $3: 1$ ratio is approximately twice the surface of the high-temperature process without recycle, the cost for the exchangers in the 3:1 recycle case would be approximately equal to that of the vessels.

In this overview evaluation of relative costs, it is recognized that all factors that may affect costs of equipment have not been considered - such as the difference in the cost of exchangers designed to produce 600 psig steam compared to $1500 \mathrm{psig}$ or the specific cost to install refractory lining. On the other hand, it must also be recognized that plant costs would depend heavily on a specific selection of parallel series flow as well as plant layout. It was not the intent to make a cost comparison of processes in this report, but merely to identify the major contributors to cost. 
Further, this study considers the single-unit operation of methanation assuming that a feed gas would be available having an acceptable ratio of $\mathrm{H}_{2}$ and $\mathrm{CO}$ for methanation. If shift conversion were required ahead of methanation for the gas recycle processes, then the small percentage advantage in costs for the high-temperature processes for methanation alone would be larger.

\section{APPLICATION TO CHEMICAL HEAT PIPE CONCEPT}

The high-temperature methanation process would lend itself to application at the exothermic end of the chemical heat pipe, since maintenance and operational problems for the small-scale users would be minimal. Reactor control is facilitated by the fact that the extent of reaction (and thus the heat release) is limited by equilibrium considerations. Any upward temperature excursion would result in a decreased conversion and a smaller amount of heat released, resulting in an inherently stable operation. In addition, the lownickel-content catalyst and refractory lining are insensitive to upward temperature excursions (of $100^{\circ} \mathrm{F}$ or more) above the design operating temperature of $1450^{\circ} \mathrm{F}$. These factors combine to make this type of methanation process easily controllable, even for an (initially) inexperienced operator employed by a typical industrial steam user.

The one- or two-shift operation of a high-temperature methanation operation would be technically feasible. However, the reactor vessel and associated equipment should be maintained at or near the reaction temperature to minimize any damage to the refractory lining of the vessel (not the catalyst) due to temperature cycling. Maintenance of the system temperature during off shifts would also help prevent carbonyl formation in the preheat exchangers during startup.

In the particularly clean (i.e., poison-free) environments expected in chemical heat pipe applications, the catalyst life would be expected to extend beyond the normal $\sim 5$ years. However, until the dominate factors limiting the catalyst life are ascertained, the extent of the catalyst life cannot be projected accurately.

Small units which could be employed by typical industrial steam units might be more economically designed by including the heat exchanger tube bundles and all catalyst beds within one shell. In this case, the costs would be likely to decrease below that estimated by the 0.6 power relationship to units of larger capacity. 
Section 4

\section{DEVELOPMENTAL PROGRAMS}

In this section, brief descriptions of methanation processes under development are presented. These have been extracted from published literature (cited as references following each process description). Proprietary information has not been solicited in the preparation of this report. If the information on any process is not considered complete, it is because details have not been published for the process.

\section{INSTITUTE OF GAS TECHNOLOGY}

This program, two-thirds funded by the U.S. Energy Research and Development Administration (ERDA) and one-third-funded by the American Gas Association (AGA), was initiated at the Institute of Gas Technology (IGT) in Chicago, Illinois, during 1971. The program covers the development of a total process (Hygas) for the conversion of solid carbonaceous materials to a substitute natural gas (SNG). Design, construction, and operation of a pilot plant have proceeded in stages.

The methanation facilities were commissioned in April 1973. The concept employed is that of two adiabatic fixed-bed reactors with split cold-gas recycle and split feed. The overall gas recycle ratio amounts to about $2.3: 1$ in the pilot plant operation. Carbon monoxide content is reduced to less than 4 vol\% entering each reactor, while temperatures in each reactor range from about $540^{\circ} \mathrm{F}$ at the inlet to about $775^{\circ} \mathrm{F}$ at the outlet. The system operates at about 900 psig. Charged into the first reactor are $15.7 \mathrm{ft}^{3}$ of catalyst, and $31.4 \mathrm{ft}^{3}$ are charged into the second. Space velocities range from 3500 to $5100 \mathrm{l} / \mathrm{h}$ in the first reactor and from 2300 to $3250 \mathrm{l} / \mathrm{h}$ in the second. space velocities are based on makeup plus recycle gas. The system has operated at a makeup feed gas rate as high as 740 MSCFD.

The catalyst charged to the reactors is of a proprietary nature, prepared by Harshaw, and consists of nickel on 1/4-in. kieselguhr pellets.

Feed gas compositions being methanated are those that are characteristic of the synthesis gas produced by the Hygas gasifier and are high in methane. The following makeup feed gas analysis is reported for the pilot plant:

$\begin{array}{lrr}\text { Component } & & \text { Vol\% } \\ \mathrm{H}_{2} & 51.6 \\ \mathrm{CH}_{4} & 23.3 \\ \mathrm{C}_{2} \mathrm{H}_{6} & 1.3 \\ \mathrm{CO} & 12.9 \\ \mathrm{CO}_{2} & & 0.0 \\ \mathrm{~N}_{2}+\mathrm{A} & & 10.9 \\ & \text { Total } & 100.0\end{array}$


Assuming complete conversion, only $26 \%$ of the methane leaving the plant is produced in the methanation equipment with the balance of $64 \%$ originating in the gasifier. In the pilot plant, when the synthesis gas contains excess carbon oxides, hydrogen is added to make a stoichiometric mix. If the hydrogen is in excess, no effort is made to adjust the $\mathrm{mix}$ and a high-hydrogen is in excess, no effort is made to adjust the $\mathrm{mix}$ and a high-hydrogen product is accepted. It is anticipated that all commercial applications of the IGT design will incorporate features to provide a 3:l hydrogen-to-carbon-monoxide mixture prior to methanation.

Purification of the makeup synthesis gas includes diglycolamine scrubbing, caustic scrubbing, and a water wash. The following are preliminary data concerning trace component analyses of the synthesis gas at several points in the train:

\begin{tabular}{|c|c|c|c|}
\hline Component & $\begin{array}{c}\text { DGA Feed } \\
\text { (Volo) } \\
\end{array}$ & $\begin{array}{l}\text { DGA Ovh } \\
\text { (ppm) }\end{array}$ & $\begin{array}{c}\text { Caustic Ovh } \\
\text { (ppm) }\end{array}$ \\
\hline $\begin{array}{l}\mathrm{CO}_{2} \\
\mathrm{H}_{2} \mathrm{~S}\end{array}$ & 6.76 & $\begin{array}{l}150 \\
0.09\end{array}$ & 50 \\
\hline $\cos$ & 0.43 & 0.07 & $0.1-0.2$ \\
\hline $\mathrm{CH}_{3} \mathrm{~S}$ & & 0.02 & \\
\hline
\end{tabular}

Recent tests produced a total pilot plant self-sustained run of 360 hours. of this period, 160 hours were under steady-state operating conditions. Most recently, instrumentation malfunctions have caused sulfur inactivation of the catalyst. The catalyst in the first bed has been replaced with zinc oxide and in the second bed with a Union Carbide molecular sieve catalyst.

\section{References}

Lee, "Status of the Hygas Program," Proceedings, Eighth Synthetic Pipeline Gas Symposium, Chicago, Illinois, October 18-20, 1976.

Lee, "Status of the Hygas Program," Proceedings, Seventh Synthetic Pipeline Gas Symposium, Chicago, Illinois, October 27-29, 1975.

Lee, "Status of Hygas Process - Operating Results," Proceedings Fifth Synthetic Pipeline Gas Symposium, Chicago, Illinois, October 29-31, 1973.

Lee and Turman, "Status of the Hygas Program," Proceedings, Sixth Synthetic Pipeline Gas Symposium, Chicago, Illinois, October 28-30, 1974.

CONOCO COAL DEVELOPMENT COMPANY - WESTFIELD

Conoco has spearheaded a joint effort by the private business sector to investigate the coupling of methanation facilities with a Lurgi gasifier at Westfield, Scotland. Members of the group included the following:

AMAX Coal Company Cities Service Gas Company Colorado Interstate Gas Company Columbia Gas Transmission Corporation 
Continental Oil Company

El Paso Natural Gas Company

Exxon Corporation

Gulf Oil Corporation

Natural Gas Pipeline Company

Northern Natural Gas Company

Panhandle Eastern Pipe Line Company

Peabody Coal Company

Rocky Mountain Energy Company

Transcontinental Gas Pipe Line Corporation

Pacific Coal Gasification Company

Transwestern Coal Gasification Company

As might be expected from the private nature of the organization, information concerning the methanation facility is sparse. The methanation process configuration employed appears to be multiple fixed-bed, adiabatic reactors employing gas recycle for temperature moderation. Operating pressure was 350 psig. Feed gas was a synthesis gas rich in methane, which is normally associated with a Lurgi operation and shifted to provide a stoichiometric mix. Purification included Rectisol wash and a zinc oxide guard.

During September and October 1974, the plant produced 2.1 MMSCFD of substitute natural gas, which displaced almost $60 \%$ of the natural gas in a local grid without producing any customer reaction.

\section{References}

Hausberger, "Methanation of Synthesis Gas Derived from Coal Passes Tests," Oil and Gas Journal, March 31, 1975.

Landers, "Review of Methanation Demonstration at Westfield, Scotland," Proceedings, Sixth Synthetic Pipeline Gas Symposium, Chicago, Illinois, October $28-30,1974$.

LURGI

The Lurgi version of methanation has been demonstrated at Schwechat, Austria and at Sasolburg, South Africa. The Lurgi concept includes two or more fixed-bed, adiabatic reactors with hot-gas recycle, interstage cooling, and split feed. Recycle gas ratio equals about 3.7:1 to 5.8:1, which results in an inlet temperature to the reactor of about $540^{\circ} \mathrm{F}$ and an outlet temperature of about $842^{\circ} \mathrm{F}$. Operating pressures run about $240 \mathrm{psig.} \mathrm{The} \mathrm{catalyst} \mathrm{is} \mathrm{a} \mathrm{pro-}$ prietary BASF 20 wt: nickel on alumina formulation.

The feed gas is a methane-rich synthesis gas, characteristic of lurgi gasification, to which steam is added. An analysis of a typical feed gas, prior to treatment and purification, is reported as follows: 


\begin{tabular}{lrr} 
Component & Vol\% \\
\hline & & 37.5 \\
$\mathrm{H}_{2}$ & & 13.1 \\
$\mathrm{CH}_{4}$ & & 19.6 \\
$\mathrm{CO}$ & & 29.2 \\
$\mathrm{CO}_{2}$ & & 0.3 \\
$\mathrm{~N}_{2}$ & & 0.3 \\
$\mathrm{H}_{2} \mathrm{~S}$ & Total & 100.0
\end{tabular}

On the basis of this analysis, $48 \%$ of the methane leaving the plant is formed in the gasifiers, while $52 \%$ is produced in the methanation equipment. The feed gas is shifted only enough to provide a slight excess of carbon oxides above a stoichiometric mix, washed with Rectisol, and passes through a zinc oxide guard.

The pilot plant single-stage reactor contained $1 \mathrm{ft}^{3}$ of catalyst and methanated up to 16 MSCFD of synthesis gas for periods up to 2600 hours. Details of the Schwechat and Sasolburg demonstration plants are not available. Each operated for 18 months, however, and provided continuous runs of up to 4000 to 5000 hours.

\section{References}

Eisenlohr, Moeller, and Dry, "Influence of Certain Reaction Parameters on Methanation of Coal Gas to SNG," Proceedings, 168th National Meeting American Chemical Society; Division of Fuel Chemistry, Atlantic City, New Jersey, September, 1974.

Moeller, Roberts, and Britz, "Methanation of Coal Gas for SNG," Hydrocarbon Processing, April 1974.

Rudolph, "The Lurgi Process - The Route to SNG from Coal," Proceedings Fourth Synthetic Pipeline Gas Symposium, Chicago, Illinois, October 30-31, 1972 .

\section{CATALYSTS AND CHEMICALS, INCORPORATED (C\&CI)}

This 3-year pilot plant program, operated by C\&CI at Louisville, Kentucky, was sponsored by the El Paso Natural Gas, Western Gasification, and Cogas Development companies. Work was concluded in March 1975. Methanation was conducted at $350 \mathrm{psig}$ in two adiabatic fixed-bed stages with two reactors in parallel in the first stage. Split-feed and split-gas recycles were used to moderate temperatures. Process temperatures were reported as follows:

$$
\text { Inlet }\left({ }^{\circ}\right) \quad \text { Outlet }\left(O_{F}\right) \text {. }
$$

$\begin{array}{lll}\text { 1st Stage } & \text { a } 550 & \text { a } 850 \\ \text { 2nd Stage } & \text { a } 490 & \text { a } 650\end{array}$


Catalyst volumes in each stage were varied from 0.3 to $5 \mathrm{ft}^{3}$. Makeup plus recycle gas space velocities of the order of 12,000 to $15,000 \mathrm{l} / \mathrm{h}$ in the first stage and 4000 to $7000 \mathrm{l} / \mathrm{h}$ in the second stage resulted essentially in equilibrium conversion.

The catalyst was a proprietary formulation containing 50 to 60 wt: nickel on alumina or silica supports. Difficulties were reported in maintaining reduction in the first stage because of the water vapor accompanying the recycle gas.

Feed gas to the test unit was a methane-rich feed gas with composition reported as follows:

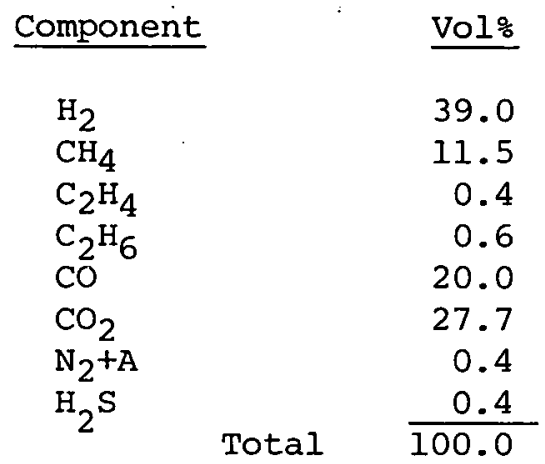

Synthesis gas was produced by steam reforming of natural gas. Purification consisted of a monoethanolamine scrub. Based upon the analysis reported, it may be concluded that only $57 \%$ of the product methane was produced in the methanation equipment.

Operating tésts included 4000- and 1000-hour runs.

\section{CONOCO COAL DEVELOPMENT COMPANY - RAPID CITY}

This developmental effort, conducted by Conoco, is sponsored by ERDA and AGA. It calls for the addition of methanation facilities to the $\mathrm{CO}_{2}$ Acceptor Process gasification facility at Rapid City, South Dakota. The concept employs shell and tube equipment with the catalyst packed into the tubes and heat rejection to vaporizing Dowtherm on the shell side. Product gas recycles ranging from $1.24: 1$ to $1.85: 1$ are employed.

Catalyst details have not been revealed.

The feed gas consists of the methane-rich synthesis gas from the $\mathrm{CO}_{2}$ Acceptor Process, which has been scrubbed of acid gases with a hot potassium carbonate system. An originally planned hydrodesulfurizer was not installed. Final purification of the gas is by means of zinc oxide. 
Composition of the stoichiometric mix feed gas is reported as follows:

\begin{tabular}{|c|c|}
\hline Component & Vol: \\
\hline $\mathrm{H}_{2}$ & 62 \\
\hline $\mathrm{CH}_{4}$ & 14 \\
\hline $\mathrm{CO}$ & 15 \\
\hline $\mathrm{CO}_{2}$ & 5 \\
\hline $\mathrm{N}_{2}$ & 4 \\
\hline
\end{tabular}

At this time, the system has operated at total (makeup and recycle) space velocities of 12,600 to $15,5001 / \mathrm{h}$, at an operating pressure of about 110 psia. Operating temperatures ran upwards of $550^{\circ} \mathrm{F}$, peaking out at $850^{\circ} \mathrm{F}$ while methanating up to 575 MSCFD.

Startup was originally scheduled for July 1974. To date, three runs have been reported, the longest lasting about 9 days.

\section{References}

Fink, "CO 2 Acceptor Process Pilot Plant - 1973," Proceedings, Fifth Synthetic Pipeline Gas Symposium, Chicago, Illinois, October 29-31, 1973.

Fink, "Status of $\mathrm{CO}_{2}$ Acceptor Process," Proceedings, Sixth Synthetic Pipeline Gas Symposium, Chicago, Illinois, October 28-30, 1974.

Fink, Curran, and Sudbury, "CO 2 Acceptor Process Pilot Plant - 1975," Proceedings, Seventh Synthetic Pipeline Gas Symposium, Chicago, Illinois, October 27-29, 1975.

McCoy, Curran, and Sudbury, "CO 2 Acceptor Process Pilot Plant - 1976," Proceedings, Eighth Synthetic Pipeline Gas Symposium, Chicago, Illinois, October $18-20,1976$.

\section{KOPPERS-IMPERIAL CHEMICAL INDUSTRIES (ICI)}

The process concept advanced by ICI is that of four fixed-bed adiabatic reactors in series with heat rejection between the catalytic stages. No gas recycle is employed. Further distinguishing this process are the relatively high operating temperatures. The first reactor is filled with a conventional water-gas shift catalyst. The feed gas is split between the first reactor and the second reactor, where the methanation reactions start. The objective of this arrangement is to adjust the composition of the synthesis gas entering the methanation stages. Methanation takes place in the presence of steam, which is added to shift and to moderate temperatures. System temperature is permitted to reach adiabatic equilibrium value at the outlet of each reactor up to a limit of $1382^{\circ} \mathrm{F}$.

Details concerning the proprietary ICI-developed catalyst have not been revealed; other than that, it consists of a $60 \%$ NiO coprecipitate. 
The feed gas is a methane-lean synthesis gas characteristic of the operation of a Koppers-Totzek gasifier. Premethanation treatment consists only of removal of particulate matter and any acid gases present at this point. Excess carbon dioxide is removed after the methanation/shift operation.

Experimental work has progressed from bench scale screening tests to the operation of a pilot plant at ICI's laboratories at Clitheroe, Lancashire, England. The pilot plant consisted of three adiabatic reactors, 1 in. id by 30 in. length, packed with 12 in. of catalyst and electrically wound to preserve the adiabatic nature of the system. Feed gas consisted of a 3:1 hydrogento-carbon-monoxide mix passed through a zinc oxide guard. The test unit operated at 425 psig and a steam-to-dry-gas ratio of 0.673 entering the first bed. The following conditions were maintained:

\begin{tabular}{|c|c|c|c|}
\hline Reactor & Inlet $\left({ }^{\circ}\right)$ & Outlet $\left({ }^{\circ} F\right)$ & Dry SV-1/Hr \\
\hline 1 & 748 & 1344 & 10000 \\
\hline 2 & 617 & 1094 & 8865 \\
\hline 3 & 572 & 802 & 7160 \\
\hline
\end{tabular}

Loss of steam caused a deposition of carbon after 1500 hours. After reestablishing steam flow, however, the first reactor recovered activity and continued operation for a total of 2200 hours.

\section{$\underline{\text { References }}$}

Franzen, "SNG Production Based on Koppers-Totzek Coal Gasification," Proceedings, Sixth Synthetic Pipeline Gas Symposium, Chicago, Illinois, October 28-30, 1974.

Woodward, "A High-Temperature Methanation Catalyst for SNG Application," Proceedings, 172nd National Meeting, American Chemical Society, Division of Fuel Chemistry (Symposium on Methanation of Synthesis Gas), San Francisco, California, August 29-September 3, 1976.

\section{THE RALPH M. PARSONS COMPANY}

The Ralph M. Parsons Company methanation concept (RMProcess) uses a series of adiabatic fixed-bed reactors with interstage heat rejection. No attempt is made to segregate the water-gas shift reaction because, under any circumstance, the water-gas shift reaction continues to take place in all reactors in either the forward or the reverse sense. Steam is added to satisfy the stoichiometry of the methanation/shift reaction, to moderate temperatures and to assist in preventing carbon deposition. Feed gas is split to maintain a maximum steamto-synthesis-gas ratio in the earlier stages of the process. The use of steam to prevent carbon deposition is coordinated with high inlet temperatures to the earlier stages for the same purpose. Reactor inlet temperatures range from about $900^{\circ} \mathrm{F}$ in the first reactor to about $600^{\circ} \mathrm{F}$ in the last reactor. Reactor outlet temperatures range from about $1500^{\circ} \mathrm{F}$ in the first reactor to about $900^{\circ} \mathrm{F}$ in the last. Process pressures range to about $1000 \mathrm{psig}$.

Catalysts used in the process are proprietary modifications of steam-methane reforming catalysts. 
All potential sources of synthesis gas, including methane-lean synthesis gases, are suitable as feedstocks. There is no requirement for feed gas composition adjustment prior to the RMProcess.

The RMProcess has undergone four years of experimental testing, ranging from bench scale to pilot plant. The latter facility, located at Texaco's. laboratories in Montebello, California, was designed to produce 250 MSCFD of methane from synthesis gas containing less than $1 \%$ methane. Six adiabatic reactors were used, each holding $5 \mathrm{ft}^{3}$ of catalyst. In operation, the pilot plant received synthesis gas produced by partial oxidation of a variety of feedstocks ranging from natural gas to solj.d carbonaceous materials. Purification included monoethanolamine scrub and a zinc oxide guard drum.

The pilot plant program was concluded late in 1975 after a calendar year of operation during which over 2000 onstream hours were accumulated.

\section{Reference}

White, Roszkowski, and Stanbridge, "The RMProcess," Proceedings, 168th National Meeting, American Chemical Society, Division of Fuel Chemistry (Symposium on Methanation of Snythesis Gas), Atlantic City, New Jersey, September $8-13,1974$.

\section{U.S. ENERGY RESEARCH AND DEVELOPMENT ADMINISTRATION (ERDA)}

The work being conducted at the Pittsburgh Energy Research Center at Bruceton, Pennsylvania is a continuation of efforts initiated by the U.S. Bureau of Mines. It is an adjunct of a larger program intended to develop a total process (Synthane) which would start with a solid carbonaceous feedstock and produce a SNG. The work is based upon the use of Raney nickel-type catalysts, flame-sprayed onto surfaces. Both adiabatic (HGR) and cooled (TWR) reactor systems have been considered. In the former case, the catalyst is coated onto flat plates of assorted shapes, which are then uniformly arranged within the reactor and temperatures controlled by means of up to a $10: 1$ gas recycle. Carbon monoxide is reduced to about 2 vol\% upon entering the reactor, while temperatures within the reactor range from $572^{\circ} \mathrm{F}$ at the inlet to $752^{\circ} \mathrm{F}$ at the outlet. As part of this work, a hot-gas recycle compressor is under study. Because the catalyst exhibits steam sensitivity, however, all or a part of the steam must be removed from the recycled gas by condensation before recycle.

The TWR design employs shell and tube equipment with the catalyst coated either inside or outside the tubes. Heat is mainly removed by direct contact of the catalyst with the tube wall and thence to circulating Dowtherm on the cold side of the heat transfer surface. Reaction zone temperatures are maintained at about $734^{\circ} \mathrm{F}$ by a moderate $3: 1$ gas recycle. Most recently, a hybrid reactor design (HYBRID) has been developed. It combines the catalyst support features of the HGR concept with the cooling arrangement of the TWR concept. Heat transfer is from the catalyst to the bulk gas, thence to the tube wall and Dowtherm. Longer catalyst life and easier replacement.are ascribed to this arrangement. Gas recycles of $5: 1$ to $8: 1$ are used. All three process concepts employ a single reactor. 
Feed gas compositions being considered are those that are characteristic of the synthesis gas produced by the Synthane gasifier and contain higi concentrations of methane. A typical methanation feed gas composition is reported as follows:

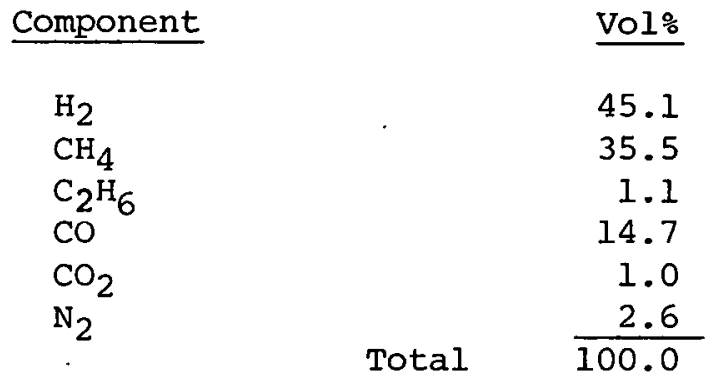

It is estimated that, of the total methane produced in the synthane process, $54 \%$ is produced in the gasifier with only $46 \%$ resulting from the methanation step. Before admission to the methanation step, the synthesis gas is shifted over CoMo catalyst and scrubbed of acid gases in a hot potassium carbonate system to yield a stoichiometric ratio of hydrogen to carbon oxides. Final purification to $0.1 \mathrm{ppm}$ hydrogen sulfide is over activated carbon.

A small HGR pilot plant, capable of methanating up to 10 MSCFD of synthesis gas, in a 3-in. Sch 40 by 10-ft, 0-in.-long pipe reactor, has been operated at 300 psig for periods up to 2300 hours. Its TWR equivalent counterpart has demonstrated runs up to 2800 hours. The HYBRID unit has been run for periods up to 3200 hours. A larger total pilot plant has been under construction at Bruceton, Pennsylvania. All types of methanation reactors are incorporated operating up to 900 psig, each being capable of methanating 170 MSCFD of synthesis gas. The HGR reactor consists of 14 in. pipe, within which 28 parallel plates of $10 \mathrm{ft}, 0$ in. length are inserted longitudinally with widths to conform to the cross section of the pipe. The TWR reactor design calls for 22 2-in. tubes $20 \mathrm{ft}, 0$ in. long, internally coated and arranged within a 16 in. shell. Details of the HYBRID unit are not available. After many delays, startup was rescheduled in early 1976. At this time, the methanation facilities of the large pilot plant have not been commissioned.

\section{References}

Carson, "Status of the Synthane Process," Proceedings, Seventh Synthetic Pipeline Gas Symposium, Chicago, Illinois, October 27-29, 1975.

Forney, "The Synthane Process - Research Results and Prototype Plant Design," Proceedings, Fourth Synthetic Pipeline Gas Symposium, Chicago, Illinois, October $30-31,1972$.

Haynes, Forney, Elliott, and Pennline, "Synthesis of Methane in a Hot Gas Recycle Reactor," Proceedings, 168th National Meeting, American Chemical Society, Division of Fuel Chemistry, Atlantic City, New Jersey, 1974.

Lewis, Clark, Haynes, and Strakey, "Operational Status of the Synthane Pilot Plant," Proceedings, Eighth Synthetic Pipeline Gas Symposium, Chicago, Illinois, October 18-20, 1976 . 
Schehl, Pennline, Strakey, and Haynes, "Pilot Plant Operation of a Nonadiabatic Methanation Reactor," Proceedings, 172nd National Meeting, American Chemical Society, Division of Fuel Chemistry (Symposium on Methanation of Synthesis Gas), San Francisco, California, August 29-September 3, 1976.

BITUMINOUS COAL RESEARCH, INCORPORATED (BCR)

The $B C R$ effort, sponsored by ERDA and $A G A$, is an adjunct of a larger program to develop a total process (Bigas) to convert solid carbonaceous materials to SNG. A novel feature of the work is the use of a fluidized catalyst bed. A single methanation reactor vessel, which is divided into zones, is used. Temperature moderation is introduced by gas recycle, injecting the feed gas at one or more elevations in the bed and by installing cooling coils at various levels. Operating pressures are in the range 1000 to $1500 \mathrm{psig}$, while operating temperatures, determined by the catalyst used, fall in the range 650 to $800^{\circ} \mathrm{F}$. Space velocities vary from 1200 to 3200 volumes of makeup and recycle gas per volume of catalyst per hour.

The catalyst is a proprietary, attrition-resistant formulation still under development.

Methanation feed gas is a methane-rich synthesis gas characteristic of the Bigas gasifier. The following typical feed gas composition has been reported:

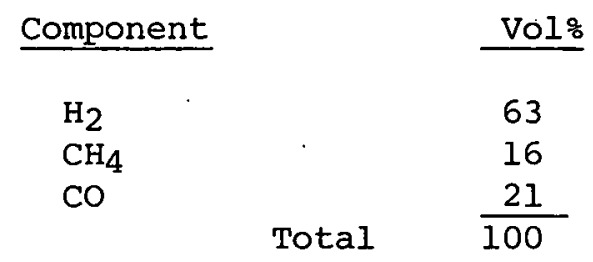

With a feed gas of this composition, 57\% of the methane in the product is produced in the methanation equipment and the balance in the gasifier. It is claimed that hydrogen-to-carbon-monoxide ratios of $2: 1$ to $10: 1$ are acceptable to the process. Sulfur effects have not yet been studied.

A small pilot plant was operated from October 1973 through September 1974. The reactor consisted of $6-\mathrm{in}$.-id pipe $20 \mathrm{ft}, 0$ in. long. A $10-\mathrm{ft}, 0-i n$. bed contained 1-1/2 $\mathrm{ft}^{3}$ of unexpanded catalyst. Two feed points were used and two cooling coils, each containing $16.6 \mathrm{ft} 2$ of finned surface, were installed. Up to 144 MSCFD of synthesis gas were methanated. Tests included two 10-day runs and five runs of 1 to 2 days' duration.

The data from the smallex pilot plant were used to design a larger pilot plant under construction at Homer City, Pennsylvania. Startup was scheduled for mid-1975. 


\section{References}

Brant, "Design of Bi-Gas SNG Process," Proceedings, Fifth Synthetic Pipeline Gas Symposium, Chicago, Illinois, October 29-31, 1973.

Diehl, "Progress in Fluidized-Bed Methanation," Proceedings, Sixth Synthetic Pipeline Gas Symposium, Chicago, Illinois, October 28-30, 1974.

Grace, "Bi-Gas Program Enters Pilot Plant Stage," Proceedings, Fourth Synthetic Pipeline Gas Symposium, Chicago, Illinois, October 30-31, 1972.

Gruboski, "Design and Operation of the BCR Fluidized-Bed Methanation PEDU," Proceedings, Fifth Synthetic Pipeline Gas Symposium, Chicago, Illinois, October $29-31,19.73$.

Hegarty and Moody, "Evaluating the Bi-Gas SNG Process," C.E.P., Volume 69, No. 3, March 1973.

Miles, "Status of the Bi-Gas Program Part I," Proceedings, Eighth Synthetic Pipeline Gas Symposium, Chicago, Illinois, October 18-20, 1976.

Streeter, Anderson, and Cobb, "Status of the Bi-Gas Program Part II," Proceedings, Eighth Synthetic Pipeline Gas Symposium, Chicago, Illinois, October 18-20, 1976.

CHEM SYSTEMS, INCORPORATED

Chem Systems' effort, sponsored by ERDA and AGA, introduces still another novelty in that an inert liquid is introduced into the reaction system for the purpose of both fluidizing the catalyst and moderating temperatures by the liquid's sensible and latent heat effects. A single reactor is used. Overhead from the reactor is cooled, condensing inert liquid and product water. The water is rejected and the inert liquid returned to the reactor. An external loop of the inert liquid is also circulated through a steam generator to reject additional heat. The inert liquid circulation rate is set to give a 5 to $10^{\circ} \mathrm{F}$ temperature rise in the reactor. No gas recycle is used. Temperatures are controlled at 527 to $662^{\circ} \mathrm{F}$ at $1000 \mathrm{psig}$.

The catalyst is a proprietary nickel catalyst that has been prepared by Catalysts and Chemicals, Harshaw, and Calsicat companies.

Hydrogen-to-carbon-monoxide mixtures of $3: 1$ have been under investigation, and a decision has recently been made to study the effect of combred methanation/shift. It has been reported that sulfur must be totally removed from the makeup syngas.

Experimental work has progressed from a bench-scale reactor measuring 0.81 in. id by $4 \mathrm{ft}$, 0 in. in length to a skid-mounted developmental unit with a reactor measuring $4.5 \mathrm{in}$. id by $7 \mathrm{ft}$, 0 in. length. The latter has methanated up to 36 MSCFD of synthesis gas. Runs of 580 hours are reported. Construction of a larger skid-mounted pilct plant has been completed. The equipment contains a reactor $2 \mathrm{ft}, 0$ in. id by $15 \mathrm{ft}, 0$ in. length, and is gapable of methanating 2 MMSCFD of synthesis gas. The large skid-mounted pilot plant has been shipped to the site of the Hygas unit. 
Blum, "Liquid Phase Methaination PDU Results," Proceedings, Sixth Synthetic Pipeline Gas Symposium, Chicago, Illinois, October 28-30, 1974.

Blum, Sherwin, and Frank, "Liquid Phase Methanation of High Concentration CO Synthesis Gas," Proceedings, 168th National Meeting, American Chemical Society, Division of Fuel Chemistry, Atlantic City, New Jersey, September, 1974 .

Frank, "Recent Developments in Liquid Phase Methanation," Proceedings, Fifth Synthetic Pipeline Gas Symposium, Chicago, Illinois, October 29-31, 1973.

Frank and Blum, "Liquid Phase Methanation/Shift," Proceedings, 172nd National Meeting, American Chemical Society, Division of Fuel Chemistry (Symposium on Methanation of Synthesis Gas), San Francisco, California, August 29-Septembex 3, 1976 .

Frank, Sherwin, Blum, and Mednick, "Liquid Phase Methanation/Shift," Proceedings, Eighth Synthetic Pipeline Gas Symposium, Chicago, Illinois, October 18-20, 1976.

Sherwin, "The Slurry Methanation Process," Proceedings, Fourth Synthetic Pipeline Gas Symposium, Chicago, Illinois, October 30-31, 1972.

Sherwin, Frank, and Blum, "Progress in Liquid Phase Methanation," Proceedings, Seventh Synthetic Pipeline Gas Symposium, Chicago, Illinois, October 27-28, 1975. 


\section{Appendix 2}

\section{THERMODYNAMIC ANALYSES}

In the analysis of energy conversion systems involving thermal, mechanical, and chemical changes the concepts of thermodynamic availability prove to be highly valuable. A general review of thermodynamic availability and computations for irreversible systems can be found in References A2-1 and A2-2.* Calculations of entropy production during thermal, mechanical, and chemical changes are described in References $\mathrm{A} 2-2$ and -3 .

The concepts of thermodynamic availability have been applied in three main areas of this report:

- Discussions of the merit of reversible chemical reactions as an energy carrier relative to other alternatives (Chapter 1 ).

- Evaluations of the net effect of thermal energy exchanges between the CHP system and the powerplant (Chapter 5).

- Evaluations of entropy production and availability losses in the CHP system (Chapter 5).

\section{A2.1 BASIC DEFINITIONS}

This report is concerned mainly with steady-flow systems. Consequently, the appropriate measure of availability is the "exergy of extraction and delivery" (Ref. A2-l) or steady-flow essergy of the system. The usual difficulties associated with the choice of an environmental "dead state" (equivalent to zero exergy) are less important in.CHP applications because of the cyclic nature of chemical changes that lead to a closed system; the overall CHP system exchanges only mechanical and thermal energy with the environment. With the exception of calculations of availability for various fuels in section 1.2 (Chapter 1) there is no need to define the chemical nature of the dead state, since the emphasis will be on changes in availability of the working fluid. Following the nomenclature in Reference A2-1, exergy of a stream is defined by

$$
E x=B-\Sigma n_{j} \mu_{j o O}=H-T_{O} S-\Sigma n_{j} \mu_{j o o}
$$

For a change from state 1 to 2 , change in exergy is given by

$$
(\Delta \mathrm{Ex})_{1 \rightarrow 2}=\mathrm{B}_{2}-\mathrm{B}_{1}=\left(\mathrm{H}_{2}-\mathrm{H}_{1}\right)-\mathrm{T}_{\mathrm{O}}\left(\mathrm{S}_{2}-\mathrm{S}_{1}\right)
$$

If changes in the state of the system occur as a result of thermal exchanges with an external fluid, one obtains the following relations:

$$
\begin{aligned}
\left(H_{\text {in }}-H_{\text {out }}\right)_{e f} & =\left(H_{\text {out }}-H_{\text {in }}\right)_{p} \\
\left(S_{\text {in }}-S_{\text {out }}\right)_{e f} & =\left(S_{\text {out }}-S_{\text {in }}\right)_{p}-\Delta S_{C} \\
\left(B_{\text {in }}-B_{\text {out }}\right)_{e f} & =\left(B_{\text {out }}-B_{\text {in }}\right)_{p}+\left(\Delta E_{X}\right)_{D} \\
& =\left(B_{\text {out }}-B_{\text {in }}\right)_{p}+T_{O} \Delta S_{C}
\end{aligned}
$$

\footnotetext{
* References cited here are listed at the end of this appendix.
} 
where subscripts $\mathrm{p}$ and ef refer to the process and external fluid, respectively; $\Delta \mathrm{S}_{\mathrm{C}}$ is the entropy creation; and $(\Delta \mathrm{Ex})_{\mathrm{D}}$ is the exergy destruction or loss. The exergy loss includes effects of all types of irreversibilities such as frictional pressure drop in process and external fluids, heat transfer, chemical reactions, and mass transfer.

More specifically, entropy creation as a result of heat transfer from an external fluid to the process is given by

$$
\begin{aligned}
\Delta S_{C} & =\left\{\frac{1}{T_{p}}-\frac{1}{T_{e f}}\right\} d Q \\
& =\frac{\Delta T}{T_{p}\left(T_{p}+\Delta T\right)} d Q
\end{aligned}
$$

where $\Delta \mathrm{T}$ is the heat transfer temperature difference $\left(\mathrm{T}_{e f}-\mathrm{T}_{\mathrm{p}}\right)$. Associated with this entropy production is an exergy destruction equal to $\left(\mathrm{T}_{\mathrm{O}} \Delta \mathrm{S}_{\mathrm{C}}\right)$. One can readily show that the exergy gained by the system is given by

$$
(\Delta \mathrm{Ex})_{\mathrm{P}}=\left\{1-\frac{\mathrm{T}_{\mathrm{O}}}{\mathrm{T}_{\mathrm{p}}}\right\} \mathrm{dQ}=\left\{1-\frac{\mathrm{T}_{\mathrm{O}}}{\mathrm{T}_{\mathrm{ef}}}\right\} \mathrm{dQ}-\mathrm{T}_{\mathrm{O}} \Delta \mathrm{S}_{\mathrm{C}}
$$

When heat is transferred to the process, the exergy required is given by

$$
(\Delta \mathrm{Ex})_{\text {ef }}=\left\{1-\frac{\mathrm{T}_{\mathrm{o}}}{\left(\mathrm{T}_{\mathrm{p}}+\Delta \mathrm{T}\right)}\right\} \mathrm{dQ} ; \Delta \mathrm{T} \geq 0
$$

when heat is transferred from the process to an external fluid, the exergy gained by the external fluid is given by

$$
(\Delta \mathrm{Ex})_{\text {ef }}=\left\{1-\frac{\mathrm{T}_{\mathrm{o}}}{\left(\mathrm{T}_{\mathrm{p}}-\Delta \mathrm{T}\right)}\right\} \mathrm{dQ} ; \Delta \mathrm{T}^{\prime} \geq 0
$$

Equations A2-8 and A2-9 will be used in section A2.3.3 to evaluate the net impact of surplus and deficits of heat shown by histograms.

The last set of exergy relationships used in this report pertain to primary and secondary energy sources. When the primary source is thermal in nature (e.g., nuclear-heated steam, helium, or liquid metal) its exergy is calculated by treating the primary heat carrier as the external fluid. When the primary (or secondary) energy source depends on a chemical reaction, the maximum exergy that can be delivered is given by Equation A2-2, where the state 1 refers to the starting mixture of reactants (e.g., fuel + air) at ambient conditions and state 2 refers to the end point of reaction products (e.g., combustion products), also at ambient conditions. It is important to note that slightly different values of exergy are obtained, depending on whether or not the mixing and demixing entropies are included (see section A2.2.1). The exergy ratio $\left(\alpha_{E}\right)$ is defined as

$$
\alpha_{E}=\frac{\Delta E x}{\Delta \mathrm{H}_{\mathrm{T}^{\prime}} \mathrm{P}_{\rho}}=\frac{\mathrm{B}_{1}-\mathrm{B}_{2}}{\mathrm{H}_{1}-\mathrm{H}_{2}}
$$


Thus, exergy ratio $\left(\alpha_{E}\right)$ represents the "quality" of the energy delivered by the source in question.

At this point energy and exergy efficiencies may be defined for a conversion from one typé of energy form to another as

$$
n_{Q}=\frac{(\Delta \mathrm{H})_{\text {out }}}{(\overline{\Delta \mathrm{H})} \text { in }} ; \eta_{\mathrm{Ex}}=\frac{(\Delta \mathrm{Ex})_{\text {out }}}{(\Delta \mathrm{Ex})_{\text {in }}}
$$

A2.2 EXERGY LOSSES DURING :ENERGY CONVERSION

In this section the following primary and secondary sources of energy are compared on the basis of their exergy ratios (Equation A2-10) and related conversion efficiencies:

$\underline{\text { Primary Sources }}$

Very High Temperature Gas Cooled Reactor (VHTR)

High Temperature Gas Cooled Reactor (HTGR)

Liquid Metal Fast Breeder Reactor (LMFBR)

Light Water Reactor (LWR)

Coal

Natural Gas

Oil

Secondary Sources

Substitute Natural Gas (SNG)

Hydrogen

Electricity

Chemical Heat Pipe (CHP)

- High temperature system (HTCHP)

- Low temperature system (LTCHP)

\section{A2.2.1 Exergy Ratios of Primary and Secondary Energy Forms}

The following assumptions were made in calculating exergy ratios:

- The nuclear reactors are treated as sources of thermal energy based on the coolant temperatures. The degradation inherent in the conversion of the nuclear reaction energy (with a high $\alpha_{\mathrm{E}}$ ) to thermal energy is considered unavoidable in view of materials limitations.

- To simplify thermodynamic calculations, coal is represented by carbon in graphitic state, natural gas and SNG by $\mathrm{CH}_{4}(\mathrm{~g})$, and oil by toluene $\left[\mathrm{C}_{7} \mathrm{H}_{8}(\ell)\right]$. 
Exergy ratios for primary and secondary energy sources are listed in Table A2-1. The following points should be especially noted:

1. Enthalpy and Gibbs' free energy changes for fuel combustion reactions have been calculated on the basis of reactants and products in their standard state (pure components at unit activity).

2. Fuel combusion enthalpies have been computed on the basis of $\mathrm{H}_{2} \mathrm{O}$ (1iquid) as the final product, and therefore represent higher heating values. The exergy ratios do change, though not markedly, if actual stoichiometric mixtures with air are used in the calculations. For example, exergy ratio for a stoichiometric mixture of air and hydrogen at atmospheric pressure is equal to 0.801 -- a $35 \%$ reduction in $\alpha_{E}$.

3. HTCHP and LTCHP exergy ratios are calculated in two different ways: one based on reactants and products in their standard states; the other based on gaseous mixtures at nominal pipeline conditions of $40 \mathrm{~atm}$ and $298 \mathrm{~K}$, with entropy of mixing included.

Table A2-1

EXERGY RATIOS FOR VARIOUS TYPES OF ENERGY SOURCES

Primary Sources

VHTR - Reformer heat
Boiler heat
Average

HTGR

LMFBR

LWR

Coal

Natural Gas

oil
(1225K to $875 \mathrm{~K})$

( $875 \mathrm{~K}$ to $625 \mathrm{~K}$ )

(1225K to $625 \mathrm{~K}$ )

(1050K to $680 \mathrm{~K})$

( $835 \mathrm{~K}$ to $670 \mathrm{~K})$

$(\sim 555 \mathrm{~K})$

$\mathrm{C}(\mathrm{s})+\mathrm{O}_{2}(\mathrm{~g})+\mathrm{CO}_{2}(\mathrm{~g})$.

$\mathrm{CH}_{4}(\mathrm{~g})+2 \mathrm{O}_{2}(\mathrm{~g}) \rightarrow \mathrm{CO}_{2}(\mathrm{~g})+2 \mathrm{H}_{2} \mathrm{O}(l)$

$\mathrm{C}_{7} \mathrm{H}_{8}(l)+9 \mathrm{O}_{2}(\mathrm{~g}) \rightarrow 7 \mathrm{CO}_{2}+4 \mathrm{H}_{2} \mathrm{O}(l)$
Exergy Ratios $\left(\alpha_{E}\right)$

0.71

0.60

0.67

0.65

0.60

0.46

1.002

0.92 .98

Secondary Sources

Electricity

SNG

Hydrogen

HTCHP : EVA-ADAM HYCO (same as natural gas)

$\mathrm{H}_{2}(g)+1 / 2-\mathrm{O}_{2} \rightarrow \mathrm{H}_{2} \mathrm{O}(\mathrm{l})$

$\mathrm{CO}(\mathrm{g})+3 \mathrm{H}_{2}(\mathrm{~g}) \rightarrow \mathrm{CH}_{4}(\mathrm{~g})+\mathrm{H}_{2} \mathrm{O}(\ell)$

$2 \mathrm{CO}(\mathrm{g})+2 \mathrm{H}_{2}(\mathrm{~g}) \rightarrow \mathrm{CH}_{4}(\mathrm{~g})+\mathrm{CO}_{2}(\mathrm{~g})$

LTCHP

$$
\mathrm{C}_{6} \mathrm{H}_{6}(l)+3 \mathrm{H}_{2}(\mathrm{~g}) \rightarrow \mathrm{C}_{6} \mathrm{H}_{12}(l)
$$

1.00

0.92

0.83

$0.60(0.70)$ *

$0.69(0.75)$ *

$0.48(0.61)$ *

$\underline{\text { Steam }}$

$$
\begin{array}{rrr}
\text { Powerplant - Superheat }(165 \mathrm{~b} ; 625 \mathrm{~K} \text { to } 813 \mathrm{~K}) & 0.57 \\
- \text { Boiler (165 b; } 523 \mathrm{~K} \text { feed }) & 0.50 \\
\text { Process Steam Condensation at }-(70 \mathrm{~b} ; 559 \mathrm{~K}) & 0.47 \\
-(35 \mathrm{~b} ; 515 \mathrm{~K}) & 0.42 \\
-(15 \mathrm{~b} ; 471 \mathrm{~K}) & 0.37
\end{array}
$$

* Numbers in parentheses are for 40.5 bars and include entropy of mixing. 
From the values of exergy ratios it is clear that all the primary fuel sources are inherently of high quality. The value of $\alpha_{E}$ greater than unity for coal is due to the fact that reversible oxidation of carbon at ambient conditions is endothermic, and therefore the theoretical work produced is greater than the enthalpy of combustion. In spite of these high exergy ratios, when in actual practice coal, oil, or gas is combusted for producing work, the maximum temperature is limited not by thermodynamic considerations but rather by materials limitations. The effective $\alpha_{E}$ under this limitation is restricted to values below 0.63 (corresponding to a temperature of $\sim 815 \mathrm{~K}$ ) for power production in a steam powerplant.

Another feature of importance is that non-CHP secondary forms all have a very high $\alpha_{E}$. The significance of this feature will become clear in section A2.3, where conversion efficiencies will be discussed.

The values of $\alpha_{E}$ for HTCHP indicate that high-temperature heat from the VHTR alone is of sufficiently high quality to supply the necessary thermal energy for methane reforming. If the exergy ratio is used as an approximate criterion to judge the suitability of a particular source, it can be concluded that VHTR, HTGR, or LMFBR is capable of supplying thermal energy of a higher quality than that needed for LTCHP. Steam from a LWR, however, is only marginally suited to the same purpose.

In addition to the primary and secondary energy, forms, Table A2-1 lists the exergy ratios for various qualities of steam ranging from typical powerplant grade steam to low-pressure process steam. These were calculated by the enthalpy and entropy changes corresponding to the superheating or condensation process.

\section{A2.2.2 Comparison of Overall Energy Efficiencies}

The objective of this section is to illustrate the improvements in overall energy efficiencies by the use of thermal transport through $\mathrm{CHP}$ (both high and low temperature systems) in comparison with alternatives (transport as electricity or SNG). The particular application considered is the delivery of thermal energy in the form of steam to industrial users from a remotely located primary source.

For the HTCHP case, the primary source is assumed to be a VHTR capable of supplying heat to a nuclear reformer. The overall efficiency for this application is compared with that for transmission as electricity. The LTCHP application is based on coal as the primary source with process conditions corresponding to the reference case example in section 13.6 (Chapter 13). The alternative for comparison is assumed to be a combination of coal gasification to SNG, transport of SNG, and a gas-fired boiler.

The energy conversion efficiencies for these four cases are shown in Figure A2-1. The overall efficiencies show clearly the enormous advantage offered by the two CHP systems. While options (b) and (d) in the figure are only two of the many alternatives for energy transport, similar results are obtained with options such as coal-electricity $\rightarrow$ steam and coal $\rightarrow$ hydrogen $\rightarrow$ steam. 
In order to understand why CHP systems offer high overall efficiencies for thermal energy delivery, it is necessary to examine the four options in Figure $\mathrm{A} 2-1$ in a thermodynamic framework. Using the exergy ratios presented earlier in Table A2-1, it is possible to construct a sketch of each conversion step on a scale of exergy ratios. Figure A2-2 shows such a sketch for each of the four options.

The first major point to note from Figure $\mathrm{A} 2-2$ is that the two CHP options provide a path, from the primary source to the ultimate user, that is devoid of an uphill climb; this is not true for options (b) and (d). Thus, a unique feature of CHP systems is that they provide a transportable secondary energy form with an exergy ratio between those of the primary source and the process steam. The alternative secondary forms (electricity, SNG, or hydrogen) are of such high thermodynamic quality that an uphill climb becomes inevitable during the primary conversion. Since an uphill climb (an energy conversion from a lower exergy ratio to a higher one) must be accompanied by some rejection of lowgrade thermal energy, the energy efficiency of such steps is inherently low -$40 \%$ for electric generation and $65 \%$ for gasification.

The second point to be noted from Figure A2-2 is that in option (b) it is theoretically possible to generate electricity at higher efficiency than that from powerplant steam. The degradation represented by a drop from the VHTR exergy ratio to the powerplant steam exergy ratio is necessitated by practical limitations (e.g., materials capability). A similar consideration applies to the coal gasification step in option (d), where the theoretical efficiency is in excess of $100 \%$ but in practice only $65 \%$ is achievable. It appears to be generally true that conversions to secondary energy forms with exergy ratios close to unity exhibit low energy efficiencies regardless of how high the primary source exergy ratio may be.

If the energy investment in producing secondary energy with high exergy ratios were fully recovered at the end-use locations, no overall penalty would accrue for the thermodynamic upgrading at the primary site. For example, if electricity in case (b) of Figure A2-2 or SNG in case (d) could be used at theoretical Carnot efficiencies for generating process steam, the overall efficiencies for both of these options would be high. In reality, however, the final conversion of high-quality secondary forms to low-quality process steam is thermodynamically not very efficient, and the effort expended in upgrading is never recovered during the downhill slide. The inefficiencies in end conversion are particularly evident wien high-grade secondary fuels such as SNG or hydrogen are combusted to generate process steam. In such instances not only the exergy efficiency but also the energy conversion efficiency is lower than $100 \%$ as a result of the stack losses.

Two important conclusions evolve from these comparisons:

- If primary energy needs to be converted, transported, and reconverted to deliver low-grade heat, it would be inefficient and wrong to generate intermediate energy forms with very high exergy ratios.

- The overall energy efficiency for CHP systems is high because both the initial conversion to chemical form and the final reconversion to heat are devoid of excessive losses, since neither step involves an upgrading of energy forms. 
a) HTCHP CASE (ci. Table 6-4)

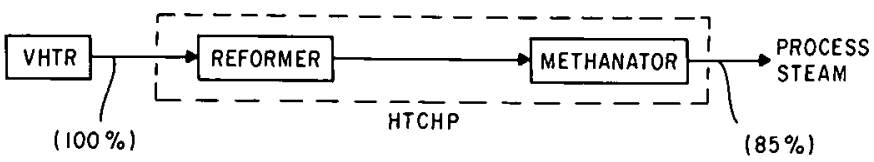

b) ELECTRICAL TRANSMISSION

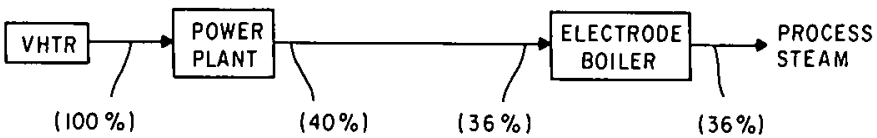

c) LTCHP CASE (cf. Section 13.8)

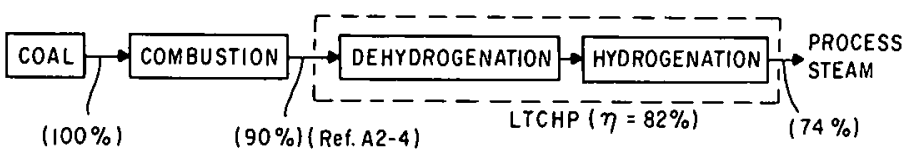

d) SNG OPTION

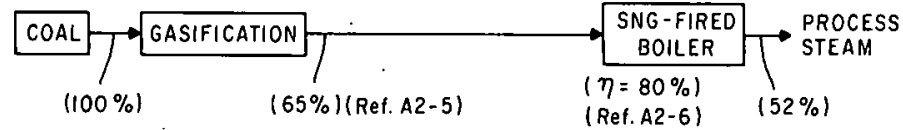

Figure A2-1. Energy Conversion Efficiencies

\section{A2.3 Powerplant Analyses}

In typical CHP designs, thermal exchanges between the reactor effluents and feed streams do not always match completely. As a consequence, these designs. often involve an interdependence between the chemical plant and the steam powerplant as described in Chapter 5 (Ref. A2-7). The purpose of this section is to develop an approximate method for evaluating the net impact of the various thermal exchanges (extraction steam, steam reheat, and feedwater preheat) without having to engage in a detailed design of the steam powerplant for each individual CHP design. The approach used is based on the concepts of exergy analyses.

The critical step in the simplification of powerplant analysis is an assumption of constant exergy efficiency of a steam powerplant. Analysis consists of an evaluation of the net exergy flow due to the various energy exchanges between the powerplant and CHP, followed by an estimation of the net impact (an increase or decrease in the total electricity produced) as a result of this exergy flow.

Section A2.3.1 deals with a typical steam powerplant analysis and contains a justification for the value of exergy efficiency used throughout this report. In section A2.3.2, the method of analysis is applied to published design (Ref. A2-7) of the reformer/steam plant. Finally, in section A2.3.3 details of the reference case (Chapter 5) histogram analysis are given. 

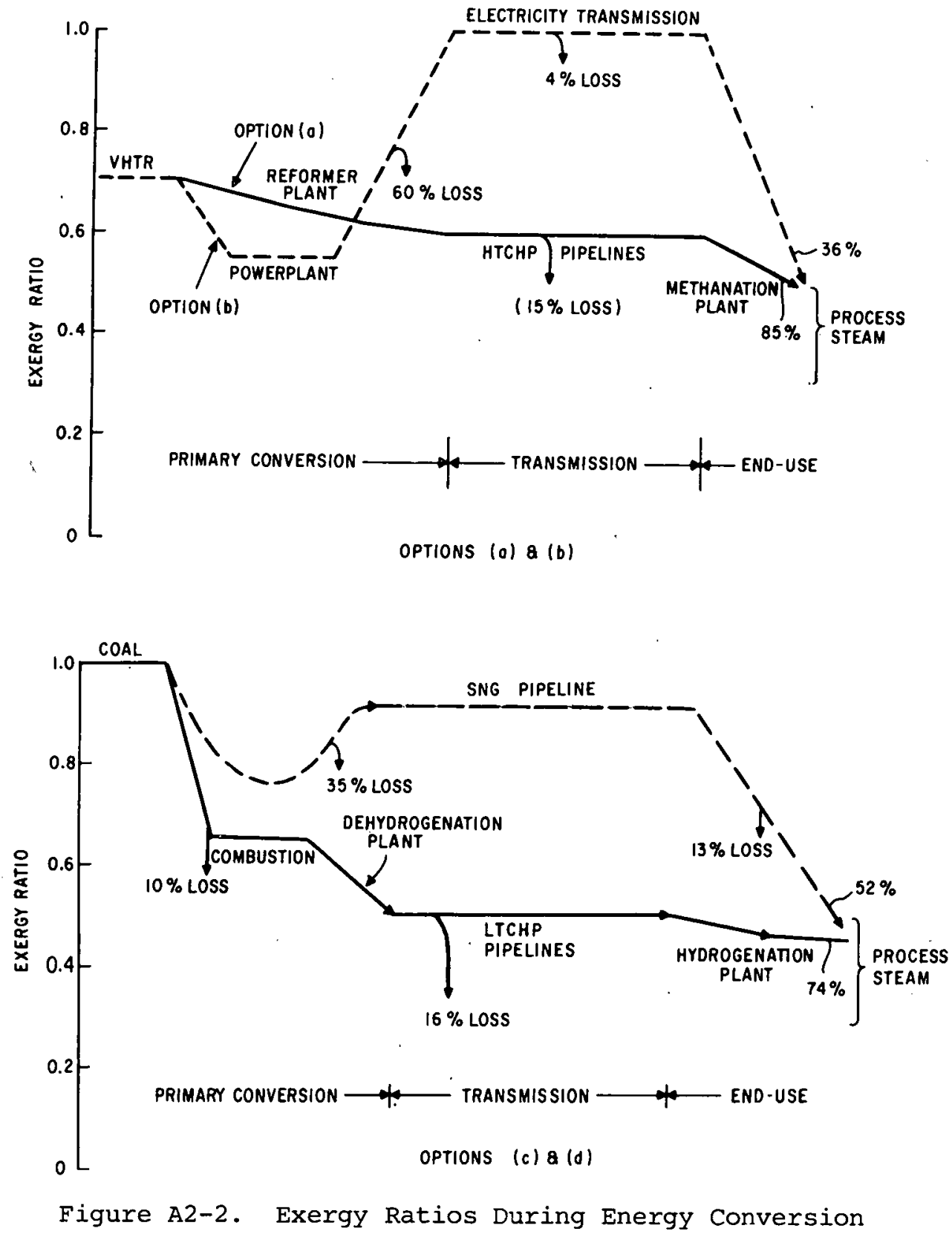

A2.3.1. Exergy Analysis of a Typical Steam Powerplant

Operating conditions of steam powerplants show large variations in pressure, peak temperature, steam reheat, and feedwater preheat (Ref. A2-8). The particular operating conditions selected for the example given here correspond to a typical fossil-fired powerplant:

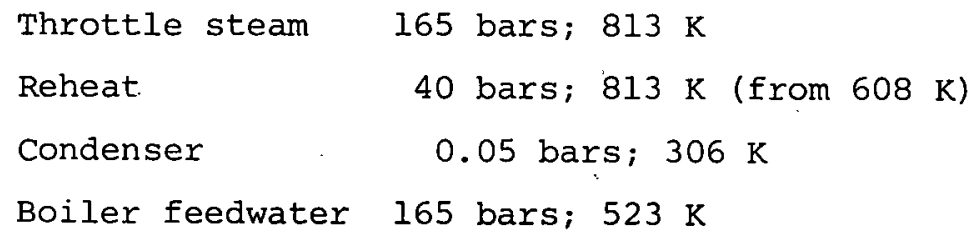


Based on these conditions, thermodynamic properties of steam (Ref. A2-9) and an assumed turbine adiabatic efficiency* of $285 \%$, the energy and exergy flows associatea with each individual step were calculated and are shown in Figure A2-3.

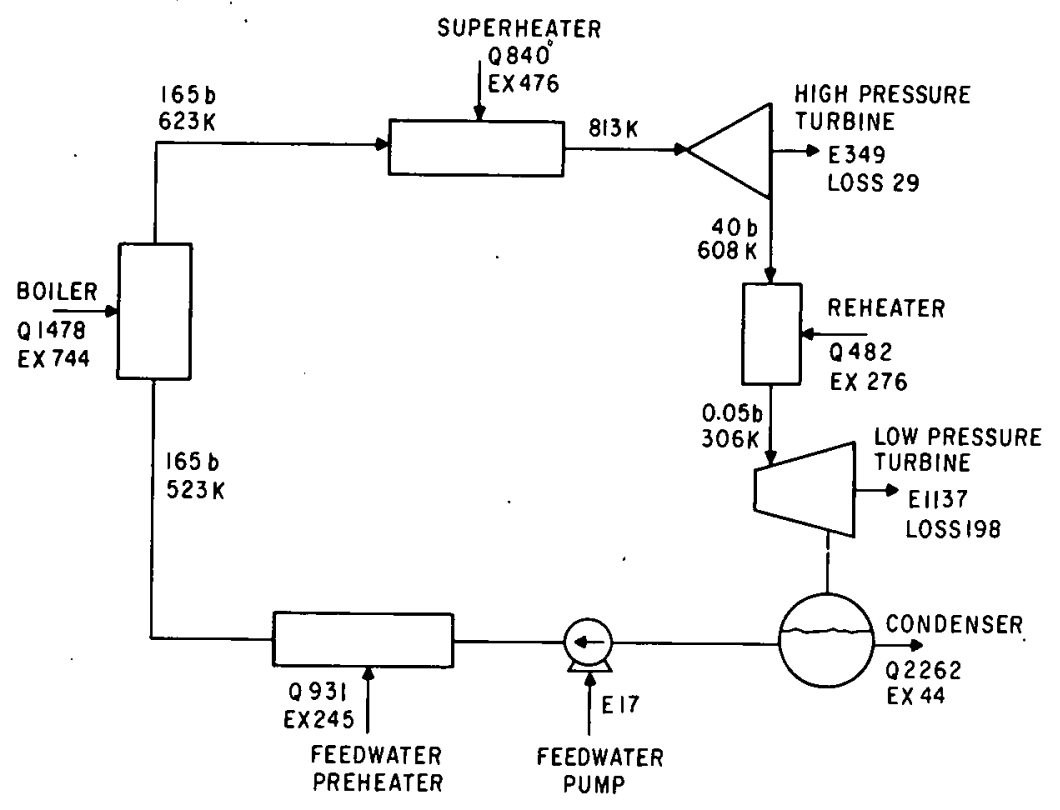

\begin{tabular}{|ccc|}
\hline Q: & ENERGY FLOW & $(\mathrm{J} / \mathrm{g})$ \\
EX: EXERGY FLOW & $(\mathrm{J} / \mathrm{g})$ \\
E: ELECTRICAL FLOW $(\mathrm{J} / \mathrm{g})$ \\
$\mathrm{b}:$ PRESSURE & $(\mathrm{b} 0 \mathrm{rs})$ \\
$\mathrm{K}:$ TEMPERATURE & $(\mathrm{K})$ \\
LOSS: EXERGY LOSS & $(\mathrm{J} / \mathrm{g})$ \\
\hline
\end{tabular}

Figure A2-3. Energy and Exergy Flows in a steam Powerplant

The following points should be noted:

- Exergy flows were calculated using enthalpy and entropy changes for steam and a datum temperature $\left(\mathrm{T}_{\mathrm{O}}\right.$ ) equal to $300 \mathrm{~K}$ (see Equation $\mathrm{A} 2-2$ ). As a result, they exclude losses associated with heat transfer.

- The Figure A2-3 shows a cycle where feedwater preheating is accomplished by an independent source. Changes due to a regenerative feedwater heating are discussed later in this section.

The overall energy efficiency of the cycle is calculated as the ratio of net work produced $(349+1137-17=1469 \mathrm{~J} / \mathrm{g})$ and the total thermal input

*Adiabatic efficiency is the ratio of actual enthalpy change or work and that for an ideal (constant entropy) change for the same pressures. 
$(1478+840+482+931=3731 \mathrm{~J} / \mathrm{g})$, equal to $39.4 \%$. On the basis of exergy flows, the exergy efficiency is obtained as the ratio of net work (1469 $\mathrm{J} / \mathrm{g}$ ) and the total exergy input $(744+476+276+245=1741 \mathrm{~J} / \mathrm{g})$, equaling $84.4 \%$.

Similar analyses performed on different steam plants show that typical exergy efficiencies are always in the vicinity of 85\%, even though the actual energy efficiencies vary over a wider range. For example, calculation of the net work produced by a cycle with regenerative feedwater heating would show a decrease from $1469 \mathrm{~J} / \mathrm{g}$ to approximately $1250 \mathrm{~J} / \mathrm{g}$; this would correspond to an increase in energy efficiency from $39.4 \%$ to $45 \%$ based on a new total energy input of only $2800 \mathrm{~J} / \mathrm{g}(1478+840+482)$. On the exergy basis, however, the work produced is still $284 \%$ of the total exergy input of $1496 \mathrm{~J} / \mathrm{g}(744+476+$ 276).

This example demonstrates that the assumption of a constant exergy efficiency is a useful approximation for evaluating the effect of plant modifications (changes involving reheat, feedwater preheat, or steam extraction) on the total work output. Admittedly, it neglects many subtleties of powerplant designs. What it does provide is a convenient rule-of-thumb procedure for estimating the net effect of thermal energy exchange with a steam plant without the need of a detailed design for each modification.

Throughout this report an exergy efficiency of $85 \%$ was assumed in evaluating changes in powerplant work output as a result of exergy changes in the powerplant steam.

\section{A2.3.2 Exergy Analysis of the KFA Reformer Design}

The method of exergy analysis described in the previous section can be applied to analysis of nuclear reformer designs. One example is the KFA design (Ref. A2-7) based on a pebble bed nuclear reactor (PBR) source. As shown in Figure A2-4, the primary thermal energy from the PBR is used for heating reformer tubes as well as for generating powerplant steam. The energy flows in the reformer and powerplants are coupled through three energy exchanges, identified in Figure $\mathrm{A} 2-4$ as

(1) A large extraction of steam after the high-pressure turbine expansion provided to the reformer plant.

(2) Reheat supplied to the powerplant for the remaining fraction of steam.

(3) Feedwater preheat provided to the powerplant.

The approach taken in analyzing these exchanges consists of decoupling the powerplant from the reformer plant with across-the-fence exchanges of thermal energy. These exchanges are visualized in Figure A2-5, where the total steam flow $(2550 \mathrm{t} / \mathrm{h})$ is divided into two separate streams -- one part $(823 \mathrm{t} / \mathrm{h})$ going through a full power. cycle, and the remainder $(1727 \mathrm{t} / \mathrm{h})$ undergoing complete extraction after the first turbine. The detailed energy. and exergy flows for both streams are shown in Figure A2-5 (a) and (b). The energy flows shown here 


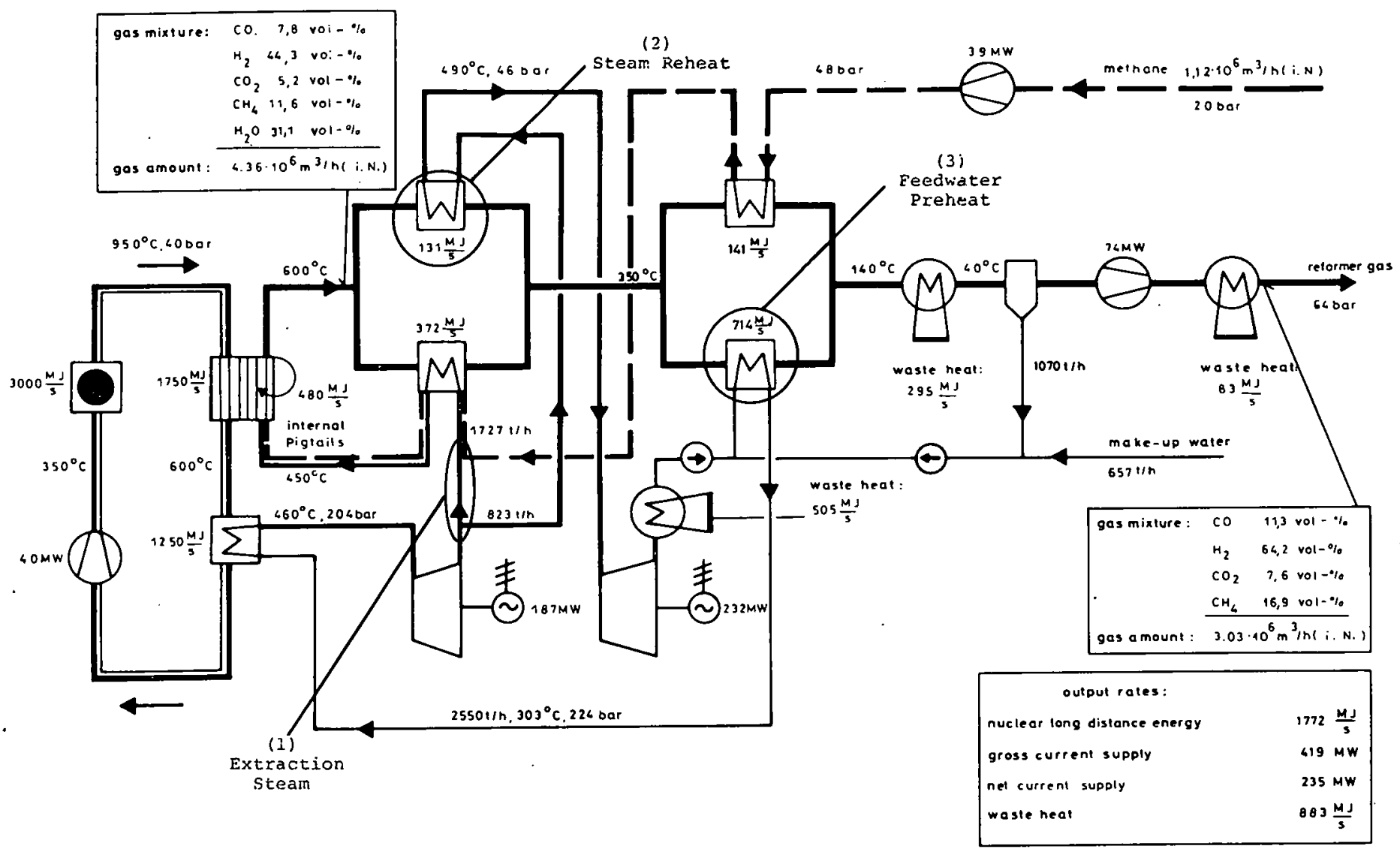

Figure A2-4. KFA Design; Reproduced from Nuclear Engineering and Design (34:1, 1975 , p. 69) with Permission of the Publishers

differ by small amounts from those in the original KFA design. In the absence of details of temperatures and pressures (Ref. A2-7) a set of conditions consistent with standard steam tables (Ref. A2-9) was used.

The reformer plant accepts $1281 \times 10^{6} \mathrm{~J} / \mathrm{sec}$ as extraction steam from the powerplant and in return provides $131 \times 10^{6} \mathrm{~J} / \mathrm{sec}$ as steam reheat as well as $(553+258=811) \times 10^{6} \mathrm{~J} / \mathrm{sec}$ as feedwater preheat. In terms of exergy flows, these exchanges amount to a net. loss of $165 \mathrm{MW}$ from the powerplant. At the assumed exergy efficiency of $85 \%$, the loss of electricity produced by the powerplant is equal to 140.5 MW. Such a detailed allocation of penalties may appear unwarranted until one realizes that the reformer penalty amounts to approximately $7 \%$ of the transported thermal energy. Since the price of electricity is usually three times that of heat, the loss of revenues due to this reduction in power output amounts to nearly $20 \%$ of the delivered thermal energy.

To countercheck the result of exergy analysis, the loss of electricity can be estimated by an alternate approach. Were it not for exchanges with the reformer plant, the powerplant could have produced electricity at an overall energy efficiency of 45\% (as shown in section A2.3). For an input of $1.25 \mathrm{GJ} /$ sec, this amounts to $562.5 \mathrm{MW}$, implying a loss of $143.5 \mathrm{MW}$ for the actual design production (419 MW). The remarkable agreement between the two estimates lends added support to the exergy analysis. 


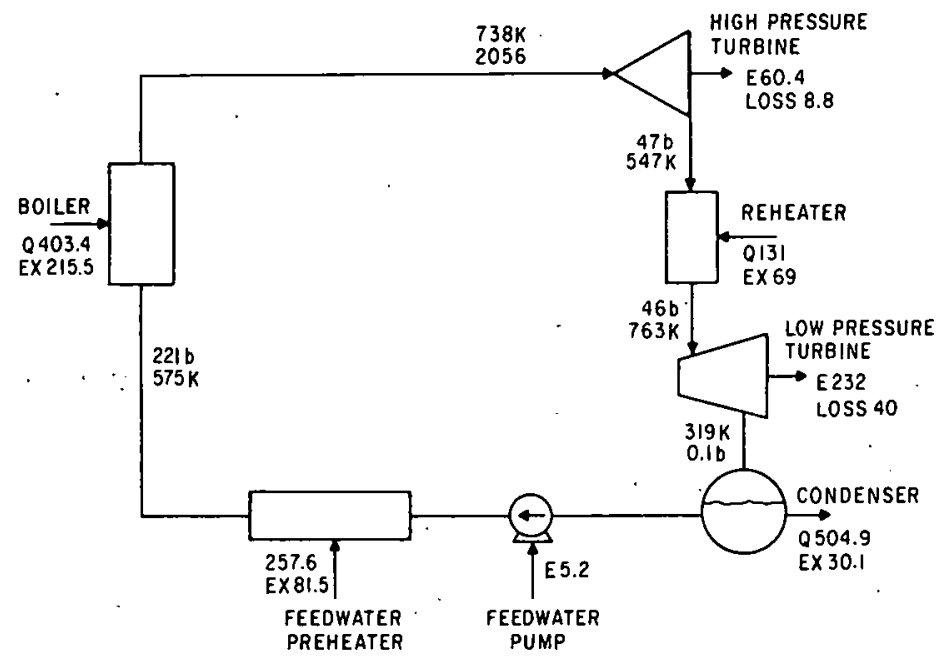

o) POWER CYCLE PART OF STEAM $\left(823 \mathrm{t} / \mathrm{h}\right.$ OR $\left.0.2286 \times 10^{6} \mathrm{~g} / \mathrm{s}\right)$

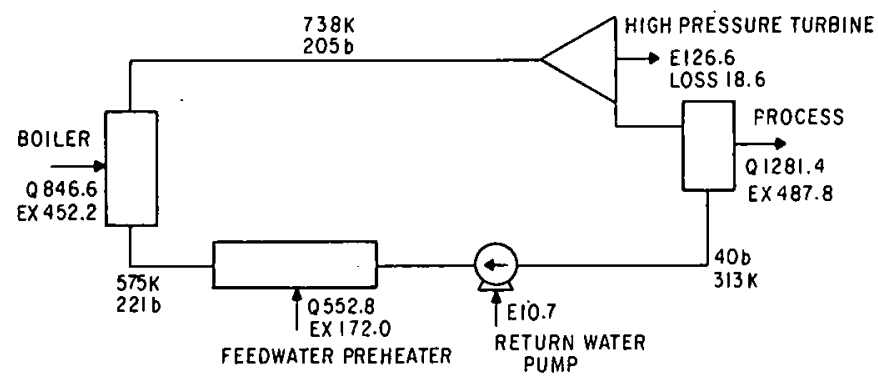

b) EXTRACTION CYCLE PART OF STEAM $\left(1727 \mathrm{t} / \mathrm{h}\right.$ OR $\left.0.4797 \times 10^{6} \mathrm{~g} / \mathrm{s}\right)$

\begin{tabular}{|lll|}
\hline Q: ENERGY FLOW. & $\left(10^{6} \mathrm{~J} / \mathrm{s}\right.$ or MW $)$ \\
EX: EXERGY FLOW & $\left(10^{6} \mathrm{~J} / \mathrm{s}\right)$ \\
E: ELECTRICAL FLOW & $\left(10^{6} \mathrm{~J} / \mathrm{s}\right)$ \\
D: PRESSURE & $\left(\mathrm{bOrr}_{\mathrm{s}}\right)$ \\
$\mathrm{K}:$ TEMPERATURE & $(\mathrm{k})$ \\
LOSS: EXERGY LOSS & $\left(10^{6} \mathrm{~J} / \mathrm{s}\right)$ \\
\hline
\end{tabular}

Figure A2-5. Energy and Exergy Flows in KFA Design

This example demonstrates the simplicity and the usefulness of exergy anal.ysis to assess the true penalty of thermal exchanges between the CHP and the powerplant. In addition, it shows that the energy exchanges to balance reformer heat duties result in a significant penalty which must be considered in calculating the overall efficiency of CHP systems.

\section{A2.3.3 Exergy Analyses Based on Histograms}

The method by which net exergy penalties were calculated for use in section 6.2. (Chapter 6) is described in this section. The reference case design with heat transfer drop $(\Delta T)$ of $20 \mathrm{~K}$ is used here as a numerical example. Table A2-2 shows the required heating and cooling duties for each $20 \mathrm{~K}$ temperature increment over the range of temperatures from 300 to $873 \mathrm{~K}$. Also shown are the exergy surplus and deficits, which were calculated by the use of Equations A2-9 and A2-8 respectively. For simplicity, arithmetic mean temperatures within each $20 \mathrm{~K}$ increment were used rather than the logarithmic mean (In). temperatures.

The following examples will demonstrate the procedure:

1. In the range 440 to $460 \mathrm{~K}, 84.42 \mathrm{MW}_{\text {th }}$ are needed. This heat can be supplied by the cooling duty in the range 460 to $480 \mathrm{~K}$ of $209.9 \mathrm{MW}_{\mathrm{th}}$. 
Table A2-2

HISTOGRAM AND EXERGY ANALYSIS FOR PEFERENCE CASE REFORMER

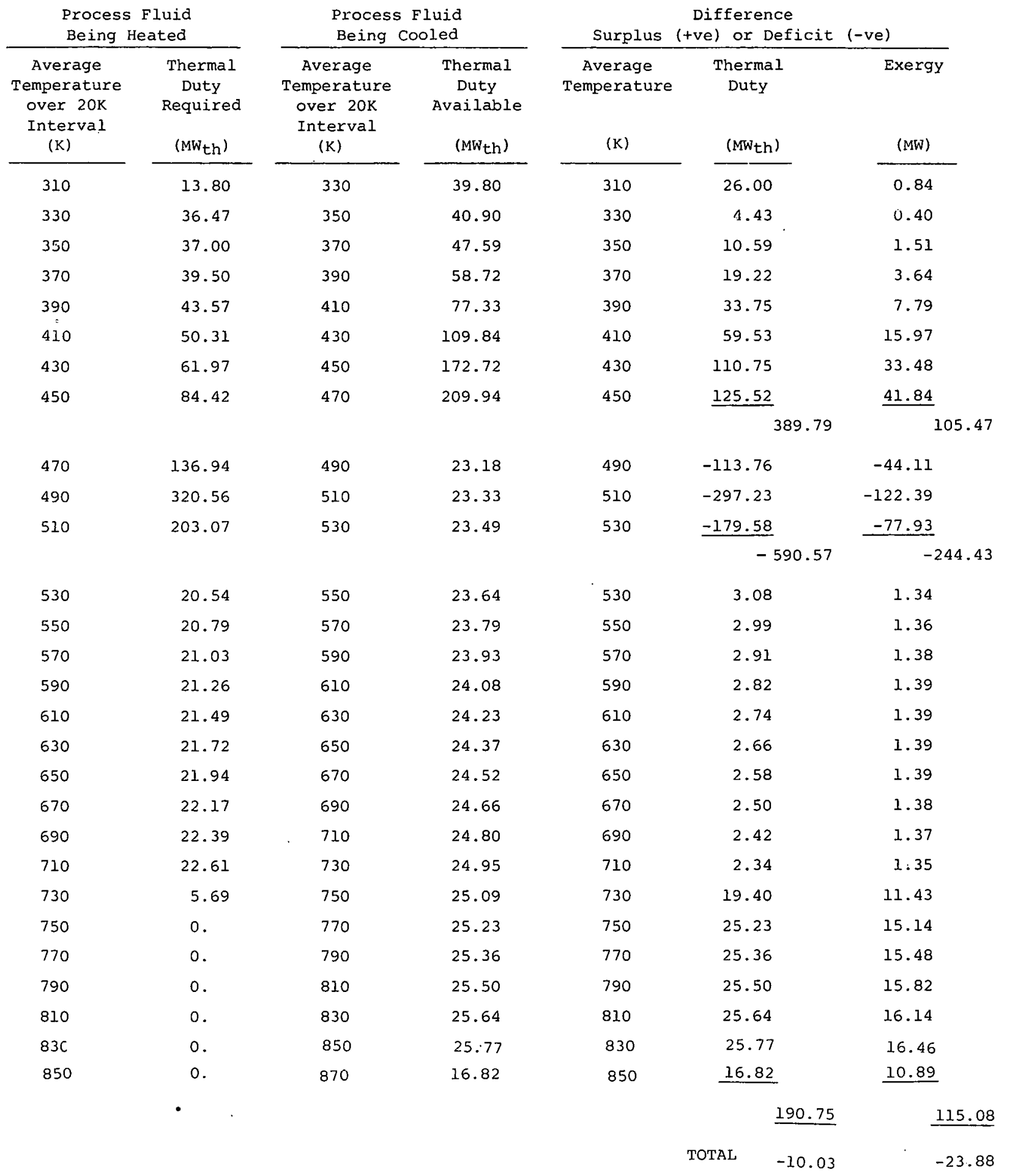


Excess energy (available to powerplant)

Temperature at which this heat is available

Exergy credit to the reformer
$=125.52 \mathrm{MW}_{\mathrm{th}}$

$=470 \mathrm{~K}-20 \mathrm{~K}=450 \mathrm{~K}$

$=125.52 \times 1-\frac{300}{450}$

$$
=41.84 \mathrm{MW}
$$

2. In the range 500 to $520 \mathrm{~K}$, the process needs $203.07 \mathrm{Mw}_{\mathrm{th}}$. Only $23.49 \mathrm{MW}_{\text {th }}$ is available at 520 to $540 \mathrm{~K}$.

Energy deficit (needed from powerplant) $=179.58 \mathrm{MW}_{\text {th }}$ Temperature at which powerplant must supply this heat $=510 \mathrm{~K}+$ $20 \mathrm{~K}=530 \mathrm{~K}$

Exergy debit $=179.58 \times\left(1-\frac{300}{530}\right)=77.93 \mathrm{MW}$

It should be noted that the net availability penalty is based on exergy changes in process streams rather than the powerplant steam, and therefore does not account for any further degradation due to a mismatch with steam heat capacity. It is difficult to estimate the magnitude of further exergy losses, since the exact steam conditions for the powerplant are not known. Furthermore, since coupled powerplants of this nature would be a new design, it is not clear which of the excess energy flows should be utilized by the powerplant in an optimum design. Sample calculations show that estimates of this additional penalty may range from 20 to $30 \mathrm{MW}$.

For the sake of completeness, detailed histograms for all of the design cases studied in Chapter 6 are included in this appendix as Figures A2-6 to A2-25.

\section{A2.4 IRREVERSIBILITIES AND ENTROPY PRODUCTION IN THE REFORMER PLANT}

The importance of thermodynamic efficiency in various aspects of CHP systems has been evident in discussions in previous sections of this appendix. In this last section, by an estimate of the magnitude of entropy production rates, a better appreciation of the impact of various irreversibilities may be gained. The reformer plant design for the reference case (see Chapters 5 and 6) will be used once again as an example. These calculations of entropy production and exergy destruction rates have proved particularly important in an improved understanding of the CHP designs and in inventing ways to imprnve the thermodynamic efficiency (cf Chapter 5).

\section{A2.4.1 Frictional Irreversibilities}

Since a detailed accounting of equipment pressure drops for each of the design cases is beyond the scope of this preliminary study, the assumed compression power requirements given in Table 6-4 (Chapter 6) will be used. For the reference case design the total compressor power requirement to overcome equipment pressure drops for reformer and methanation plants was $19.3 \times 10^{6} \mathrm{~J} / \mathrm{s}$. If half of this is attributed to the reformer plant, the rate of entropy production equals $32.2 \mathrm{~kJ} / \mathrm{K}$ sec. Based on an average molar flow rate of $110 \times 10^{6}$ gmoles/hr or $30.6 \times 10^{3}$.gmoles/sec, this would correspond to a pressure drop of approximately 5.6 bars. 


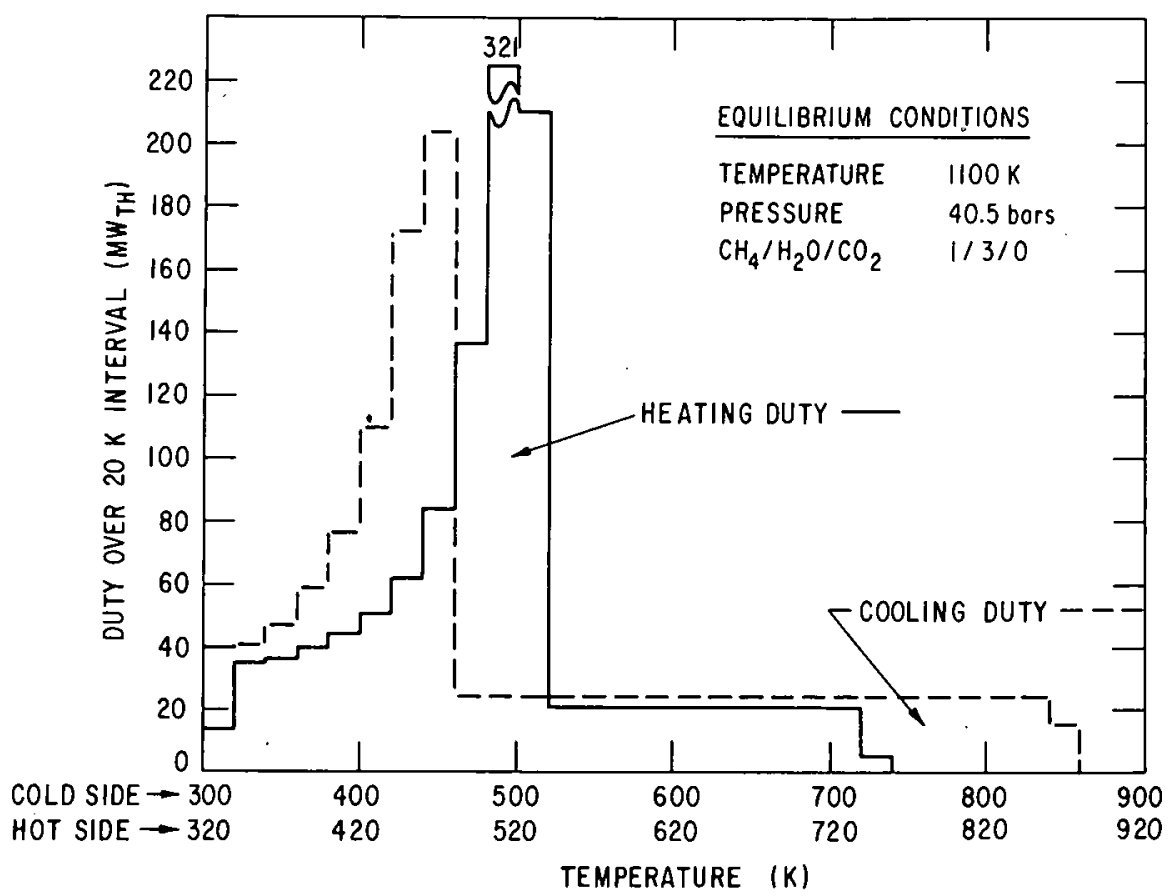

Figure A2-6. Reference Case - Reformer Plant

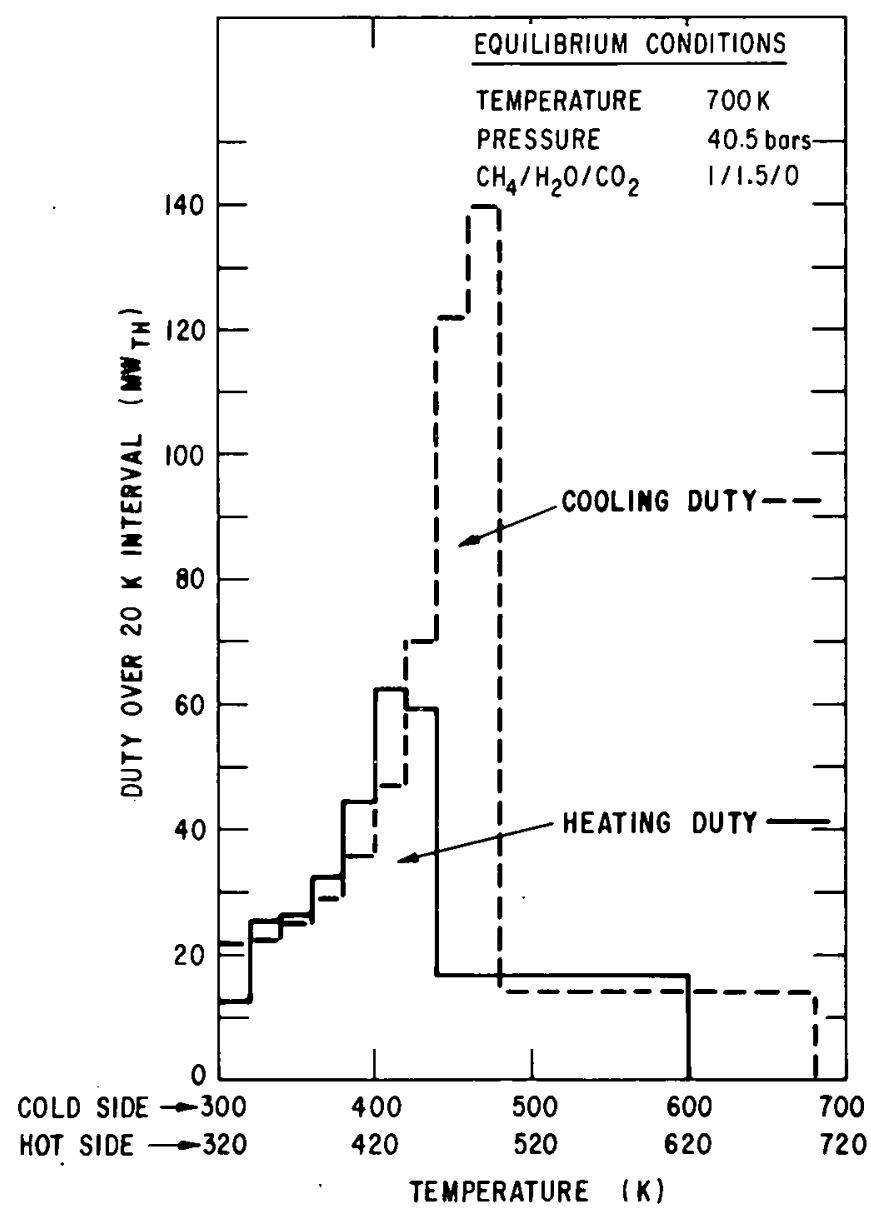

Figure A2-7. Reference Case - Methanation Plant 


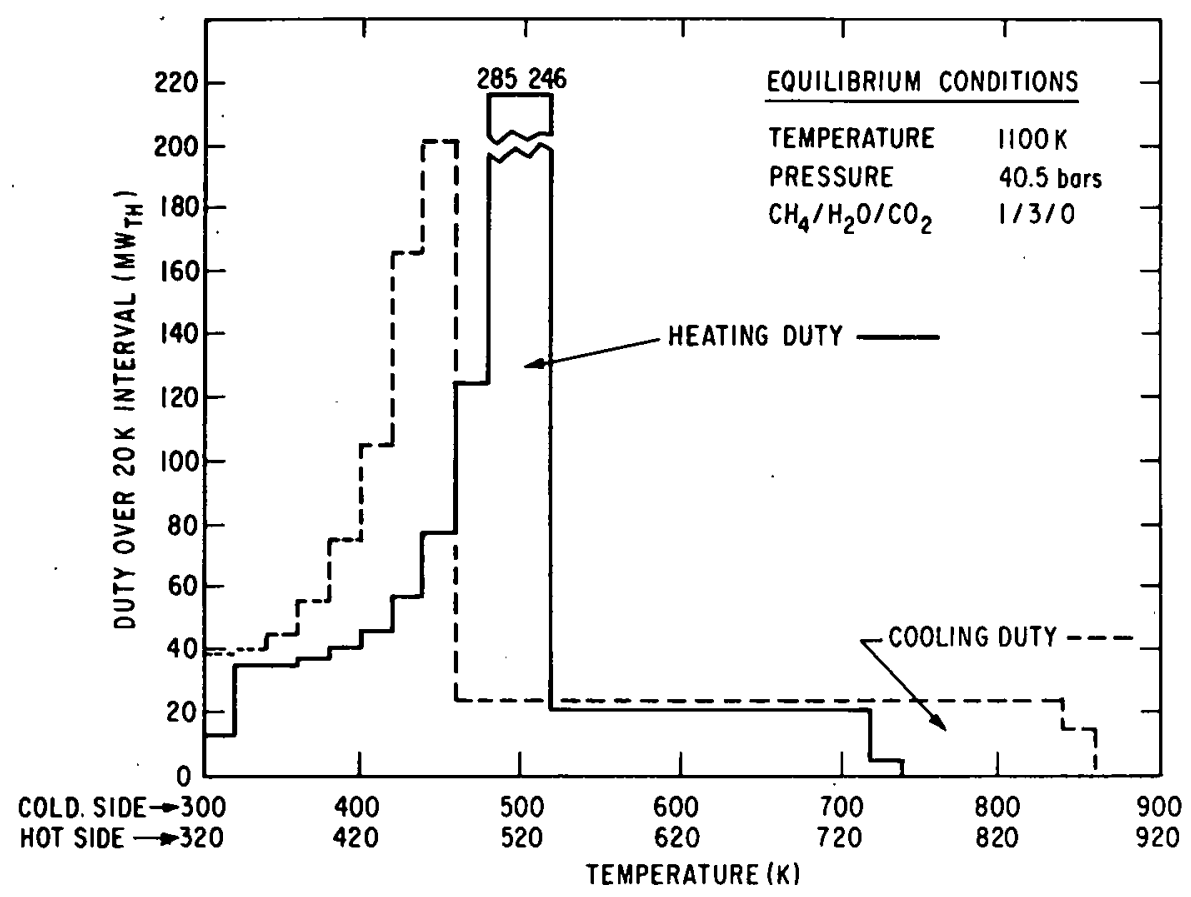

Figure A2-8. Case A - Reformer Plant

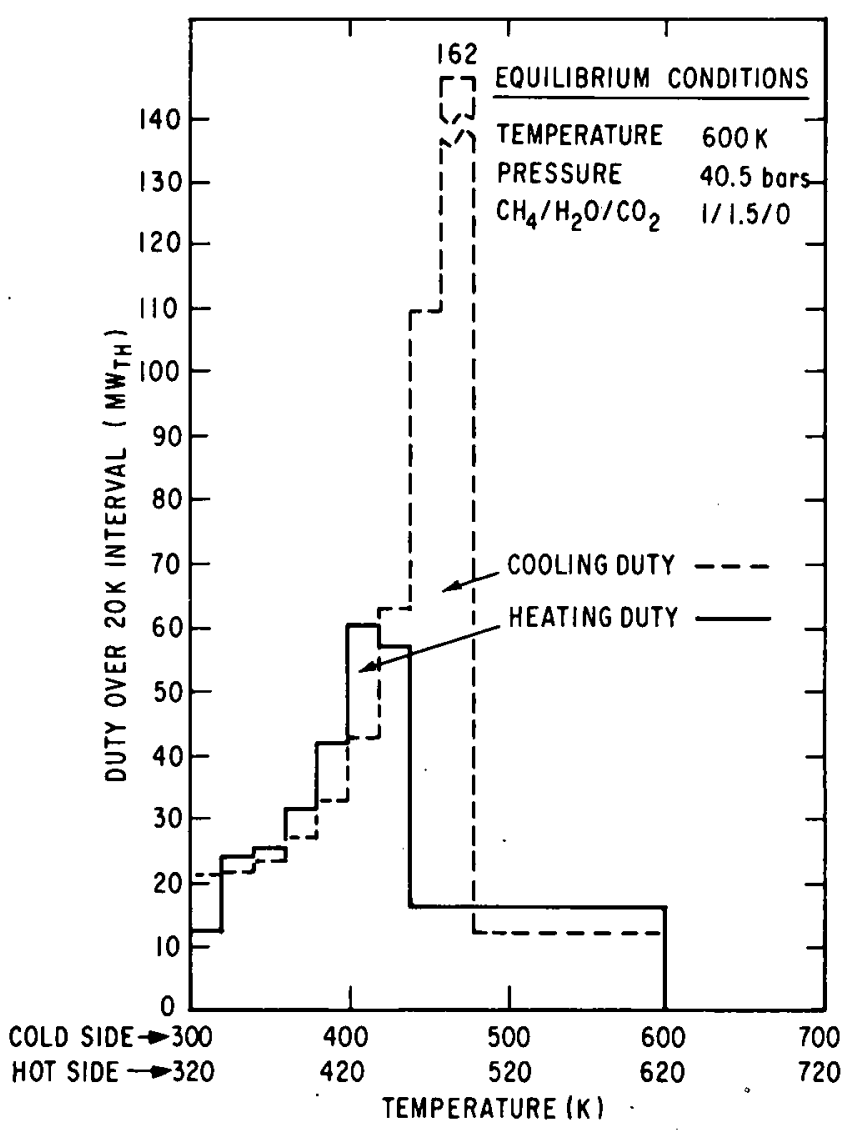

Figure A2-9. Case: A - Methanation Plant 


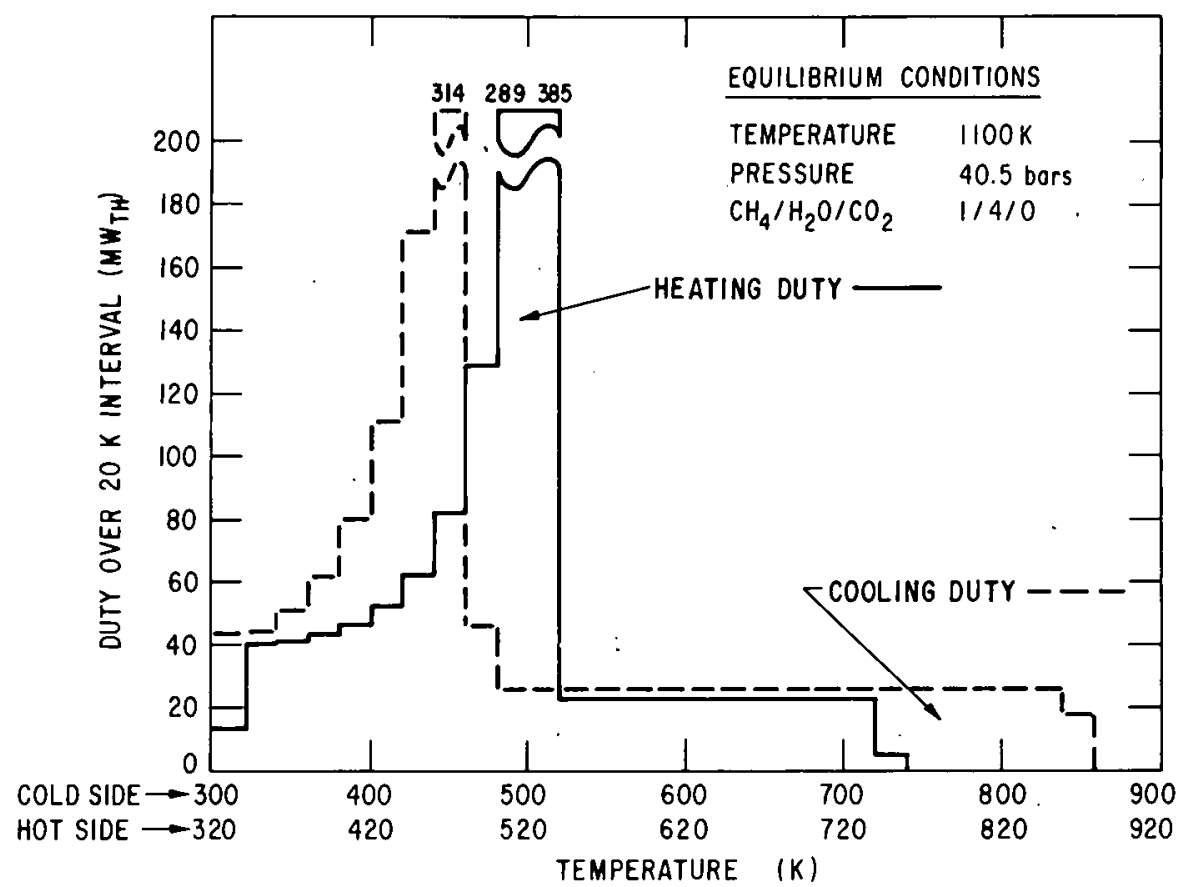

Figure A2-10. Case B - Reformer Plant

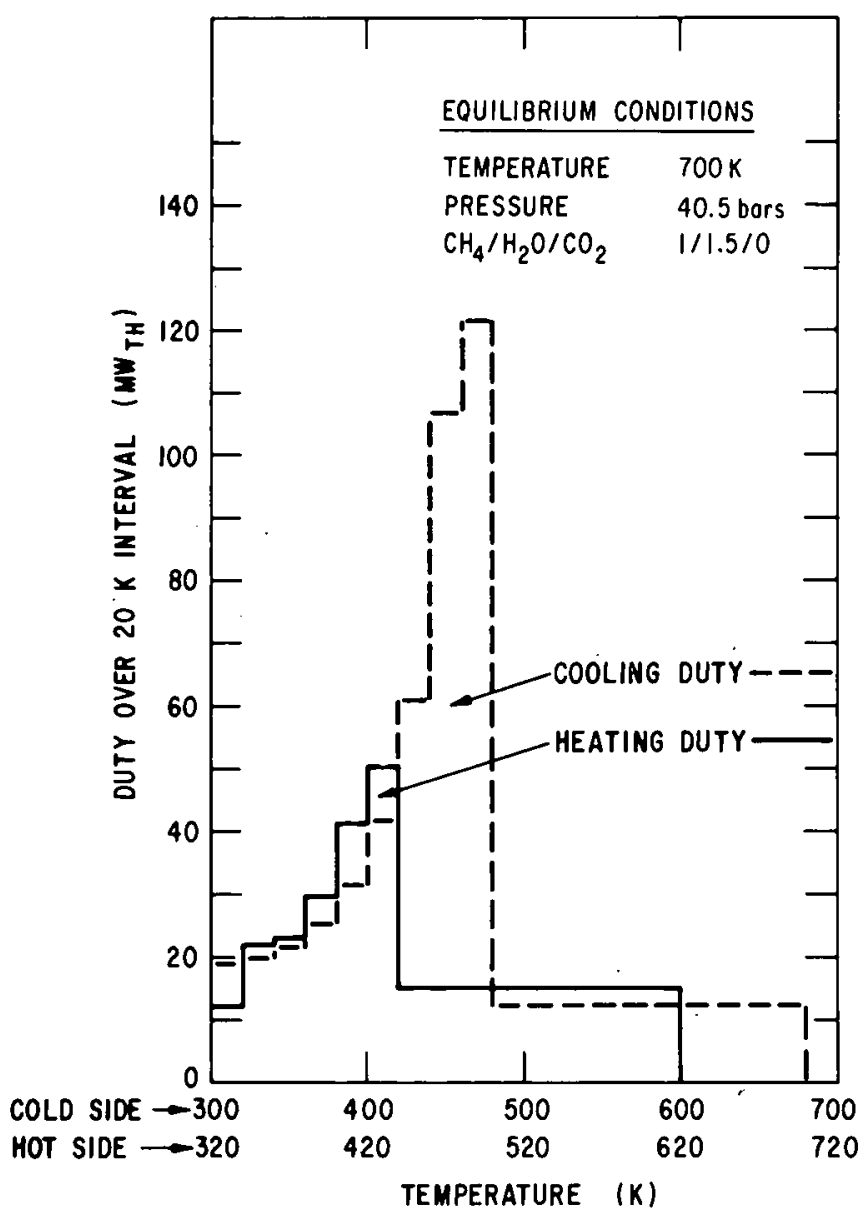

Figure A2-11. Case B - Methanation Plant 


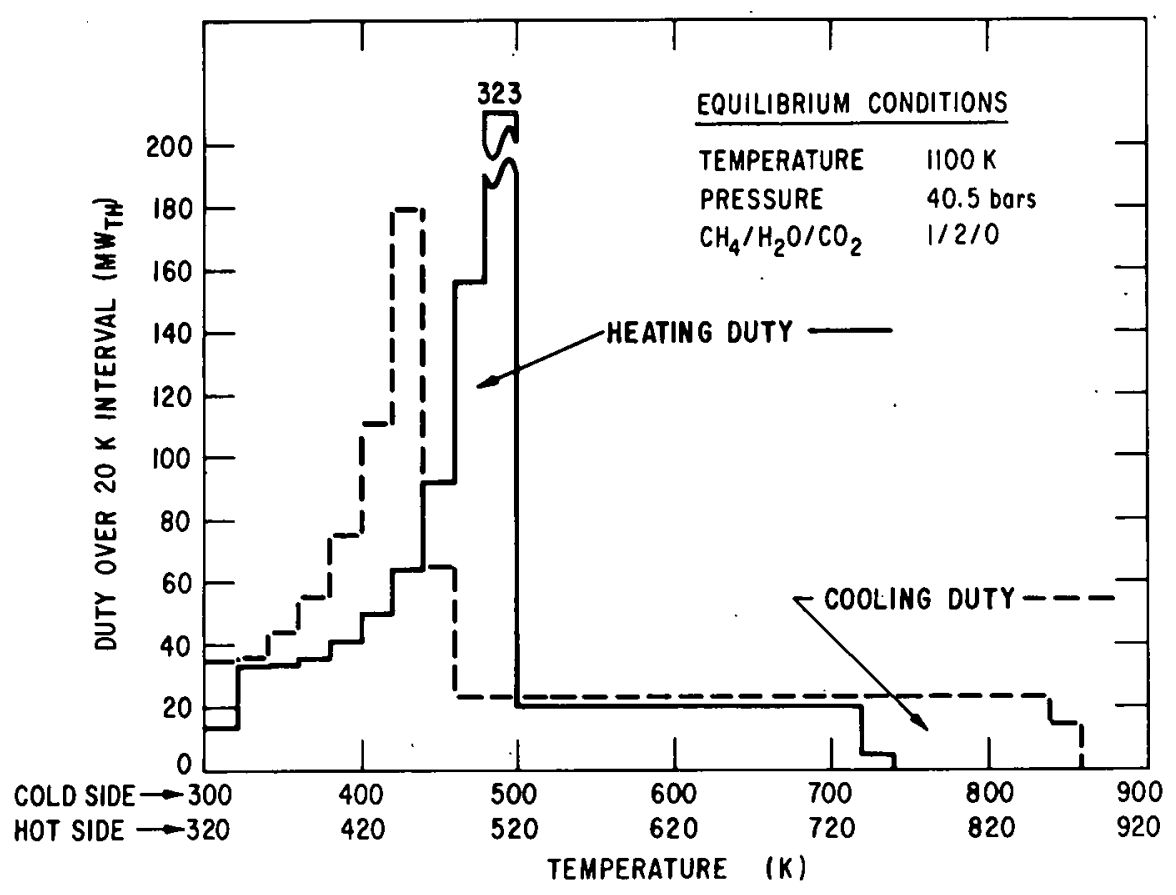

Figure A2-12. Case C - Reformer Plant

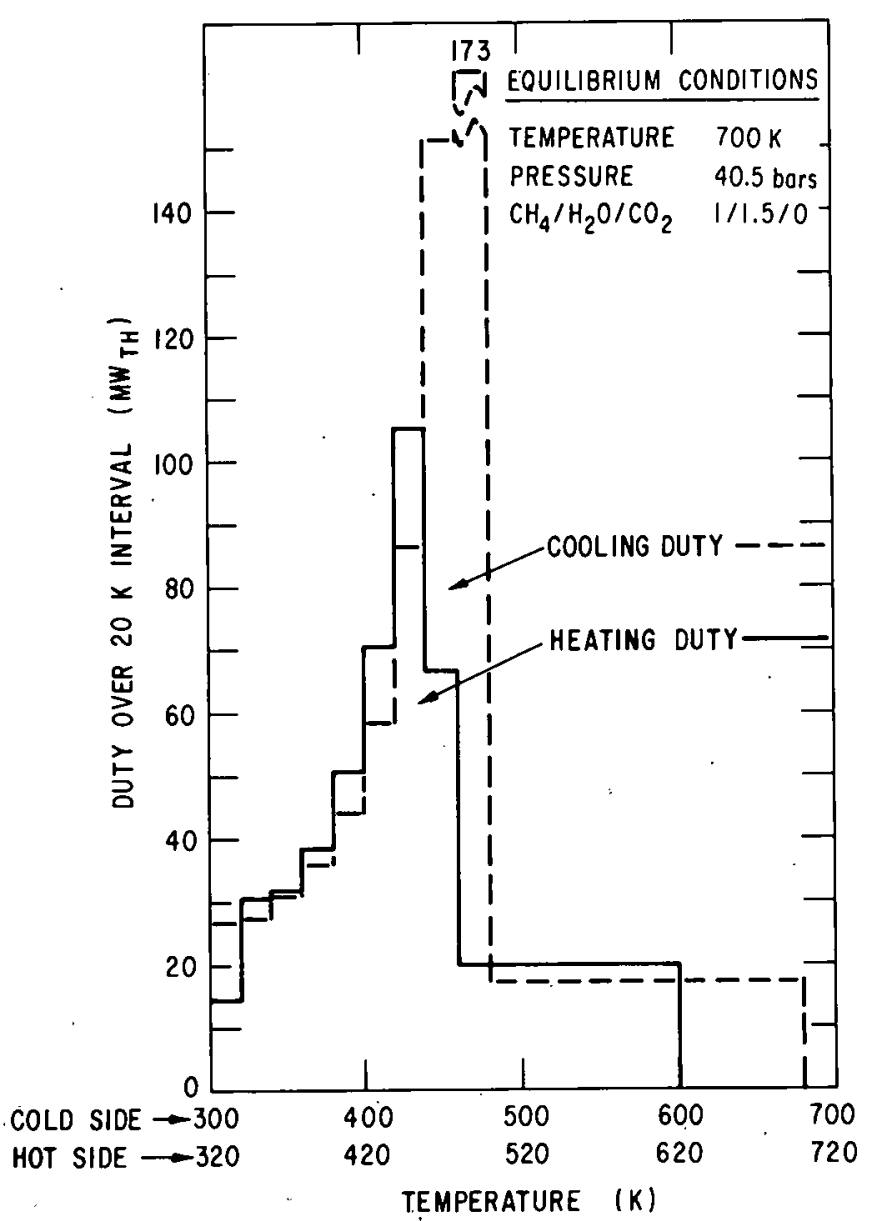

Figure A2-13. Case C - Methanation Plant 


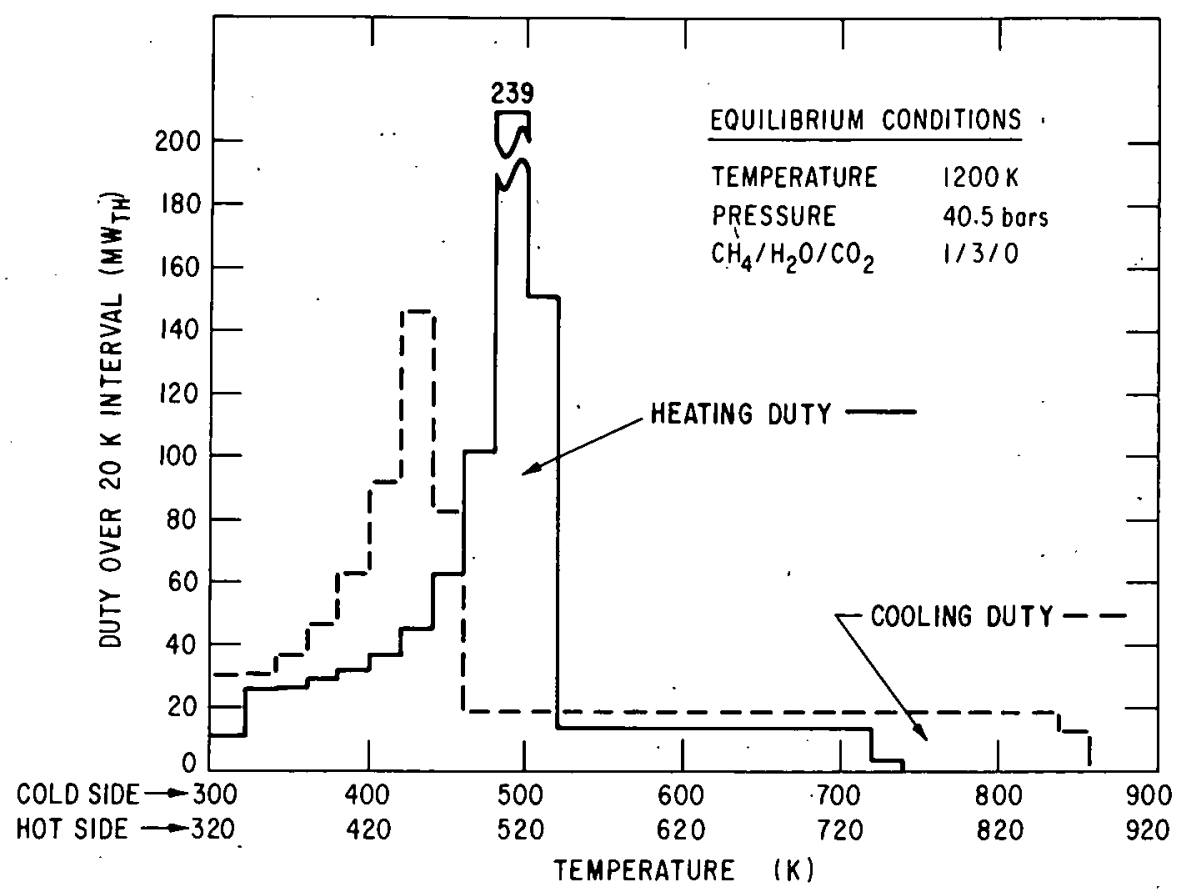

Figure A2-14. Case D - Reformer Plant

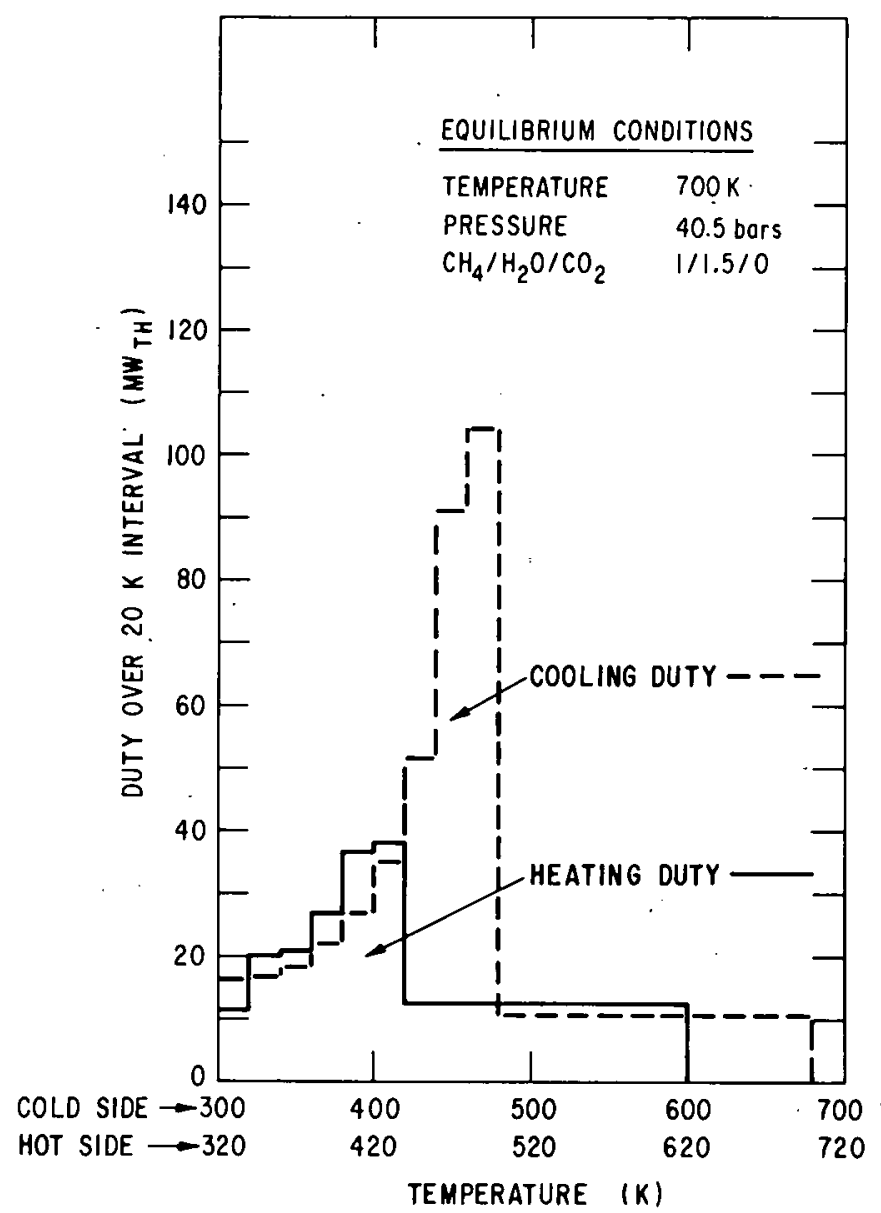

Figure A2-15. Case D - Methanation Plant 


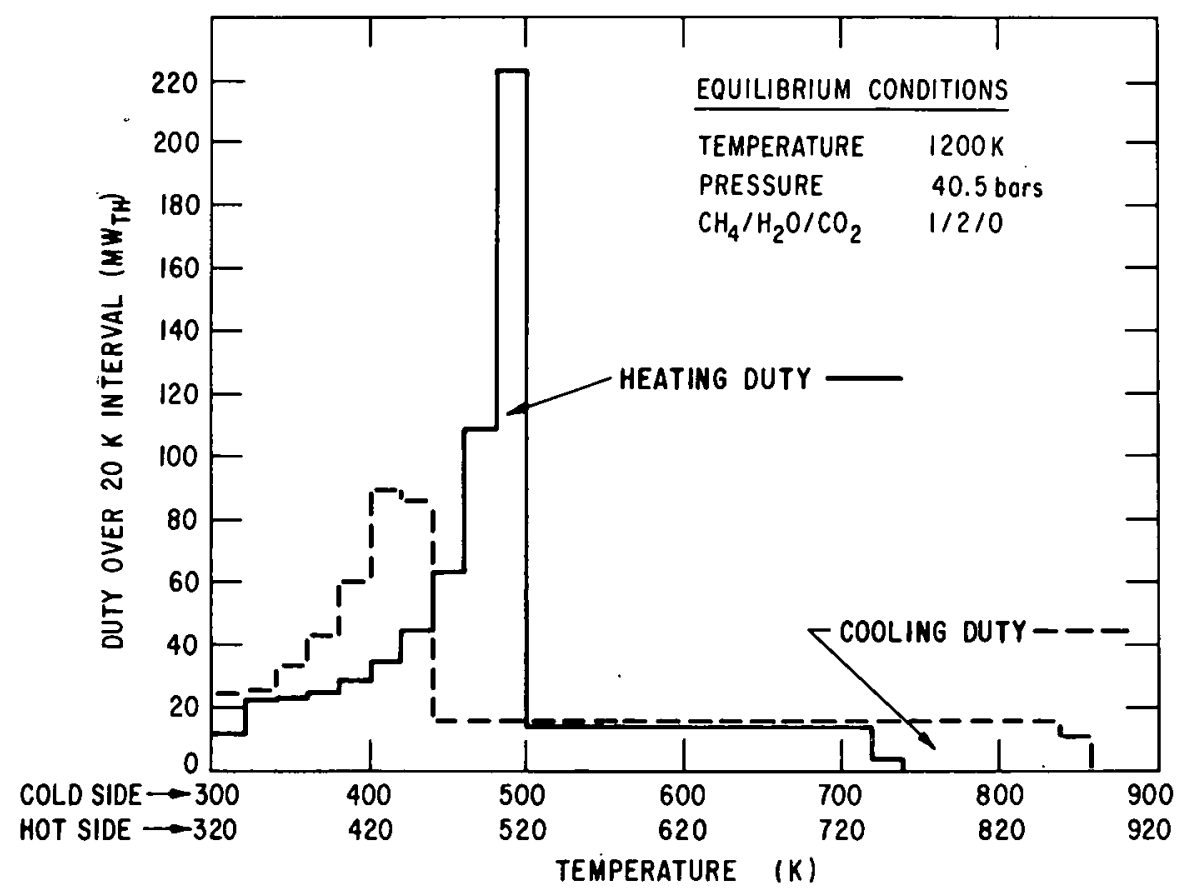

Figure A2-16. Case E - Reformer Plant

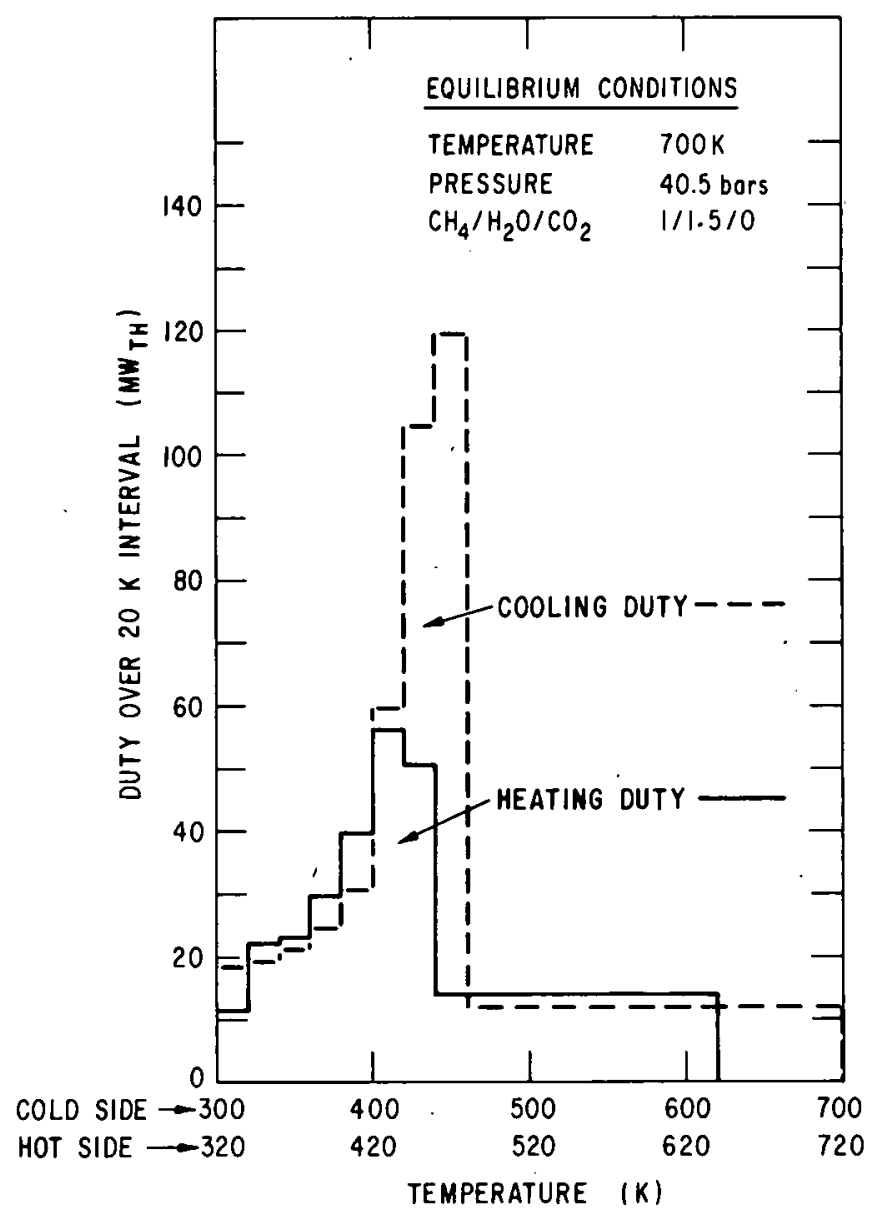

Figure A2-17. Case E - Methanation Plant 


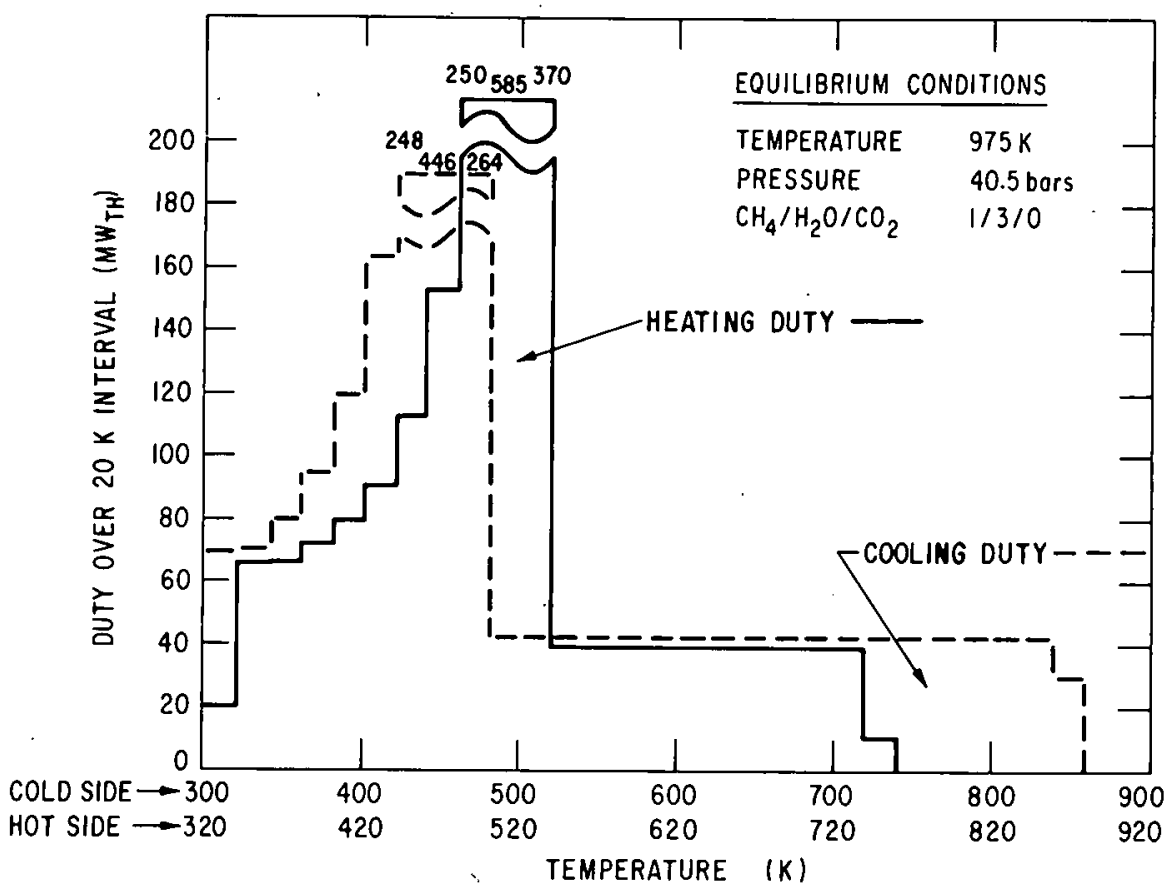

Figure A2-18. Case F - Reformer Plant

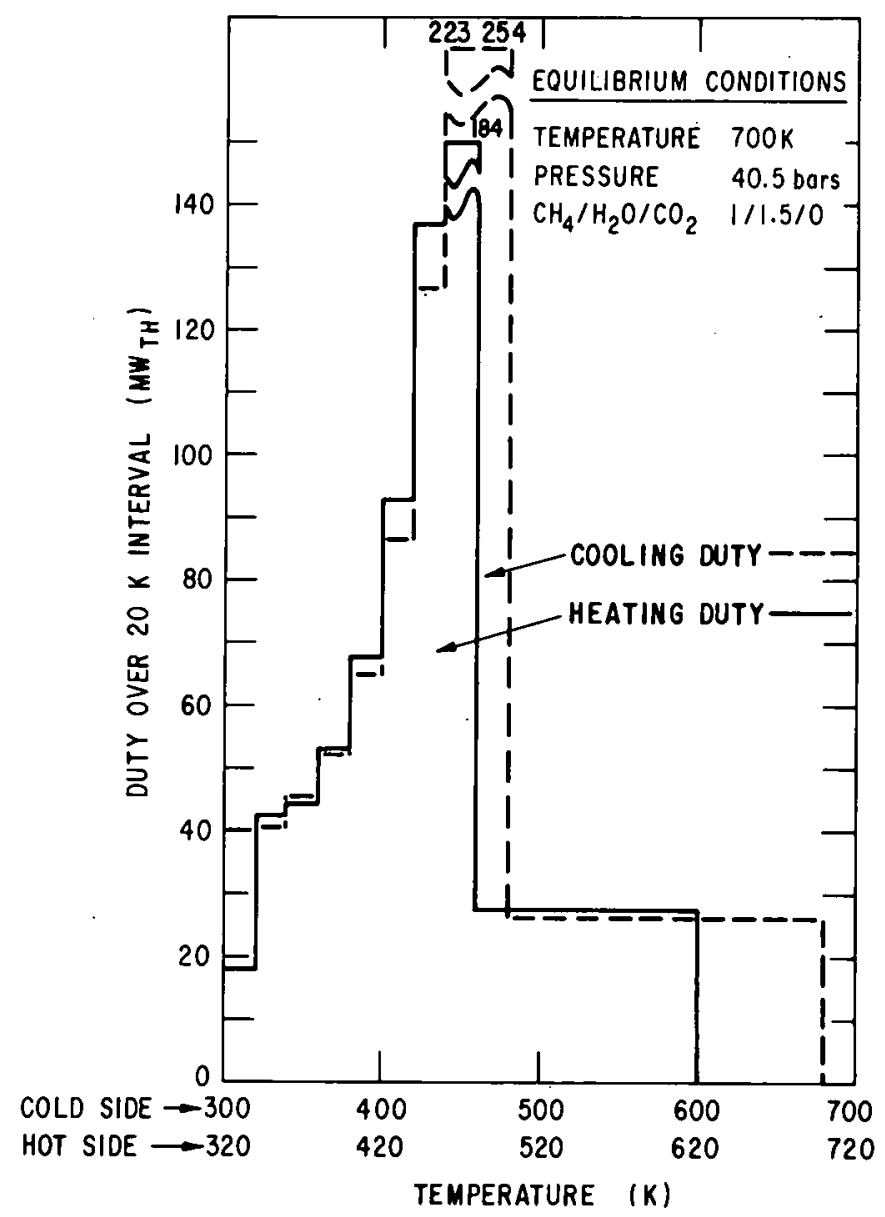

Figure A2-19: Case F - Methanation Plant 


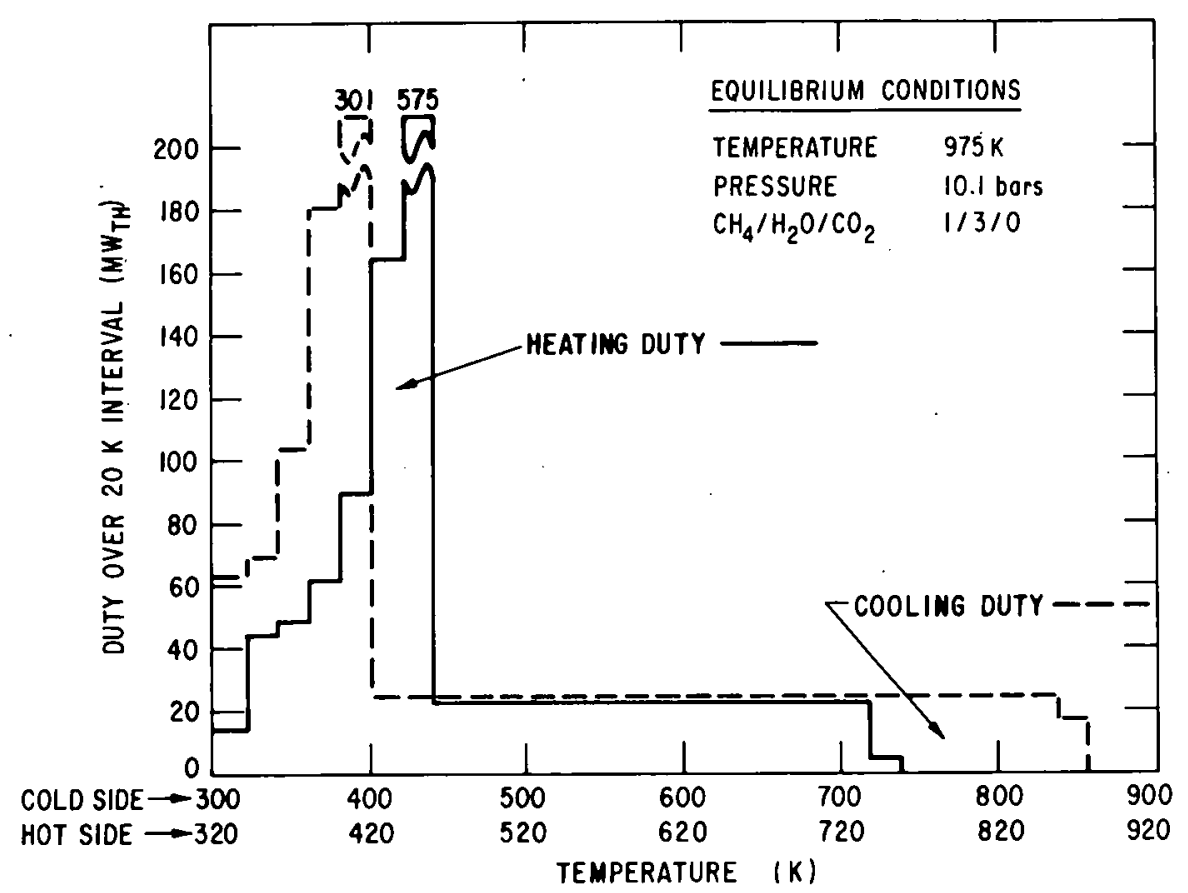

Figure A2-20. Case G - Reformer Plant

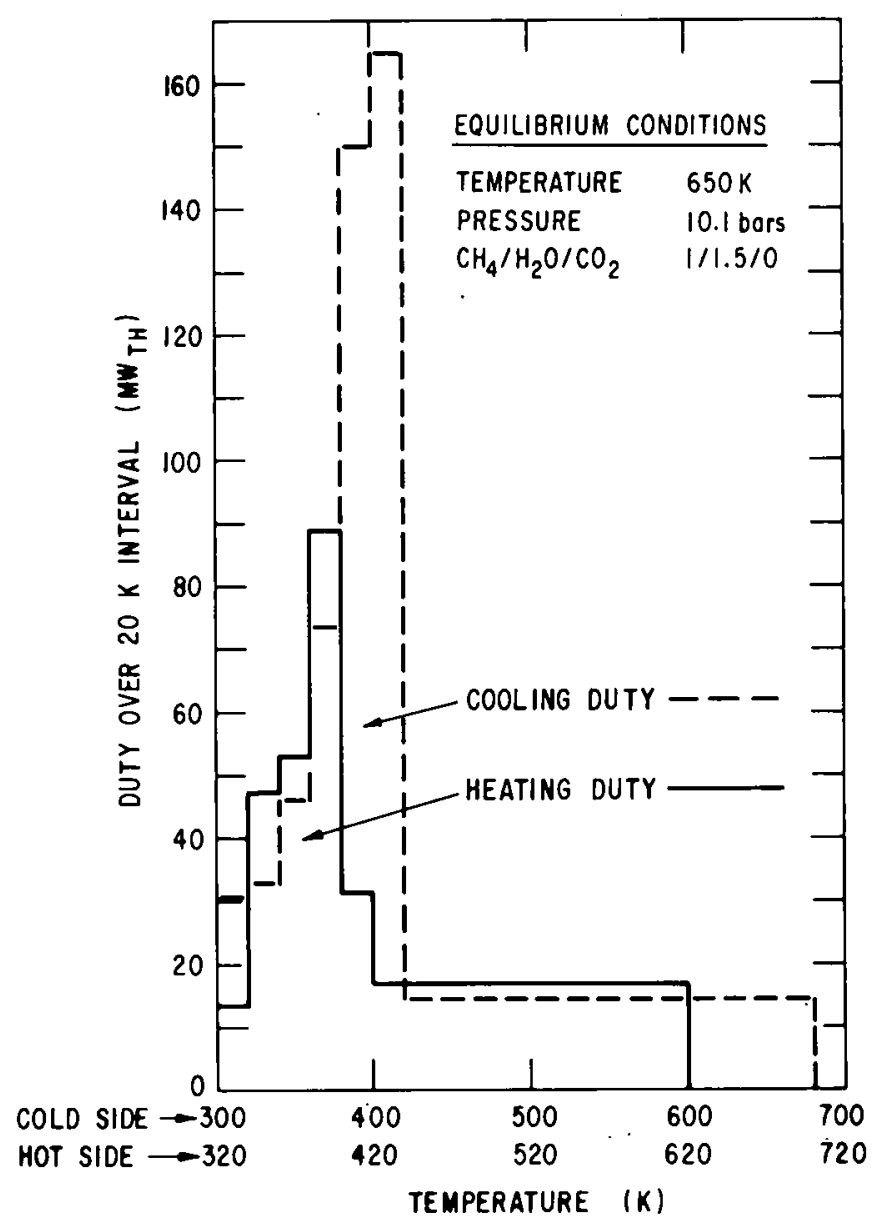

Figure A2-21. Case G - Methanation Plant 


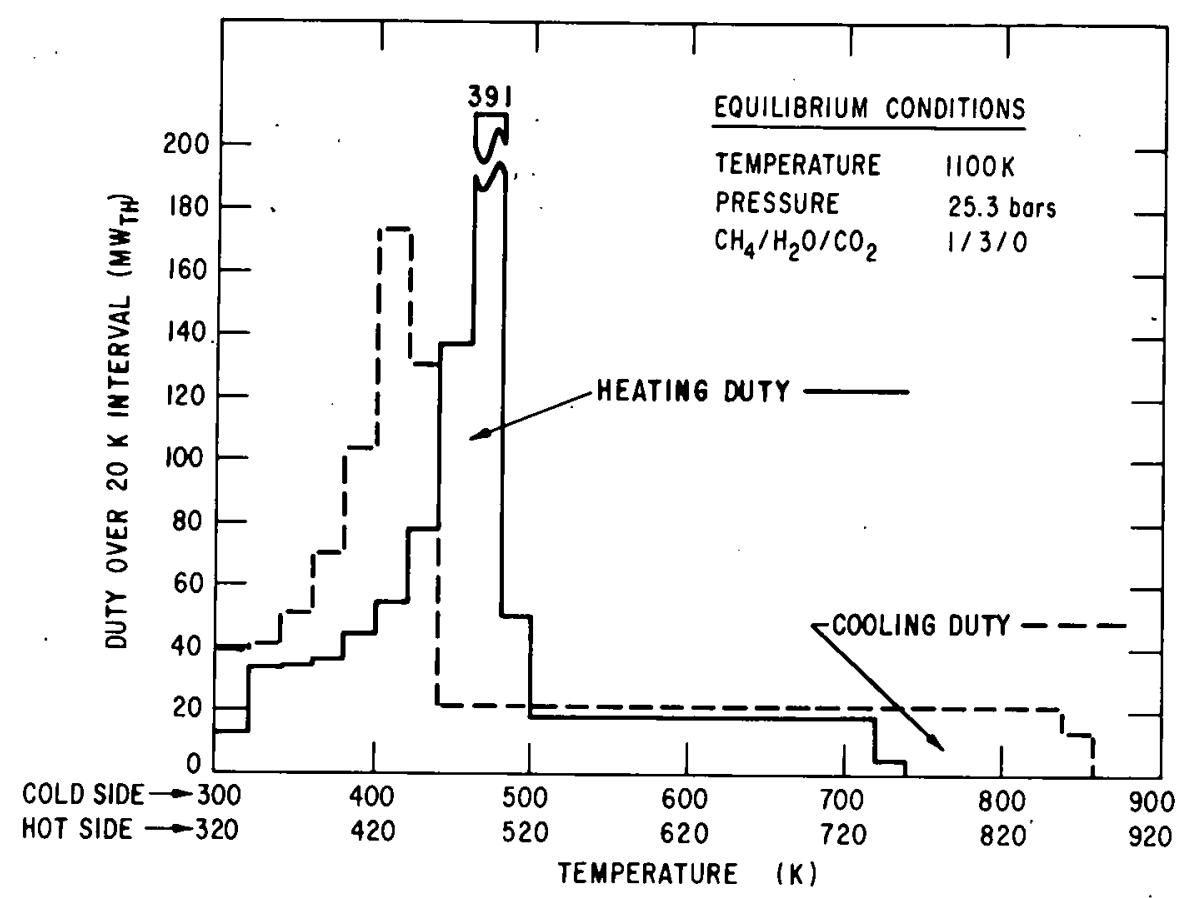

Figure A2-22. Case H - Reformer Plant

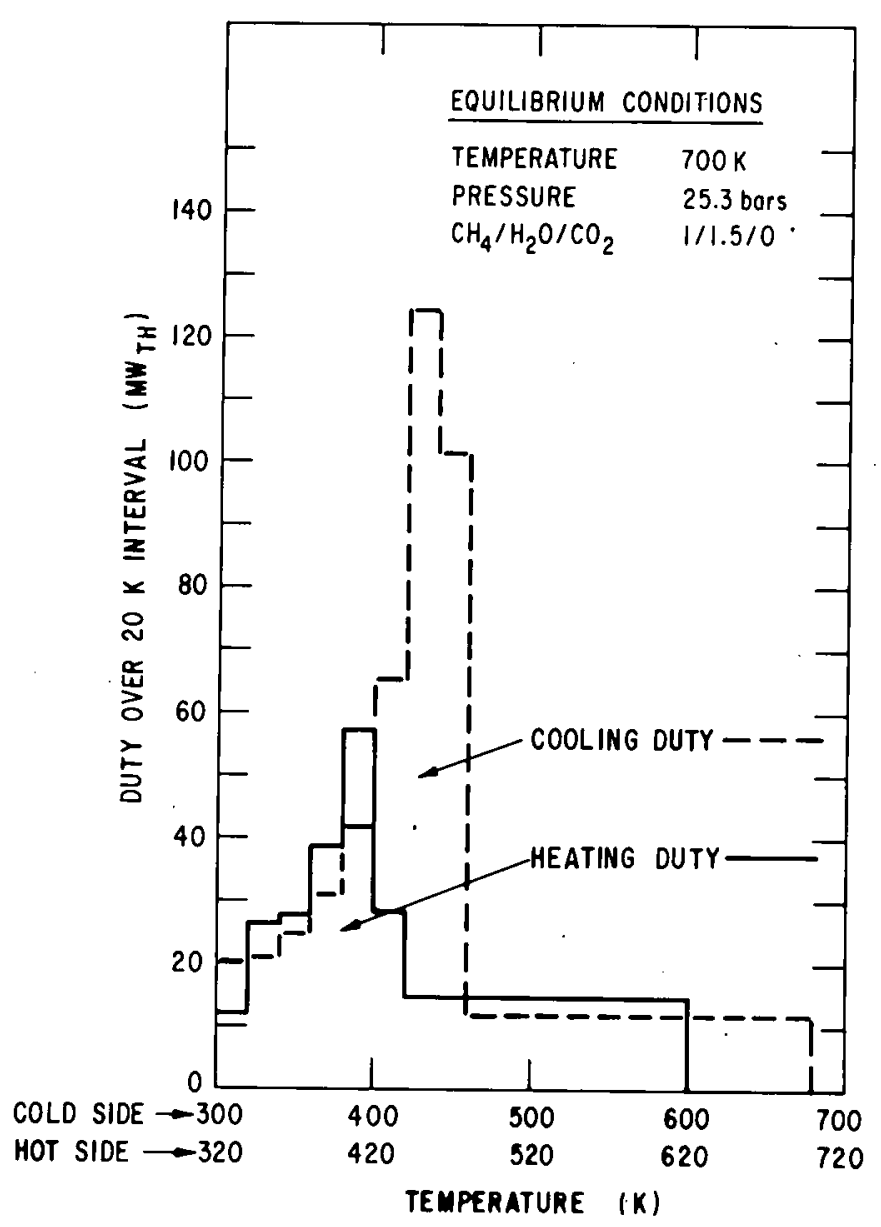

Figure A2-23. Case $\mathrm{H}$ - Methanation Plant 


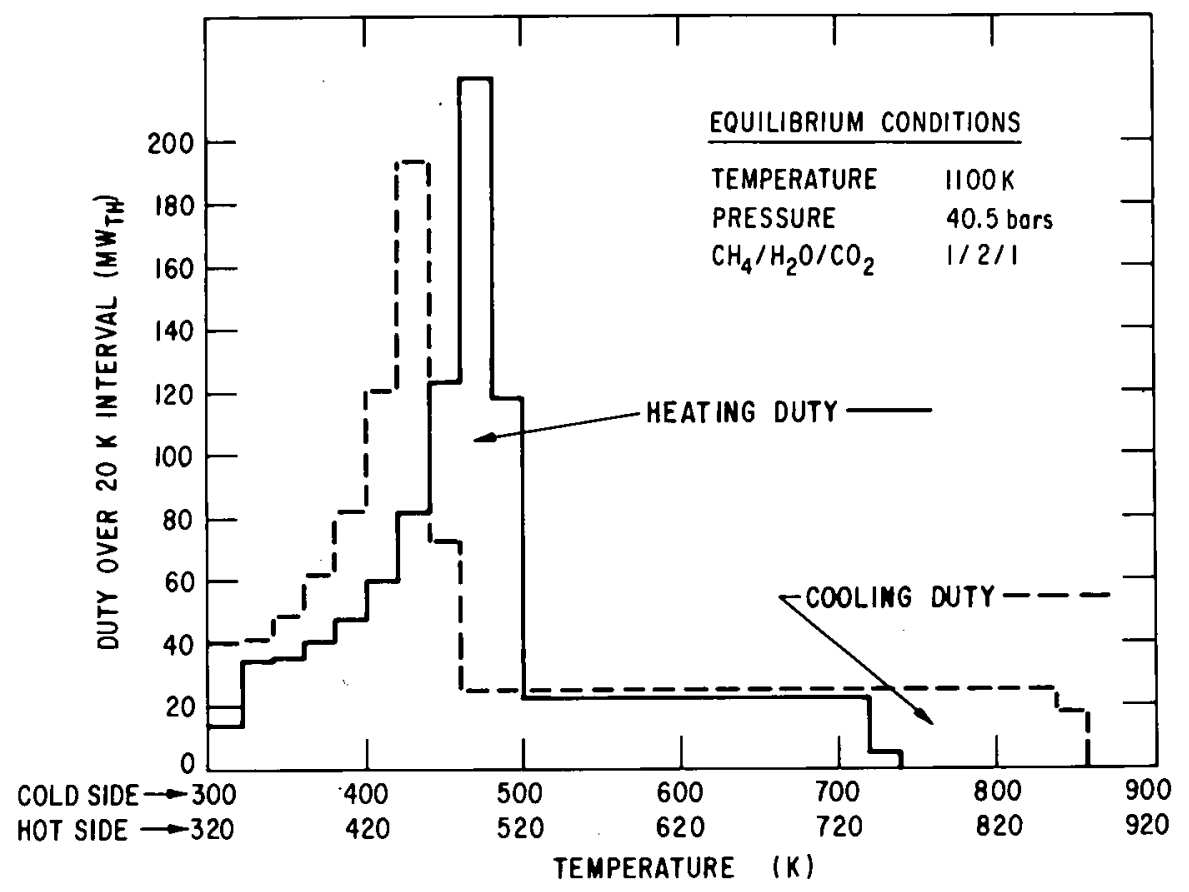

Figure A2-24. Case I - Reformer Plant

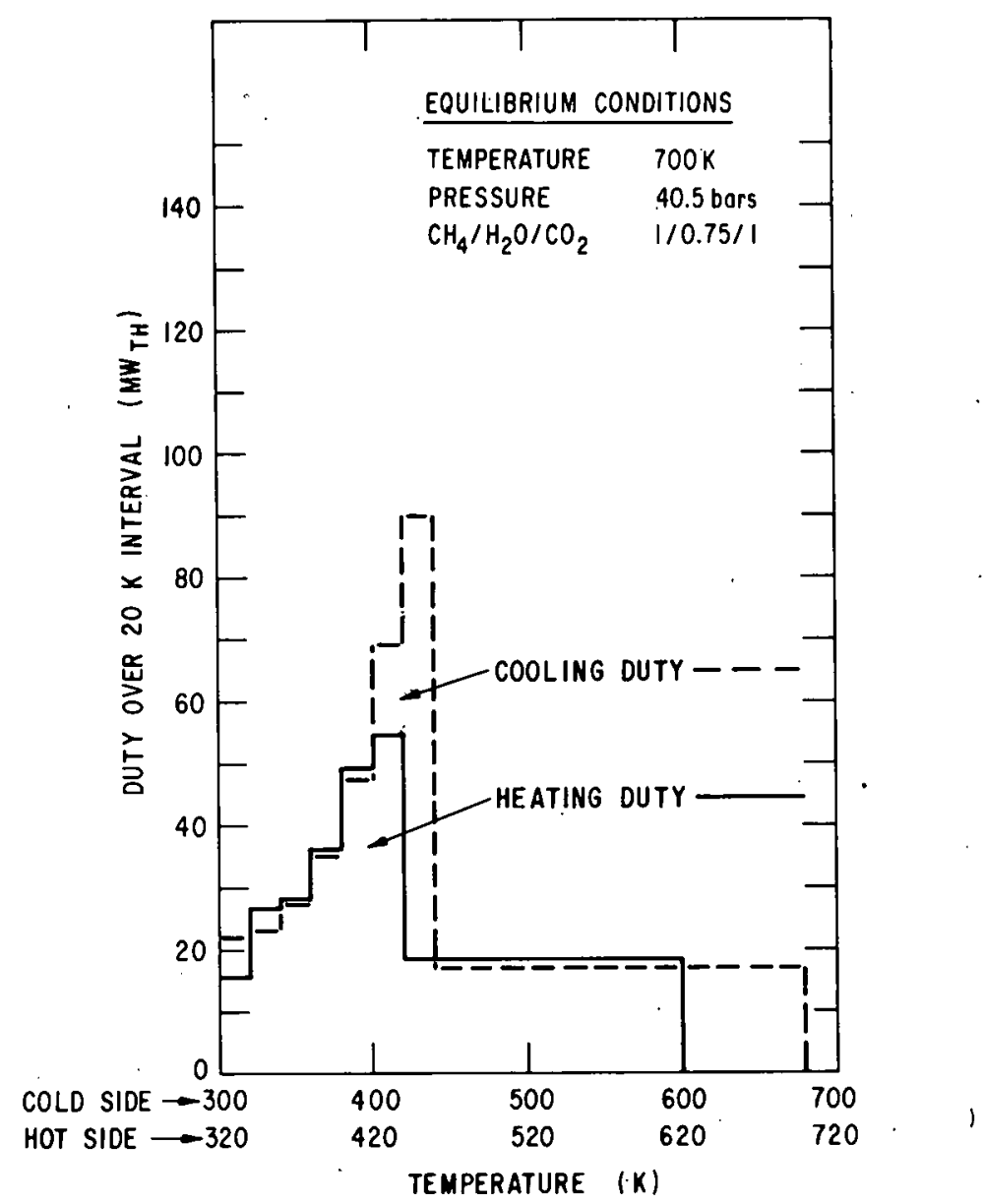

Figure A2-25. Case I - Methanation Plant 


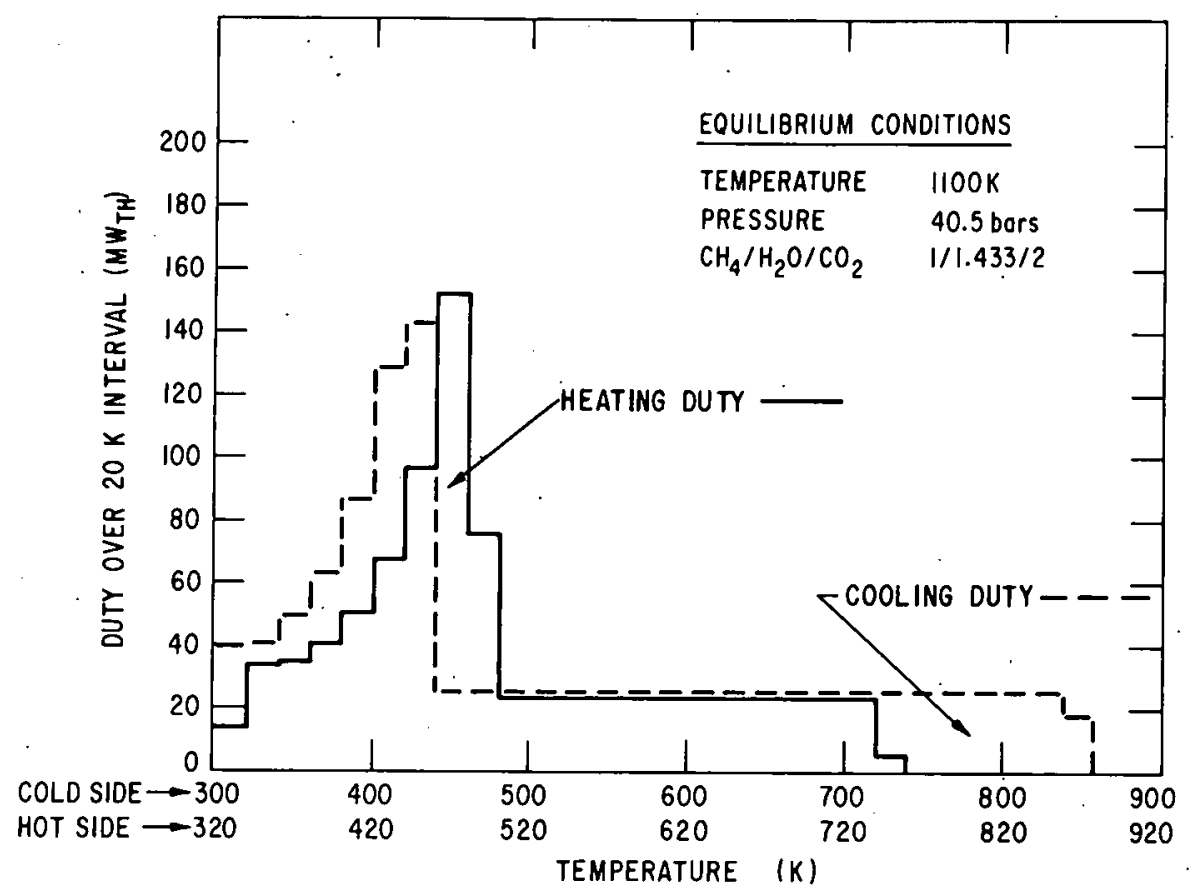

Figure A2-26. Case J - Reformer Plant

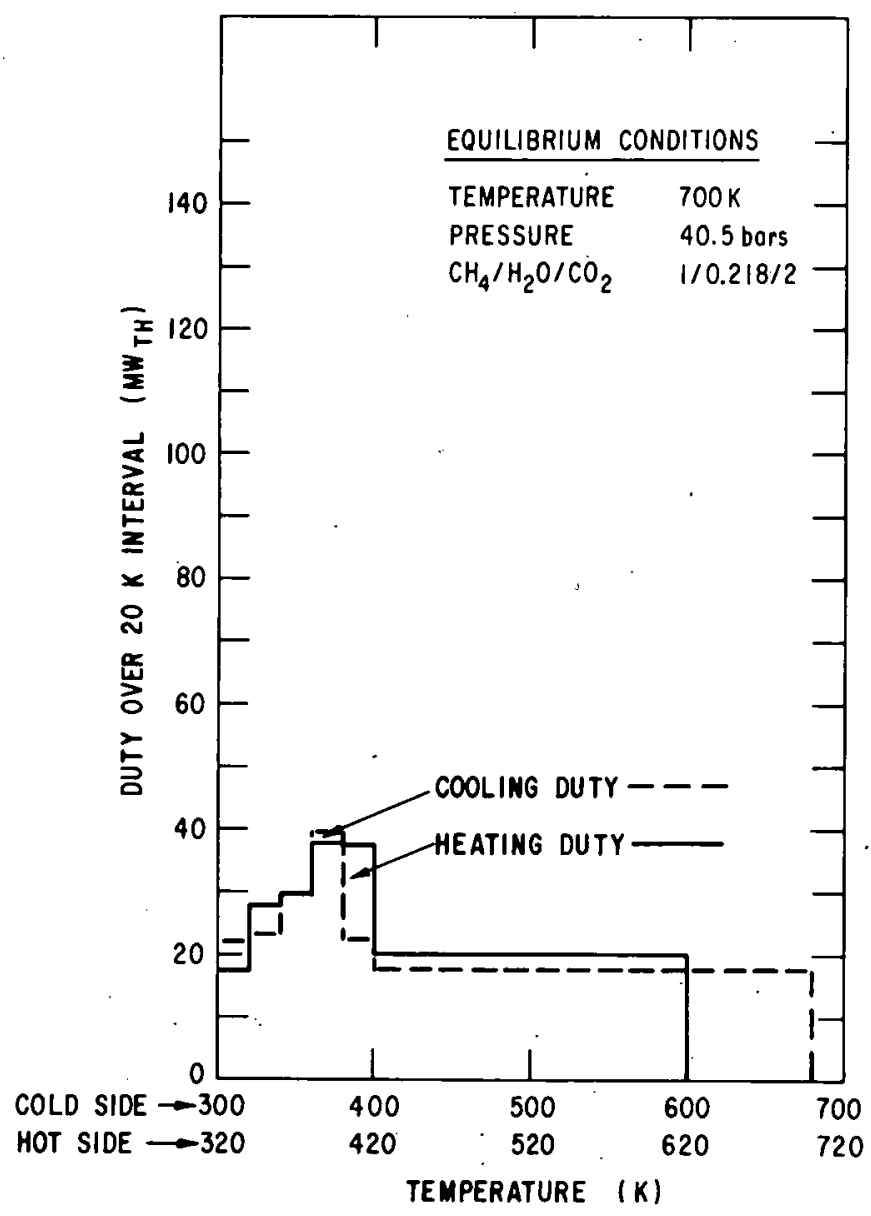

Figure A2-27. Case J - Methanation Plant 
For the total pipeline compressor power of $19.3 \times 10^{6} \mathrm{~J} / \mathrm{sec}$ the entropy production rate due to friction in pipelines $160 \mathrm{~km}$ long is found to be $64.3 \mathrm{KJ} / \mathrm{K}$ sec.

\section{A2.4.2 Irreversibilities Due to Heat Exchanges}

One way to estimate the exergy loss associated with heat exchangers is to compare the net exergy penalties with temperature drops of zero to $20 \mathrm{~K}$. In Table 5- the difference is found to equal $48.6 \times 10^{6} \mathrm{~J} / \mathrm{sec}(64.3-15.7 \mathrm{MW})$. The corresponding entropy production rate equals $162 \mathrm{~kJ} / \mathrm{K} \mathrm{sec}$. It should be noted that this entropy production rate includes the effect of $20 \mathrm{~K}$ temperature drop for internal heat exchanges as well as for exchanges with the powerplant. The only heat exchanges not included in this estimate are the heliumto-process-gas and pigtail-to-process-gas heat transfers in the reformer tubes.

\section{A2.4.3 Irreversibilities in the Reformer Tubes}

Entrony production rates and the associated exergy losses for each of the four irreversibilities are shown in Table A2-3. Also shown are the exergy and entropy flows corresponding to an energy flux of $10^{9} \mathrm{j} / \mathrm{s}(1000 \mathrm{MW} t \mathrm{~h})$ pipeline conditions. The tabulated values show clearly that the manner in which the reformer feed stream is prepared is of considerable importance and can lead to a major loss of exergy, comparable in magnitude to the heat transfer and reactor losses.

$\begin{aligned} \text { Logarithmic mean temperature } & =1038 \mathrm{~K} \\ \text { Entropy drop in helium } & =\frac{1.003 \times 10^{9} \mathrm{~J} / \mathrm{sec}}{1038 \mathrm{~K}} \\ & =966 \mathrm{~kJ} / \mathrm{K} \mathrm{sec}\end{aligned}$

Entropy increase in the process gas from $725 \mathrm{~K}$ at the inlet composition to $873 \mathrm{~K}$ at the exit composition equals $1108 \mathrm{KJ} / \mathrm{K}$ sec. Therefore,

total entropy production rate $=1108-966=142 \mathrm{~kJ} / \mathrm{K} \mathrm{sec}$

It should be noted that this $142 \mathrm{KJ} / \mathrm{K}$ sec includes the effect of heat transfer as well as of irreversible chemical reaction. If an average heat transfer $\Delta \mathrm{T}$ of $100 \mathrm{~K}$ is assumed, the helium-to-process heat exchange alone would contribute an entropy production rate of $103 \mathrm{KJ} / \mathrm{sec} \mathrm{K}$.

\section{A2.4.4 Irreversibility Due to Steam/Gas Mixing}

The type of entropy production considered in this section is somewhat less obvious than the previous three types. As shown in Figure 5-2, the preparation of reformer feed consists of mixing two gaseous streams -- one from the boiler, and the other from the gas preheater. The thermodynamic price to be paid as a result of this irreversible mixing of two gaseous streams is not evident until one has to "undo" the process by separating out the unreacted water molecules in the partial condenser. At this stage one realizes that the invested evaporation energy in the boiler is recovered at considerably lower temperatures. 
Entropy production rate for this irreversible process is calculated as follows :

$$
\Delta_{i} S=n_{\mathrm{H}_{2} \mathrm{O}} \mathrm{Rln}\left[\frac{\mathrm{n}_{\mathrm{H}_{2} \mathrm{O}^{+n_{G}}}}{\mathrm{n}_{\mathrm{H}_{2} \mathrm{O}}}\right]+n_{\mathrm{G}} \mathrm{R} \ln \left[\frac{\mathrm{n}_{\mathrm{H}_{2} \mathrm{O}^{+n_{G}}}^{n_{G}}}{n_{\mathrm{G}}}\right]
$$

where ${ }^{n_{2}}$ and ${ }^{n_{G}}$ are the mqlar flowrates of steam and gas streams respectively, and $\mathrm{R}$ is the gas. constant.

For the example case, molar flow rates are $27 \times 10^{6}$ gmoles $/ \mathrm{hr}\left(7.5 \times 10^{3}\right.$ gmoles/ sec) of gas and $68.7 \times 10^{6}$ gmoles $/ \mathrm{hr}\left(19 \times 10^{3}\right.$ gmoles/sec $)$ of steam. The resultant entropy production is $131.6 \mathrm{~kJ} / \mathrm{sec} \mathrm{K}$ and the associated exergy loss - is 39.5 MW.

Entropy production rates and the associated exergy losses for each of the four irreversibilities are shown in Table A2-3.

$$
\text { Table } \mathrm{A} 2-3
$$

IRREVERSIBILITIES FOR THE REFERENCE CASE

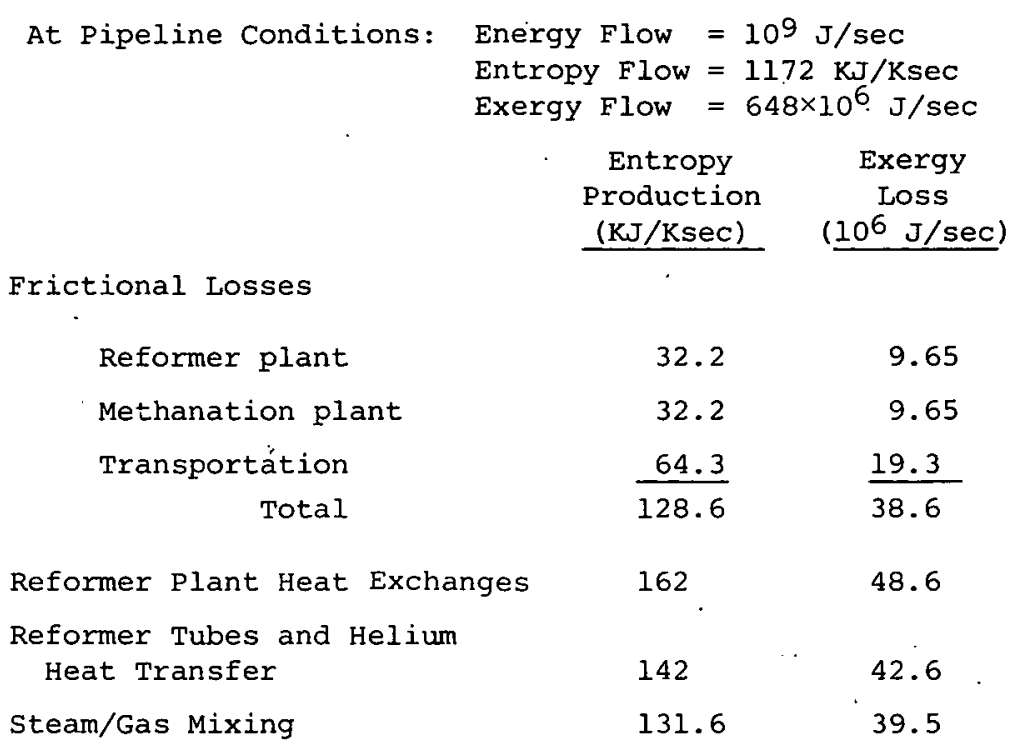

\section{A2.5 SUMMARY}

Relevant background for performing thermodynamic analyses of CHP systems was presented in the framework of exergy flows. These methods were used to analyze overall energy delivery efficiencies, interactions between the chemical plant and the powerplant, and effects of irreversibilities in a CHP system.

The major conclusions were as follows:

- Thermodynamic losses play an important role at several levels of CHP designs. 
- The concept of thermodynamic availability (as represented by exergy or steady-flow essergy) is of considerable value in CHP analyses.

- For delivering thermal energy to process steam users from remotely located primary sources, overall efficiencies for CHP systems are significantly higher (75 to $85 \%$ ) than those for current alternatives (35 to 50\%). The ability of CHP systems to provide an intermediate energy carrier with an exergy ratio between those of the primary source and the end use is identified as the key reason for these high overall efficiencies.

- Exergy efficiency of powerplants remains approximately constant at $85 \%$ for a variety of designs with different energy efficiencies. This permits a convenient evaluation of the net effect of thermal exchanges with powerplants.

- Application of exergy analyses to thermal exchanges with the powerplant shows the hidden penalty to the reformer plant to be approximately $7 \%$ of the transported thermal energy.

- Computation of entropy production shows that a seemingly innocuous step in the reformer feed preparation -- irreversible mixing of steam and methane-rich gases -- is a major source of thermodynamic loss. It leads to an exergy destruction equal to $39.5 \times 10^{6} \mathrm{~J} / \mathrm{sec}$, which is comparable to the other two major sources of exergy loss, namely thermal degradation in heat exchangers and thermal and chemical irreversibilities in reformer tubes.

\section{REFERENCES}

RA2-1 Haywood, R.W., "A Critical Review of the Theorems of Thermodynamic Availability, with Concise Formulations," Journal of Mechanical Engineering Science, Vol. 6, 1974, No. 3, p. 160 and No. 4, p. 258.

RA2-2 VanRysselberghe, P., Thermodynamics of Irreversible Processes, Hermann, Paris, 1963.

RA2-3 Denbigh, K.G., "The Second-law Efficiency of Chemical Processes," Chemical Engineering Science, Vo1. 6, No. 1, October 1956, p. 1.

RA2-4 "Energy Conversion Alternatives Study - Phase 2," Final Report NASACR-134949, Contract NAS3-19406, General Electric Corporate Research and Development, Schenectady, N.Y., December 1976.

RA2-5 Wen, C.Y., et a1., "Factors Affecting the Thermal Efficiency of a Gasification Process," American Chemical Society (Fuel Division) 170th National Meeting, Vol. 20, No. 4, 1975, p. 219.

RA2-6 "Patterns of Energy Consumption in the United States," : Stanford Research Institute, Menlo Park, Calif.; U.S. Government Printing Office stock No. 4106-0034, 1972 . 
RA2-7 Kugeler, K., et al., "Transport of Nuclear Energy by Means of Chemical Energy," Nuclear Engineering and Design, Vol. 34, No. 1, 1975, p. 65.

RA2-8 Haywood, R.W., Analysis of Engineering Cycles, Pergamon Press, New York, 1975.

RA2-9 Keenan, J.H., et al., Steam Tables, John Wiley \& Sons, New York, 1969.

B

Ex

$(\Delta \mathrm{Ex})_{\mathrm{D}}$

$\mathrm{H}$

$n_{j}$

$\mathrm{dQ}$

S

$\Delta S_{C}$

To

$\mu_{j o 0}$

Subscript

ef

$\mathrm{p}$
NOMENCLATURE

(See also Reference A2-1)

Steady-flow availability function ( $\left(\mathrm{H}_{\mathrm{H}}-\mathrm{T}_{\mathrm{O}} \mathrm{S}\right.$ )

Exergy of extraction and delivery

Exergy destruction due to irreversibilities

Enthalph

Number of moles of component $j$

Heat flow to or from the control volume

Entropy

Entropy creation due to irreversibilities

Thermodynamic temperature of the environment (dead state)

Chemical potential of component $j$ in the environmental

(dead state) mixtrue

External fluid

Process fluid 


\section{Appendix 3 \\ HEAT EXCHAANGER TRAIN DESIGN FOR $1000 \mathrm{MW}_{\text {th }}$ METHANE REFORMER FOR HTCHP}

\section{A.1 INTRODUCTION}

In this appendix, a detailed design is given for the process gas heat exchangers required in the base case analysis of the HTCHP methane reforming process. This "first order" design was undertaken to establish reasonable estimates for design parameters such as heat transfer coefficients, exchanger areas, and costs. This design is based on $25 \mathrm{~K}$ minimum temperature differences and $3 / 1$ stoichiometry in the methanator process. The areas and heat loads have been modified in the analyses performed in the main text to conform to a $20 \mathrm{~K} \Delta \mathrm{T}$ and a $1.5 / 1$ stoichiometry in the methanation process.

The remainder of this appendix is divided into two sections; the first reviews the overall heat exchanger process designs and summarizes the detail analyses, the second gives the detailed design for each of the seven exchangers. The analysis of exchanger 7 is based on the Mixed Feed Evaporator concept discussed in Chapter 5.

\section{A3.2 DESIGN SUMMARY FOR METHANE REFORMING HEAT EXCHANGERS}

The reformer plant consists of a series of heat exchangers arranged by duty, temperature difference, and function. The standard reformer plant ( $1000 \mathrm{MW}_{\mathrm{th}}, 40 \mathrm{~atm}, 1100 \mathrm{~K}, 3 / 1 \mathrm{H}_{2} \mathrm{O} / \mathrm{CH}_{4}$ ) will require the seven heat exchangers specified in Figure A3-1. (The functions of these exchangers (HX) are listed in Table A3-1.) The Specified Process Temperatures are the assumed feed streams of the thermodynamic analysis. The Design Approach Temperatures are estimated for a $25^{\circ} \mathrm{C} \Delta \mathrm{T}$ with the corresponding countercurrent flow stream. Where possible all inlet and outlet $\Delta \mathrm{Ts}$ are taken as $25^{\circ} \mathrm{C}$ except as required by the additional constraint of the overall energy balance.

\section{A3.2.1 Dimension}

For each of the seven heat exchangers the following information is calculated:

- Heat exchange area

- Shell side pressure drop

- Tube side pressure drop

- Shell diameter

- Tube length

- Cost based on a nominal $\$ 25 / f t^{2}$ of inside tube area

These calculations are only estimates; they are not of sufficient detail to specify manufacture. In each case a purely countercurrent heat exchange process is specified at 40 atmospheres of pressure. A part of the baffle arrangement shown as Figure $A 3-2$ allows this and is called orifice Baffling. Nominal spacing of 2 feet is assumed, allowing for a $\Delta$ pitch of tubes. All of these heat exchangers will be assumed to have similar geometries - $60^{\circ}$ 


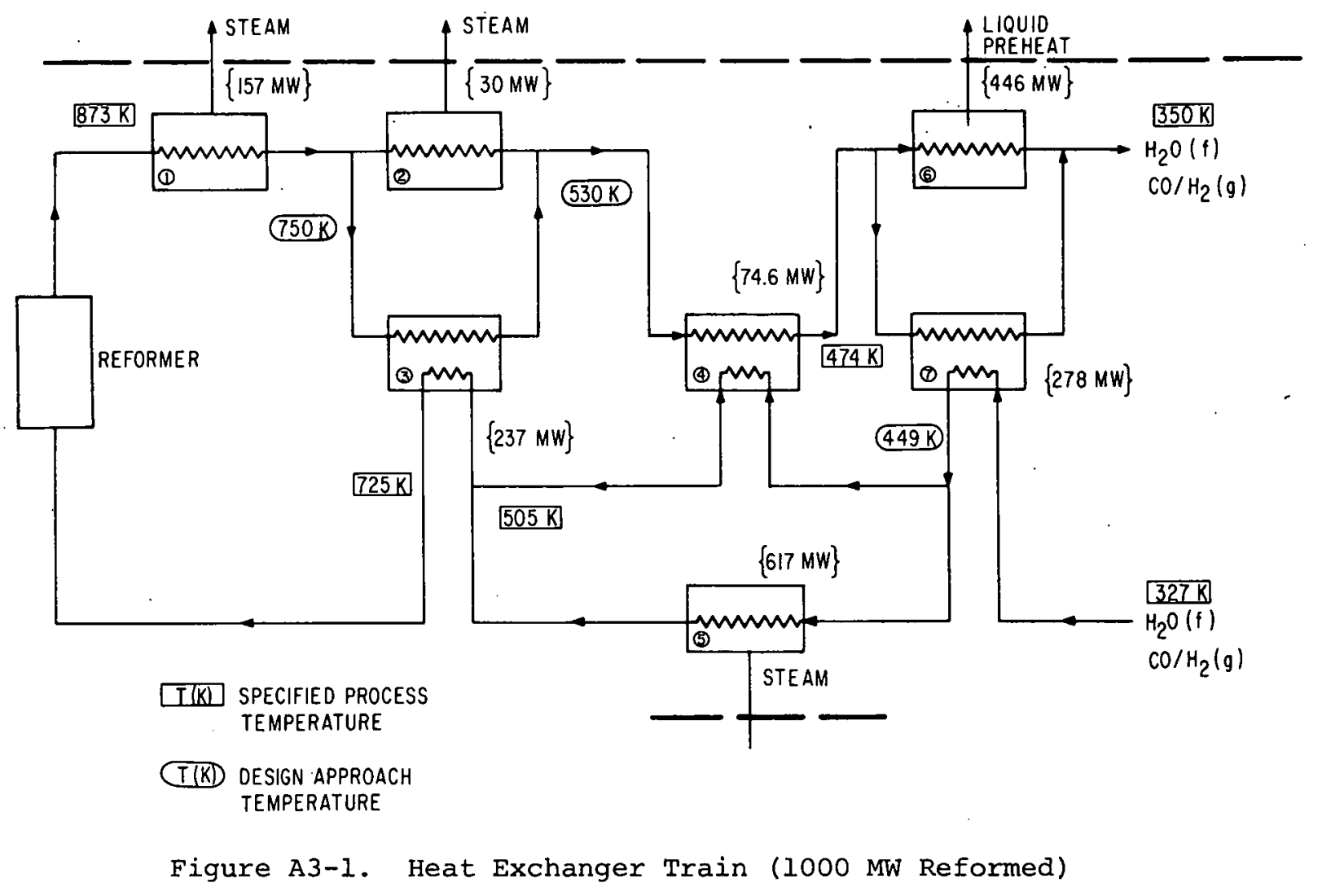

Table A3-1

HEAT EXCHANGER TYPES

\begin{tabular}{cl} 
HX No. & \multicolumn{1}{c}{ Function } \\
1 & Output cooler/powerplant steam superheater \\
2 & Output cooler/powerplant steam superheater \\
3 & Output cooler/final feed heater \\
4 & Output cooler/feed boiler \\
5 & Feed boiler \\
6 & Condenser/powerplant feed heater \\
7 & Combined condenser/evaporator
\end{tabular}

equilateral pitch $1-1 / 8$ inches with standard $3 / 4$ inch $0 . d$. tubes of i.d. 0.652 inch, as shown in Figure A3-3. In necessary cases these will be assumed finned to an effective area multiplier over the inside tube area of 2.5 to 1 .

Assuming there are $\mathrm{n}$ tubes in the heat exchanger, then:

(1) Tube side flow area $=0.00232 \mathrm{n} \mathrm{ft}^{2}$

(2) Heat exchange area per unit shell length $0.1706 \mathrm{n} \mathrm{ft}^{2} / \mathrm{ft}$ of shell 

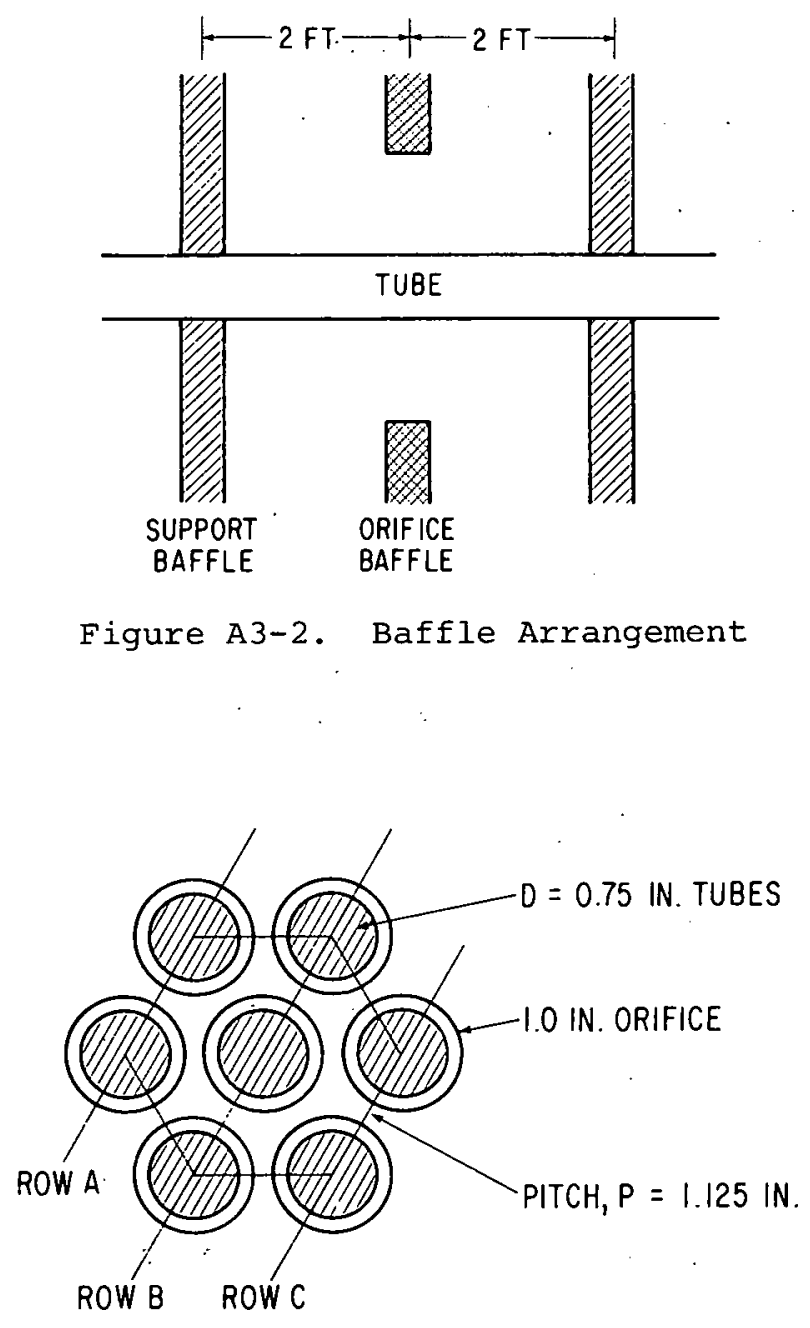

Figure A3-3. Orifice and Tube Pitch Geometry, or Flow Cell for a Typical Tube (at any Baffle, Alternate Rows $A$ and $C$ Open or $B$, but Not Both)

(3) Cell flow area (flow area for a single heat exchanger tube):

$$
\begin{aligned}
a & =\frac{3}{2} \mathrm{p}^{2} \cot \frac{\pi}{6}-\frac{3}{4} \pi \mathrm{D}^{2} \\
& =0.0136 \mathrm{ft}^{2} / \operatorname{cell}
\end{aligned}
$$

(4) Total shell side flow area -- Each cell passes the flow for 1 central tube and $6 \times 1 / 3$ peripheral tubes:

$\therefore$ shell side flow area $=\frac{0.0136}{3} \times \mathrm{n}=0.0045 \mathrm{ft}^{2}$

(5) Shell cross section:

$$
\begin{aligned}
\frac{\pi D^{2}}{4} \text { shell } & =\frac{n \pi}{4} d_{\text {tube }}^{2}+0.0045 n \\
& =0.0076 n \mathrm{ft}^{2}
\end{aligned}
$$


(6) Hydraulic diameter of typical flow cell:

Wetted surface area $=\pi D+6 \times \frac{2 \pi}{3} \times \frac{D}{2}=3 \pi D$

$D_{e}=\frac{4 a}{3 \pi D}=\frac{4 \times 0.0136 \times 12}{3 \times \pi \times 0.75}$

(7) Orifice flow area

$$
\begin{aligned}
A_{O} & =\frac{n}{2} \frac{\pi}{4}\left(D_{0}^{2}-d_{\text {tube }}^{2}\right) \\
& =0.00119 n \mathrm{ft}^{2} \\
& =\frac{1}{3.77} \text { shell side flow area }
\end{aligned}
$$

(8) Shell side pressure drop -- For a heat exchanger of length $L$ ft, there are $\mathrm{N}=\mathrm{L} / 2$ baffles. The mass velocity in the shell is

$$
G_{s}=\frac{W_{s}}{0.0045 n} \frac{1 b_{m}}{h r f t^{2}}
$$

if $\mathrm{w}_{\mathrm{S}}$ is the total shell-side mass flow rate.

Thus the axial pressure drop for a smooth tube is

$$
\Delta \mathrm{p}=2 \times 0.005 \times \frac{\mathrm{L}}{\mathrm{D}} \times \frac{\mathrm{G}_{\mathrm{S}}^{2}}{\rho_{\mathrm{S}}}+\mathrm{N}\left\{\frac{\left(3.77 \mathrm{G}_{\mathrm{S}}\right)^{2}}{2 \rho_{\mathrm{S}} \mathrm{C}_{\mathrm{D}}^{2}}\right\}
$$

where $\rho_{S}$ is the shell-side mass density and $C_{D}$ is the coefficient of discharge through the orifice. To a reasonable approximation, $C_{D}=0.62$ over a very large orifice Reynolds number range:

$$
\Delta p=\frac{G_{S}^{2}}{\rho_{S}}\left\{0.01 \frac{\mathrm{L}}{D}+18.5 \mathrm{~N}\right\}
$$

For roughened heat exchanger tubes the friction factor rises from 00.005 to 0.02 and then remains constant. For these

$$
\begin{aligned}
\Delta \mathrm{P} & =\left\{2 \times 0.02 \times \frac{\mathrm{L}}{\mathrm{D}} \frac{\mathrm{G}_{\mathrm{S}}^{2}}{\rho_{\mathrm{S}}}+\frac{\mathrm{N}}{2 \rho_{\mathrm{S}}}\left(\frac{3.77 \mathrm{G}_{\mathrm{S}}}{\mathrm{C}_{\mathrm{D}}}\right)^{2}\right\} \\
& =\frac{\mathrm{G}_{\mathrm{S}}^{2}}{\rho_{\mathrm{S}}}\left\{0.004 \frac{\mathrm{L}}{\mathrm{D}}+18.5 \mathrm{~N}\right\}
\end{aligned}
$$

To this it may be necessary to add an additional acceleration-related pressure drop if boiling occurs, or a pressure recovery term if condensation occurs.

$$
\left.\Delta \mathrm{p}_{\text {momentum }}= \pm\left|\mathrm{G}_{\mathrm{s}}^{2}\right| \frac{1}{\rho_{\mathrm{s}_{\text {in }}}}-\frac{1}{\rho_{\mathrm{s}_{\text {out }}}}\right\}
$$

Unless the heat exchanger is short, this is a small term compared with the viscous or mbmentum terms previously noted. 
(9)

Inside tube heat transfer coefficient

$$
\frac{\mathrm{hd}_{\mathrm{ID}}}{\mathrm{k}}=0.023\left(\frac{\mathrm{Gube}_{\text {id }}}{\mathrm{G}}\right)^{0.8^{\prime}} \mathrm{Pr}^{0.4}
$$

This will not be applicable with change of phase.

(10) Shell side coefficient -- In the abserice of change of phase on smooth tubes the Colburn $j$ factor analogy is used:

$$
\begin{aligned}
& \frac{h}{G_{S} C_{p}}\left(\frac{\mu C_{p}}{k}\right)^{0.6}=\frac{f}{2} \\
& \text { or } \frac{h D_{e}}{k}=\frac{f}{2}\left(\frac{G D e}{\mu}\right)\left(\frac{\mu C_{p}}{k}\right)^{0.4} \\
& \text { For smooth tubes } f=\frac{0.046}{\operatorname{Re}^{0.2}} \\
& \frac{{ }^{h D_{e}}}{k}=0.023\left(\frac{{ }_{s} D_{e}}{\mu}\right)^{0.8}:\left(\frac{\mu_{p}}{k}\right)^{0.4} \\
& \text { For rough tubes. } \\
& \frac{\mathrm{hD}_{\mathrm{e}}}{\mathrm{k}} \rightarrow\left(\frac{{ }_{\text {rough }}}{\mathrm{f}_{\text {smooth }}}\right)\left(\frac{\mathrm{hD}_{\mathrm{e}}}{\mathrm{k}}\right)_{\text {smooth }} \\
& \text { or } \frac{\mathrm{hD}_{\mathrm{e}}}{\mathrm{k}} \approx, 4 \times, 0.023 \mathrm{Re}^{0.8} \mathrm{Pr}^{0.4}
\end{aligned}
$$

\section{Fouling Factors}

In each case the recommendation of API for clean heat exchangers will be followed: 0.0005 (Btu/hr ft2OF.) $)^{-1}$ for each side.

\section{A3.2.2 Results}

The total pressure drop from the methane feed to the reformer is about $34 \mathrm{psi}$. The total pressure drop from the reformer to the hydrogen feed is about $24 \mathrm{psi}$. Therefore an overall $\Delta \mathrm{p}$ of $58 \mathrm{psi}$ exclusive of the reformer has been achieved. Note that parallel heat exhcangers do not match in pressure drop. This could be (wastefully) accomplished by throttling, for example.

The sizes of the heat exchangers, summarized in Table A3-2, are calculated in the appendixes. The final costs are $\$ 12.3 \mathrm{million}$ for $1840 \mathrm{MW}_{\mathrm{T}}$ or $\$ 6.67 /$ $k w_{t}$.

\section{A.3.3 DETAILED DESIGN OF HEAT EXCHANGERS}

\section{A.3.3.1 Heat Exchanger 1}

Output Cooler/Steam Super Heater

In this case, because the heat transfer coefficients on both sides will be of similar magnitude the hydrocarbon side was placed on the tube side to facilitate cleaning of any possible condensed fraction:

$$
\text { A3-5 }
$$


Table A3-2

SUMMARY OF CALCULATED HEAT TRANSFER COEFFICIENTS, PRESSURE DROPS, AREAS, AND COSTS

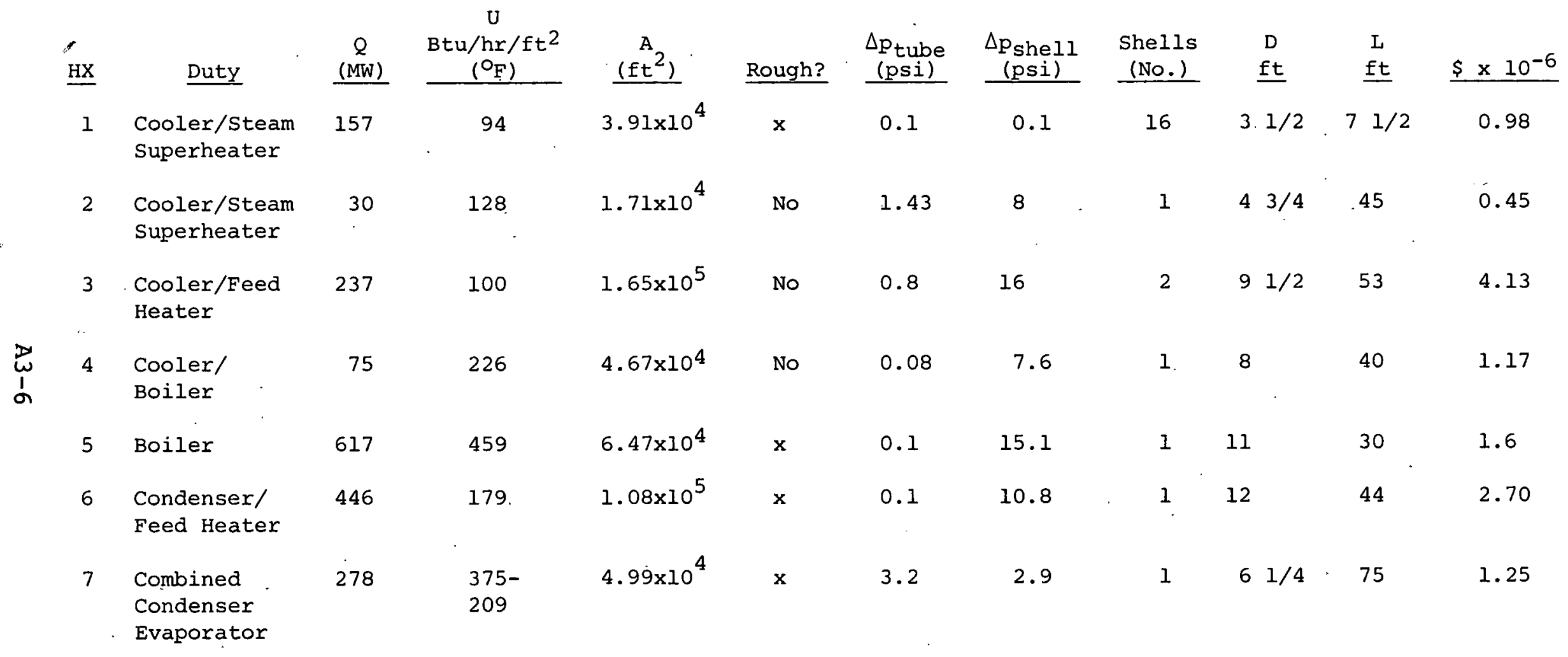



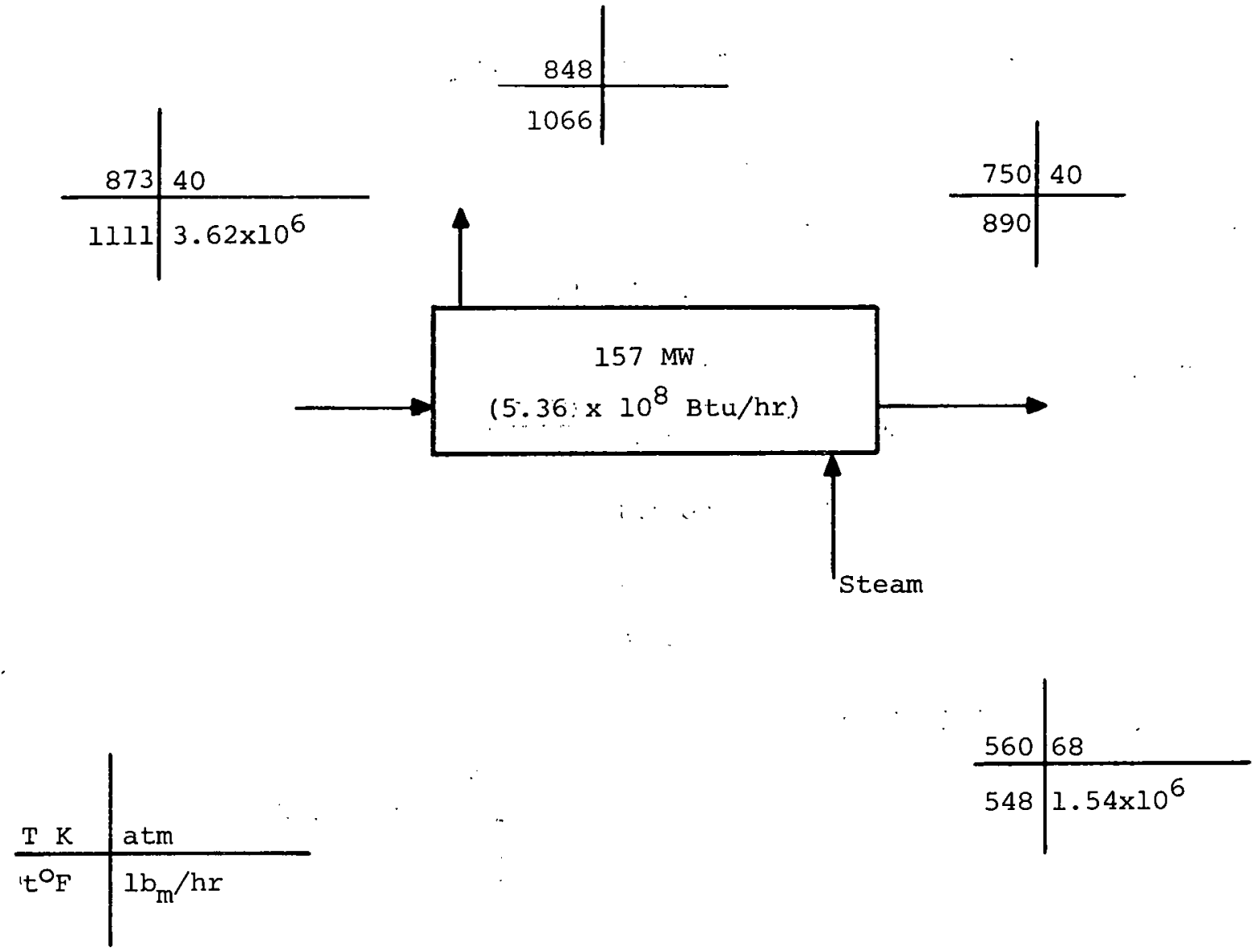

Assume: Tube-side mass velocity is $5 \times 10^{4} 1 \mathrm{~b}_{\mathrm{m}} / \mathrm{hr} \mathrm{ft}^{2}$ :

$$
\begin{aligned}
A_{\text {tube }} & =72.6 \mathrm{ft}^{2} \\
\mathrm{n} & =\underline{31293} \\
\mathrm{~A}_{\text {shell }} & =141 \mathrm{ft}^{2} \\
\mathrm{D}_{\text {shell }} & =17.4 \mathrm{ft} \quad \text { (Too large) }
\end{aligned}
$$

The maximum is 12 feet.

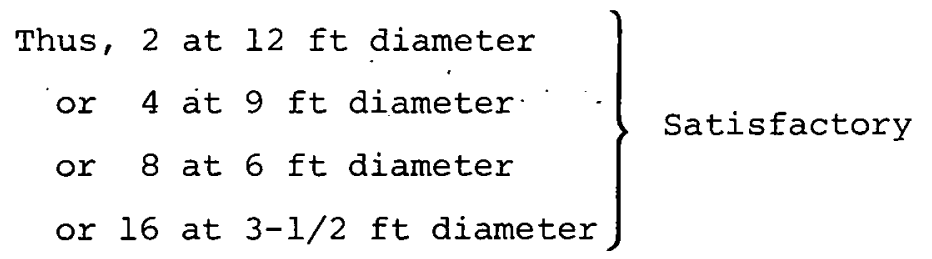

The heat exchange area is $5339 \mathrm{ft}^{2} / \mathrm{ft}$ of combined shells.

$$
G_{\text {shell }}=1.09 \times 10^{4} 1 \mathrm{~b}_{\mathrm{m}} / \mathrm{hr} \mathrm{ft}^{2}
$$


Tube Side

$$
\begin{aligned}
R e & =5 \times 10^{4} \times \frac{0.652}{12} \times \frac{1}{0.0335} \\
R e & =3.25 \times 10^{4} \\
\mathrm{Nu} & =81.5 \\
\mathrm{~h} & =148 \mathrm{Btu} / \mathrm{hr} \mathrm{ft}^{2} \mathrm{O}_{\mathrm{F}} .
\end{aligned}
$$

Shell side

$$
\begin{aligned}
\mathrm{Re} & =\frac{1.09 \times 10^{4} \times 0.0924}{0.078}=1.29 \times 10^{4} \\
\mathrm{Nu} & =38.9 \quad(\text { smooth) } \\
\mathrm{Nu} & =155.5 \text { (rough) } \\
\mathrm{h} & =123 \mathrm{Btu} / \mathrm{hr} \mathrm{ft}^{2} \mathrm{OF}
\end{aligned}
$$

Overall - based on $d_{\text {tube }}\left(i . d_{.}\right)$

$$
\begin{aligned}
& \frac{1}{U}=\frac{1}{2.5 \times 123}=0.0005+0.0005 \times \frac{0.652}{2.5 \times 0.75}+\frac{1}{148} \\
& \mathrm{U}=94 \mathrm{Btu} / \mathrm{hr} \mathrm{ft}^{2} \mathrm{O}_{\mathrm{F}}
\end{aligned}
$$

\section{Area}

Log mean temperature difference

$$
\begin{aligned}
\Delta \mathrm{T}_{\mathrm{LM}} & =146^{\circ} \mathrm{F} \\
\mathrm{A} & =3.91 \times 10^{4} \mathrm{ft}^{2}
\end{aligned}
$$

Hence, 16 shells $31 / 2 \mathrm{ft}$ diameter $\times 71 / 2 \mathrm{ft}$ would be satisfactory. Cost would be about $\$ 0.98$ million.

\section{Pressure Drop}

Tube Side

$$
\begin{aligned}
\Delta p & =2 \times 0.005 \times \frac{7.5 \times 12}{0.652} \times \frac{1}{0.524} \times\left(\frac{5 \times 10^{4}}{3600}\right)^{2} \times \frac{1}{144} \times \frac{1}{32.2} \\
& =0.10 \mathrm{psi}
\end{aligned}
$$

Shell Side

$$
\begin{aligned}
& \Delta p=\left(\frac{1.09 \times 10^{4}}{3600}\right)^{2} \times \frac{1}{1.38} \times \frac{1}{144} \times \frac{1}{32.2} \\
&\left\{0.04 \times \frac{7.5}{0.0924}+18.5 \times \frac{7.5}{21}\right\} \\
& \Delta p=0.11 \mathrm{psi}
\end{aligned}
$$




\section{A.3.3.2 Heat Exchanger 2}

Output Cooler/Steam Superheater:

Superheat steam will be generated on the shell side:

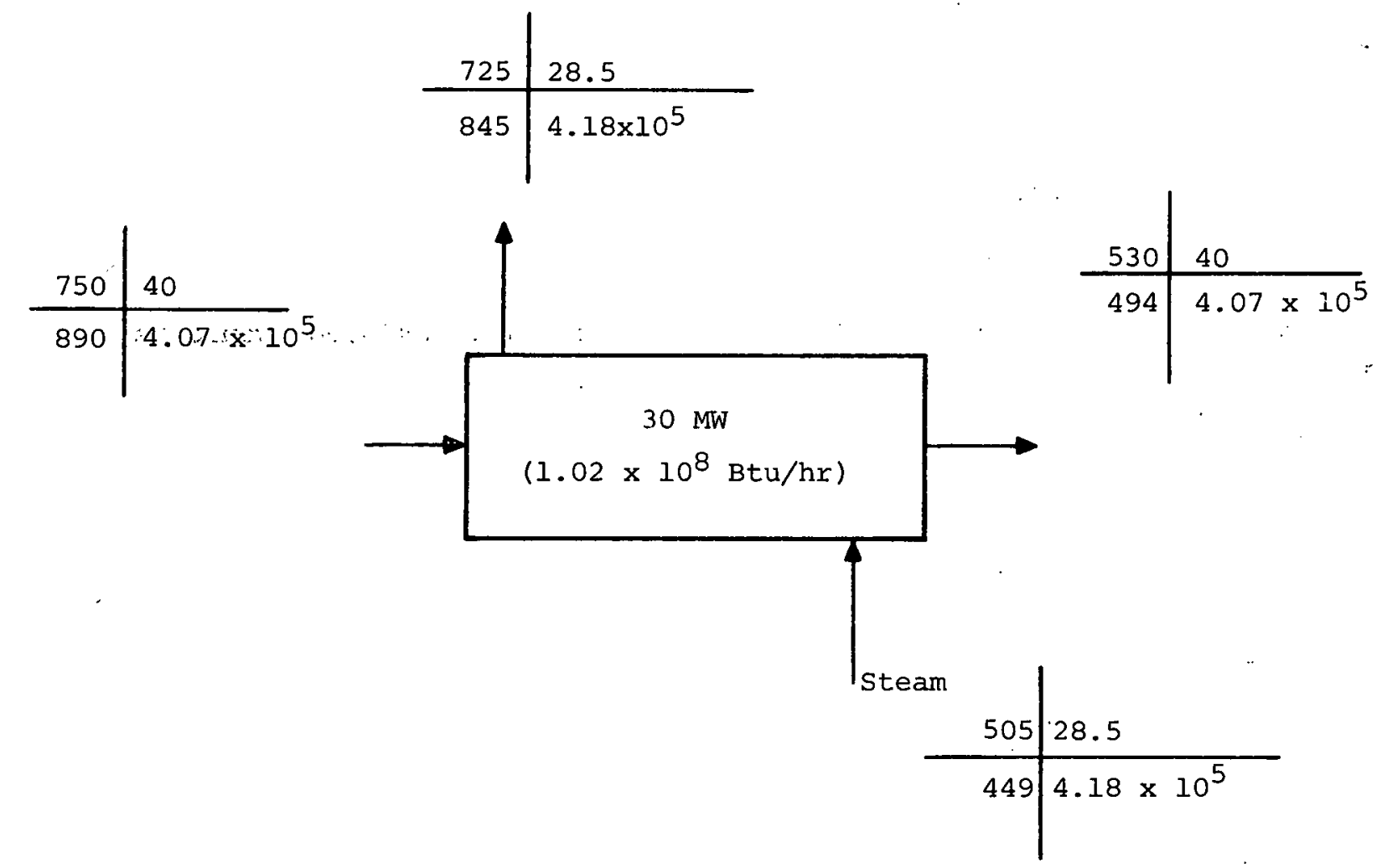

Assume: Tube-side mass velocity is $7.5 \times 10^{4} \mathrm{lb} / \mathrm{hr} \mathrm{ft}^{2}$

$$
\begin{aligned}
A_{\text {tube }} & =5.43 \mathrm{ft}^{2} \\
\mathrm{n} & =2339 \\
\mathrm{~A}_{\text {shell }} & =10.5 \mathrm{ft}^{2} \\
D_{\text {shell }} & =4.75 \mathrm{ft}
\end{aligned}
$$

The heat exchange area is $399 \mathrm{ft}^{2} / \mathrm{ft}$ of shell

$$
\mathrm{G}_{\text {shell }}=3.98 \times 10^{4} 1 \mathrm{~b}_{\mathrm{m}} / \mathrm{hr} \mathrm{ft}^{2}
$$

Tube Side

$$
\mathrm{h}=205 \mathrm{Btu} / \mathrm{hr} \mathrm{ft}^{2} \mathrm{O}_{\mathrm{F}}
$$

Shell side

$$
\begin{aligned}
\mathrm{Re} & =6.23 \times 10^{4} \\
\mathrm{Nu} & =137 \text { (smooth) } \\
\mathrm{h} & =433 \mathrm{Btu} / \mathrm{hr} \mathrm{ft}^{2} \mathrm{o}_{\mathrm{F}}
\end{aligned}
$$


Overall

$$
\begin{aligned}
& \frac{1}{U}=\frac{0.652}{0.72} \times \frac{1}{433}+\frac{1}{205}+0.0005\left(1+\frac{0.652}{0.72}\right) \\
& U=128 \mathrm{Btu} / \mathrm{hr} \mathrm{ft}^{2} \mathrm{O}_{\mathrm{F}}
\end{aligned}
$$

Area

Log mean temperature difference

$$
\begin{aligned}
\Delta \mathrm{T}_{\mathrm{LM}} & =45^{\circ} \mathrm{F} \quad \text { (constant) } \\
\mathrm{A} & =1.77 \times 10^{4} \mathrm{ft}^{2}
\end{aligned}
$$

Therefore, one $\times 4.75 \times 441 / 2$ ft shell costing $\sim \$ 440,000$ would be satisfactory.

Pressure Drop

Tube Side

$$
\Delta \mathrm{p}=1.43 \mathrm{psi}
$$

Shell Side

$$
\Delta \mathrm{p}=8 \mathrm{psi}
$$




\section{A3.3.3 Heat Exchanger 3}

Output Cooler/Final Feed Heater
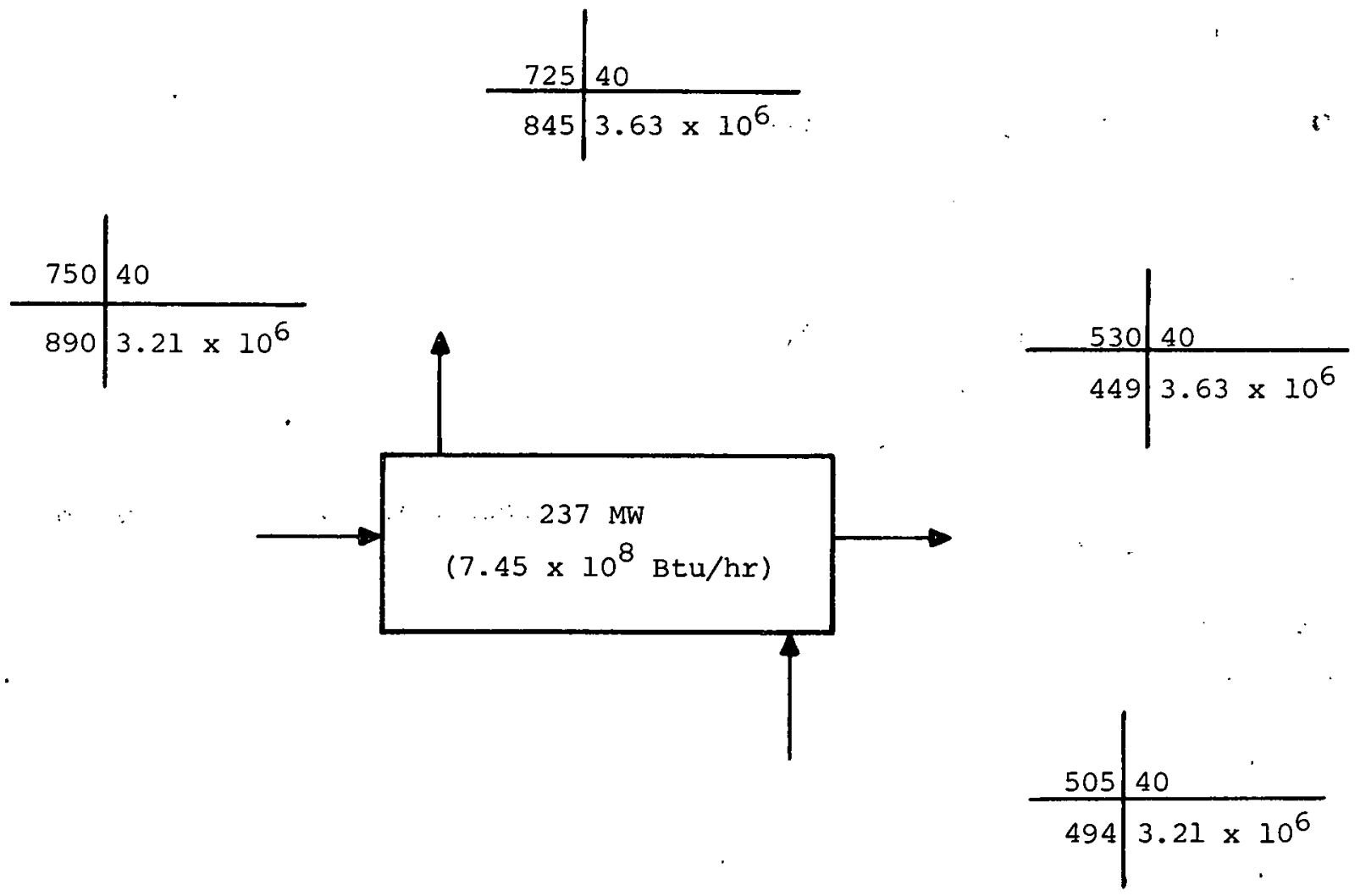

Assume: Tube-side mass velocity is $7.5 \times 10^{4} 1 \mathrm{~b}_{\mathrm{m}} / \mathrm{hr} \mathrm{ft}^{2}$

$$
\begin{aligned}
A_{\text {tube }} & =42.8 \mathrm{ft}^{2} \\
\mathrm{n} & =18448 \\
\mathrm{~A}_{\text {shell }} & =83 \mathrm{ft}^{2} \\
D_{\text {shell }} & =13.4
\end{aligned}
$$

or two 9.5-ft-diameter shells. The heat exchange area is $3147 \mathrm{ft}^{2} / \mathrm{ft}$ of combined shells:

$$
\mathrm{G}_{\text {shell }}=4.37 \times 10^{4} 1 \mathrm{~b}_{\mathrm{m}} / \mathrm{hr} \mathrm{ft}^{2}
$$

\section{Tube Side}

$$
\mathrm{h}=205 \mathrm{Btu} / \mathrm{ft} \mathrm{t}^{2} \mathrm{hr}
$$

\section{Shell Side}

$$
\begin{aligned}
& \mathrm{Re}=4.96 \times 10^{4} \\
& \mathrm{Nu}=114 \quad(\text { smooth })
\end{aligned}
$$

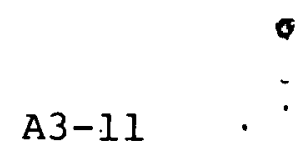


or $\mathrm{Nu}=285$ (rough)

$$
\mathrm{h}=227 \mathrm{Btu} / \mathrm{hr} \mathrm{ft}^{2} \mathrm{O}_{\mathrm{F}}
$$

Overall

$$
\begin{aligned}
& \frac{1}{U}=\frac{1}{227}+\frac{1}{205}+0.0005(1+0.35) \\
& U=100 \mathrm{Btu} / \mathrm{hr} \mathrm{ft}^{2} \mathrm{O}_{\mathrm{F}}
\end{aligned}
$$

Area

$$
\begin{aligned}
& \text { Log mean temperature difference } \\
& \begin{aligned}
\Delta \mathrm{T}_{\mathrm{LM}} & =45^{\circ} \mathrm{F} \text { (constant) } \\
\mathrm{A} & =1.65 \times 10^{5} \mathrm{ft}^{2}
\end{aligned}
\end{aligned}
$$

Therefore, two 9.5-ft-shells, $\times 52.4-\mathrm{ft}$ long heat exchangers costing a total of $\sim \$ 4.13$ million would suffice.

\section{Pressure Drop}

Tube Side

$$
\Delta \mathrm{p}=0.8 \mathrm{psi}
$$

Shell Side

$$
\Delta p=16 \text { psi }
$$




\section{A3.3.4 Heat Exchanger 4 .}

Output Cooler/Feed Boiler

On the evaporation side, the heat transfer resistance is very small. Therefore, this side is passed through the tubes:

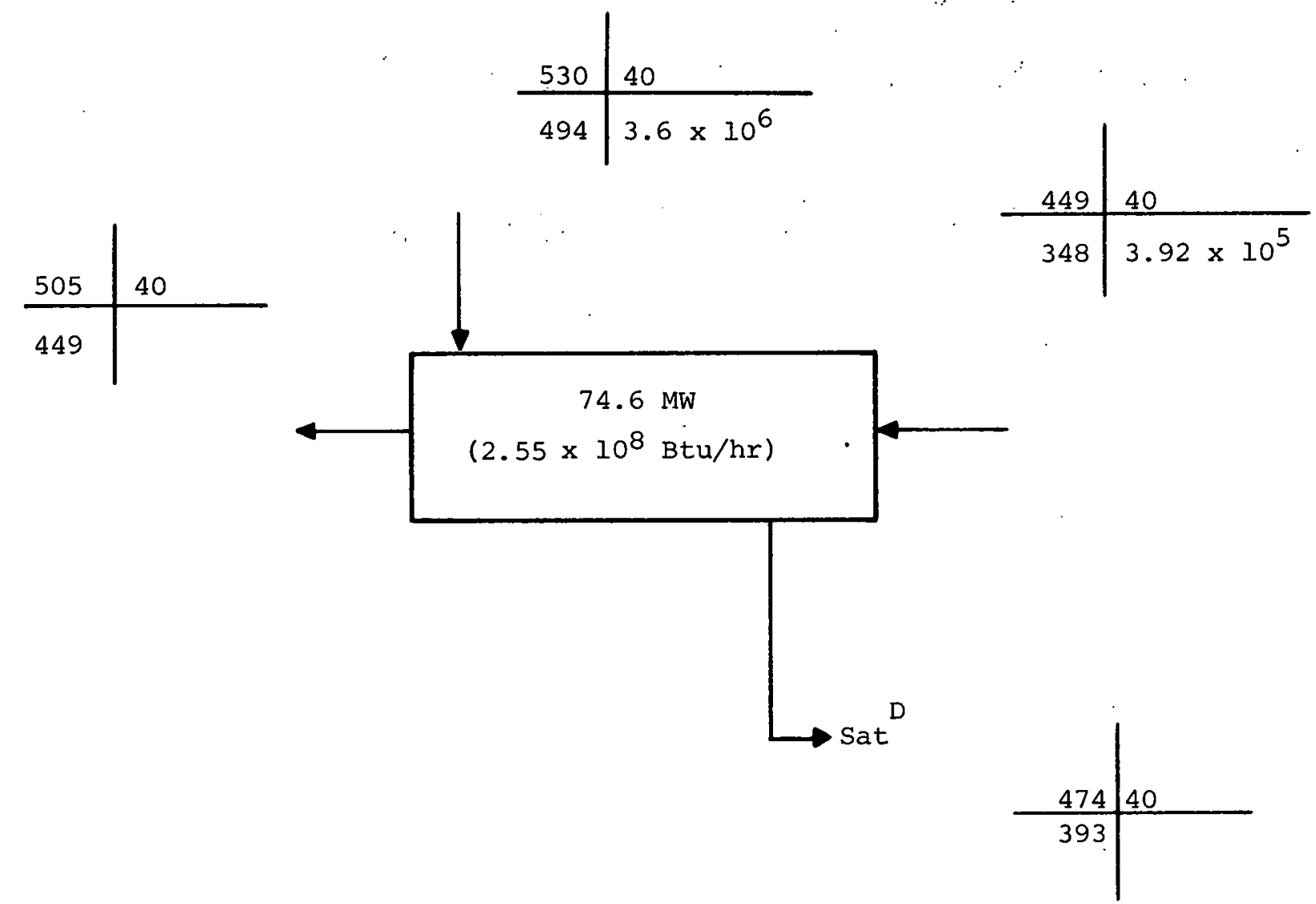

Total gas moles to reformer $=5.96 \times 10^{4} \mathrm{lb} / \mathrm{moles} / \mathrm{hr}$, of which 0.108 fraction (6429 $\mathrm{lb}$ moles/hr) enters this evaporator. Thus gas mass flow is $97080 \mathrm{lb} \mathrm{m} / \mathrm{hr}$. On the reformer feed side, the total water flow is $1.514 \times 10^{5} \mathrm{lb} / \mathrm{moles} / \mathrm{hr}$, of which $1.637 \times 10^{4} \mathrm{lb}$ moles/hr are fed to heat exchanger 4 . The total flow to this exchanger is then $\left(97080+2.946 \times 10^{5}\right)=3.92 \times 10^{5} \mathrm{lb} / \mathrm{hr}$. Since the evaporator (Heat Exchanger 7) does not evaporate all the incoming moisture, the inlet steam content is 0.223 mole fraction (saturated vapor at $348^{\circ} \mathrm{F}$ ). Hence the steam content is $4700 \mathrm{lb}$ moles/hr or $8.46 \times 10^{4} 1 \mathrm{bm} / \mathrm{hr}$ with the remaining flow of water in the liquid phase:

The tube-side energy balance may be expressed by

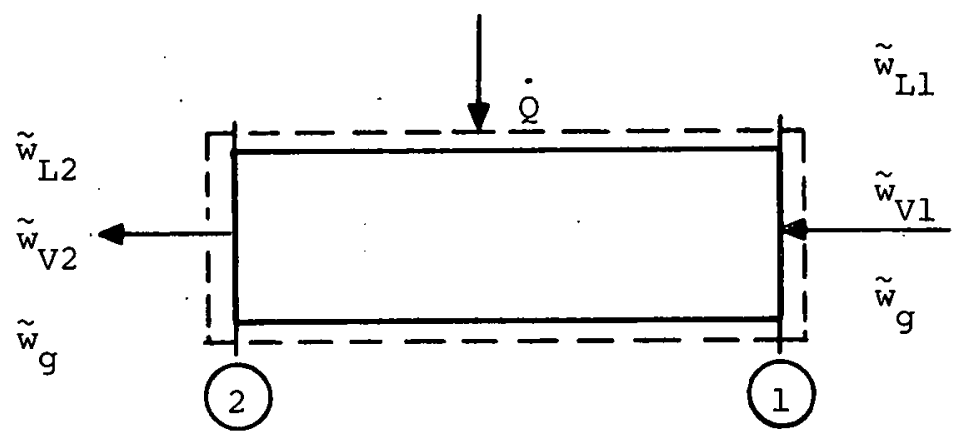




$\begin{array}{llr}\tilde{\mathrm{w}}_{\mathrm{L}} & \text { moles } / \mathrm{hr} \text { liquid } & \begin{array}{r}\left(1.167 \times 10^{4} \mathrm{lb} \text { moles } / \mathrm{hr}\right. \\ \text { at entrance })\end{array} \\ \widetilde{\mathrm{w}}_{\mathrm{V}} & \text { moles } / \mathrm{hr} \text { vapor (steam) } & (4700 \mathrm{lb} \text { moles } / \mathrm{hr} \text { at entrance }) \\ \tilde{\mathrm{w}}_{\mathrm{g}} & \text { moles } / \mathrm{hr} \text { gas } & (6429 \mathrm{lb} \text { moles } / \mathrm{hr})\end{array}$

Then between (1) and (2)

$$
\tilde{\mathrm{w}}_{L 1} \mathrm{H}_{L 1}+\tilde{\mathrm{w}}_{\mathrm{Vl}} \mathrm{H}_{\mathrm{Vl}}+\tilde{\mathrm{w}}_{\mathrm{G}} \mathrm{H}_{\mathrm{Gl}}+\dot{\mathrm{Q}}=\tilde{\mathrm{w}}_{\mathrm{L} 2}{ }_{\mathrm{H} 2}+\tilde{\mathrm{w}}_{\mathrm{V} 2}{ }_{\mathrm{H} 2}+\tilde{\mathrm{w}}_{\mathrm{G}} \mathrm{H}_{\mathrm{G} 2}
$$

Hence,

$$
\begin{gathered}
\dot{Q}=\tilde{\mathrm{w}}_{G}\left\{\mathrm{C}_{\mathrm{pg}}\left(\mathrm{T}_{2}-\mathrm{T}_{1}\right)+\frac{1}{\tilde{\mathrm{w}}_{G}}\left(\tilde{\mathrm{w}}_{L 0}-\tilde{\mathrm{w}}_{\mathrm{V} 2}\right) \mathrm{H}_{L 2}-\frac{1}{\tilde{\mathrm{w}}_{G}}\left(\tilde{\mathrm{w}}_{L O}-\tilde{\mathrm{w}}_{L 1}\right)\right. \\
\left.\mathrm{H}_{L 1}+\frac{\tilde{\mathrm{w}}_{\mathrm{V} 2}}{\tilde{\mathrm{w}}_{G}} \mathrm{H}_{\mathrm{V} 2}-\frac{\tilde{\mathrm{w}}_{\mathrm{V} 1}}{\tilde{\tilde{w}}_{\mathrm{G}}} \mathrm{H}_{\mathrm{V}}\right\}
\end{gathered}
$$

since

$$
\frac{\tilde{w}_{L}}{\tilde{w}_{G}}=\frac{1}{\tilde{w}_{G}} \quad\left(\tilde{w}_{L O}-\tilde{w}_{V}\right)
$$

and

$$
\tilde{\mathrm{w}}_{\mathrm{LO}}=1.167 \times 10^{4} \mathrm{Ib} / \mathrm{moles} / \mathrm{hr}
$$

Noting

$$
\frac{\tilde{w}_{V}}{\tilde{w}_{G}}=\frac{y_{V}}{1-y_{V}}
$$

where $\mathrm{y}_{\mathrm{V}}$ is the mole fraction of steam in vapor phase,

$$
\begin{aligned}
& \frac{\dot{Q}}{\tilde{\mathrm{w}}_{G}}=\left[\frac{\tilde{\mathrm{w}}_{\mathrm{LO}}}{\tilde{\mathrm{w}}_{\mathrm{G}}}-\left(\frac{\mathrm{y}_{\mathrm{v}}}{1-\mathrm{y}_{\mathrm{V}}}\right)_{2}\right]^{\mathrm{H}_{\mathrm{L} 2}}+\left(\frac{\mathrm{y}_{\mathrm{v}}}{1-\mathrm{y}_{\mathrm{v}}}\right)^{\mathrm{H}_{\mathrm{V}} 2} \text {. } \\
& -\left(\frac{y_{v}}{1-y_{v}}\right)_{2}^{H}-\left[\frac{\tilde{w}_{L O}}{\tilde{w}_{G}}-\left(\frac{y_{v}}{1-y_{v}}\right)_{l}\right]^{{ }^{H} L l} \text {. } \\
& +\widetilde{C}_{P G}\left(T_{2}-T_{1}\right) \\
& \text { or } \frac{\dot{\dot{Q}}}{\mathrm{w}_{G}}=\frac{\tilde{\mathrm{w}}_{\mathrm{LO}}}{\tilde{\tilde{w}}_{G}} \tilde{\mathrm{C}}_{\mathrm{PL}}\left(\mathrm{T}_{2}-\mathrm{T}_{1}\right)+\tilde{\mathrm{C}}_{\mathrm{PG}}\left(\mathrm{T}_{2}-\mathrm{T}_{1}\right) \\
& +\cdot \frac{\mathrm{Y}_{\mathrm{V}}}{1-\mathrm{y}_{\mathrm{v}}} 2\left(\mathrm{H}_{\mathrm{V} 2}-\mathrm{H}_{\mathrm{L} 2}\right)-\frac{\mathrm{Y}_{\mathrm{V}}}{1-\mathrm{y}_{\mathrm{V}}}{ }_{1}\left(\mathrm{H}_{\mathrm{V} 1}-\mathrm{H}_{\mathrm{Ll}}\right) \text {. }
\end{aligned}
$$




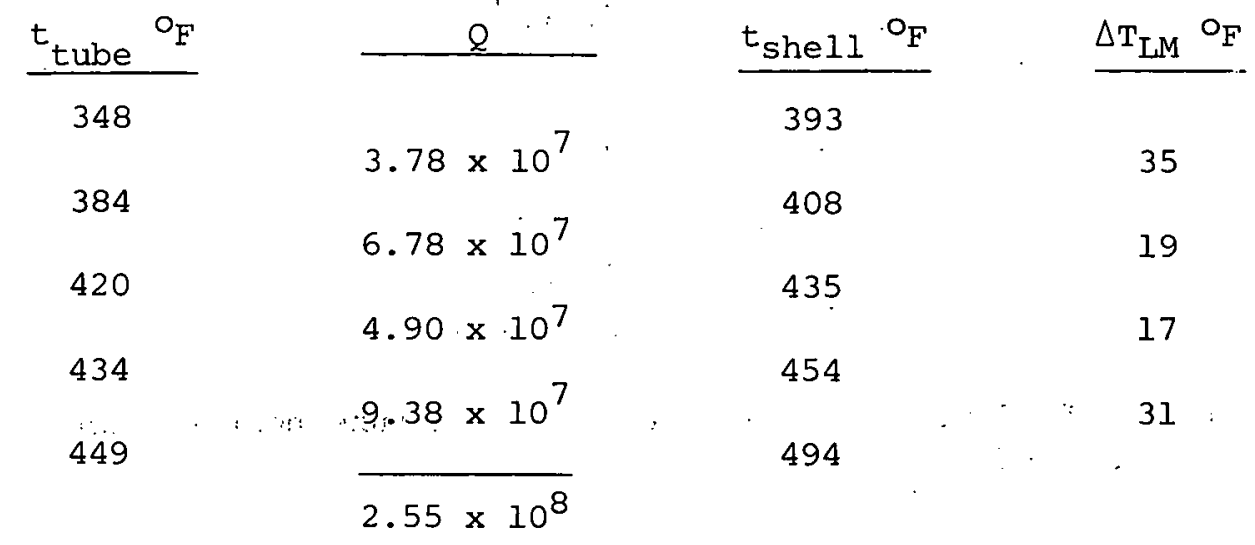

Using Equation (A3.3.4-1), the table above was generated for several segments of the evaporator. The shell side temperature was calculated from the incremental load and a logarithmic temperature difference,calculated for each of the increments.

$$
\begin{aligned}
& \text { Assume tube side }=2.5 \times \cdot 10^{4} \mathrm{lbm} / \mathrm{hr} \mathrm{ft}^{2} \text { at the outlet: } \\
& A_{\text {tube }}=15.7 \mathrm{ft}^{2} \\
& \mathrm{n}=6759 \\
& A_{\text {shell }}=30.4 \mathrm{ft}^{2} \\
& \mathrm{D}_{\text {shell }}=8.1 \mathrm{ft}
\end{aligned}
$$

The heat exchange area is $1153 \mathrm{ft}^{2} / \mathrm{ft}$ of shell:

$$
\mathrm{G}_{\text {shell }}=1.19 \times 10^{5} \mathrm{lbm} / \mathrm{hr} \mathrm{ft}^{2}
$$

\section{Tube Side}

Evaporation $\mathrm{h} \geq 2000 \mathrm{Btu} / \mathrm{hr} \mathrm{ft}^{2} \mathrm{O}_{\mathrm{F}}$

Shell Side

$$
\begin{aligned}
\operatorname{Re}=1.47 \times 10^{5} & \\
\mathrm{Nu} & =272 \\
\mathrm{~h} & =290 \text { (smooth) }
\end{aligned}
$$

\section{Overall}

$$
\begin{aligned}
& \frac{1}{U} \leq \frac{1}{290} \times \frac{0.652}{0.75} \times \frac{1}{2000}+0.00051+\left[\frac{0.652}{0.75}\right] \\
& \mathrm{U} \geq 226 \mathrm{Btu} / \mathrm{hr} \mathrm{ft}^{2} \mathrm{O}_{\mathrm{F}}
\end{aligned}
$$

(This can be improved by finning, but the shell-side pressure drop will be then excessive.) 


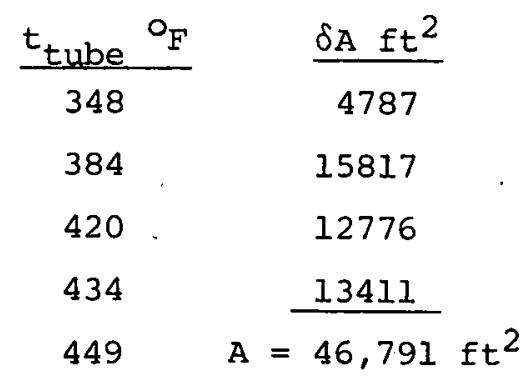

Thus, one 8.1-ft-diameter $\times 40.5$ ft shell-and-tube heat-exchanger costing $\$ 1.17$ million will suffice.

Pressure Drop

Tube side $-\Delta p=0.08 \mathrm{psi}$

Shell side $-\Delta \mathrm{p}=7.60 \mathrm{psi}$

\section{A3.3.5 Heat Exchanger 5}

\section{Feed Boiler}
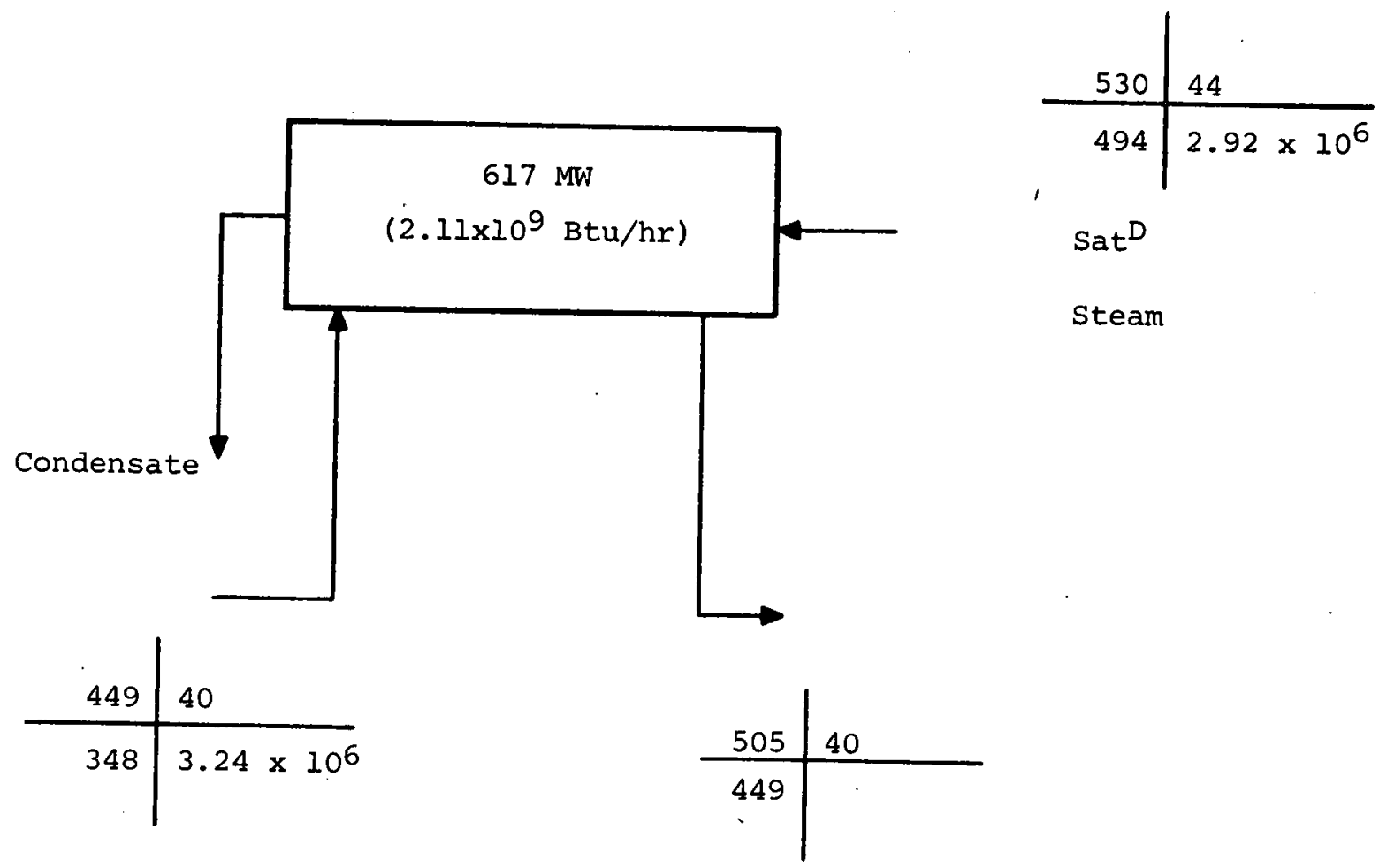

Because the condensing steam will have a high $h$, it will be condensed on the tube side.

$$
\text { Assume } G_{t u b e}=10^{5} \mathrm{lb} \text { moles } / \mathrm{hr} \mathrm{ft}^{2}
$$


Then

$$
\begin{aligned}
A_{\text {tube }} & =29.2 \mathrm{ft}^{2} \\
\mathrm{n} & =12593 \\
\mathrm{~A}_{\text {shell }} & =57.9 \mathrm{ft}^{2} \\
\mathrm{D}_{\text {shell }} & =11 \mathrm{ft} \\
\mathrm{G}_{\text {shell }} & =5.60 \times 10^{4} \mathrm{lbm} / \mathrm{hr} \mathrm{ft}^{2}
\end{aligned}
$$

\begin{tabular}{|c|c|c|c|c|}
\hline$t_{\text {shel1 }}{ }^{\circ}$ & $\begin{array}{c}\mathrm{Q} \\
\mathrm{Btu} / \mathrm{hr} \\
\end{array}$ & $t_{\text {tube }}{ }^{O_{F}}$ & $\underline{\mathrm{TM}}^{\mathrm{O}_{\mathrm{F}}}$ & $\delta \mathrm{A}\left(\mathrm{ft} \mathrm{t}^{2}\right)$ \\
\hline \multirow[t]{2}{*}{348} & & 494 & & \\
\hline & $3.13 \times 10^{8}$ & & 127 & 5365 \\
\hline \multirow[t]{2}{*}{384} & & 494 & & \\
\hline & $5.61 \times 10^{8}$ & & 91 & 13421 \\
\hline \multirow[t]{2}{*}{420} & & 494 & & \\
\hline & $4.05 \times 10^{8}$ & & 67 & 1315.9 \\
\hline \multirow[t]{2}{*}{434} & & 494. & & \\
\hline & $7.82 \times 10^{8}$ & : & 52 & 32738 \\
\hline 449 & & 494 & & \\
\hline & $2.11 \times 10^{9}$ & & & $6.47 \times 10$ \\
\hline
\end{tabular}

The heating load varies according to the inset table below:

\section{Tube Side}

For condensing steam, assume $\mathrm{h} \simeq 1200 \mathrm{Btu} / \mathrm{hr} \mathrm{ft}^{2} \mathrm{FF}$

Shell side

$$
\begin{aligned}
\mathrm{Re} & =8.77 \times 10^{4} \\
\mathrm{Nu} & =721 \quad \text { (roughened) } \\
\mathrm{h} & =585 \quad \mathrm{Btu} / \mathrm{hr} \quad \mathrm{ft}^{2} \quad \text { oF }
\end{aligned}
$$

Overall

$$
\begin{aligned}
& \frac{1}{U}=\frac{1}{1200}+\frac{1}{2.5 * 585}+0.0005\left(1+\frac{0.652}{2.5 \times 0.75}\right) \\
& \mathrm{U}=459 \mathrm{Btu} / \mathrm{hr} \mathrm{ft^{2 }} \text { OF }
\end{aligned}
$$

$\underline{\text { Area }}$

The total area (Table A3-2) is $6.47 \times 10^{4} \mathrm{ft}^{2}$. Hence, one $11-\mathrm{ft}$-diameter shell $30 \mathrm{ft}$ long, costing $\$ 1.6$ million would be adequate. 
Pressure Drop

Tube Side $-\Delta \mathrm{p} \quad 0.1 \mathrm{psi}$

Shell side $-\Delta \mathrm{p}=15.1 \mathrm{psi}$

\section{A3.3.6 Heat Exchanger 6}

\section{Condenser/Powerplant Feed Heater}

The water side will be in the tube:

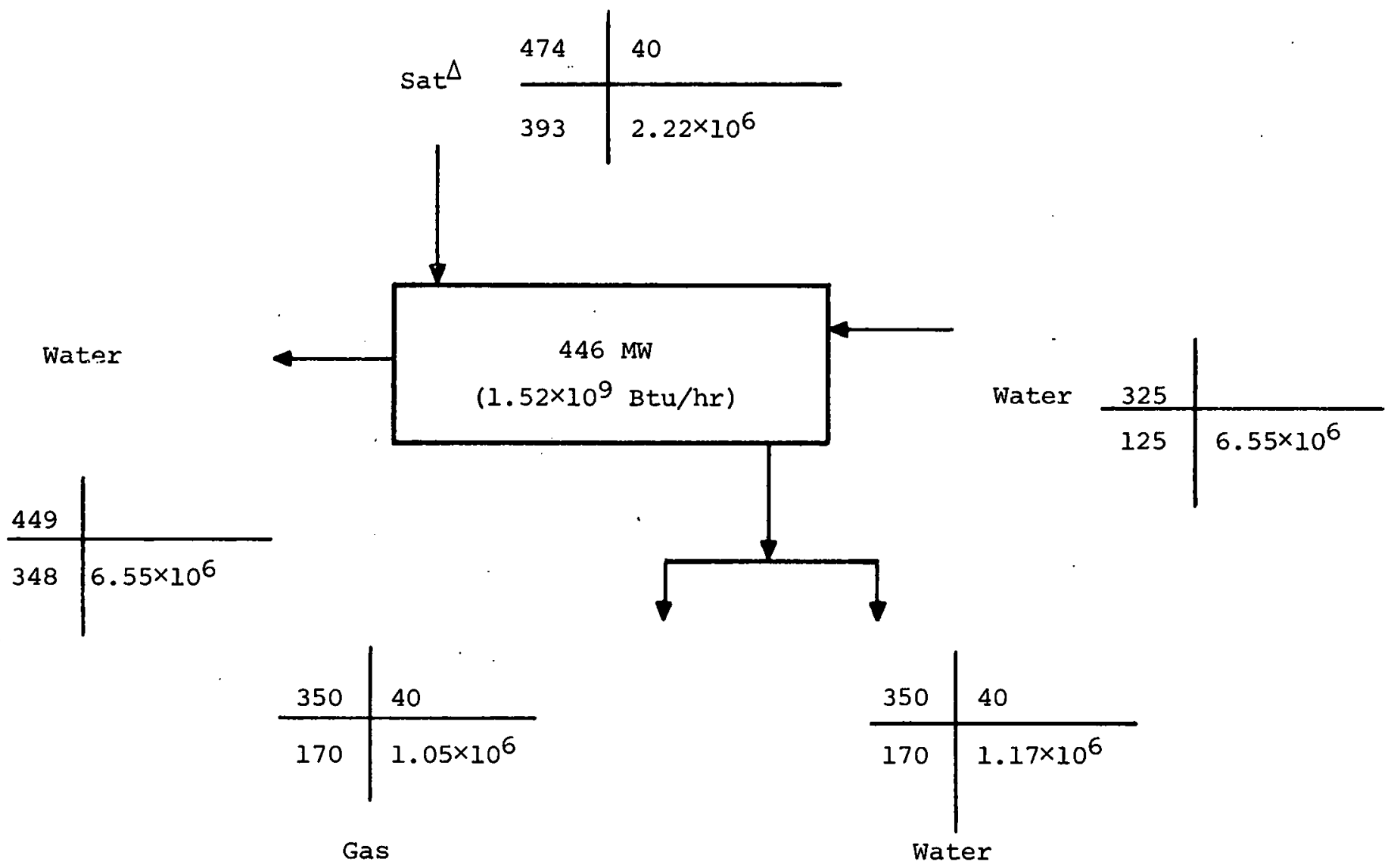




\begin{tabular}{|c|c|c|c|}
\hline${ }_{\text {shell }}{ }^{\circ} \mathrm{F}$ & $\begin{array}{c}\mathrm{Q} \\
\text { Btu/hr } \\
\end{array}$ & $t_{\text {tube }} o_{F}$ & $\mathrm{~T}_{\mathrm{LM}}{ }^{\circ} \mathrm{F}$ \\
\hline \multirow[t]{2}{*}{393} & & 348 & \\
\hline & $4.37 \times 10^{8}$ & & 62.5 \\
\hline \multirow[t]{2}{*}{368} & & 284 & \\
\hline & $2.83 \times 10^{8}$ & & 92 \\
\hline \multirow[t]{2}{*}{343} & & 242 & \\
\hline & $2.09 \times 10^{8}$ & $\vdots$ & 103.5 \\
\hline \multirow[t]{2}{*}{318} & & 212 & \\
\hline & $1.58 \times 10^{8}$ & & 105 \\
\hline \multirow[t]{2}{*}{293} & 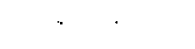 & 188.5 & \\
\hline & $2.12 \times 10^{8}$ & . & 95 \\
\hline \multirow[t]{2}{*}{243} & & 157.4 & \\
\hline & $2.21 \times 10^{8}$ & & 63 \\
\hline 170 & & 125 & \\
\hline
\end{tabular}

The above table was generated by an enthalpy balance corresponding to that used with Heat Exchanger 4; the tube-side temperature (water) was calculated by a corresponding enthalpy balance.

$$
\begin{aligned}
G_{\text {tube }} & =2 \times 10^{5} 1 \mathrm{~b} / \mathrm{hr} \mathrm{ft}^{2} \\
A_{\text {tube }} & =32.75 \mathrm{ft}^{2} \\
\mathrm{n} & =14116 \\
\mathrm{~A}_{\text {shell }} & =63.5 \mathrm{ft}^{2} \\
D_{\text {shell }} & =11.7 \mathrm{ft}
\end{aligned}
$$

The heat transfer area $\leq 2408 \mathrm{ft}^{2} / \mathrm{ft}$ of shell

$$
\mathrm{G}_{\text {shell }}=3.50 \times 10^{4} \mathrm{lbm} / \mathrm{hr} \mathrm{ft}^{2} \text { (entrance total) }
$$

Tube Side

$$
\begin{aligned}
\mathrm{Re} & =1.84 \times 10^{4} \\
\mathrm{Nu} & =67.6 \\
\mathrm{~h} & =498 \mathrm{Btu} / \mathrm{hr} \mathrm{ft}^{2} \text { oF }
\end{aligned}
$$


Shell Side.

Worst Case

Gas: $\quad G_{\text {shell }}=1.65 \times 10^{4}$ (gas only)

This is applicable near the exit.

$\mathrm{G}_{\text {shell }}=3.50 \times 10^{4} \quad($ gas + steam $)$

This is applicable near the entrance:

\begin{tabular}{|c|c|}
\hline Entrance & Exit \\
\hline $\mathrm{Nu}=630$ & 346 \\
\hline $\mathrm{h}=601$ & 491 \\
\hline
\end{tabular}

Water: Condensate will be retained in the roughness geometry. Assuming a mean fin of 0.02 inch covering $50 \%$ of the tube:

$$
\begin{aligned}
& \text { Mean water thickness }=0.01 \text { inch } \\
& \text { Resistance }=0.00208\left(\mathrm{Btu} / \mathrm{hr} \mathrm{ft} \mathrm{OF}^{2}\right)
\end{aligned}
$$

Overall

$$
\begin{aligned}
& \frac{1}{U_{\text {in }}}=\frac{1}{498}+1.35 \times 0.0005+\frac{1}{601 \times 2.5}+0.00208 \\
& U_{\text {in }}=184 \mathrm{Btu} / \mathrm{hr} \mathrm{ft}^{2} \mathrm{O}_{\mathrm{F}} \\
& \frac{1}{\mathrm{U}_{\text {out }}}=\frac{1}{495}+1.35 \times 0.0005+\frac{1}{491 \times 2.5}+0.00208 \\
& \mathrm{U}_{\text {out }}=179 \mathrm{Btu} / \mathrm{hr} \mathrm{ft}^{2} \text { oF } \\
& \text { Assume Uut }=179 \mathrm{Btu} / \mathrm{hr} \mathrm{ft}^{2} \text { oF }_{\text {out }} \text { for conservatism. }
\end{aligned}
$$


Area

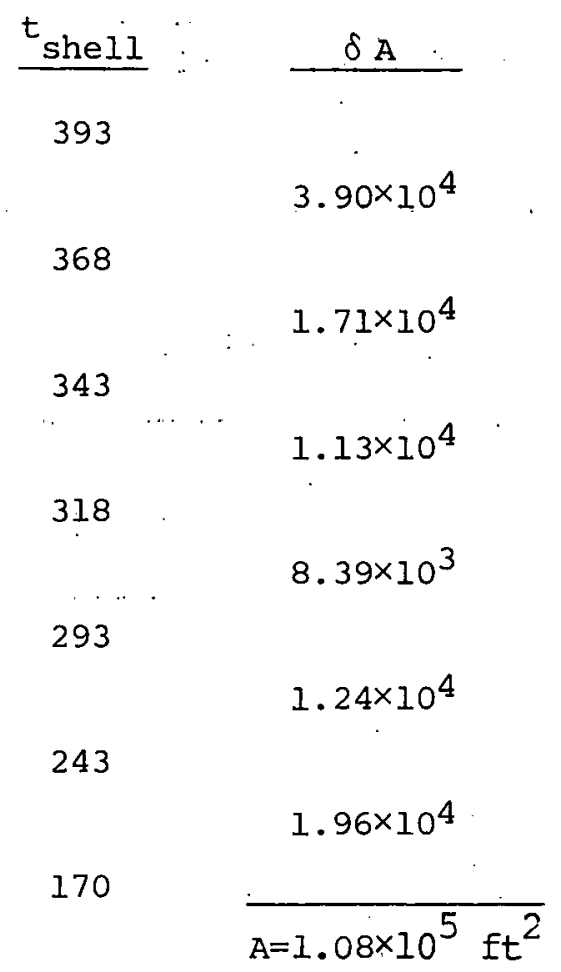

Therefore, one 11.7-ft-diameter, 44-ft-long shell-and-tube heat exchanger costing $\sim \$ 2.70$ million would be satisfactory.

Pressure Drop

$$
\begin{array}{rlrl} 
& \text { Tube side } & \Delta \mathrm{p} & =0.1 \mathrm{psi} \\
\text { Shell side } & \Delta \mathrm{p}=10.8 \mathrm{psi}
\end{array}
$$

A3.3.7 Heat Exchanger 7

Combined Condenser/Evaporator

To facilitate the reduction in the noncondensable thermal resistance, condensation will be done on the shell side: 


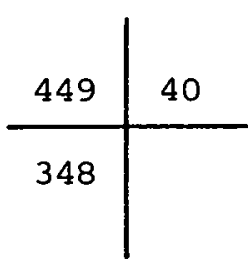

Water + Gas

+ Steam

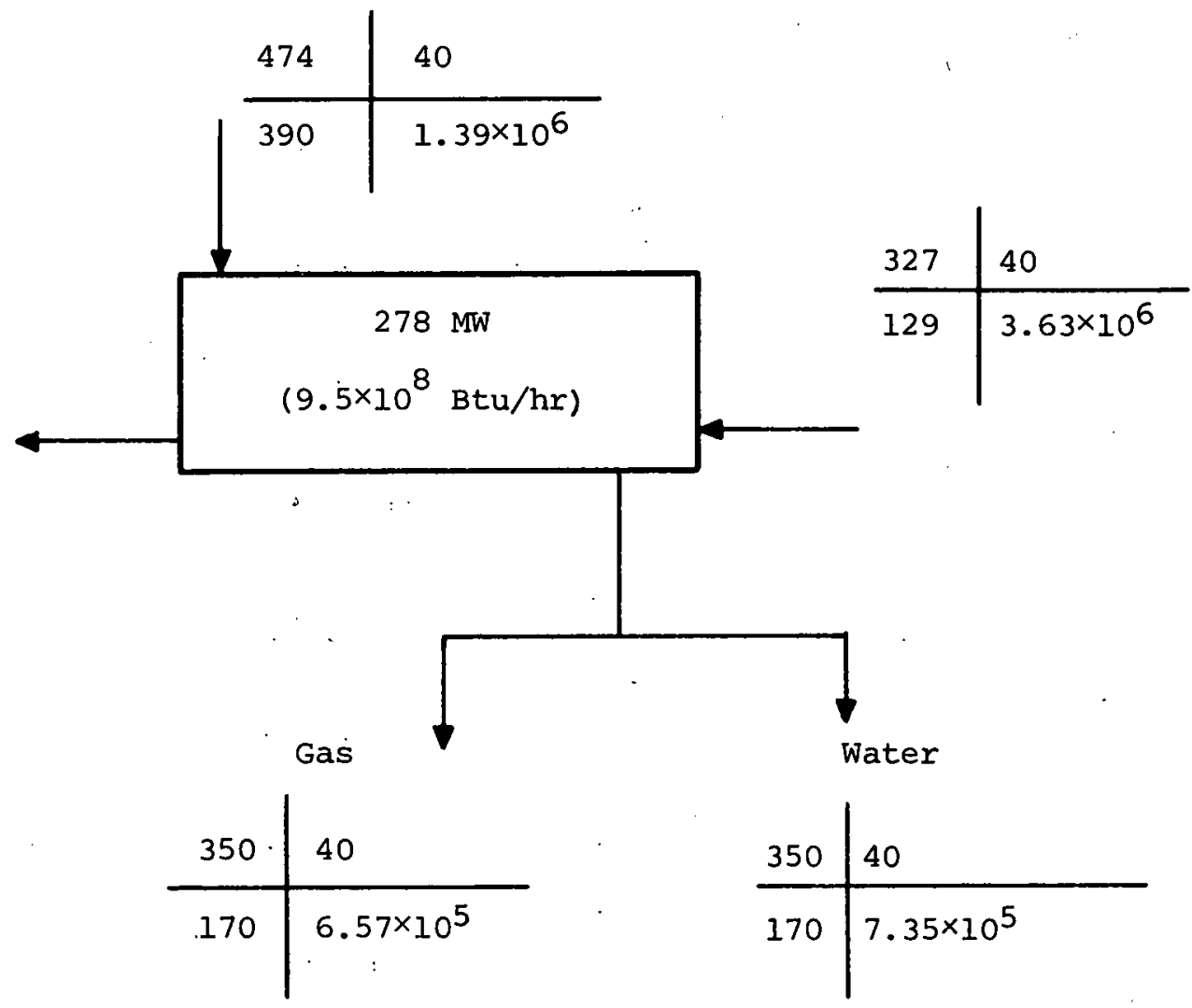

Two diphasic problems must be solved here simultaneously, at several points, to obtain the local \& vs T relationships. Summarized, these yield

\begin{tabular}{|c|c|c|c|}
\hline tube ${ }^{O F}$ & $\begin{array}{c}Q \\
\mathrm{Btu} / \mathrm{hr} \\
\end{array}$ & $t_{\text {shell }}{ }^{\circ}$ & $\mathrm{T}_{\mathrm{LM}} \mathrm{O}_{\mathrm{F}}$ \\
\hline 348 & & 393 & \\
\hline & $1.65 \times 10^{8}$ & & 51 \\
\hline 323 & & 380 & \\
\hline & $1.35 \times 10^{8}$ & & $62^{:}$ \\
\hline 298 & & 365 &.$:$ \\
\hline & $1.20 \times 10^{8}$ & & 73 \\
\hline 273 & $1.07 \times 10^{8}$ & 352 & 83 \\
\hline 248 & & 336 & \\
\hline & $9.78 \times 10^{7}$ & & 90.5 \\
\hline 223 & & 316 & \\
\hline & $9.98 \times 10^{7}$ & & 86 \\
\hline 198 & & 288 & \\
\hline & $2.26 \times 10^{8}$ & & 58 \\
\hline 129 & - & 170 & \\
\hline & $9.50 \times 10^{8}$ & & \\
\hline
\end{tabular}


Tube Exit

$$
\begin{aligned}
& \text { Assume } \quad G_{\text {tube }}=4 \times 10^{5} \mathrm{lbm} / \mathrm{hr} \mathrm{ft}^{2} \\
& \therefore A_{\text {tube }}=9.1 \mathrm{ft}^{2} \\
& \mathrm{n}=3922 \\
& \therefore{ }^{A_{\text {shell }}}=17.7 \mathrm{ft}^{2} \\
& D_{\text {shell }}=6.16 \mathrm{ft} \\
& G_{\text {shell }}^{:}=7.67 \times 10^{4} \mathrm{lbm} / \mathrm{hr} \mathrm{ft}^{2} \text { combined }
\end{aligned}
$$

The heat transfer area $=669 \mathrm{ft}^{2} / \mathrm{ft}$ of shell.

$\underline{\text { Inside }}$

Void fraction $\sim 0.96$

Flow is shear-dominated.

Water fraction is constant.

$$
\begin{aligned}
\therefore R e=\frac{G_{W} d}{\mu_{L}} & =3 \times 10^{5} \times \frac{0.652}{12} \times \frac{1}{0.48}=3.39 \times 10^{4} \\
G_{W} & =\text { mass velocity liquid water }
\end{aligned}
$$

Nondimensional film thickness:

$$
\delta^{+}=0.0504 \mathrm{Re}^{7 / 8}=464
$$

Average viscous shear gradient caused by vapor and gas flow:

$$
\begin{aligned}
\left(-\frac{\mathrm{dp}}{\mathrm{dz}}\right)_{F r} & =2 * 0.005 \times \frac{12}{0.652} \times\left(\frac{1.2 \times 10^{6}}{9.4 \times 3600}\right)^{[2} \times \frac{1}{1.17} \times \frac{1}{32.2} \\
& =6.12 \mathrm{lb} / \mathrm{ft}^{3}
\end{aligned}
$$

Shear velocity

$$
\begin{aligned}
U_{*} & =\sqrt{\frac{d_{\text {tube }}}{4 \rho_{L}}\left(\frac{d p}{d z}\right.} F r \\
& =\left(\frac{0.652}{4^{*} 12} \times \frac{6.12}{55} \times 32.2\right)^{1 / 2} \\
U_{*} & =0.232 \mathrm{ft} / \mathrm{sec}
\end{aligned}
$$


Nondimensional resistance:

$$
\left.\mathrm{T}^{+}=5 \operatorname{Pr}+\ln (1+5 \mathrm{Pr})+0.495 \ln \delta^{+} / 30\right\}
$$

Water: $\operatorname{Pr}=1.2$

$$
\mathrm{T}^{+}=22.0
$$

Martinelli analogy:

$$
\begin{aligned}
\frac{\mathrm{h}}{\rho_{L} C_{L} U_{\star}} & =\frac{1}{T^{\star}} \\
\cdot h & =1960 \mathrm{Btu} / \mathrm{hr} \mathrm{ft}^{2} \circ_{\mathrm{F}}
\end{aligned}
$$

Shell side

To condensing water interface

Assume the water interface at $T_{i}$, with water thickness of 0.01 inch held on fins:

$$
\begin{aligned}
& \frac{1}{U}=\frac{1}{1960}+0.0005 \times 1.35+\frac{2.08}{2.5} \times 10^{-3} \\
& U=489
\end{aligned}
$$

Therefore, referring to the inside area:

$$
\frac{\dot{Q}}{A_{I N}}=489\left(T_{i}-T_{\text {tube }}\right)
$$

To condensing stream

Gas coefficient

$$
\begin{aligned}
& \operatorname{Re}=8.06 \times 10^{4}: \text { (uncondensed) } \\
& \text { (7666 condensed) } \\
& \text { Uncondensed Condensed } \\
& \mathrm{Nu} \quad 671 \quad 103 \quad \text { (Roughened) } \\
& h_{G}^{\prime} \quad 640 \quad 146 \\
& h_{G} \quad 1600 \quad 365 \quad \text { (Referred to i.d.) }
\end{aligned}
$$

Mass Transfer

$$
\frac{Q}{A_{\text {in }}}=h_{G^{\prime}}\left(T_{\text {shell }}-T_{i}\right)+h_{f g^{K}} K^{\prime} \quad\left(p_{v}-p_{i}\right)
$$


where $\mathrm{T}_{\text {shell }}$ is the temperature of the condensing steam, and $\mathrm{p}$ is the partial pressure of vapor in the bulk stream (sub $v$ ) and at the interface (sub $i$ ).

$$
\frac{\mathrm{K}^{\prime} \mathrm{P}_{\text {LM }}}{2.5 \mathrm{G}_{\text {shell }}}\left(\frac{\mu}{\rho^{\mathrm{D}}}\right)_{\mathrm{V}}^{0.6}:=\frac{0.02}{2} \cdot \text { (Colburn analogy) }
$$

where $\mathrm{p}_{L M}$ is the logarithmic-mean gaseous partial pressure difference.

Since $\mathrm{T}_{\text {shell }}$ and $\mathrm{T}_{\text {tube }}$ are known at any location (tabled data), $\mathrm{T}_{i}$ and $Q / A_{\text {in }}$ can be calculated from equations (3.3.7-1 and (3.3.7-2). In the worst case, when the vapor is almost totally condensed,

$$
\mathrm{T}_{i}-\mathrm{T}_{\text {shel1 }} \simeq 3^{\circ} \mathrm{F}
$$

so the socal.jed noncondensable resistance can be neglected in this case. The overall $U$ then varies from inlet to outlet as the steam is condensed, primarily through the reduction in $h^{\prime}{ }_{G}$ by shell-side mass velocity:

$$
\begin{aligned}
& \text { Uncondensed } \frac{1}{U}=\frac{1}{489}+\frac{1}{1600} ; \text { or } U=375 \\
& \text { Condensed } \frac{1}{U}=\frac{1}{489}+\frac{1}{365} ; \text { or } U=209
\end{aligned}
$$

It can therefore be assumed that for the dependence of $\mathrm{U}$,

$$
\begin{aligned}
& \frac{1}{\mathrm{U}}=2.66 \times 10^{-3}+\frac{393-t_{\text {shell }}}{223} \times 2.12 \times 10^{3} \\
& t_{\text {shell }} \text { OF } \quad \text { Btu/hr } \mathrm{Et}^{2} \text { OF }_{\mathrm{F}} \quad \delta \mathrm{At} \mathrm{ft}^{2}
\end{aligned}
$$

$$
375 \quad 8.51 \times 10^{3}
$$

380

$$
352 \quad 6.20 \times 10^{3}
$$

365

$343 \quad 4.81 \times 10^{3}$

352

336

$321 \quad 4.00 \times 10^{3}$

$298 \quad 3.63 \times 10^{3}$

316

282

$4.12 \times 10^{3}$

288

209

$1.86 \times 10^{4}$

170

$$
\overline{A=49,900 \mathrm{ft}^{2}}
$$

This can be accommodated in a 6.25-ft-diameter shell, $75 \mathrm{ft}$ long at an approximate cost of $\$ 1.25$ million. 
Pressure Drop

Tube Side

$$
\begin{aligned}
\Delta_{\mathrm{p}} & =\frac{18 \times 6.12}{144} \mathrm{psi} \\
\Delta \mathrm{p} & =3.19 \mathrm{psi}
\end{aligned}
$$

Shell Side

$$
\Delta \mathrm{p}<2.9 \mathrm{psi}
$$




\section{Appendix 4}

\section{ANALYSIS OF TRANSMISSION COSTS FOR CHEMICAL HEAT PIPE APPLICATIONS}

\section{A4. 1 INTRODUCTION}

The chemical heat pipe has been proposed as a means of transporting thermal energy. The operation is completed in four steps:

1. Absorption of heat from location 1 by an endothermic chemical reaction.

2. Transmission of the energy-rich products from location 1 to location 2 . through the pipeline.

3. Liberation of heat at location 2 by reversing the chemical reaction of step 1.

4. Return of the energy-poor reactants by pipeline to repeat the cycle.

In this appendix a cost analysis for steps 2 and 4 - transmission of the chemicals involved - is given. This analysis is independent of the reactor operations described in steps 1 and 3 , since the chemicals enter and exit from the pipelines at a specific temperature and pressure (chosen to be $300 \mathrm{~K}$ and 40 bars for this analysis) regardless of reactor operations.

This analysis differs from traditional investigations for the transport of hydrogen or natural gas because

- Transmission distances are shorter.

- Transmission involves multiple pipelines located on the same right of way.

- The pipeline must be integrated into chemical process plants at both ends.

The transport of the chemicals used in two reaction systems is evaluated in this study. The high-temperature chemical heat pipe (HTCHP) operation involves the reforming of methane and the subsequent methanation of carbon monoxide as shown in Equations (A4-1) and (A4-2):

$$
\begin{array}{cc}
\mathrm{CH}_{4}+\mathrm{H}_{2} \mathrm{O} \rightarrow \mathrm{CO}+3 \mathrm{H}_{2} \quad \Delta \mathrm{H}_{\text {reaction }}^{298}=+206 \mathrm{KJ} / \mathrm{mole} \\
\mathrm{CO}+3 \mathrm{H}_{2} \rightarrow \mathrm{CH}_{4}+\mathrm{H}_{2} \mathrm{O} \quad \Delta \mathrm{H}_{\text {reaction }}^{298}=-206 \mathrm{KJ} / \mathrm{mole}
\end{array}
$$

Carbon dioxide is also generated in this system by the water-gas shift reaction (Equation $\mathrm{A} 4-3$ ).

$$
\mathrm{H}_{2} \mathrm{O}(\mathrm{g})+\mathrm{CO} \rightarrow \mathrm{H}_{2}+\mathrm{CO}_{2} \Delta \mathrm{H}_{\text {reaction }}^{298}=-4 \mathrm{KJ} / \mathrm{mole}
$$

The low-temperature chemical heat pipe (LTCHP) employs the dehydrogenation of cyclohexane and the hydrogenation of benzene as shown in Equations (A4-4) and $(A 4-5)$ : 


$$
\begin{aligned}
& \mathrm{C}_{6} \mathrm{H}_{12} \rightarrow \mathrm{C}_{6} \mathrm{H}_{6}+3 \mathrm{H}_{2} \quad \Delta \mathrm{H}_{\text {reaction }}^{298}=206 \mathrm{KJ} / \mathrm{mole} \\
& \mathrm{C}_{6} \mathrm{H}_{6}+3 \mathrm{H}_{2} \rightarrow \mathrm{C}_{6} \mathrm{H}_{12} \quad \Delta \mathrm{H}_{\text {reaction }}^{298}=-206 \mathrm{KJ} / \mathrm{mole} \quad(\mathrm{A} 4-5)
\end{aligned}
$$

other reactions may also take place in this system, particularly the isomerization of cyclohexane to methylcyclopentane. The LTCHP analyses presented here are based on the assumption that reactions to form side products can be suppressed by proper catalyst formulation and control of reaction conditions.

Table A4-1 shows the equilibrium reactor outlet concentrations for these two reaction systems.

Table A4-1

REACTOR OUTLET MOLAR COMPOSITIONS FOR HTCHP

AND LTCHP TRANSPORTATION ANALYSES

HTCHP System:

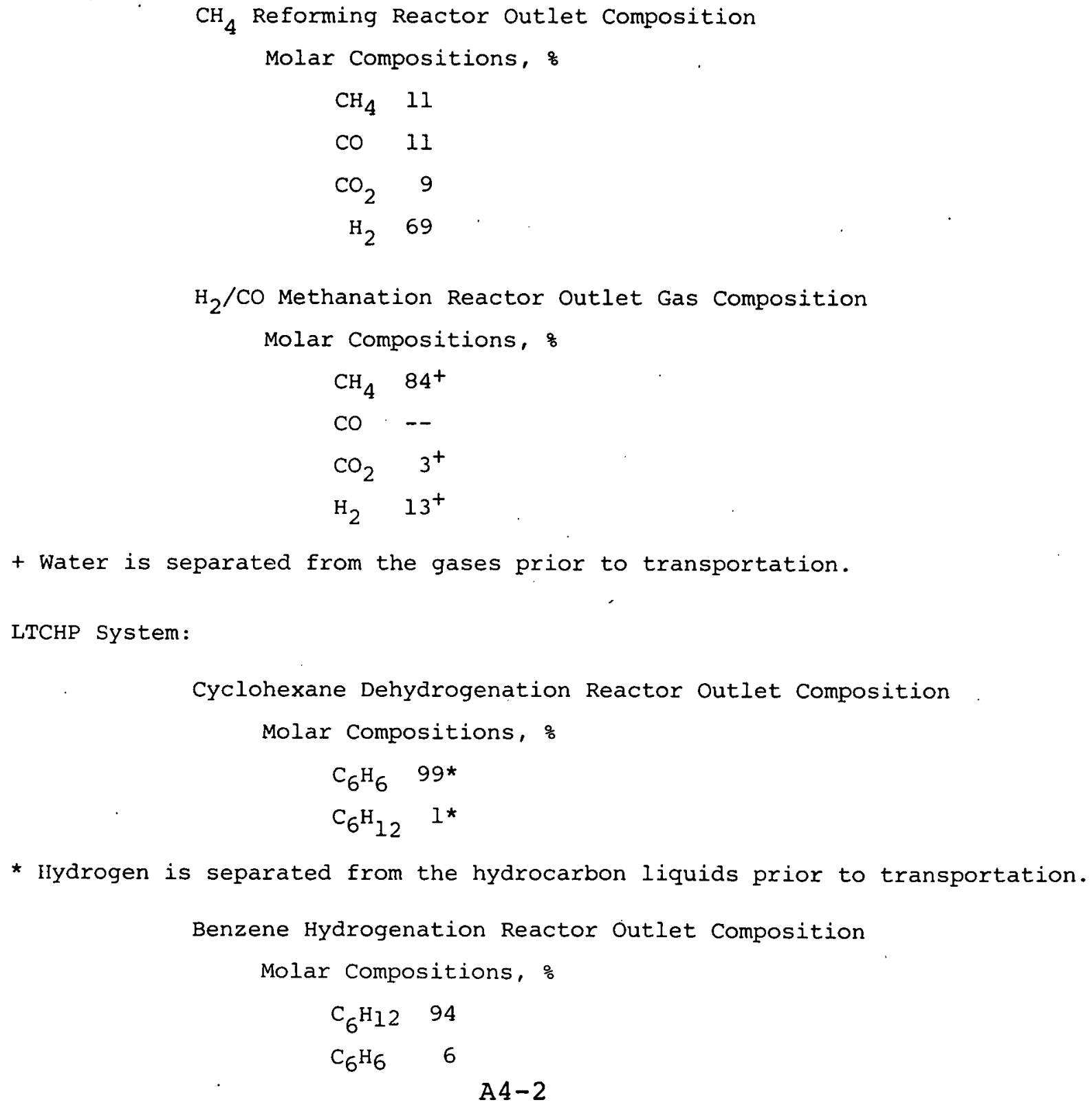

* Hydrogen is separated from the hydrocarbon liquids prior to transportation.

Benzene Hydrogenation Reactor Outlet Composition

Molar Compositions, :

$$
\mathrm{C}_{6} \mathrm{H}_{6} \quad 6
$$$$
\mathrm{C}_{6} \mathrm{H}_{12} \quad 94
$$ 
These outlet compositions define the composition of the six streams whose costs will be investigated in this report. The HTCHP transportation system is used to transmit three of these streams:

- Reformer outlet gases consisting mainly of hydrogen (69\%) with $\mathrm{CO}_{2}$, $\mathrm{CO}$, and $\mathrm{CH}_{4}$ also present.

- Methanator outlet gases consisting primarily of methane with hydrogen and carbon dioxide also present.

- Liquid water from the methanation unit.

The LTCHP transportation system involves the transmission of the other three types of fluids:

- Hydrogen gas from the dehydrogenation unit.

- The liquid products from the dehydrogenation unit, consisting mainly of benzene.

- The liquid products from the hydrogenation unit, consisting mainly of cyclohexane.

The remainder of this appendix will be devoted to analysis of the costs of transportation of these fluids. The approach that will be used is development of the equations that relate pressure drop in the transmission lines, work requirements of gas and Iiquid compression, cost of the transmission lines, and total cost of transmission to economic and design parameters. In section A4.3, the cost of transmission for the six fluids will be shown as expected when installed, on the basis of one line per right of way.

The sensitivity of the transmission cost to changes in various economic and design parameters will be explored in section A4.4, and the effect of various design philosophies on transportation cost will be investigated in section A4.5. In section A4.6, the cost of transportation for the two groups of fluids used in the HTCHP and LTCHP systems will be determined on the basis of multiple lines installed on one right of way. The economics of transport of compression work and pipeline storage of gases are given in section A4.7 and section A4.8 summarizes the findings of this appendix.

\section{A4.2 PRESSURE DROP, WORK, AND COST RELATIONSHIPS}

\section{A4.2.1 Introduction}

The goal of this section is to develop equations that allow calculation of the total cost of transporting liquids and gases present in the chemical heat pipe systems. To accomplish this development, first, the relationship for pressure drop for flow in the pipelines as a function of design parameters will be presented; then this relationship will be used in expressing the work requirements for liquid and gas transport. The costs for compressors, pumps, and transmission pipelines will be presented; and they will be combined with the work equations to yield equations which can be used to calculate the total cost of transportation as a function of the economic and design parameters.

\section{A4.2.2 Pressure Drop for Flow in Pipeline}

Equation (A4-6) gives the relationship between the pressure drop and the various design parameters for transmission of a fluid through a pipeline

$$
\text { A } 4-3
$$




$$
\frac{\Delta \mathrm{P}}{\mathrm{I}}=\frac{2 \mathrm{f} \rho \mathrm{v}^{2}}{\mathrm{~g}_{\mathrm{C}} \mathrm{D}}
$$

where $\Delta \mathrm{P} / \mathrm{L}=$ pressure drop per unit length

$$
\begin{aligned}
f & =\text { friction factor } \\
\rho & =\text { fluid density } \\
v & =\text { fluid velocity } \\
g_{C} & =\text { gravitational constant } \\
D & =\text { pipeline diameter }
\end{aligned}
$$

The friction factor, $f$, has been correlated with the Reynolds number, $\mathrm{N}_{\mathrm{Re}}=\rho D v / \mu(\mu=$ viscosity $)$, for various fluids and has a typical value of 0.005 for flow of fluid in the turbulent region $\left(\mathrm{N}_{\mathrm{Re}}>2100\right)$.

For the flow of ideal gases (compressible fluids)

$$
V=\frac{8 N R T}{\pi D^{2}\left(P_{1}+P_{2}\right)}
$$

and

$$
\rho=\frac{M\left(P_{I}+P_{2}\right)}{2 R T}
$$

where $\mathrm{N}=$ molar flowrate

$\mathrm{R}=$ gas constant

$\mathrm{T}=$ temperature

$\mathrm{P}_{1}=$ pressure at outlet of pipe

$\mathrm{P}_{2}=$ pressure at inlet of pipe

Substitution of Equations (A4-7) and (A4-8) into (A4-6) yields

$$
\mathrm{P}_{2}{ }^{2}-\mathrm{P}_{1}{ }^{2}=\frac{64 \mathrm{f1N} \mathrm{N}^{2} \mathrm{MRT}^{*}}{\pi^{2} \mathrm{~g}_{\mathrm{C}} \mathrm{D}^{5}}
$$

Equation (A4-10) shows the equivalent relationship for the flow of liquids:

$$
P_{2}-P_{1}=\frac{32 f N^{2} M^{2} L}{\pi^{2} g_{C} \rho D^{5}}
$$

\footnotetext{
*Equation (A4-9) can be derived rigorously from the integration of the differential form of Equation (A4-6), utilizing rigorous definitions of $v$ and $\rho$.
} 


\section{A4.2.3 Work Requirements for Gas Compression}

The work required to compress $\mathrm{N}$ moles of a gas from an initial pressure, $P_{i}$, to a final pressure, $P_{f^{\prime}}$. is given by. Equation (A4-II):

$$
w_{C}=\frac{N R T \gamma}{\eta_{C}(\gamma-1)}\left[\left(\frac{P_{f}}{P_{i}}\right)^{\frac{\gamma-1}{\gamma}}-1\right]
$$

where $\gamma=$ ratio of heat capacity at constant pressure to the heat capacity at constant volume

$\eta_{C}=$ adiabatic efficiency of the compressor

It is assumed in this study that the compression work done during transmission is sufficient to account for the pressure drop experienced by the gas as it flows through the line; thus, the outlet pressure from the transmission line is equivalent to the inlet pressure. Therefore, Equations (A4-9) and (A4-11) can be combined to yield the work requirement per unit length for gas transmission as a function of the design parameters:

$$
\frac{W_{C}}{L}=\frac{N R T \gamma}{\eta_{C} L(\gamma-1)}\left[\left(\frac{64 f L N^{2} M R T}{\pi^{2} g_{C} D^{5} P^{2}}+1\right)^{\frac{\gamma-1}{2 \gamma}}-1\right]
$$

This derivation assumes that the numerical compression ratio $\left(P_{f} / P_{i}\right)$ is small; therefore the ratio of the heat capacities, $\gamma$, is constant during the compression.

\section{A4.2.4 Work Requirements for Liquid Pumping}

The work required to pump $N$ moles of a liquid from $P_{i}$ to $P_{f}$ is

$$
W_{p}=\frac{N M}{\rho}\left(P_{f}-P_{i}\right)
$$

The work per unit length required to recover the pressure drop during flow of a liquid in a pipeline can be determined by the combination of Equations $(A 4-10)$ and $(A 4-13)$ :

$$
\frac{W_{p}}{L}=\frac{32 \mathrm{fN}^{3} M^{3}}{\pi 2 g_{C} \rho^{2} D^{5}}
$$

\section{A4.2.5 Compressor Costs}

Either axial or centrifugal compressors with ratings of the order of $10 \mathrm{MW}$ are most suitable for CHP applications. Reported installed costs (RA4-1) for pipeline station compressors range from approximately $\$ 240 / \mathrm{KW}_{e}$ to $\$ 900 / \mathrm{KW}_{e}$ and average about $\$ 500 / \mathrm{KW}_{e}$. On the other hand, large axial compressors installed in inplant locations are considerably les's expensive with installed costs of the order of $\$ 65 / \mathrm{KW}_{e}(\mathrm{RA} 4-2)$. 
For an inplant location where land and services are available, lower cost might be expected. In CHP applications, an inplant compressor could be coupled directly to the main steam turbine to eliminate the intermediate generator, switchgear, and electric motor. This configuration would result in a lower capital cost and smaller effective cost of energy. With these costs in mind, the compressor capital cost was chosen as $\$ 100 / \mathrm{KW}$ for plant site locations and $\$ 350 / \mathrm{KW}$ for substation locations. The annualized cost was considered to be $20 \%$ of the total capital cost.

The cost of energy, which will be shown to dominate the total compressor and pump costs, will be assumed independent of location. Scaling the compressor cost by a power-law relationship made no significant difference in the results presented here. In view of the preliminary cost estimates used, the effect of variations in compressor and electrical cost on transmission cost will be explored parametrically in section $\mathrm{A} 4-4.3$.

\section{A4.2.6 Pump Costs}

The cost of the pumps for liquid transportation were taken from Guthrie (RA4-3), updated to mid-1976 costs by the Chemical Engineering Plant cost Index (RA4-4), and approximated by the following equation, assuming a fixed capital rate of $20 \%$ year for

$$
\begin{aligned}
& \begin{aligned}
W_{\text {pump }}< & 400 \mathrm{KW}_{e} \\
& \operatorname{cost}\left(\$ / K W_{e}-\mathrm{yr}\right)=-0.8765 * \mathrm{~W}_{\mathrm{p}}+116.091+\frac{467.16}{W_{p}}
\end{aligned} \\
& \begin{aligned}
\mathrm{w}_{\text {pump }}> & 400 \mathrm{~kW} \\
& \operatorname{cost}\left(\$ / \mathrm{KW}_{\mathrm{e}}-\mathrm{yr}\right)=235.975 \mathrm{~W}_{\mathrm{p}}^{-0.176}
\end{aligned}
\end{aligned}
$$

For example, these equations yield a total capital cost rate of $\$ 395 / \mathrm{KW}$ for a $500 \mathrm{KW}_{\mathrm{e}}$ unit and $\$ 605 / \mathrm{KW}$ e for a $50 \mathrm{KW}_{e}$ unit.

\section{A4.2.7 Pipeline Costs}

Reported pipeline costs for FY 1976 (RA4-1) were fitted to the empirical relationship

$$
\operatorname{cost}=a+b D+c P D^{2}
$$

The three terms approximate the cost contributions of the right of way, the installation, and the pipeline materials, respectively. It was assumed that the reported costs were for pipelines designed for a maximum pressure of 70 bar ( 1000 psia). The variation of material costs (pipeline thickness) with pressure is accounted for by the inclusion of the pressure dependence in the second order term. The annualized cost of the line (including depreciation, interest, taxes) was assumed to be $20 \%$ of the total cost of the line.

For analyses using multiple lines on one right of way, it was assumed that the costs for the materials of construction (c term) were additive and the cost of the right of way (a term) was constant, independent of the number of lines installed. The installation cost ( $b$ term) was assumed to be a function of the pipeline diameters: 


$$
\text { installation cost }=b\left(D_{1}^{2}+D_{2}^{2} \cdot \cdot .\right)^{1 / 2}
$$

In this case, the diameter term represents a diameter of a single line having the same area as the sum of the areas of the constituent pipelines.

Figure (A4-1) shows the range of annualized costs of pipelines constructed during FY 1976 in $\$ / \mathrm{km}-\mathrm{Yr}$, as a function of pipeline diameter. Also shown are the average costs for each diameter and the curve representing the relationship used in this investigation with $\mathrm{a}=2500, \mathrm{~b}=16,096$ and $\mathrm{c}=238$ for $\mathrm{P}$ in bars and $D$ in meters. The large range of pipeline costs results from variation in factors. such as the type of terrain, weather during construction, and local labor conditions. .

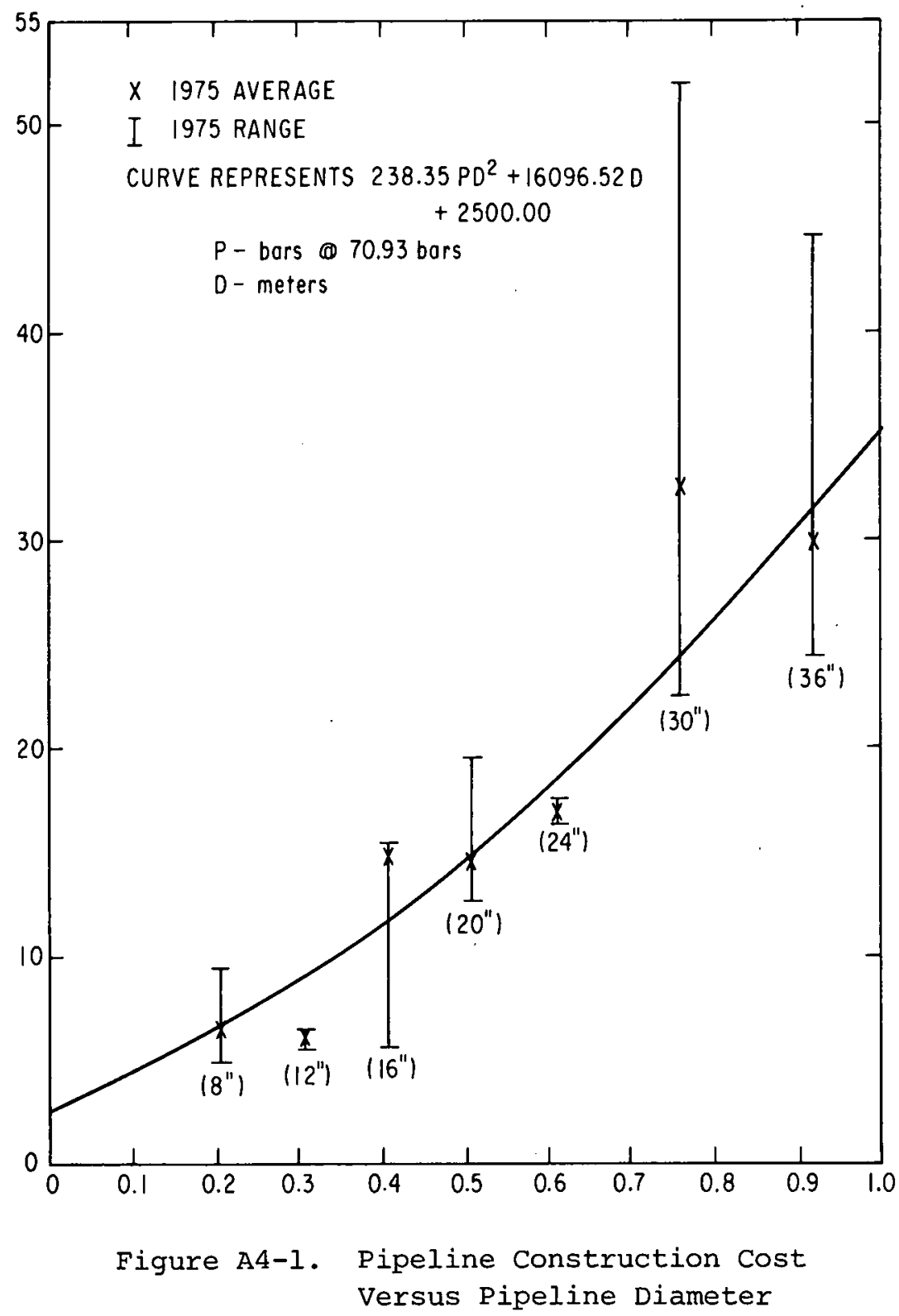


Other available data (RA4-5, $-6,-7$ ) are in reasonably good agreement with the costs used here. For example, Konopka and Wurm (RA4-5) present data for pipeline costs as a function of diameter and design pressure from actual. quotes. When these data are updated to an equivalent time period, using appropriate factors (RA4-8), agreement is within $10 \%$ of the costs used in this report. Considering the approximate nature of the correlation and the update factor, this agreement was considered satisfactory. It should be noted that the data of Konopka show that the line design is based on an allowable design stress of 0.5 times the yield strength. This is important in determining wall thicknesses and total line costs, given the cost of steel.

The use of Equation (A4-1:7) to describe pipeline costs is a simplification which does not account for the fact that pipelines are manufactured in discrete diameters ( 36 or 42 inches). The impact of this quantization should be evaluated in more detailed investigations.

\section{A4.2.8 Total Cost of Transmission}

The total cost per year of transmitting a fluid through a pipeline, $\mathrm{C}_{\mathrm{T}}$, is determined by

1. Annualized pipeline capital cost, $C_{P}$

2. Annualized capital cost for the pump or compressor, $C_{M}$

3. Annual operating cost of energy to run the machinery

If a yearly operating period of 8000 hours and electric drives is assumed, then the total annual cost of transmitting the fluid per unit length and per unit flow is

$$
C_{T}=\frac{C_{p}}{N}+\frac{W}{N L}\left(C_{M}+8000 C_{e}\right) \$ / k m-y r-t-m o l e / h r
$$

where $\mathrm{C}_{e}=$ cost of electricity, $\$ / \mathrm{kWh}$

The relationships developed previously can be substituted in Equation (A4-19) to yield Equation (A4-20) for gas transport and Equation (A4-2l) for liquid transport, giving the total cost of transmission as a function of the economic and design parameters.

Total Cost for Gas Transmission

$$
C_{T^{\prime}} \text { gas }=\frac{a}{b}+\frac{b D}{N}+\frac{C_{D P}^{2}}{N}+\left(C_{M}+8000 C_{e}\right)^{2} \frac{\gamma \cdot R T}{(\gamma-1) \eta_{C}^{L}}\left[\left(\frac{64 f_{L N}^{2} M R T}{\pi^{2} g_{C} D^{5}}\right)^{\frac{\gamma-1}{2 \gamma}}-1\right](A 4-20)
$$

\section{Total Cost for Liquid Transmission}

$$
C_{T^{\prime} \text { liquid }}=\frac{a}{N}+\frac{b D}{N}+\frac{C P D}{N}+\left(C_{M}+8000 C_{e}\right) \frac{32 f N^{2} M^{3}}{n_{p} \pi^{2} g_{C} \rho^{2} D^{5}}
$$


Equations (A4-20) and (A4-21) have been used as the basis for computer programs which calculate transmission costs as a function of the various design and economic parameters. Examples of these programs are given in Appendix 6 . (see TGLI, TLP1). Other programs were written to calculate optimum line diameters as a function of design and economic parameters (see Appendix 6, DOPGL, DOPTLP).

\section{A4.3 TRANSPORTATION COSTS FOR CHEMICAL HEAT PIPE MATERIALS}

\section{A4.3.1 Introduction}

The relationships developed in the previous section will be used in this section to determine the costs of transmitting the various. product streams present in the proposed high- and low-temperature chemical heat pipe systems. These cost analyses are performed assuming one pipeline per trench.

\section{A4.3.2 Design Basis}

The base case values for the design and economic parameters used in these analyses are given in Table $44-2$.

Table A4-2

BASE CASE VALUES FOR ECONOMIC AND DESIGN PARAMETERS FOR CHEMICAL HEAT PIPE REACTANTS

Compositions, HTCHP (moles)

\begin{tabular}{|c|c|c|c|}
\hline \multirow{3}{*}{\multicolumn{2}{|c|}{ Reformer Exit Gases }} & $\mathrm{CO}_{2}$ & 98 \\
\hline & & $\mathrm{H}_{2}$ & 698 \\
\hline & & co & 118 \\
\hline & $\therefore$ & $\mathrm{CH}_{4}$ & 118 \\
\hline & Methanator Exit Gases & $\mathrm{CH}_{4}$ & 848 \\
\hline & & $\mathrm{H}_{2}$ & 138 \\
\hline & & $\mathrm{CO}_{2}$ & 38 \\
\hline & Methanator Exit Liquice & $\mathrm{H}_{2} \mathrm{O}$ & 1008 \\
\hline \multicolumn{4}{|c|}{ Compositions, LTCHP (moles } \\
\hline \multirow{6}{*}{ ' } & Dehydrogenation Exit Gas & $\mathrm{H}_{2}$ & 1008 \\
\hline & $\begin{array}{l}\text { Dehydrogenation Exit } \\
\text { Liquid }\end{array}$ & $\mathrm{C}_{6} \mathrm{H}_{6}$ & 998 \\
\hline & & $\mathrm{C}_{6} \mathrm{H}_{12}$ & 18 \\
\hline & $\begin{array}{l}\text { Hydrogenation Exit } \\
\text { Liquid }\end{array}$ & $\mathrm{C}_{6} \mathrm{H}_{6}$ & 68 \\
\hline & & $\mathrm{C}_{6}{ }^{\mathrm{H}} 12$ & 948 \\
\hline & Pressure & & \\
\hline & Transportation System Inlet & & 40 bars \\
\hline & Transportation System Oulet & & 40 bars \\
\hline & Maximum Pressure & & 70 bars \\
\hline & Minimum Pressure & & 5 bars \\
\hline Temperatur & e - Transportation System I & nlet & $300 \mathrm{~K}$ \\
\hline Transmissi & Lon Distance & & $160 \mathrm{~km}$ (100 miles) \\
\hline Compressor & and Pump Efficiences & & 0.8 \\
\hline Cost of $\mathrm{El}$ & Lectricity & & $20 \mathrm{mills} / \mathrm{kWh}$ \\
\hline Operating & Factor & & $8000 \mathrm{hr} / \mathrm{yr}$ \\
\hline $\begin{array}{l}\text { Gamna (rat } \\
\text { capacit }\end{array}$ & $\begin{array}{l}\text { =io of heat capacity at cons } \\
\text { Ey at constant volume) }\end{array}$ & tant pres & ssure to heat \\
\hline & Reformer Exit Gases & & 1.43 \\
\hline & Methanator Exit Gases & & 1.30 \\
\hline & Dehydrogenation Exit Hydro & & 1.41 \\
\hline
\end{tabular}


The base case design philosophy utilizes compressor stations located only at the reactor sites. Liquid pumping follows the philosophy of allowing the liquid entering the system to flow through the pipeline until the minimum pressure of 5 bars is reached; then the liquid is again pumped to 40 bars and the cycle repeated until the total transmission distance is reached. The liquid is then pumped to 40 bars and exits from the transmission system.

\section{A4.3.3 Results and Discussion}

Figure A4-2 through A4-7 show transportation costs for the $\mathrm{H}_{2} / \mathrm{CO}, \mathrm{CH}_{4}$, $\mathrm{H}_{2} \mathrm{O}, \mathrm{C}_{6} \mathrm{H}_{6}, \mathrm{C}_{6} \mathrm{H}_{14}$, and $\mathrm{H}_{2}$ streams, respectively, as a function of flowrate for various pipeline diameters. In each case, the transportation cost for a given pipeline diameter goes through a minimum as a function of flowrate. At flowrates below the optimal rate, the rate of decrease in pipeline capital cost with flowrate is greater than the rate of increase in compression cost. At flowrates above the optimum, the rate of increase of compression cost per unit flow dominates the rate of savings in pipeline capital cost per unit flow. As expected, these figures show that an optimal pipeline diameter exists at any given flowrate. Figure A4-8 shows this optimum line diameter for CHP materials transport as a function of power delivered by the chemical heat pipe. The flowrates for the various streams are directly proportional to the power de- . livered; the relationship between the various flows and the power delivered is specified in Table A4-3.

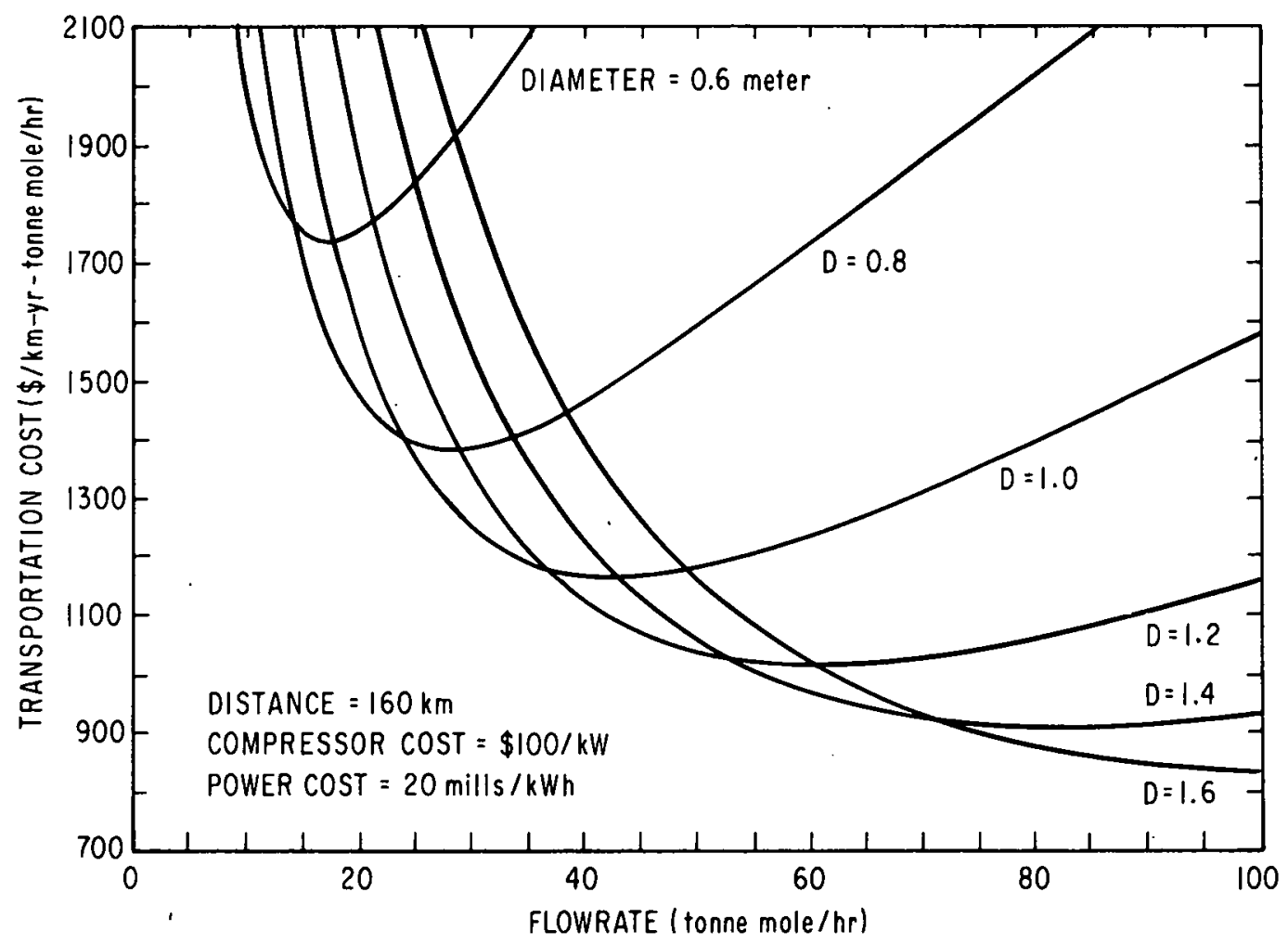

Figure A4-2. Transportation Cost of Reformer Exit Gases Versus Flowrate 


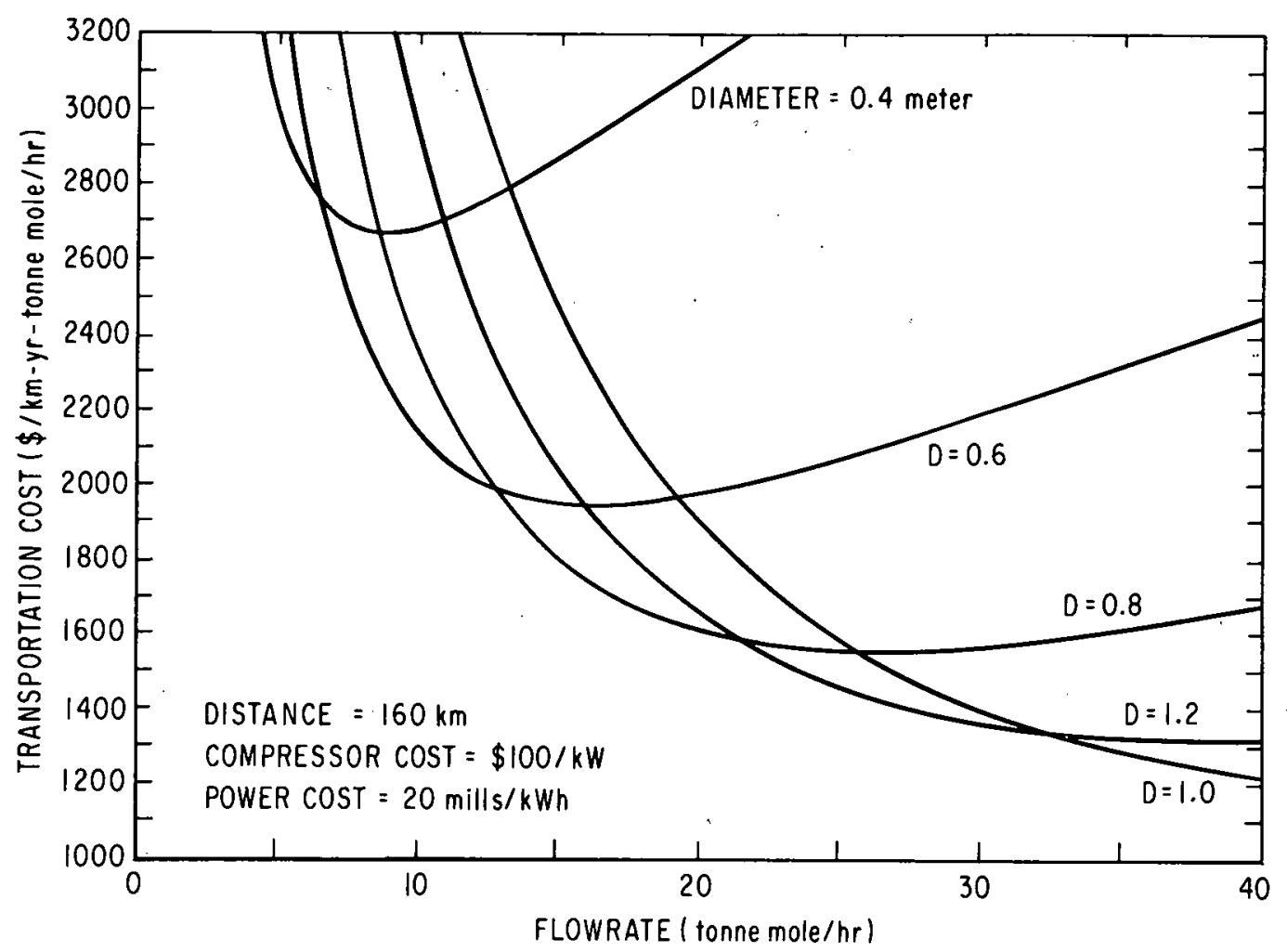

Figure A4-3. Transportation Cost of Methanator Exit Gases Versus Flowrate

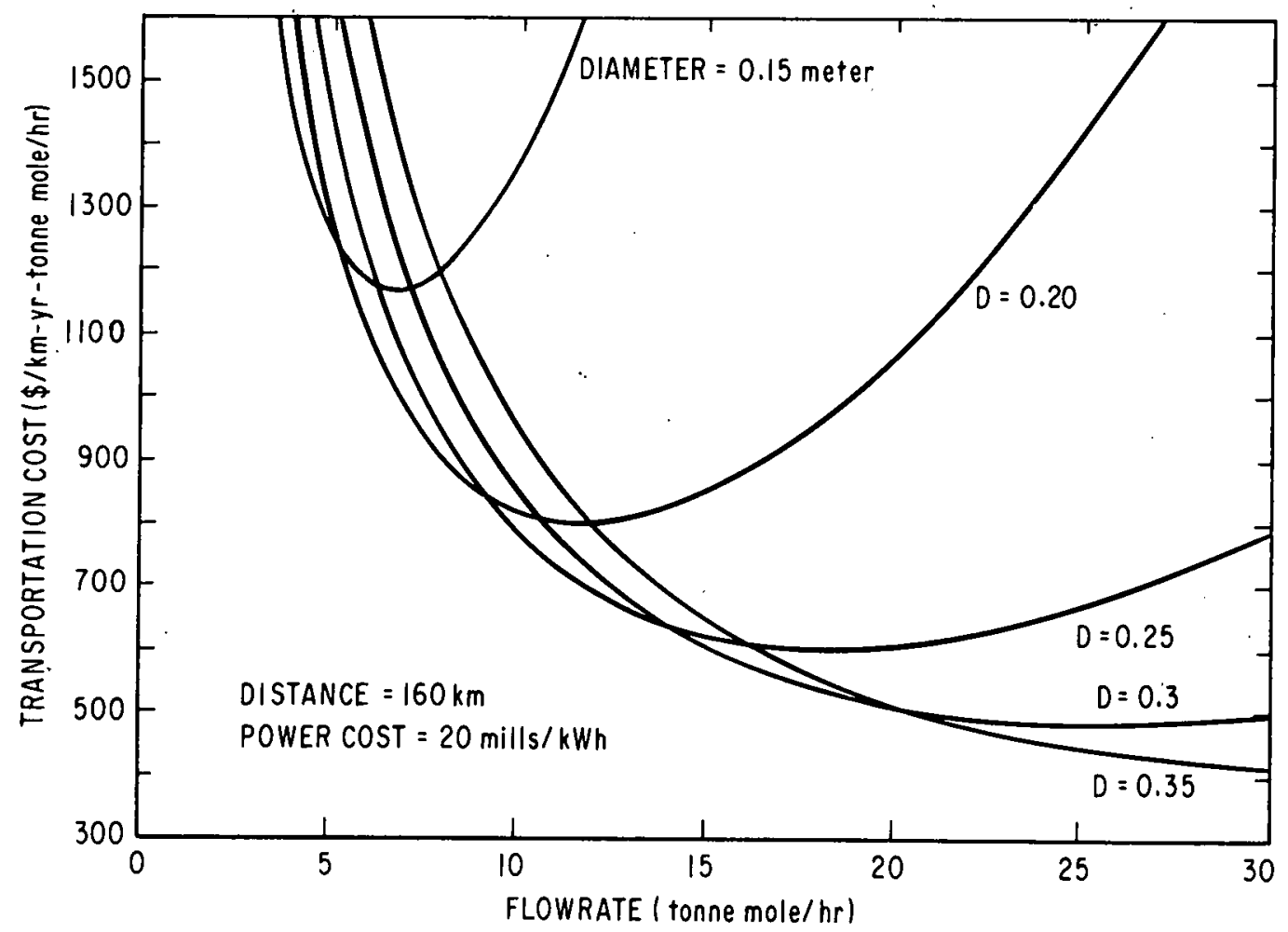

Figure A4-4. Transportation Cost of Water Versus Flowrate 


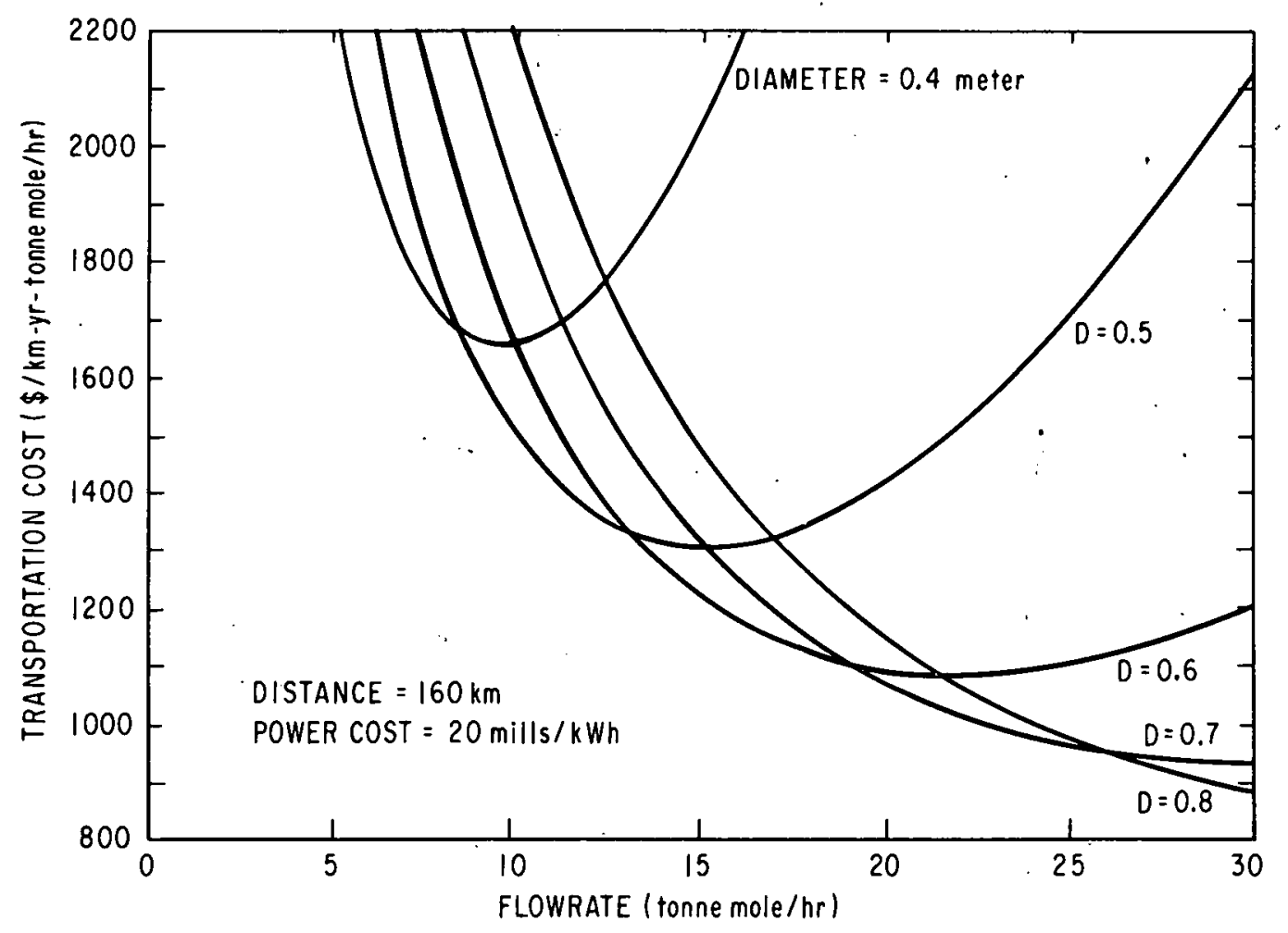

Figure A4-5. Transportation Cost of Benzene Versus Flowrate

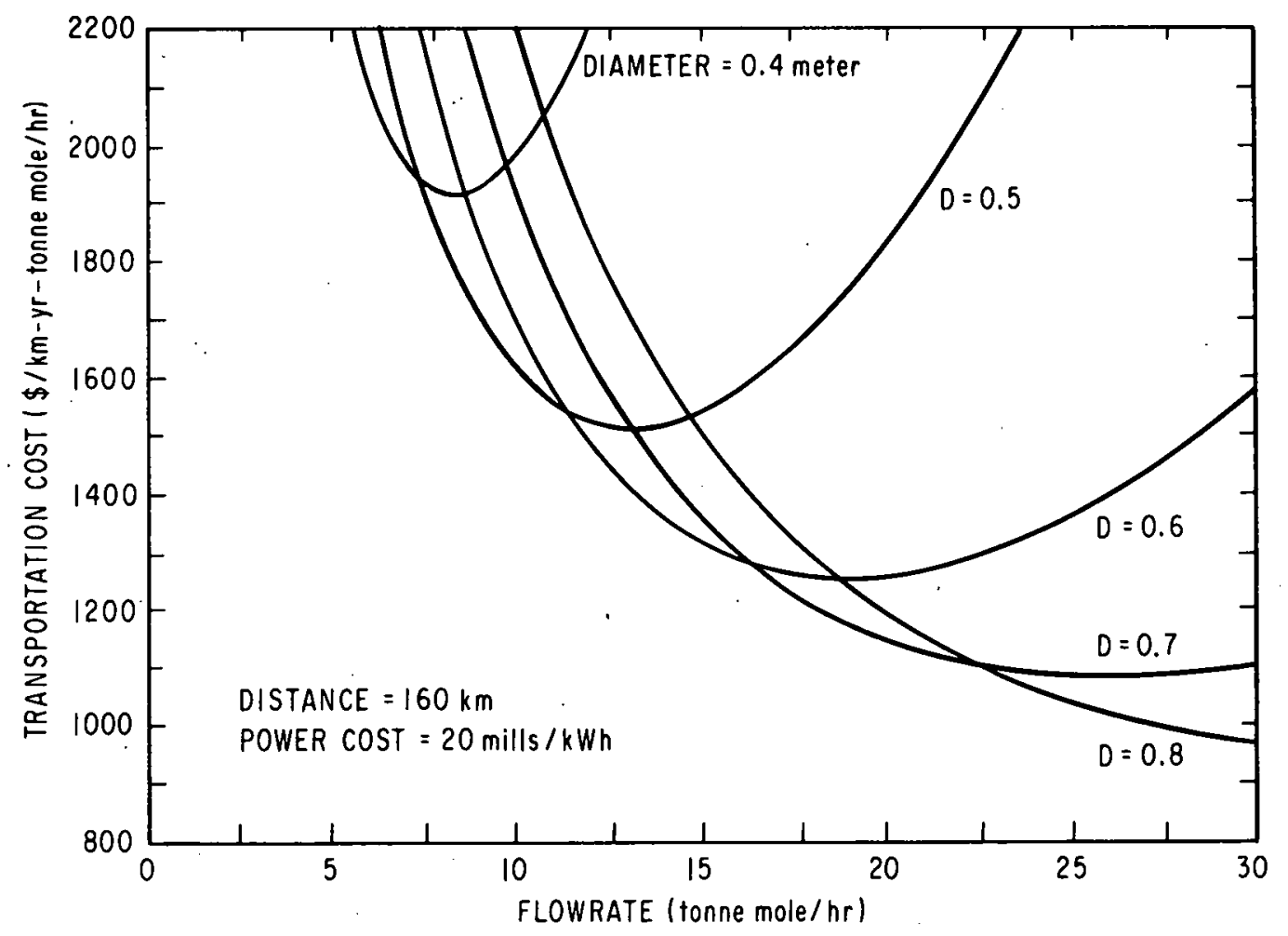

Figure A4-6. Transportation Cost of Cyclohexane Versus Flowrate 


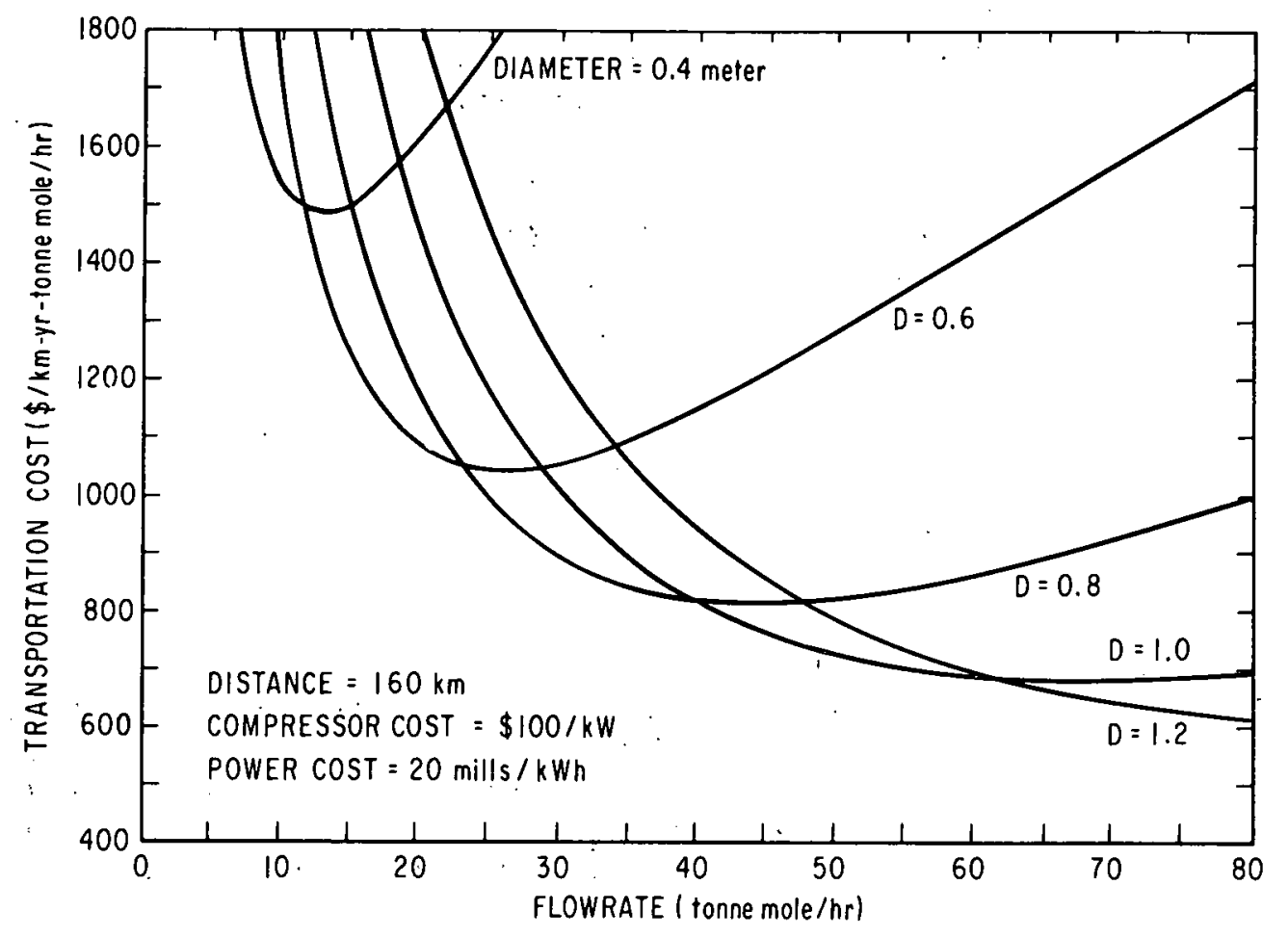

Figure A4-7. Transportation Cost of Hydrogen Versus Flowrate

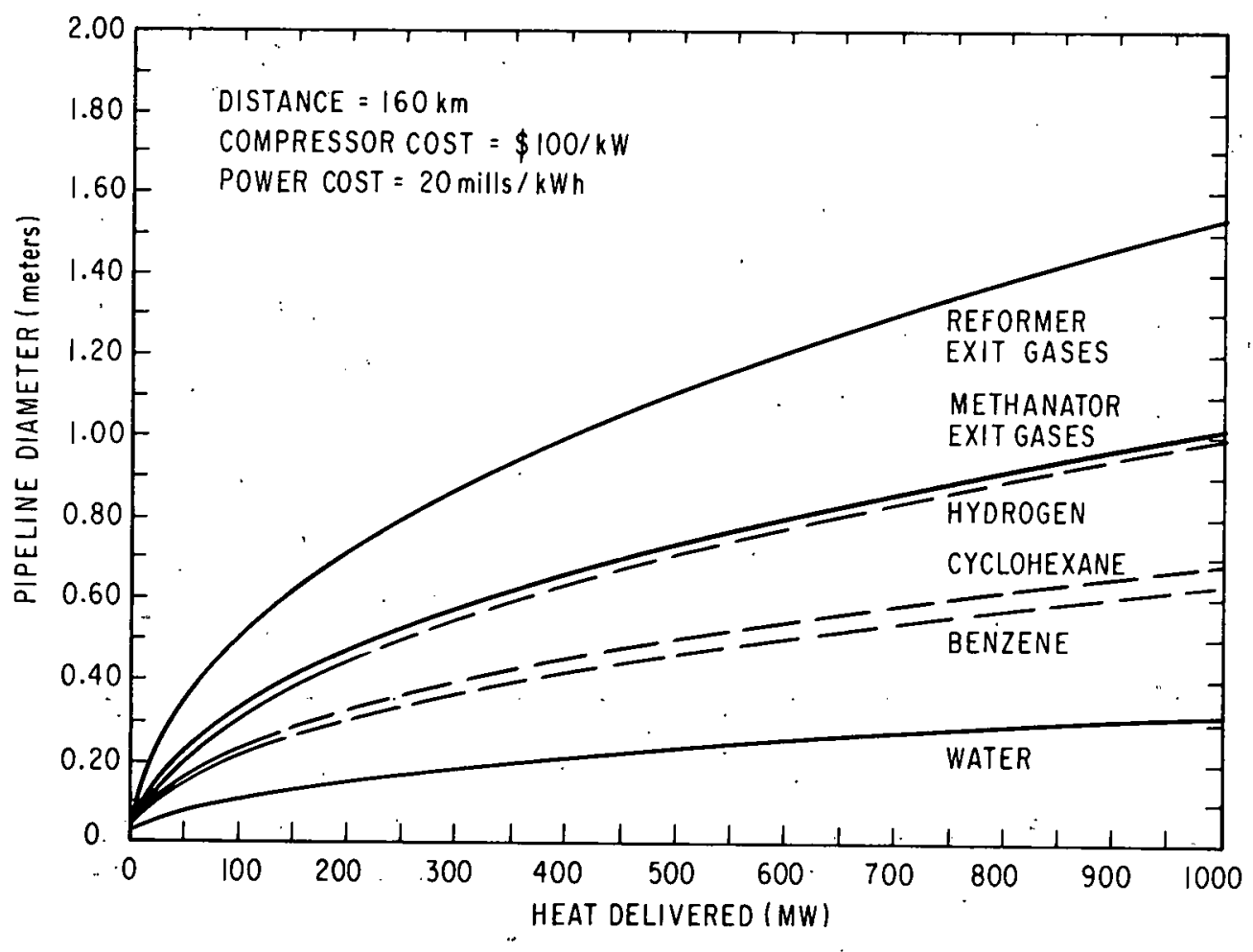

Figure A4-8. Optimum Pipeline Diameter Versus Heat Delivered for Chemical Heat Pipe Fluids 
Table A4-3

FLOWRATES FOR CHP MATERIALS

AT 1000 MW POWER DELIVERED

Flowrate

Stream tonne moles/hr

$\mathrm{H}_{2} / \mathrm{CO} \quad 76$

$\mathrm{CH}_{4} \quad 27$

$\mathrm{H}_{2} \mathrm{O} \quad 20$

$\mathrm{C}_{6} \mathrm{H}_{6} \quad 18.7$

$\mathrm{C}_{6} \mathrm{H}_{12} \quad$ i8.7

$\mathrm{H}_{2}$

52.7

Transportation costs (single line per trench) at the optimum pipeline diameter for these same materials are shown in Figure A4-9 as a function of power delivered. Both costs and line diameters are lower for liquid transport as would be expected from the higher densities of liquids. Others (RA4-9, -10) who have investigated transportation of similar fluids have generally calculated lower costs (due mainly to lower pipeline and energy costs).

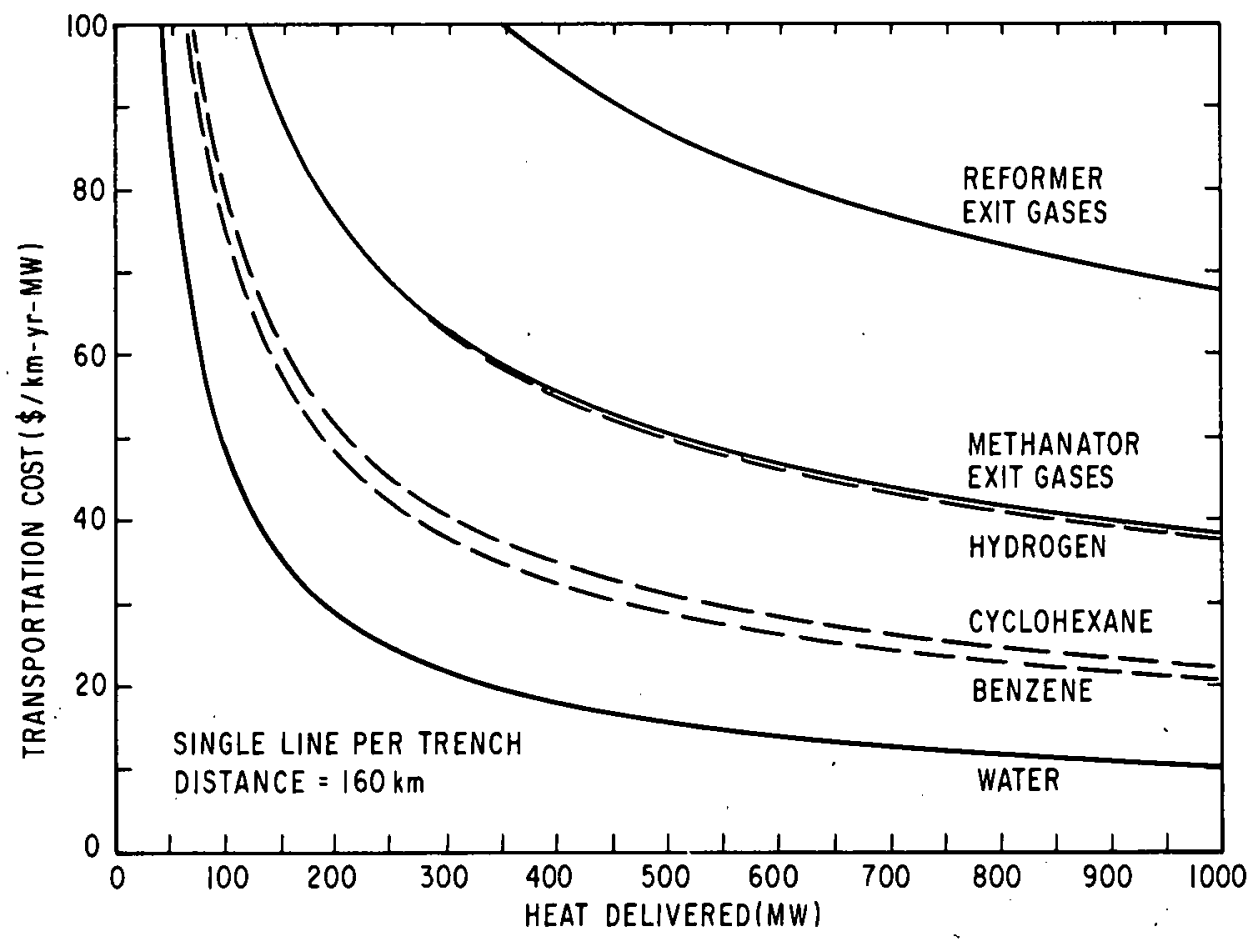

Figure A4-9. Transportation Cost (single line per trench) Versus Heat Delivered for Chemical Heat Pipe Fluids 
The contributions of the individual costs (e.g., pipeline materials, energy) to the total cost of transportation are shown in Table A4-4 for each pipeline, for 1000 MW delivered, the base case design parameters, and the optimum line diameters. This breakdown of costs shows that over $80 \%$ of the total transmission cost results from the pipeline capital cost. Only about $2 \%$ of the total cost of gas transmission results from compressor capital cost, and $5 \%$ of liquid transmission results from pump capital costs. The remainder ( $\sim 17 \%$ for gas transmission and $\sim 12 \%$ for liquid transmission) results from energy costs. It can be concluded from this breakdown that the transmission costs are most sensitive to pipeline costs and least sensitive to compressor and pump capital costs. To better quantify these statements, a parametric analysis of total transportation cost versus individual component costs will be given in the following section.

\section{A4.4 SENSITIVITY OF TRANSMISSION COSTS TO VARIATIONS IN DESIGN AND ECONOMIC PARAMETERS}

\section{A4.41. Introduction}

In a preliminary study such as this, uncertainties exist in the various design parameters due to effects of inflation, equipment substitution, and variations in design conditions. A complete analysis requires investigation of the variation in these design and economic parameters to determine the magnitude of these variations and their effect on the cost of the system. In this section, effects of variation in pipeline, capital costs, compressor and pump costs, energy costs, transmission pressure, and transmission distance are investigated. Since the effects of each variable will be qualitatively the same, $\mathrm{H}_{2} / \mathrm{CO}$ transmission will be used as an example.

\section{A4.4.2 Effect of Pipeline Capital Cost on Overall Transportation Cost}

The costs developed in the previous section showed that the pipeline costs represent $80 \%$ or more of the transportation cost for both gases and liquids. Hence, variation in pipeline capital costs can be expected to have a major impact on overall transmission costs. Variation in the cost of the transmission line can be accounted for by an adjustment of the coefficients of Equation A4-17, used to approximate pipeline costs. Since the coefficients used as the basis for the previous calculations represent well the actual costs for fiscal 1976 Federal Power Commission data, they would not be expected to decrease in the future in a period of inflating steel, labor, and land costs. Therefore, the effect of increases in these coefficients will be explored to estimate the effect of increases in future prices.

Figure A4-10 shows the effect of increase in the pipeline cost coefficients on the total transportation cost of the $\mathrm{H}_{2} / \mathrm{CO}$ gas mixture at the optimum line diameter for each cost. The costs are shown as a function of the percentage increase in the coefficients. Each of the coefficients $(a, b, c)$ is increased by the same percentage, so no attempt is made to estimate the variations in the individual rate of inflation of steel, labor, or land costs. The results show that for every percent increase in pipeline cost coefficients, the total transportation cost increases by about $0.75 \%$. This proportionality can be explained by the fact that only about $80 \%$ of the total cost of transportation resuits from the pipeline capital costs. 
Table $\cdot A 4-4$

INDIVIDUAL COMPONENTS OF OVERALL TRANSMISSION COST AT $1000 \mathrm{MW}$ TRANSPORTED

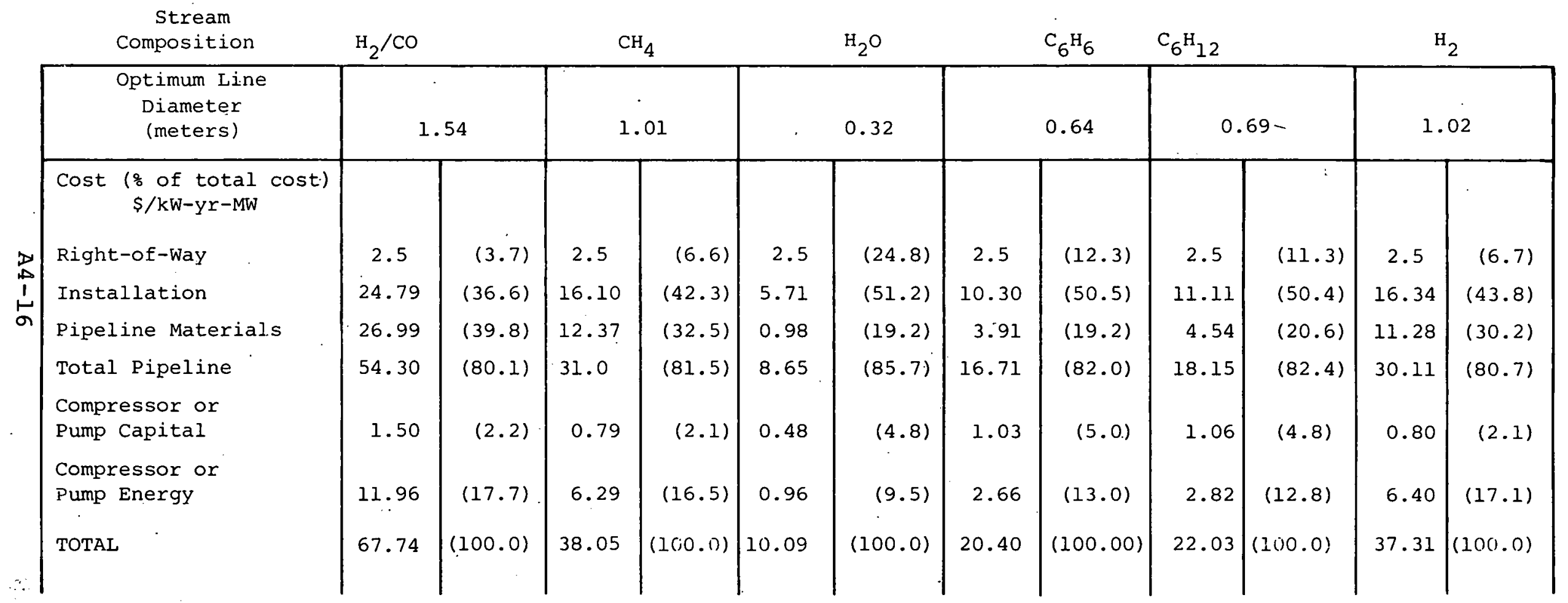




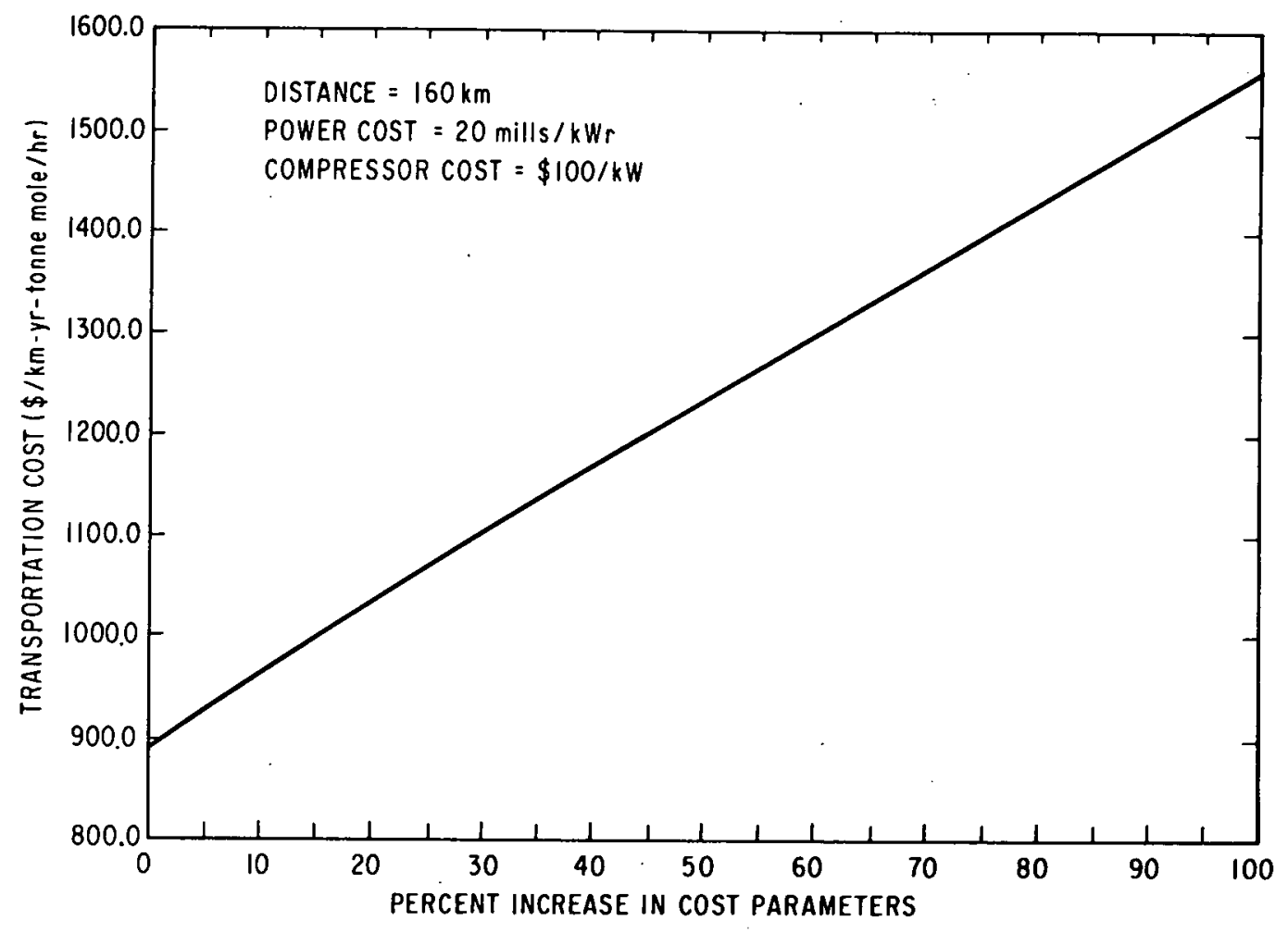

Figure A4-10. Transportation Cost Versus Percent Increase in cost Parameters

In addition, the optimum diameter decreases with increasing pipeline cost such that a slightly smaller line is required and, hence, lower costs result than would be expected from a constant diameter comparison. Since the pipeline cost amounts to about $80 \%$ of the total cost of transportation in all of the cases investigated here, a ratio of about 0.75 for the increase in transportation cost to increase in pipeline cost can be expected for all cases considered.

\section{A4.4.3 Effect of Compressor Capital Cost on Overall Transmission Cost}

The effect of compressor capital cost on transportation cost can be evaluated by variation of the coefficient $C_{M}$ in Equation $(A 4-20)$. This effect is shown in Figure A4-11 for compressor costs ranging from $\$ 100 / \mathrm{kW}$ to $\$ 1000 / \mathrm{kW}_{e}$. Examination of this figure shows that overall transmission costs vary only about $2 \%$ with each increase of $\$ 100 / \mathrm{kW}_{\mathrm{e}}$ in compressor cost. Even the entire tenfold increase of compressor cost results in only a $15 \%$ increase in total transmission cost. It can be concluded from this study that inflation or inaccuracies in compressor costs will have little effect on the overall transmission cost.

\section{A4.4.4 Effect of Power Cost on Overall Transmission Cost}

Figure A4-12 shows tramsission cost as a function of power cost at the optimum line diameter. This figure demonstrates that an increase of $200 \%$ in power cost results in an increase in transportation cost of only about $20 \%$. This indicates that transmission cost is more sensitive to change in power 


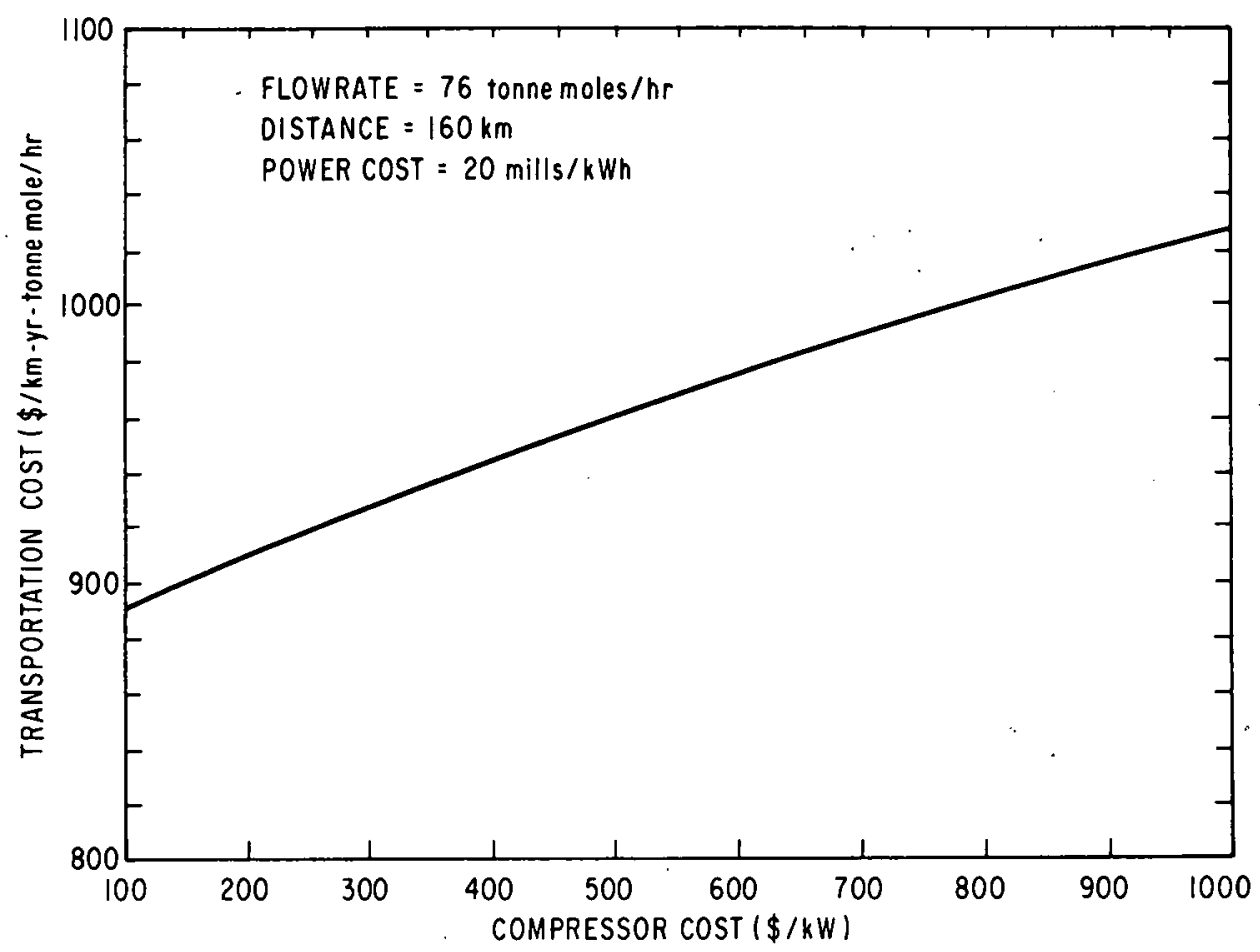

Figure A4-11. Transportation Cost of Reformer Exit Gases Versus Compressor Cost

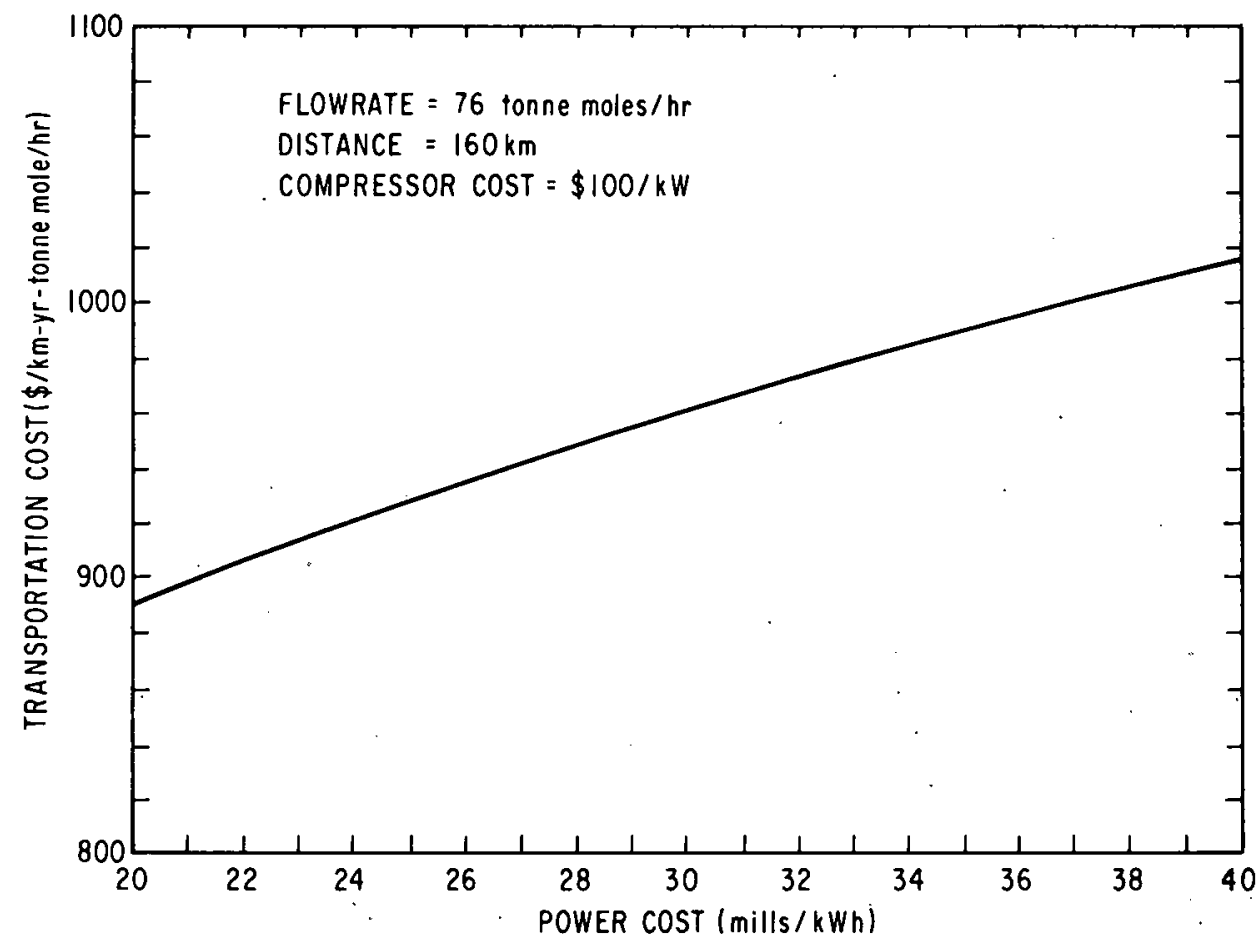

Figure A4-12. Transportation Cost of Reformer Exit Gases Versus Electricity' Cost 
cost than to change in compressor capital cost, although the sensitivity to both of these costs is significantly less than the sensitivity to pipeline capital.cost. Again, this can easily be explained by examination of the breakdown of individual components of the overall transportation costs given in Table A4-4. Since pipeline capital cost dominates $(\sim 80 \%)$ the total cost, sensitivity of total cost of transmission to power and compressor costs should be less than to pipeline costs as it is in these examples. For similar reasons, variation in compressor efficiency also contributes only slightly to overall transmission costs.

\section{A4.4.5 Effect of Transmission Pressure on Transportation Cost}

Figure A4-13 shows the effect of transmission pressure on the transmission cost for the base case flowrate of the reformer exit gases $\left(\mathrm{H}_{2} / \mathrm{CO}\right)$. As can be seen from this figure, an optimum transmission pressure exists which is coupled to the optimum diameter because the transmission length is fixed. It may be desirable to operate the transmission line at pressure other than optimum during transients due to storage of gases in the line. In this case, the increase in cost of transmission must be absorbed as one component of the cost of storage. This topic is discussed in more detail in Section A4.7 of this appendix.

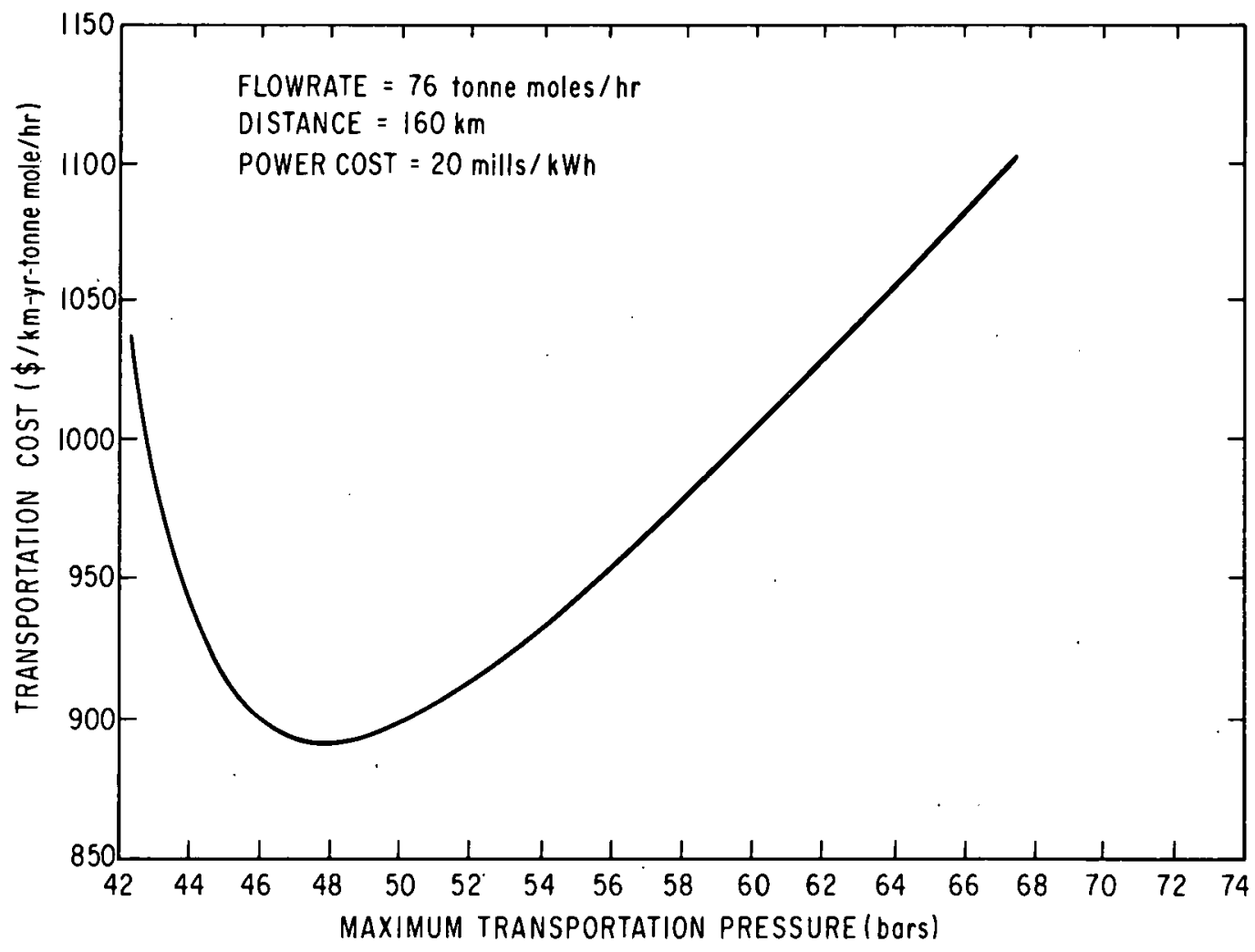

Figure A4-13. Transmission Cost of Reformer Exit Gases Versus Maximum Transmission Line Pressure 


\section{A4.4.6 Effect of Transmission Distance on Transportation Cost}

Figure A4-14 demonstrated the effect of transmission distance on the an-. nualized cost of transmission per unit flow and per unit distance at the optimum line diameter. This diagram shows that the transmission cost per unit length remains essentially constant. Therefore, for preliminary estimates, the total cost of transmission can be scaled directly with transmission distance.

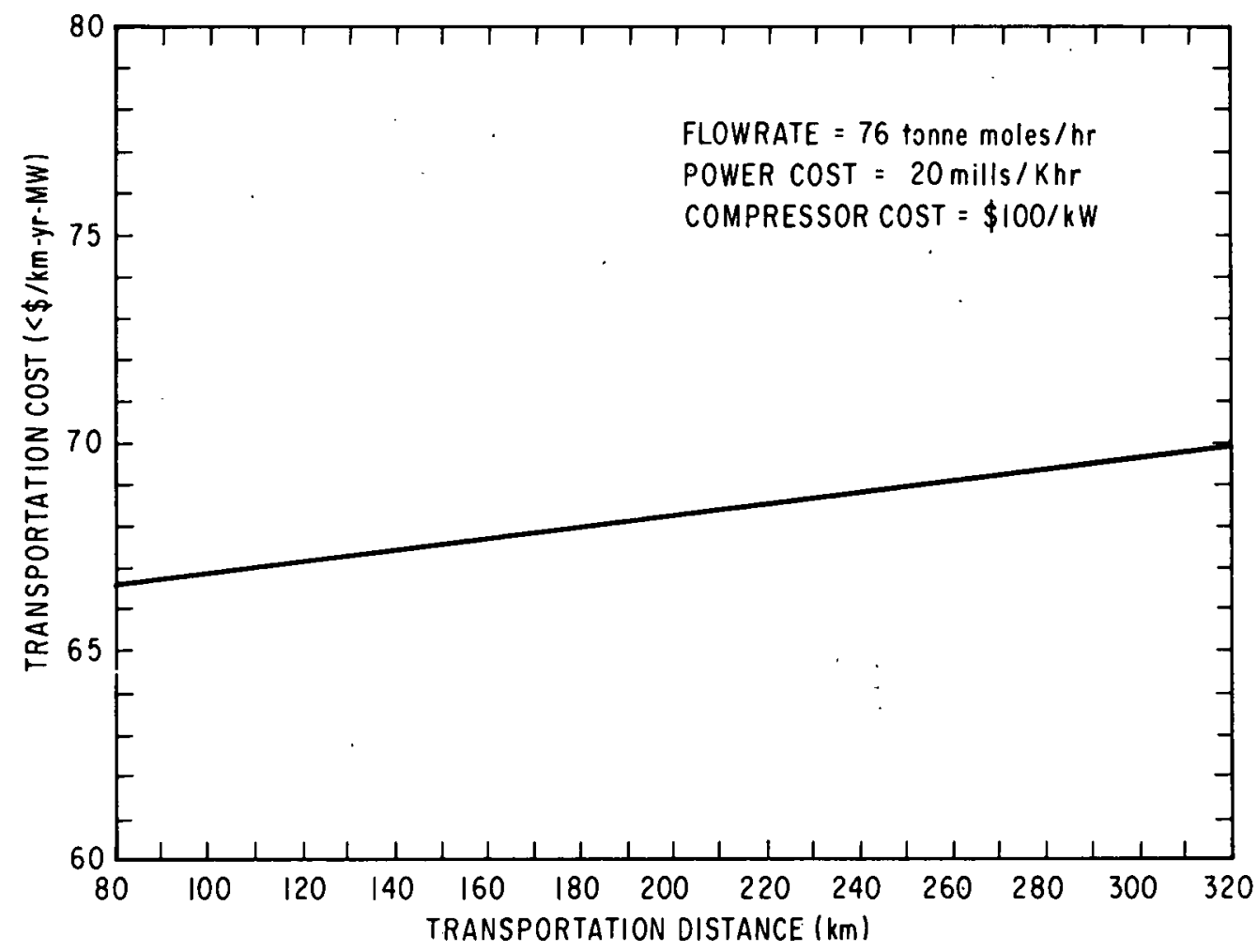

Figure A4-14. Transportation Cost Versus Transportation Distance Reformer Exit Gases at Optimum Pipeline Diameter

\section{A4.5 EFFECT OF TRANSPORTATION PHILOSOPHY ON TRANSMISSION COST}

\section{A4.5.1 Introduction}

As mentioned previously, the question of pump or compressor siting is complicated by the uncertainties in compressor costs when installed in various locations. Of course, a detailed design of a CHP process would eliminate these uncertainties. However, in view of the preliminary nature of the design used in this study, the following examples are given to explore the tradeoffs involved in compressor siting and pump in design philosophy.

\section{A4.5.2 Gaseous Transport}

The analyses presented in the previous sections of this appendix assume that the gaseous transport is accomplished by on-site compression at either the reformer or methanator site. In these cases, the gas is compressed to a pressure which allows it to flow the required distance and exit the transmission line at 40 bars. This philosophy is different from the natural gas 
industry, in which compressor substations are used at periodic intervals to boost the transmission pressure back to the desired level. For transmission over extended distances, high transmission pressures are economically more viable; the increased capital requirements for the thicker walled high-pressure pipeline are more than compensated by the decreased work requirements per unit pressure drop needed at higher pressures.

Over the limited transmission distances visualized for CHP applications, the use of high transmission pressures is unfavorable, since the work required to compress the gas to the desired pressure (above a compression ratio of $\sim 1.1$ ) is not compensated by the smaller (but thicker walled) transmission line.

Another option would be to use substations at intervals along the shorter CHP line to maintain a lower compression ratio during gas transmission, thus lowering the work requirement per unit pressure drop. One drawback to this approach is the expected increased capital cost for compressor units installed at substation locations over those installed on-site at either the reformer or methanator locations. Table A4-5 shows a comparison of transmission costs for the transmission of 76 tonne moles of the reformer exit gases $\left(\mathrm{H}_{2} / \mathrm{CO}\right)$ over a distance of $160 \mathrm{~km}$. Included are the transmission costs for the cases of compressors installed at:

1. The reformer site at $\$ 100 / \mathrm{kW}_{\mathrm{e}}$

2. Substation locations at $\$ 350 / \mathrm{kW}_{\mathrm{e}}$

3. Substation locations at $\$ 100 / \mathrm{kW}_{\mathrm{e}}$

Table A4-5

COMPARISON OF TRANSMISSION COSTS OF REFORMER EXIT GASES FOR VARIOUS COMPRESSOR LOCATIONS AND CAPITAL COSTS

Case No.

Compressor Location

Compressor Capital Cost, $\$ / \mathrm{kW}_{\mathrm{e}}$ Compression Ratio

Pipeline Cost, $\$ / \mathrm{km}-\mathrm{yr}$-tomme-mole/hr

Compressor Total cost

TOTAL COST

$\$ / \mathrm{km}-\mathrm{yr}$ tonne mole/hr
1

2

3 Reformer Substation Substation 100 350 100

1.194

1.1

1.1

714.1

718.5

680.0

177.1

207.5

196.8

891.2

926.0

876.8

Table A4-5 shows that for the assumed compressor costs the use of substations is not economical. However, if the compressor substations could be built at a cost comparable to the on-site cost, then the substation alternative would become more attractive. 


\section{A4.5.3 Liquid Transportation Philosophy}

The data presented in Figure A4-4 for the cost of water transmission were based on the use of the PV work available in 40 bar water entering the transportation system to transport it through the pipeline until minimum pressure of 5 bars is reached. At this point, the water is again pumped to 40 bars and the process repeated until the water reaches the outlet of the transportation system. An alternative approach would be to let the water fall from 40 bars to 5 bars and then repeatedly pump the water to some pressure below 40 bars until it has reached the outlet of the transportation section, where it would again be pumped to 40 bars. The advantage to this method would be that low-pressure transportation could be accomplished in a thinner walled, more inexpensive pipeline. Table A4-6 is a comparison of the economics of these two transportation schemes.

Table A4-6

COMPARATIVE ECONOMICS FOR HIGH AND LOW PRESSURE LIQUID TRANSPORTATION PHILOSOPHIES

[ $\$ 1000 / \mathrm{km}-\mathrm{yr}$-tonne mole $\left(\mathrm{H}_{2} \mathrm{O}\right) \mathrm{hr}=\$ 0.1 \mathrm{l} / \mathrm{GJ}$ delivered $8000 \mathrm{hr} / \mathrm{yr}$ and $160 \mathrm{~km}]$

\section{Case}

Upper Pressure, bars

Pipeline Diameter, meters

Pipeline Cost,

$\$ / \mathrm{km}$-yr-tonne mole/hr

Pumping Cost,

$\$ / \mathrm{km}$-yr-tonne mole/hr

TOTAL COST

$\$ / k w-y r-t o n n e ~ m o l e / h r$
Low Pressure

8.4

0.34

421.8

64.5

486.3
High Pressure

40.0

0.32

432.5

71.7

$504: 2$

Table A4-6 shows that the low-pressure method is somewhat (3.6\%) less expensive. Even though the low-pressure line has a slightly larger diameter the materials cost is smaller, since thinner walls are required to contain the lower pressure. The slightly larger diameter also allows savings in pumping costs.

In general, a wide variety of transportation philosophies could be proposed for both liquid and gas transport. However, none of those explored during this investigation (either those presented in this report or others) change the transportation cost by more than about 10\%. Therefore, it is concluded that the preliminary transportation costs presented here represent fairly well the actual costs for the given values of the design parameters, and any change in design philosophy will not significantly improve the transportation economics. 


\section{A4.6 TRANSMISSION COSTS FOR CHEMICAL HEAT PIPE APPLICATIONS}

(MULTIFLE LINES PER TRENCH)

\section{A4.6.1 Introduction}

In an application of the CHP concept, the transmission system would comprise several pipelines, all installed on the same right of way, to transport gases and liquids both to and from the user site. The installation of multiple lines in a single trench offers both advantages and disadvantages in comparison with single-line systems. As mentioned previously, multiple lines should result in savings in both right-of-way costs and installation costs. Considerations of safety and ease of repair probably favor single line per trench configurations. These tradeoffs will have to be addressed in a more detailed design. In this section, the economic effect of installation of multiple lines per trench for CHP applications will be investigated; a comparison will be made between water recycle and water treatment in the HTCHP system, and the transportation costs of the two systems will be compared. The effect of hydrocarbon inventories on transportation cost will also be explored.

\section{A4.6.2 HTCHP Multiple Line per. Trench -- Transmission Costs}

Figure A4-15 shows the cost of transportation over $160 \mathrm{~km}$ as a function of energy delivered for the multiple line per trench.HTCHP. The pipeline costs are evaluated, as mentioned previously, by assuming the right-of-way cost. to be constant, independent of the number of lines present, and the installation. cost proportional to the diameter of a line with the same crosssectional area as the sum of the three lines. The line diameters used in this case are the optimal diameters evaluated in the single line per trench.

Also shown in Figure $A 4-15$ is the summation of the costs of the single line per trench, for the three lines as a function of the energy delivered over $160 \mathrm{~km}$. As expected, the cost of transportation calculated for three lines per trench is significantly less than that for one line per trench. At $1000 \mathrm{MW}_{\text {th }}$ delivered, the three line cost is approximately $19 \%$ less than the one line cost; at $10 \mathrm{MW}_{\text {th }}$ delivered (where the right-of-way costs are more important because of the small pipelines), the three line cost is about $46 \%$ less than the cost of one line per trench.

The costs of three lines per trench must be considered preliminary because of the assumptions made concerning the effect of multiple lines on the installation costs. However, this analysis does indicate that significant savings can' be expected over the simple summation of costs of single lines per trench, and that these factors must be considered in more detailed designs of the transportation system for the chemical heat pipe.

These costs of three lines per trench are converted to cost per unit of energy delivered, in Figure A4-16, which shows the transportation cost over $160 \mathrm{~km}$ increases from about $\$ 0.58 / \mathrm{GJ}$ (for $1000 \mathrm{MW}$ delivered) to $\$ 4.66 / \mathrm{GJ}$ (for $10 \mathrm{MW}$ delivered). Both the power requirement and the capital cost per unit of energy delivered increase significantly for small systems. It can be concluded that the use of many small lines over an extended distance would not be an economically attractive system.

A detailed design of the layout of a transportation system would require specific knowledge of the user site locations and the magnitude of each user's 


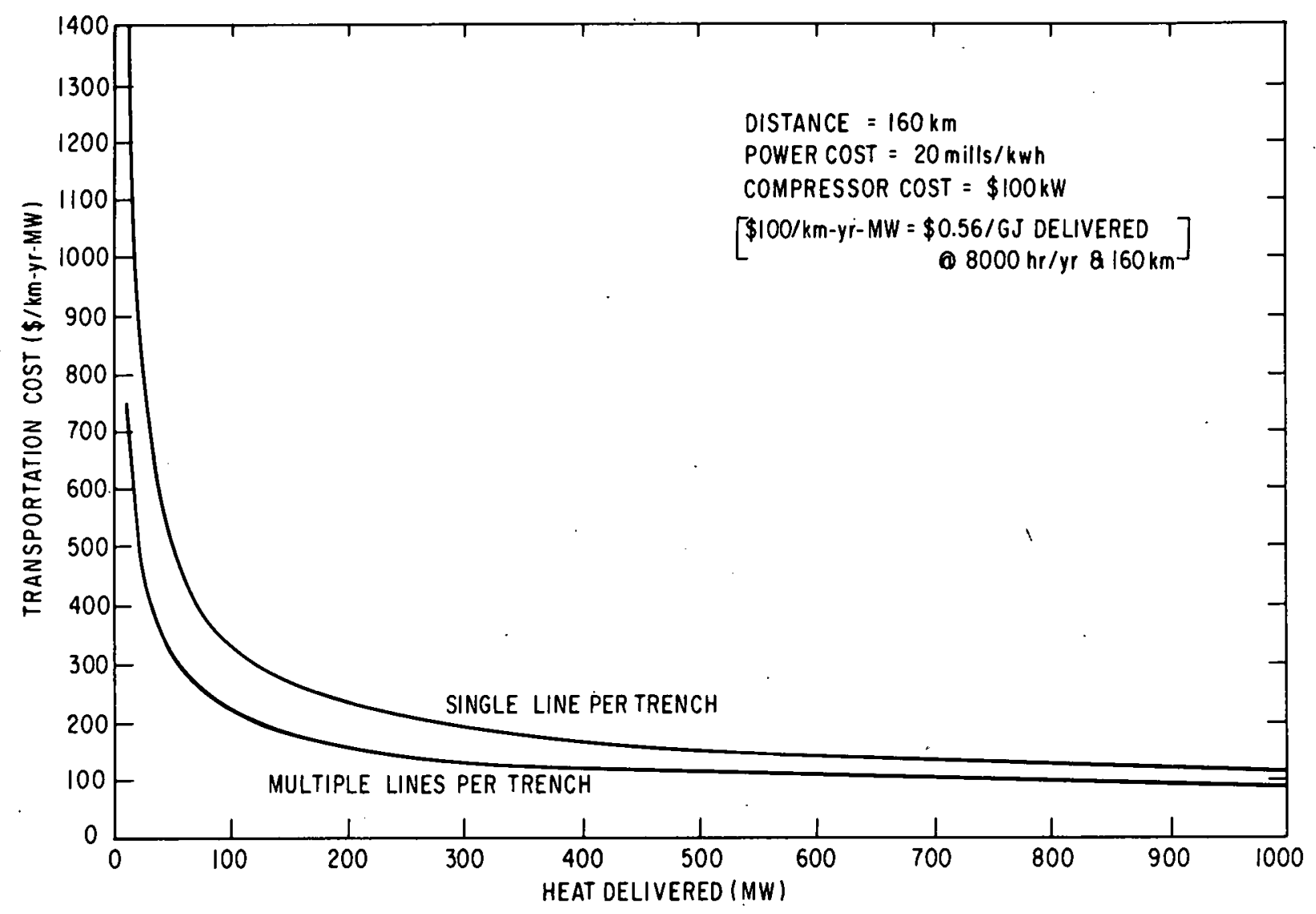

Figure A4-15. Transportation Cost Versus Heat Delivered for Methane-Based CHP Single Line per Trench and Multiple Lines Per Trench

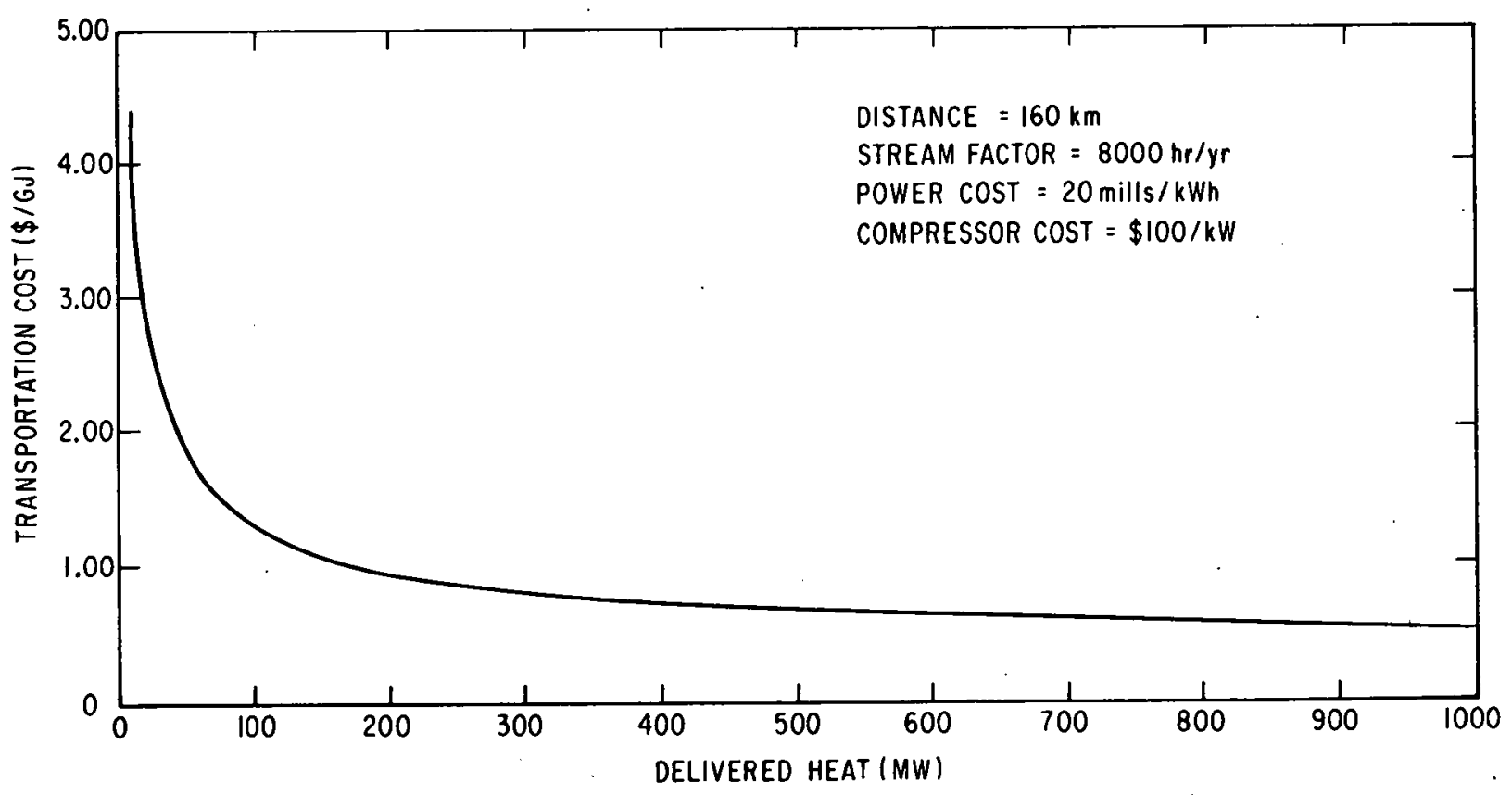

Figure A4-16. Cost per GJ Delivered Versus Heat Delivered for Methane-Based Chemical Heat Pipe 
energy needs before any optimization of line routes could be undertaken. However, a simplified analysis of the problem shows that total cost for one set of lines to deliver the entire $1000 \mathrm{MW}_{\mathrm{th}}$ over a $160 \mathrm{~km}$ distance is a good first approximation for the total transportation cost for users totaling 1000 $\mathrm{MW}_{\mathrm{th}}$ within a $160 \mathrm{~km}$ radius of the primary reformer plant.

A4.6.3 Economic Tradeoff Between Water Recycle and Water Treatment in the HTCHP System

The HTCHP system can be designed so that water is transported from the methanator site back to the reformer plant to maintain a closed system, or so that water is brought into the system at the reformer site and disposed of at the methanator site. When considering "opening up" the closed cycle, several factors must be considered. First, the introduction of noncycling water into the system requires that it be treated to eliminate any impurities which would tend to build up in the system as the other components continue to cycle around the closed loop. In particular, molecular oxygen and carbon dioxide should be eliminated so that the correct carbon-to-oxygen ratios in the reactions can be maintained. Nitrogen should be avoided, to prevent the requirement for a bleed stream.

Mineral components should be eliminated to prevent fouling of heat exchanger surfaces. Rejection of the water at the methanator site would require treatment to eliminate potentially environmental polluting chemicals and to recover other components in the steady-state mixture so that makeup chemical would not be required. The cost of treatment at both ends could total approximately $\$ 0.30$ to $\$ 2$ per 1000 gallons of water (RA4-11), depending on the final degree of treatment decided on.

For comparison, the incremental cost of transporting water in the design for three lines per trench is shown in Figure A4-17 as a function of energy delivered. This figure shows that the cost of water from the transmission system is competitive with the cost of treating water for an open system. If the water supplied to the open system must be piped over any significant distance the closed system prices would look more favorable, since the piping cost is most. likely to be on the basis of one line per trench and not just the incremental cost of adding a third pipeline to the existing right of way as in the closed system.

\section{A4.6.4 LTCHP Transportation Cost for Multiple Lines Per Trench Installation}

The transportation cost for the LTCHP components, based on multiple lines per trench, can be calculated by using the assumption that installation cost is proportional to the square root of the sum of the diameters of the lines squared. Figure A4-18 shows this cost as a function of the energy delivered by the system. For comparison, the summation of the costs of a single line per trench determined in section $A 4.3$ is included in the figure. The LTCHP transportation cost is approximately $\$ 0.66 / \mathrm{km}-\mathrm{yr}$-MW $(38 \mathrm{k} / \mathrm{GJ}$, at $8000 \mathrm{hr} / \mathrm{yr}$ and $160 \mathrm{~km}$ ) for $1000 \mathrm{MW}$ delivered in the case of a multiple line per trench. This is a decrease of approximately 25\% from the summation of costs for the single line per trench. The multiple line per trench cost increases to $\$ 1.00 /$ $\mathrm{km}-\mathrm{yr}-\mathrm{MW}_{\mathrm{th}}$ at $100 \mathrm{MW}$ transported, and at this power delivery is about 58\% of the single line per trench cost. 


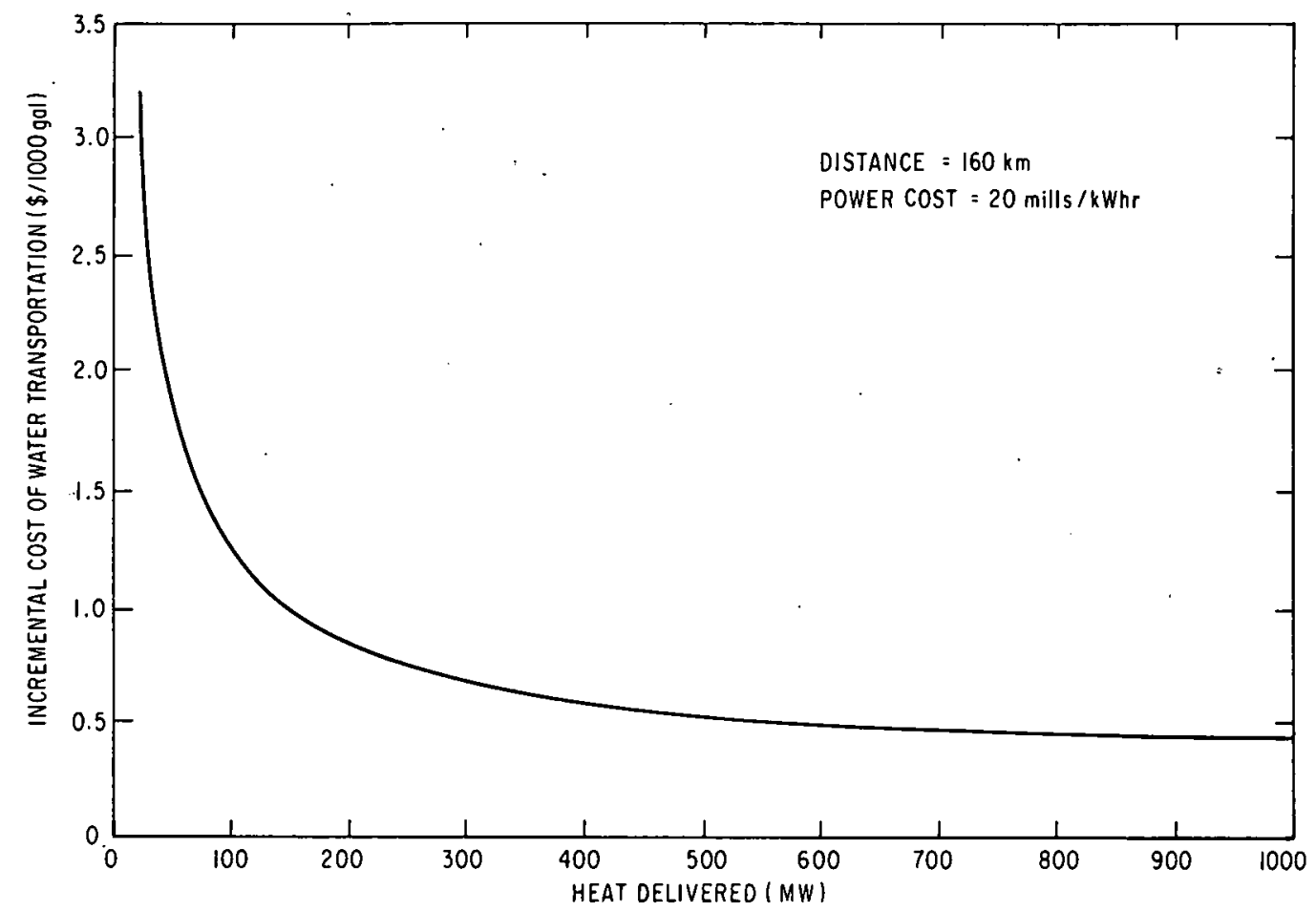

Figure A4-17. Incremental Cost of Water Transportation Versus Heat Delivered for Methane-Based Chemical Heat Pipe

Two facts concerning this cost should be mentioned. First, the transportation costs are relatively insensitive to the rate of energy transport over a wide range of values of power delivered (pipeline flowrates). This means that the cost for a single $160 \mathrm{~km}$ line is a good approximation of the summation of costs of a network grid to deliver power to a variety of small users. An exact analysis of the network grid transportation costs is beyond the scope of this work, but Figure A4-18 indicates that total grid transportation cost should not exceed the total straight line cost derived here by a significant amount:: Secondly, a material balance shows that the amount of hydrogen transported is independent of the conversion in the reactor systems. This implies. that the cost for hydrogen transport will be independent of the conversion in the exothermic reactor.

In the LTCHP, the inventory of liquids in the transmission line is considerable. Hence, the cost of this inventory will affect the design. For a given transmission distance, the volume and cost of the liquid inventory is proportional to the square of the diameter of the line. In effect, the inventory cost adds an incremental amount to the materials cost of the line. In the HTCHP, the density of the gases is relatively low, and therefore the value of the inventory is small. In the LTCHP, the amount of benzene and cyclohexane contained in the Iine is significant and affects the optimal line diameter. That is, the optimal line diameter will tend to be smaller so that a less expensive product will be stored in the line.

Figure A4-19 shows the line diameter and associated increase in transportation cost as a function of cost of benzene for the LTCHP dehydrogenation liquid flowrate at 1000 MW delivered. The benzene inventory adds approximately $\$ 0.06 / G J$ delivered at a benzene cost of $\$ 0.75 / \mathrm{gal}$ (R4A-12). A similar increase 


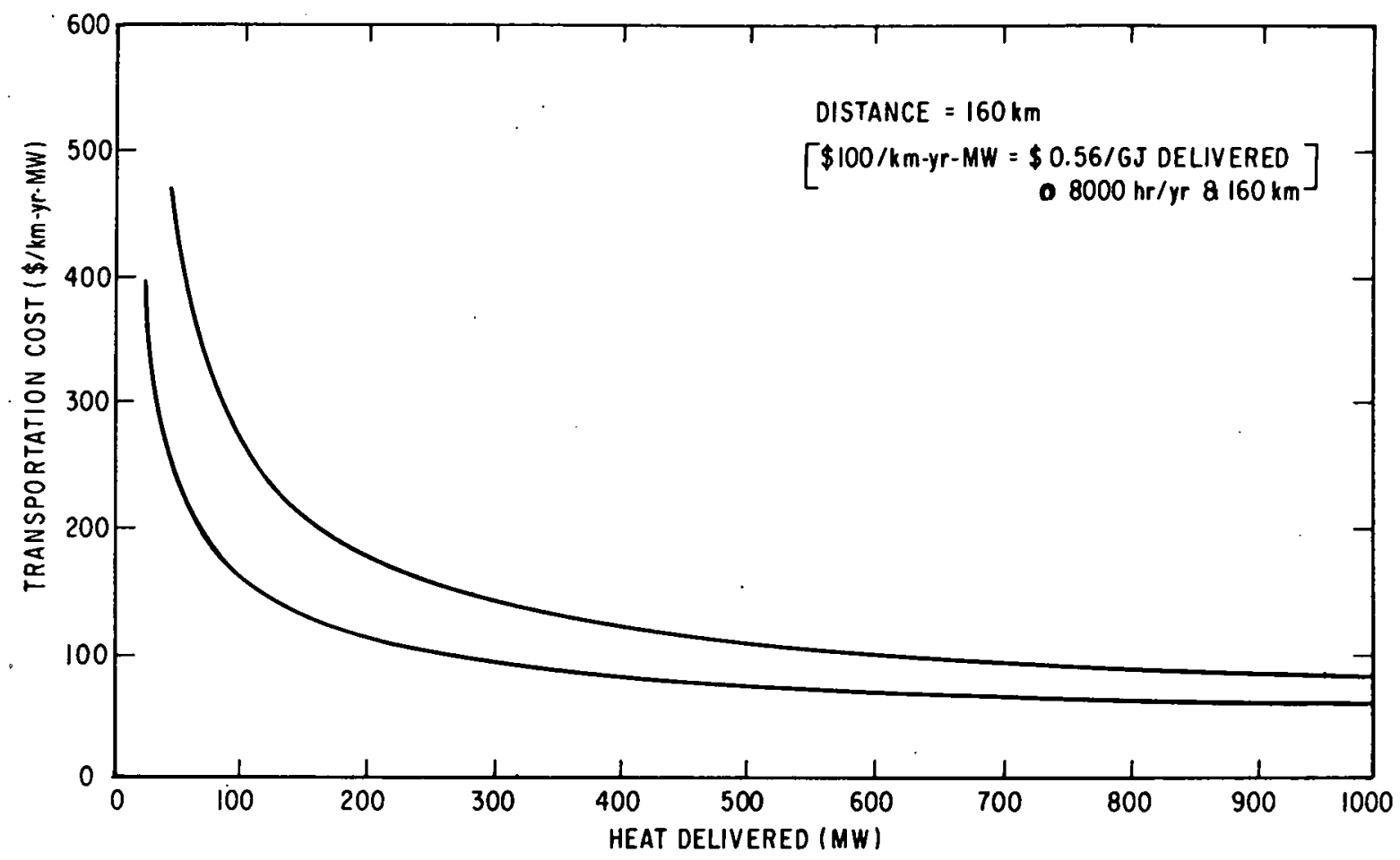

Figure A4-18. Transportation Costs at Single Line per Trench and Three Lines per Trench Versus Heat Delivered for the Benzene/Cyclohexane-Based CHP

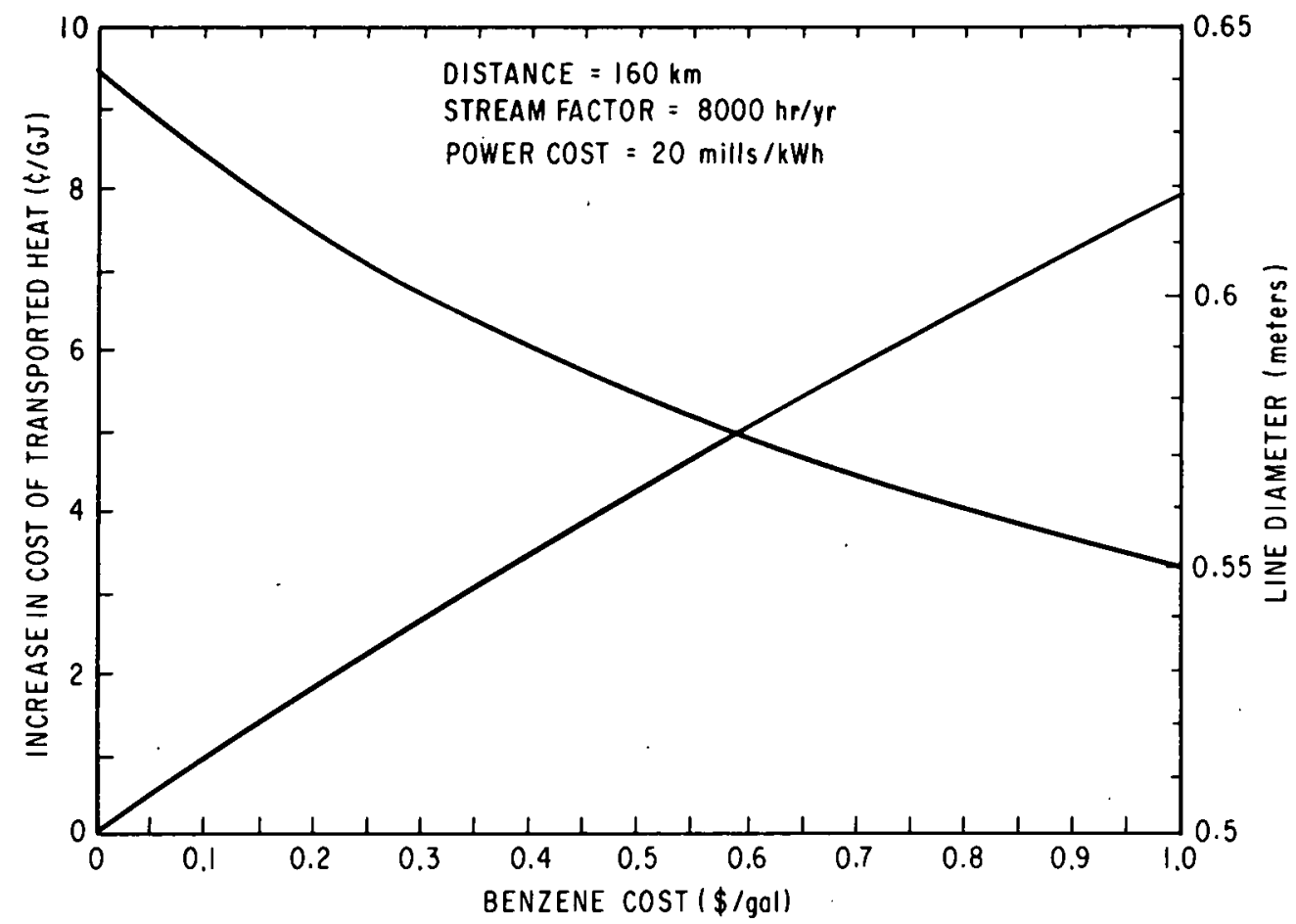

Figure A4-19. Increase in Cost of Transported Heat and Optimum Pipeline Diameter Versus Cost of Benzene 
can be expected in the cost of returning the cyclohexane to the dehydrogenation reactor; hence, the total effect of inventory cost is approximately $\$ 0.12 / G J$. The relationship between the cost of inventory and the increased cost of transportation is not linear because of the change in optimum line diameter.o

\section{A4.6.5 Comparison of LTCHP and HTCHP Transportation Costs}

Figure A4-20 shows a comparison of transportation costs of three lines per trench for the methane-based chemical heat pipe and the benzene/cyclohexanebased heat pipe, both with and without the cost of benzene and cyclohexane inventory included. The lower energy requirements and higher energy density of the benzene system are reflected in the lower overall transportation cost of the benzene system. The increased energy density is a result of the fact that the benzene system is predominantly liquid transport and because of the higher conversions in the benzene system as compared with the methane system.

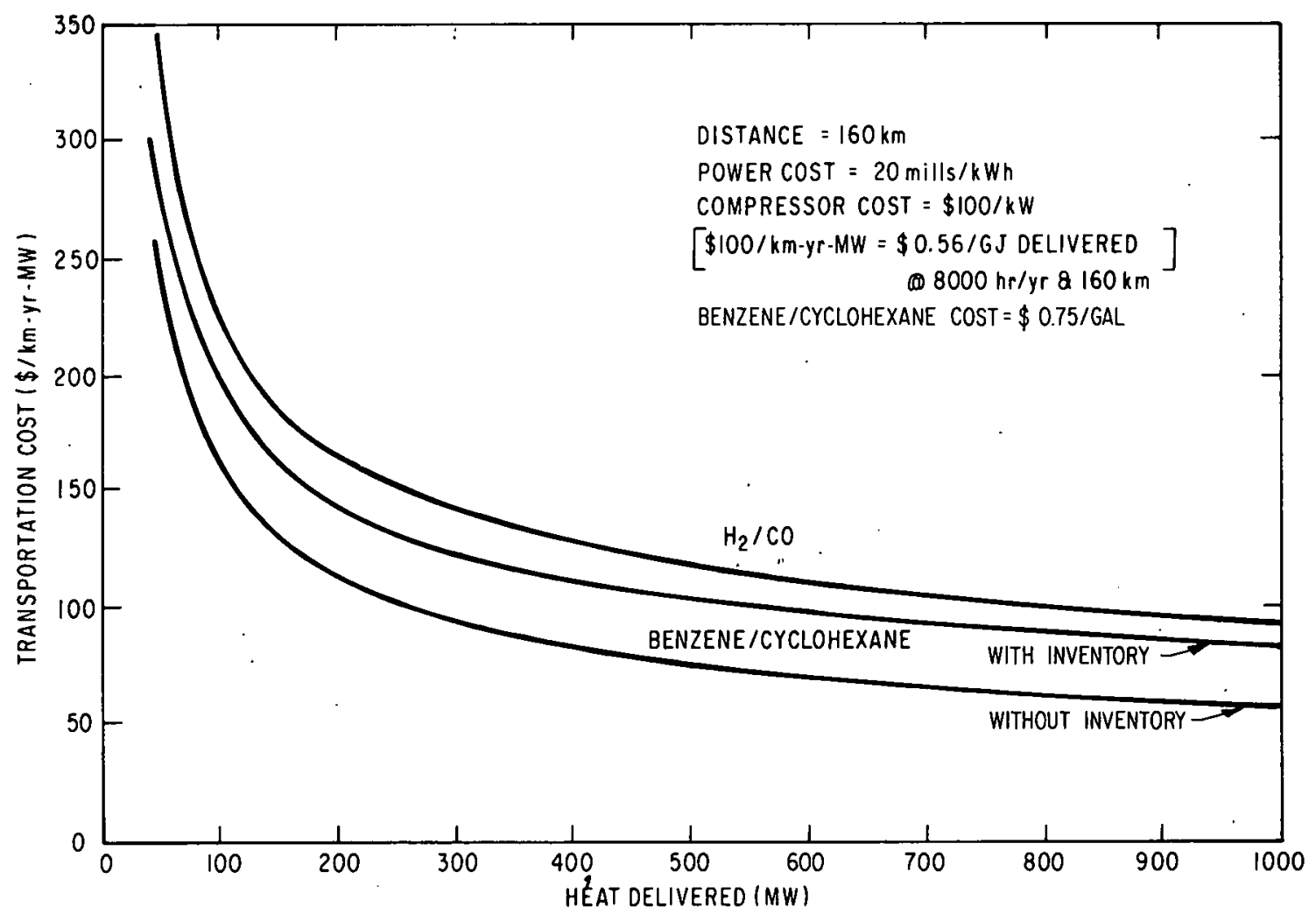

Figure A4-20. Three Line per Trench Transportation Costs Versus Heat Delivered Methane-Based and Benzene/Cyclohexane-Based Chemical Heat Pipes

\section{A4.7: INVESTIGATION OF ALTERNATIVE USES OF CHP TRANSPORTATION SYSTEM}

\section{A4.7.1 Introduction}

The ability of the chemical heat pipe to both store and transmit energy offers several options not available in competing systems. In this section, two of these options for using the CHP concept to transport PV work and to store energy in the form of chemical bonds in the pipeline will be explored. 


\section{A4.7.2 Use of Pipelines to Transport PV Work}

Base load electricity (or high pressure steam) at the site of the endothermic reaction (reformer) could be used to compress the exit gas to a high pressure. 'The gas would then be transmitted through the pipeline at the elevated pressure, and then expanded in a turbine at the exothermic reaction (methanator) site to recover the transmitted PV work on a peaking basis.

A simplified economic analysis of this concept can be developed as follows. For the system shown in Figure $\mathrm{A} 4-21$, assume $1 \mathrm{~kW}_{e}$ is produced by the expansion of the gas. Therefore, $1 / \eta_{e} \mathrm{~kW} W_{P V}$ must be transported $\left(\eta_{e}\right.$ equals the expander efficiency) and $1 / \eta_{e} \times \eta_{c}$ ( $\eta_{c}$ equals compressor efficiency) must be consumed by the compressor at the reformer site. Neglecting the incremental cost of the transmission line due to the higher transmission pressure, the cost of transmission can be related to the compressor and expander capital costs as follows:

$$
\operatorname{cost}_{\text {transmission }}=\frac{\text { cost } \text { expander }^{(\$ / \mathrm{kW})}}{\eta_{e}}+\frac{\operatorname{cost}_{\operatorname{comp}}(\$ / \mathrm{kW})}{\eta_{c}}
$$

Assuming that the efficiencies of both expander and compressor are 0.8 and their annualized capital cost is $\$ 20 / \mathrm{kW}-\mathrm{yr}$, then the transmission cost becomes

$$
\begin{aligned}
\text { CT } & =(\$ 20 / \mathrm{kW}-\mathrm{yr}) \cdot\left(\frac{1}{0.8}+\frac{.1}{0.64} \frac{\mathrm{kW}_{\mathrm{e}}}{\mathrm{kW}_{\mathrm{e}}}\right. \\
& =\$ 56.25 \mathrm{~kW}-\mathrm{yr}
\end{aligned}
$$

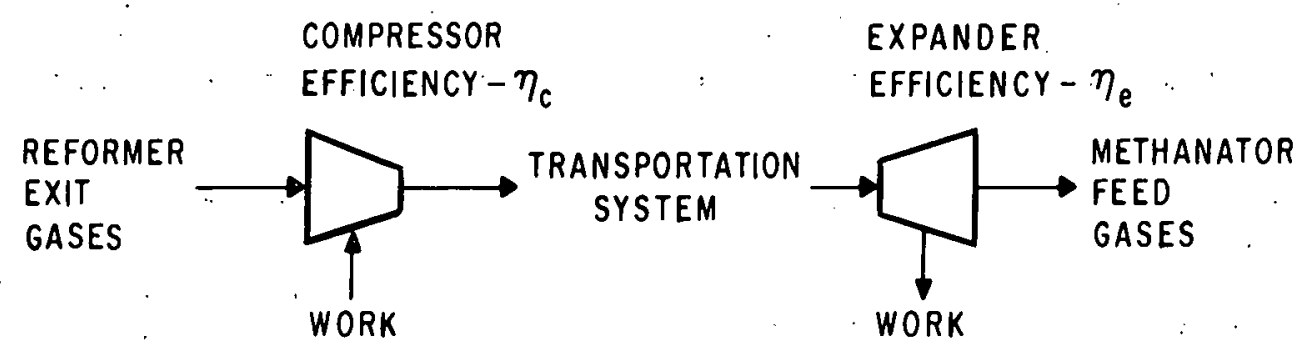

Figure A4-21. Compressor/Expander System for Transporting PV Work

For. 8000 hours per year, $C_{T}$ equals approximately 7 mills per $\mathrm{kWh}$. However, to supply $1 \mathrm{~kW}$ at the methanator site, $1 / 0.64 \mathrm{~kW}_{e}$ must be consumed at the reformer site. Therefore, the breakdown cost of electricity at the methanator site can be related to the cost of electricity at the reformer site by

$$
c_{e}^{\dot{M}}=\frac{c_{e}^{R}}{0.64}+7(\operatorname{mills} / \mathrm{kWh})
$$

Table A4-7 shows the cost of electricity at the methanator (user site) as a function of the cost at the reformer site. It is obvious from the data presented in Table A4-7 that this concept adds significantly to the cost of energy and probably is not economically viable except under certain specialized conditions. 
Table A4-7

COST OF ELECTRICITY AT USER SITE AS A FUNCTION OF COST

AT GENERATION SITE FOR TRANSPORTATION : VIA PV WORK

\begin{tabular}{|c|c|c|}
\hline $\begin{array}{l}\text { Generation Site } \\
\text { Cost (mills } / \mathrm{kW}_{e}-\mathrm{hr} \text { ) }\end{array}$ & $\begin{array}{c}\text { Users Site } \\
\text { Cost (mills } / \mathrm{kWh})\end{array}$ & $\begin{array}{l}\text { Total Conversion } \\
\text { and Transportation } \\
\text { Cost (mills/kWh) }\end{array}$ \\
\hline 10 & 22.6 & 12.6 \\
\hline 15 & 30.4 & 15.4 \\
\hline 20 & 38.3 & 18.3 \\
\hline 25 & 46.1 & $21: 1$ \\
\hline 30 & 53.9 & 23.9 \\
\hline
\end{tabular}

\section{A4.7.3 Utilization of Transmission Line for Storage}

The "packing" of natural gas transmission lines is common practice during winter months in order to store additional gas for nonuniform usage rates during periodic cold spells. This same concept could be applied to the CHP in order to store the baseload thermal energy from the endothermic reactor for utilization on a one- or two-shift basis at the exothermic reactor.

Several variations of pipeline diameter and pressure could be employed to produce this storage capability. The simplest procedure would be to allow the outlet pressure in the pipeline to drop as the shift users come on line and withdraw a portion of the inventory in the line. Additional storage capacity could be obtained by paying an incremental cost to expand the transmission line beyond the optimum diameter, resulting in additional volume which could be utilized for storage.

A detailed investigation of this concept would require an analysis of the transients in the line (requiring a knowledge of use location and thermal requirements as a function of time) which is beyond the scope of this work. However, the storage capability of the line can be calculated as a function of the decrease in average line pressure below the inlet pressure.

For this example calculation, the $\mathrm{H}_{2} / \mathrm{CO}$ gas mixture exiting from the reformer in the HTCHP will be used. The reference case conditions pertinent to this calculation are:

$\begin{array}{ll}\text { Flowrate } & 76 \text { tonne moles } / \mathrm{hr} \\ & \left(76 \times 10^{6} \text { gmoles } / \mathrm{hr}\right) \\ & 48 \mathrm{bars} \text { (peak) } \\ \text { Transportation line pressure } & 1.54 \text { meters } \\ \text { Optimum line diameter } & \end{array}$

The total volume of the line can be calculated as follows:

$$
V=\frac{\pi D^{2} L}{4}
$$


For a 1.54-meter-diameter line $160 \mathrm{~km}$ long,

$$
\mathrm{V}=2.98 \times 10^{5} \mathrm{~m}^{3}
$$

Assuming ideal gases, the number of moles contained in the line at any pressure is

$$
\mathrm{n}=\frac{\mathrm{PV}}{\mathrm{RT}}
$$

The change in the number of moles in the line as the pressure changes is

$$
\Delta \mathrm{n}=\Delta \mathrm{P} \quad\left(\frac{\mathrm{V}}{\mathrm{RT}}\right)
$$

and for this. case,

$$
\begin{aligned}
\frac{\mathrm{V}}{\mathrm{RT}} & =\frac{2.98 \times 10^{5}}{(83.14)(300)} \frac{\text { tonne moles }}{\text { bars }} \\
& =11.95 \frac{\text { tonne moles }}{\mathrm{bar}}
\end{aligned}
$$

Therefore, the amount of gas stored in the line as a function of pressure is

$$
\Delta \mathrm{n}=11.95 \Delta \mathrm{P} \text { tonne moles }
$$

and the hours of storage, based on the reference case flowrate, is

$$
\begin{gathered}
\text { hours of storage }=\frac{11.95}{76} \Delta \mathrm{P} \\
=0.16 \Delta \mathrm{P}
\end{gathered}
$$

for variations in the diameter, these equations can be combined to yield

$$
\Delta \mathrm{n}=5.04 \mathrm{D}^{2} \Delta \mathrm{P}
$$

$$
\text { Hours of storage }=0.0663 \mathrm{D}^{2} \Delta \mathrm{P}
$$

The amount of storage calculated from Equations.(A4-29) and (A4-30) is shown in Figure A4-22 for various line diameters above and including the optimum for the reference case flowrate in the $\mathrm{H}_{2} / \mathrm{CO}$ line. The incremental cost for the additional storage volume in the pipeline is shown in Figure A4-23. This cost includes both the additional cost of the larger transmission line and the credit for the decreased pumping cost resulting from larger pipeline diameters. Not included is the cost of the compressor at the outlet end, required to compress the gas to methanator inlet pressure.

An alternate approach that might be employed for utilizing the transmission line as storage would be to allow the pipeline pressure to build up above the steady-state value as the one- and two-shift users go off-line. Again the line diameter could be expanded to give additional storage capability. The high pressure pipeline approach has some advantages and disadvantages as compared with the lower pressure approach. The additional pressure would require 


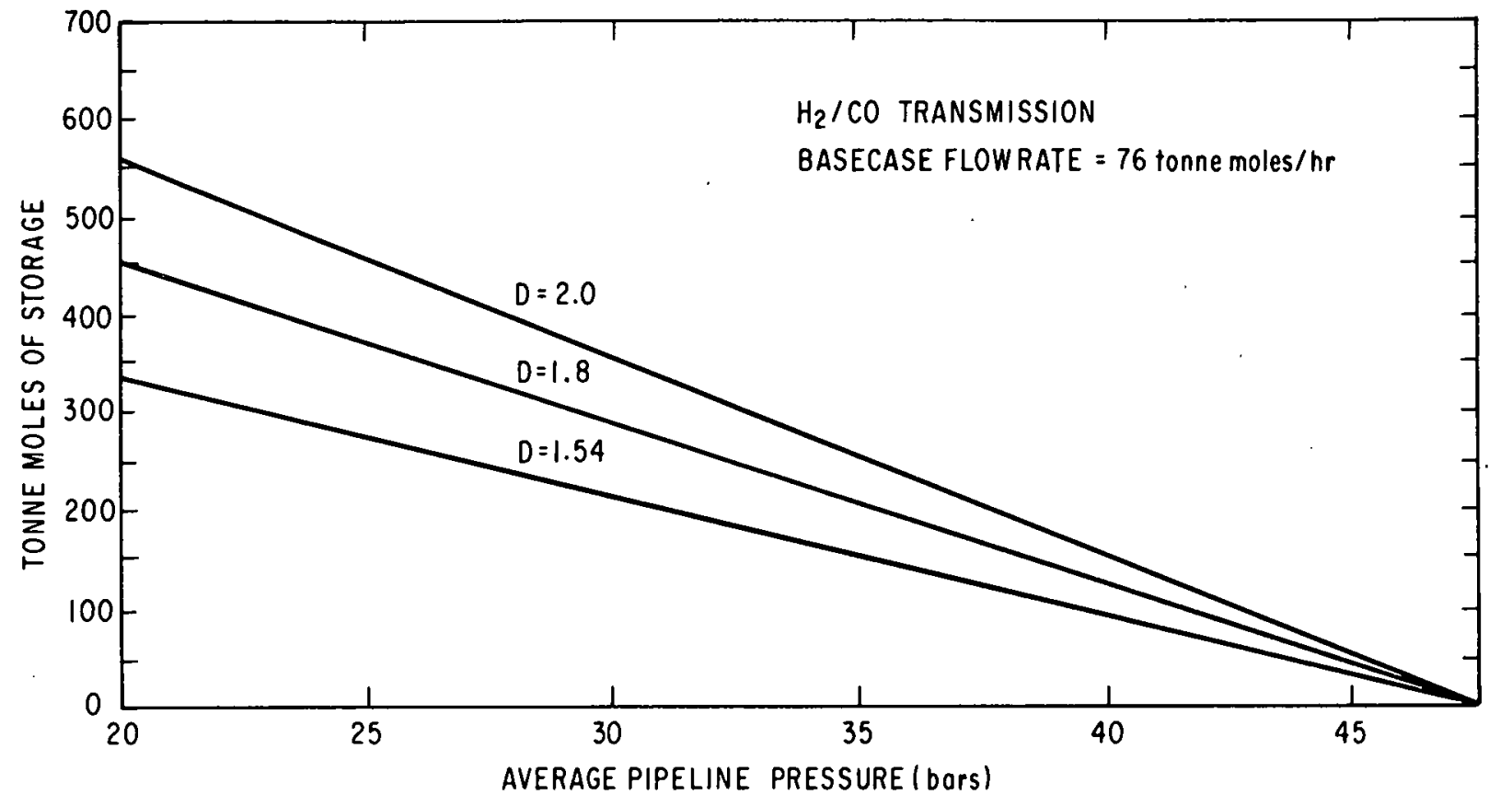

Figure A4-22. Storage Capability of Transmission Line Versus Average Line Pressure

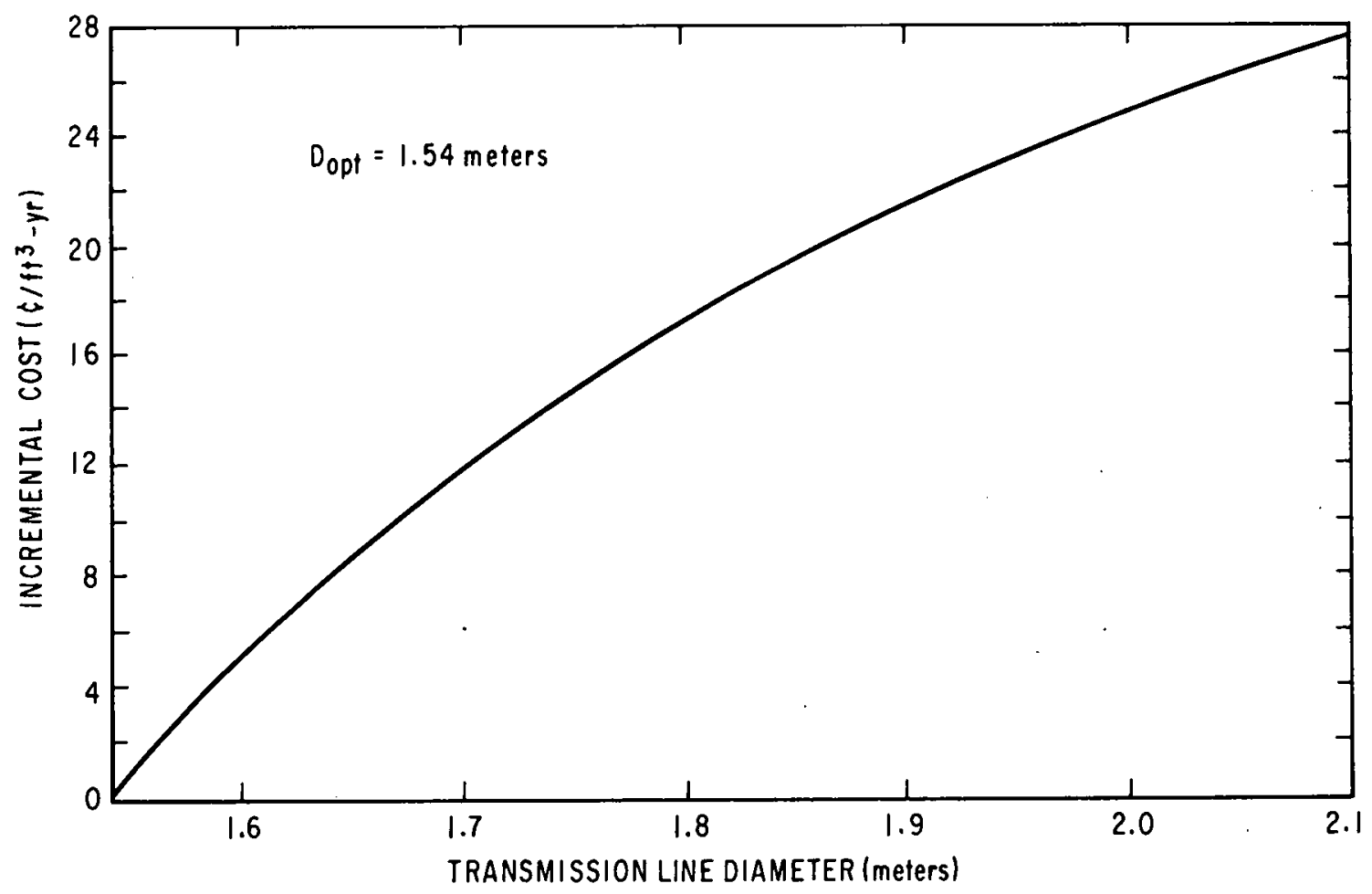

Figure A4-23. Incremental Cost for Storage Versus Transmission Line Diameter for $\mathrm{H}_{2} / \mathrm{CO}$ Storage 
a larger pipeline wall thickness and, hence, more material for pipeline construction. This would be partially offset by the decreased pressure drop experienced by the gas at the higher pressure.

A comparison of the compressor requirements shows that the high-pressure compressor at the endothermic site would require less work to compress the gas over a given pressure differential as compared with the low-pressure compressor located at the exothermic reaction site. If the high-pressure compressor were coupled with an expander recovering a portion of the work in periods in which the outlet pressure was above that required for the exothermic reactor. This might be one situation in which the transmission of PV work discussed earlier might become economically viable. In any case, the interaction of the various components in the pipeline during utilization of pressure transients for storage is complicated and could only be analyzed in detail after a time-distance distribution, is set for the various users.

It should be pointed out that such a time distribution would undoubtedly show an "averaging" effect for the entire transmission grid, in which various uses would be on stream at different periods during the day, minimizing the peak loads at the exothermic sites.

In general, one can conclude that the costs of storage in the pipeline do not appear to be excessive. If large-diameter pipelines can be built, pipeline storage appears to be one competitive approach to storage of gases.

The noncompressible nature of liquids makes the utilization of the transmission line impractical for liquid storage. However, initial estimates of aboveground, atmospheric tanks show that for the base case flowrate approximately eight hours of storage ( 150 tonne moles, $5.2 \times 10^{5} \mathrm{ft}^{3}$ ) would cost approximately $\$ 0.28 / \mathrm{ft}^{3}-\mathrm{yr}$ at a $20 \% / \mathrm{yr}$ capitalization rate (RA4-3, -4). Assuming eight hours of storage capability is required at both the hydrogenation and dehydrogenation ends of the transmission line, the additional cost to the delivered heat would amount to approximately $\$ 0.01 / \mathrm{GJ}$.

\section{A4.8 SUMARY}

In this appendix, transportation costs, sensitivity of transportation costs to design parameters, and various transportation philosophies have been explored. The major conclusions can be summarized as follows:

- HTCHP transportation costs amount to approximately $60 \mathrm{k} / \mathrm{J}$ delivered.

- LTCHP transportation costs amount to approximately 40\%/GJ delivered, not considering inventory cost; and 53k/ $\mathrm{J}$ considering inventory cost.

- Transportation cost is sensitive to the cost of pipeline and insensitive to compressor and energy costs. It is directly proportional to transmission distance.

- In the HTCHP, recycling of water from the methanator to the reformer appears to be economically competitive with the treatment of water required in an open system.

- Transportation costs are insensitive to compressor siting and liquid design pressure. 
- Multiple lines per trench offer significant savings over single line per trench.

- PV work transmission by means of compressed gases does not appear to be economical.

- Use of the pipelines to store gases for peak or one-shift usage appears economically attractive.

Finally, it should be recognized that the analyses performed in this appendix are not intended to be final. They have been used only to obtain some feeling for important economic and design parameters on total costs. As work continues on the CHP and related concepts, these analyses should be updated and carried out in greater detail.

\section{REFERENCES - APPENDIX A4}

RA4-1 Federal Power Commission, Bureau of Natural Gas, Staff Report, "Cost of Pipeline and Compressor Station Construction Under Non-Budget Pipeline Certificate Authorizations as Reported by Pipeline Companies in Fiscal Year 1976," Washington D.C., 1976.

RA4-2 Vosburgh, K.G. et al., "Conceptual Design for a Pilot/Demonstration Compressed Air Storage Facility Employing a Solution-Mined Salt Cavern," Final Report, EPRI EM-391 (Research Project 737-1), Appendix B, General Electric Corporate Research and Development, Schenectady, NY, June 1977.

RA4-3 Guthrie, K.M., "Data and Techniques for Preliminary Capital Cost Estimating," Chemical Engineering, Vol. 76, No. 6, 1969, p. 114.

RA4-4 Anon., "Economic Indicators," Chemical Engineering, Vol. 80, No. 23, 1976.

RA4-5 Konopka, A., and Wurm, J., Paper No. 749015, Proceedings of the Ninth Intersociety Energy Conversion Conference, 1974, p. 405.

RA4-6 Reynolds, R.A. and Slagyex, W.L., "Transportation and Storage of Hydrogen for Eco-Energy," General Electric Company, Report 72TMP-54, Santa Barbara, California, 1972 .

RA4-7 Coates, D.L., "Computer Analyses -- Pipeline Design," Oil and Gas Journal, December 21,1970, p. 41.

RA4-8 Congram, G.E., "U.S. Pipeline Investment Expands by $\$ 4.26$ Billion," Oil and Gas Journal, August 22, 1977, p. 73.

RA4-9 Icerman, L., "Relative Costs of Energy Transmission for Hydrogen, Natural Gas, and Electricity," Energy Sources, Vol. 1, 1974, p. 435.

R̊ 4-10 Beghi, G., et al., "Hydrogen, Oxygen, and Natural Gas By Pipelines: Comparative Transportation Costs," Report EUR-51031, Commission of the European Communities, Joint Nuclear Research Center, 1974. 
RA4-11 Peters, M.S., and Timmerhaus, K.D., Plant Design and Economics for Chemical Engineers; McGraw-Hill, New York, 1968, p. 772 .

RA4-12 Anon., Chemical Marketing Reporter, Schnell Publishing Company, December 5,1977 . 


\title{
Appendix 5
}

\section{REVIEW OF THE STATE-OF-THE-ART OF HYDROGENATION-DEHYDROGENATION PROCESSES AND REACTOR DESIGNS}

\author{
By UOP Inc.*
}

\author{
BENZENE-CYCLOHEXANE REACTIONS REVIEW
}

HYDROGENATION OF BENZENE TO CYCLOHEXANE

General

The selective catalytic hydrogenation of benzene to cyclohexane is a wellestablished industrial process, which has been carried out commercially for about 25 years. In 1976, nine companies produced cyclohexane by this route in the United States in eleven plants, with a total production of 2.2 billion pounds (Rl). The cyclohexane growth rate has been $4.5 \%$ per year for the period 1971-1976.

Because nearly all the cyclohexane produced is converted to intermediates for polyamide production - adipic acid, caprolactom or hexamethylenediamine the purity requirements for cyclohexane are very exacting; even small amounts of the isomeric methylcyclopentane impart undesirable properties to the ultimate product. The catalysts and processes developed for cyclohexane production have therefore been designed to minimize byproduct formation.

\section{Process Scheme}

The hydrogenation is generally carried out in liquid phase (although there is a vapor-phase process, practiced abroad) (R2) at temperatures in the range 135 to $275^{\circ} \mathrm{C}$ and pressures of about $500 \mathrm{psi}$. Because the reaction is highly exothermic, with a heat of reaction of $51.2 \mathrm{kcal} / \mathrm{mol}$ at $200^{\circ} \mathrm{C}$ (R3), a principal concern of the reactor design is the effective removal of the heat evolved, to maintain the temperature in the desired range. In an adiabatic system, each percent of benzene converted raises the temperature 7 to $8^{\circ} \mathrm{C}$. As shown in Figure A5-1 (R4), the amount of residual benzene rises with increase of temperature; in addition, high temperatures are likely to induce cracking and isomerization, with the latter side reaction increasingly favored thermodynamically as the temperature increases. The reaction rate is zero order with respect to benzene (R3).

Several flow schemes are employed to control temperature. In one (R5), the catalyst is suspended in the liquid-phase reactants and the slurry is circulated through an indirect heat exchanger, where the heat is removed by pressurized water. Depressurizing of the cooling water produces low-pressure steam. In other processes, a multitubular reactor (R6) is used which is water cooled (and also generates low-pressure steam). The tubes are filled with granular catalyst which constitute fixed beds.

\footnotetext{
*This appendix was not prepared under funding from this contract. It is included with permission of UOI Inc. to add depth to the background of the hydrogenation/ dehydrogenation processes that serve as a basis for the ITCHP.
} 


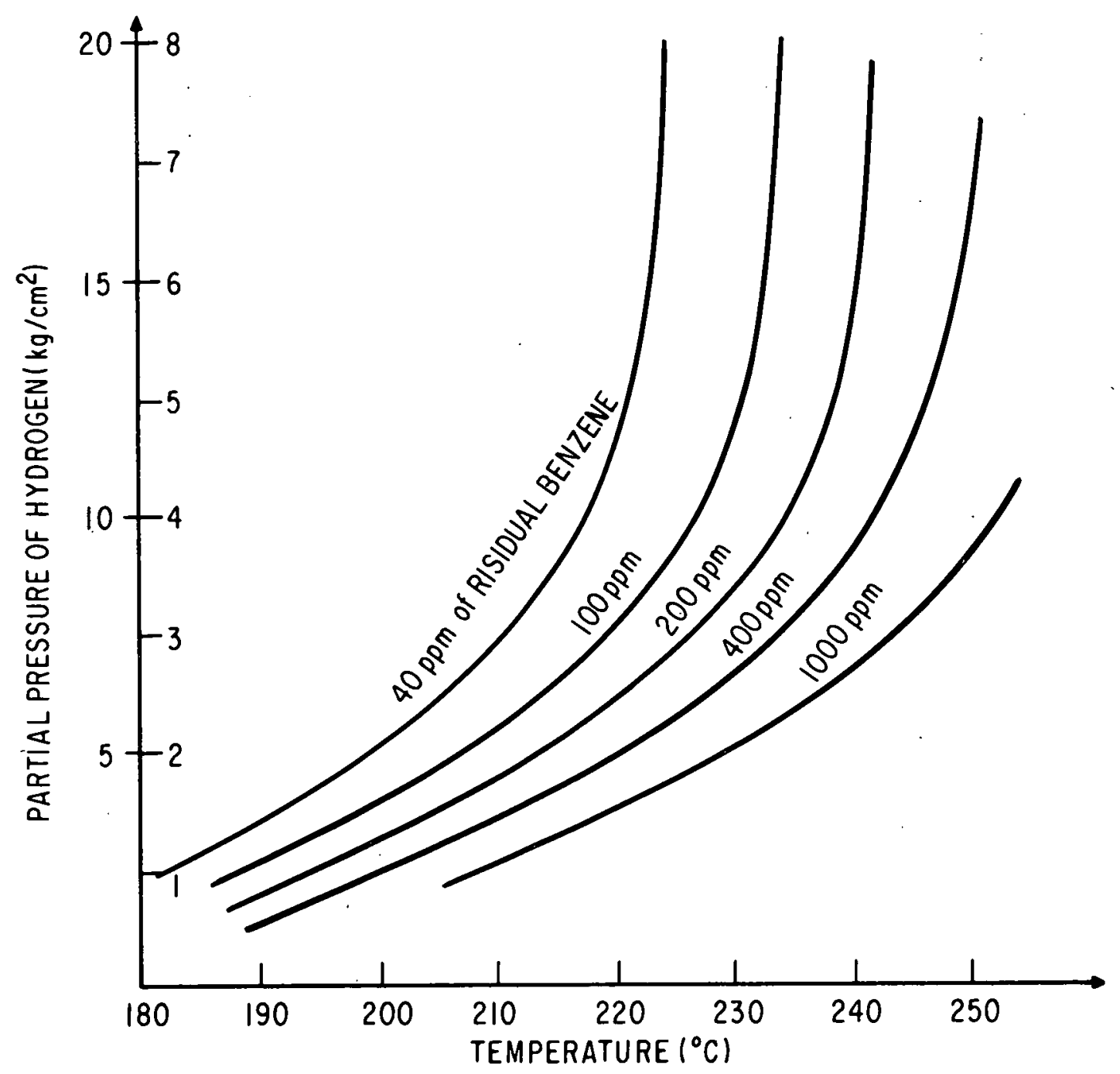

Figure A5-1. Residual Benzene Concentration in Hydrogenation Reaction as a Function of Temperature and Hydrogen Partial Pressure

The preferred industrial processes employ several fixed-bed adiabatic reactors in series (generally two, three, or four) in which partial reaction occurs in each reactor; the temperature is controlled in inter-reactor cooling, by recirculation of product cyclohexane (which acts as a heat sink), or by a combination of these. Two flow schemes for such processes are shown in Figures A5-2 (R7) and -3 (R8). In the first of these, recirculated cyclohexane flows concurrently with the fresh benzene feed through three reactors in series; in the second, benzene is fed through the four reactors in parallel, while cyclohexane and hydrogen flow through the reactors in series. Various other reactors, heat exchangers, and flow arrangements are possible for control of temperature with minimum overall circulation of cyclohexane, cooling water, and hydrogen.

\section{Hydrogen}

The circulated hydrogen need not be pure, and where the cyclohexane facility is part of a refinery operation, hydrogen from a catalytic reforming unit

$$
\text { A5-2 }
$$




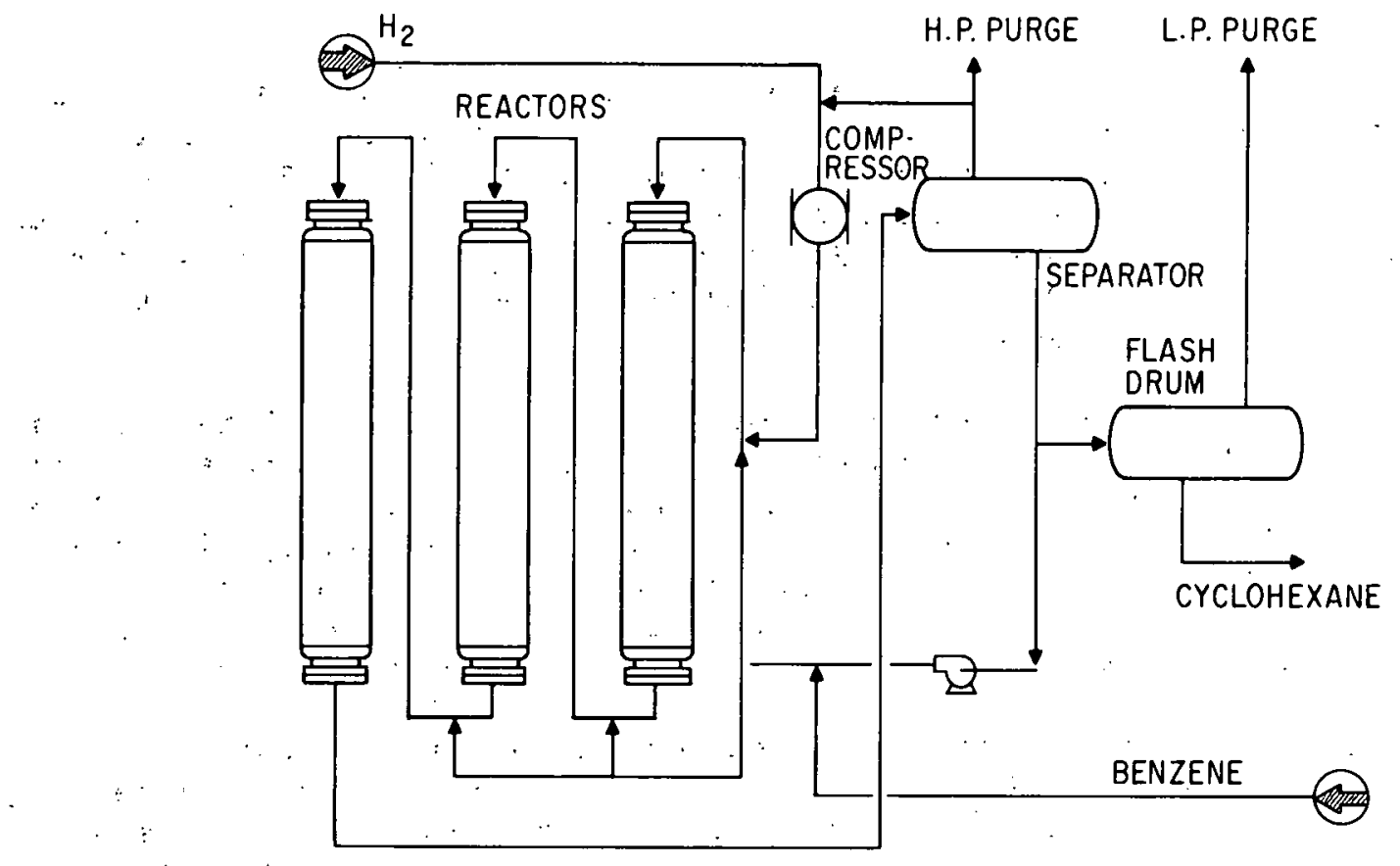

Figure A5-2. Flow Scheme for Benzene Hydrogenation Process

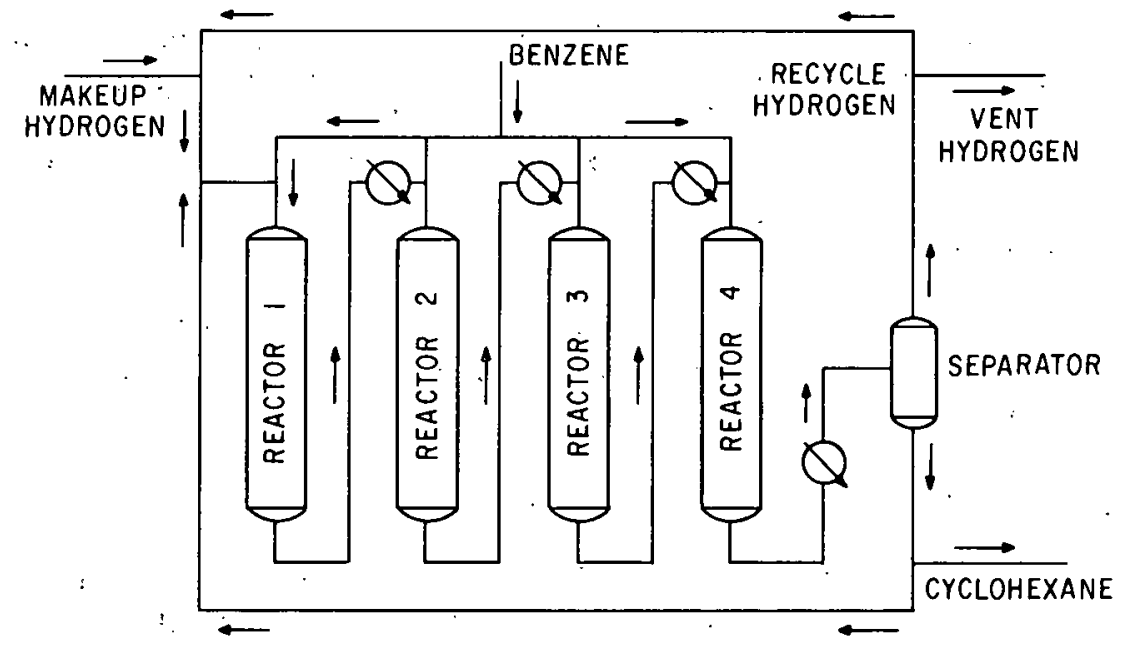

Figure A5-3. Modified Flow Scheme for Benzene Hydrogenation Process

or hydrodealkylation unit may be used for benzene hydrogenation (R9) with a hydrogen purity sometimes under $70 \%$. Likewise, if cracking occurs during the latter process so that the circulating hydrogen must be bled off, makeup hydrogen is supplied at a rate and purity required to keep the hydrogen concentration above 30\% (R5). Typical hydrogen/benzene mol ratios vary from 3-3.8:1 to as high as 8:1 (RI0); or, expressed in terms of hydrogen/total hydrocarbon mol ratio, $2: 1$ (R5). Liquid flow rates for the fixed-bed operation correspond to liquid hourly space velocities of 0.5 to 4.0 (Rlo). 
Catalysts used for benzene hydrogenation usually comprise a Group VIII metal as the active component, a refractory oxide support, and an alkaline modifier. The active metal may be platinum, palladium, ruthenium, or nickel (R3). The noble metals are generally used in a concentration of 0.375 to $2 \%$ on the support, ,while nickel is used in large amounts - 25 to $60 \%$ by weight (RI1). The support may be silica, alumina (particularly gamma alumina), magnesia, zirconia, or mixtures of these, or similar oxides.

Because the principal side reaction (isomerization to methylcyclohexane) is known to be acid-catalyzed, alkaline components are included in the catalyst system to suppress acjdity. These may be added to the reactant stream (e.g., 10 to $1000 \mathrm{ppm}$ of basic nitrogen compounds such as ammonia, alkylamines, cyclic amines, or alkylene polyamines) (RII) or they may be incorporated as a component of the solid catalyst. One such catalyst of the latter type, for example, comprises platinum on alumina with a small amount of a lithium salt as alkalizing agent (R8).

Nickel catalysts are irreversibly poisoned by sulfur, and reversibly poisoned by $\mathrm{CO}$ and $\mathrm{CO}_{2}$ (RI2); such impurities must therefore be removed from feeds used with nickel catalysts. On the other hand, platinum catalysts can tolerate up to $300 \mathrm{ppm}$ of sulfur compounds in the benzene feed (R5), and are preferred when such impurities are present.

Product Purity

Cyclohexane of 99.98 purity, containing less than $100 \mathrm{ppm}$ of benzene (typically as little as $10 \mathrm{ppm}$ ), can easily be made by benzene hydrogenation (R3, R13, R14), and product purities as high as $99.99 \%$ have been reported (R15). The purity of the product cyclohexane is closely related to that of the feed benzene.

\section{Utility Requirements}

Some idea of the utility requirements and other economic data is afforded by the tabulation for seven processes shown in Table A5-1. These data were published in 1975 (RI6).

Considerations for General Electric Project

Since complete conversion is not necessary, the lower portions of the temperature range cited may be used, and/or higher space velocities, and/or fewer reactors, and/or lower pressure, depending on the economics of these variations. Use of relatively low temperatures should minimize side reactions, particularly cracking. Since there will be essentially a single batch of cycled feed, with little makeup, feed impurities should not be a major consideration, and nickel catalysts should be feasible.

DEHYDROGENATION OF CYCLOHEXANE TO BENZENE

\section{General}

In contrast to the relatively well-defined commercial operations for selective hydrogenation of benzene to cyclohexane, the reverse reaction, as an isolated operation, is not practiced industrially. However, the conversion of 
Table A5-1

PROCESSES FOR THE PRODUCTION OF CYCLOHEXANE: ECONOMIC DATA

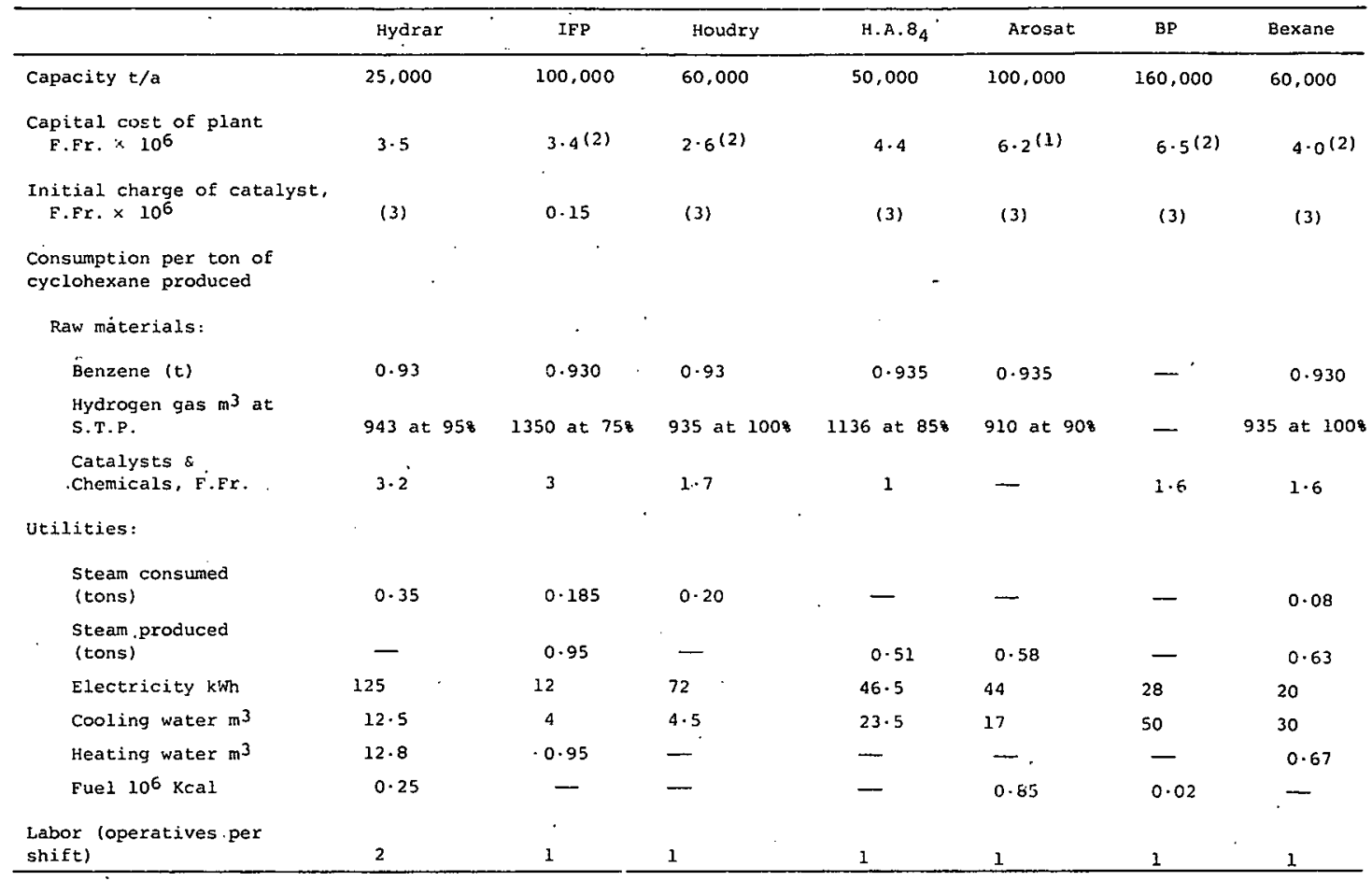

(1) Complete plants including any pretreatment, additional compressors and, if neçessary, the equipment for preparing the catalyst is included.

(2) Plants limited solely to the hydrogenation of the benzene.

(3) Included in capital cost of plant.

cyclohexane to benzene occurs on a huge scale as part of the complex of reactions collectively known as catalytic reforming, by which low-grade naphthas are converted to higher octane reformates considerably richer in aromatic hydrocarbons, including benzene.

In 1976, 1.71 billion gallons of benzene were extracted from catalytic reformates in the U.S., constituting approximately 95\% of the total benzene production. The nation's catalytic reforming capacity at the beginning of this year was 3.67 million barrels per day (R1) (56 billion gal./yr), so that the recovered benzene constituted slightly over $3 \%$ of total reformate production.

The benzene found in reformate is formed by several reactions (Rl8) - cyclohexane dehydrogenation, dehydro-isomerization of methylcyclopentane, cyclodehydrogenation of $\mathrm{n}$-hexane, and hydrodealkylation of toluene and higher alkylbenzenes. The higher aromatics are of similar varied origin; and because it is desired to produce substantial quantities of aromatics in the reforming process, the reforming catalyst is designed to promote not only simple naphthene dehydrogenation, but other reactions as well - isomerization of paraffins and naphthenes, cracking, and cyclization. Reforming catalysts are therefore duofunctional in composition: a balanced composite of a noble metal which catalyzes cyclization, hydrogenation-dehydrogenation, and some isomerization, as well as keeping the surface clean by hydrogenating potential foulants; and an acidic support which catalyzes most of the isomerization and cracking, including dealkylation. The two functions are so intimately composited that they act in concert. 
Cyclohexane Dehydrogenation - Thermodynamics and Kinetics

For the simple dehydrogenation of cyclohexane, the endothermic heat of reaction is $+51.2 \mathrm{kcal} / \mathrm{mol}$ (liquid phase); in vapor phase, $\Delta \mathrm{H}=+49.2 \mathrm{kcal} / \mathrm{mol}$, and

$$
\Delta G=52,610-94.6 \mathrm{~T}
$$

$\Delta G=0$ at $282^{\circ} \mathrm{C}$, and above that temperature, equilibrium favors complete dehydrogenation (RI9). This reaction is also favored by low pressures, but if higher pressures are necessary "for operational reasons" or preservation of catalyst activity, higher temperatures are used to maintain favorable equilibrium conditions.

The activation energy for cyclohexane dehydrogenation is $18.1 \mathrm{kcal} / \mathrm{mol}$, corresponding to a doubling of the reaction rate for each $41^{\circ} \mathrm{C}$ temperature rise (RI8). (The corresponding figures for methylcyclohexane conversion to benzene over a hydroreforming catalyst are $32.8 \mathrm{kcal} / \mathrm{mol}$ and $23^{\circ} \mathrm{C}$.) The reaction has been found to be zero order in cyclohexane at 150 to $225^{\circ} \mathrm{C}$ and 0.4 to 1 atm cyclohexane partial pressure, but first order at 300 to $416^{\circ} \mathrm{C}(\mathrm{R} 20, \mathrm{R} 21)$.

Process Conditions

In catalytic reforming for aromatics production, temperatures are generally in the range 480 to $510^{\circ} \mathrm{C}$ and pressures 200 to $300 \mathrm{psi}$; under these conditions, over 90\% of the naphthenes (including 5-membered rings) are converted to aromatics (RI9). Because the reaction is endothermic, the catalyst is usually disposed in a series of adiabatic reactors with interheaters between beds as well as provision for preheating the feed. One such reaction system with three beds in series is shown in Figure A5-4 (RI8).

The operating conditions for reforming, however, are determined more by the requirements of the other reactions discussed above then by those for cyclohexane dehydrogenation. For this reaction, dehydrogenation is virtually complete at $300^{\circ} \mathrm{C}$ and atmospheric pressure (R22).

\section{Catalysts}

Reforming catalysts generally comprise a noble metal (usually platinum) on an acidic support (usually halided alumina or silica-alumina) (R23). Platinum modifiers of various types have also been employed - other noble metals, transition metal oxides, and the like, generally for purposes of increasing stability under severely deactivating reforming conditions. The reasons for such modifications do not appear applicable to simple cyclohexane dehydrogenation.

It has been shown that isomerization of cyclohexane to methylcyclopentane occurs at a rate 0.005 to 0.01 as fast as dehydrogenation to benzene with a platinum-alumina catalyst (unhalided) at $520^{\circ} \mathrm{C}, 300 \mathrm{psig}, \mathrm{H}_{2} / \mathrm{HC}=6$, and space velocities of 1000 to 32,000 , corresponding to cyclohexane conversions of 32 to 3\% (R22). When the platinum became sulfur-poisoned, dehydrogenation was retarded, allowing isomerization to become a principal reaction ( $\mathrm{MCP} /$ benzene ratio $=1.5$ at 4000 LHSV). It is apparent that such systems are extremely sensitive to poisons, and that even the slight acidity inherent in alumina is 


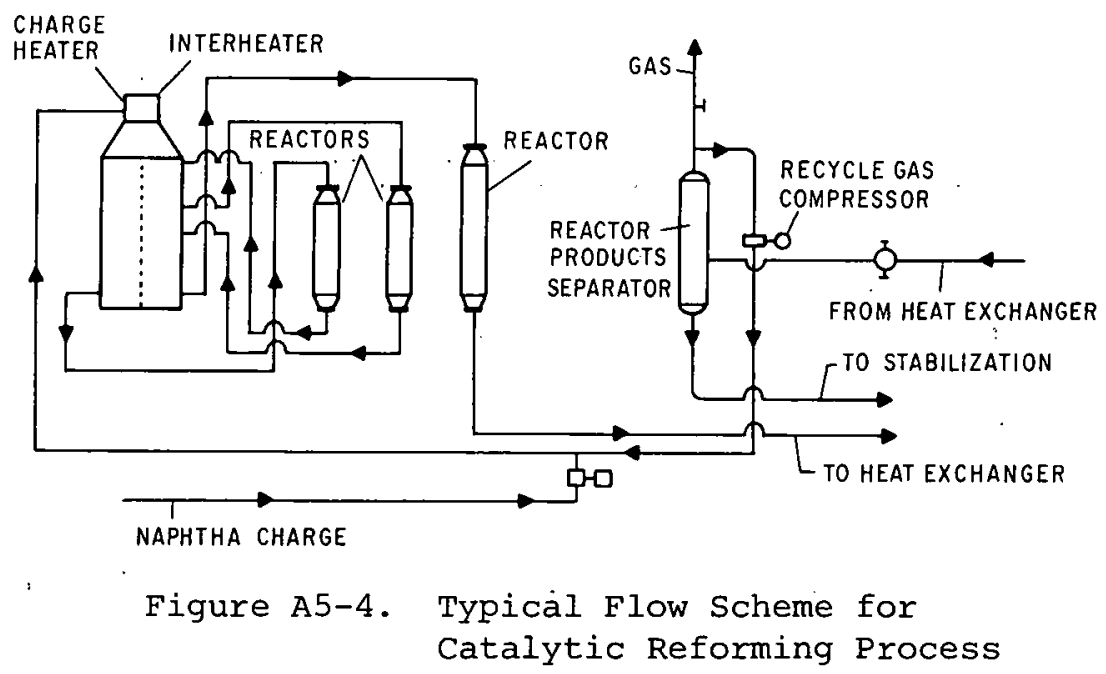

sufficient to produce appreciable naphthene isomerization at higher temperatures.

Modification of platinum with palladium on an alumina support has been shown to have little effect on overall catalyst activity; for the single metals, platinum was 60 times as active as palladium (R24). The addition of rhenium was found to increase the stability of a platinum-alumina catalyst for cyclohexane dehydrogenation but not its activity (R25). However, platinum-alumina catalysts (unmodified) have been claimed to be active for this reaction for 1.5 years, with little change in activity after an initial decline of some $15 \%$ during the first 100 hours of use at $350^{\circ} \mathrm{C}, 20$ to $30 \mathrm{~atm}, \mathrm{H}_{2} / \mathrm{HC}=6$ (R26). In studies made with a chromia-alumina catalyst, the ratio of methylcyclopentane to benzene formed was $1: 20$; but when potassium was added to the catalyst no methylcyclopentane was formed (R21). With such catalysts, the rate-limiting step was found to be the abstraction of the first hydrogen (R27) in studies at 400 to $520^{\circ} \mathrm{C}$.

\section{Considerations for General Electric Project}

It appears likely that catalysts similar to those developed for benzene hydrogenation will operate well for the reverse reaction; that the latter will be highly selective at temperatures near or slightly above $300^{\circ} \mathrm{C}$, and that reasonable stability can be maintained in the presence of hydrogen. The exact operating conditions for such a process remain to be determined.

\section{References}

Rl Chemical Engineering News, Vol. 55, May 2, 1977, p. 37.

R2 Benzene and Its Industrial Derivatives (E.G. Hancock, ed.), John Wiley and Sons, New York, 1975, p. 227.

R3 ibid., p. 224 .

R4 ibid., p. 225 .

R5 ibid., p. 229 . 
R7 ibid., p. 228 .

R8 Kirk-Othmer, Encyclopedia of Chemical Technology, 2d ed., Interscience Publishers, New York, 1965, Vol. 6, p. 679.

R9 Sittig, M. , Aromatic Hydrocarbons - Manufacture and Technology, Noyes Data Corp., Park Ridge, N.J., 1976, p. 242.

R10 Sittig, ibid., pp. 245, 250.

Rll Sittig, ibid., p. 243

R12 Benzene and Its Industrial Derivatives (E.G. Hancock, ed.), John Wiley and.Sons; New York, 1975, p. 226.

R13 Benzene and Its Industrial Derivatives (E.G. Hancock, ed.), John Wiley and Sons, New York, 1975, p. 222.

R14 Craig, R.G., "Route Gives Volume Output of High-Purity Cyclohexane," Chemical Engineering, Vol. 77, June 1, 1970, pp. 108-110.

R15 Benzene and Its Industrial Derivätives (E.G. Hancock, ed.), John Wiley and Sons, New York, 1975, p. 232.

Rl6 Benzene and Its Industrial Derivatives (E.G. Hancock, ed.), John Wiley and Sons, New York, 1975, p. 235.

R17 Oil and Gas Journal, March 28, 1977, p. 98.

R18 Haensel, V. and Berger, C.V., Advances in Petroleum Chemistry and Refining (edited by K.A. Kobe and John J. McKetta, Jr.), Interscience Publishers, New York, 1958, Vol. 1, pp. 387-427.

R19 Goldstein and Waddams, "Petroleum Chemicals Industry", 3d ed., E. and F. Spon Ltd., London, 1967, pp. 261-262.

R20 Maatman, R., et al., Journal of Catalysis, Vol. 31, No. 3, 1973, pp. 384-88.

R21 MacIver, D.S., et,al., Journal of Physical Chemistry, Vol. 66, 1962, p. 871.

R22 Haensel, V., Donaldson, G.R., and Riedl, F.J., "Mechanisms of Cyclohexane Conversion over Platinum Catalysts," Proceedings of Third International Congress on Catalysis, Amsterdam, Netherlands, 1964.

R23 Kirk-othmer, Encyclopedia of Chemical Technology, 2d ed., Interscience Publishers, New York; 1965, Vol. 3, pp. 376-378.

R24 Haro, J., et al., Journal of Catalysis, Vol. 45, No. 3, 1976, pp. 326-31,

R25 Kozlov, N.S., et al., Neftekhimiya, Vol. 15, No. 1, 1975, pp. 69-73.

R26 Kozorezov, Yu I., Neftepererab Neftekhim, No. 6, 1973, pp. $26-28$.

R27 Uchijima, T., et al., Journal of Catalysis, Vol. 17, 1970, pp. 287-96. 
For some rime, chemical engineering methods have been available for the modeling and optimization of chemical reactors; however, routine application of these methods became practical only with increased computer capabil.ities. Mathematical modeling of reactors has now become standard practice, making it possible to manipulate reactor variables to define the configuration that is theoretically most profitable for a given process objective. Commercially, however, the sophisticated reactor systems required to attain these objectives have not always been justified, for reasons normally characterized as "practical engineering considerations."

The thermodynamics of chemical reactions are orderly in that equilibrium and thermal effects are well defined, but kinetics, subject to highly variable catalytic behavior, can introduce extreme cumplexitiea. Rates vary widely, and catalysts can be very stable or be rapidily deactivated, requiring continuous or intermittent reactivation. Many types of reactor systems have been developed and standardized to some extent for normal combinations cf reaction characteristics, but the choice often involves compromises.

A brief review of trends in reactor design in the refining and petrochemical industry will serve as a limited illustration of reduction to practice. The early catalytic cracking reactors used for petroleum conversion were fixed bed with molten salt in heating and colling pipes, to supply heat of reaction during processing and to remove heat of catalyst deposit combustion during regeneration. The early dehydrogenation reactors employed catalysts in tubes surrounded by heating and cooling fluids (such as flue gas or molten salt) to perform similar functions. Nonregenerative exothermic reactions, such as catalytic polymerization, oxidation, and hydrogenation, often used similar tubular reactors with heat removal by steam generation or fluids circulation.

The largest volume petrochemical intermediate, syngas, used for production of hydrogen, ammonia, methanol, and other common products is still largely produced in directly fired catalyst tubes operating close to the metallurgical temperature limit, demonstrating a satisfactory, although costly, solution to a complex reactor design problem. In the subsequent production of ammonia and methanol, exothermic reactions favored by high pressure and the lowest practical operating temperatures, ingenious integration of heat exchangers and catalytic reactors was widely used until the largest practical sizes of these types of integrated reactors became inadequate for the size of single trains required for world-scale economical operation. For these purposes multiple-bed, adiabatic reactors with intermediate gas quench are now used, and even these are approaching their maximum design limits.

Similarly, with increased size of petroleum and petrochemical processes, using particulate catalysts, the trend has been toward adiabatic reactors with either interheating or intercooling as well as recycle for delta temperature control. Where pressure drop is a problem, radial flow or multipass flow is ofren used, and continuously regenerated moving bed systems have been developed for specific process requirements. 
In catalytic oil conversion two processes dominate. One employs hydrocracking, generally using adiabatic reactors with intermediate gas quench with some variants, such as ebullition, in limited use. The other employs catalyst circulation between a regenerator, which also serves as a heat source, and a reactor where heavy distillates and sometimes residual oils are converted to lighter products.

There was an excursion into moving bed systems, but fluidized systems have become dominant and, while the technology is over 35 years old, significant improvements have been made only recently. The regenerator or catalyst deposit burner, which can also be a fluidized boiler, is normally dense or turbulent phase with various special arrangements to improve burning, and the preferred reactor is of the short-time transport type termed a "riser reactor."

Two major dehydrogenation processes, ethylbenzene to styrene and butanebutylene to butadiene, present an interesting contrast in that, typically, EB is dehydrogenated in large continuous, multiple adiabatic bed reactors with interheating, while $\mathrm{BB}$ is dehydrogenated in large adiabatic bed reactors in a cyclic manner, utilizing a diluted fixed catalyst bed as a heat sink during regeneration and a heat source during processing. The difference here, of course, is that although the processes have similar thermodynamics, a steam resistant catalyst is used in the former, while the butadiene catalyst would be deactivated by steam -- thus the added complications.

Oxidation and ammoxidation processes, also widely practiced, are now generally carried out in gas or liquid fluidized solids systems (although homogeneous catalysts are sometimes used). However, equilibrium limited type reactions are severely penalized by backmixing, and plug flow reactors are preferred.

Electrical heating is now used to only a limited extent, although in some applications it would have attractive features.

While recent sharp increases in energy costs have required reexamination of thermal efficiencies of reactor systems, few major reactor revisions have resulted. The overriding considerations have tended to be conversion/selectivity, operability at large capacities, and other economic considerations that tend to outweigh differential energy costs.

For the use of reversible chemical reactions in energy storage and transmission, with thermodynamic efficiency the primary consideration, it seems rather unlikely, then, that an off-the-shelf reactor system would be acceptable, and fundamental rethinking appears appropriate.

Maximizing thermodynamic efficiency, measured by approach to reversibility at all points in the process, involves not only minimizing end losses, as is presumably done to the economic limit in present reactor designs, but also carrying out the reaction countercurrent to the heating or. cooling medium with optimally uniform temperature differences at all points in the reactors. In present design practice, as noted, this is a task of selecting the maximum economic stages, since countercurrent reactors have seldom been used. For energy conversion processes, however, the advantages of a constant gradient, countercurrent reactor would justify considerable design effort. 
Again, the problem of scale-up arises. Reversible chemical reaction, energy conversion reactors would tend to be quite large, relative to the largest existing units, and already scale-up has compromised thermal efficiency. This would tend, for example, to eliminate tubular heat exchanger reactors, since an unreasonable number of tubes would be required as a consequence of high radial temperature gradients in large tubes and high pressure drop in long tubes.

This suggests reexamination of gas and liquid fluidized heat exchanger reactor systems, which conceivably could be built for any required capacity if adequately staged or if counterflow concepts could be implemented. While not completely understood, the science of fluidized systems has advanced to the point where designs are quite reliable, and this technology will undoubtedly play a large part in alternate energy processes.

An investigation of adequate scope, in addition to evaluating the above, would undoubtedly reveal alternatives that would justify similar consideration with a high probability of advancing the science of chemical reactor design, especially with regard to thermodynamic efficiency. 


\section{Appendix 6}

\section{COMPUTER PROGRAMS}

Computer programs used in the design analyses of the HTCHP and LTCHP are given in this, appendix. The programs included are as follows:

- HTCHP Design Program: Used to determine material and energy balances for the HTCHP system design analysis.

- LTCHP Equilibrium Program: Used to calculate the chemical reaction equilibrium for the dehydrogenation of cyclohexane and its isomerization to methylcyclopentane. The program can calculate the equilibrium of both reactions in parallel or of just the dehydrogenation reaction.

- LTCHP Energy Balance Program: Used to determine vapor-liquid equilibrium for four-component mixture of benzene, cyclohexane, methylcyclopentane, and hydrogen and the enthalpies of the gas and liquid streams.

- Liquid Optimum Diameter Transportation Program: Used to calculate the cost of liquid transport at the optimum pipeline diameter as a function of composition, flowrate, power cost, and transmission pressure.

- Gas Transportation Program: Used to calculate the cost of the transport of gases as a function of composition, flowrate, power cost, and transmission distance. 
* THIS PROgRAM CALCUlates both THE ENERgy AND MATERIAL

* BALANCES FOR THE DESIGN OF THE METHANE STEAM REFORMING

* BASED CHEMICAL hEAT PIPE (HTCHP).

* INPUTS REQUIRED ARE: THE REFORMING TEMPERATURE AND PRESSURE,

* AND the moles of the fEed Gas. at the methanation end,

* TEMPERATURE, PRESSURE, AND THE CHANGE IN WATER

* STOICHIOMETRY ARE REQUIRED. THE ORDER OF THE VARIABLES IS

$\star \mathrm{CH} 4, \mathrm{H} 2 \mathrm{O}, \mathrm{CO}, \mathrm{CO} 2$, AND H2.

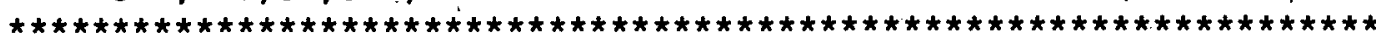

DIMENSION ENU $(5,2), \operatorname{ENIO}(5), \operatorname{ENI}(5), \operatorname{ENUT}(2), C P(5)$,

$\& X I(2), A O(2)$

CHARACTER $\star 5 S P(5), Y * 1$

DIMENSION QI $(40) / 40 * 0 . /, \mathrm{QO}(40) / 40 * 0 . /$

DIMENSION T (10), ENRO (5), ENR (5), Q(15),W(2)

NAMELIST/D/P1, P2,T

DATA ENUT $/ 2,0 . /$, ENU $/ 2 *-1,1,1,0,3 ., 0 ., 2 *-1,, 2 * 1 . /$

DATA SP/"CH4", "H2O","CO","CO2","H2"/

COMMON $R, T 0$

$\mathrm{R}=1.9872 ; \mathrm{T} 0=298.16$

$\mathrm{NS}=5 ; \mathrm{ND}=5$

ENERT $=0$.

1000 CONTINUE

PRINT," TR, PR,ENR0?"

READ, TR , PR , ENRO

IF (TR.LE. O.) GO TO 2000

CALL EQUIL (NS , ND , ENRO ,ENTRO , TR , PR , ENU , ENUT , AO , XI , ENR , ENTR , SP, $\&(P)$

PRINT, ENR (2)

DO $1 \mathrm{I}=1,5$

$1 \operatorname{ENIO}(I)=\operatorname{ENR}(I)$

PRINT, "TM, PM, DEL-H2OM?"

READ, TM, PM , ENIO (2)

ENIO (2) $=\operatorname{ENIO}(2)+\operatorname{ENR}(2)$

CALL EQUIL (NS, ND , ENIO , ENTO , TM , PM , ENU , ENUT , AO , XI , ENI , ENT , SP , CP \&)

5 FORMAT (/)

PRINT 5 ; PRINT, "MATERIAL BALANCE"

PRINT 5

PRINT," INPUT OUTPUT FRACTION D

\&RY FRACTION"

PRINT," METHANATOR:"

PRINT 10, (SP (I), ENI0 (I) ,ENI (I), ENI (I) /ENT, ENI (I) /(ENT-ENI (2))

$\&, I=1, N S)$

WMM $=(16$ *ENI $(1)+28 * \operatorname{ENI}(3)+44 \star \operatorname{ENI}(4)+2 \star \operatorname{ENI}(5)) /(\operatorname{ENT}-\operatorname{ENI}(2))$

PRINT 10, "TOTAL" , ENT0, ENT

10 FORMAT ( $5 \mathrm{X}, \mathrm{A5}, \mathrm{F} 9.3, \mathrm{~F} 15.3, \mathrm{~F} 14.4, \mathrm{~F} 12.4)$

DO $2 I=1,5$

$2 \operatorname{ENRO}(I)=\operatorname{ENI}(I)$

ENRO (2) = ENI (2) - ENIO (2) +ENR (2)

ENTR0 $=E N T-E N I O(2)+E N R(2)$ 


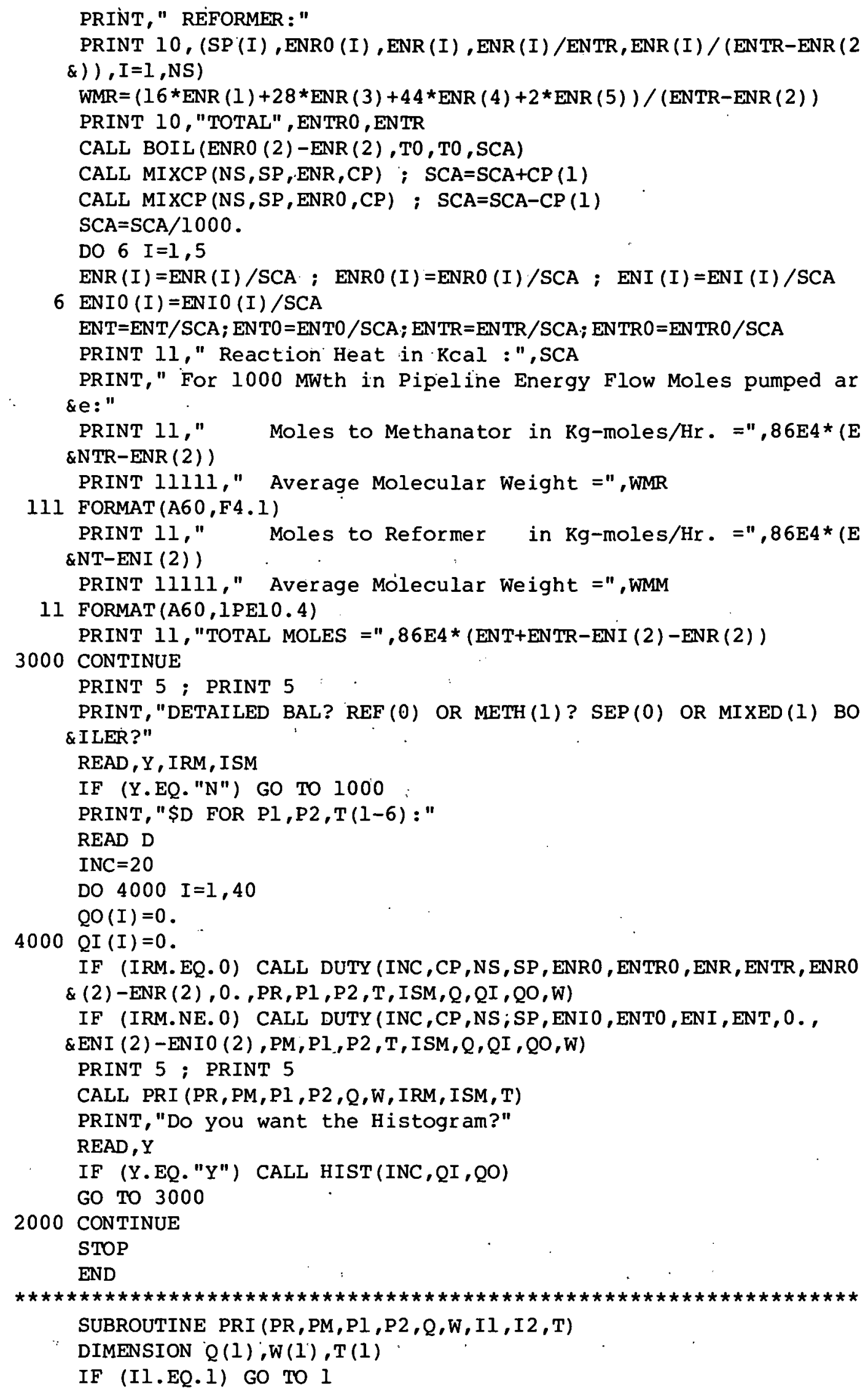


PRINT, "Detailed Energy Balance for the Reformer Plant:"

PRINT 21," Reformer Pressure (atm) =",PR

21 FORMAT (A30,F5.1)

PRINT 5

GO TO 2

1 PRINT, "Detailed Energy Balance for the Methanator Plant:" PRINT 2l," Methanator Pressure (atm) =",PM

5 FORMAT (" ")

PRINT 5

2 IF (I2.EQ.1) GO TO 3

PRINT, "Feed Boiler is separate from the Gas Heater"

GO TO 4

3 PRINT, "Feed Boiler is mixed with the Gas Heater"

4 PRINT 21," Pipeline Inlet Pressure (atm) =",P1

PRINT 21," Pipeline Exit Pressure (atm) =",P2

PRINT," UNIT Temp RANGE

PRINT," $\quad$ deg $K$

Duty"

\&"

PRINT 5

PRINT, "Heat Inputs:"

PRINT 5

PRINT 10,"Input preheater", T(1),T(2), $Q(1)$

10 FORMAT (A20,F10.1," to",F6.1,F16.1)

PRINT 10,"Feed Water Preheat",T(1),T(5), Q(2)

100 FORMAT (A20,6X," Work Input", 2X,F16.1)

PRINT 100,"Gas Expander",W(1)

PRINT 10,"Gas Heater", T(7), T (8), Q(3)

PRINT 10,"Feed Boiler", $T(5), T(8), Q(4)$

PRINT 10,"Final Feed Heater", $T(8), T(3), Q(5)$

PRINT 5

PRINT 20,"Reactor Heat Input", Q(6)

PRINT 5 ; PRINT 5

20 FORMAT (A20,15X,F20.1)

PRINT, "Heat Outputs:"

PRINT 5

PRINT 10, "Output Cooler",T(4),T(9), Q(7)

PRINT 10, "Partial Condensor", $T(9), T(5), Q(8)$

PRINT 100,"Gas Compressor",W(2)

PRINT 10, "Final Gas Cooler", $T(10), T(6), Q(9)$

PRINT 10,"Gas Heat Loss", T(6), T(1),Q(10)

PRINT 10,"Water Heat Loss", T(5), T(1),Q(11)

PRINT 5

. $\mathrm{HTH}=0 . ; \mathrm{HTC}=0$.

DO $6 I=1,5$

$\mathrm{H} T \mathrm{TH}=\mathrm{HTH}+\mathrm{Q}(\mathrm{I})$

$H T C=H T C+Q(6+I)$

6 CONTINUE

$\mathrm{HTH}=\mathrm{HTH}+\mathrm{W}(1) ; \mathrm{HTC}=\mathrm{HTC}-\mathrm{W}(2)-Q(10)-Q(11)$

PRINT 20,"TOTAL HEATING DUTY",HTH

PRINT 20,"TOTAL COOLING DUTY",HTC

RETURN

END

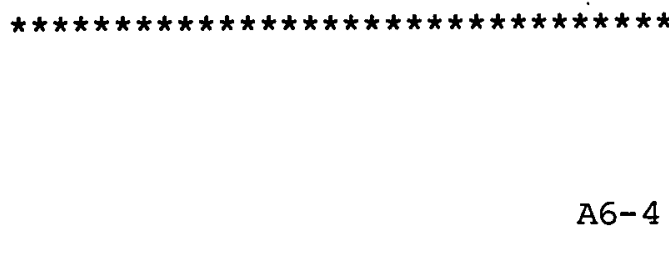




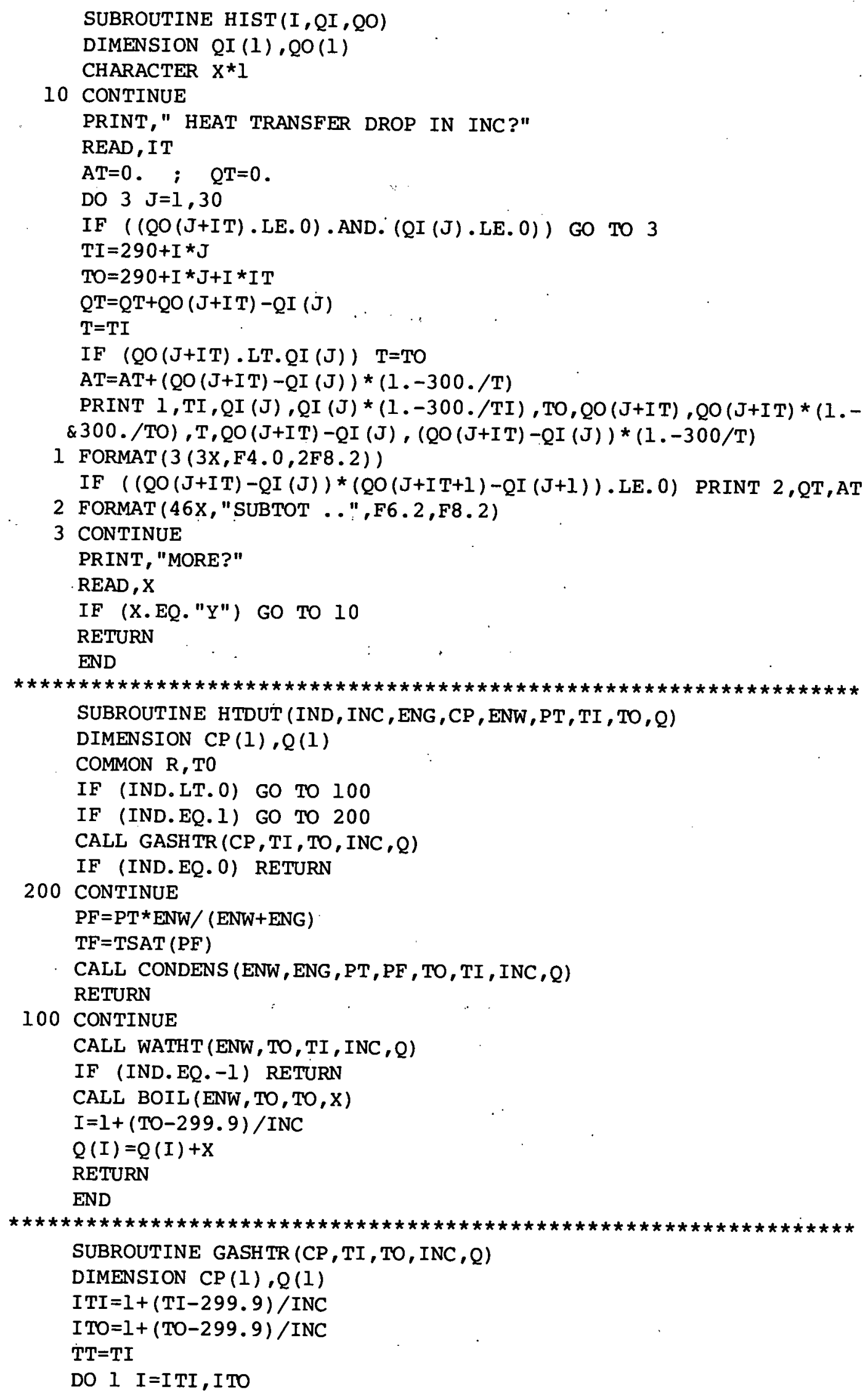


$T=300 .+I * I N C$

IF (T.GT. TO) $T=T O$

$Q(I)=Q(I)+D E L H(C P, T T, T)$

$\mathrm{TT}=\mathrm{T}$

1 CONTINUE

RETURN

END

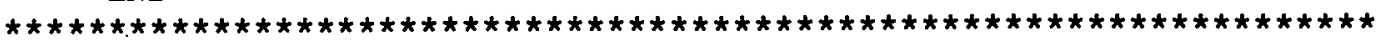

SUBROUTINE WATHT (EN, TO, TI , INC , Q)

DIMENSION $Q(1)$

$I 1=1+(T I-299.9) / I N C$

I $2=1+($ TO-299.9) / INC

$Q(I I)=Q(I 1)+E N * 18 . *(300 .+I N C * I I-T I)$

DO I $I=I 1+1, I 2$

IF (I.GE.I2) GO TO 2

$Q(I)=Q(I)+E N * 18 * I N C$.

1 CONTINUE

$2 Q(\dot{I} 2)=Q(I 2)+E N * 18 . *(T O-300 .-I N C *(I 2-1))$

RETURN

END

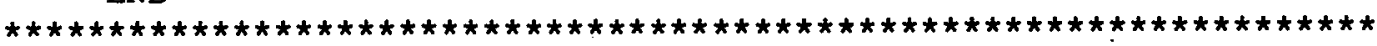

SUBROUTINE CPDATA (NAME, CP)

CHARACTER *5NAME, $V(5)$

DIMENSION $A(5,5), C P(5)$

DATA V/"CH4", "H2O", "CO", "CO2", "H2"/

DATA $A /-17889 ., 44.5,3.38,17.905 \mathrm{E}-3,-4.188 \mathrm{E}-6$,

$\& \quad \quad \quad-57798 ., 45.106,6.89,3.283 \mathrm{E}-3,-.343 \mathrm{E}-6$,

$-26416 ., 47.301,6.25,2.091 \mathrm{E}-3,-.459 \mathrm{E}-6$,

$-94052 ., 51.061,6.85,8.533 \mathrm{E}-3,-2.475 \mathrm{E}-6$,

$\& \quad-94052,51.061,6.85,8.533 \mathrm{E}-3,-2,4$

DO $1 \quad I=1,5$

IF $(V(I)$. EQ.NAME) GO TO 2

1 CONTINUE

2 CONTINUE

DO $3 \mathrm{~J}=1,5$

$C P(J)=A(J, I)$

3 CONTINUE

RETURN

END

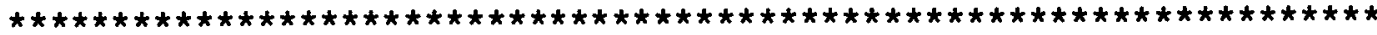

SUBROUTINE HOSO (T, CP, HO, SO)

COMMON R, TO

DIMENSION CP (1)

$\mathrm{HO}=\mathrm{CP}(1)+\mathrm{CP}(5) *(\mathrm{~T} * * 3-\mathrm{TO} * * 3) / 3$

$\mathrm{S} 0=\mathrm{CP}(2)+\mathrm{CP}(3) * \mathrm{ALOG}(\mathrm{T} / \mathrm{T} 0)$

DO $1 \quad I=1,2$

$\mathrm{X}=(\mathrm{T} * * \mathrm{I}-\mathrm{T} 0 * \star \mathrm{I}) / \mathrm{I}$

$\mathrm{HO}=\mathrm{H} 0+\mathrm{CP}(I+2) * \mathrm{X}$

$\mathrm{S} 0=\mathrm{S} 0+\mathrm{CP}(\mathrm{I}+3) \star \mathrm{X}$

1 CONTINUE

RETURN

END

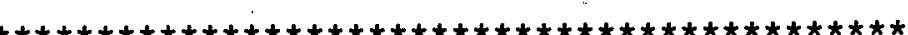




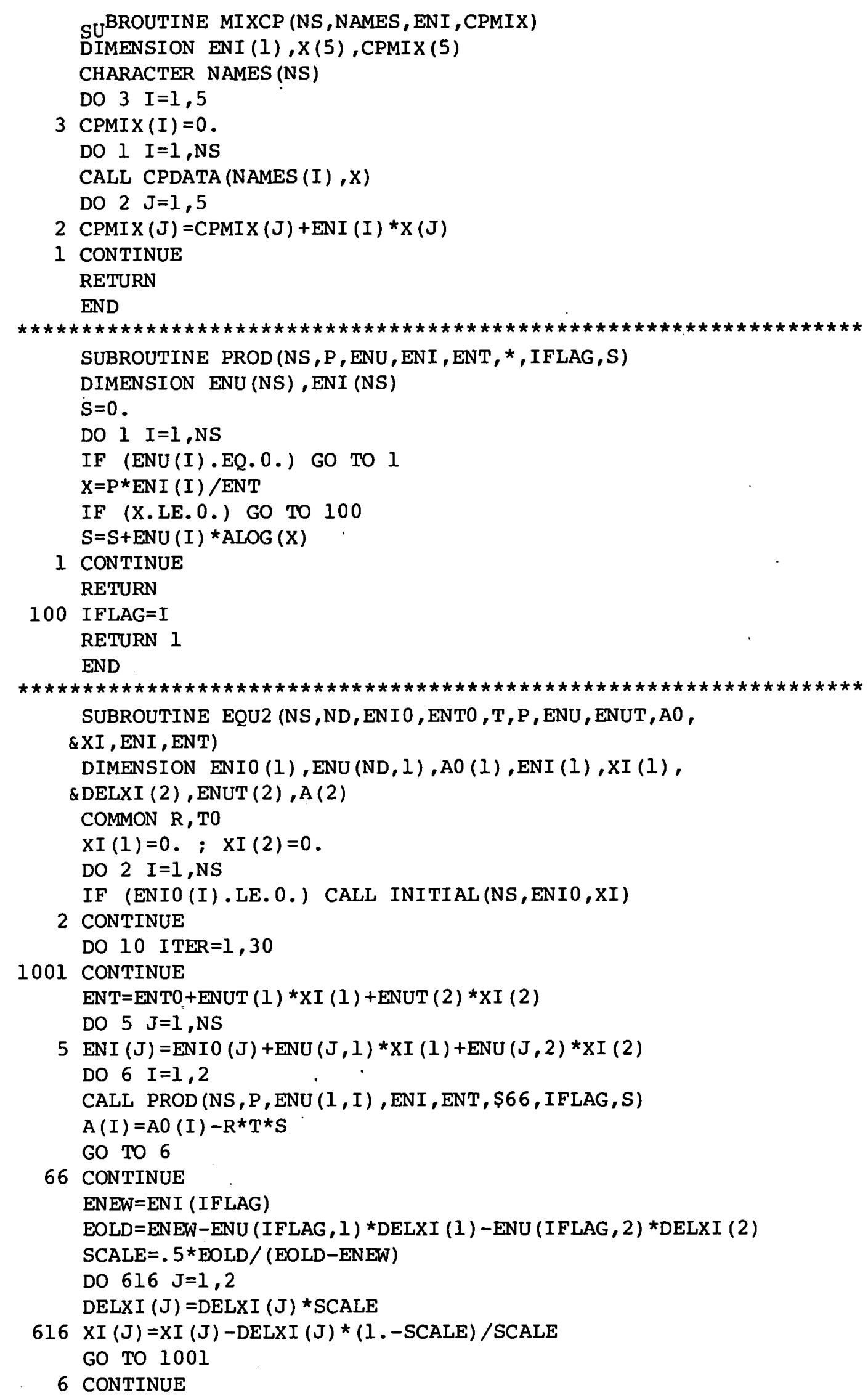




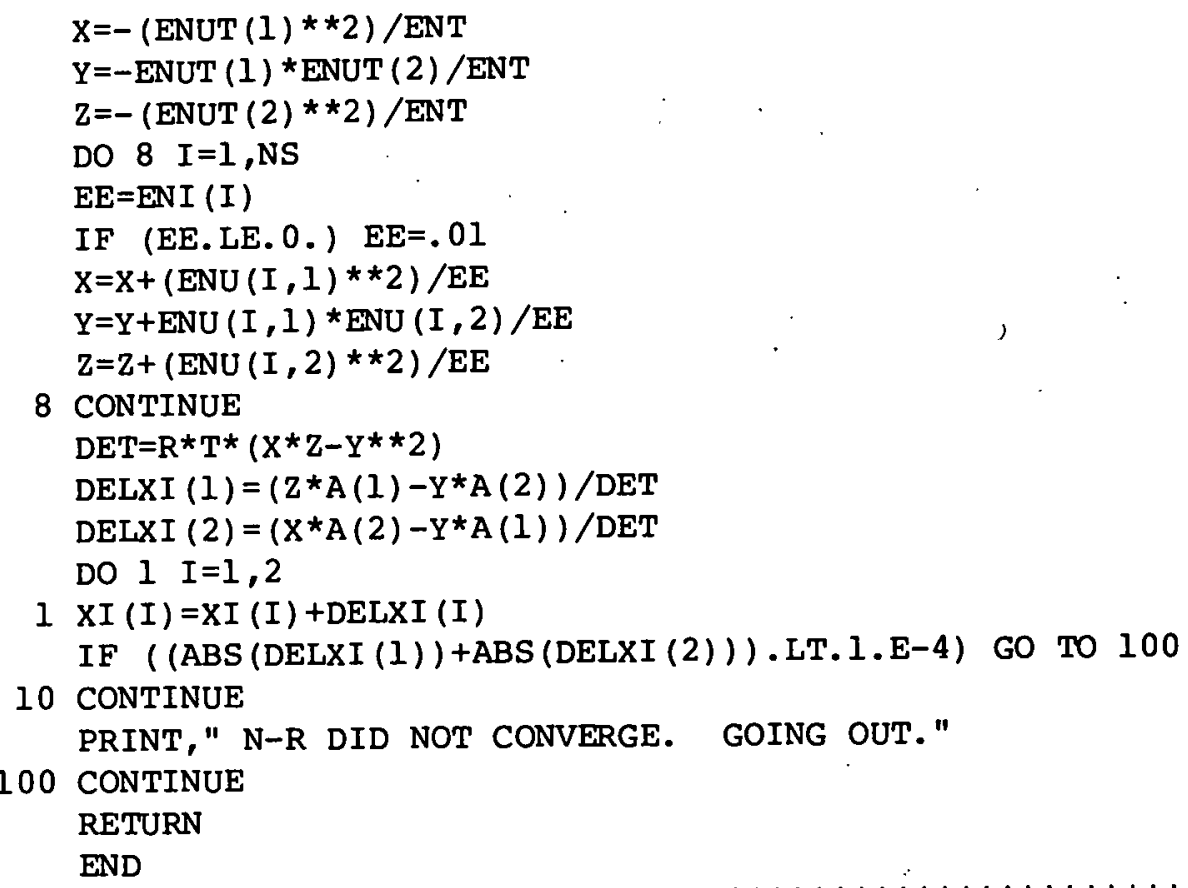

$1 \mathrm{XI}(2)=.5 *(\operatorname{AMIN} \mathrm{E}(\mathrm{E}(2), \mathrm{E}(3))-\operatorname{AMINI}(\mathrm{E}(4), \mathrm{E}(5)))$

$C=E(3)-X I(2) ; D=E(5)+X I(2)$

$X I(1)=-.5 * A M I N 1(C, D / 3$.

RETURN

END

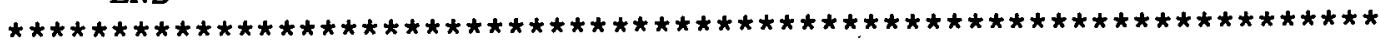

SUBROUTINE EQUIL(NS, ND, ENIO, ENTO , T, P, ENU, ENUT , AO , XI , ENI , ENT, S $\& \mathrm{P}, \mathrm{CP})$

DIMENSION ENIO (1), ENU (ND, 1), ENUT (1), A0 (1),XI (1), ENI (1), CP (1) 
CHARACTER SP(NS) .

ENERT $=0$.

ENTO $=$ ENERT

DO $1 \mathrm{I}=1$, NS

1 ENTO $=$ ENTO+ENIO (I)

DO $4 I=1,2$

CALL MIXCP (NS, SP, ENU $(I, I), C P$ )

CALL HOSO ( T, CP, HO, SO)

$\mathrm{AO}(\mathrm{I})=\mathrm{T} * \mathrm{SO}-\mathrm{HO}$

4 CONTINUE

C=ENIO (1) + ENIO (3) + ENIO (4)

DO $6 I=1, N S$

$6 \operatorname{ENIO}(\mathrm{I})=\operatorname{ENIO}(\mathrm{I}) / \mathrm{C}$

$\mathrm{ENTO}=\mathrm{ENTO} / \mathrm{C}$

CALL EQU2 (NS,ND, ENIO , ENTO , T, P , ENU , ENUT , AO , XI , ENI , ENT)

PRINT $101, C \star X I(1), C \star X I(2)$

101 FORMAT( "Equilibrium Conversions are:"/

$\& 5 \mathrm{X}$, "Reforming $=$ ",F7.4,5X,"Shift $=$ ",F7.4/)

DO $7 \mathrm{I}=1$, NS

$\operatorname{ENIO}(I)=C * \operatorname{ENIO}(I)$

$7 \operatorname{ENI}(I)=C * \operatorname{ENI}(I)$

ENTO $=\mathrm{C} *$ ENTO

$\mathrm{ENT}=\mathrm{ENT}^{*} \mathrm{C}$

RETURN

END

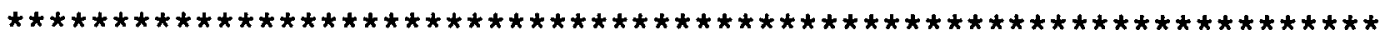

FUNCTION TSAT (P)

$\mathrm{PSI}=14.696 * \mathrm{P}$

$\mathrm{PL}=\mathrm{ALOG} 10$ (PSI)

IF (PSI.GT.1080) GO TO 1

IF (PL.GT.1.653) GO TO 2

$\mathrm{TF}=2634.7 /(6.026-\mathrm{PL})-335.486+4.484 * \mathrm{PL}$

GO TO 3

$2 \mathrm{TF}=105.802+65.14 * \mathrm{PL}+24.859 * \mathrm{PL} \star \star 2-4.3391 * \mathrm{PL} \star * 3+1.6889 * \mathrm{PL} * \star 4$

GO TO 3

$1 \mathrm{TF}=620.994+.05538 * \mathrm{PSI}-3.516 \mathrm{E}-6 * \mathrm{PSI} * * 2-226805 /(\mathrm{PSI}+768.85)$

$3 \mathrm{TSAT}=(\mathrm{TF}+459.67) / 1.8$

RETURN

END

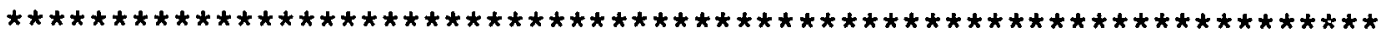

SUBROUTINE DUTY (INC, CP,NS, SP, EO ,ETO , E , ET, HI ,HO , PO , P1 , P2 , T , ID, $\& Q, Q I, Q O, W)$

DIMENSION QI (1), QO (1)

DIMENSION EO (1),E(1), T(1),Q(1),CP (1),W(1)

CHARACTER *5SP (1)

COMMON $R, T 0$

$\mathrm{Z}=\mathrm{EO}(2) ; \mathrm{EO}(2)=0 . ; \mathrm{ETA}=0.85$

CALL MIXCP (NS, SP, E0,CP)

$Q(1)=\operatorname{DELH}(C P, T(1), T(2))$

ENG=ETO $-Z$

CALL HTDUT (0, INC, ENG , CP , 0, ,PO, T (1), T (2), QI)

$\mathrm{Q}(2)=\mathrm{HI} * 18$. * $(\mathrm{T}(5)-\mathrm{T}(1))$

CALL HTDUT (-1, INC, ENG , CP, HI , PO , T (1) , T (5) , QI)

$C P P=Q(1) \%(T(2)-T(1))$ 
$\mathrm{G}=\mathrm{CPP} /(\mathrm{CPP}-\mathrm{R}$ * $(\mathrm{ETO}-\mathrm{Z}))$

CALL EXPAND $(T(7)(, T(2), P 0, P 1, G, E T A, C P P, W(1))$

$W(1)=\operatorname{DELH}(C P, T(2), T(7))$

$\mathrm{X}=\mathrm{PO}$; IF (ID.NE.0) $\mathrm{X}=\mathrm{X} \star \mathrm{Z} / \mathrm{ETO}$

$\mathrm{T}(8)=\mathrm{TSAT}(\mathrm{X})$

$\mathrm{Q}(3)=\mathrm{DELH}(\mathrm{CP}, \mathrm{T}(7), \mathrm{T}(8))$

CALL HTDUT $(0$, INC, ENG , CP , 0. , PO, T (7), T (8), QI)

CALL BOIL $(Z, T(5), T(8), Q(4))$

$I=1$; IF (ID. EQ. 0) $I=-2$

CALL HTDUT ( $I, I N C, E N G, C P, Z, P O, T(5), T(8), Q I)$

$\mathrm{EO}(2)=\mathrm{Z}$

CALL MIXCP (NS, SP, EO,CP)

$Q(5)=\operatorname{DELH}(C P, T(8), T(3))$

CALL HTDUT $(0, I N C, E T 0, C P, 0,, P O, T(8), T(3), Q I)$

CALL HOSO ( T (3), CP, HO, SO)

CALL MIXCP (NS, SP, E, CP)

CALL HOSO (T (4), CP,H, S)

$\mathrm{Q}(6)=\mathrm{H}-\mathrm{HO}$

$\mathrm{T}(9)=\mathrm{TSAT}(\mathrm{PO} * \mathrm{E}(2) / \mathrm{ET})$

$Q(7)=\operatorname{DELH}(C P, T(9), T(4))$

CALL HTDUT $(0$, INC, ET, CP, 0, , PO , T (9), T (4), QO)

$\mathrm{Z}=\mathrm{E}(2) ; \mathrm{E}(2)=0$.

CALL MIXCP (NS, SP, E, CP)

$\mathrm{E}(2)=\mathrm{Z}$

CALL BOIL $(\mathrm{Z}, \mathrm{T}(5), \mathrm{T}(9), \mathrm{Q}(8))$

$Q(8)=Q(8)+D E L H(C P, T(5), T(9))$

CALL HTDUT (2, INC, ET-Z, CP, Z, PO , T (5) , T (9) , QO)

$\mathrm{CPP}=\mathrm{DELH}(\mathrm{CP}, \mathrm{T}(5), \mathrm{T}(9)) /(\mathrm{T}(9)-\mathrm{T}(5))$

$\mathrm{G}=\mathrm{CPP} /\left(\mathrm{CPP}-\mathrm{R}^{*}(\mathrm{ET}-\mathrm{Z})\right)$

CALL EXPAND(T(10), T(5), P2,P0, G, ETA, CPP, W(2))

$\mathrm{W}(2)=\mathrm{DELH}(\mathrm{CP}, \mathrm{T}(5), \mathrm{T}(10))$

$Q(9)=\operatorname{DELH}(C P, T(6), T(10))$

CALL HTDUT $(0, I N C, E T-Z, C P, 0, ~, P O, T(6), T(10), Q 0)$

$Q(10)=$ DELH $(C P, T \cdot(1), T(6))$

$\mathrm{Q}(11)=\mathrm{HO} * 18 . *(\mathrm{~T}(5)-\mathrm{T}(1))$

RETURN

END

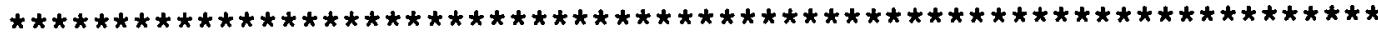

SUBROUTINE EXPAND (TO, TI , PO, PI , GAMMA, ETA, CP , WID)

. $1 \mathrm{X}=($ GAMMA-1. ) /GAMMA

$\mathrm{TO}=((\mathrm{PO} / \mathrm{PI}) * \star \mathrm{X}) * \mathrm{TI}$

$W I D=C P *(T I-T O)$

IF (PO.GT.PI) GO TO 1

$\mathrm{TO}=((1 .-\mathrm{ETA}) * \mathrm{WID} / \mathrm{CP})+\mathrm{TO}$

WI $D=E T A * W I D$

RETURN

$1 \mathrm{TO}=\mathrm{TO}-((1 .-\mathrm{ETA})$ *WID $/ \mathrm{ETA}) / \mathrm{CP}$

WID $=$ WID $/ E T A$

RETURN

END

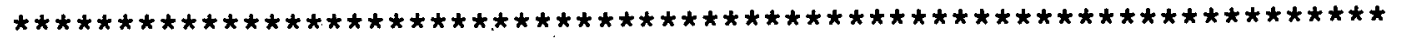

FUNCTION DELH (C,T,TO)

DIMENSION $\mathrm{C}(1)$

$\mathrm{DELH}=\mathrm{C}(3) *(\mathrm{TO}-\mathrm{T})+\mathrm{C}(4) *(\mathrm{TO} * \star 2-\mathrm{T} * * 2) / 2 .+\mathrm{C}(5) *(\mathrm{TO} * * 3-\mathrm{T} * * 3) / 3$. 


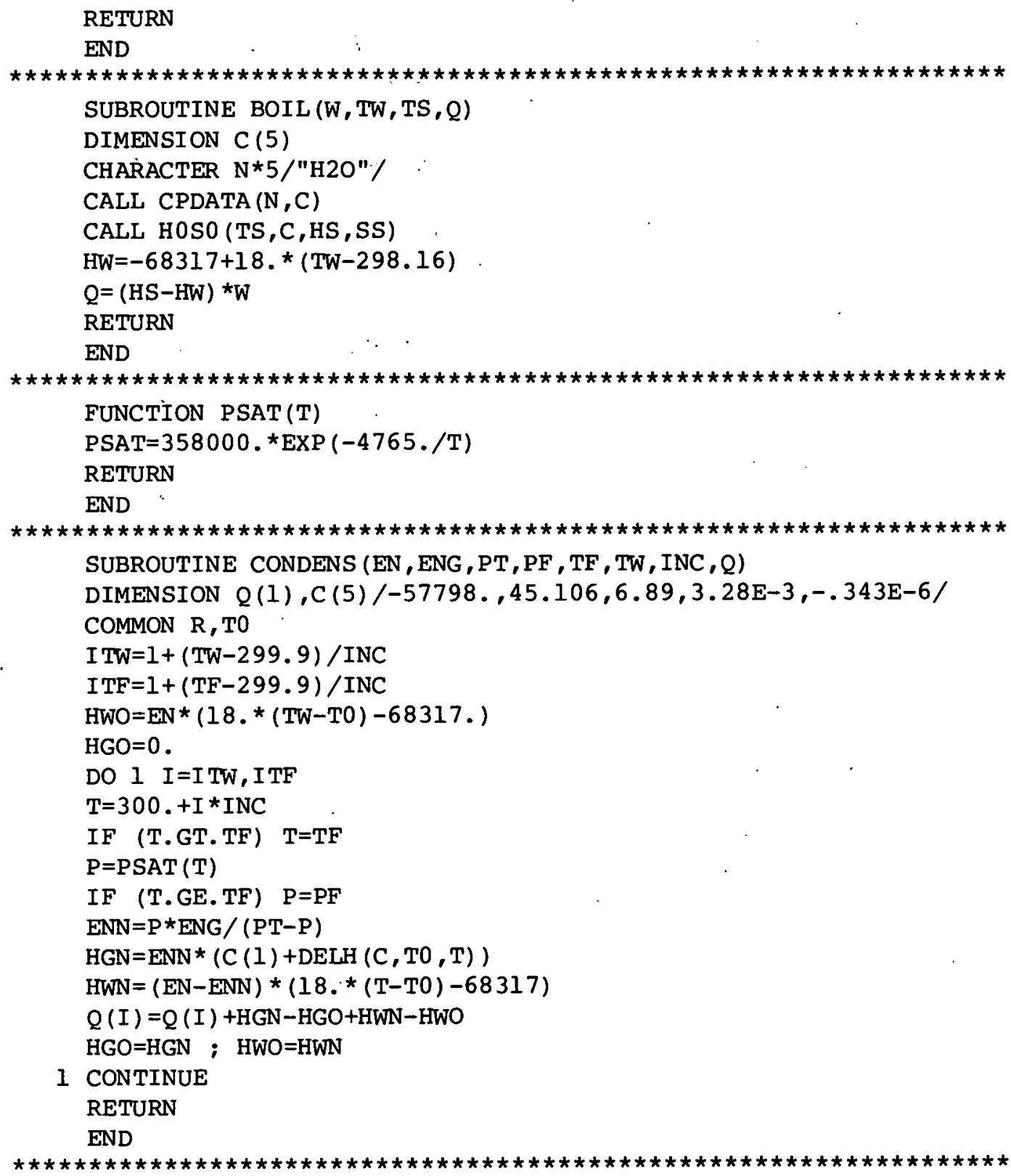

LTCHP EQUILIBRIUM PROGRAM

* EQUil PROG FOR LOW TEMP CHP

* THIS PROgRam CALCULATES THE CHEMICAL REACTION EQUilibra FOR

* THE CYCLOHEXANE DEHYDROGENATION AND ISOMERIZATION REACTIONS

* THE OPTION EXISTS TO INVESTIGATE ONLY THE DEHYDROGENATION

* REACTION BY SETTING THE FLAG (IFLAG) TO 0

* THE REACTION EQUILIBRIUM IS CALCULATED AS A FUNCTION OF

* TEMPERATURE, PRESSURE, AND MOLAR COMPOSITION.

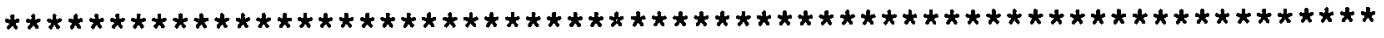




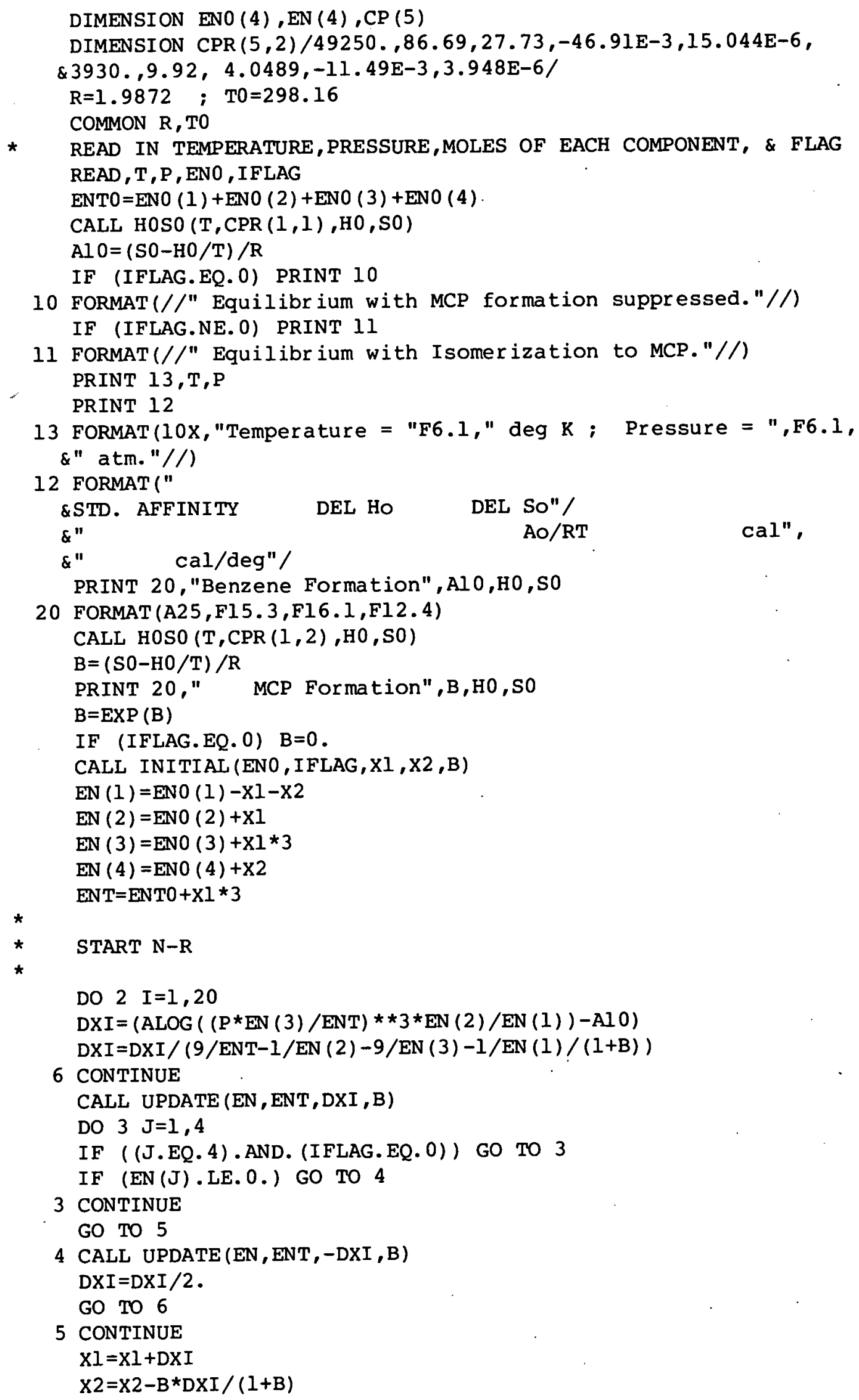


IF (DXI**2.LE.1.E-I0) GO TO 7

2 CONTINUE

PRINT," N-R DID NOT CONVERGE"

7 CONTINUE

PRINT $30, \mathrm{Xl}, \mathrm{X} 2$

30 FORMAT (//"Equilibrium Conversions are:"//"Benzene Formed = ", \&F7.4," ; MCP Formed = ",F7.4///) PRINT 31

31 FORMAT (35x, "Inlet",9x, "Outlet" ,7x," Mole "/

$\& 35 \mathrm{x}$, "Moles" , 9x," Moles" ,7X, "Fraction"//)

32 FORMAT (A25, 3F15.4)

PRINT 32,"CYclohexane" ,ENO (1), EN (1), EN (1)/ENT

PRINT 32 ,"Benzene", ENO (2), EN (2), EN (2)/ENT

PRINT 32, "Hydrogen", ENO (3) , EN (3) ,EN (3)/ENT

PRINT 32,"MCP", ENO (4), EN (4), EN (4)/ENT

PRINT 33

33 FORMAT ( $34 \mathrm{X}, "$ $", 9 \mathrm{X}, "$ $", 9 x, "$ ")

PRINT $32, "$ TOTAL", ENTO, ENT

STOP

END

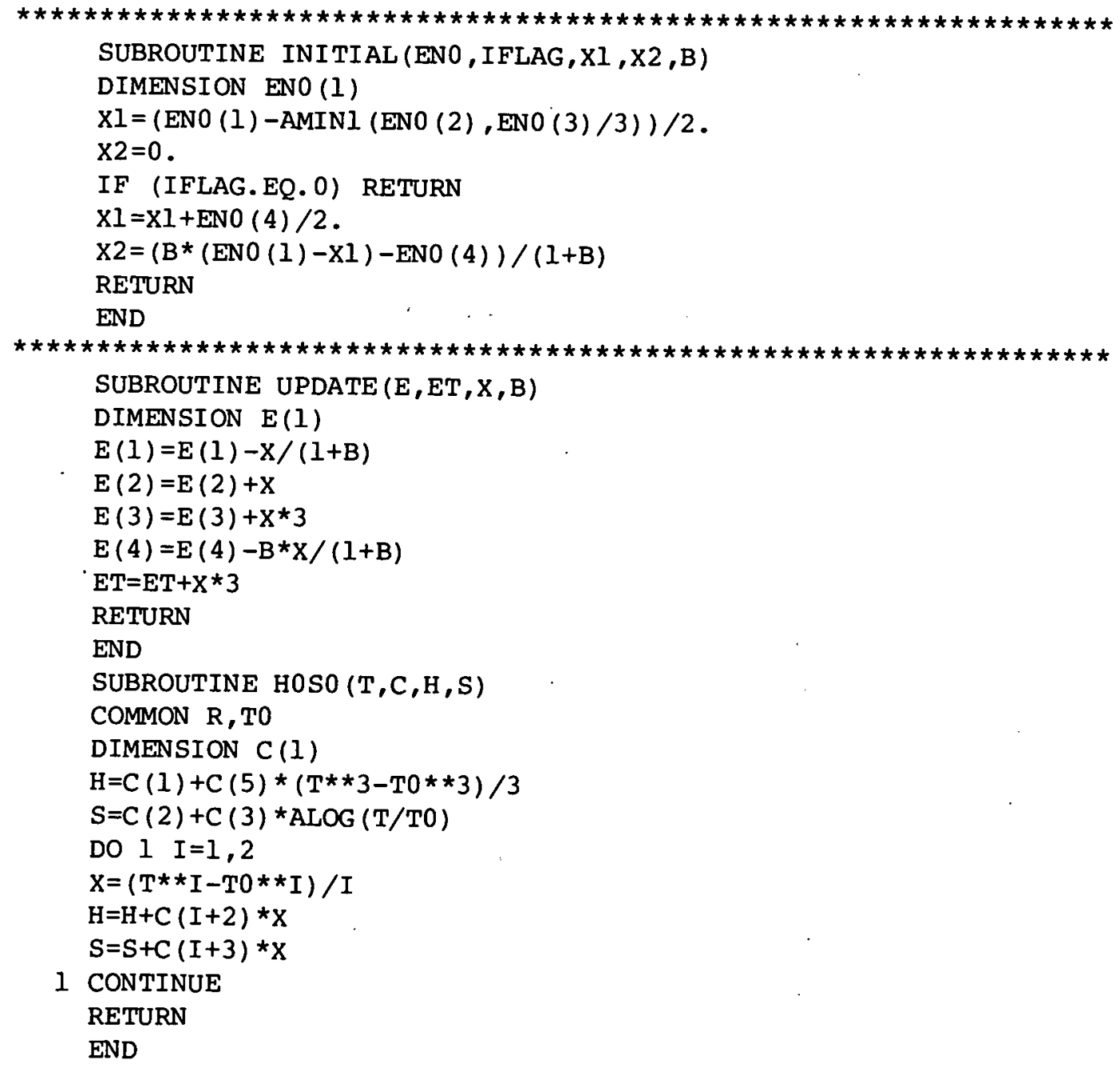

1 CONTINUE

RETURN

END 
* THIS PROGRAM CALCULATES THE VAPOR LIQUID EQUILIBRIUM FOR

* THE FOUR COMPONENT SYSTEM OF CYCLOHEXANE, BENZENE, HYDROGEN,

* AND METHYLCYCLOPENTANE AND THE ENTHALPIES OF THE GAS AND

* LIQUID STREAMS.

* AMV=MOLES IN VAPOR

* AML=MOLES IN LIQUID

* AMC=MOLES CONDENSED

* HLIQ=MOLAR LIQUID ENTHALPY AT TEMPERATURE T

* T=TEMPERATURE, $\mathrm{K}$

* P=PRESSURE, ATMS

* COMPONENT 1 - CYCLOHEXANE

* COMPONENT 2 - BENZENE

* COMPONENT 3 - HYDROGEN

* COMPONENT 4 - METHYLCYCLOPENTANE

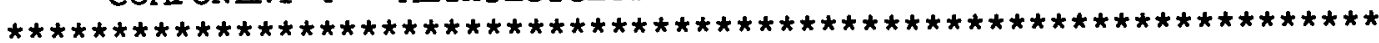

DIMENSION AMV (4), AML (4), AMC (4), HLIQ (4)

DIMENSION CPV (5)

DIMENSION $\mathrm{H}(40)$

$\operatorname{TOT}(A)=A(1)+A(2)+A(3)+A(4)$

$\operatorname{PROD}(X, Y)=X(1) * Y(1)+X(2) * Y(2)+X(3) * Y(3)+X(4) * Y(4)$

* READ IN START POINT--CAN BE SWItChed to a sUb later

10 CONTINUE

PRINT, "VAP MOLES, LIQ MOLES?"

READ, AMV , AML

20 CONTINUE

PRINT, "P (ATM) , TSTART, TFIN, DELT, PMDEL"

READ , $P$, TS , TF , DT , PMDEL

$\mathrm{T}=\mathrm{TS}$

JCOUN T $=0$

SUMH $=0.0 ;$ SUMA $=0.0 ;$ SUMAP $=0.0 ;$ SUMAM $=0.0$

1 IF (T.GT.TF) GO TO 2

JCOUNT $=$ JCOUNT+1

CALL FLASH (AMV, AML, AMC, HLIQ, T, P)

CALL CPMIX (AMV, CPV)

CALL HOSO (T, CPV, HV, SV)

$\mathrm{HL}=\mathrm{PROD}$ (AML , HLIQ)

$\mathrm{HT}=\mathrm{HV}+\mathrm{HL}$

IF (JCOUNT. GT. I) GO TO 100

HSTORE $=$ HT

100 DELH=HT-HSTORE

HSTORE $=\mathrm{HT}$

DELH $=$ DELH $* 1.1622 \mathrm{E}-3$

TMINUS $=\mathrm{T}-. .5 * \mathrm{DT}-\mathrm{PMDEL}$

AMINUS=DELH * $($ TMINUS-300.) $/$ TMINUS

$A=D E L H *(T-.5 * D T-300) /.(T-.5 * D T)$

TPLUS $=\mathrm{T}-.5 * \mathrm{DT}+\mathrm{PMDEL}$

APLUS $=$ DELH* $($ TPLUS -300.$) /$ TPLUS

SUMAP $=$ SUMAP+APLUS

SUMAM $=$ SUMAM+AMINUS

SUMH $=$ SUMH + DELH 


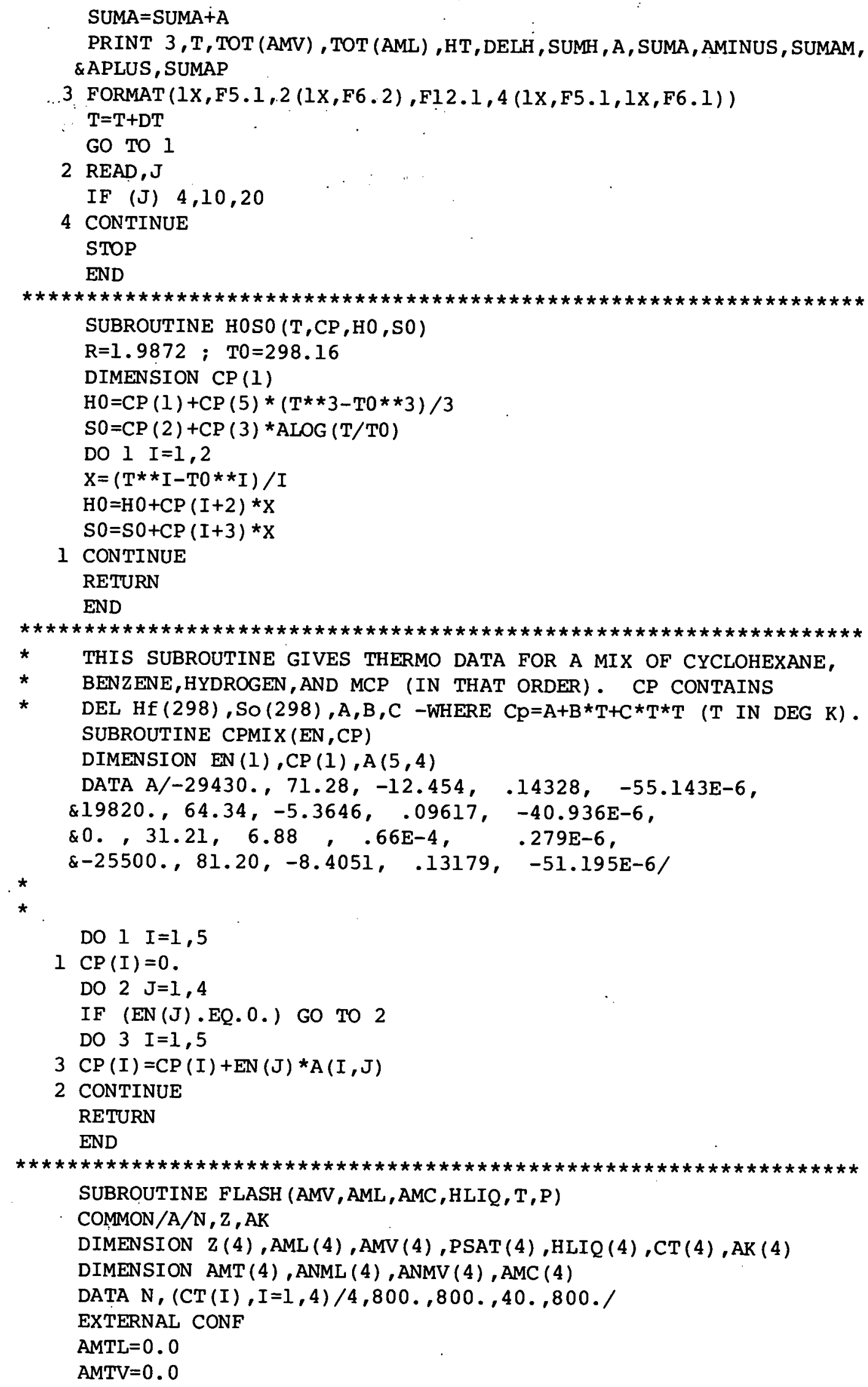

1 CONTINUE

RETURN END

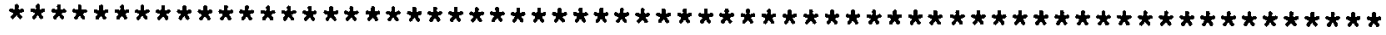

* THIS SUBROUTINe GIVES THERMO DATA FOR A MIX OF CYCLOHEXANE,

* BENZENE, HYDROGEN, AND MCP (IN THAT ORDER). CP CONTAINS

* DEL Hf (298), So (298), A,B,C -WHERE CP=A+B*T+C*T*T ( $T$ IN DEG K). SUBROUTINE CPMIX (EN, CP)

$1 \mathrm{CP}(\mathrm{I})=0$.

DO $2 \mathrm{~J}=1,4$

IF (EN (J).EQ.0.) GO TO 2

DO $3 \quad I=1,5$

$3 \mathrm{CP}(\mathrm{I})=\mathrm{CP}(\mathrm{I})+\mathrm{EN}(\mathrm{J}) * \mathrm{~A}(\mathrm{I}, \mathrm{J})$

2 CONTINUE

RETURN

END

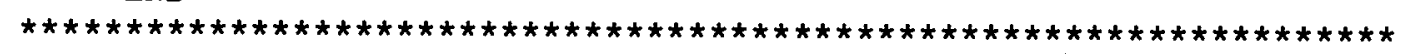

SUBROUTINE FLASH (AMV, AML, AMC, HLIQ, T, P)

COMMON $/ \mathrm{A} / \mathrm{N}, \mathrm{Z}, \mathrm{AK}$

DIMENSION $2(4), A M L(4), A M V(4), \operatorname{PSAT}(4), \mathrm{HLIQ}(4), \mathrm{CT}(4), \mathrm{AK}(4)$

DIMENSION AMT (4), ANML (4), ANMV (4), AMC (4)

DATA N, (CT (I) , I =1,4) $/ 4,800,800 ., 40 ., 800 . /$

EXTERNAL CONF

AMTL $=0.0$

AMTV $=0.0$ 


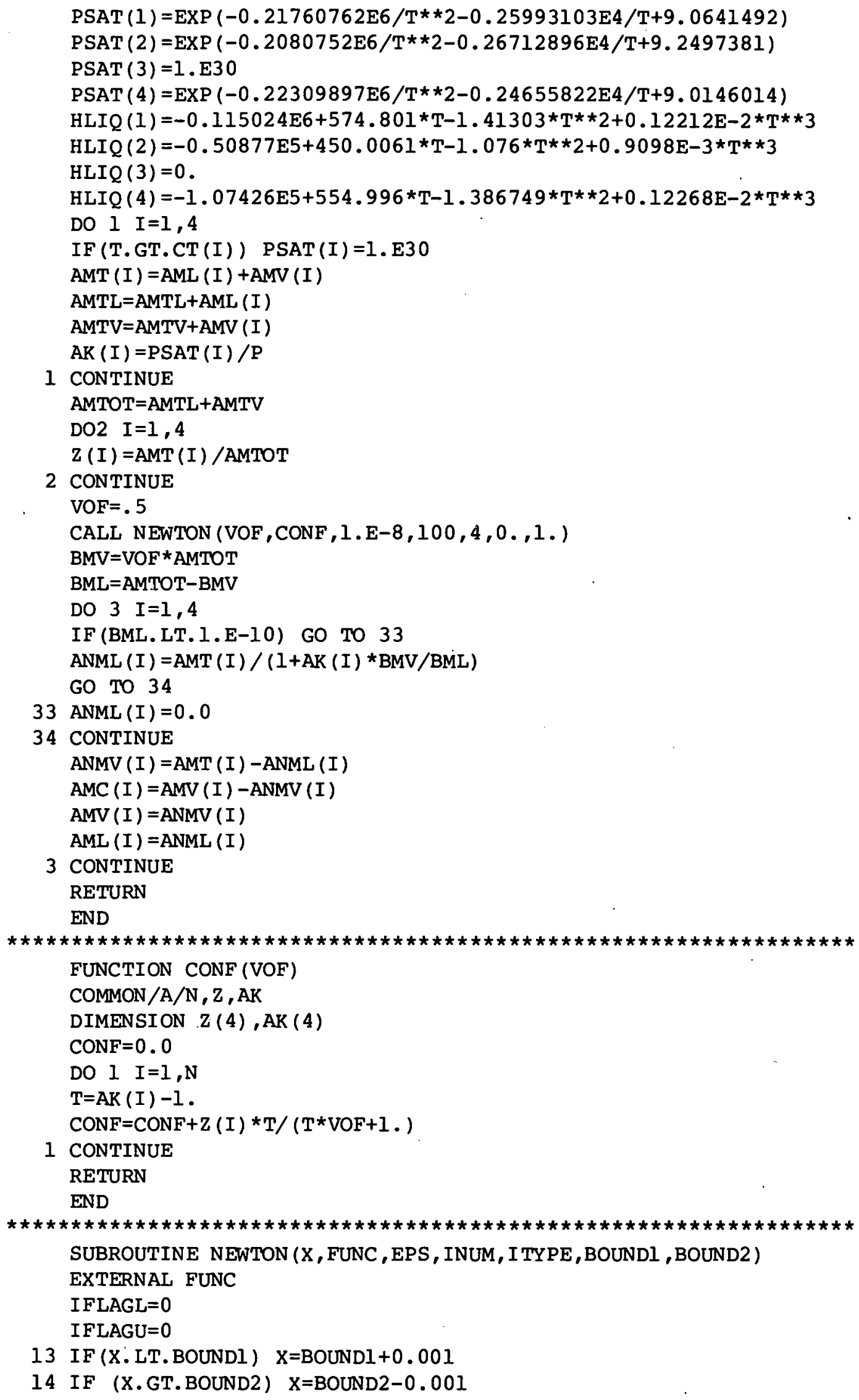




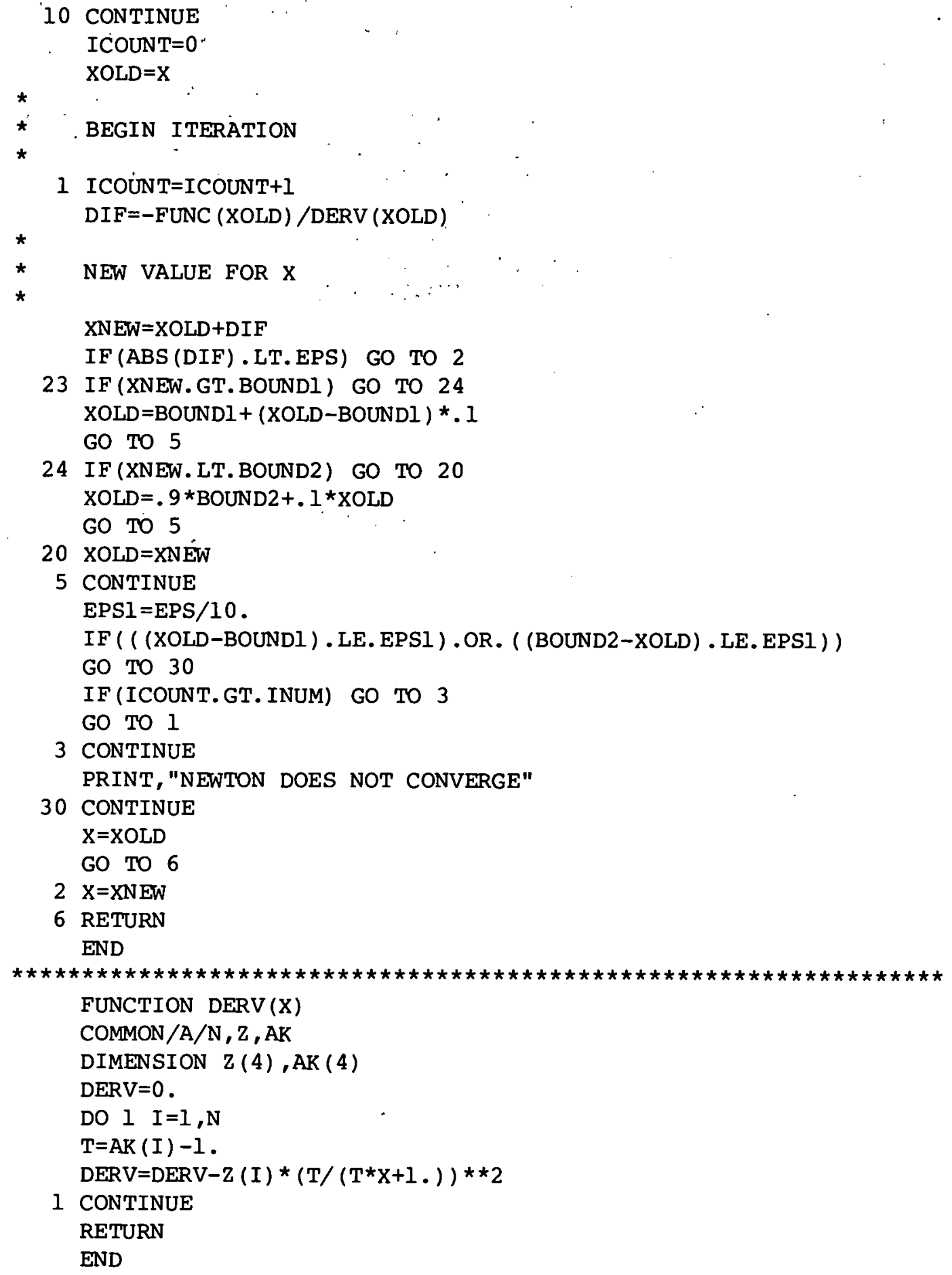

* THIS PROGRAM CALCULATES THE OPTIMUM PIPELINE DIAMETER FOR 
* THE FLOW OF A LIQUID AT GIVEN INLET AND OUTLET PRESSURES.

* DIFFERENT LIQUIDS, FLOWRATES, POWER COSTS , AND PRESSURES

* CAN BE INVESTIGATED.

* THE PROGRAM ITERATES ON THE TOTAL COST OF TRANSMISSION USING

* A NEWTON-RAPHSON TECNIQUE. THE FIRST AND SECOND DERIVATIVES

* ARE CALCULATED BY FINITE DIFFERENCE TECHNIQUES.

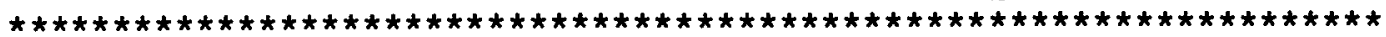

REAL ND,NDD, MW,NDI, L, LD , LI

DATA A,B,C,F/238.3465,16096.52,2500 . .005/

COMMON / A/RE , RP , L , MW , F , ND , P2 , R , A, B , C , ETA , RHO , PDROP

COMMON/B/P, COSTl, COST2, COST3, PLCOST, CCOST

EXTERNAL TCOST

4 CONTINUE

* INPUT DATA

PRINT, "NEW MW, ETA, RHO"

READ, MW, ETA, RHO

REAL ND,NDD, MW, NDI , L , LD, LI

550 CONTINUE

PRINT, " $P$, ND , NDD , NDT , P2 , P2D , NP2T , R, RD , NRT, D , DD , MDT"

$R E A D, P, N D, N D D, N D T, P 2, P 2 D, N P 2 T, R, R D, N R T, D, D D, M D T$

* BEGIN PRIMARY ITERATION

$\mathrm{ND}=\mathrm{ND}-\mathrm{NDD}$

$\mathrm{P} 2=\mathrm{P} 2-\mathrm{P} 2 \mathrm{D}$

$\mathrm{R}=\mathrm{R}-\mathrm{RD}$

$\mathrm{D}=\mathrm{D}-\mathrm{DD}$

$\mathrm{DI}=\mathrm{D}$

$\mathrm{NDI}=\mathrm{ND}$

$\mathrm{P} 2 \mathrm{I}=\mathrm{P} 2$

DO $10 \mathrm{Il}=1$,NRT

$\mathrm{R}=\mathrm{R}+\mathrm{RD}$

$\mathrm{RE}=\mathrm{R} * 8$.

$\mathrm{P} 2=\mathrm{P} 2 \mathrm{I}$

DO 12 I $3=1, N P 2 T$

$\mathrm{P} 2=\mathrm{P} 2+\mathrm{P} 2 \mathrm{D}$

$\mathrm{PDROP}=\mathrm{P}-\mathrm{P} 2$

$\mathrm{D}=\mathrm{DI}$

$\mathrm{ND}=\mathrm{NDI}$

DO 14 I5=1,NDT

$\mathrm{ND}=\mathrm{ND}+\mathrm{NDD}$

CALL NEWTON (D, TCOST, $1 . \mathrm{E}-6,100,4,0 ., 5$.

$Q=T C O S T(D)$

$\mathrm{RT}=\mathrm{RE}+\mathrm{RP}$

$\mathrm{Cl}=\operatorname{CCOST}{ }^{*} \mathrm{RP} / \mathrm{RT}$

$\mathrm{C} 2=\mathrm{CCOST}-\mathrm{Cl}$

PRINT $200, R E, R P, P, P 2, D$

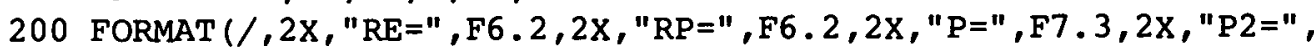
$\& F 6: 2,2 X, " D=", F 6,3)$

PRINT $201, N D, L, C O S T 1, \operatorname{cosT2}, \cos 33, \mathrm{PLCOST}, \mathrm{Cl}, \mathrm{C} 2, \mathrm{CCOST}, \mathrm{Q}$

201 FORMAT (2X, "FLOWRATE =" ,F6 . 2,/, 2X, "L=" ,F6 . 2,/,2X, \& "ROW COST $=", F 9.3, /, 2 \mathrm{x}$, "INSTALLATION COST =",F9.3,/,2x, \& "MATERIALS COST =",F9.3,/,2X,"TOTAL PIPELINE COST =" ,F9.3,/, $\& 2 \mathrm{X}$, "PUMP COST =" ,F9.3,/,2X, "ENERGY COST =" F9.3,/,2X,

\&"TOTAL PUMP COST =",F9.3,/,2X,"TOTAL TRANSMISSION COST=",F9.3)

14 CONTINUE 
12 CONTINUE

11 CONTINUE

10 CONTINUE

PRINT, " $\mathrm{K}=$ "

READ, $K$

GO TO $(4,550,21), \mathrm{K}$

21 STOP

END

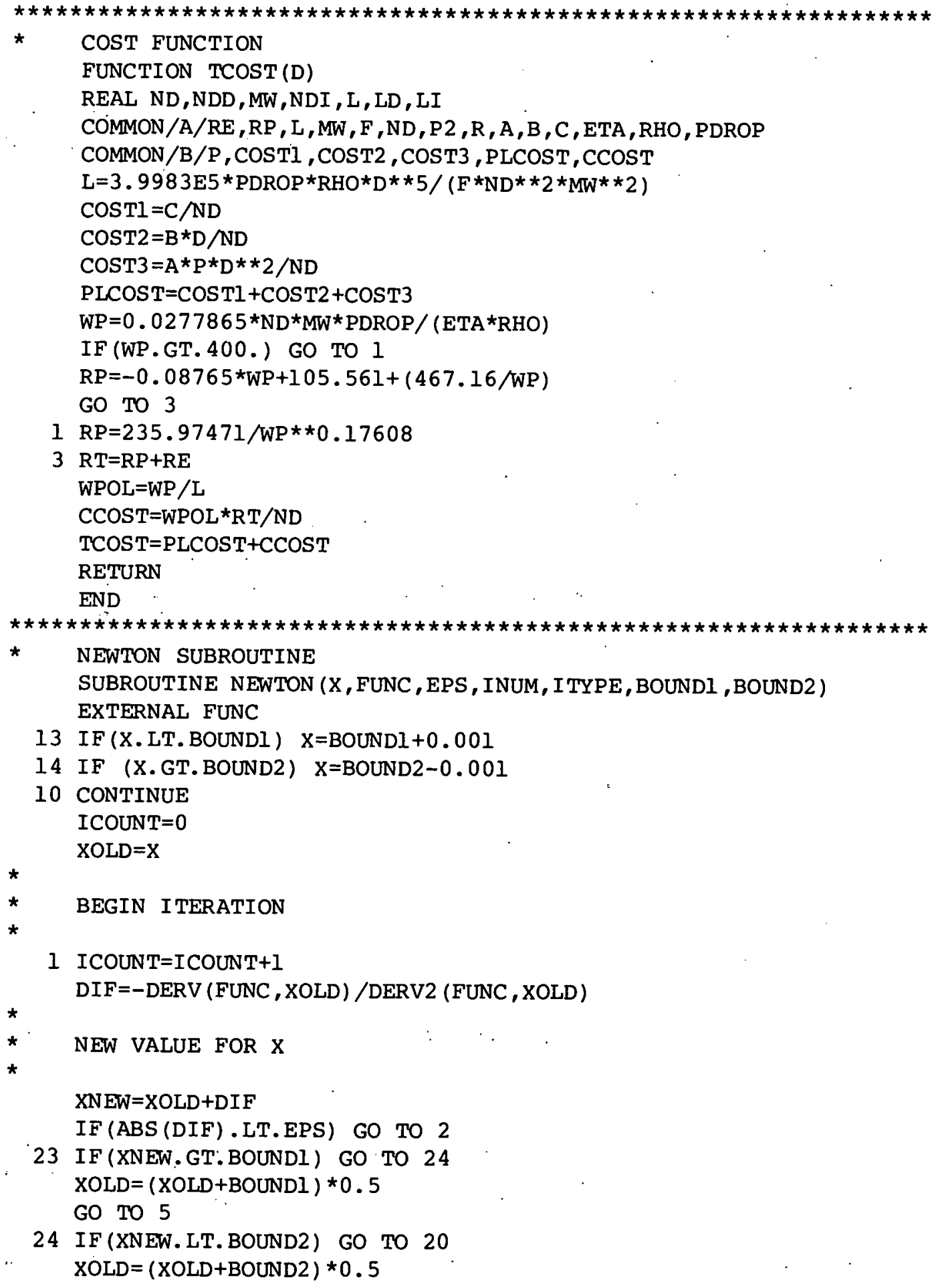

1 ICOUNT=ICOUNT+1 


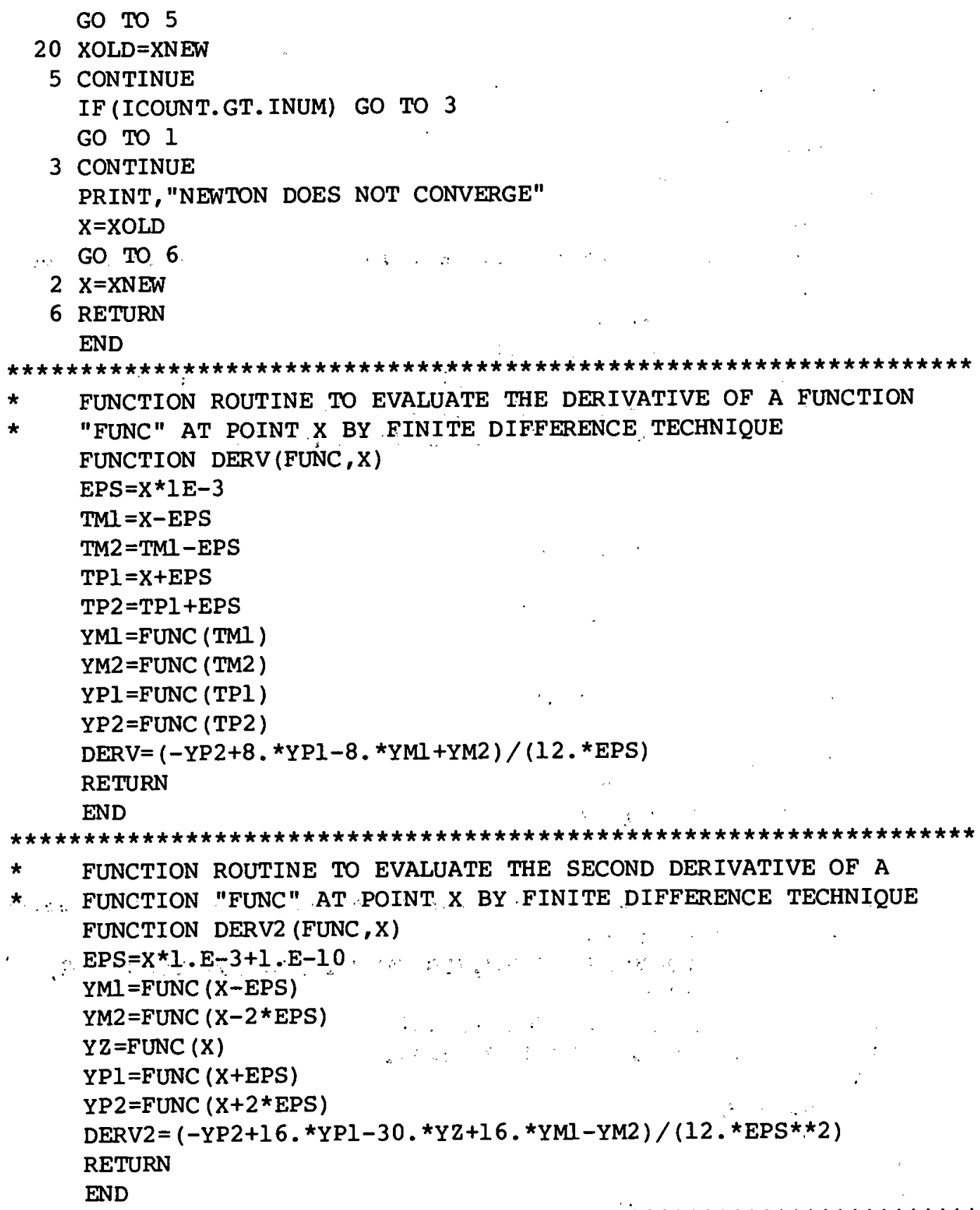

PROGRAM FOR GAS PIPELINE

* program used to calculate transportation costs as a function * OF GAS FLOWRATE AND COMPOSTION FOR VARIOUS LINE DIAMETERS, * TRANSMISSION DISTANCES, AND COSTS OF POWER.

* Each VARIABLE MAY BE INCREMENTED BY A DESIRED DELTA AS MANY

$$
\text { A6-20 }
$$


FUNCTION COEFF (GAMMA, P , P2)

$A=($ GAMMA-1.0) $/$ GAMMA

COEFF $=((P / P 2) \star \star A-1.0) / A$

RETURN

END

A6-22

"U.S. GOVERNMENT PRINTING OFFICE: 1979-740-092/368 
* TIMES AS THE USER WISHES.

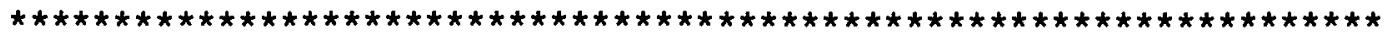

REAL ND,NDD,MW,NDI , L , LD, LI

DATA A,B, C, F/238.3465,16096.52,2500 ,0.005/

4 CONTINUE

* READ INPUT VARIABLES

PRINT, "NEW MW, ETA, GAMMA, T"

READ, MW , ETA, GAMMA, T

550 CONTINUE

PRINT, "L , LD , LT, ND, NDD, NDT , R , RD, NRT , D, DD, MDT"

$R E A D, L, L D, L T, N D$, NDD , NDT , R , RD , NRT , D , DD , MDT

* BEGIN ITERATIONS

$\mathrm{ND}=\mathrm{ND}-\mathrm{NDD}$

$\mathrm{LI}=\mathrm{L}-\mathrm{LD}$

$\mathrm{P} 2=40$

$\mathrm{D}=\mathrm{D}-\mathrm{DD}$

$\mathrm{DI}=\mathrm{D}$

$\mathrm{NDI}=\mathrm{ND}$

$\mathrm{R}=\mathrm{R}-\mathrm{RD}$

DO $10 \mathrm{Il}=1$,NRT

$\mathrm{R}=\mathrm{R}+\mathrm{RD}$

$\mathrm{L}=\mathrm{LI}$

DO 12 I $3=1, L T$

$\mathrm{L}=\mathrm{L}+\mathrm{LD}$

$\mathrm{D}=\mathrm{DI}$

DO I3 I $4=1$, MDT

$D=D+D D$

$\mathrm{ND}=\mathrm{NDI}$

PRINT $200, R, D, L$

200 FORMAT $(/, " R A T E=", F 6.2,2 \mathrm{X}, " \mathrm{D}=$ " ,F6.3,2X,"L= ",F6.2)

DO 14 I $5=1$,NDT

$\mathrm{ND}=\mathrm{ND}+\mathrm{NDD}$

* PRESSURE DROP CALCUlation

$\mathrm{PS}=\mathrm{L} * \mathrm{MW} * \mathrm{~F} * \mathrm{~T} * \mathrm{ND} * * 2 /(2.4042 \mathrm{E} 3 * \mathrm{D} * * 5)$

$\mathrm{P}=\mathrm{SQRT}(\mathrm{P} 2 * \star 2+\mathrm{PS})$

$\star \quad$ COST CALCULATION

$\operatorname{cosTl}=\mathrm{C} / \mathrm{ND}$

$\cos T 2=B * D / N D$

$\cos T 3=A * P * D * * 2 / N D$

$\mathrm{PLCOST}=\operatorname{Cos} \mathrm{T} 1+\operatorname{COST} 2+\cos \mathrm{T} 3$

$\operatorname{CCOST}=2.3109 * R * T * \operatorname{COEFF}($ GAMMA $, P, P 2) /(\mathrm{L} \star E T A)$

$T \operatorname{COST}=\mathrm{CCOST}+\mathrm{PLCOST}$

PRINT $201, \mathrm{ND}, \mathrm{P}, \operatorname{COSTl}, \cos \mathrm{T} 2, \mathrm{COST} 3, \mathrm{PLCOST}, \mathrm{CCOST}, \mathrm{TCOST}$

201 FORMAT (F6. 2, 1X, 4 (F8. 2,1X), 3 (F9. 2,1X))

14 CONTINUE

13 CONTINUE

12 CONTINUE

10 CONTINUE

PRINT, " $K="$

READ, $K$

GO TO $(4,550,21), \mathrm{K}$

21 STOP

END 